RECEIVED FFA 03 25:

\section{O S.T।}

\title{
January 1998
}

Energy intormation Administration

\section{Energy Plug:}

Performance Profiles 1996 


\section{DOE/EIA-0035(98/01)}

Monthly Energy Review

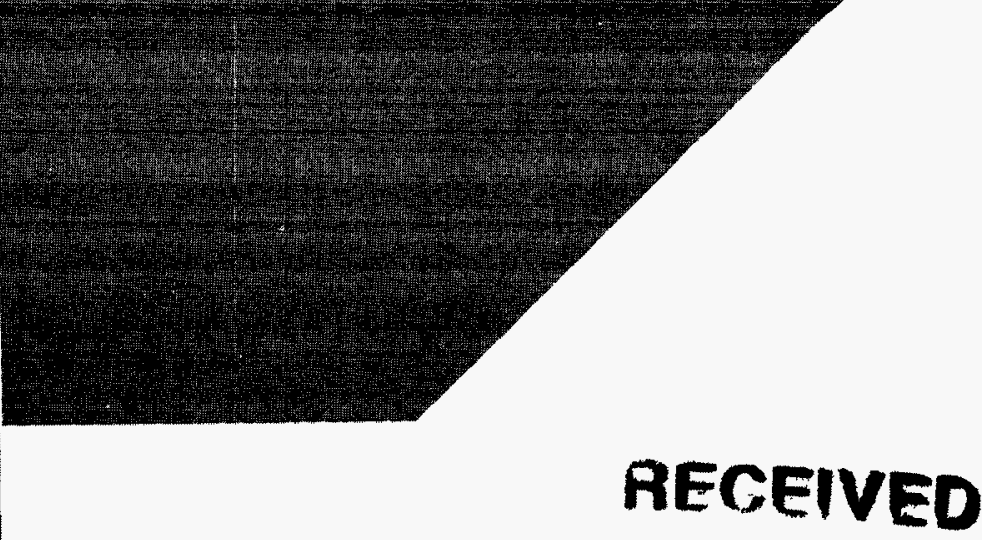

FFR 03 100

0.71

\section{January 1998}

\section{Energy Plug:}

Performance Profiles 1996 


\section{Monthly Energy Review}

The Monthly Energy Review (MER) presents an overview of the Energy Information Administration's recent monthly energy statistics. The statistics cover the major activities of U.S. production, consumption, trade, stocks, and prices for petroleum, natural gas, coal, electricity, and nuclear energy. Also included are international energy and thermal and metric conversion factors.

Publication of this report is in keeping with responsibilities given to the Energy Information Administration (EIA) in Public Law 95-91 (Department of Energy Organization Act), which states, in part, in Section 205(a)(2), that:

\begin{abstract}
"The Administrator shall be responsible for carrying out a central, comprehensive, and unified energy data and information program which will collect, evaluate, assemble, analyze, and disseminate data and information...."
\end{abstract}

The $M E R$ is intended for use by Members of Congress, Federal and State agencies, energy analysts, and the general public. EIA welcomes suggestions from readers regarding data series in the $M E R$ and in other EIA publications.

Related publications: Other monthly EIA reports are Petroleum Supply Monthly, Petroleum Marketing Monthly, Natural Gas Monthly, Electric Power Monthly, and International Petroleum Statistics Report.

Readers of the $M E R$ may also be interested in EIA's Annual Energy Review, where many of the same data series are provided annually beginning with 1949. Contact our National Energy Information Center at 202-586-8800 for more information.

\section{Ordering Information}

Complimentary subscriptions and single issues are available to certain groups of subscribers, such as public and academic libraries; Federal, State, local, and foreign governments; EIA survey respondents; and the media. For further informatior and for answers to questions on energy statistics, contact:

National Energy Information Center, EI-231
Energy Information Administration
Forrestal Building, Room 1F-048
Washington, DC 20585
$202-586-8800$
Fax: 202-586-0727
Internet E-Mail: infoctr@eia.doe.gov
TTY: For people who are deaf
$\quad$ or hard of hearing: 202-586-1181
9 a.m. to 5 p.m., eastern time, M-F

This publication and other EIA publications may be purchased from the Superintendent of Documents, U.S. Government Printing Office. Orders may be directed to:

\author{
Superintendent of Documents \\ U.S. Government Printing Office \\ P.O. Box 371954 \\ Pittsburgh, PA 15250-7954 \\ 202-512-1800
}

Fax: 202-512-2250

8 a.m. to $4: 30$ p.m., eastern time, M-F

The Monthly Energy Review (ISSN 0095-7356) sells for $\$ 88.00$ per year (price is subject to change without advance notice). Second-class postage rates are paid at Washington, DC 10066-9998, and at additional mailing offices. POSTMASTER: Send address changes to Monthly Energy Review, Energy Information Administration, EI-231, 1000 Independence Avenue, S.W., Washington, DC 20585.

\section{Electronic Access}

Monthly Energy Review (MER) data are also available through these electronic means:

- ASCII text, Lotus (wk1), and Excel (xls) versions of the MER tables are available through EIA's Internet homepage at:

http://www.eia.doe.gov/emeu/mer/contents.htm/

- A portable document format (pdf) file of the complete MER including text, tables, and graphs can be downloaded via the homepage at: http://www.eia.doe.gov/bookshelf/multi.html

- MER data series in ASCll comma delimited file format (previously available on diskettes) can be downloaded via EIA's ftp site at ftp://ftp.eia.doe.gov/pub/energy.overview/monthly .energy/current.mer

- For information about the Energy Info Disc, call 1-800-STAT-USA. This CD-ROM contains over 200 reports, databases, and models.

Timing of Release: $M E R$ data are normally released in the afternoon of the third-from-the-last workday of each month and are usually available electronically late that day.

Released for Printing: January 27, 1998

Printed with soy ink on recycled paper 


\section{DISCLAIMER}

Portions of this document may be illegible electronic image products. Images are produced from the best available original document. 


\section{DISCLAIMER}

This report was prepared as an account of work sponsored by an agency of the United States Government. Neither the United States Government nor any agency thereof, nor any of their employees, makes any warranty, express or implied, or assumes any legal liability or responsibility for the accuracy, completeness, or usefulness of any information, apparatus, product, or process disclosed, or represents that its use would not infringe privately owned rights. Reference herein to any specific commercial product, process, or service by trade name, trademark, manufacturer, or otherwise does not necessarily constitute or imply its endorsement, recommendation, or favoring by the United States Government or any agency thereof. The views and opinions of authors expressed herein do not necessarily state or reflect those of the United States Government or any agency thereof. 


\title{
Monthly Energy Review
}

\section{January 1998}

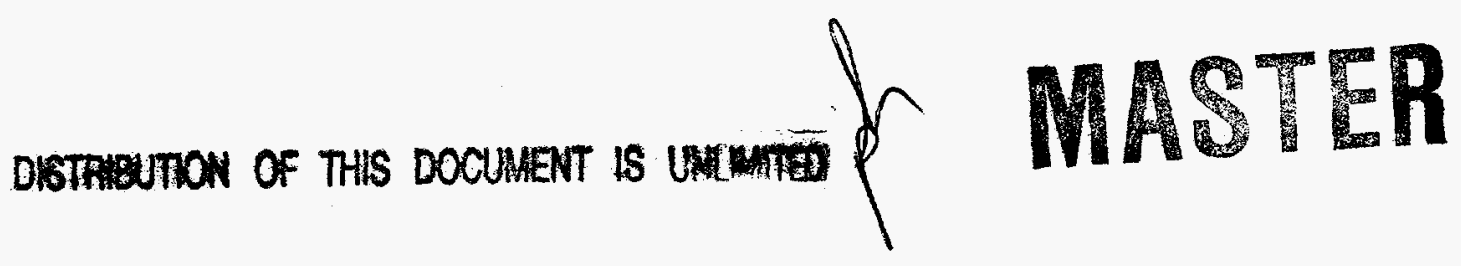

\author{
Energy Information Administration \\ Office of Energy Markets and End Use \\ U.S. Department of Energy \\ Washington, DC 20585
}

This report was prepared by the Energy Information Administration, the independent statistical and analytical agency within the U.S. Department of Energy. The information contained herein should be attributed to the Energy information Administration and should not be construed as advocating or reflecting any policy of the Department of Energy or any other organization. 


\section{Contacts}

The Monthly Energy Review is prepared in the Integrated Energy Statistics Division of the Office of Energy Markets and End Use, Energy Information Administration, under the direction of Katherine E. Seiferlein, 202586-5695 (kitty.seiferlein@eia.doe.gov). Questions and comments about the Monthly Energy Review may be directed to Chuck Allen, 202-586-5828 (chuck.allen@eia.doe.gov), or Diane D. Perritt, 202-586-2788 (diane.perritt@eia.doe.gov), or to the following subject specialists:

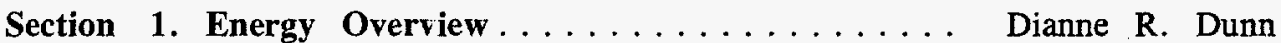

202-586-2792 dianne.dunn@eia.doe.gov

Section 2. Energy Consumption ............. Dianne R. Dunn

202-586-2792 dianne.dunn@eia.doe.gov

Section 3. Petroleum................. Michael Conner

202-586-1795 michael.conner@eia.doe.gov

Section 4. Natural Gas. ................. Ann $M$. Ducca

202-586-6137 ann.ducca@eia.doe.gov

Section 5. Oil and Gas Resource Development ...... Robert F. King

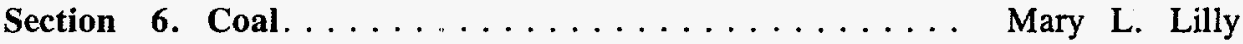

202-586-4787 robert.king@eia.doe.gov

202-426-1154 mary.lilly@eia.doe.gov

Section 7. Electricity

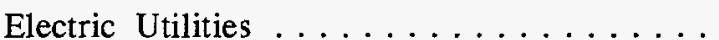

Melvin E. Johnson

$202-426-1172$ melvin.johnson@eia.doe.gov

Retail Sales............... Linda M. Bromley

Nonutility Power Producers ......... Betty L. Williams 202-426-1164

202-426-1269 betty.williams@eia.doe.gov

Section 8. Nuclear Energy $\ldots \ldots \ldots \ldots \ldots \ldots \ldots \ldots$ John R. Moens

202-426-1247 john.moens@eia.doe.gov

Section 9. Energy Prices

Petroleum . . . . . . . . . . . . .

Claudia Hernandez

202-586-4323 claudia.hernandez@eia.doe.gov

Natural Gas. . . . . . . . . . . . Roy Kass

202-586-4790 roy.kass@eia.doe.gov

Electricity Retail Prices ............ Linda M. Bromley

202-426-1164 linda.bromley@eia.doe.gov

Electricity Fossil-Fuel Receipts .........

Kenneth M. McClevey

202-426-1144 kenneth.mcclevey@eia.doe.gov

Section 10. International Energy

Petroleum Production.............. Patricia Smith

202-586-6925

patricia.smith@eia.doe.gov

Petroleum Consumption and Stocks ........

H. Vicky McLaine

202-586-9412

harriet.mclaine@eia.doe.gov

Nuclear Electricity Gross Generation ...... John R. Moens

202-426-1247

john.moens@eia.doe.gov

Requests for additional information on other energy statistics available from the Energy Information Administration and questions concerning subscriptions and report distribution may be directed to the National Energy Irformation Center, 202-586-8800 (TTY, for people who are deaf or hard of hearing, 202-586-1181). 


\section{Contents}

Energy Plug: Performance Profiles of Major Energy Producers 1996. . ix

Section 1. Energy Overview $\ldots \ldots \ldots \ldots \ldots \ldots \ldots \ldots \ldots \ldots \ldots, 1$

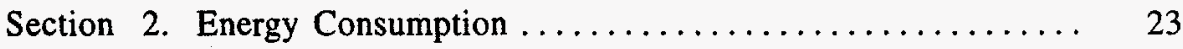

Section 3. Petroleum............................ 41

Section 4. Natural Gas .......................... 71

Section 5. Oil and Gas Resource Development .............. 81

Section 6. Coal ................................... 85

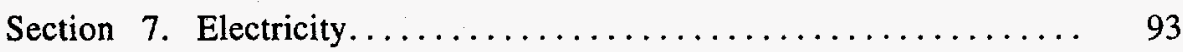

Section 8. Nuclear Energy ......................... 103

Section 9. Energy Prices .............................. 109

Section 10. International Energy .................... 129

Appendix A. Thermal Conversion Factors $\ldots \ldots \ldots \ldots \ldots \ldots \ldots \ldots, 145$

Appendix B. Metric and Other Physical Conversion Factors........ 155

Appendix C. Carbon Dioxide Emission Factors for Coal .......... 159

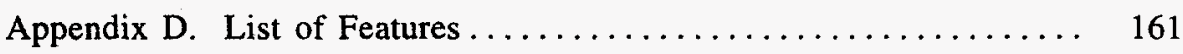

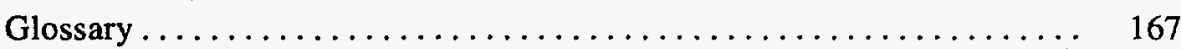


Section 1. Energy Overview

Energy Summary for October $1997 \ldots \ldots \ldots \ldots \ldots \ldots \ldots \ldots \ldots \ldots \ldots \ldots \ldots$

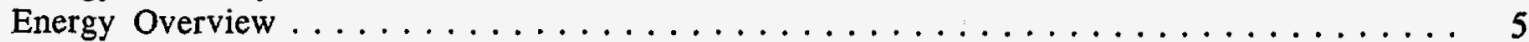

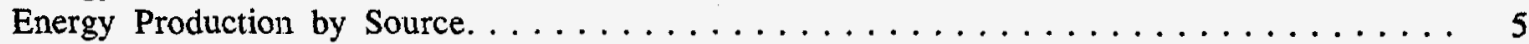

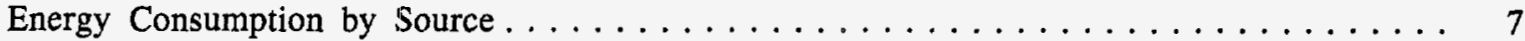

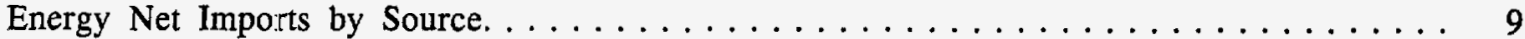

Merchandise Trade value . . . . . . . . . . . . . . . . . . . . . . 11

Cost of Fuels to End Users in Constant (1982-1984) Dollars . . . . . . . . . . . . . . 13

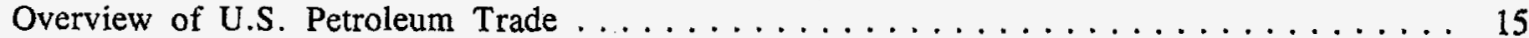

Energy Consumption per Dollar of Gross Domestic Product................ 16

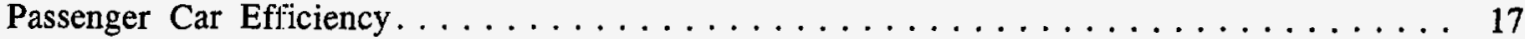

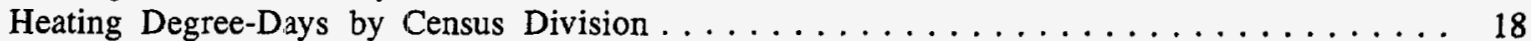

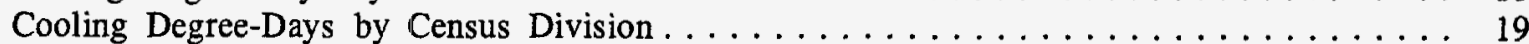

Section 2. Energy Consumption

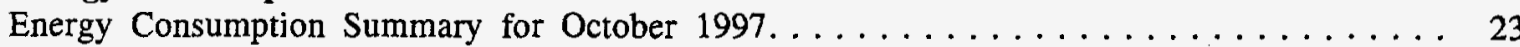

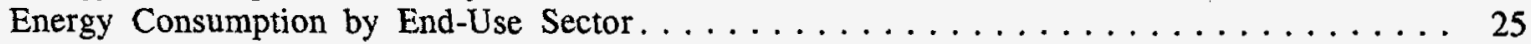

Residential and Commercial Energy Consumption .................. 27

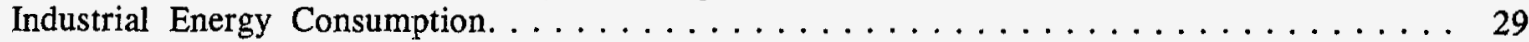

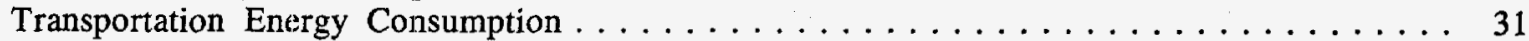

Energy Input at Electric Utilities $\ldots \ldots \ldots \ldots \ldots \ldots \ldots \ldots \ldots \ldots \ldots \ldots \ldots \ldots$

Section 3. Petroleum

3.1 Petroleum Overview

3.1a Field Production, Stock Change, Petroleum Products Supplied, and Ending Stocks .

$3.1 \mathrm{~b}$ Imports, Exports, and Net Imports . . . . . . . . . . . . . . .

Crude Oil Supply and Disposition

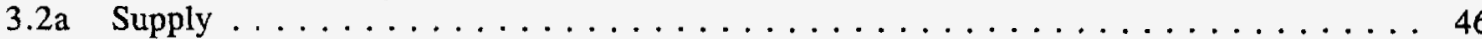

$3.2 \mathrm{~b}$ Disposition and Ending Stocks $\ldots \ldots \ldots \ldots \ldots \ldots \ldots \ldots \ldots \ldots \ldots \ldots \ldots \ldots \ldots$

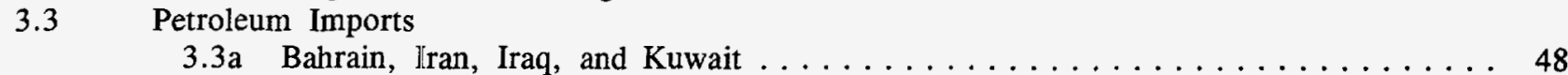

3.3b Qatar, Saudi Arabia, U.A.E., and Total Persian Gulf . . . . . . . . . . . . . 49

$3.3 \mathrm{c}$ Algeria, Ecuador, Gabon, Indonesia, and Libya ................ 50

$3.3 \mathrm{~d}$ Nigeria, Venezuela, Total Other OPEC, and Total OPEC ........... 51

3.3e Angola, Australia, Bahama Islands, Brazil, Canada, and China. . . . . . . . . 52

$3.3 \mathrm{f}$ Colombia, Ecuador, Gabon, Italy, Malaysia, and Mexico ............ 53

$3.3 \mathrm{~g}$ Netherlands, Netherlands Antilles, Norway, Puerto Rico, Russia, and Spain ... 54

3.3h Trinidad and Tobago, United Kingdom, Virgin Islands, Other Non-OPEC,

Total Non-OPEC, and Total Imports. . . . . . . . . . . . . . 55

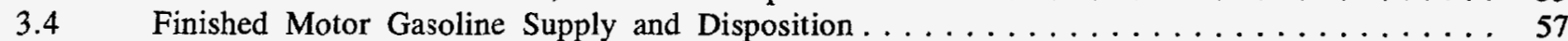

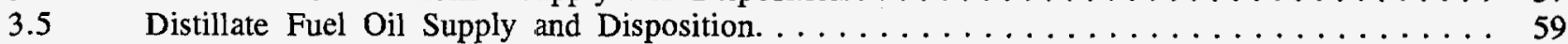

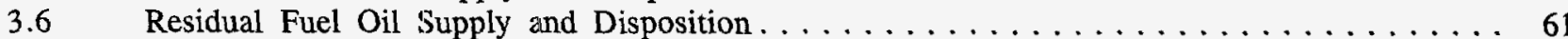

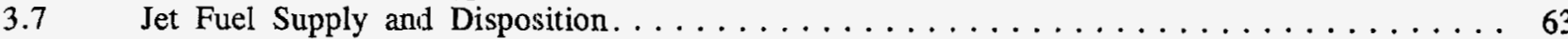

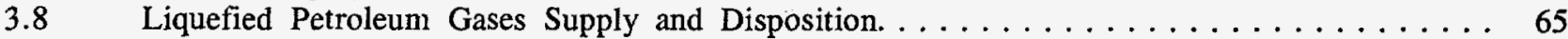

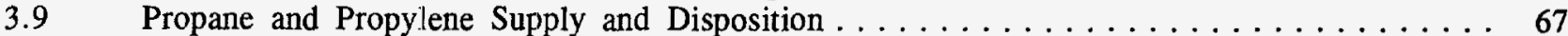

$3.10 \quad$ Other Petroleum Products Supply and Disposition $\ldots \ldots \ldots \ldots \ldots \ldots \ldots \ldots \ldots$

Section 4. Natural Gas

Natural Gas Overview. ........................... 73

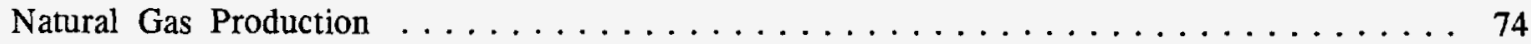

Natural Gas Trade by Country. . . . . . . . . . . . . . . . . . 75

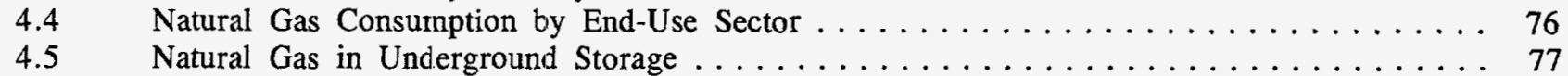


Section 5. Oil and Gas Resource Development

Page

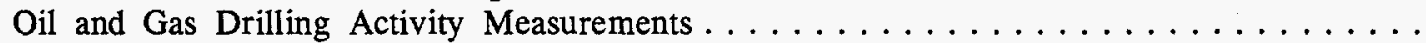

Oil and Gas Wells Drilled.

Section 6. Coal

6.1

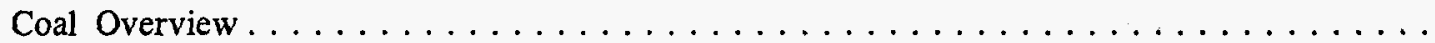

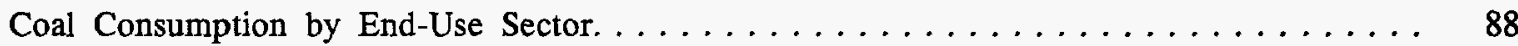

6.2

Coal Stocks, End of Period.

\section{Section 7. Electricity}

Electric Power Industry Net Generation $\ldots \ldots \ldots \ldots \ldots \ldots \ldots \ldots \ldots$

Electric Utility Retail Sales of Electricity by End-Use Sector . . . . . . . . . . . . . . . . 97

Electric Utility Consumption of Fossil Fuels To Generate Electricity. . . . . . . . . .

Electric Utility Stocks of Coal and Petroleum, End of Period. . . . . . . . . . . . . 100

Nonutility Power Net Generation of Electricity . . . . . . . . . . . . . . .

\section{Section 8. Nuclear Energy}

Nuclear Power Plant Operations. . . . . . . . . . . . . . . . . . . .

\section{Section 9. Energy Prices}

Crude Oil Price Summary.

Landed Costs of Crude Oil Imports from Selected Countries . . . . . . . . . . . . . . 113

Motor Gasoline Retail Prices, U.S. City Average . . . . . . . . . . . . . . . . . . 114

Refiner Prices of Residual Fuel Oil . . . . . . . . . . . . . . . . . .

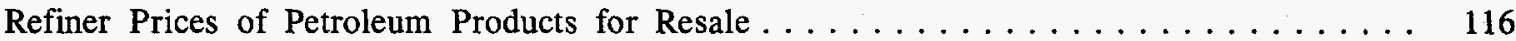

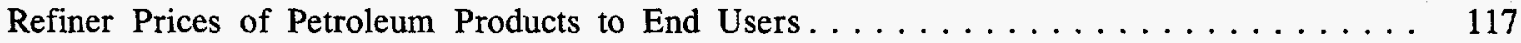

$9.8 \quad$ No. 2 Distillate Prices to Residences

$9.8 \mathrm{a}$ Northeastern States. . . . . . . . . . . . . . . . . . . . . . . . . . . 118

$9.8 \mathrm{~b}$ Selected South Atlantic and Midwestern States . . . . . . . . . . . . 119

$9.8 \mathrm{c}$ Selected Western States and U.S. Average . . . . . . . . . . . . . . . 120

$9.9 \quad$ Retail Prices of Electricity Sold by Electric Utilities . . . . . . . . . . . . . . . . . 122

9.10 Quantity and Cost of Fossil-Fuel Receipts at Steam-Electric Utility Plants . . . . . . . 123

$9.11 \quad$ Natural Gas Prices . . . . . . . . . . . . . . . . . . . . . . . . . 125

\section{Section 10. International Energy}

10.1 World Crude Oil Production

10.1a Algeria Through Venezuela. . . . . . . . . . . . . . . . . . . . . . 130

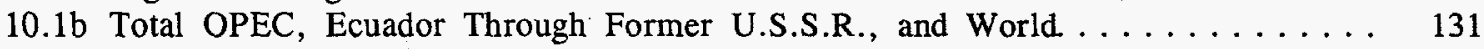

$10.2 \quad$ Petroleum Consumption in OECD Countries $\ldots \ldots \ldots \ldots \ldots \ldots \ldots \ldots \ldots \ldots \ldots \ldots$

10.3 Petroleum Stocks in OECD Countries, End of Period $\ldots \ldots \ldots \ldots \ldots \ldots \ldots \ldots \ldots \ldots \ldots$

10.3 Petroleum Stocks in OECD Countries, End of Period $\ldots \ldots \ldots \ldots \ldots \ldots \ldots \ldots \ldots$

10.4 Nuclear Electricity Gross Generation

$10.4 a$ Regions and World ............................ 139

10.4b North, Central, and South America . . . . . . . . . . . . . . . 140

$10.4 \mathrm{c}$ Western Europe............................. 141

$10.4 \mathrm{~d}$ Far East and Africa.......................... 142

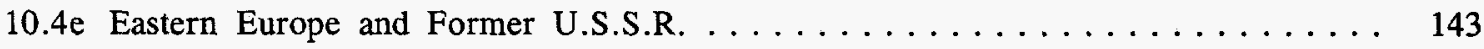


Appendix A. Thermal Conversion Factors

Al. Approximate Heat Content of Petroleum Products

A2. Approximate Heat Content of Crude Oil, Crude Oil and Products, and Natural Gas Plant Liquids

A3. Approximate Heat Content of Petroleum Products, Weighted Averages. Approximate Heat Content of Natural Gas.

Appendix B. Metric and Other Physical Conversion Factors

B1.

Metric Conversion Factors.

B2.

Metric Prefixes

Other Physical Conversion Factors.

B3.

Appendix C. Carbon Dioxide Emission Factors for Coal

C1. Average Carbon Dioxide Emission Factors for Coal by Coal-Consuming Sector 
Section 1. Energy Overview Page

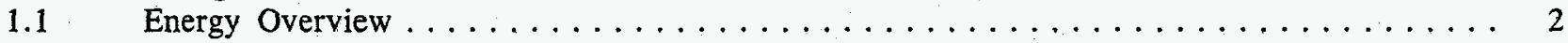

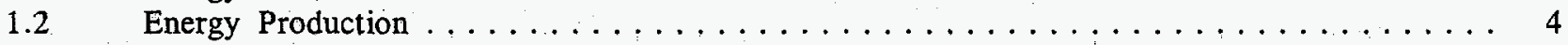

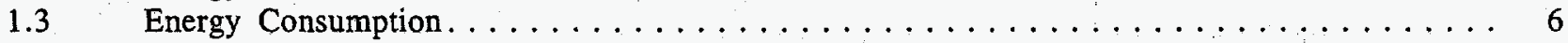

$1.4 \quad$ Energy Net Imports $\ldots \ldots \ldots \ldots \ldots \ldots \ldots \ldots \ldots \ldots \ldots \ldots \ldots \ldots \ldots \ldots$

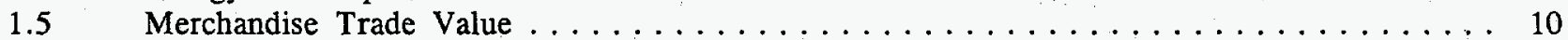

$1.6 \quad$ Cost of Fuels to End Users in Constant $(1982-1984)$ Dollars . . . . . . . . . . . . . 12

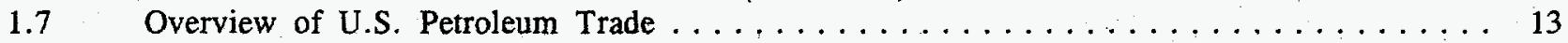

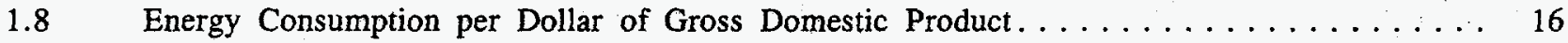

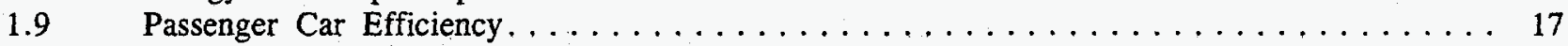

Section 2. Energy Consumption

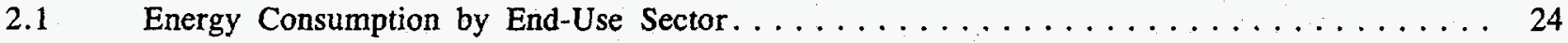

$2.2 \quad$ Residential and Commercial Energy Consumption $\ldots \ldots \ldots \ldots \ldots \ldots \ldots \ldots$

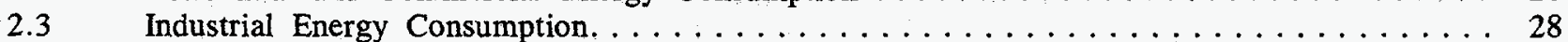

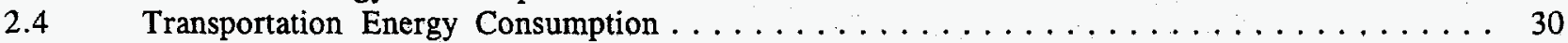

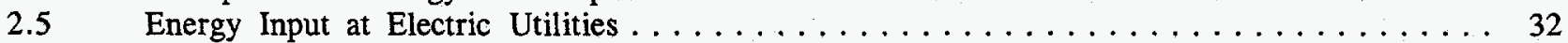

Section 3. Petroleum

$3.1 \quad$ Petroleum Overview $\ldots \ldots \ldots \ldots \ldots \ldots \ldots \ldots \ldots \ldots \ldots \ldots \ldots \ldots \ldots \ldots \ldots$

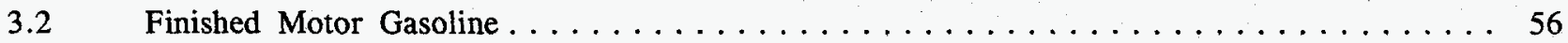

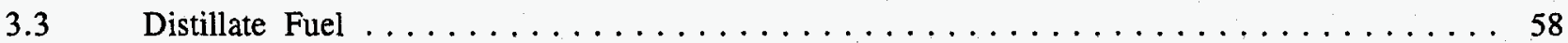

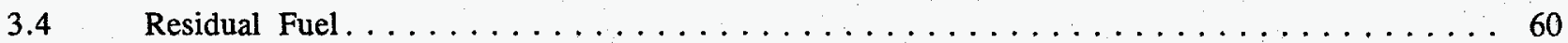

$3.5 \quad$ Jet Fuel. . . . . . . . . . . . . . . . . . . . . . . . . . 62

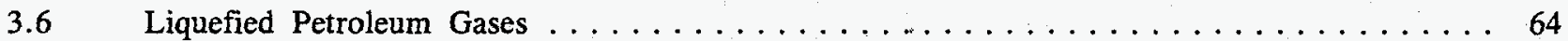

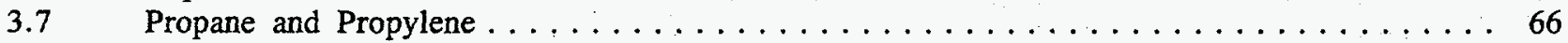

Section 4. Natural Gas

$4.1 \quad$ Natural Gas . . . . . . . . . . . . . . . . . . . . . . . 72

Section 5. Oil and Gas Resource Development

$5.1 \quad$ Oil and Gas Resource Development Indicators. . . . . . . . . . . . . . 81

Section 6. Coal

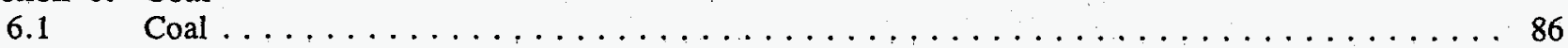

Section 7. Electricity

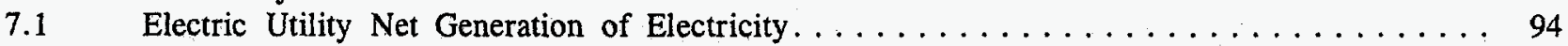

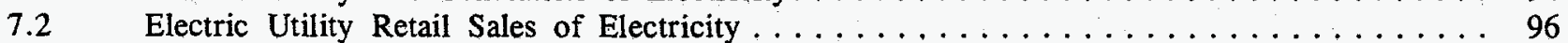

$7.3 \quad$ Electric Utility Consumption and Stocks of Fossil Fuels. . . . . . . . . . . . . . 98

Section 8. Nuclear Energy

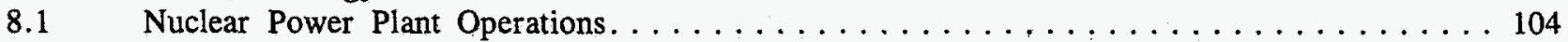

Section 9. Energy Prices

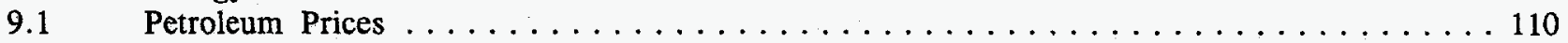

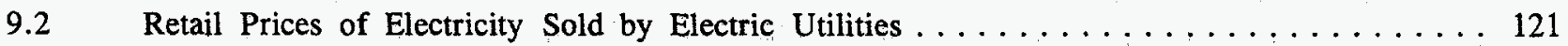

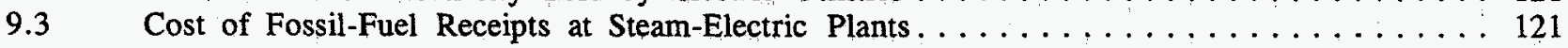

$9.4 \quad$ Natural Gas Prices . . . . . . . . . . . . . . . . . . . . . . . . . . . . . 124

Section 10. International Energy

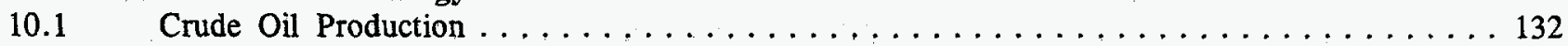

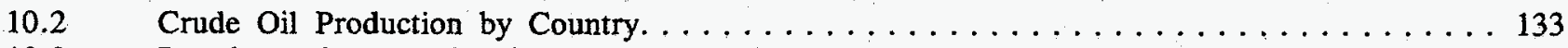

10.3 Petroleum Consumption in OECD Countries $\ldots \ldots \ldots \ldots \ldots \ldots \ldots \ldots \ldots \ldots \ldots \ldots$

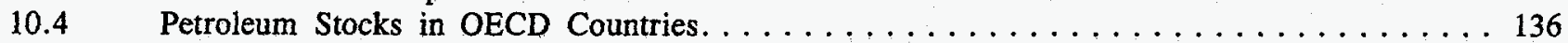

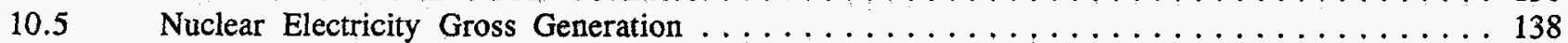




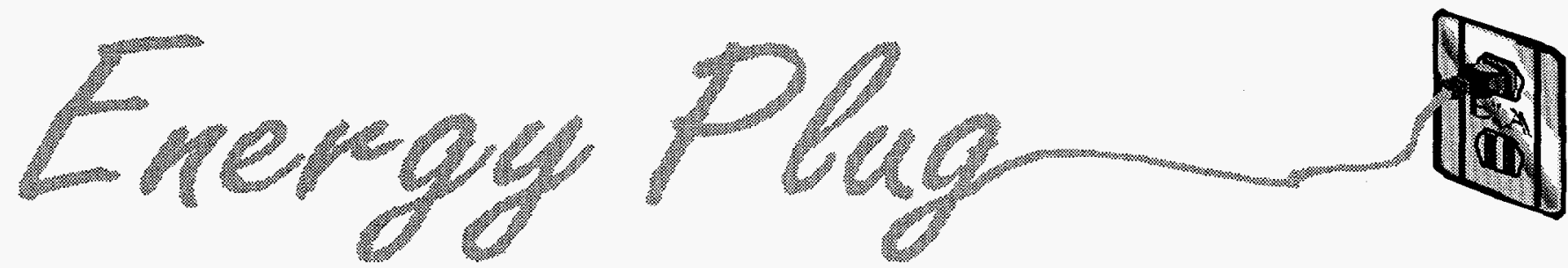

\section{Performance Profiles of Major Energy Producers 1996}

High demand and tight supplies in petroleum markets helped boost the 1996 aggregate net income of two dozen major U.S. energy producers by 52 percent over 1995 levels, to a record $\$ 32$ billion. The year marked the fourth increase in a row. Overall profitability (as return on equity) also rose, to 18 percent. The 24 companies are surveyed annually via the Energy Information Adminstration's Financial Reporting System (FRS) and the results published in Performance Profiles of Major Energy Producers.

Three sources contributed heavily to the FRS companies' 1996 rise in income:

- Net income from domestic and foreign oil and gas production operations rose $\$ 8$ billion in 1996 , driven mainly by higher prices. Income from U.S. crude oil and natural gas production doubled, while profitability tripled, reaching the highest level since 1985.

- Income from U.S. refining and marketing operations doubled to $\$ 2.5$ billion, albeit from a historically low level in 1995.

- The FRS companies took advantage of 1996 's record revenues to reduce their long-term debt to the lowest level in 13 years. Debt reduction accounted for nearly all of the $\$ 1$ billion decline in interest expenditures in 1996.

Several forces, including higher oil and gas prices and such cost-reducing factors as 3-D seismic exploration, horizontal drilling, and new offshore production technologies, encouraged the FRS companies to increase their investment in oil and gas exploration and development by 24 percent in 1996. The companies spent $\$ 6.7$ billion on offshore domestic exploration (mostly in the Gulf of Mexico), 42 percent more than in 1995. In contrast, investment in refining operations fell 41 percent, apparently due to the completion of projects related to compliance with environmental standards. Investment in other lines of business was also generally down.

In individual chapters, the report reviews key financial developments; oil and gas exploration, development, and production; downstream petroleum operations; coal and alternative energy; and foreign direct investment in U.S. energy. Appendices include a description of the structure of the Financial Reporting System and detailed statistical tables.

\section{Performance Profiles}

of Major Energy Producers 1996

185 pages, 104 tables, 30 figures.

To order a hard copy of the report, use the order form in the back of this publication. To access the report via the Internet, go to http://www.eia.doe.gov and click on "Finance." For help with problems accessing the report, call 202-586-1130 or contact wmaster@eia.doe.gov. If you have technical questions about the report, contact Jon A. Rasmussen, Office of Energy Markets and End Use, at 202-586-1449 or jon.rasmussen @eia.doe.gov. For general information about energy, contact the National Energy Information Center at infoctr@eia.doe.gov or 202-586-8800. 


\section{Section 1. Energy Overview}

Energy production during October 1997 totaled 5.8 quadrillion Btu, a 0.7 -percent increase from the level of production during October 1996. Coal production increased 2.2 percent, natural gas production increased 1.9 percent, and production of crude oil and natural gas plant liquids decreased 1.2 percent. All other forms of energy production combined were down 2.4 percent from the level of production during October 1996.

Energy consumption during October 1997 totaled 7.4 quadrillion Btu, 2.1 percent above the level of consumption during October 1996. Consumption of coal rose 6.3 per- cent, consumption of natural gas increased 4.6 percent, while consumption of petroleum products decreased 0.3 percent. Consumption of all other forms of energy combined decreased 2.5 percent from the level 1 year earlier.

Net imports of energy during October 1997 totaled 1.8 quadrillion Btu, 6.8 percent above the level of net imports 1 year earlier. Net imports of petroleum increased 6.6 percent, but net imports of natural gas were down 2.7 percent. Net exports of coal fell 7.5 percent from the level in October 1996.

\section{Table 1.1 Energy Summary for October 1997}

$$
\text { (Quadrillion Btu) }
$$

\begin{tabular}{|c|c|c|c|c|c|c|c|c|}
\hline & \multicolumn{3}{|c|}{ October } & \multicolumn{5}{|c|}{ Cumulative January Through October } \\
\hline & 1997 & 1996 & $\begin{array}{l}\text { Percent } \\
\text { Change }\end{array}$ & 1997 & $\begin{array}{l}1997 \\
\text { Daily } \\
\text { Rate }\end{array}$ & 1996 & $\begin{array}{l}1996 \\
\text { Daily } \\
\text { Rate }\end{array}$ & $\begin{array}{l}\text { Percent } \\
\text { Change }\end{array}$ \\
\hline Production & 5.816 & $\mathbf{5 . 7 7 7}$ & 0.7 & 57.680 & 0.190 & 57.555 & 0.189 & 0.5 \\
\hline Coal & 2.060 & 2.016 & 2.2 & 19.399 & .064 & 18.937 & .062 & 2.8 \\
\hline Natural Gas (Dry) & $E_{1.631}$ & 1.600 & 1.9 & $E_{16.178}$ & E.053 & 16.103 & .053 & .8 \\
\hline 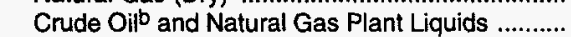 & $E_{1.372}$ & 1.389 & -1.2 & E 13.415 & $E .044$ & 13.520 & .044 & -.4 \\
\hline 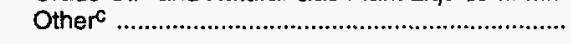 & .753 & .771 & -2.4 & 8.689 & .029 & 8.996 & .029 & -3.1 \\
\hline Consumption & 7.383 & 7.233 & 2.1 & 74.778 & .246 & 74.399 & .244 & .8 \\
\hline Coal & $E_{1.786}$ & 1.680 & 6.3 & $E_{17.364}$ & $\mathrm{E} .057$ & 16.936 & .056 & 2.9 \\
\hline 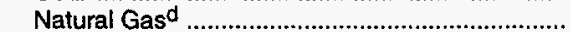 & F 1.645 & 1.572 & 4.6 & $E_{18.243}$ & E.060 & 18.285 & .060 & .1 \\
\hline Petroleum Products ${ }^{e}$ & 3.172 & 3.181 & -.3 & 30.150 & .099 & 29.846 & .098 & 1.3 \\
\hline 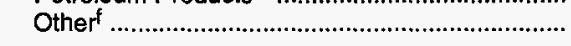 & .780 & .799 & -2.5 & 9.021 & .030 & 9.332 & .031 & -3.0 \\
\hline Net Imports .... & 1.781 & 1.668 & 6.8 & 17.075 & .056 & 16.100 & .053 & 6.4 \\
\hline Coal9 & -.181 & -.195 & -7.5 & -1.698 & -.006 & -1.818 &. .006 & -6.3 \\
\hline Natural Gas & E.234 & .241 & -2.7 & $E_{2} .380$ & E.008 & 2.339 & .008 & 2.1 \\
\hline Petroleum $h$ & 1.701 & 1.595 & 6.6 & 16.060 & .053 & 15.242 & .050 & 5.7 \\
\hline 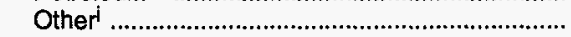 & .027 & .028 & -4.5 & .332 & .001 & .336 & .001 & -.8 \\
\hline
\end{tabular}

a Based on daily rates prior to rounding.

b Includes lease condensate.

c "Other" is hydroelectric and nuclear electric power, and electricity generated for distribution from wood, waste, geothermal, wind, photovoltaic, and solar thermal energy.

d Includes supplemental gaseous fuels.

e Products obtained from the processing of crude oil (including lease condensate), natural gas, and other hydrocarbon compounds.

$f$ "Other" is hydroelectric and nuclear electric power; electricity generated for distribution from wood, waste, geothermal, wind, photovoltaic, and solar thermal energy; and net imports of electricity and coal coke.
9 Minus sign indicates exports are greater than imports.

h Crude oil, lease condensate, petroleum products, pentanes plus, unfinished oils, gasoline blending components, and imports of crude oil for the Strategic Petroleum Reserve.

$i$ "Other" is net imports of electricity and coal coke.

E=Estimate. F=Forecast.

Notes: - Totals may not equal sum of components due to independent rounding. - Geographic coverage is the 50 States and the District of Columbia.

Sources: Tables 1.3,1.4, and 1.5.

Please Read: Due to a lack of consistent monthly historical data, some renewable energy sources are not included in production and consumption. In 1996, for example, 3.9 quadrillion Btu of renewable energy used by electric utilities to generate electricity for distribution is included, but an estimated 3.4 quadrillion Btu used by residential, commercial, and industrial consumers is not. See Note 12 at the end of Section 2 for details. 
Figure 1.1 Energy Overview

(Quadrillion Btu)

Consumption, Production, and Imports, 1973-1996

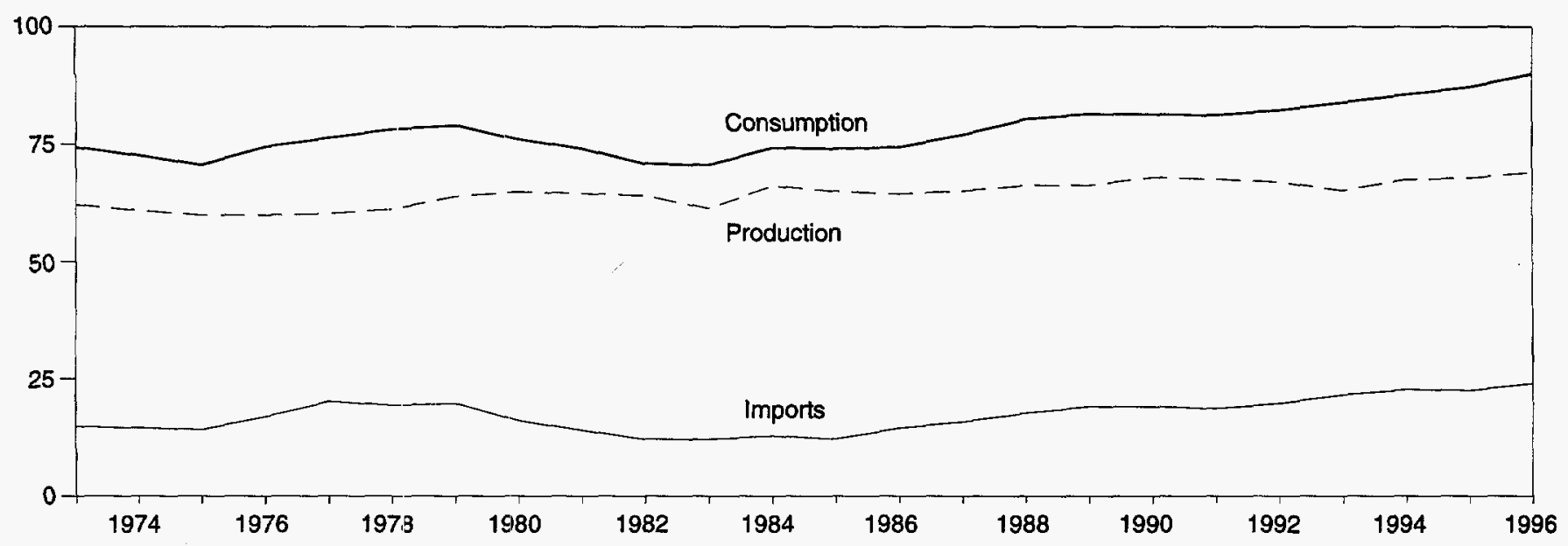

Consumption, Production, and Irnports, Monthly

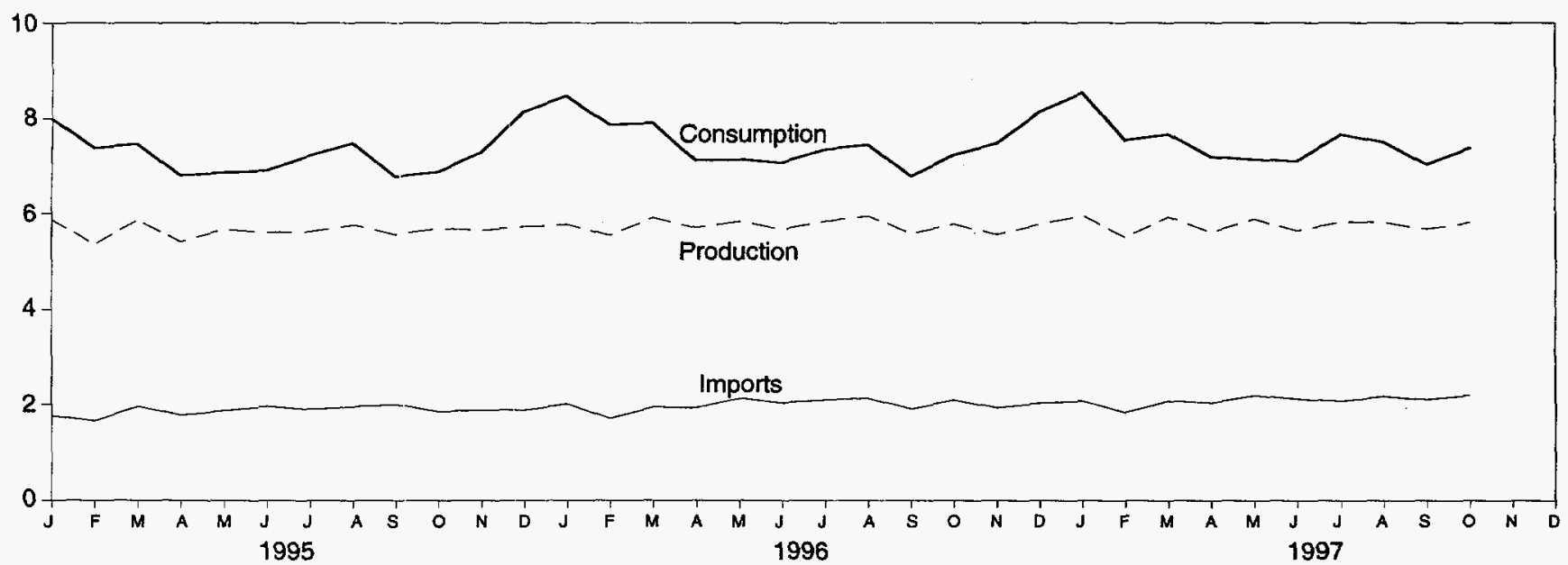

Overview, October 1997

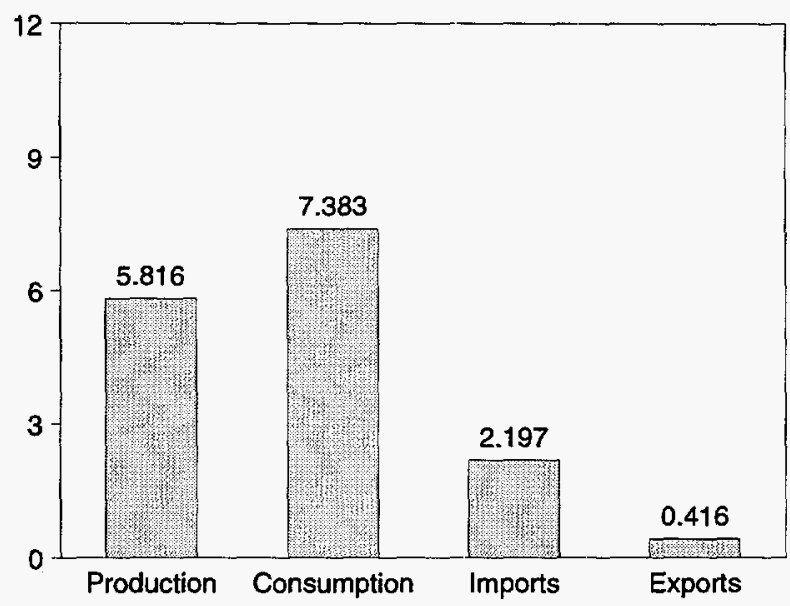

Net Imports, January-October

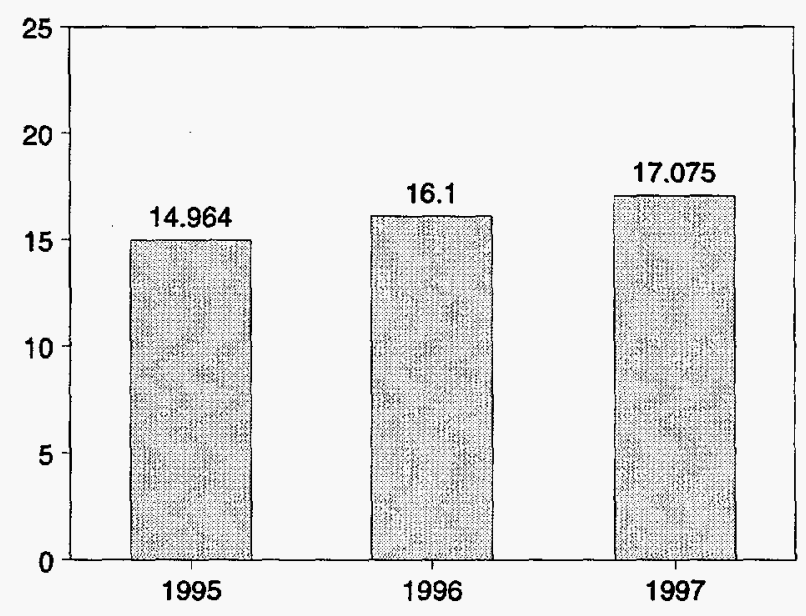

Note: Because vertical scales differ, graphs should not be compared.

Source: Table 1.2. 
Table 1.2 Energy Overview

(Quadrillion Btu)

\begin{tabular}{|c|c|c|c|c|c|}
\hline & Production & Consumption ${ }^{a}$ & Imports & Exports & Net Imports \\
\hline 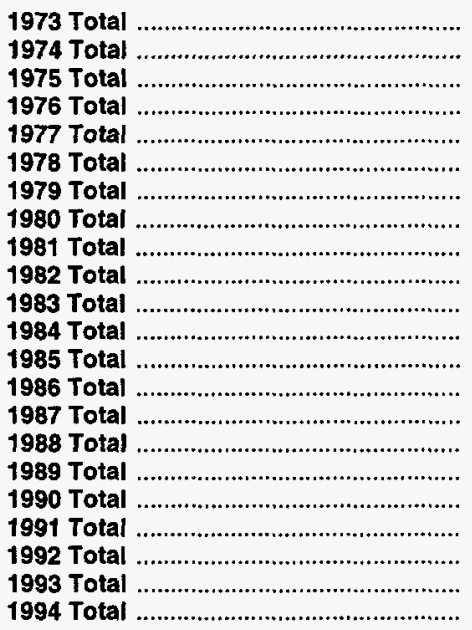 & $\begin{array}{l}62.060 \\
60.835 \\
59.860 \\
59.892 \\
60.219 \\
61.103 \\
63.801 \\
64.761 \\
64.421 \\
63.962 \\
61.279 \\
65.962 \\
64.871 \\
64.350 \\
64.952 \\
66.105 \\
66.129 \\
67.853 \\
67.484 \\
66.853 \\
65.163 \\
67.448\end{array}$ & $\begin{array}{l}74.282 \\
72.543 \\
70.546 \\
74.362 \\
76.288 \\
78.089 \\
78.898 \\
75.955 \\
73.990 \\
70.848 \\
70.524 \\
74.144 \\
73.981 \\
74.297 \\
76.894 \\
80.218 \\
81.325 \\
81.265 \\
81.116 \\
82.144 \\
83.863 \\
85.587\end{array}$ & $\begin{array}{l}14.731 \\
14.413 \\
14.111 \\
16.837 \\
20.090 \\
19.254 \\
19.616 \\
15.971 \\
13.975 \\
12.092 \\
12.027 \\
12.767 \\
12.103 \\
14.438 \\
15.764 \\
17.564 \\
18.947 \\
18.987 \\
18.577 \\
19.650 \\
21.530 \\
22.695\end{array}$ & $\begin{array}{l}2.051 \\
2.223 \\
2.359 \\
2.188 \\
2.071 \\
1.931 \\
2.870 \\
3.723 \\
4.329 \\
4.633 \\
3.717 \\
3.804 \\
4.231 \\
4.055 \\
3.853 \\
4.415 \\
4.765 \\
4.910 \\
5.220 \\
5.017 \\
4.350 \\
4.125\end{array}$ & $\begin{array}{r}12.680 \\
12.190 \\
11.752 \\
14.648 \\
18.019 \\
17.323 \\
16.746 \\
12.247 \\
9.646 \\
7.460 \\
8.310 \\
8.963 \\
7.872 \\
10.382 \\
11.911 \\
13.149 \\
14.181 \\
14.077 \\
13.357 \\
14.633 \\
17.180 \\
18.570\end{array}$ \\
\hline 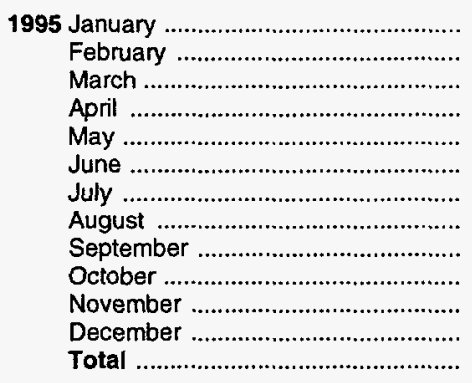 & $\begin{array}{r}5.874 \\
5.363 \\
5.861 \\
5.418 \\
5.665 \\
5.605 \\
5.614 \\
5.754 \\
5.558 \\
5.681 \\
5.644 \\
5.720 \\
67.759\end{array}$ & $\begin{array}{r}R_{7.983} \\
R_{7.376} \\
R_{7.467} \\
R_{6.813} \\
6.871 \\
6.912 \\
7.216 \\
R_{7.478} \\
6.780 \\
\text { R }_{6.884} \\
R_{7.281} \\
R_{8.139} \\
R_{87.199}\end{array}$ & $\begin{array}{r}1.766 \\
R_{1} 1.660 \\
R_{1} 1.959 \\
R_{1} .780 \\
R_{1} 1.873 \\
1.962 \\
R 1.899 \\
R 1.953 \\
R 1.999 \\
R 1.853 \\
R_{1} 1.882 \\
R_{1} 1.882 \\
R_{22} 2.469\end{array}$ & $\begin{array}{r}\text { R. } .357 \\
\text { R. } .348 \\
\text { R. } .383 \\
\text { R. } .383 \\
\text { R. } .389 \\
.394 \\
\text { R. } .357 \\
\text { R. } .366 \\
\text { R. } .368 \\
\text { R. } .397 \\
\text { R. } .388 \\
\text { R. } .450 \\
\text { R } 4.579\end{array}$ & $\begin{array}{r}R_{1.409} \\
R_{1.312} \\
R_{1.576} \\
R_{1.398} \\
1.485 \\
R_{1.567} \\
1.542 \\
R_{1.588} \\
R_{1.630} \\
R_{1.456} \\
1.494 \\
R_{1.432} \\
R_{17.889}\end{array}$ \\
\hline 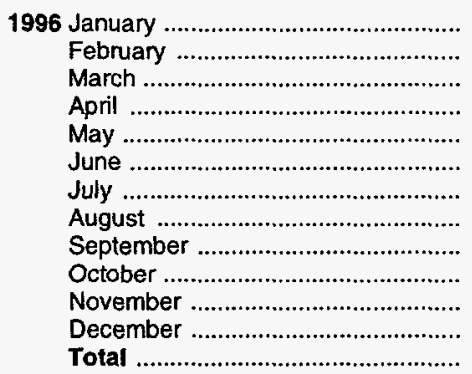 & $\begin{array}{r}5.764 \\
5.546 \\
5.907 \\
5.699 \\
5.834 \\
5.666 \\
5.832 \\
5.942 \\
5.587 \\
5.777 \\
5.567 \\
5.775 \\
68.897\end{array}$ & $\begin{array}{r}8.476 \\
7.863 \\
7.905 \\
7.120 \\
7.141 \\
7.075 \\
7.344 \\
7.450 \\
6.793 \\
7.233 \\
7.474 \\
8.132 \\
\mathbf{9 0 . 0 0 5}\end{array}$ & $\begin{array}{r}2.010 \\
1.714 \\
1.947 \\
1.934 \\
2.130 \\
2.033 \\
2.094 \\
2.129 \\
1.911 \\
2.093 \\
1.935 \\
2.029 \\
23.959\end{array}$ & $\begin{array}{r}.389 \\
.376 \\
.359 \\
.378 \\
.378 \\
.387 \\
.396 \\
.381 \\
.428 \\
.425 \\
.412 \\
.399 \\
4.706\end{array}$ & $\begin{array}{r}1.621 \\
1.338 \\
1.588 \\
1.556 \\
1.753 \\
1.647 \\
1.698 \\
1.747 \\
1.484 \\
1.668 \\
1.523 \\
1.630 \\
19.253\end{array}$ \\
\hline 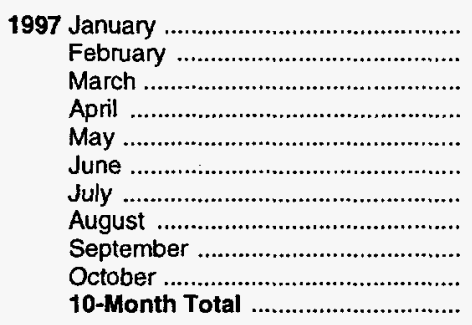 & $\begin{array}{r}5.952 \\
5.512 \\
5.925 \\
5.617 \\
5.884 \\
5.645 \\
R 5.828 \\
R \mathbf{5} 521 \\
\text { R } 5.680 \\
5.816 \\
\mathbf{5 7 . 6 8 0}\end{array}$ & $\begin{array}{l}R_{8.544} \\
R_{7.541} \\
R_{7.668} \\
R_{7.196} \\
R_{7.145} \\
R_{7.105} \\
R_{7.660} \\
R_{7.498} \\
R_{7.037} \\
7.383 \\
\mathbf{7 4 . 7 7 8}\end{array}$ & $\begin{array}{r}R_{2} .073 \\
1.838 \\
2.077 \\
R_{2} .036 \\
R_{2} .188 \\
2.117 \\
2.072 \\
R_{2} .171 \\
R_{2.105} \\
2.197 \\
\mathbf{2 0 . 8 7 3}\end{array}$ & $\begin{array}{l}.397 \\
.337 \\
.372 \\
.359 \\
.363 \\
.359 \\
.376 \\
.439 \\
.380 \\
.416 \\
3.799\end{array}$ & $\begin{array}{r}1.676 \\
1.501 \\
1.705 \\
1.676 \\
1.824 \\
1.758 \\
1.697 \\
\mathrm{~A}_{1.732} \\
\mathrm{R} 1.724 \\
1.781 \\
17.075\end{array}$ \\
\hline 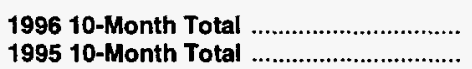 & $\begin{array}{l}57.555 \\
56.394\end{array}$ & $\begin{array}{l}74.399 \\
71.779\end{array}$ & $\begin{array}{l}19.996 \\
18.705\end{array}$ & $\begin{array}{l}3.896 \\
3.741\end{array}$ & $\begin{array}{l}16.100 \\
14.964\end{array}$ \\
\hline
\end{tabular}

a The sum of domestic energy production and net imports of energy does not equal domestic energy consumption. The difference is attributed to stock changes; losses and gains in conversion, transportation, and distribution; the addition of blending compounds shipments of anthracite to U.S. Armed Forces in Europe; and adjustments to account for discrepancies between reporting systems.

$\mathrm{R}=$ Revised data
Notes: - For definitions, see Notes 1 through 4 at end of section. - Totals may not equal sum of components due to independent rounding. - Geographic coverage is the 50 States and the District of Columbia.

Sources: • Production: Table 1.3. - Consumption: Table 1.4. - Imports and Exports: Tables 3.1b, 4.2, 6.1, A2-A8, and Section 2, "Energy Consumption Notes and Sources," Notes 8 and 9. - Net Imports: Table 1.5.

Please Read: Due to a lack of consistent monthly historical data, some renewable energy sources are not included in production and consumption. In 1996, for example, 3.9 quadrillion Btu of renewable energy used by electric utilities to generate electricity for distribution is included, but an estimated 3.4 quadrillion Btu used by residential, commercial, and industrial consumers is not. See Note 12 at the end of Section 2 for details. 
Figure 1.2 Energy Production

(Quadrillion Eitu)

Total, 1973-1996

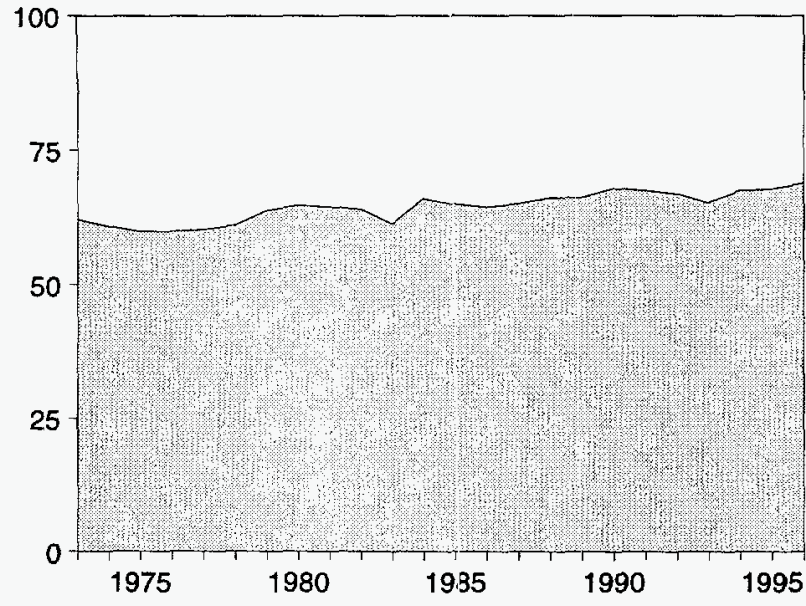

By Major Sources, 1973-1996

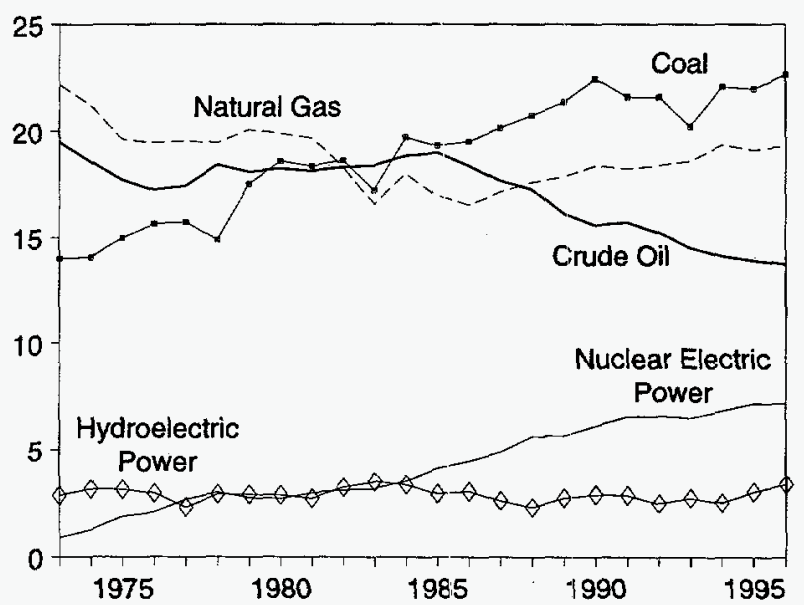

Total, January-October

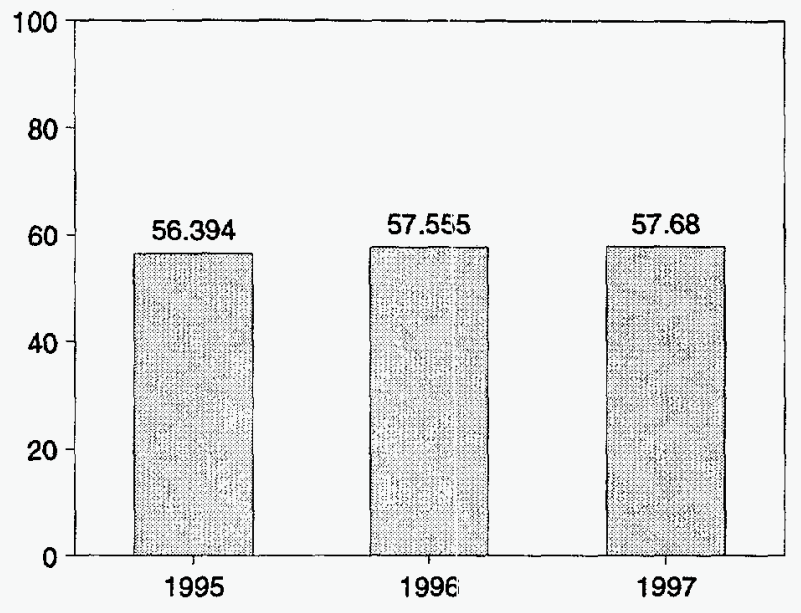

Total, Monthly

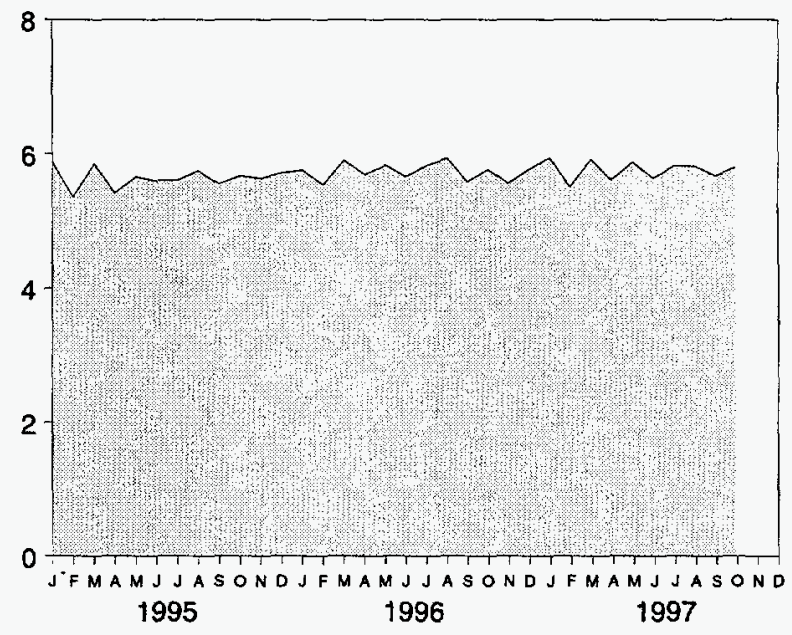

By Major Sources, Monthly

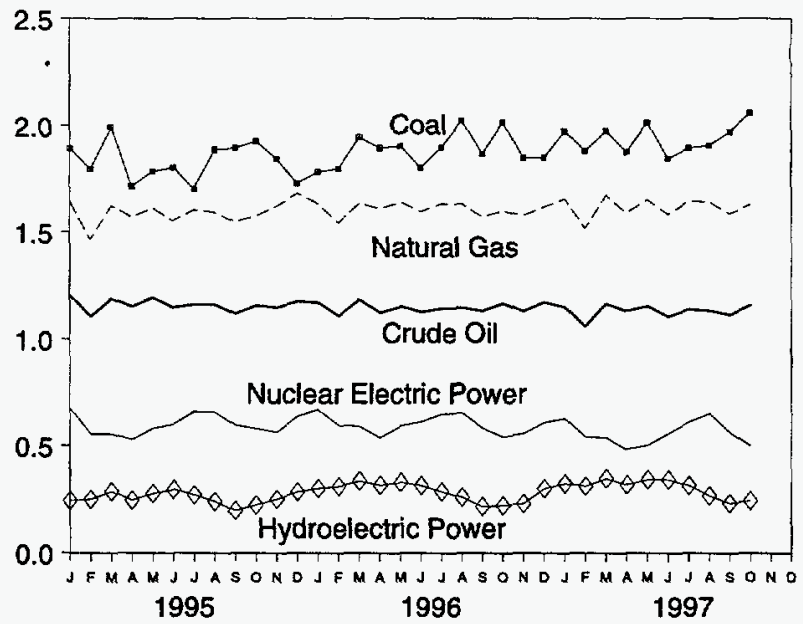

By Major Sources, October 1997

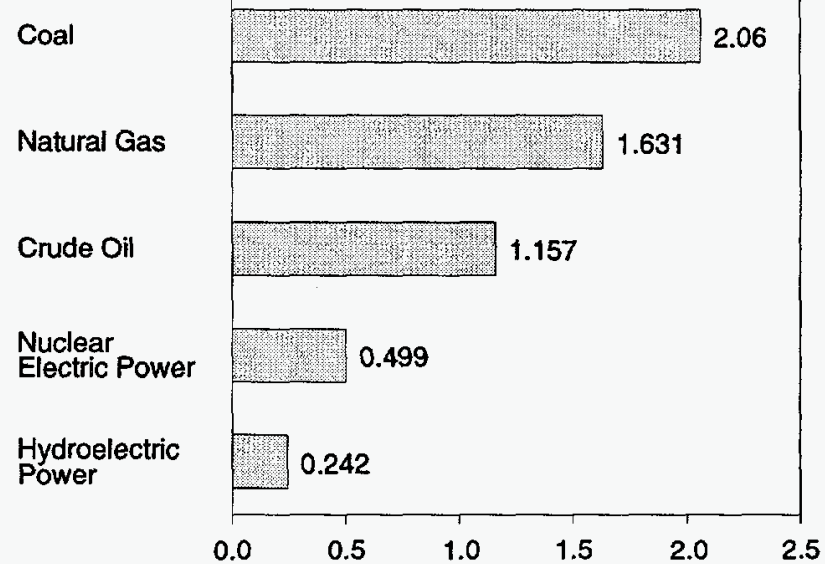

Note: Because vertical scales differ, graphs should not be compared. Source: Table 1.3. 
Table 1.3 Energy Production by Source

(Quadrillion Btu)

\begin{tabular}{|c|c|c|c|c|c|c|c|c|c|}
\hline & Coal & $\begin{array}{c}\text { Natural } \\
\text { Gas } \\
\text { (Dry) }\end{array}$ & $\begin{array}{c}\text { Crude } \\
\text { Oila }\end{array}$ & $\begin{array}{c}\text { Natural } \\
\text { Gas Plant } \\
\text { Liquids }\end{array}$ & $\begin{array}{l}\text { Nuclear } \\
\text { Electric } \\
\text { Power }\end{array}$ & $\begin{array}{l}\text { Hydro- } \\
\text { electric } \\
\text { Powerb }\end{array}$ & $\begin{array}{c}\text { Geothermal } \\
\text { Energy }\end{array}$ & Otherc & Total \\
\hline 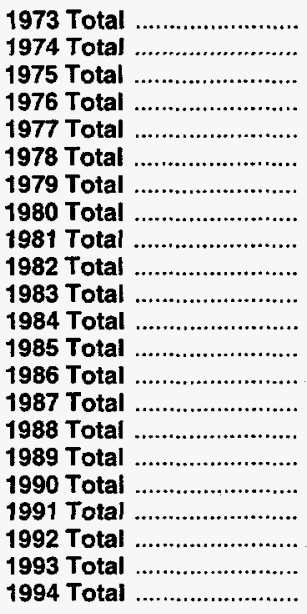 & $\begin{array}{l}13.993 \\
14.074 \\
14.990 \\
15.654 \\
15.755 \\
14.910 \\
17.539 \\
18.597 \\
18.376 \\
18.639 \\
17.246 \\
19.719 \\
19.325 \\
19.510 \\
20.142 \\
20.737 \\
21.345 \\
22.456 \\
21.594 \\
21.593 \\
20.221 \\
22.068\end{array}$ & $\begin{array}{l}22.187 \\
21.210 \\
19.640 \\
19.480 \\
19.565 \\
19.485 \\
20.076 \\
19.908 \\
19.699 \\
18.319 \\
16.593 \\
18.008 \\
16.980 \\
16.541 \\
17.136 \\
17.599 \\
17.847 \\
18.362 \\
18.229 \\
18.375 \\
18.584 \\
19.348\end{array}$ & $\begin{array}{l}19.493 \\
18.575 \\
17.729 \\
17.262 \\
17.454 \\
18.434 \\
18.104 \\
18.249 \\
18.146 \\
18.309 \\
18.392 \\
18.848 \\
18.992 \\
18.376 \\
17.675 \\
17.279 \\
16.117 \\
15.571 \\
15.701 \\
15.223 \\
14.494 \\
14.103\end{array}$ & $\begin{array}{l}2.569 \\
2.471 \\
2.374 \\
2.327 \\
2.327 \\
2.245 \\
2.286 \\
2.254 \\
2.307 \\
2.191 \\
2.184 \\
2.274 \\
2.241 \\
2.149 \\
2.215 \\
2.260 \\
2.158 \\
2.175 \\
2.305 \\
2.363 \\
2.408 \\
2.391\end{array}$ & $\begin{array}{l}0.910 \\
1.272 \\
1.900 \\
2.111 \\
2.702 \\
3.024 \\
2.776 \\
2.739 \\
3.008 \\
3.131 \\
3.203 \\
3.553 \\
4.149 \\
4.471 \\
4.906 \\
5.661 \\
5.677 \\
6.161 \\
6.579 \\
6.607 \\
6.519 \\
6.837\end{array}$ & $\begin{array}{l}2.861 \\
3.177 \\
3.155 \\
2.976 \\
2.333 \\
2.937 \\
2.931 \\
2.900 \\
2.758 \\
3.266 \\
3.527 \\
3.386 \\
2.970 \\
3.071 \\
2.635 \\
2.334 \\
2.767 \\
2.926 \\
2.885 \\
2.501 \\
2.757 \\
2.536\end{array}$ & $\begin{array}{l}0.043 \\
.053 \\
.070 \\
.078 \\
.077 \\
.064 \\
.084 \\
.110 \\
.123 \\
.105 \\
.129 \\
.165 \\
.198 \\
.219 \\
.229 \\
.217 \\
.197 \\
.181 \\
.170 \\
.169 \\
.158 \\
.145\end{array}$ & $\begin{array}{l}0.003 \\
.003 \\
.002 \\
.003 \\
.005 \\
.003 \\
.005 \\
.005 \\
.004 \\
.003 \\
.004 \\
.009 \\
.015 \\
.012 \\
.016 \\
.017 \\
.020 \\
.021 \\
.021 \\
.022 \\
.021 \\
.020\end{array}$ & $\begin{array}{l}62.060 \\
60.835 \\
59.860 \\
59.892 \\
60.219 \\
61.103 \\
63.801 \\
64.761 \\
64.421 \\
63.962 \\
61.279 \\
65.962 \\
64.871 \\
64.350 \\
64.952 \\
66.105 \\
66.129 \\
67.853 \\
67.484 \\
66.853 \\
65.163 \\
67.448\end{array}$ \\
\hline 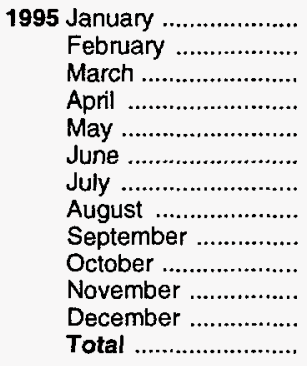 & $\begin{array}{r}1.893 \\
1.797 \\
1.994 \\
1.716 \\
1.785 \\
1.805 \\
1.704 \\
1.888 \\
1.895 \\
1.927 \\
1.846 \\
1.730 \\
21.978\end{array}$ & $\begin{array}{r}1.642 \\
1.464 \\
1.625 \\
1.571 \\
1.614 \\
1.554 \\
1.605 \\
1.594 \\
1.548 \\
1.577 \\
1.623 \\
1.683 \\
19.101\end{array}$ & $\begin{array}{r}1.201 \\
1.103 \\
1.187 \\
1.149 \\
1.192 \\
1.145 \\
1.159 \\
1.159 \\
1.116 \\
1.155 \\
1.146 \\
1.174 \\
13.887\end{array}$ & $\begin{array}{l}.210 \\
.189 \\
.209 \\
.204 \\
.211 \\
.198 \\
.206 \\
.204 \\
.200 \\
.207 \\
.205 \\
.199 \\
2.442\end{array}$ & $\begin{array}{l}.675 \\
.553 \\
.553 \\
.526 \\
.580 \\
.601 \\
.661 \\
.657 \\
.594 \\
.579 \\
.562 \\
.638 \\
7.177\end{array}$ & $\begin{array}{l}.243 \\
.249 \\
.286 \\
.245 \\
.277 \\
.296 \\
.270 \\
.239 \\
.196 \\
.223 \\
.250 \\
.284 \\
3.057\end{array}$ & $\begin{array}{l}.009 \\
.006 \\
.007 \\
.006 \\
.005 \\
.006 \\
.006 \\
.011 \\
.008 \\
.013 \\
.012 \\
.011 \\
.099\end{array}$ & $\begin{array}{l}.001 \\
.001 \\
.001 \\
.002 \\
.001 \\
.001 \\
.002 \\
.002 \\
.002 \\
.002 \\
.002 \\
.001 \\
.017\end{array}$ & $\begin{array}{r}5.874 \\
5.363 \\
5.861 \\
5.418 \\
5.665 \\
5.605 \\
5.614 \\
5.754 \\
5.558 \\
5.681 \\
5.644 \\
5.720 \\
67.759\end{array}$ \\
\hline 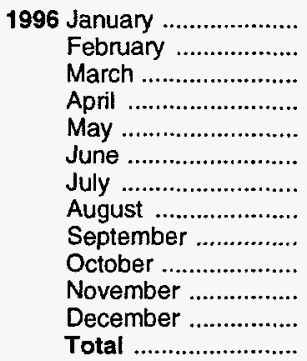 & $\begin{array}{r}1.783 \\
1.799 \\
1.945 \\
1.896 \\
1.905 \\
1.803 \\
1.899 \\
2.023 \\
1.868 \\
2.016 \\
1.849 \\
1.850 \\
22.635\end{array}$ & $\begin{array}{r}1.634 \\
1.544 \\
1.635 \\
1.612 \\
1.641 \\
1.597 \\
1.634 \\
1.633 \\
1.572 \\
1.600 \\
1.578 \\
1.618 \\
19.300\end{array}$ & $\begin{array}{r}1.168 \\
1.106 \\
1.182 \\
1.121 \\
1.150 \\
1.124 \\
1.140 \\
1.144 \\
1.128 \\
1.165 \\
1.127 \\
1.170 \\
13.723\end{array}$ & $\begin{array}{l}.201 \\
.184 \\
.212 \\
.209 \\
.212 \\
.208 \\
.214 \\
.218 \\
.212 \\
.224 \\
.217 \\
.220 \\
.2 .530\end{array}$ & $\begin{array}{l}.669 \\
.594 \\
.589 \\
.535 \\
.591 \\
.611 \\
.648 \\
.653 \\
.580 \\
.538 \\
.554 \\
.607 \\
7.168\end{array}$ & $\begin{array}{l}.300 \\
.310 \\
.335 \\
.316 \\
.329 \\
.314 \\
.285 \\
.258 \\
.215 \\
.220 \\
.228 \\
.299 \\
.411\end{array}$ & $\begin{array}{l}.007 \\
.008 \\
.007 \\
.008 \\
.005 \\
.008 \\
.012 \\
.012 \\
.010 \\
.011 \\
.011 \\
.010 \\
.110\end{array}$ & $\begin{array}{l}.002 \\
.001 \\
.002 \\
.001 \\
.001 \\
.002 \\
.002 \\
.002 \\
.002 \\
.002 \\
.002 \\
.002 \\
.020\end{array}$ & $\begin{array}{r}5.764 \\
5.546 \\
5.907 \\
5.699 \\
5.834 \\
5.666 \\
5.832 \\
5.942 \\
5.587 \\
5.777 \\
5.567 \\
5.775 \\
68.897\end{array}$ \\
\hline 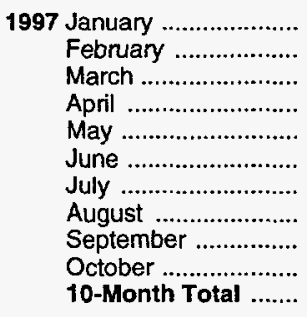 & $\begin{array}{r}1.974 \\
1.881 \\
1.974 \\
1.878 \\
2.014 \\
1.846 \\
R 1.895 \\
R 1.907 \\
R 1.970 \\
2.060 \\
19.399\end{array}$ & $\begin{array}{r}1.657 \\
1.515 \\
1.673 \\
1.591 \\
1.653 \\
1.583 \\
R_{1} .646 \\
R_{1.642} \\
1.586 \\
E_{1.631} \\
E_{16.178}\end{array}$ & $\begin{array}{l}E_{1} .148 \\
E_{1} .058 \\
E_{1} .163 \\
E_{1} .128 \\
E_{1} 1.151 \\
E_{1} 103 \\
E_{1} 1.136 \\
E_{1} 1.130 \\
E_{1} 1111 \\
E_{1} 157 \\
E_{11.285}\end{array}$ & $\begin{array}{l}.212 \\
.201 \\
.223 \\
.210 \\
.214 \\
.209 \\
.217 \\
.217 \\
.212 \\
.215 \\
.2 .130\end{array}$ & $\begin{array}{l}.626 \\
.538 \\
.536 \\
.481 \\
.500 \\
.553 \\
.609 \\
.649 \\
.559 \\
.499 \\
5.550\end{array}$ & $\begin{array}{l}.323 \\
.310 \\
.346 \\
.317 \\
.341 \\
.341 \\
.313 \\
.265 \\
.230 \\
.242 \\
3.028\end{array}$ & $\begin{array}{l}.009 \\
.006 \\
.009 \\
.010 \\
.010 \\
.008 \\
.011 \\
.011 \\
.010 \\
.010 \\
.094\end{array}$ & $\begin{array}{l}.002 \\
.002 \\
.002 \\
.002 \\
.002 \\
.002 \\
.002 \\
.002 \\
.002 \\
.002 \\
.017\end{array}$ & $\begin{array}{r}5.952 \\
5.512 \\
5.925 \\
5.617 \\
5.884 \\
5.645 \\
\text { R } 5.828 \\
\text { R } 5.821 \\
\text { R } 5.680 \\
5.816 \\
\mathbf{5 7 . 6 8 0}\end{array}$ \\
\hline $\begin{array}{l}1996 \text { 10-Month Total ....... } \\
1995 \text { 10-Month Total ....... }\end{array}$ & $\begin{array}{l}18.937 \\
18.402\end{array}$ & $\begin{array}{l}16.103 \\
15.795\end{array}$ & $\begin{array}{l}11.426 \\
11.567\end{array}$ & $\begin{array}{l}2.093 \\
2.038\end{array}$ & $\begin{array}{l}6.007 \\
5.978\end{array}$ & $\begin{array}{l}2.883 \\
2.524\end{array}$ & $\begin{array}{l}.089 \\
.077\end{array}$ & $\begin{array}{l}.017 \\
.014\end{array}$ & $\begin{array}{l}\mathbf{5 7 . 5 5 5} \\
\mathbf{5 6 . 3 9 4}\end{array}$ \\
\hline
\end{tabular}

a Includes lease condensate.

b Electric utility and industrial generation.

c "Other" production is electricity generated for distribution from wood, waste, wind, photovoltaic, and solar thermal energy.

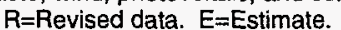

Notes: - See Note 1 at end of section. - Totals may not equal sum of components due to independent rounding. - Geographic coverage is the $\mathbf{5 0}$

States and the District of Columbia.

Sources: - Coal: Tables 6.1 and A5-A7. - Natural Gas (Dry): Tables 4.1 and A4. Crude Oil and Natural Gas Plant Liquids: Tables 3.1a and A2. - Nuclear Electric Power: Tables 7.1 and A8. Hydroelectric Power: Table 7.1; Section 2, "Energy Consumption Notes and Sources," Note 8; and Table A8. - Geothermal Energy and Other: Section 2, "Energy Consumption Notes and Sources," Note 7, and Table A8.

Please Read: Due to a lack of consistent monthly historical data, some renewable energy sources are not included in total production. In 1996, for example, 3.9 quadrillion Btu of renewable energy used by electric utilities to generate electricity for distribution is included, but an estimated 3.4 quadrillion Btu used by residential, commercial, and industrial consumers is not. See Note 12 at the end of Section 2 for details. 
Figure 1.3 Energy Consumption

(Quadrillion Btu)

Total, 1973-1996

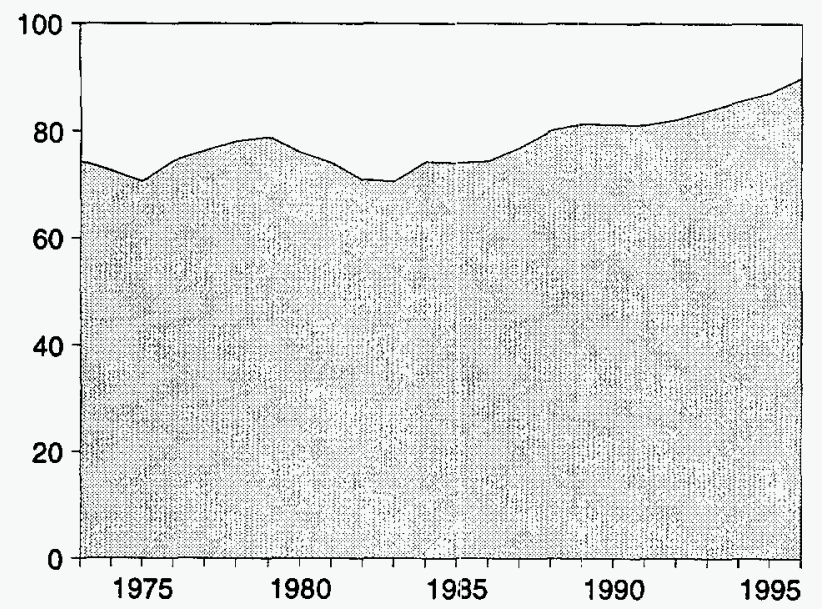

By Major Sources, 1973-1996

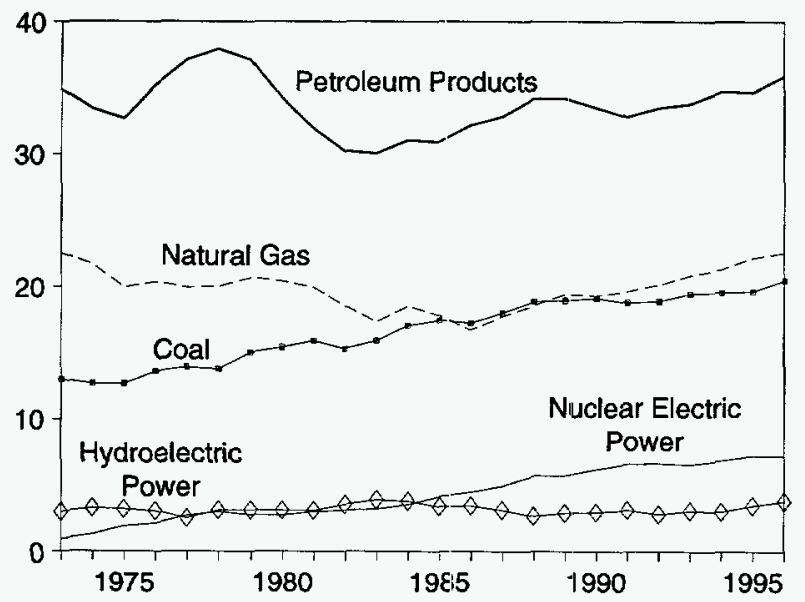

Total, January-October

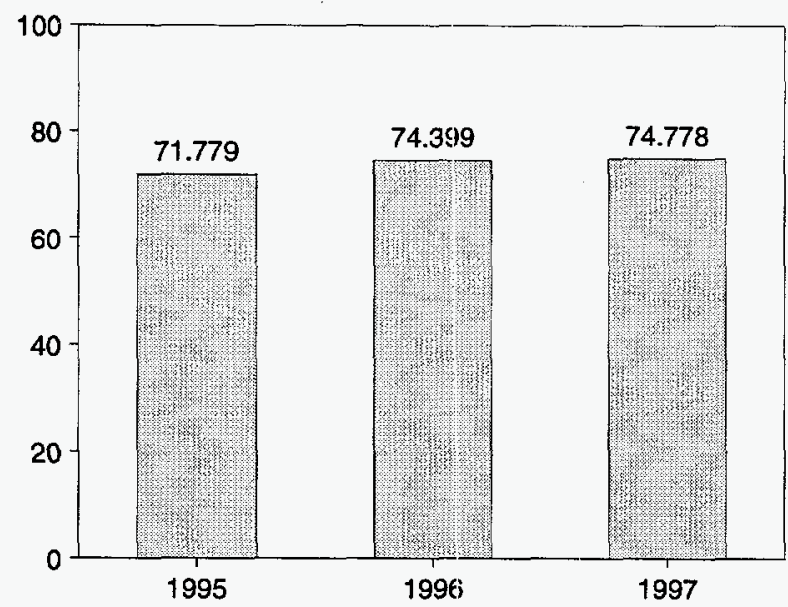

Total, Monthly

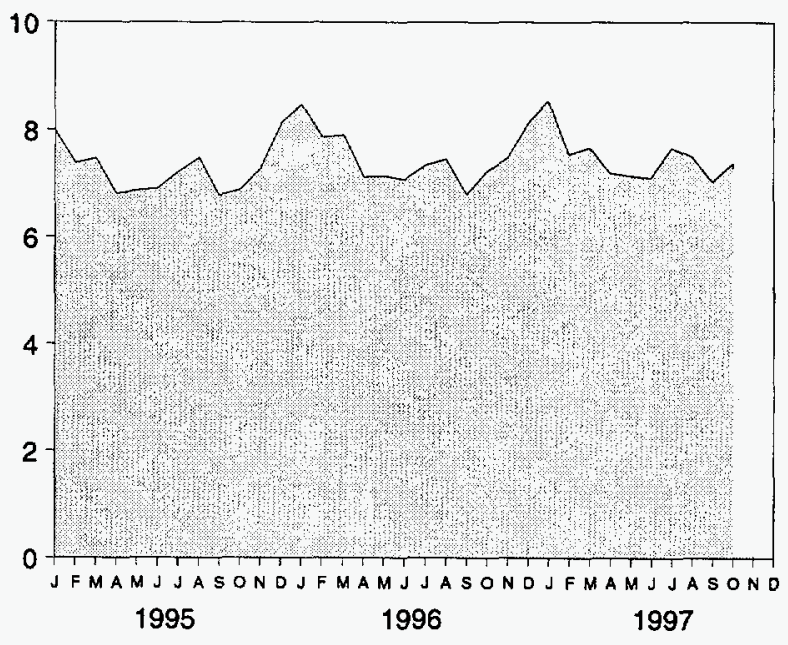

By Major Sources, Monthly

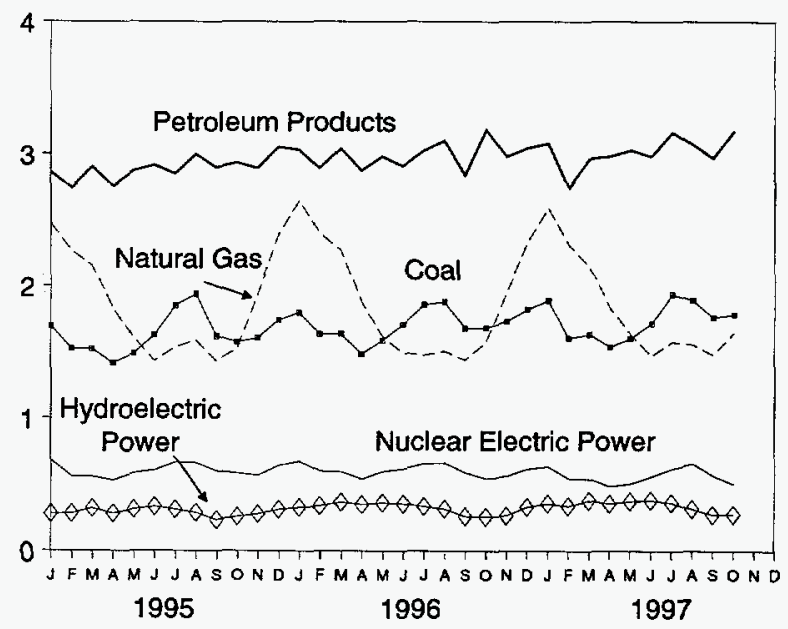

By Major Sources, October 1997

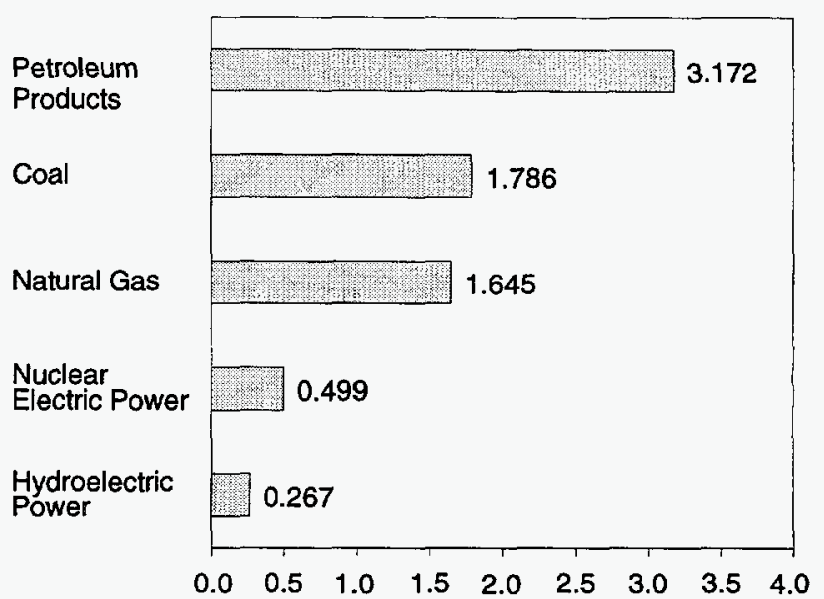

Note: Because vertical scales differ, graphs should not be compared. Source: Table 1.4. 
Table 1.4 Energy Consumption by Source (Quadrillion Btu)

\begin{tabular}{|c|c|c|c|c|c|c|c|c|}
\hline & Coal & $\begin{array}{l}\text { Natural } \\
\text { Gas }^{\mathrm{a}}\end{array}$ & $\begin{array}{l}\text { Petraleum } \\
\text { Products }\end{array}$ & $\begin{array}{l}\text { Nuclear } \\
\text { Electric } \\
\text { Power }\end{array}$ & $\begin{array}{l}\text { Hydro- } \\
\text { electric } \\
\text { Power }\end{array}$ & $\begin{array}{l}\text { Geothermal } \\
\text { Energy }\end{array}$ & Otherd & Total \\
\hline 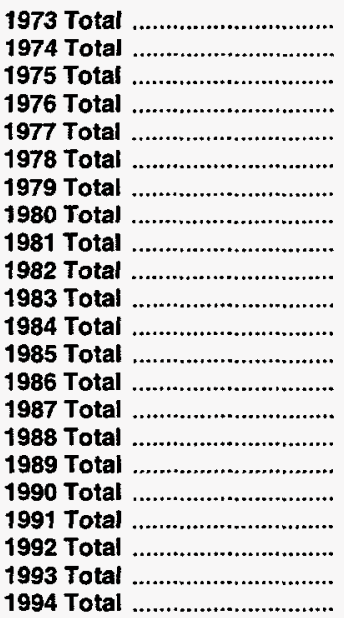 & $\begin{array}{l}12.971 \\
12.663 \\
12.663 \\
13.584 \\
13.922 \\
13.765 \\
15.039 \\
15.423 \\
15.907 \\
15.322 \\
15.894 \\
17.071 \\
17.478 \\
17.261 \\
18.008 \\
18.846 \\
18.925 \\
19.101 \\
18.770 \\
18.868 \\
19.430 \\
19.544\end{array}$ & $\begin{array}{l}22.512 \\
21.732 \\
19.948 \\
20.345 \\
19.931 \\
20.000 \\
20.666 \\
20.394 \\
19.928 \\
18.505 \\
17.357 \\
18.507 \\
17.834 \\
16.708 \\
17.744 \\
18.552 \\
19.384 \\
19.296 \\
19.606 \\
20.131 \\
20.827 \\
21.288\end{array}$ & $\begin{array}{l}\mathbf{3} 4.840 \\
\mathbf{3 3 . 4 5 5} \\
\mathbf{3 2 . 7 3 1} \\
\mathbf{3 5 . 1 7 5} \\
\mathbf{3 7 . 1 2 2} \\
\mathbf{3 7 . 9 6 5} \\
\mathbf{3 7 . 1 2 3} \\
\mathbf{3 4 . 2 0 2} \\
\mathbf{3 1 . 9 3 1} \\
\mathbf{3 0 . 2 3 1} \\
\mathbf{3 0 . 0 5 4} \\
\mathbf{3 1 . 0 5 1} \\
\mathbf{3 0 . 9 2 2} \\
\mathbf{3 2 . 1 9 6} \\
\mathbf{3 2 . 8 6 5} \\
\mathbf{3 4 . 2 2 2} \\
\mathbf{3 4 . 2 1 1} \\
\mathbf{3 3 . 5 5 3} \\
\mathbf{3 2 . 8 4 5} \\
\mathbf{3 3 . 5 2 7} \\
\mathbf{3 3 . 8 4 1} \\
\mathbf{3 4 . 7 3 5}\end{array}$ & $\begin{array}{l}0.910 \\
1.272 \\
1.900 \\
2.111 \\
2.702 \\
3.024 \\
2.776 \\
2.739 \\
3.008 \\
3.131 \\
3.203 \\
3.553 \\
4.149 \\
4.471 \\
4.906 \\
5.661 \\
5.677 \\
6.161 \\
6.579 \\
6.607 \\
6.519 \\
6.837\end{array}$ & $\begin{array}{l}3.010 \\
3.309 \\
3.219 \\
3.066 \\
2.515 \\
3.141 \\
3.141 \\
3.118 \\
3.105 \\
3.572 \\
3.899 \\
3.800 \\
3.398 \\
3.446 \\
3.117 \\
2.662 \\
2.881 \\
2.946 \\
3.115 \\
2.793 \\
3.050 \\
2.994\end{array}$ & $\begin{array}{l}0.043 \\
.053 \\
.070 \\
.078 \\
.077 \\
.064 \\
.084 \\
.110 \\
.123 \\
.105 \\
.129 \\
.165 \\
.198 \\
.219 \\
.229 \\
.217 \\
.197 \\
.181 \\
.170 \\
.169 \\
.158 \\
.145\end{array}$ & $\begin{array}{r}-0.004 \\
.059 \\
.016 \\
.003 \\
.020 \\
.128 \\
.068 \\
-.031 \\
-.012 \\
-.018 \\
-.012 \\
-.002 \\
.001 \\
-.004 \\
.024 \\
.057 \\
.051 \\
.026 \\
.030 \\
.049 \\
.038 \\
.044\end{array}$ & $\begin{array}{l}74.282 \\
72.543 \\
70.546 \\
74.362 \\
76.288 \\
78.089 \\
78.898 \\
75.955 \\
73.990 \\
70.848 \\
70.524 \\
74.144 \\
73.981 \\
74.297 \\
76.894 \\
80.218 \\
81.325 \\
81.265 \\
81.116 \\
82.144 \\
83.863 \\
85.587\end{array}$ \\
\hline 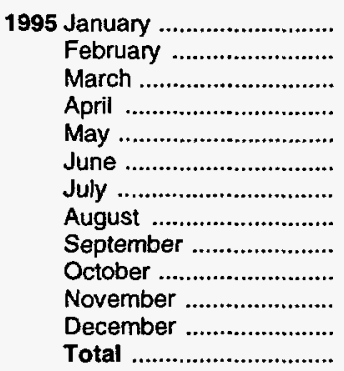 & $\begin{array}{r}1.693 \\
1.527 \\
1.525 \\
1.417 \\
1.489 \\
1.626 \\
1.851 \\
1.936 \\
1.619 \\
1.577 \\
1.604 \\
1.743 \\
19.608\end{array}$ & $\begin{array}{r}2.467 \\
2.267 \\
2.155 \\
1.828 \\
1.609 \\
1.433 \\
1.537 \\
1.590 \\
1.431 \\
1.526 \\
1.937 \\
2.384 \\
22.163\end{array}$ & $\begin{array}{r}2.860 \\
2.742 \\
2.904 \\
2.755 \\
2.872 \\
2.914 \\
2.848 \\
2.997 \\
2.897 \\
2.932 \\
2.890 \\
3.051 \\
34.663\end{array}$ & $\begin{array}{l}.675 \\
.553 \\
.553 \\
.526 \\
.580 \\
.601 \\
.661 \\
.657 \\
.594 \\
.579 \\
.562 \\
.638 \\
7.177\end{array}$ & $\begin{array}{r}\text { R. } .274 \\
\text { R. } 278 \\
R .318 \\
\text { R. } .278 \\
.309 \\
.330 \\
.309 \\
\text { R. } 284 \\
.228 \\
\text { R. } .253 \\
.273 \\
\text { R. } .309 \\
\text { R.445 }\end{array}$ & $\begin{array}{l}.009 \\
.006 \\
.007 \\
.006 \\
.005 \\
.006 \\
.006 \\
.011 \\
.008 \\
.013 \\
.012 \\
.011 \\
.099\end{array}$ & $\begin{array}{l}.005 \\
.003 \\
.004 \\
.003 \\
.006 \\
.002 \\
.003 \\
.003 \\
.004 \\
.004 \\
.004 \\
.003 \\
.044\end{array}$ & $\begin{array}{r}R_{7.983} \\
R_{7.376} \\
R_{7.467} \\
R_{6.813} \\
6.871 \\
6.912 \\
7.216 \\
R_{7.478} \\
6.780 \\
R_{6.884} \\
R_{7.281} \\
R_{8.139} \\
R_{87.199}\end{array}$ \\
\hline 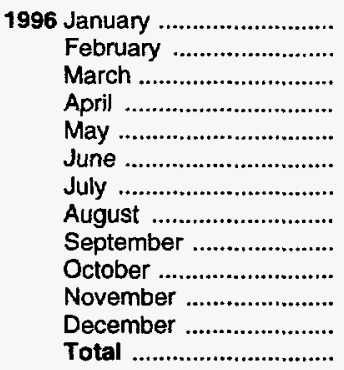 & $\begin{array}{r}1.801 \\
1.634 \\
1.636 \\
1.484 \\
1.587 \\
1.706 \\
1.856 \\
1.876 \\
1.677 \\
1.680 \\
1.728 \\
1.822 \\
20.486\end{array}$ & $\begin{array}{r}2.643 \\
2.398 \\
2.269 \\
1.875 \\
1.619 \\
1.493 \\
1.474 \\
1.504 \\
1.437 \\
1.572 \\
1.948 \\
2.327 \\
22.560\end{array}$ & $\begin{array}{r}3.030 \\
2.890 \\
3.036 \\
2.872 \\
2.979 \\
2.907 \\
3.021 \\
3.096 \\
2.835 \\
3.181 \\
2.976 \\
3.042 \\
\mathbf{3 5 . 8 6 4}\end{array}$ & $\begin{array}{r}.669 \\
.594 \\
.589 \\
.535 \\
.591 \\
.611 \\
.648 \\
.653 \\
.580 \\
.538 \\
.554 \\
.607 \\
7.168\end{array}$ & $\begin{array}{l}.324 \\
.335 \\
.364 \\
.346 \\
.359 \\
.351 \\
.330 \\
.310 \\
.252 \\
.249 \\
.256 \\
.323 \\
3.798\end{array}$ & $\begin{array}{l}.007 \\
.008 \\
.007 \\
.008 \\
.005 \\
.008 \\
.012 \\
.012 \\
.010 \\
.011 \\
.011 \\
.010 \\
.110\end{array}$ & $\begin{array}{r}.003 \\
.004 \\
.005 \\
.000 \\
.001 \\
-.001 \\
.002 \\
-.001 \\
.002 \\
.002 \\
.002 \\
.001 \\
.020\end{array}$ & $\begin{array}{r}8.476 \\
7.863 \\
7.905 \\
7.120 \\
7.141 \\
7.075 \\
7.344 \\
7.450 \\
6.793 \\
7.233 \\
7.474 \\
8.132 \\
90.005\end{array}$ \\
\hline 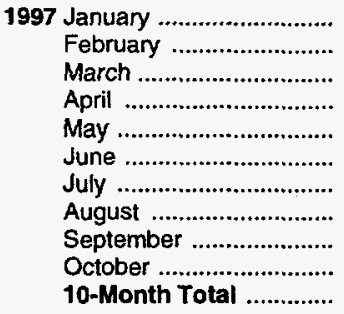 & $\begin{array}{r}1.888 \\
1.605 \\
1.633 \\
1.539 \\
1.603 \\
1.716 \\
R 1.937 \\
R 1.894 \\
R 1.762 \\
E_{1.786} \\
E_{17.364}\end{array}$ & $\begin{array}{r}2.587 \\
R_{2} .314 \\
R_{2} 2.148 \\
R_{1} 1.832 \\
R_{1} 1.635 \\
R_{1} 1.470 \\
R_{1} 1.578 \\
R_{1} 1.557 \\
R_{1} 1.478 \\
F_{1.645} \\
E_{1} 18.243\end{array}$ & $\begin{array}{r}3.079 \\
2.744 \\
2.965 \\
2.982 \\
3.027 \\
2.981 \\
3.163 \\
3.070 \\
2.967 \\
3.172 \\
30.150\end{array}$ & $\begin{array}{r}.626 \\
.538 \\
.536 \\
.481 \\
.500 \\
.553 \\
.609 \\
.649 \\
.559 \\
.499 \\
\mathbf{5 . 5 5 0}\end{array}$ & $\begin{array}{l}.351 \\
.331 \\
.374 \\
.349 \\
.367 \\
.375 \\
.358 \\
.308 \\
.263 \\
.267 \\
3.344\end{array}$ & $\begin{array}{l}.009 \\
.006 \\
.009 \\
.010 \\
.010 \\
.008 \\
.011 \\
.011 \\
.010 \\
.010 \\
.094\end{array}$ & $\begin{array}{l}.003 \\
.003 \\
.003 \\
.002 \\
.004 \\
.003 \\
.003 \\
.009 \\
-.001 \\
.004 \\
.033\end{array}$ & $\begin{array}{l}R_{8.544} \\
R_{7.541} \\
R_{7.668} \\
R_{7.196} \\
R_{7.145} \\
R_{7.105} \\
R_{7.660} \\
R_{7.498} \\
R_{7.037} \\
7.383 \\
74.778\end{array}$ \\
\hline $\begin{array}{l}1996 \text { 10-Month Total .............. } \\
1995 \text { 10-Month Total .................. }\end{array}$ & $\begin{array}{l}16.936 \\
16.260\end{array}$ & $\begin{array}{l}18.285 \\
17.843\end{array}$ & $\begin{array}{l}29.846 \\
28.723\end{array}$ & $\begin{array}{l}6.007 \\
5.978\end{array}$ & $\begin{array}{l}3.219 \\
2.863\end{array}$ & $\begin{array}{l}.089 \\
.077\end{array}$ & $\begin{array}{l}.017 \\
.037\end{array}$ & $\begin{array}{l}74.399 \\
71.779\end{array}$ \\
\hline
\end{tabular}

a Includes supplemental gaseous fuels.

b Products obtained from the processing of crude oil (including lease condensate), natural gas, and other hydrocarbon compounds.

c Electric utility and industrial generation and net imports of electricity.

d Net imports of coal coke and electricity generated for distribution from wood, waste, wind, photovoltaic, and solar thermal energy.

$R=$ Revised data. E=Estimate. F=Forecast.

Notes: - See Note 2 at end of section. - Totals may not equal sum of components due to independent rounding. - Geographic coverage is the $\mathbf{5 0}$ States and the District of Columbia.

Sources: - Coal: Tables 6.1 and A5-A7. - Natural Gas: Tables 4.2 and A4. - Petroleum: Tables 3.1a and A3. - Nuclear Electric Power:

Tables 7.1 and A8. - Hydroelectric Power: Table 7.1: Section 2, "Energy Consumption Notes and Sources," Note 8; and Table A8. - Geothermal Energy and Other: Section 2, "Energy Consumption Notes and Sources," Note 7 , and Table A8.

Please Read: Due to a lack of consistent monthly historical data, some renewable energy sources are not included in total consumption. In 1996, for example, 3.9 quadrillion Btu of renewable energy used by electric utilities to generate electricity for distribution is included, but an estimated 3.4 quadrillion Btu used by residential, commercial, and industrial consumers is not. See Note 12 at the end of Section 2 for details. 
Figure 1.4 Energy Net Imports

(Quadrillion Btu, Except as Noted)

Total, 1973-1996

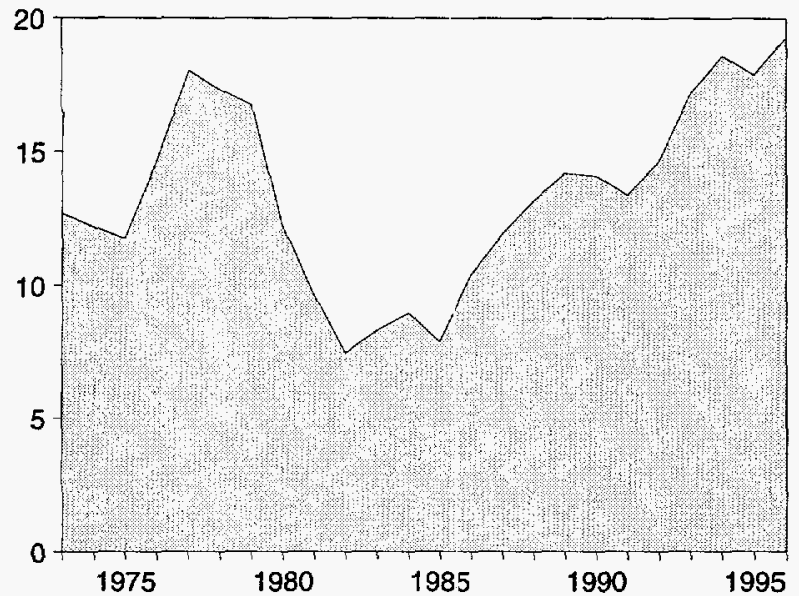

By Major Sources, 1973-1996

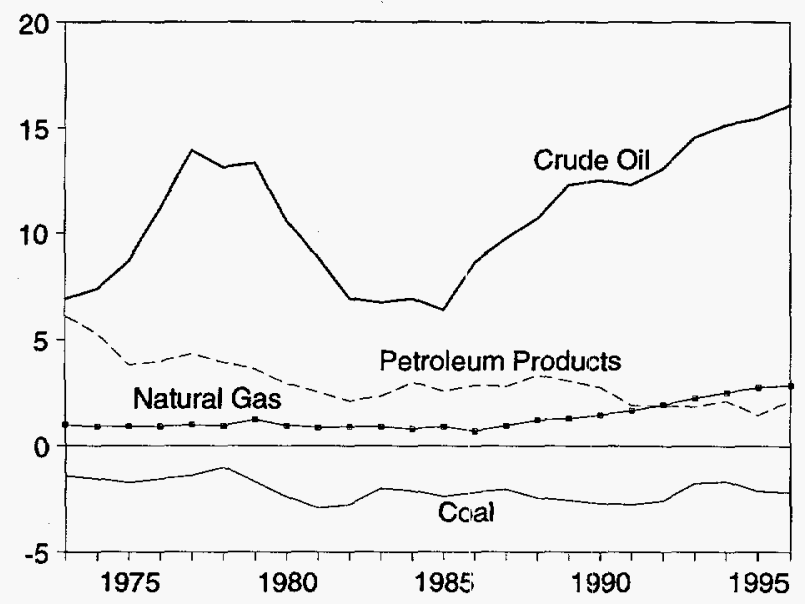

By Major Sources, October 1997

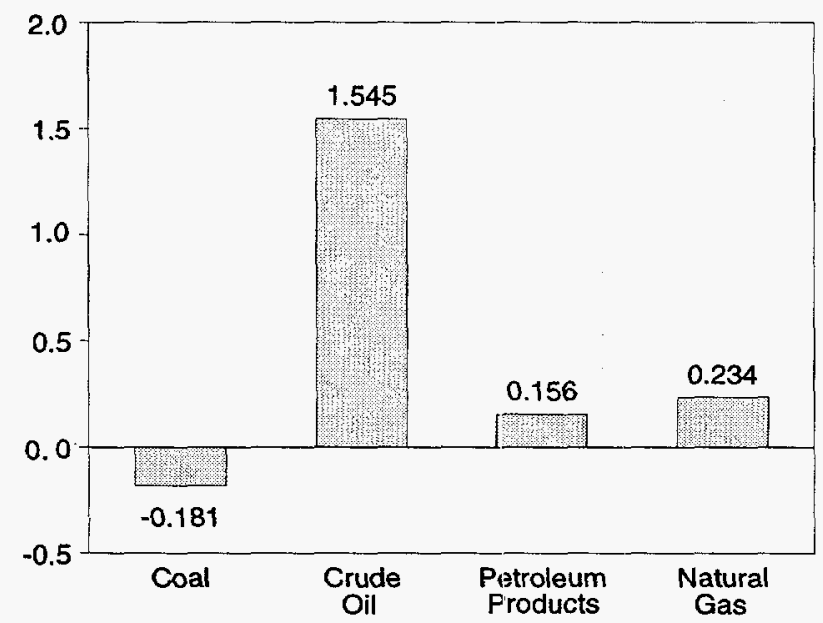

Total, Monthly

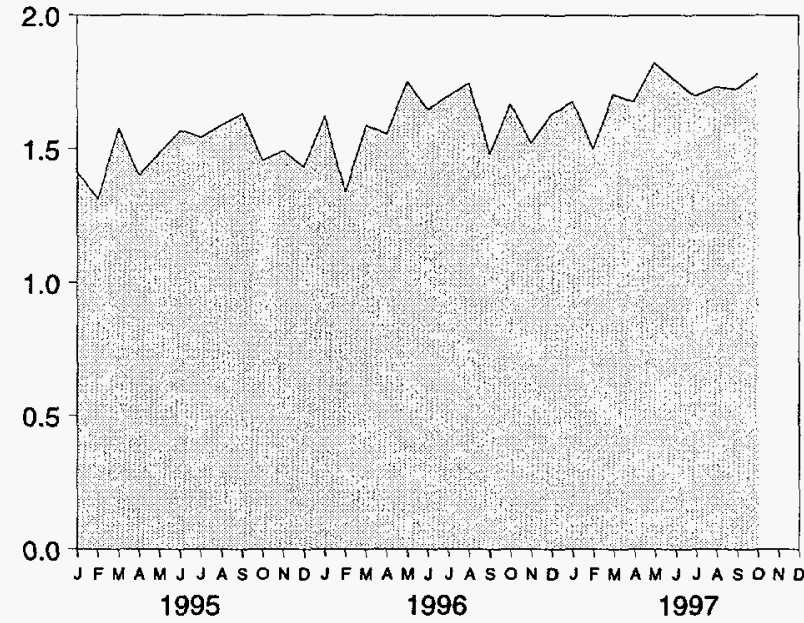

By Major Sources, Monthly

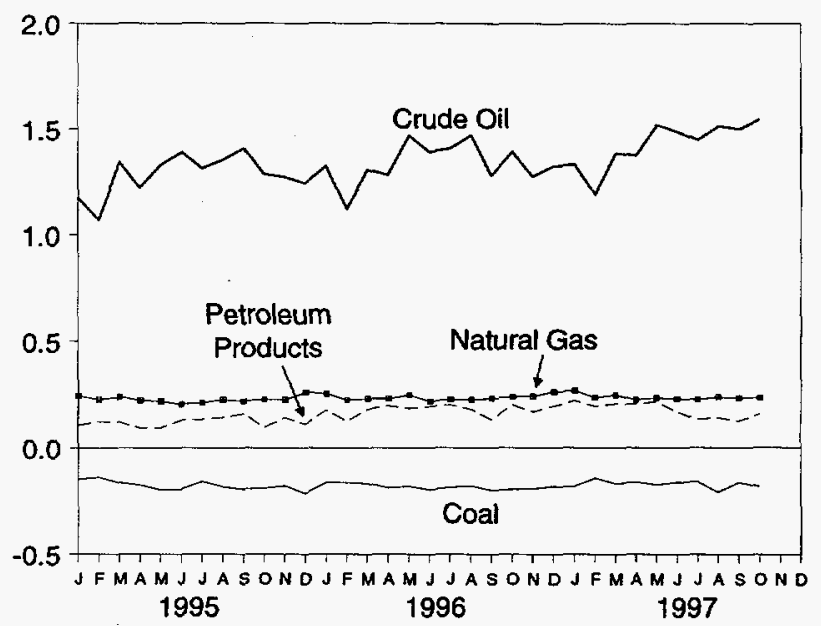

As Share of Consumption, January-October

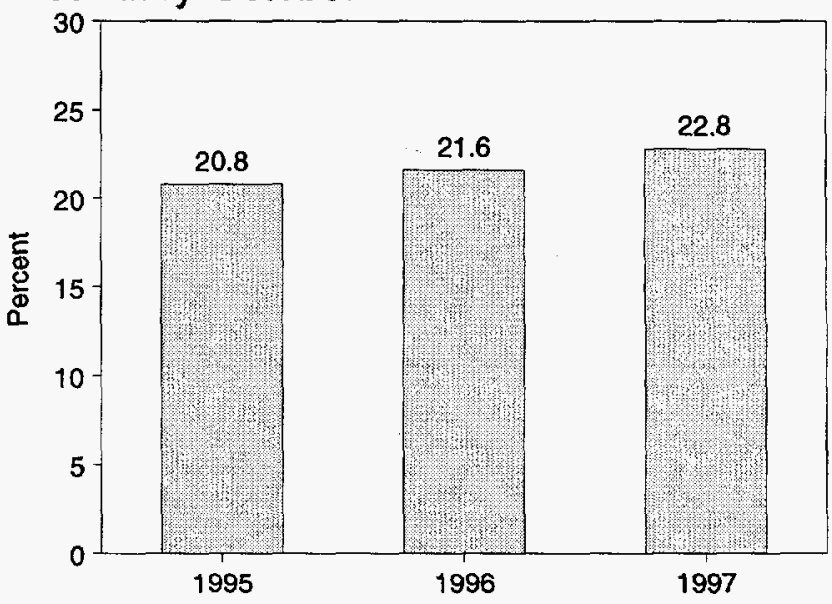

Note: Because vertical scales differ, graphs should not be compared. Sources: Tables 1.4 and 1.5. 


\begin{tabular}{|c|c|c|c|c|c|c|c|}
\hline & Coal & $\begin{array}{c}\text { Natural } \\
\text { Gas }\end{array}$ & $\begin{array}{c}\text { Crude } \\
\text { Oila }\end{array}$ & $\begin{array}{l}\text { Petroleum } \\
\text { Products }^{b}\end{array}$ & Electricityc & $\begin{array}{l}\text { Coal } \\
\text { Coke }\end{array}$ & Total \\
\hline $\begin{array}{l}1973 \text { Total } \\
1974 \text { Total } \\
1975 \text { Total } \\
1976 \text { Total } \\
1977 \text { Total } \\
1978 \text { Total } \\
1979 \text { Total } \\
1980 \text { Total } \\
1981 \text { Total } \\
1982 \text { Total } \\
1983 \text { Total } \\
1984 \text { Total } \\
1985 \text { Total } \\
1986 \text { Total } \\
1987 \text { Total } \\
1988 \text { Total } \\
1989 \text { Total } \\
1990 \text { Total } \\
1991 \text { Total } \\
1992 \text { Total } \\
1993 \text { Total } \\
1994 \text { Total }\end{array}$ & $\begin{array}{l}-1.422 \\
-1.568 \\
-1.738 \\
-1.567 \\
-1.401 \\
-1.004 \\
-1.702 \\
-2.391 \\
-2.918 \\
-2.768 \\
-2.013 \\
-2.119 \\
-2.389 \\
-2.193 \\
-2.049 \\
-2.446 \\
-2.566 \\
-2.705 \\
-2.769 \\
-2.587 \\
-1.780 \\
-1.689\end{array}$ & $\begin{array}{r}0.981 \\
.907 \\
.904 \\
.922 \\
.981 \\
.941 \\
1.243 \\
.957 \\
.857 \\
.898 \\
.885 \\
.792 \\
.896 \\
.686 \\
.937 \\
1.221 \\
1.278 \\
1.464 \\
1.666 \\
1.941 \\
2.255 \\
2.518\end{array}$ & $\begin{array}{r}6.883 \\
7.389 \\
8.708 \\
11.221 \\
13.921 \\
13.125 \\
13.328 \\
10.586 \\
8.854 \\
6.917 \\
6.731 \\
6.918 \\
6.381 \\
8.676 \\
9.748 \\
10.698 \\
12.296 \\
12.536 \\
12.308 \\
13.065 \\
14.542 \\
15.131\end{array}$ & $\begin{array}{l}6.097 \\
5.273 \\
3.800 \\
3.982 \\
4.321 \\
3.932 \\
3.603 \\
2.912 \\
2.522 \\
2.128 \\
2.351 \\
2.970 \\
2.570 \\
2.855 \\
2.784 \\
3.308 \\
3.029 \\
2.757 \\
1.912 \\
1.895 \\
1.854 \\
2.128\end{array}$ & $\begin{array}{l}0.148 \\
.133 \\
.064 \\
.089 \\
.182 \\
.204 \\
.211 \\
.217 \\
.347 \\
.306 \\
.372 \\
.414 \\
.428 \\
.375 \\
.483 \\
.328 \\
.113 \\
.020 \\
.231 \\
.292 \\
.292 \\
.459\end{array}$ & $\begin{array}{r}-0.007 \\
.056 \\
.014 \\
(\mathrm{~s}) \\
.015 \\
.125 \\
.063 \\
-.035 \\
-.016 \\
-.022 \\
-.016 \\
-.011 \\
-.013 \\
-.017 \\
.009 \\
.040 \\
.030 \\
.005 \\
.009 \\
.027 \\
.017 \\
.024\end{array}$ & $\begin{array}{r}12.680 \\
12.190 \\
11.752 \\
14.648 \\
18.019 \\
17.323 \\
16.746 \\
12.247 \\
9.646 \\
7.460 \\
8.310 \\
8.963 \\
7.872 \\
10.382 \\
11.911 \\
13.149 \\
14.181 \\
14.077 \\
13.357 \\
14.633 \\
17.180 \\
18.570\end{array}$ \\
\hline 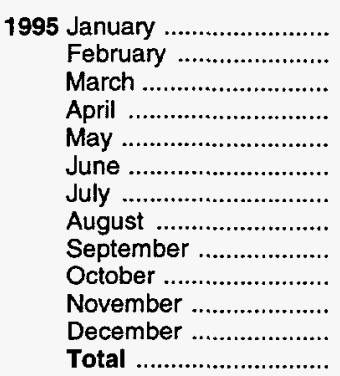 & $\begin{array}{l}-.149 \\
-.139 \\
-.165 \\
-.176 \\
-.197 \\
-.194 \\
-.159 \\
-.183 \\
-.194 \\
-.190 \\
-.178 \\
-.214 \\
-2.138\end{array}$ & $\begin{array}{l}.245 \\
.228 \\
.241 \\
.224 \\
.220 \\
.206 \\
.213 \\
.228 \\
.221 \\
.229 \\
.228 \\
.262 \\
2.745\end{array}$ & $\begin{array}{r}1.174 \\
1.070 \\
1.345 \\
1.224 \\
1.332 \\
1.391 \\
1.316 \\
1.355 \\
1.410 \\
1.290 \\
1.277 \\
1.247 \\
15.432\end{array}$ & $\begin{array}{l}.104 \\
.122 \\
.119 \\
.091 \\
.093 \\
.129 \\
.132 \\
.142 \\
.160 \\
.094 \\
.141 \\
.110 \\
1.437\end{array}$ & $\begin{array}{l}\mathrm{R} .031 \\
\mathrm{R} .029 \\
\mathrm{R} .033 \\
\mathrm{R} .033 \\
.032 \\
.034 \\
.039 \\
\mathrm{R} .045 \\
.032 \\
\mathrm{R} .030 \\
\mathrm{R} .023 \\
\mathrm{R} .025 \\
\mathrm{R} .387\end{array}$ & $\begin{array}{l}.004 \\
.002 \\
.003 \\
.001 \\
.004 \\
.001 \\
.002 \\
.001 \\
.002 \\
.003 \\
.002 \\
.002 \\
.026\end{array}$ & $\begin{array}{r}R_{1} .409 \\
R_{1} .312 \\
R_{1} .576 \\
R_{1} .398 \\
1.485 \\
R_{1.567} \\
1.542 \\
R_{1} 1.588 \\
R_{1} 1.630 \\
R_{1.456} \\
1.494 \\
R_{1.432} \\
R_{1} 1.889\end{array}$ \\
\hline 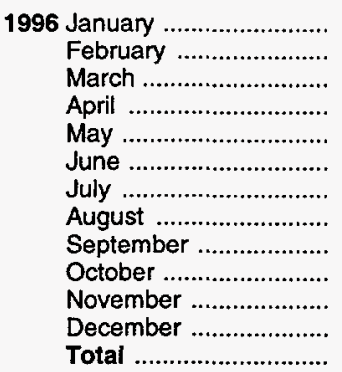 & $\begin{array}{l}-.163 \\
-.163 \\
-.168 \\
-.188 \\
-.181 \\
-.196 \\
-.186 \\
-.179 \\
-.199 \\
-.195 \\
-.192 \\
-.181 \\
-2.190\end{array}$ & $\begin{array}{l}.255 \\
.226 \\
.232 \\
.232 \\
.249 \\
.219 \\
.228 \\
.226 \\
.232 \\
.241 \\
.243 \\
.264 \\
2.847\end{array}$ & $\begin{array}{r}1.328 \\
1.123 \\
1.311 \\
1.287 \\
1.471 \\
1.394 \\
1.410 \\
1.472 \\
1.284 \\
1.393 \\
1.278 \\
1.327 \\
16.075\end{array}$ & $\begin{array}{l}.177 \\
.124 \\
.182 \\
.197 \\
.185 \\
.195 \\
.201 \\
.180 \\
.130 \\
.202 \\
.167 \\
.196 \\
2.135\end{array}$ & $\begin{array}{l}.024 \\
.025 \\
.029 \\
.029 \\
.030 \\
.037 \\
.046 \\
.052 \\
.036 \\
.029 \\
.027 \\
.024 \\
.387\end{array}$ & $\begin{array}{r}.001 \\
.003 \\
.003 \\
-.001 \\
-.001 \\
-.002 \\
(s) \\
-.003 \\
(s) \\
(s) \\
(s) \\
-.001 \\
(s)\end{array}$ & $\begin{array}{r}1.621 \\
1.338 \\
1.588 \\
1.556 \\
1.753 \\
1.647 \\
1.698 \\
1.747 \\
1.484 \\
1.668 \\
1.523 \\
1.630 \\
19.253\end{array}$ \\
\hline 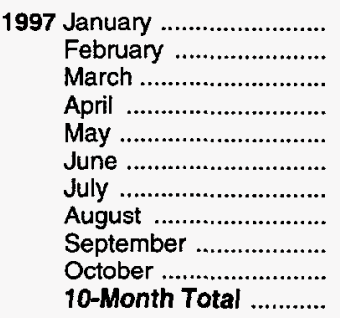 & $\begin{array}{l}-.181 \\
-.143 \\
-.167 \\
-.161 \\
-.174 \\
-.162 \\
-.159 \\
-.208 \\
-.163 \\
-.181 \\
-1.698\end{array}$ & $\begin{array}{r}E .270 \\
E .236 \\
E .249 \\
E .226 \\
\text { E. } 235 \\
E .231 \\
.227 \\
\text { RE .238 } \\
\text { RE .234 } \\
\text { E. } 234 \\
\text { E } 2.380\end{array}$ & $\begin{array}{r}1.335 \\
1.190 \\
1.386 \\
1.375 \\
1.519 \\
1.486 \\
1.448 \\
1.513 \\
1.499 \\
1.545 \\
14.294\end{array}$ & $\begin{array}{l}.222 \\
.195 \\
.207 \\
.205 \\
.217 \\
.168 \\
.133 \\
.139 \\
.124 \\
.156 \\
1.766\end{array}$ & $\begin{array}{l}\text { E.028 } \\
\text { E.021 } \\
\text { E.028 } \\
\text { E.032 } \\
\text { E.026 } \\
\text { E.034 } \\
\text { E.046 } \\
\text { E.043 } \\
\text { E.033 } \\
\text { E.025 } \\
\text { E.316 }\end{array}$ & $\begin{array}{r}.002 \\
.002 \\
.002 \\
(s) \\
.002 \\
.001 \\
.002 \\
.007 \\
-.003 \\
.002 \\
.016\end{array}$ & $\begin{array}{r}1.676 \\
1.501 \\
1.705 \\
1.676 \\
1.824 \\
1.758 \\
1.697 \\
R 1.732 \\
R 1.724 \\
1.781 \\
17.075\end{array}$ \\
\hline $\begin{array}{l}1996 \text { 10-Month Total ........... } \\
1995 \text { 10-Month Total ........... }\end{array}$ & $\begin{array}{l}-1.818 \\
-1.746\end{array}$ & $\begin{array}{l}2.339 \\
2.255\end{array}$ & $\begin{array}{l}13.470 \\
12.908\end{array}$ & $\begin{array}{l}1.772 \\
1.186\end{array}$ & $\begin{array}{l}.335 \\
.339\end{array}$ & $\begin{array}{r}(s) \\
.022\end{array}$ & $\begin{array}{l}16.100 \\
14.964\end{array}$ \\
\hline
\end{tabular}

a Crude oil, lease condensate, and imports of crude oil for the Strategic Petroleum Reserve.

b Petroleum products, unfinished oils, pentanes plus, and gasoline blending components

c Assumed to be hydroelectricity and estimated at the average input heat rate for fossil-fuel steam-electric power plant generation, which has ranged from 10.2 thousand Btu to 10.5 thousand Btu per kilowatthour since 1973. Actual heat rates applied in converting kilowatthours to Btu are listed by year in Table A8.

$R=$ Revised data. $E=E s t i m a t e .(s)=L e s s$ than +0.5 trillion Btu and greater than -0.5 trillion Btu.

Notes: - See Notes 3 and 4 at end of section. - Net imports equal imports minus exports. Minus sign indicates exports are greater than imports. - Totals may not equal sum of components due to independent rounding. - Geographic coverage is the $\mathbf{5 0}$ States and the District of Columbia.

Sources: - Coal: Tables 6.1 and A5-A7. - Natural Gas: Tables 4.2 and A4. - Crude Oil and Petroleum Products: Tables 3.1b and A2. - Electricity: Section 2, "Energy Consumption Notes and Sources," Note 8, and Table A8. - Coal Coke: Section 2, "Energy Consumption Notes and Sources," Note 9, and Table A7. 
Figure 1.5 Merchandise Trade Value

(Billion Dollars)

Imports and Exports, 1974-1996

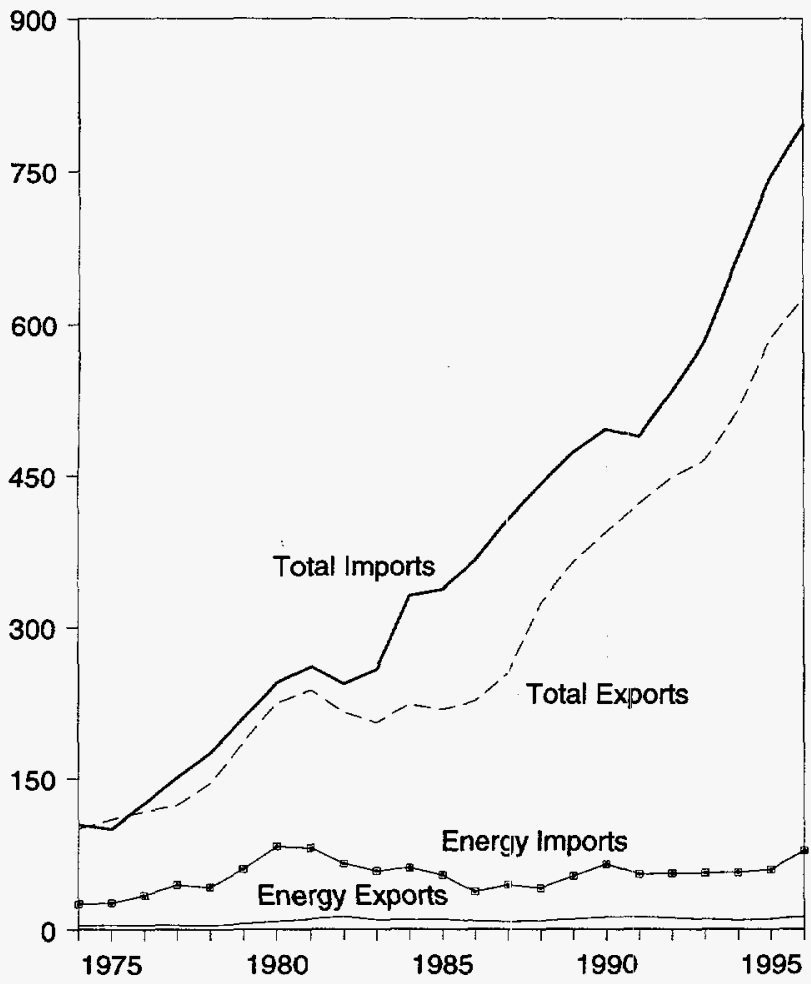

Trade Balance, 1974-1996

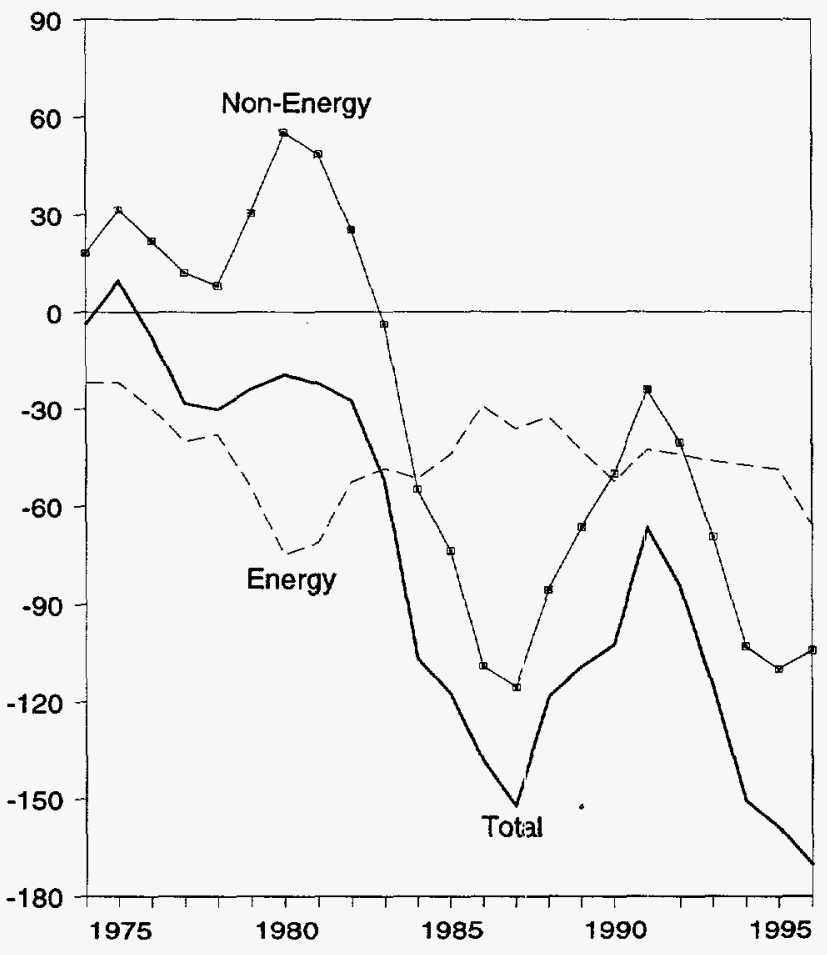

Imports and Exports, Monthly

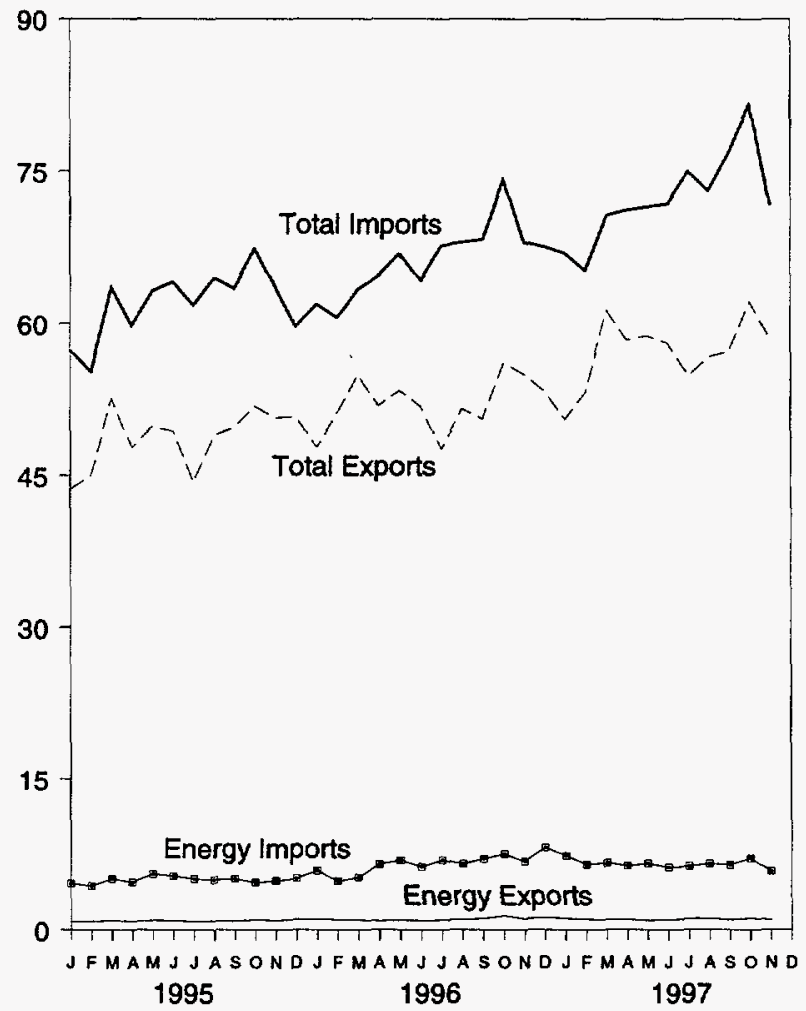

Trade Balance, Monthly

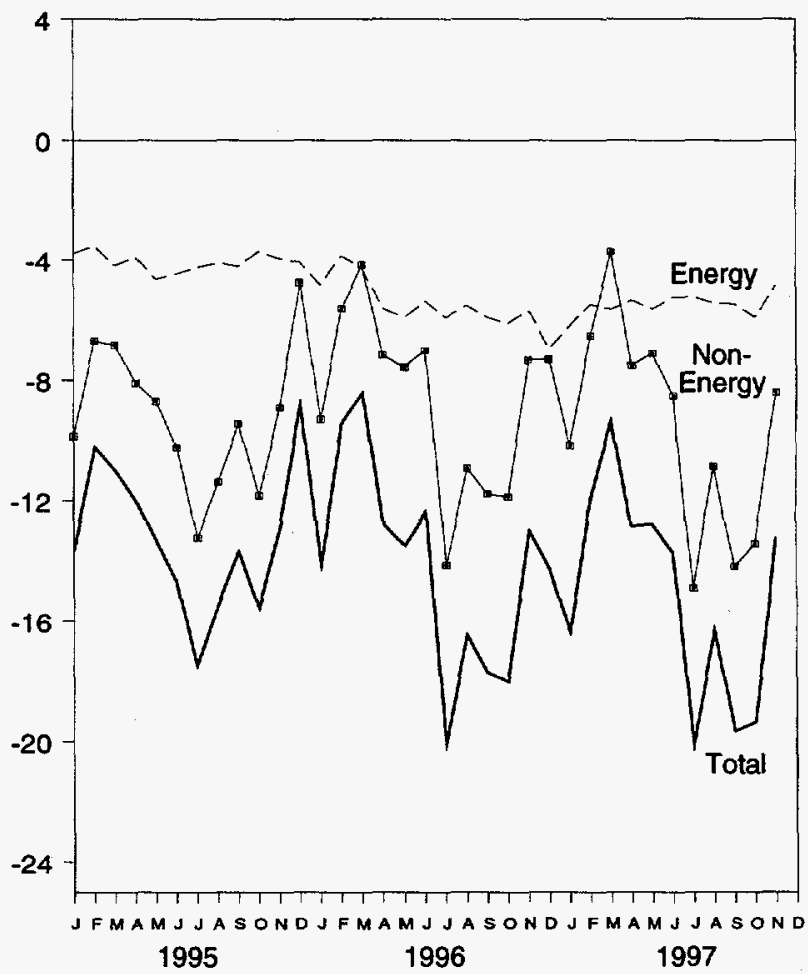

Note: Because vertical scales differ, graphs should not be compared.

Source: Table 1.6 


\begin{tabular}{|c|c|c|c|c|c|c|c|c|c|c|}
\hline & \multicolumn{3}{|c|}{ Petroleuma } & \multicolumn{3}{|c|}{ Energy $b$} & \multirow{2}{*}{$\begin{array}{c}\text { Non- } \\
\text { Energy } \\
\text { Balance }\end{array}$} & \multicolumn{3}{|c|}{ Total Merchandise } \\
\hline & Exports & Imports & Balance & Exports & Imports & Balance & & Exports & Imports & Balance \\
\hline 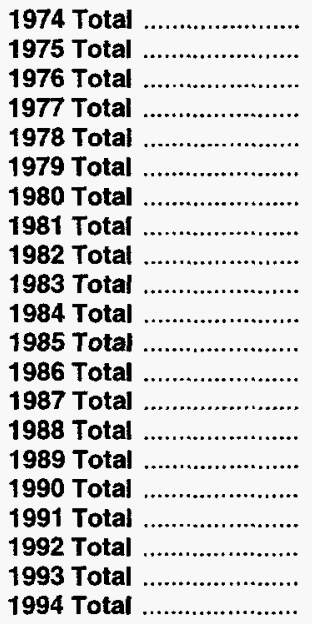 & $\begin{array}{r}792 \\
907 \\
998 \\
1,276 \\
1,561 \\
1,914 \\
2,833 \\
3,696 \\
5,947 \\
4,557 \\
4,470 \\
4,707 \\
3,640 \\
3,922 \\
3,693 \\
5,021 \\
6,901 \\
6,954 \\
6,412 \\
6,215 \\
5,659\end{array}$ & $\begin{array}{l}24,668 \\
25,197 \\
32,226 \\
42,368 \\
39,526 \\
56,715 \\
78,637 \\
76,659 \\
60,458 \\
53,217 \\
56,924 \\
50,475 \\
35,142 \\
42,285 \\
38,787 \\
49,704 \\
61,583 \\
51,350 \\
51,217 \\
51,046 \\
50,835\end{array}$ & $\begin{array}{l}-23,876 \\
-24,289 \\
-31,228 \\
-41,093 \\
-37,965 \\
-54,801 \\
-75,803 \\
-72,963 \\
-54,511 \\
-48,659 \\
-52,454 \\
-45,768 \\
-31,503 \\
-38,363 \\
-35,094 \\
-44,683 \\
-54,682 \\
-44,396 \\
-44,805 \\
-44,831 \\
-45,176\end{array}$ & $\begin{array}{r}3,444 \\
4,470 \\
4,226 \\
4,184 \\
3,881 \\
5,621 \\
7,982 \\
10,279 \\
12,729 \\
9,500 \\
9,311 \\
9,971 \\
8,115 \\
7,713 \\
8,235 \\
9,869 \\
12,233 \\
12,081 \\
11,254 \\
9,756 \\
8,911\end{array}$ & $\begin{array}{l}25,454 \\
26,476 \\
33,996 \\
44,537 \\
42,096 \\
59,998 \\
82,924 \\
81,360 \\
65,409 \\
57,952 \\
60,980 \\
53,917 \\
37,310 \\
44,220 \\
41,042 \\
52,779 \\
64,661 \\
54,629 \\
55,256 \\
55,900 \\
56,391\end{array}$ & $\begin{array}{l}-22,010 \\
-22,006 \\
-29,770 \\
-40,354 \\
-38,215 \\
-54,377 \\
-74,942 \\
-71,081 \\
-52,680 \\
-48,452 \\
-51,669 \\
-43,946 \\
-29,195 \\
-36,506 \\
-32,806 \\
-42,910 \\
-52,428 \\
-42,548 \\
-44,002 \\
-46,144 \\
-47,480\end{array}$ & $\begin{array}{r}18,126 \\
31,557 \\
21,950 \\
12,001 \\
8,010 \\
30,455 \\
55,246 \\
48,814 \\
25,170 \\
-3,957 \\
-55,033 \\
-73,765 \\
-109,084 \\
-115,613 \\
-85,720 \\
-66,490 \\
-50,068 \\
-24,175 \\
-40,500 \\
-69,425 \\
-103,149\end{array}$ & $\begin{array}{r}99,437 \\
108,856 \\
116,794 \\
123,182 \\
145,847 \\
186,363 \\
225,566 \\
238,715 \\
216,442 \\
205,639 \\
223,976 \\
218,815 \\
227,159 \\
254,122 \\
322,426 \\
363,812 \\
393,592 \\
421,730 \\
448,164 \\
465,091 \\
512,626\end{array}$ & $\begin{array}{r}103,321 \\
99,305 \\
124,614 \\
151,534 \\
176,052 \\
210,285 \\
245,262 \\
260,982 \\
243,952 \\
258,048 \\
330,678 \\
336,526 \\
365,438 \\
406,241 \\
440,952 \\
473,211 \\
496,088 \\
488,453 \\
532,665 \\
580,659 \\
663,256\end{array}$ & $\begin{array}{r}-3,884 \\
9,551 \\
-7,820 \\
-28,353 \\
-30,205 \\
-23,922 \\
-19,696 \\
-22,267 \\
-27,510 \\
-52,409 \\
-106,703 \\
-117,712 \\
-138,279 \\
-152,119 \\
-118,526 \\
-109,399 \\
-102,496 \\
-66,723 \\
-84,501 \\
-115,568 \\
-150,629\end{array}$ \\
\hline 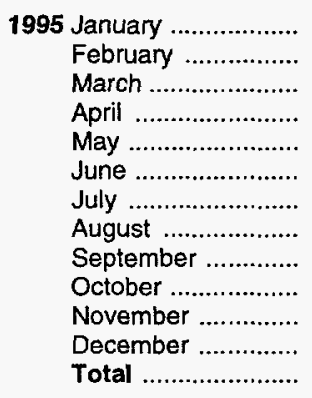 & $\begin{array}{r}491 \\
528 \\
552 \\
504 \\
538 \\
508 \\
476 \\
469 \\
444 \\
587 \\
529 \\
696 \\
6,321\end{array}$ & $\begin{array}{r}4,148 \\
3,948 \\
4,654 \\
4,344 \\
5,115 \\
4,955 \\
4,687 \\
4,567 \\
4,648 \\
4,278 \\
4,423 \\
4,601 \\
54,368\end{array}$ & $\begin{array}{r}-3,657 \\
-3,420 \\
-4,102 \\
-3,840 \\
-4,577 \\
-4,447 \\
-4,211 \\
-4,098 \\
-4,204 \\
-3,691 \\
-3,894 \\
-3,905 \\
-48,047\end{array}$ & $\begin{array}{r}792 \\
793 \\
882 \\
818 \\
883 \\
865 \\
815 \\
844 \\
820 \\
954 \\
883 \\
1,011 \\
10,358\end{array}$ & $\begin{array}{r}4,572 \\
4,321 \\
5,064 \\
4,715 \\
5,511 \\
5,325 \\
5,053 \\
4,933 \\
5,031 \\
4,665 \\
4,830 \\
5,089 \\
59,109\end{array}$ & $\begin{array}{r}-3,780 \\
-3,528 \\
-4,182 \\
-3,897 \\
-4,628 \\
-4,460 \\
-4,238 \\
-4,089 \\
-4,211 \\
-3,711 \\
-3,947 \\
-4,078 \\
-48,751\end{array}$ & $\begin{array}{r}-9,881 \\
-6,690 \\
-6,822 \\
-8,087 \\
-8,715 \\
-10,237 \\
-13,226 \\
-11,391 \\
-9,482 \\
-11,851 \\
-8,920 \\
-4,748 \\
-110,050\end{array}$ & $\begin{array}{r}43,633 \\
44,999 \\
52,579 \\
47,808 \\
49,855 \\
49,393 \\
44,390 \\
48,972 \\
49,723 \\
51,828 \\
50,710 \\
50,853 \\
584,742\end{array}$ & $\begin{array}{r}57,293 \\
55,217 \\
63,583 \\
59,792 \\
63,198 \\
64,090 \\
61,854 \\
64,452 \\
63,417 \\
67,390 \\
63,577 \\
59,679 \\
743,543\end{array}$ & $\begin{array}{r}-13,661 \\
-10,218 \\
-11,004 \\
-11,984 \\
-13,343 \\
-14,697 \\
-17,464 \\
-15,480 \\
-13,693 \\
-15,562 \\
-12,867 \\
-8,826 \\
-158,801\end{array}$ \\
\hline 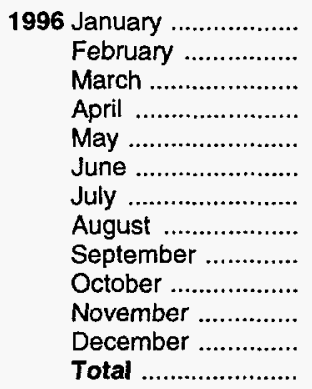 & $\begin{array}{r}722 \\
611 \\
612 \\
517 \\
574 \\
498 \\
592 \\
640 \\
695 \\
961 \\
724 \\
839 \\
7,984\end{array}$ & $\begin{array}{r}5,327 \\
4,315 \\
4,679 \\
6,004 \\
6,421 \\
5,787 \\
6,407 \\
6,006 \\
6,557 \\
7,021 \\
6,147 \\
7,351 \\
72,022\end{array}$ & $\begin{array}{r}-4,605 \\
-3,704 \\
-4,067 \\
-5,487 \\
-5,847 \\
-5,289 \\
-5,815 \\
-5,366 \\
-5,862 \\
-6,060 \\
-5,423 \\
-6,512 \\
-64,038\end{array}$ & $\begin{array}{r}1,032 \\
932 \\
941 \\
864 \\
921 \\
867 \\
942 \\
993 \\
1,071 \\
1,353 \\
1,080 \\
1,185 \\
12,181\end{array}$ & $\begin{array}{r}5,842 \\
4,791 \\
5,197 \\
6,472 \\
6,846 \\
6,217 \\
6,869 \\
6,492 \\
6,993 \\
7,480 \\
6,747 \\
8,141 \\
78,086\end{array}$ & $\begin{array}{r}-4,810 \\
-3,859 \\
-4,256 \\
-5,608 \\
-5,925 \\
-5,350 \\
-5,927 \\
-5,499 \\
-5,922 \\
-6,127 \\
-5,667 \\
-6,956 \\
-65,905\end{array}$ & $\begin{array}{r}-9,332 \\
-5,609 \\
-4,156 \\
-7,184 \\
-7,573 \\
-7,025 \\
-14,157 \\
-10,951 \\
-11,788 \\
-11,883 \\
-7,333 \\
-7,318 \\
-104,309\end{array}$ & $\begin{array}{r}47,767 \\
51,112 \\
54,952 \\
51,872 \\
53,359 \\
51,821 \\
47,598 \\
51,575 \\
50,598 \\
56,107 \\
55,016 \\
53,295 \\
625,075\end{array}$ & $\begin{array}{r}61,910 \\
60,580 \\
63,364 \\
64,664 \\
66,857 \\
64,196 \\
67,682 \\
68,025 \\
68,309 \\
74,118 \\
68,016 \\
67,570 \\
795,289\end{array}$ & $\begin{array}{r}-14,142 \\
-9,468 \\
-8,412 \\
-12,792 \\
-13,498 \\
-12,375 \\
-20,084 \\
-16,450 \\
-17,710 \\
-18,010 \\
-13,000 \\
-14,274 \\
-170,214\end{array}$ \\
\hline 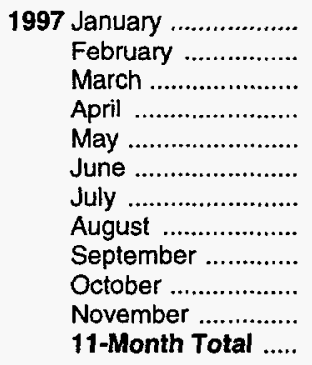 & $\begin{array}{r}763 \\
681 \\
639 \\
677 \\
590 \\
637 \\
761 \\
722 \\
656 \\
758 \\
626 \\
7,510\end{array}$ & $\begin{array}{r}6,694 \\
5,773 \\
6,018 \\
5,686 \\
6,098 \\
5,713 \\
5,780 \\
6,002 \\
5,901 \\
6,479 \\
5,193 \\
65,337\end{array}$ & $\begin{array}{l}-5,931 \\
-5,092 \\
-5,379 \\
-5,009 \\
-5,508 \\
-5,076 \\
-5,019 \\
-5,280 \\
-5,245 \\
-5,721 \\
-4,567 \\
-57,827\end{array}$ & $\begin{array}{r}1,096 \\
1,009 \\
973 \\
992 \\
907 \\
956 \\
1,074 \\
1,112 \\
976 \\
1,120 \\
956 \\
11,170\end{array}$ & $\begin{array}{r}7,287 \\
6,474 \\
6,614 \\
6,313 \\
6,538 \\
6,166 \\
6,287 \\
6,532 \\
6,423 \\
7,034 \\
5,784 \\
71,452\end{array}$ & $\begin{array}{l}-6,191 \\
-5,465 \\
-5,641 \\
-5,321 \\
-5,631 \\
-5,210 \\
-5,213 \\
-5,420 \\
-5,447 \\
-5,914 \\
-4,828 \\
-60,282\end{array}$ & $\begin{array}{r}-10,168 \\
-6,528 \\
-3,729 \\
-7,516 \\
-7,128 \\
-8,520 \\
-14,903 \\
-10,877 \\
-14,199 \\
A-13,436 \\
-8,399 \\
-105,403\end{array}$ & $\begin{array}{r}50,544 \\
53,202 \\
61,275 \\
58,341 \\
58,719 \\
58,037 \\
54,829 \\
56,705 \\
57,221 \\
R 62,158 \\
58,408 \\
629,439\end{array}$ & $\begin{array}{r}66,903 \\
65,196 \\
70,645 \\
71,178 \\
71,478 \\
71,767 \\
74,945 \\
73,001 \\
76,868 \\
R 81,509 \\
71,635 \\
795,125\end{array}$ & $\begin{array}{r}-16,359 \\
-11,993 \\
-9,370 \\
-12,837 \\
-12,759 \\
-13,730 \\
-20,116 \\
-16,297 \\
-19,646 \\
R-19,350 \\
-13,227 \\
-165,685\end{array}$ \\
\hline $\begin{array}{l}1996 \text { 11-Month Tofal ..... } \\
1995 \text { 11-Month Total ..... }\end{array}$ & $\begin{array}{l}7,146 \\
5,626\end{array}$ & $\begin{array}{l}64,671 \\
49,767\end{array}$ & $\begin{array}{l}-57,525 \\
-44,141\end{array}$ & $\begin{array}{r}10,996 \\
9,349\end{array}$ & $\begin{array}{l}69,946 \\
54,020\end{array}$ & $\begin{array}{l}-58,950 \\
-44,671\end{array}$ & $\begin{array}{r}-96,991 \\
-105,302\end{array}$ & $\begin{array}{l}571,777 \\
533,890\end{array}$ & $\begin{array}{l}727,721 \\
683,863\end{array}$ & $\begin{array}{l}-155,941 \\
-149,973\end{array}$ \\
\hline
\end{tabular}

a Crude oil, petroleum preparations, liquefied propane and butane, and other mineral fuels.

b Petroleum, coal, natural gas, and electricity.

$R=$ Revised data.

Notes: - Monthly data are not adjusted for seasonal variations. - See Note 5 at end of section. - Totals may not equal sum of components due to independent rounding. - The U.S. import statistics reflect both government and nongovernment imports of merchandise from foreign countries into the U.S. customs territory, which comprises the 50 States, the District of Columbia, Puerto Rico, and the Virgin Islands.

Sources: - U.S. Department of Commerce, Bureau of the Census, Foreign Trade Division. For details, see "Sources for Table 1.6" at the end of this section. 
Figure 1.6 Cost of Fuels to End-Users in Constant (1982-1984) Dollars

Costs, 1973-1996

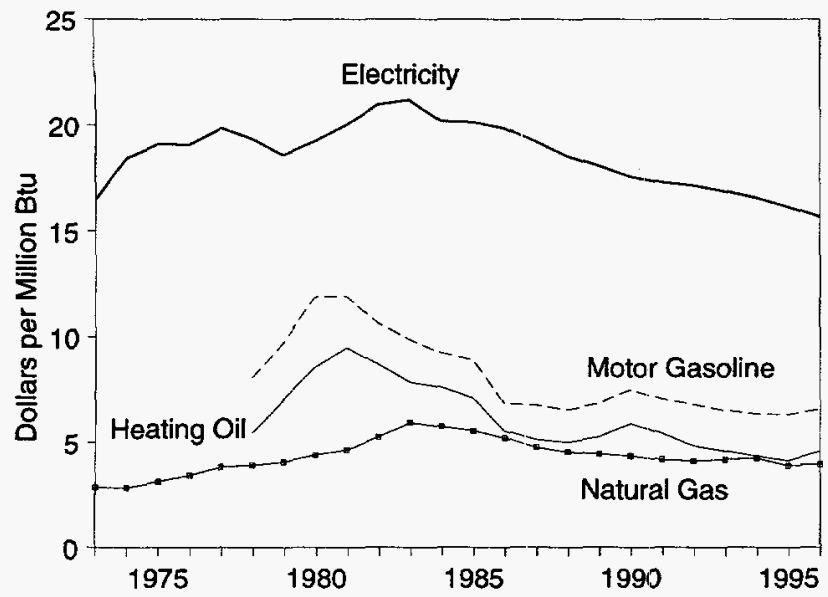

Electricity, Monthly

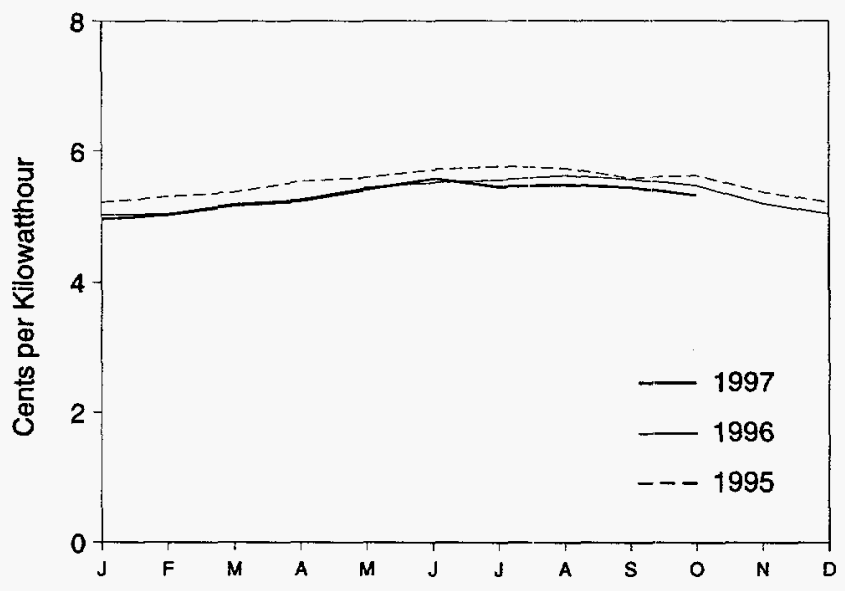

Heating Oil, Monthly

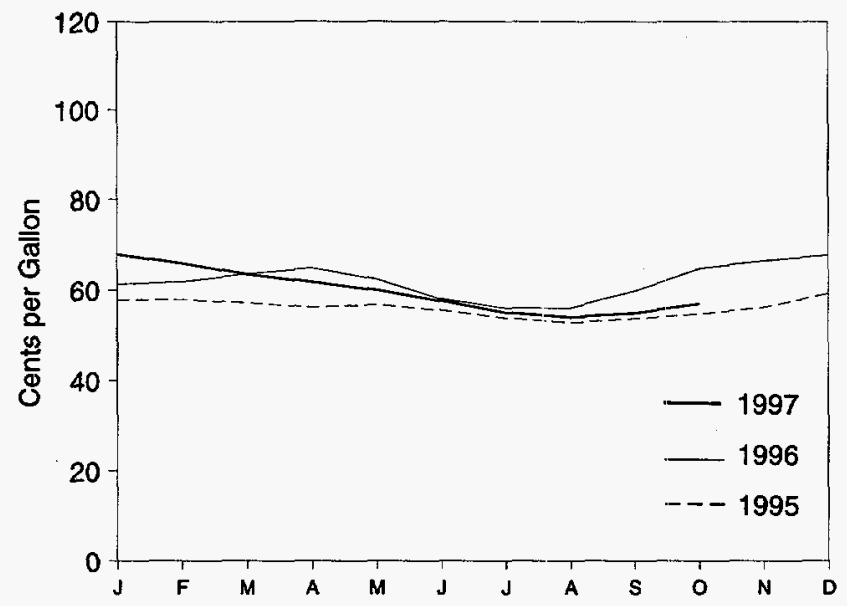

Costs, October 1997

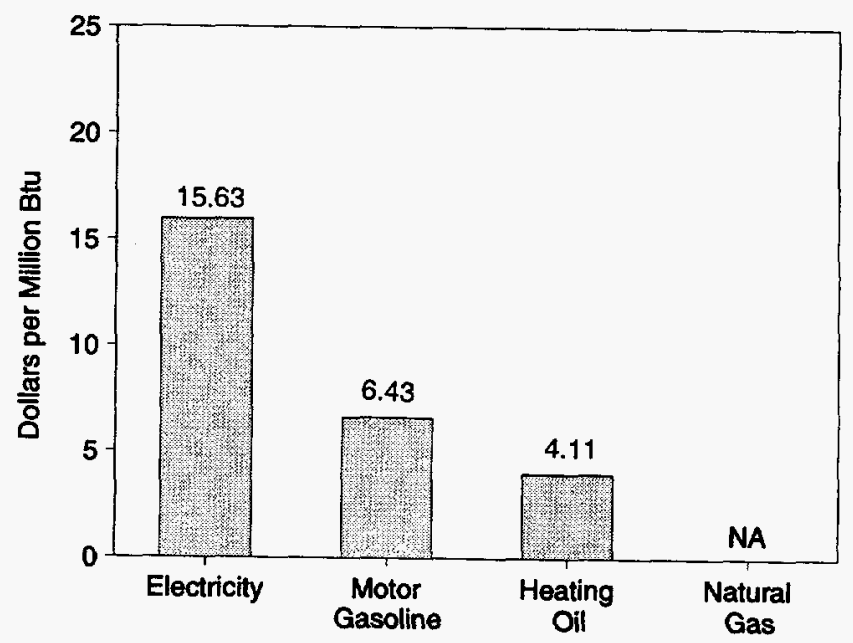

Motor Gasoline, Monthly

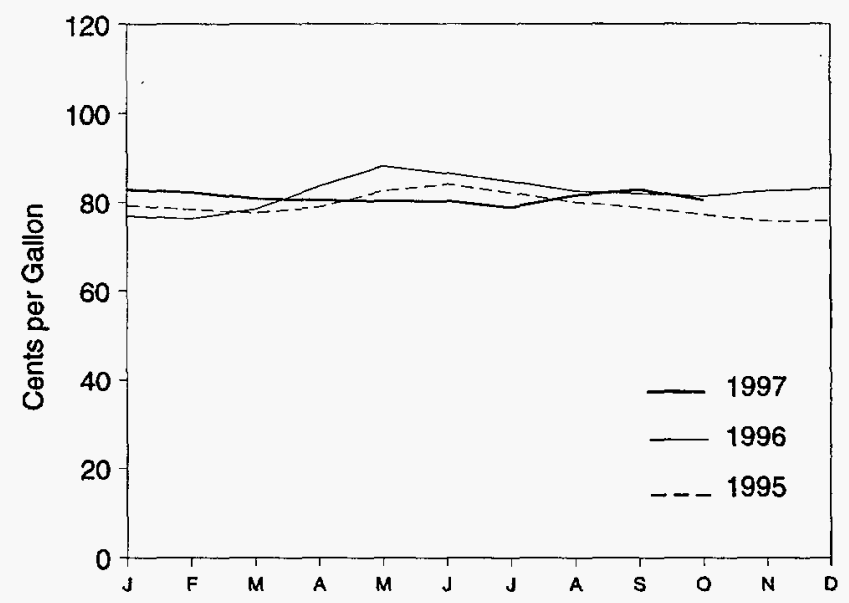

Natural Gas, Monthly

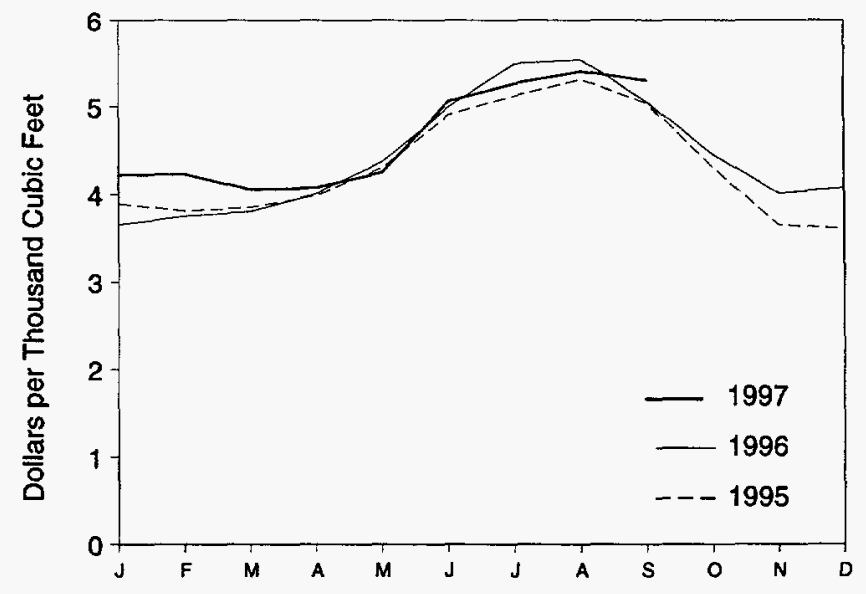

NA=Not available.

Source: Table 1.7 . 
Table 1.7 Cost of Fuels to End Users in Constant (1982-84) Dollars

\begin{tabular}{|c|c|c|c|c|c|c|c|c|c|}
\hline & \multirow{2}{*}{$\begin{array}{c}\begin{array}{c}\text { Consumer } \\
\text { Price Index } \\
\text { (Urban) }\end{array} \\
\text { Index } \\
1982-1984=100\end{array}$} & \multicolumn{2}{|c|}{$\begin{array}{c}\text { Motor Gasoline } \\
\text { (All Types) }\end{array}$} & \multicolumn{2}{|c|}{$\begin{array}{l}\text { Residential } \\
\text { Heating Oil }\end{array}$} & \multicolumn{2}{|c|}{$\begin{array}{l}\text { Residential } \\
\text { Natural Gas }\end{array}$} & \multicolumn{2}{|c|}{$\begin{array}{c}\text { Residential } \\
\text { Electricity }\end{array}$} \\
\hline & & $\begin{array}{l}\text { Cents per } \\
\text { Gallon }\end{array}$ & $\begin{array}{l}\text { Dollars per } \\
\text { Million Btu }\end{array}$ & $\begin{array}{l}\text { Cents per } \\
\text { Gallon }\end{array}$ & $\begin{array}{l}\text { Dollars per } \\
\text { Million Btu }\end{array}$ & $\begin{array}{l}\text { Cents per } \\
\text { Thousand } \\
\text { Cubic Feet }\end{array}$ & $\begin{array}{l}\text { Dollars per } \\
\text { Million Btu }\end{array}$ & $\begin{array}{c}\text { Cents per } \\
\text { Kilowatthour }\end{array}$ & $\begin{array}{l}\text { Dollars per } \\
\text { Million Btu }\end{array}$ \\
\hline 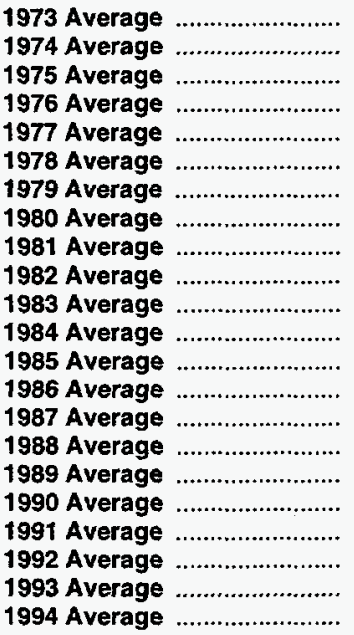 & $\begin{array}{r}44.4 \\
49.3 \\
53.8 \\
56.9 \\
60.6 \\
65.2 \\
72.6 \\
82.4 \\
90.9 \\
96.5 \\
99.6 \\
103.9 \\
107.6 \\
109.6 \\
113.6 \\
118.3 \\
124.0 \\
130.7 \\
136.2 \\
140.3 \\
144.5 \\
148.2\end{array}$ & $\begin{array}{c}\text { NA } \\
\text { NA } \\
\text { NA } \\
\text { NA } \\
\text { NA } \\
100.0 \\
121.5 \\
148.2 \\
148.8 \\
132.7 \\
123.0 \\
115.3 \\
111.2 \\
84.9 \\
84.2 \\
81.4 \\
85.5 \\
93.1 \\
87.8 \\
84.8 \\
81.2 \\
79.2\end{array}$ & $\begin{array}{c}\text { NA } \\
\text { NA } \\
\text { NA } \\
\text { NA } \\
\text { NA } \\
8.00 \\
9.71 \\
11.85 \\
11.90 \\
10.61 \\
9.83 \\
9.22 \\
8.89 \\
6.79 \\
6.74 \\
6.51 \\
6.83 \\
7.44 \\
7.02 \\
6.78 \\
6.49 \\
6.33\end{array}$ & $\begin{array}{c}\text { NA } \\
\text { NA } \\
\text { NA } \\
\text { NA } \\
\text { NA } \\
75.2 \\
97.0 \\
118.2 \\
131.4 \\
120.2 \\
108.2 \\
105.0 \\
97.9 \\
76.3 \\
70.7 \\
68.7 \\
72.6 \\
81.3 \\
74.8 \\
66.6 \\
63.0 \\
59.6\end{array}$ & $\begin{array}{l}\text { NA } \\
\text { NA } \\
\text { NA } \\
\text { NA } \\
\text { NA } \\
5.42 \\
6.99 \\
8.52 \\
9.47 \\
8.67 \\
7.80 \\
7.57 \\
7.06 \\
5.50 \\
5.10 \\
4.96 \\
5.23 \\
5.86 \\
5.39 \\
4.80 \\
4.55 \\
4.30\end{array}$ & $\begin{array}{l}290.5 \\
290.1 \\
317.8 \\
348.0 \\
387.8 \\
392.6 \\
410.5 \\
446.6 \\
471.9 \\
535.8 \\
608.4 \\
589.0 \\
568.8 \\
531.9 \\
487.7 \\
462.4 \\
454.8 \\
443.8 \\
427.3 \\
419.8 \\
426.3 \\
432.5\end{array}$ & $\begin{array}{l}2.85 \\
2.83 \\
3.12 \\
3.41 \\
3.81 \\
3.86 \\
4.03 \\
4.36 \\
4.60 \\
5.22 \\
5.90 \\
5.72 \\
5.52 \\
5.17 \\
4.73 \\
4.49 \\
4.41 \\
4.31 \\
4.14 \\
4.07 \\
4.15 \\
4.20\end{array}$ & $\begin{array}{l}5.6 \\
6.3 \\
6.5 \\
6.5 \\
6.8 \\
6.6 \\
6.3 \\
6.6 \\
6.8 \\
7.2 \\
7.2 \\
6.88 \\
6.87 \\
6.77 \\
6.56 \\
6.32 \\
6.17 \\
5.99 \\
5.90 \\
5.85 \\
5.76 \\
5.65\end{array}$ & $\begin{array}{l}16.50 \\
18.43 \\
19.07 \\
19.06 \\
19.83 \\
19.33 \\
18.57 \\
19.21 \\
19.99 \\
20.96 \\
21.19 \\
20.17 \\
20.13 \\
19.84 \\
19.22 \\
18.53 \\
18.08 \\
17.56 \\
17.30 \\
17.15 \\
16.88 \\
16.57\end{array}$ \\
\hline 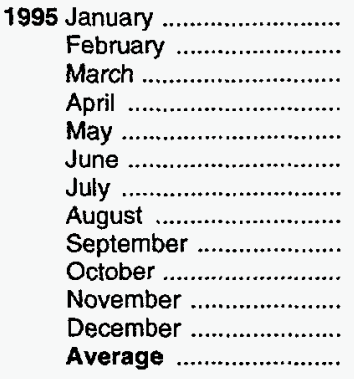 & $\begin{array}{l}150.3 \\
150.9 \\
151.4 \\
151.9 \\
152.2 \\
152.5 \\
152.5 \\
152.9 \\
153.2 \\
153.7 \\
153.6 \\
153.5 \\
152.4\end{array}$ & $\begin{array}{l}79.2 \\
78.3 \\
77.5 \\
78.8 \\
82.5 \\
84.0 \\
82.1 \\
79.9 \\
78.7 \\
77.1 \\
75.6 \\
75.6 \\
79.1\end{array}$ & $\begin{array}{l}6.33 \\
6.26 \\
6.19 \\
6.30 \\
6.60 \\
6.72 \\
6.56 \\
6.39 \\
6.29 \\
6.16 \\
6.04 \\
6.04 \\
6.32\end{array}$ & $\begin{array}{l}57.8 \\
57.9 \\
57.2 \\
56.2 \\
56.8 \\
55.5 \\
53.8 \\
52.8 \\
53.7 \\
54.7 \\
56.2 \\
59.3 \\
56.9\end{array}$ & $\begin{array}{l}4.17 \\
4.18 \\
4.12 \\
4.05 \\
4.09 \\
4.00 \\
3.88 \\
3.81 \\
3.87 \\
3.94 \\
4.05 \\
4.28 \\
4.10\end{array}$ & $\begin{array}{l}389.2 \\
381.7 \\
385.7 \\
398.9 \\
429.7 \\
491.1 \\
512.8 \\
531.7 \\
504.6 \\
430.7 \\
365.2 \\
360.9 \\
397.6\end{array}$ & $\begin{array}{l}3.79 \\
3.72 \\
3.76 \\
3.88 \\
4.18 \\
4.78 \\
4.99 \\
5.18 \\
4.91 \\
4.19 \\
3.56 \\
3.51 \\
3.87\end{array}$ & $\begin{array}{l}5.22 \\
5.31 \\
5.38 \\
5.54 \\
5.60 \\
5.72 \\
5.77 \\
5.74 \\
5.59 \\
5.63 \\
5.38 \\
5.22 \\
5.51\end{array}$ & $\begin{array}{l}15.31 \\
15.56 \\
15.76 \\
16.23 \\
16.43 \\
16.76 \\
16.91 \\
16.83 \\
16.40 \\
16.49 \\
15.76 \\
15.31 \\
16.15\end{array}$ \\
\hline 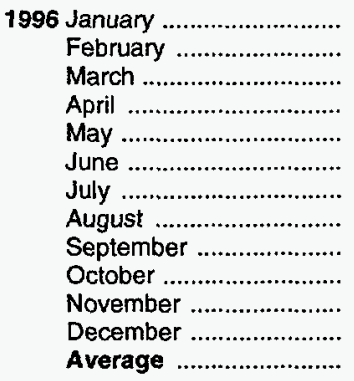 & $\begin{array}{l}154.4 \\
154.9 \\
155.7 \\
156.3 \\
156.6 \\
156.7 \\
157.0 \\
157.3 \\
157.8 \\
158.3 \\
158.6 \\
158.6 \\
156.9\end{array}$ & $\begin{array}{l}76.8 \\
76.2 \\
78.3 \\
83.5 \\
88.0 \\
86.4 \\
84.6 \\
82.5 \\
81.9 \\
81.3 \\
82.5 \\
83.1 \\
\mathbf{8 2 . 1}\end{array}$ & $\begin{array}{l}6.14 \\
6.10 \\
6.26 \\
6.68 \\
7.04 \\
6.91 \\
6.76 \\
6.60 \\
6.55 \\
6.50 \\
6.59 \\
6.64 \\
6.56\end{array}$ & $\begin{array}{l}61.3 \\
61.9 \\
63.6 \\
64.9 \\
62.5 \\
58.1 \\
56.0 \\
56.0 \\
59.9 \\
64.8 \\
66.5 \\
67.8 \\
63.0\end{array}$ & $\begin{array}{l}4.42 \\
4.46 \\
4.59 \\
4.68 \\
4.50 \\
4.19 \\
4.04 \\
4.04 \\
4.32 \\
4.67 \\
4.79 \\
4.89 \\
4.54\end{array}$ & $\begin{array}{l}365.3 \\
375.7 \\
380.9 \\
401.2 \\
436.8 \\
499.7 \\
550.3 \\
555.0 \\
506.3 \\
445.4 \\
401.6 \\
407.9 \\
404.1\end{array}$ & $\begin{array}{l}3.56 \\
3.66 \\
3.71 \\
3.91 \\
4.25 \\
4.87 \\
5.36 \\
5.40 \\
4.93 \\
4.34 \\
3.91 \\
3.97 \\
3.93\end{array}$ & $\begin{array}{l}R_{5.02} \\
R_{5.04} \\
R_{5.20} \\
R_{5.27} \\
R_{5.45} \\
R_{5.52} \\
R_{5.56} \\
R_{5.63} \\
R_{5.57} \\
R_{5.48} \\
R_{5.20} \\
R_{5.04} \\
R_{5.33}\end{array}$ & $\begin{array}{l}R_{14.71} \\
R_{14.78} \\
R_{15.23} \\
R_{15.45} \\
R_{15.98} \\
R_{16.18} \\
R_{16.30} \\
R_{16.51} \\
R_{16.33} \\
R_{16.05} \\
R_{15.25} \\
R_{14.77} \\
R_{15.62}\end{array}$ \\
\hline 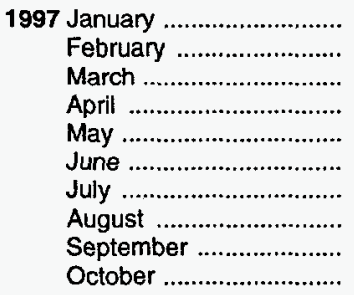 & $\begin{array}{l}159.1 \\
159.6 \\
160.0 \\
160.2 \\
160.1 \\
160.3 \\
160.5 \\
160.8 \\
161.2 \\
161.6\end{array}$ & $\begin{array}{l}82.8 \\
82.2 \\
80.8 \\
80.4 \\
80.2 \\
80.2 \\
78.7 \\
81.5 \\
82.8 \\
80.4\end{array}$ & $\begin{array}{l}6.62 \\
6.57 \\
6.46 \\
6.43 \\
6.41 \\
6.41 \\
6.29 \\
6.51 \\
6.62 \\
6.43\end{array}$ & $\begin{array}{r}67.8 \\
65.9 \\
63.5 \\
61.9 \\
60.1 \\
57.6 \\
55.0 \\
54.0 \\
\text { R } 54.9 \\
57.0\end{array}$ & $\begin{array}{l}4.89 \\
4.75 \\
4.58 \\
4.46 \\
4.34 \\
4.15 \\
3.97 \\
3.90 \\
3.96 \\
4.11\end{array}$ & $\begin{array}{r}R_{421.7} \\
R_{422.9} \\
405.6 \\
R_{407.6} \\
R_{424.7} \\
R_{506.6} \\
R_{527.1} \\
R_{541.0} \\
R_{530.4} \\
\text { NA }\end{array}$ & $\begin{array}{r}4.11 \\
4.12 \\
3.95 \\
\text { R } 3.97 \\
R_{4.14} \\
R_{4.93} \\
R_{5.13} \\
R_{5.27} \\
\text { R } 5.16 \\
\text { NA }\end{array}$ & $\begin{array}{l}4.96 \\
5.02 \\
5.17 \\
5.24 \\
5.42 \\
5.58 \\
5.46 \\
5.49 \\
5.45 \\
5.33\end{array}$ & $\begin{array}{l}14.53 \\
14.71 \\
15.17 \\
15.37 \\
15.89 \\
16.35 \\
16.01 \\
16.09 \\
15.96 \\
15.63\end{array}$ \\
\hline
\end{tabular}

a Consumer Price Index, All Urban Consumers, All Items, 1982-1984= 100.0 .

$\mathrm{R}=$ Revised data. $\mathrm{NA}=$ Not available.

Notes: - Fuel costs are calculated by using the Urban Consumer Price Index (CPI) developed by the Bureau of Labor Statistics. - Annual averages may not equal average of months due to independent rounding. - Geographic coverage is the $\mathbf{5 0}$ States and the District of Columbia.
Sources: - Annual Data: Annual prices in Tables 9.4 (All Types), 9.8c, 9.11, and 9.9 , adjusted by the CPI. Monthly Data: Monthly prices in Tables 9.4 (All Types), 9.8c, 9.11 , and 9.9 (Monthly Series), adjusted by the CPI. - CPI: 1973-1993-Economic Report of the President, February 1997, Table B-59. 1994 forward-Council of Economic Advisers, Economic Indicators, December 1997, "Consumer Prices - All Urban Consumers." - Conversion Factors: Tables A1, A4, and A8. 
Figure 1.7 Overview of U.S. Petroleum Trade

(Quadrillion Btu)

Overview, November 1997

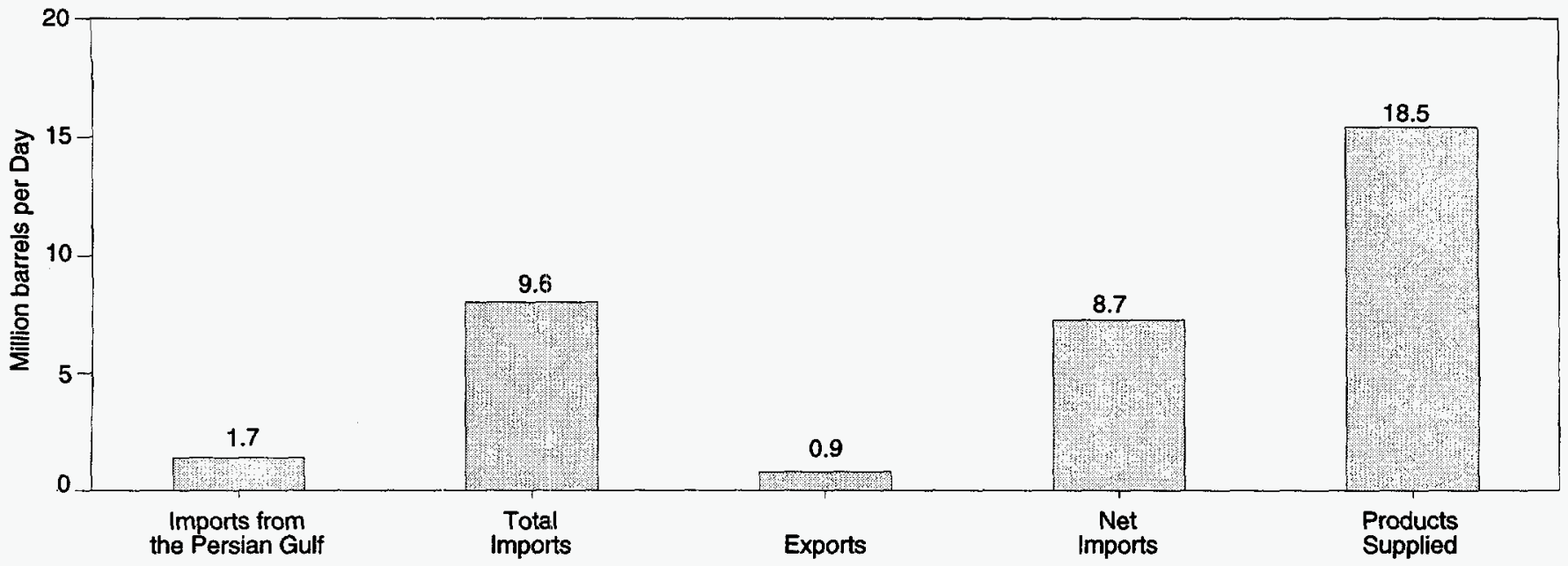

Imports from the Persian Gulf as a Share of Total Imports
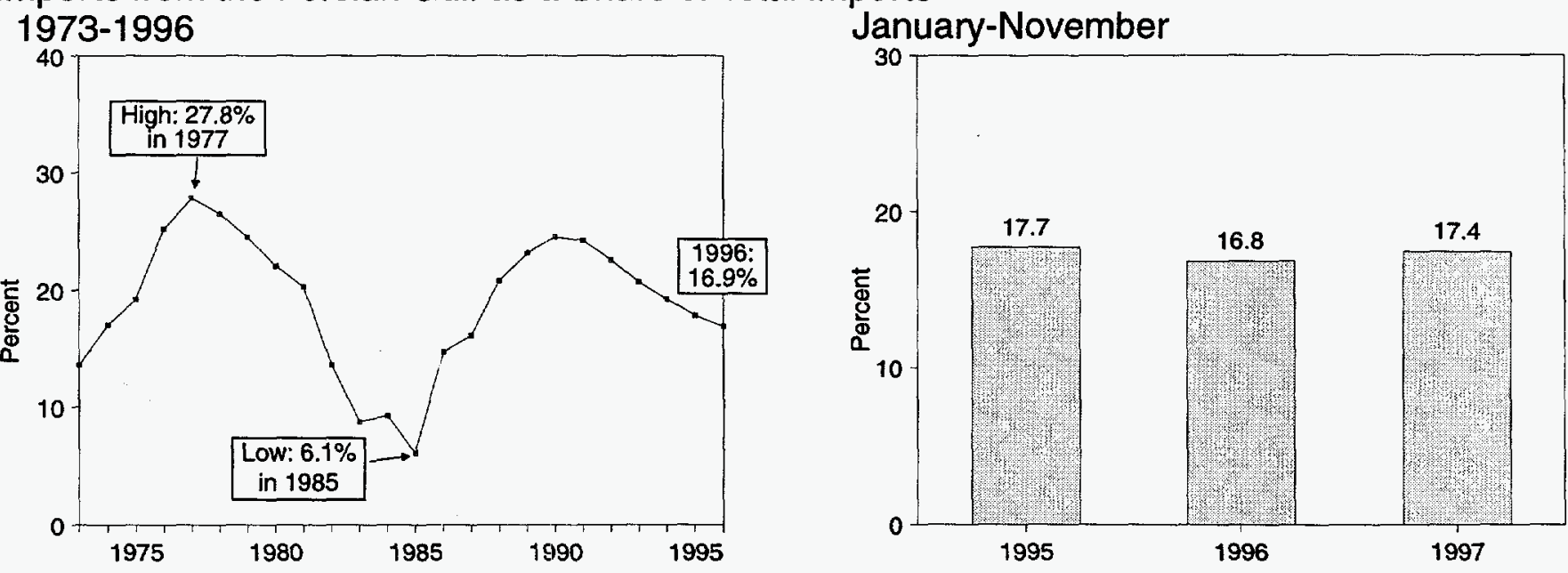

Net Imports as Share of Product Supplied
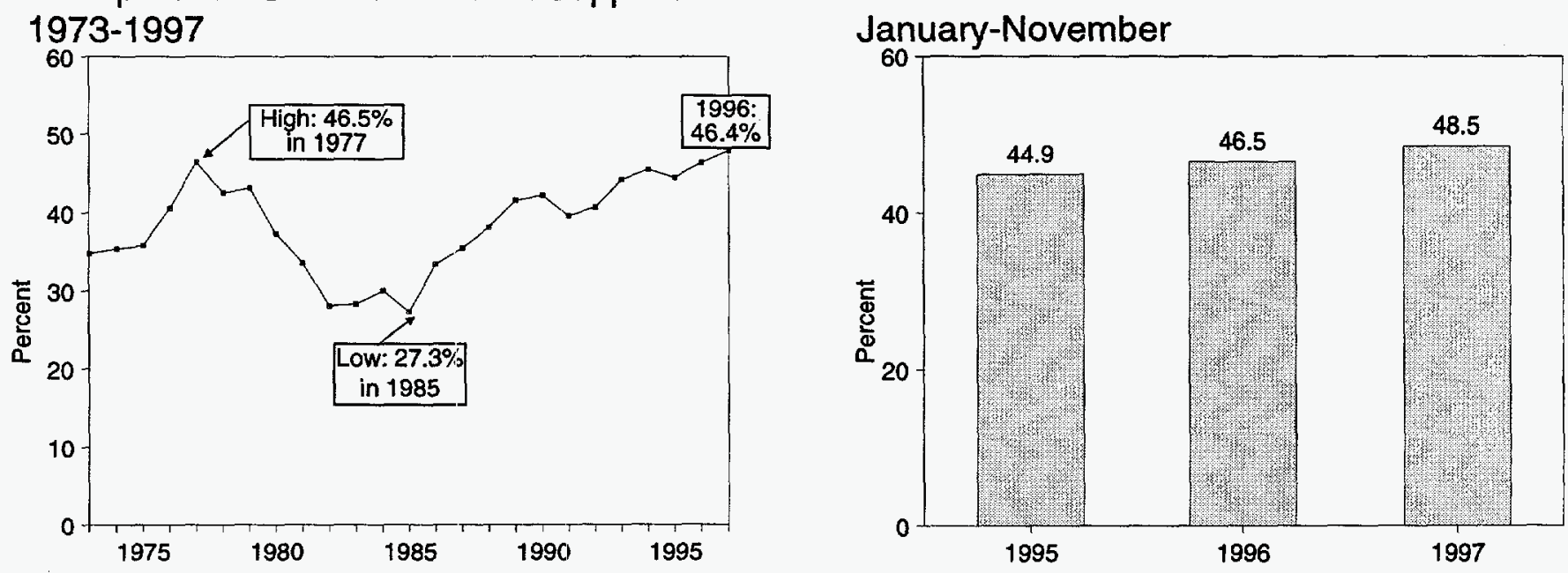

Note: Because vertical scales differ, graphs stiould not be compared.

Source: Table 1.8 . 
Table 1.8 Overview of U.S. Petroleum Trade

\begin{tabular}{|c|c|c|c|c|c|c|c|c|c|}
\hline & \multirow{2}{*}{$\begin{array}{c}\text { Imports } \\
\text { from the } \\
\text { Persian } \\
\text { Gulfa }\end{array}$} & \multirow[b]{2}{*}{$\begin{array}{l}\text { Total } \\
\text { Imports }\end{array}$} & \multirow[b]{2}{*}{ Exports } & \multirow[b]{2}{*}{$\begin{array}{c}\text { Net } \\
\text { Imports }\end{array}$} & \multirow[b]{2}{*}{$\begin{array}{l}\text { Products } \\
\text { Supplied }\end{array}$} & \multicolumn{3}{|c|}{ As Share of Products Supplied } & \multirow{2}{*}{$\begin{array}{l}\text { Imports from } \\
\text { the Persian Gulfa } \\
\text { as a Share } \\
\text { of Total Imports }\end{array}$} \\
\hline & & & & & & $\begin{array}{l}\text { Imports from the } \\
\text { Persian Gulfa }\end{array}$ & $\begin{array}{c}\text { Total } \\
\text { Imports }\end{array}$ & $\begin{array}{c}\text { Net } \\
\text { Imports }\end{array}$ & \\
\hline & \multicolumn{5}{|c|}{ Thousand Barrels per Day } & \multicolumn{4}{|c|}{ Percent } \\
\hline $\begin{array}{l}1973 \text { Average } \\
1974 \text { Average } \\
1975 \text { Average } \ldots \ldots \ldots \ldots \ldots \ldots \ldots \ldots \\
1976 \text { Average } . . . \ldots \ldots \ldots \ldots \ldots \ldots \\
1977 \text { Average } \ldots \ldots \ldots \ldots \ldots \ldots \ldots \\
1978 \text { Average } \\
1979 \text { Average } \\
1980 \text { Average } \\
1981 \text { Average } \\
1982 \text { Average }\end{array}$ & $\begin{array}{r}848 \\
1,039 \\
1,165 \\
1,840 \\
2,448 \\
2,219 \\
2,069 \\
1,519 \\
1,219 \\
696 \\
442 \\
506 \\
311 \\
912 \\
1,077 \\
1,541 \\
1,861 \\
1,966 \\
1,845 \\
1,778 \\
1,782 \\
1,728\end{array}$ & $\begin{array}{l}6,256 \\
6,112 \\
6,056 \\
7,313 \\
8,807 \\
8,363 \\
8,456 \\
6,909 \\
5,996 \\
5,113 \\
5,051 \\
5,437 \\
5,067 \\
6,224 \\
6,678 \\
7,402 \\
8,061 \\
8,018 \\
7,627 \\
7,888 \\
8,620 \\
8,996\end{array}$ & $\begin{array}{r}231 \\
221 \\
209 \\
223 \\
243 \\
362 \\
471 \\
544 \\
595 \\
815 \\
739 \\
722 \\
781 \\
785 \\
764 \\
815 \\
859 \\
857 \\
1,001 \\
950 \\
1,003 \\
942\end{array}$ & $\begin{array}{l}6,025 \\
5,892 \\
5,846 \\
7,090 \\
8,565 \\
8,002 \\
7,985 \\
6,365 \\
5,401 \\
4,298 \\
4,312 \\
4,715 \\
4,286 \\
5,439 \\
5,914 \\
6,587 \\
7,202 \\
7,161 \\
6,626 \\
6,938 \\
7,618 \\
8,054\end{array}$ & $\begin{array}{l}17,308 \\
16,653 \\
16,322 \\
17,461 \\
18,431 \\
18,847 \\
18,513 \\
17,056 \\
16,058 \\
15,296 \\
15,231 \\
15,726 \\
15,726 \\
16,281 \\
16,665 \\
17,283 \\
17,325 \\
16,988 \\
16,714 \\
17,033 \\
17,237 \\
17,718\end{array}$ & $\begin{array}{r}4.9 \\
6.2 \\
7.1 \\
10.5 \\
13.3 \\
11.8 \\
11.2 \\
8.9 \\
7.6 \\
4.5 \\
2.9 \\
3.2 \\
2.0 \\
5.6 \\
6.5 \\
8.9 \\
10.7 \\
11.6 \\
11.0 \\
10.4 \\
10.3 \\
9.8\end{array}$ & $\begin{array}{l}36.1 \\
36.7 \\
37.1 \\
41.9 \\
47.8 \\
44.4 \\
45.7 \\
40.5 \\
37.3 \\
33.4 \\
33.2 \\
34.6 \\
32.2 \\
38.2 \\
40.1 \\
42.8 \\
46.5 \\
47.2 \\
45.6 \\
46.3 \\
50.0 \\
50.8\end{array}$ & $\begin{array}{l}34.8 \\
35.4 \\
35.8 \\
40.6 \\
46.5 \\
42.5 \\
43.1 \\
37.3 \\
33.6 \\
28.1 \\
28.3 \\
30.0 \\
27.3 \\
33.4 \\
35.5 \\
38.1 \\
41.6 \\
42.2 \\
39.6 \\
40.7 \\
44.2 \\
45.5\end{array}$ & $\begin{array}{r}13.6 \\
17.0 \\
19.2 \\
25.2 \\
27.8 \\
26.5 \\
24.5 \\
22.0 \\
20.3 \\
13.6 \\
8.8 \\
9.3 \\
6.1 \\
14.7 \\
16.1 \\
20.8 \\
23.1 \\
24.5 \\
24.2 \\
22.5 \\
20.7 \\
19.2\end{array}$ \\
\hline 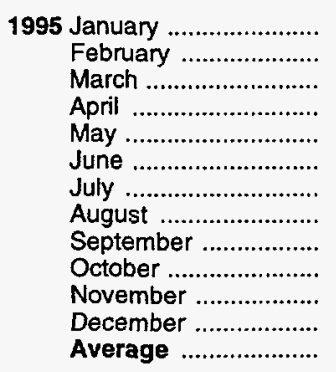 & $\begin{array}{l}1,459 \\
1,550 \\
1,788 \\
1,547 \\
1,490 \\
1,558 \\
1,460 \\
1,541 \\
1,691 \\
1,524 \\
1,677 \\
1,593 \\
1,573\end{array}$ & $\begin{array}{l}8,015 \\
8,345 \\
9,006 \\
8,465 \\
8,709 \\
9,558 \\
8,863 \\
9,061 \\
9,736 \\
8,577 \\
9,074 \\
8,612 \\
8,835\end{array}$ & $\begin{array}{r}978 \\
1,062 \\
948 \\
998 \\
876 \\
919 \\
895 \\
821 \\
805 \\
962 \\
1,002 \\
1,135 \\
949\end{array}$ & $\begin{array}{l}7,037 \\
7,283 \\
8,059 \\
7,467 \\
7,832 \\
8,639 \\
7,969 \\
8,240 \\
8,930 \\
7,615 \\
8,072 \\
7,477 \\
7,886\end{array}$ & $\begin{array}{l}17,219 \\
18,279 \\
17,484 \\
17,142 \\
17,293 \\
18,131 \\
17,147 \\
18,044 \\
18,026 \\
17,651 \\
17,979 \\
18,366 \\
17,725\end{array}$ & $\begin{array}{r}8.5 \\
8.5 \\
10.2 \\
9.0 \\
8.6 \\
8.6 \\
8.5 \\
8.5 \\
9.4 \\
8.6 \\
9.3 \\
8.7 \\
8.9\end{array}$ & $\begin{array}{l}46.5 \\
45.7 \\
51.5 \\
49.4 \\
50.4 \\
52.7 \\
51.7 \\
50.2 \\
54.0 \\
48.6 \\
50.5 \\
46.9 \\
49.8\end{array}$ & $\begin{array}{l}40.9 \\
39.8 \\
46.1 \\
43.6 \\
45.3 \\
47.6 \\
46.5 \\
45.7 \\
49.5 \\
43.1 \\
44.9 \\
40.7 \\
44.5\end{array}$ & $\begin{array}{l}18.2 \\
18.6 \\
19.8 \\
18.3 \\
17.1 \\
16.3 \\
16.5 \\
17.0 \\
17.4 \\
17.8 \\
18.5 \\
18.5 \\
17.8\end{array}$ \\
\hline 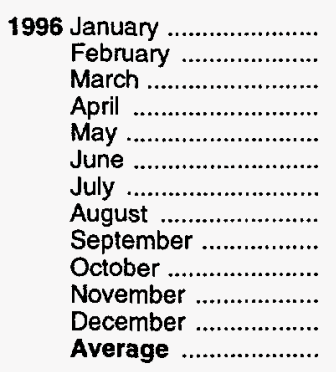 & $\begin{array}{l}1,546 \\
1,344 \\
1,549 \\
1,506 \\
1,748 \\
1,537 \\
1,819 \\
1,747 \\
1,591 \\
1,635 \\
1,525 \\
1,675 \\
1,604\end{array}$ & $\begin{array}{r}9,364 \\
8,390 \\
9,092 \\
9,429 \\
10,007 \\
9,938 \\
9,820 \\
9,986 \\
9,142 \\
9,837 \\
9,244 \\
9,417 \\
9,478\end{array}$ & $\begin{array}{r}1,070 \\
1,048 \\
867 \\
976 \\
891 \\
895 \\
945 \\
896 \\
1,104 \\
1,045 \\
1,024 \\
1,013 \\
981\end{array}$ & $\begin{array}{l}8,294 \\
7,342 \\
8,225 \\
8,453 \\
9,116 \\
9,043 \\
8,876 \\
9,090 \\
8,038 \\
8,792 \\
8,220 \\
8,404 \\
8,498\end{array}$ & $\begin{array}{l}18,261 \\
18,620 \\
18,301 \\
17,885 \\
17,957 \\
18,107 \\
18,211 \\
18,658 \\
17,655 \\
19,171 \\
18,535 \\
18,334 \\
18,309\end{array}$ & $\begin{array}{r}8.5 \\
7.2 \\
8.5 \\
8.4 \\
9.7 \\
8.5 \\
10.0 \\
9.4 \\
9.0 \\
8.5 \\
8.2 \\
9.1 \\
\mathbf{8 . 8}\end{array}$ & $\begin{array}{l}51.3 \\
45.1 \\
49.7 \\
52.7 \\
55.7 \\
54.9 \\
53.9 \\
53.5 \\
51.8 \\
51.3 \\
49.9 \\
51.4 \\
51.8\end{array}$ & $\begin{array}{l}45.4 \\
39.4 \\
44.9 \\
47.3 \\
50.8 \\
49.9 \\
48.7 \\
48.7 \\
45.5 \\
45.9 \\
44.3 \\
45.8 \\
46.4\end{array}$ & $\begin{array}{l}16.5 \\
16.0 \\
17.0 \\
16.0 \\
17.5 \\
15.5 \\
18.5 \\
17.5 \\
17.4 \\
16.6 \\
16.5 \\
17.8 \\
16.9\end{array}$ \\
\hline 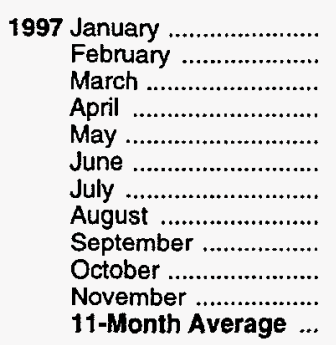 & $\begin{array}{l}1,553 \\
1,533 \\
1,641 \\
1,862 \\
1,706 \\
1,785 \\
1,719 \\
1,850 \\
1,873 \\
1,882 \\
1,686 \\
1,736\end{array}$ & $\begin{array}{r}9,633 \\
9,475 \\
9,712 \\
9,934 \\
10,442 \\
10,357 \\
9,703 \\
10,155 \\
10,201 \\
10,414 \\
9,639 \\
9,973\end{array}$ & $\begin{array}{r}1,038 \\
1,015 \\
932 \\
937 \\
876 \\
955 \\
1,012 \\
1,074 \\
997 \\
1,066 \\
934 \\
985\end{array}$ & $\begin{array}{l}8,595 \\
8,460 \\
8,780 \\
8,997 \\
9,565 \\
9,402 \\
8,691 \\
9,081 \\
9,204 \\
9,347 \\
8,705 \\
8,988\end{array}$ & $\begin{array}{l}18,560 \\
18,308 \\
17,869 \\
18,572 \\
18,244 \\
18,563 \\
19,065 \\
18,506 \\
18,480 \\
19,121 \\
18,498 \\
18,528\end{array}$ & $\begin{array}{r}8.4 \\
8.4 \\
9.2 \\
10.0 \\
9.4 \\
9.6 \\
9.0 \\
10.0 \\
10.1 \\
9.8 \\
9.1 \\
9.4\end{array}$ & $\begin{array}{l}51.9 \\
51.8 \\
54.4 \\
53.5 \\
57.2 \\
55.8 \\
50.9 \\
54.9 \\
55.2 \\
54.5 \\
52.1 \\
\mathbf{5 3 . 8}\end{array}$ & $\begin{array}{l}46.3 \\
46.2 \\
49.1 \\
48.4 \\
52.4 \\
50.6 \\
45.6 \\
49.1 \\
49.8 \\
48.9 \\
47.1 \\
48.5\end{array}$ & $\begin{array}{l}16.1 \\
16.2 \\
16.9 \\
18.7 \\
16.3 \\
17.2 \\
17.7 \\
18.2 \\
18.4 \\
18.1 \\
17.5 \\
17.4\end{array}$ \\
\hline $\begin{array}{l}1996 \text { 11-Month Average ... } \\
1995 \text { 11-Month Average ... }\end{array}$ & $\begin{array}{l}1,597 \\
1,571\end{array}$ & $\begin{array}{l}9,484 \\
8,856\end{array}$ & $\begin{array}{l}978 \\
932\end{array}$ & $\begin{array}{l}8,507 \\
7,924\end{array}$ & $\begin{array}{l}18,307 \\
17,665\end{array}$ & $\begin{array}{l}8.7 \\
8.9\end{array}$ & $\begin{array}{l}51.8 \\
50.1\end{array}$ & $\begin{array}{l}46.5 \\
44.9\end{array}$ & $\begin{array}{l}16.8 \\
17.7\end{array}$ \\
\hline
\end{tabular}

a Bahrain, Iran, Iraq, Kuwait, Qatar, Saudi Arabia, and the United Arab Emirates.

Notes: - Readers of Table 1.8 may be interested in a feature article, "Measuring Dependence on Imported Oil," that was published in the August 1995 Monthly Energy Review. - Petroleum is crude oil, lease condensate, unfinished oils, petroleum products, natural gas plant liquids, and nonhydrocarbon compounds blended into finished petroleum products. - Beginning in October 1977, petroleum imported for the Strategic Petroleum Reserves is included. - Annual averages may not equal average of months due to independent rounding. - U.S. geographic coverage is the 50 States and the District of Columbia. U.S. exports include shipments to U.S. territories, and imports include receipts from U.S. territories.

Sources: - Column 1: Table 3.3b. - Columns 2 - 4: Table 3.1b - Column 5: Table 3.1a. - Column 6: Column 1 divided by column 5 times 100. Column 7: Column 2 divided by column 5 times 100. - Column 8: Column 4 divided by column 5 times 100. Column 9: Column 1 divided by column 2 times 100 . 


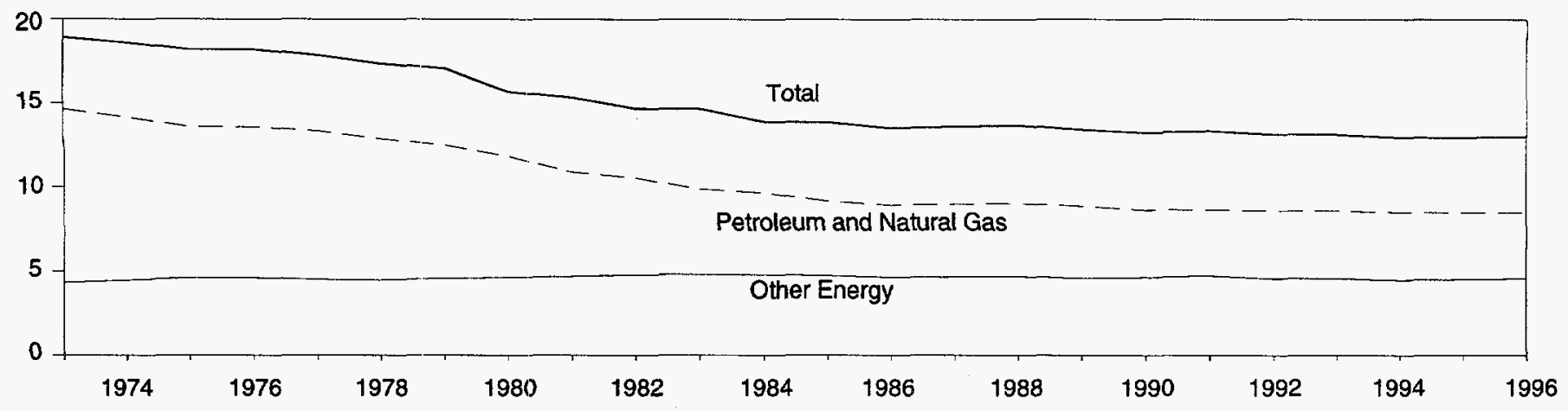

Table 1.9 Energy Consumption per Dollar of Gross Domestic Product (Seasonally Adjusted at Annual Rates)

\begin{tabular}{|c|c|c|c|c|c|c|c|}
\hline \multirow{3}{*}{. } & \multicolumn{3}{|c|}{ Energy Consumption } & \multirow{3}{*}{$\begin{array}{c}\begin{array}{c}\text { Gross } \\
\text { Domestic } \\
\text { Product } \\
\text { (GDP) }\end{array} \\
\begin{array}{c}\text { Billion Chained } \\
\text { (1992) Dollars }\end{array}\end{array}$} & \multicolumn{3}{|c|}{ Energy Consumption per Dollar of GDP } \\
\hline & $\begin{array}{c}\begin{array}{c}\text { Petroleum } \\
\text { and } \\
\text { Natural Gas }\end{array} \\
\end{array}$ & $\begin{array}{c}\text { Other } \\
\text { Energy }\end{array}$ & Totala & & $\begin{array}{c}\begin{array}{c}\text { Petroleum } \\
\text { and } \\
\text { Natural Gas }\end{array} \\
\end{array}$ & $\begin{array}{c}\text { Other } \\
\text { Energya }\end{array}$ & Totala \\
\hline & \multicolumn{3}{|c|}{ Quadrillion Btu } & & \multicolumn{3}{|c|}{ Thousand Btu per Chained (1992) Dollar } \\
\hline 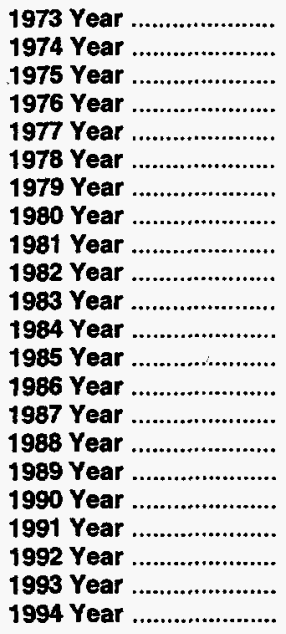 & $\begin{array}{l}\mathbf{5 7 . 3 5 2} \\
\mathbf{5 5 . 1 8 7} \\
\mathbf{5 2 . 6 7 8} \\
\mathbf{5 5 . 5 2 0} \\
\mathbf{5 7 . 0 5 3} \\
\mathbf{5 7 . 9 6 6} \\
\mathbf{5 7 . 7 8 9} \\
\mathbf{5 4 . 5 9 6} \\
\mathbf{5 1 . 8 5 9} \\
\mathbf{4 8 . 7 3 6} \\
\mathbf{4 7 . 4 1 1} \\
\mathbf{4 9 . 5 5 8} \\
\mathbf{4 8 . 7 5 6} \\
\mathbf{4 8 . 9 0 4} \\
\mathbf{5 0 . 6 0 9} \\
\mathbf{5 2 . 7 7 4} \\
\mathbf{5 3 . 5 9 5} \\
\mathbf{5 2 . 8 4 9} \\
\mathbf{5 2 . 4 5 2} \\
\mathbf{5 3 . 6 5 7} \\
\mathbf{5 4 . 6 6 8} \\
\mathbf{5 6 . 0 2 2}\end{array}$ & $\begin{array}{l}16.930 \\
17.356 \\
17.867 \\
18.842 \\
19.236 \\
20.123 \\
21.108 \\
21.359 \\
22.131 \\
22.111 \\
23.114 \\
24.586 \\
25.225 \\
25.393 \\
26.285 \\
27.443 \\
27.731 \\
28.416 \\
28.665 \\
28.487 \\
29.195 \\
29.565\end{array}$ & $\begin{array}{l}74.282 \\
72.543 \\
70.546 \\
74.362 \\
76.288 \\
78.089 \\
78.898 \\
75.955 \\
73.990 \\
70.848 \\
70.524 \\
74.144 \\
73.981 \\
74.297 \\
76.894 \\
80.218 \\
81.325 \\
81.265 \\
81.116 \\
82.144 \\
83.863 \\
85.587\end{array}$ & $\begin{array}{l}3,916.3 \\
3,891.2 \\
3,873.9 \\
4,082.9 \\
4,273.6 \\
4,503.0 \\
4,630.6 \\
4,615.0 \\
4,720.7 \\
4,620.3 \\
4,803.7 \\
5,140.1 \\
5,323.5 \\
5,487.7 \\
5,649.5 \\
5,865.2 \\
6,062.0 \\
6,136.3 \\
6,079.4 \\
6,244.4 \\
6,389.6 \\
6,610.7\end{array}$ & \begin{tabular}{|l|}
14.64 \\
14.18 \\
13.60 \\
13.60 \\
13.35 \\
12.87 \\
12.48 \\
11.83 \\
10.89 \\
10.55 \\
9.87 \\
9.64 \\
9.16 \\
8.91 \\
8.96 \\
9.00 \\
8.84 \\
8.61 \\
8.63 \\
8.59 \\
8.56 \\
8.47
\end{tabular} & $\begin{array}{l}4.32 \\
4.46 \\
4.61 \\
4.61 \\
4.50 \\
4.47 \\
4.56 \\
4.63 \\
4.69 \\
4.79 \\
4.81 \\
4.78 \\
4.74 \\
4.63 \\
4.65 \\
4.68 \\
4.57 \\
4.63 \\
4.72 \\
4.56 \\
4.57 \\
4.47\end{array}$ & $\begin{array}{l}18.97 \\
18.64 \\
18.21 \\
18.21 \\
17.85 \\
17.34 \\
17.06 \\
15.67 \\
15.33 \\
14.68 \\
14.66 \\
13.90 \\
13.88 \\
13.53 \\
13.61 \\
13.68 \\
13.42 \\
13.24 \\
13.34 \\
13.15 \\
13.12 \\
12.95\end{array}$ \\
\hline $\begin{array}{r}19951^{\text {st }} \text { Quarter ............ } \\
2^{\text {nd }} \text { Quarter ........... } \\
3^{\text {rd }} \text { Quarter ........... } \\
4^{\text {th }} \text { Quarter ........... } \\
\text { Year ...................... }\end{array}$ & $\begin{array}{l}\mathbf{5 6 . 5 3 7} \\
\mathbf{5 7 . 1 0 1} \\
\mathbf{5 6 . 8 1 3} \\
\mathbf{5 6 . 8 5 4} \\
\mathbf{5 6 . 8 2 7}\end{array}$ & $\begin{array}{l}R_{29.781} \\
R_{30.1335} \\
R_{30.972} \\
R_{30.1586} \\
R_{30.1373}\end{array}$ & $\begin{array}{l}R_{86.317} \\
R_{87.136} \\
R_{87.785} \\
R_{87.540} \\
R_{87.199}\end{array}$ & $\begin{array}{l}6,703.7 \\
6,708.8 \\
6,759.2 \\
6,796.5 \\
6,742.1\end{array}$ & $\begin{array}{l}8.43 \\
8.51 \\
8.41 \\
8.37 \\
8.43\end{array}$ & $\begin{array}{r}R_{4.44} \\
4.48 \\
R_{4.58} \\
R_{4.51} \\
4.50\end{array}$ & $\begin{array}{r}\mathrm{R}_{12.88} \\
12.99 \\
\mathrm{R} 12.99 \\
12.88 \\
12.93\end{array}$ \\
\hline $\begin{array}{r}19961^{\text {st }} \text { Quarter ............ } \\
2^{\text {nd }} \text { Quarter ........... } \\
3^{\text {rd }} \text { Quarter ........... } \\
4^{\text {th }} \text { Quarter ............ } \\
\text { Year ..................... }\end{array}$ & $\begin{array}{l}\mathbf{5 9 . 2 8 2} \\
\mathbf{5 8 . 5 9 1} \\
\mathbf{5 7 . 4 4 2} \\
\mathbf{5 8 . 3 9 2} \\
\mathbf{5 8 . 4 2 4}\end{array}$ & $\begin{array}{l}31.1663 \\
31.1390 \\
31.060 \\
31.718 \\
31.581\end{array}$ & $\begin{array}{l}90.945 \\
90.480 \\
88.501 \\
90.110 \\
90.005\end{array}$ & $\begin{array}{l}6,826.4 \\
6,926.0 \\
6,943.8 \\
7,017.4 \\
6,928.4\end{array}$ & $\begin{array}{l}8.68 \\
8.46 \\
8.27 \\
8.32 \\
8.43\end{array}$ & $\begin{array}{l}4.64 \\
4.60 \\
4.47 \\
4.52 \\
4.56\end{array}$ & $\begin{array}{l}13.32 \\
13.06 \\
12.75 \\
12.84 \\
12.99\end{array}$ \\
\hline $\begin{array}{r}19971^{\text {st }} \text { Quarter ........... } \\
2^{\text {nd }} \text { Quarter .......... } \\
3^{\text {rd }} \text { Quarter ........... }\end{array}$ & $\begin{array}{l}R_{58.187} \\
R_{59.165} \\
R_{59.260}\end{array}$ & $\begin{array}{l}R_{31.534} \\
R_{31.505} \\
R_{31.1549}\end{array}$ & $\begin{array}{l}{ }^{R} 89.722 \\
R_{90.670} \\
\text { P }_{90.909}\end{array}$ & $\begin{array}{r}7,101.6 \\
7,159.6 \\
R 7,214.0\end{array}$ & $\begin{array}{r}8.19 \\
\text { म } 8.26 \\
8.21\end{array}$ & $\begin{array}{l}4.44 \\
4.40 \\
4.39\end{array}$ & $\begin{array}{r}12.63 \\
R_{12.66} \\
P_{12.60}\end{array}$ \\
\hline
\end{tabular}

a Due to a lack of consistent monthly historical data, some renewable energy sources are not included in other eriergy or total consumption. For example, in 1995, 3.4 quadrillion Btu of renewable energy used by electric utilities to generate electricity for distribution is included, but an estimated 3.3 quadrillion Btu used by residential, commercial, and industrial consumers is not. See Note 12 at the end of Section 2 for cletails.

$R=$ Revised data.

Notes: - Quarterly data are seasonally adjusted and shown at annual rates. Yearly data may not equal average of quarters due to seasonality adjustments and independent rounding. - Totals may not equal sum of components due to independent rounding. - Geographic coverage is the 50 States and the District of Columbia.

Sources: - Energy Consumption: Table 1.4. - Gross Domestic Product: 1973-1995-U.S. Department of Commerce, Bureau of Economic Analysis, Survey of Current Business, May 1997, Table 2A. 1996 forward-U.S. Department of Commerce, Bureau of Economic Analysis, United States Department of Commerce News, December 23, 1997, Table 2. 
Figure 1.9 Passenger Car Efficiency

(Index, 1973 = 100)

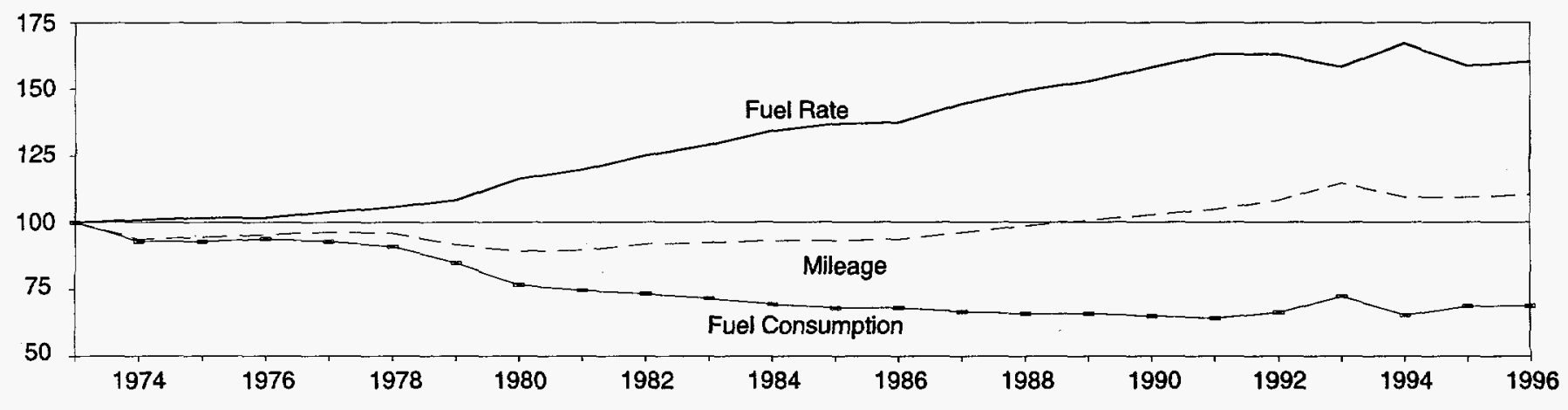

Table 1.10 Passenger Car Efficiency

\begin{tabular}{|c|c|c|c|c|c|c|}
\hline & \multicolumn{2}{|c|}{ Mileage } & \multicolumn{2}{|c|}{ Fuel Consumption } & \multicolumn{2}{|c|}{ Fuel Rate } \\
\hline & $\begin{array}{l}\text { Miles } \\
\text { per Car }\end{array}$ & $\begin{array}{c}\text { Index } \\
1973=100.0\end{array}$ & $\begin{array}{l}\text { Gallons } \\
\text { per Car }\end{array}$ & $\begin{array}{c}\text { Index } \\
1973=100.0\end{array}$ & $\begin{array}{c}\text { Miles } \\
\text { per Gallon }\end{array}$ & $\begin{array}{c}\text { Index } \\
1973=100.0\end{array}$ \\
\hline $\begin{array}{l}1973 \\
1974 \\
1975 \\
1976 \\
1977 \\
1978 \\
1979 \\
1980 \\
1981 \\
1982 \\
1983 \\
1984 \\
1985 \\
1986 \\
1987 \\
1988 \\
1989 \\
1990 \\
1991 \\
1992 \\
1993 \\
1994 \\
1995 \\
1996\end{array}$ & $\begin{array}{r}10,256 \\
9,606 \\
9,690 \\
9,785 \\
9,879 \\
9,835 \\
9,403 \\
9,141 \\
9,186 \\
9,428 \\
9,475 \\
9,558 \\
9,560 \\
9,608 \\
9,878 \\
10,121 \\
10,332 \\
10,548 \\
10,757 \\
11,100 \\
11,760 \\
11,210 \\
11,203 \\
11,314\end{array}$ & $\begin{array}{r}100.0 \\
93.7 \\
94.5 \\
95.4 \\
96.3 \\
95.9 \\
91.7 \\
89.1 \\
89.6 \\
91.9 \\
92.4 \\
93.2 \\
93.2 \\
93.7 \\
96.3 \\
98.7 \\
100.7 \\
102.8 \\
104.9 \\
108.2 \\
114.7 \\
109.3 \\
109.2 \\
110.3\end{array}$ & $\begin{array}{l}\mathbf{7 7 1} \\
\mathbf{7 1 6} \\
716 \\
\mathbf{7 2 3} \\
\mathbf{7 1 6} \\
\mathbf{7 0 1} \\
\mathbf{6 5 3} \\
\mathbf{5 9 1} \\
\mathbf{5 7 6} \\
\mathbf{5 6 6} \\
\mathbf{5 5 3} \\
\mathbf{5 3 6} \\
\mathbf{5 2 5} \\
\mathbf{5 2 6} \\
\mathbf{5 1 4} \\
\mathbf{5 0 9} \\
\mathbf{5 0 9} \\
\mathbf{5 0 2} \\
\mathbf{4 9 6} \\
\mathbf{5 1 2} \\
\mathbf{5 5 9} \\
\mathbf{5 0 4} \\
\mathbf{5 3 0} \\
\mathbf{5 3 1}\end{array}$ & $\begin{array}{r}100.0 \\
92.9 \\
92.9 \\
93.8 \\
92.9 \\
90.9 \\
84.7 \\
76.7 \\
74.7 \\
73.4 \\
71.7 \\
69.5 \\
68.1 \\
68.2 \\
66.7 \\
66.0 \\
66.0 \\
65.1 \\
64.3 \\
66.4 \\
72.5 \\
65.4 \\
68.7 \\
68.9\end{array}$ & $\begin{array}{l}13.30 \\
13.42 \\
13.52 \\
13.53 \\
13.80 \\
14.04 \\
14.41 \\
15.46 \\
15.94 \\
16.65 \\
17.14 \\
17.83 \\
18.20 \\
18.27 \\
19.20 \\
19.87 \\
20.31 \\
21.02 \\
21.69 \\
21.68 \\
21.04 \\
22.24 \\
21.10 \\
21.30\end{array}$ & $\begin{array}{l}100.0 \\
100.9 \\
101.7 \\
101.7 \\
103.8 \\
105.6 \\
108.3 \\
116.2 \\
119.8 \\
125.2 \\
128.9 \\
134.1 \\
136.8 \\
137.4 \\
144.4 \\
149.4 \\
152.7 \\
158.0 \\
163.1 \\
163.0 \\
158.2 \\
167.2 \\
158.6 \\
160.2\end{array}$ \\
\hline
\end{tabular}

a Preliminary data.

Note: Geographic coverage is the 50 States and the District of Columbia.

Sources: Indices are prepared from statistics published by the U.S.

Department of Transportation, Federal Highway Administration, Federal
Highway Statistics Division. 1973-1985: Highway Statistics Summary to 1985, Table VM-201A. - 1986 forward: Highway Statistics, annual, Table VM-1. 


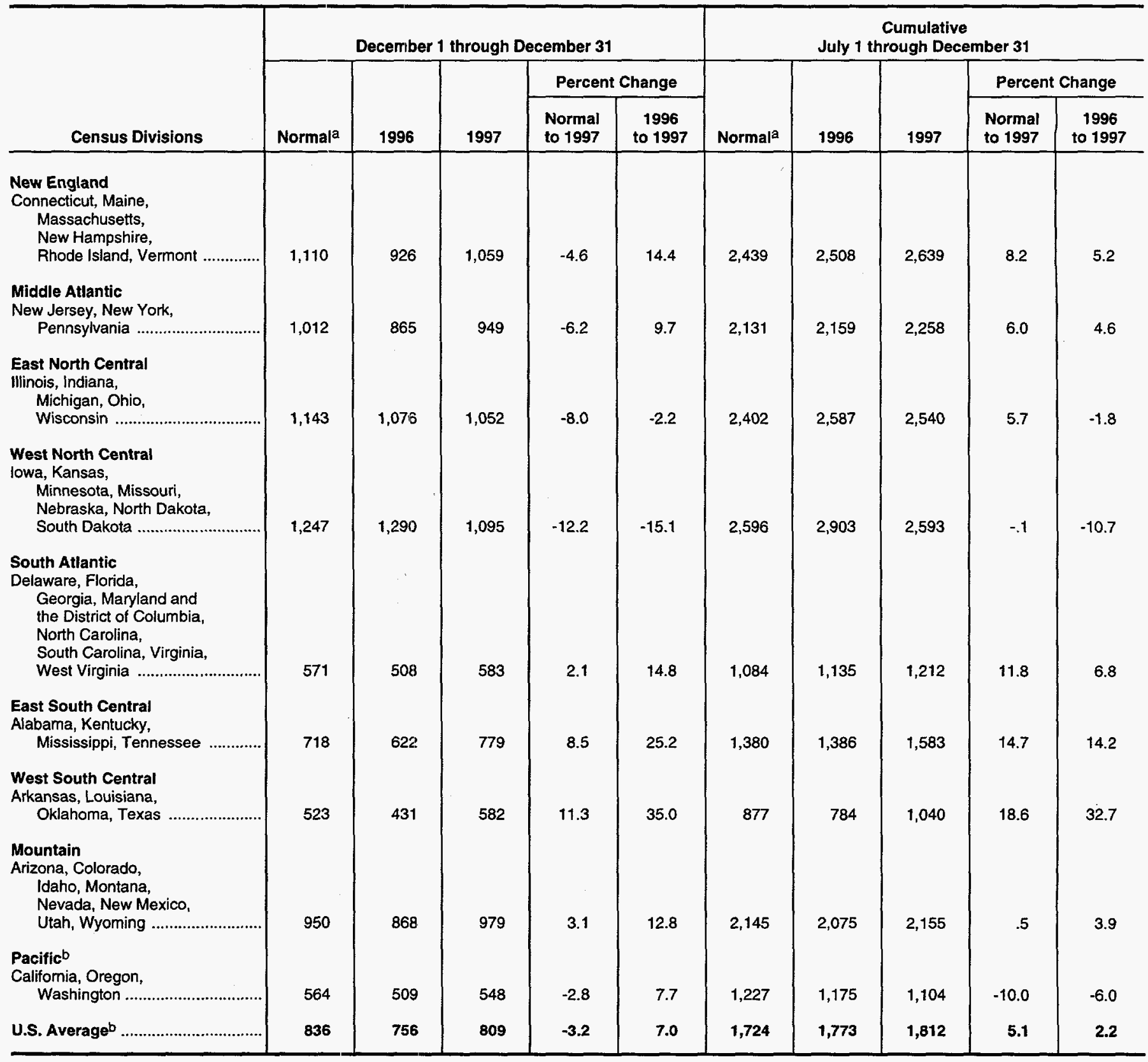

a "Normal" is based on calculations of data from 1961 through 1990.

b Excludes Alaska and Hawaii.

Notes: Degree-days are relative measurements of outcioor air temperature used as an index for heating and cooling eriergy requirements. Heating degree-days are the number of degrees that the daily average temperature falls below $65^{\circ} \mathrm{F}$. Cooling degree-days are the number of degrees that the daily average temperature rises above $65^{\circ} \mathrm{F}$. The daily average temperature is the mean of the maximum and minimum temperatures in a 24-hour period. For example, a weather station recording an average daily temperature of $40^{\circ}$ $F$ would report 25 heating degree-days for that day (and 0 cooling degree-days). If a weather station recorded an average daily temperature of $78^{\circ} \mathrm{F}$, cooling degree-days for that station would be 13 (and 0 heating degree days).

Sources: See end of section. 


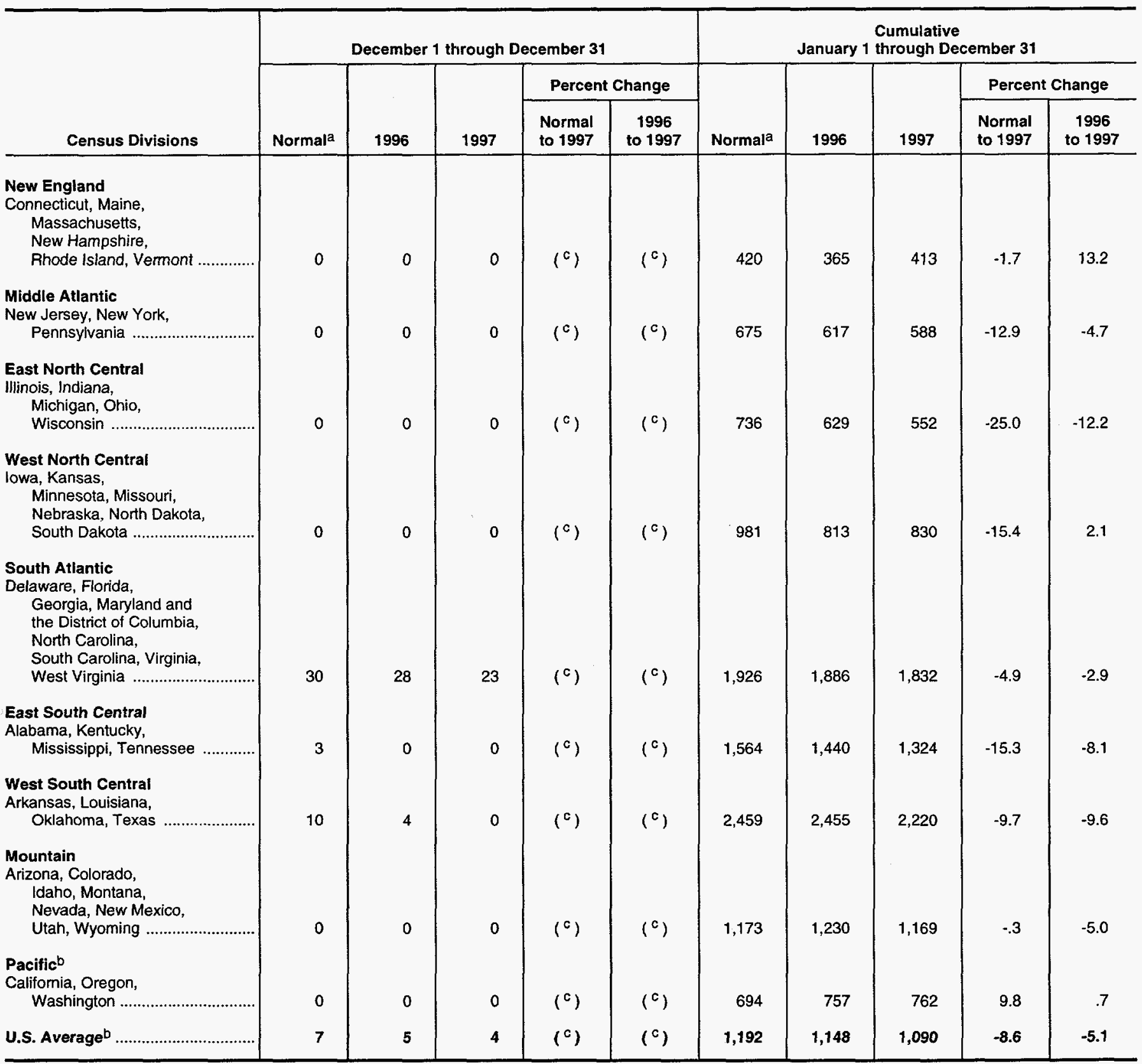

a "Normal" is based on calculations of data from 1961 through 1990.

b Excludes Alaska and Hawaii.

c Percent change is not meaningful: normal is less than 100 or ratio is incalculable.

Notes: Degree-days are relative measurements of outdoor air temperature used as an index for heating and cooling energy requirements. Cooling degree-days are the number of degrees that the daily average temperature rises above $65^{\circ} \mathrm{F}$. Heating degree-days are the number of degrees that the daily average temperature falls below $65^{\circ} \mathrm{F}$. The daily average temperature is the mean of the maximum and minimum temperatures in a 24-hour period. For example, if a weather station recorded an average daily temperature of $78^{\circ} \mathrm{F}$, cooling degree-days for that station would be 13 (and 0 heating degree-days). A weather station recording an average daily temperature of $40^{\circ} \mathrm{F}$ would report 25 heating degree-days for that day (and 0 cooling degreedays).

Sources: See end of section. 


\section{Energy Summary Notes}

1. Energy Production: Production of energy includes production of coal, crude oil and lease condensate, natural gas plant liquids, natural gas (dry), electric utility and industrial production of hydroslectric power, and electricity generated from nuclear power. Production also includes electricity generated for distribution from wood, waste, geothermal, wind, photovoltaic, and solar thermal energy but excludes other energy obtained from those sources because consistent historical data are not available. Approximate heat contents (Btu values) are derived by using the conversion factors provided in Appendix A.

2. Energy Consumption: Consumption of energy includes consumption of coal, natural gas (including supplemental gaseous fuels), petroleum products supplied, electric utility and industrial production of hydroelectric power, net imports of electricity (assumed to be hydroelectricity), net imports of coal coke, and electricity generated from nuclear power. Consumption also includes electricity generated for distribution from geothermal, wood, waste, wind, photovoltaic, and solar thermal energy but excludes other energy obtained from those sources because consistent historical data are not available. Approximate heat contents (Btu values) are derived by using the conversion factors provided in Appendix A.

3. Energy Imports: Energy imports include imports of coal, crude oil (including crude oil imported for the Strategic Petroleum Reserve), petroleum products, natural gas, electricity (assumed to be hydroelectricity), and coal coke. Approximate heat contents (Btu values) are derived by using the conversion factors provided in Appendix A. For further information on electricity, see "Note for imports and exports of electricity" under Note 8 of Section 2, Energy Consumption Section Notes and Sources.

4. Energy Exports: Energy exports include coal, crude oil, petroleum products, natural gas, electricity produced from hydroelectric power, and coal coke. Approximate heat contents (Btu values) are derived by using the conversion factors provided in Appendix A. For more information on electricity, see "Note for imports and exports of electricity" under Note 8 of Section 2, Energy Consumption Section Notes and Sources.

5. Merchandise Trade Value: Import data presented are based on the customs value. That value does not include insurance and freight and is consequently lower than the cost, insurance, and freight (CIF) value, which is also reported by the Bureau of the Census. All export data, and import data prior to 1981 , are on a free alongside ship (f.a.s.) basis.

"Balance" is exports minus imports; a positive balance indicates a surplus trade value and a negative balance indicates a deficit trade value. "Energy" includes mineral fuels, lubricants, and related material. "Non-Energy Balance" and "Total Merchandise" include foreign exports (i.e., re-exports) and nonmonetary gold and Department of Defense Grant-Aid shipments. The
"Non-Energy Balance" is calculated by subtracting the "Energy" from the "Total Merchandise Balance."

"Imports" consist of government and nongovernment shipments of merchandise into the 50 States, the District of Columbia, Puerto Rico, the U.S. Virgin Islands, and the U.S. Foreign Trade Zones. They reflect the total arrival from foreign countries of merchandise that immediately entered consumption channels, warehouses, the Foreign Trade Zones, or the Strategic Petroleum Reserve. They exclude shipments between the United States, Puerto Rico, and U.S. possessions, shipments to U.S. Armed Forces and diplomatic missions abroad for their own use, U.S. goods returned to the United States by its Armed Forces, and in-transit shipments.

\section{Sources for Table 1.6}

U.S. Department of Commerce, Bureau of the Census, Foreign Trade Division:

\section{Petroleum Exports}

1974-1987: "U.S. Exports," FT410, December issues. 1988: "Report on U.S. Merchandise Trade, 1988 Final Revisions."

1989: "Report on U.S. Merchandise Trade, 1989 Revisions."

1990: "U.S. Merchandise Trade, 1990 Final Report."

1991: "U.S. Merchandise Trade, 1991 Final Report," May 13, 1992.

1992: "U.S. Merchandise Trade, 1992 Final Report," May 12, 1993.

1993: "U.S. International Trade in Goods and Services, Annual Revision for 1993."

1994: "U.S. International Trade in Goods and Services, Annual Revision for 1994."

1995: "U.S. International Trade in Goods and Services, Annual Revision for 1995."

1996: "U.S. International Trade in Goods and Services, Annual Revision for 1996."

1997: "U.S. International Trade in Goods and Services," FT-900, monthly.

\section{Petroleum Imports}

1974-1987: "U.S. Merchandise Trade," FT900, December issues, 1975-1988.

1988: "Report on U.S. Merchandise Trade, 1988 Final Revisions."

1989: "Report on U.S. Merchandise Trade, 1989 Revisions."

1990: "U.S. Merchandise Trade, 1990 Final Report." 1991: "U.S. Merchandise Trade, 1991 Final Report," May 13, 1992, and "U.S. Merchandise Trade, October 1992," December 17, 1992, page 3.

1992: "U.S. Merchandise Trade, 1992 Final Report," May 12, 1993.

1993: "U.S. International Trade in Goods and Services, Annual Revision for 1993."

1994: "U.S. International Trade in Goods and Services, Annual Revision for 1994."

1995: "U.S. International Trade in Goods and Services, 
Annual Revision for 1995."

1996: "U.S. International Trade in Goods and Services, Annual Revision for 1996."

1997: "U.S. International Trade in Goods and Services," FT-900, monthly.

\section{Energy Exports and Imports}

1974-1987: U.S. merchandise trade press releases and database printouts for adjustments.

1988: January-July, monthly FT-900 supplement, 1989 issues. August-December, monthly FT-900, 1989 issues. 1989: Monthly FT-900, 1990 issues.

1990: "U.S. Merchandise Trade, 1990 Final Report." 1991: "U.S. Merchandise Trade, 1991 Final Report," May 13, 1992, and "U.S. Merchandise Trade, October 1992," December 17, 1992, page 3.

1992: "U.S. Merchandise Trade, 1992 Final Report," May $12,1993$.

1993: "U.S. International Trade in Goods and Services, Annual Revision for 1993."

1994: "U.S. International Trade in Goods and Services, Annual Revision for 1994."

1995: "U.S. International Trade in Goods and Services, Annual Revision for 1995."

1996: "U.S. International Trade in Goods and Services, Annual Revision for 1996."

1997: "U.S. International Trade in Goods and Services," FT-900, monthly.

\section{Energy and Non-Energy Balances}

Calculated by the Energy Information Administration.

\section{Total Merchandise}

1974-1987: U.S. merchandise trade press releases and database printouts for adjustments.

1988: "Report on U.S. Merchandise Trade, 1988 Final Revisions," August 18, 1989.

1989: "Report on U.S. Merchandise Trade, 1989 Revi- sions," July 10, 1990.

1990: "U.S. Merchandise Trade, 1990 Final Report," May 10, 1991, and "U.S. Merchandise Trade, December 1992," February 18, 1993, page 3.

1991: "U.S. Merchandise Trade, 1992 Final Report," May 12, 1993.

1992: "U.S. International Trade in Goods and Services, Annual Revision for 1994."

1993-1994: "U.S. International Trade in Goods and Services, Annual Revision for 1995."

1995-1996: "U.S. International Trade in Goods and Services, Annual Revision for 1996."

1997: "U.S. International Trade in Goods and Services," FT-900, monthly.

\section{Sources for Tables 1.11 and 1.12}

There are several degree-day databases maintained by the National Oceanic and Atmospheric Administration. The information published here is developed by the $\mathrm{Na}$ tional Weather Service Climate Analysis Center, Camp Springs, MD. The data are available weekly with monthly summaries and are based on mean daily temperatures recorded at about 200 major weather stations around the country. The temperature information recorded at those weather stations is used to calculate statewide degree-day averages based on population.

The State figures are then aggregated into Census Divisions and into the national average. The population weights currently used represent resident State popula. tion data estimated for 1990 by the U.S. Department of Commerce, Bureau of the Census. The data provided here are available sooner than the Historical Climatology Series 5-1 (heating degree-days) and 5-2 (cooling degree-days) developed by the National Climatic Data Center, Asheville, NC, which compiles data from some 8,000 weather stations. 


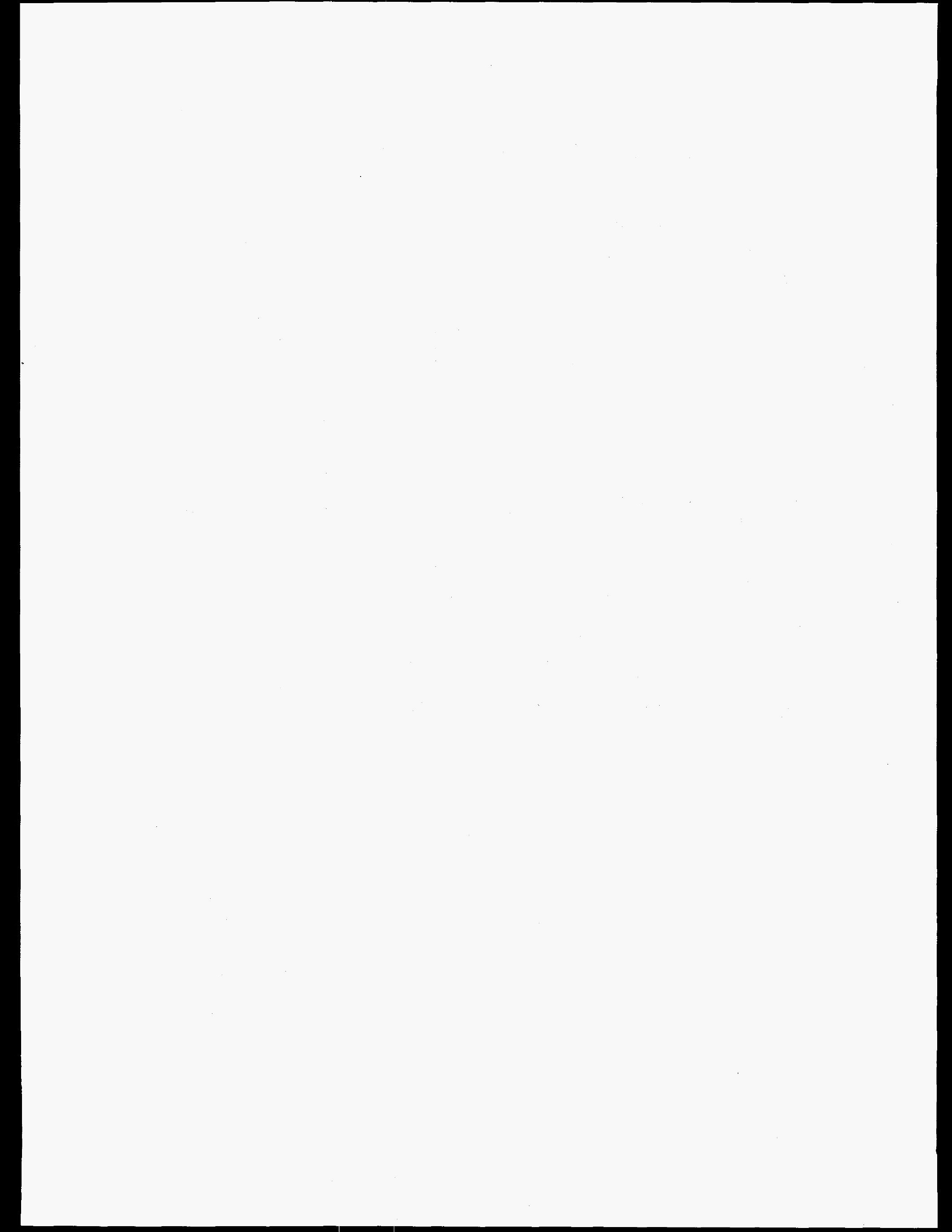




\section{Section 2. Energy Consumption}

U.S. total energy consumption in October 1997 was 7.4 quadrillion Btu. Petroleum products accounted for 43 percent of the energy consumed in October 1997, while coal accounted for 24 percent, and natural gas accounted for 22 percent.

Residential and commercial sector consumption was 2.4 quadrillion Btu in October 1997, up 7 percent from the October 1996 level. The sector accounted for 32 percent of October 1997 total consumption, up 1 percentage point from its 31 percent share in October 1996.

Industrial sector consumption was 2.8 quadrillion Btu in October 1997, up slightly from the October 1996 level. The industrial sector accounted for 39 percent of October 1997 total consumption, about the same share as in October 1996.
Transportation sector consumption of energy was 2.1 quadrillion Btu in October 1997, down 1 percent from the October 1996 level. The sector accounted for 29 percent of October 1997 total consumption, down 1 percentage point from its 30-percent share in October 1996.

Electric utility consumption of energy totaled 2.7 quadrillion Btu in October 1997, up 5 percent from the October 1996 level. Coal contributed 59 percent of the energy consumed by electric utilities in October 1997, while nuclear electric power contributed 19 percent; hydroelectric 10 percent; natural gas 9 percent; petroleum 3 percent; and geothermal, wood, waste, wind, photovoltaic, and solar thermal energy, less than 1 percent.

\section{Table 2.1 Energy Consumption Summary for October 1997}

(Quadrillion Btu)

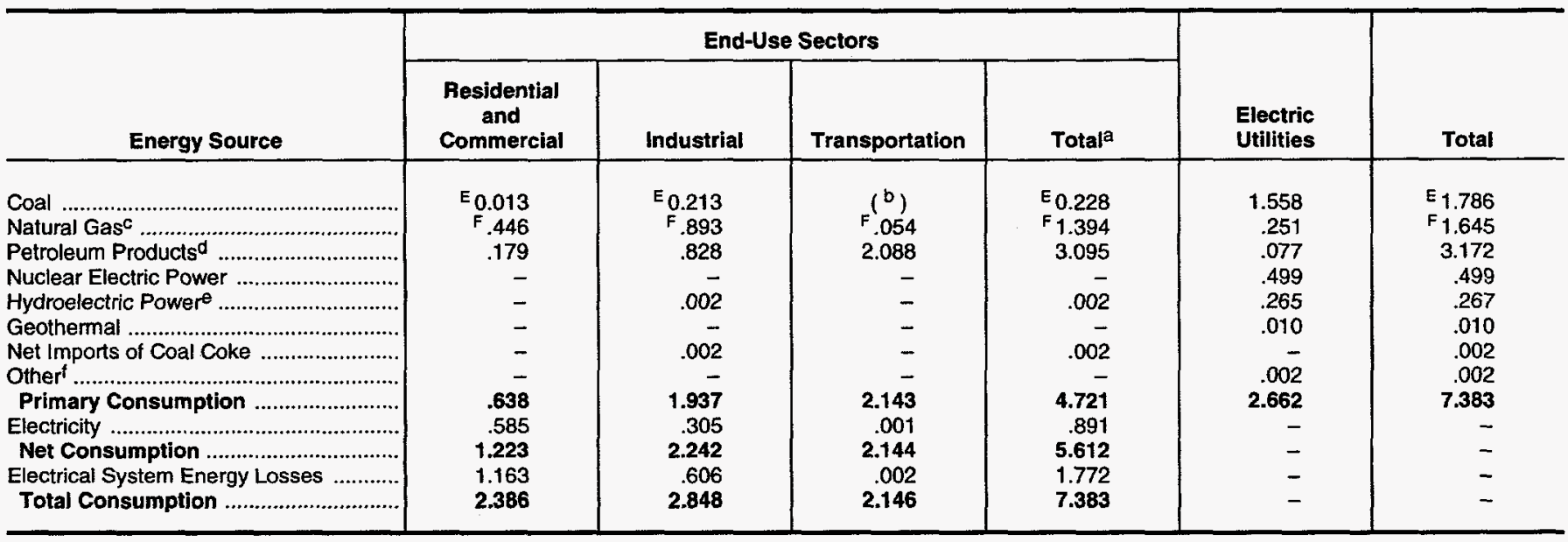

a Totals for coal and natural gas may not equal sum of sectors due to the use of sector-specific conversion factors.

b Small amounts of coal consumed for transportation are reported as industrial sector consumption.

c Includes supplemental gaseous fuels. Transportation sector is pipeline fuel only.

d Products obtained from the processing of crude oil (including lease condensate), natural gas, and other hydrocarbon compounds.

$e$ includes net imports of electricity. $f$ "Other" is electricity generated for distribution from wood, waste, wind, photovoltaic, and solar thermal energy.

$-=$ Not applicable. (s) $=$ Less than +0.5 trillion Btu and greater than -0.5

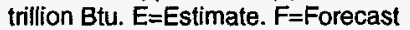

Notes: - Totals may not equal sum of components due to independent rounding. - Geographic coverage is the 50 States and the District of Columbia.

Additional Notes and Sources: See Tables 2.2-2.6 and end of section.

Please Read: Due to a lack of consistent monthly historical data, some renewable energy sources are not included in total consumption. For the full year of 1996, for example, 3.9 quadrillion Btu of renewable energy used by electric utilities to generate electricity for distribution is included, but an estimated 3.4 quadrillion Btu used by residential, commercial, and industrial consumers is not. See Note 12 at the end of section for details. 
Figure 2.1 Energy Consumption by End-Use Sector

(Quadrillion Btu)

Oveniew, 1973-1996

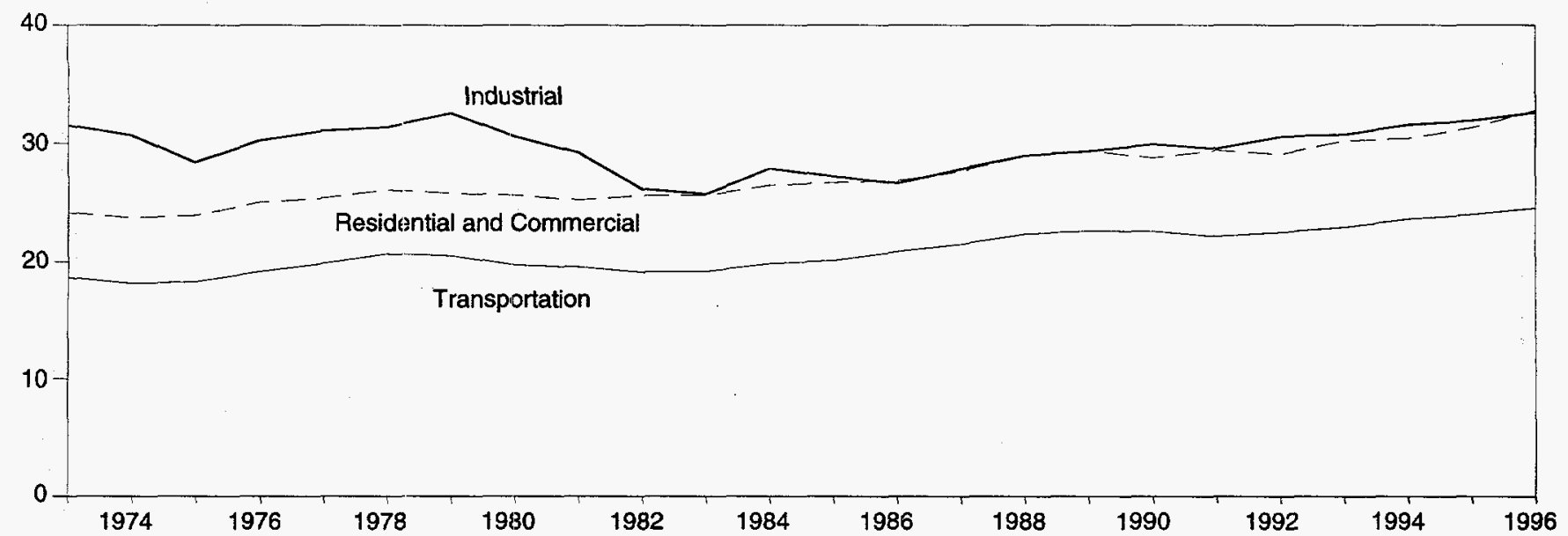

\section{Overview, Monthly}

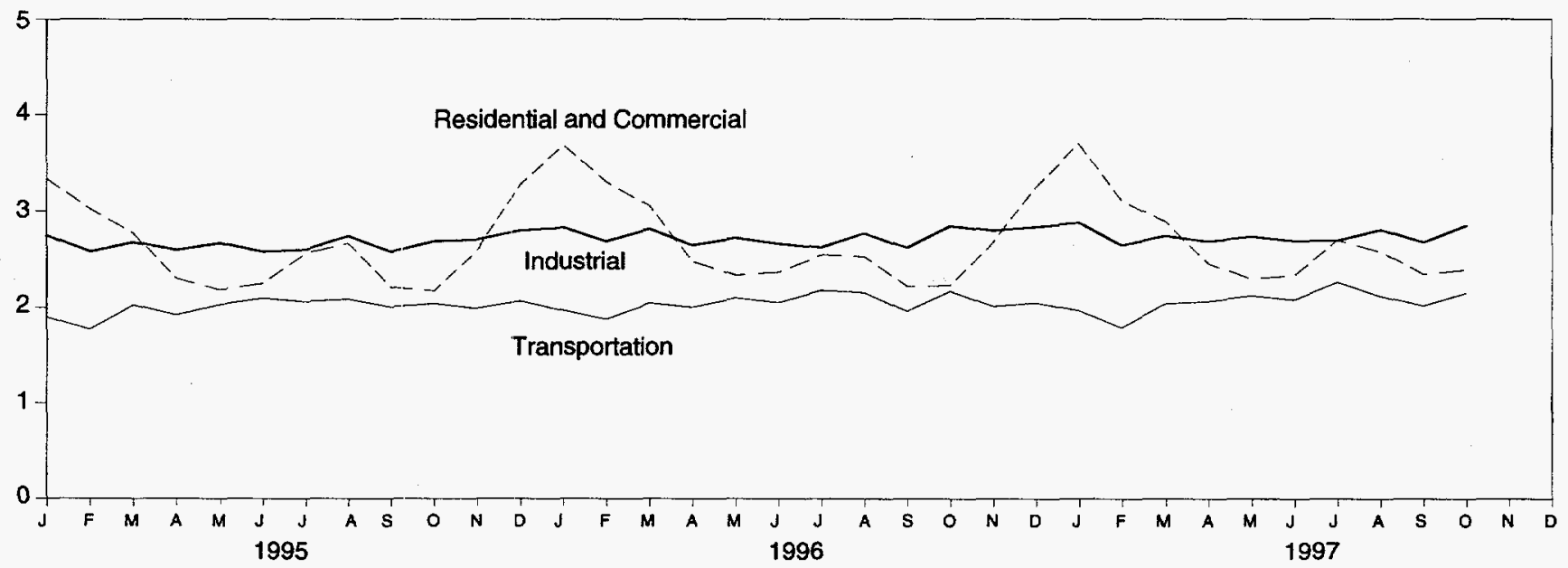

\section{Overview, October}

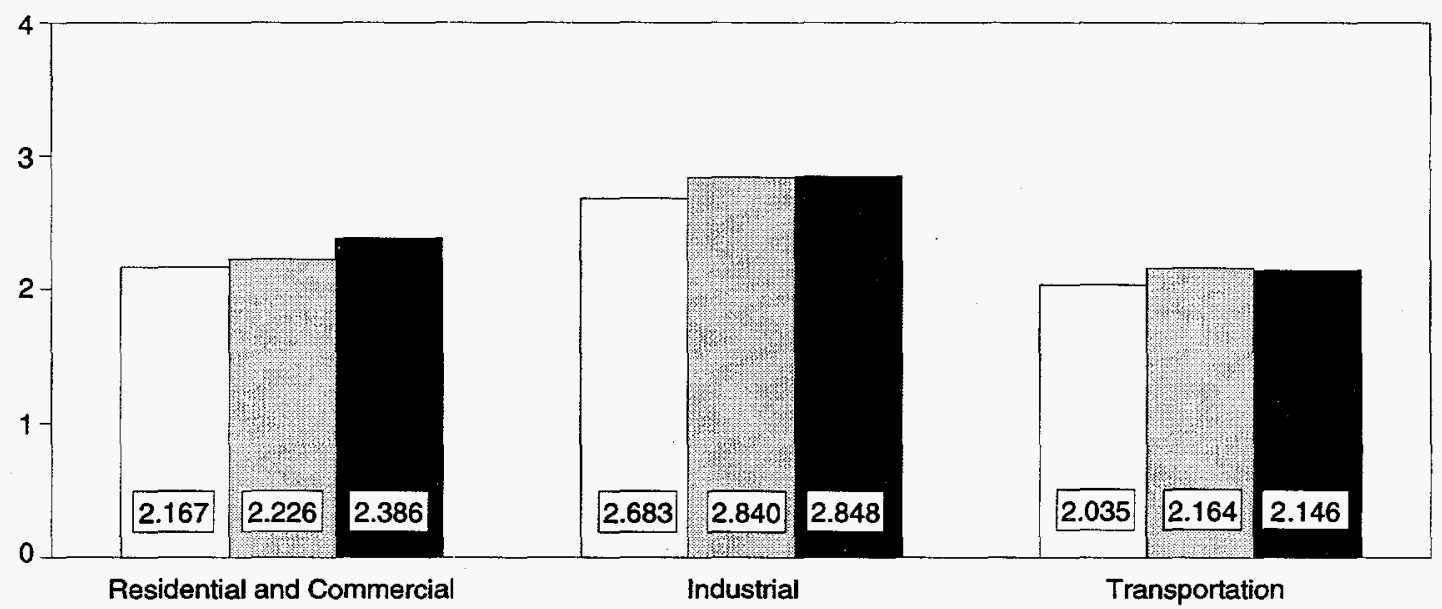

October 1995

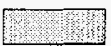

October 1996

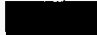

October 1997

Note: Because vertical scales differ, graphs should not be compared. Source: Table 2.2. 
Table 2.2 Energy Consumption by End-Use Sector (Quadrillion Btu)

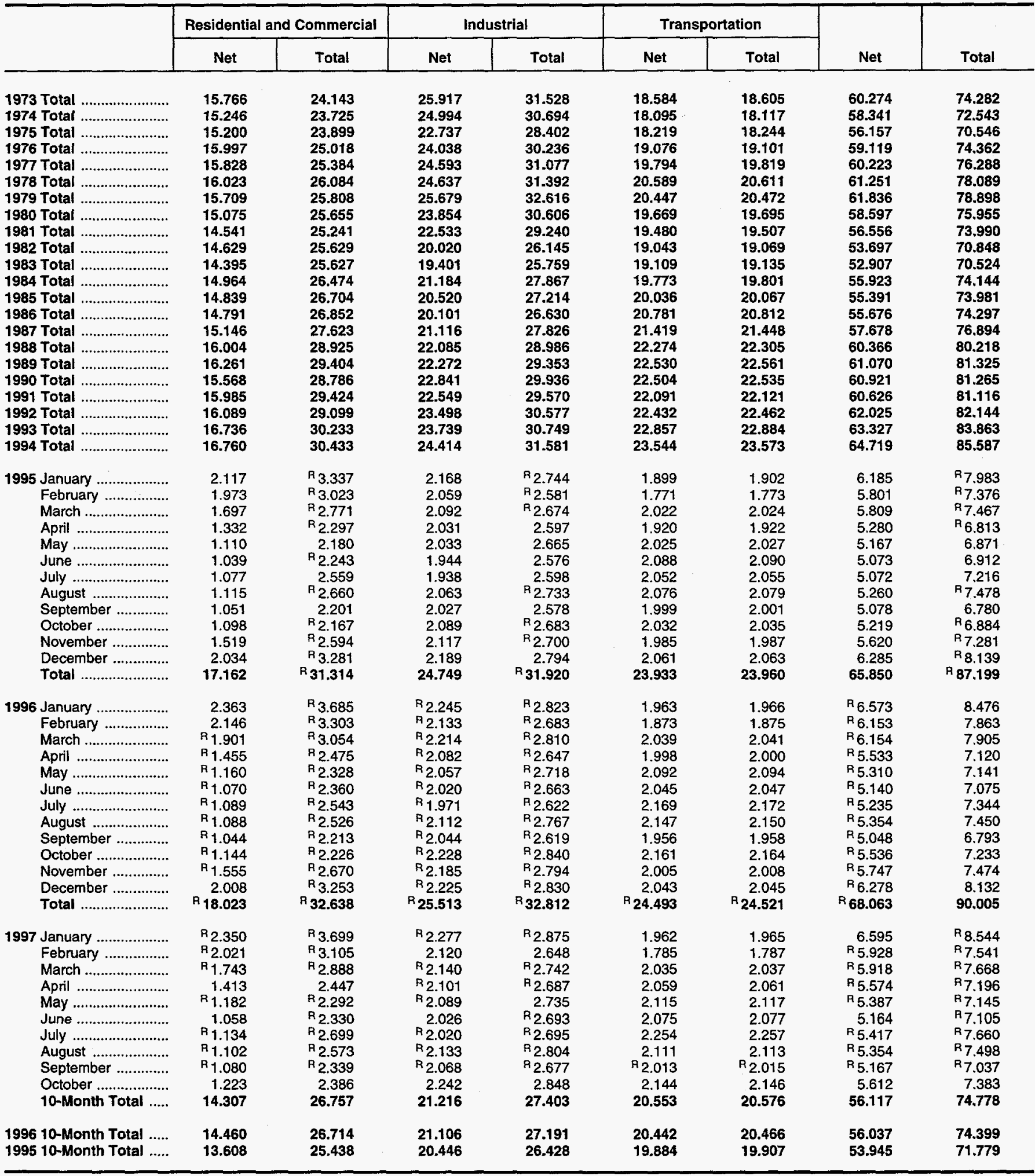

R=Revised data.

Notes: - Totals may not equal sum of components due to independent rounding and the use of sector-specific conversion factors for natural gas and coal. - Geographic coverage is the 50 States and the District of Columbia. Additional Notes and Sources: See end of section.

Please Read: Due to a lack of consistent monthly historical data, some renewable energy sources are not included in total consumption. In 1996, for example, 3.9 quadrillion Btu of renewable energy used by electric utilities to generate electricity for distribution is included, but an estimated 3.4 quadrillion Btu used by residential, commercial, and industrial consumers is not. See Note 12 at the end of section for details. 
Figure 2.2 Residential and Commercial Energy Consumption

(Quadrillion Btu)

By Major Sources, 1973-1996

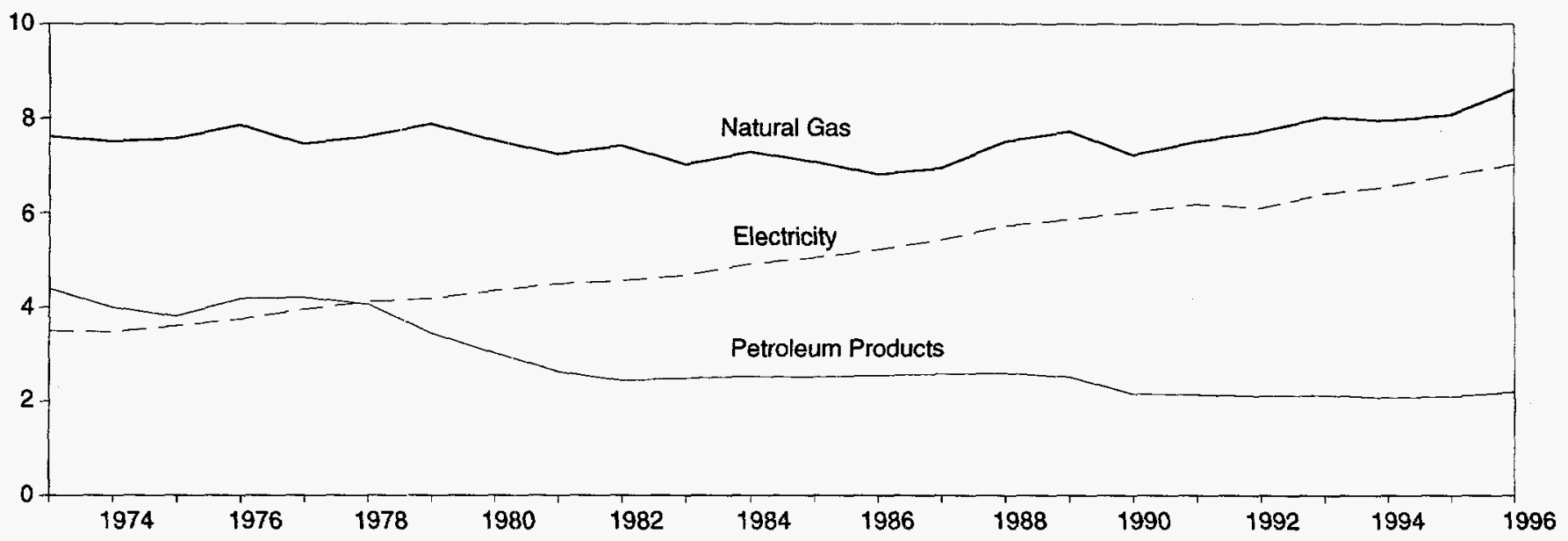

By Major Sources, Monthly

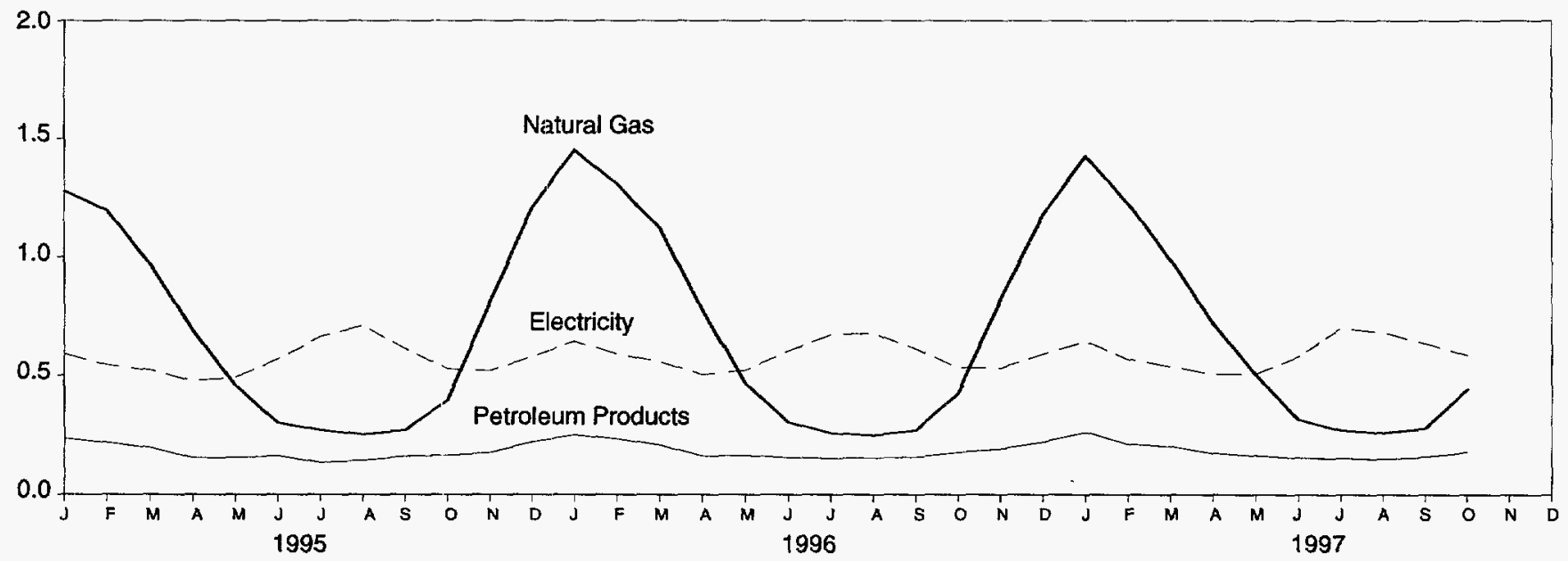

Total, January-October

By Major Sources, October 1997
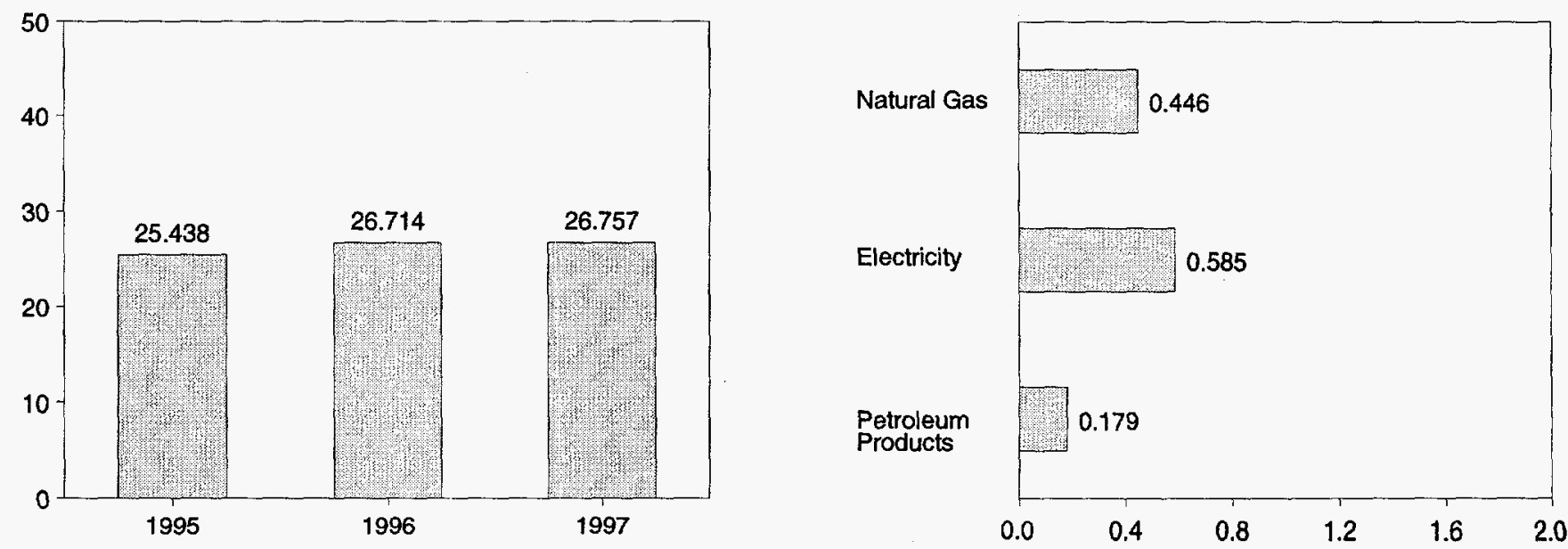

Note: Because vertical scales differ, graphs should not be compared.

Source: Table 2.3. 
Table 2.3 Residential and Commercial Energy Consumption

(Quadrillion Btu)

\begin{tabular}{|c|c|c|c|c|c|c|c|c|}
\hline & Coal & $\begin{array}{c}\text { Natural } \\
\text { Gasa }\end{array}$ & $\begin{array}{l}\text { Petroleum } \\
\text { Products }^{b}\end{array}$ & $\begin{array}{c}\text { Primary } \\
\text { Consumption }\end{array}$ & Electricity & $\begin{array}{c}\text { Net } \\
\text { Consumption }\end{array}$ & $\begin{array}{c}\text { Electrical } \\
\text { System } \\
\text { Energy } \\
\text { Losses }\end{array}$ & $\begin{array}{c}\text { Total } \\
\text { Consumption }\end{array}$ \\
\hline 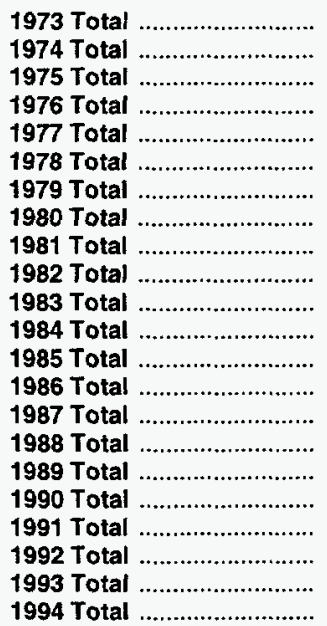 & $\begin{array}{l}0.254 \\
.257 \\
.209 \\
.203 \\
.205 \\
.214 \\
.187 \\
.145 \\
.167 \\
.187 \\
.192 \\
.209 \\
.176 \\
.176 \\
.162 \\
.168 \\
.146 \\
.156 \\
.141 \\
.142 \\
.143 \\
.139\end{array}$ & $\begin{array}{l}7.626 \\
7.518 \\
7.581 \\
7.866 \\
7.461 \\
7.624 \\
7.891 \\
7.540 \\
7.243 \\
7.427 \\
7.024 \\
7.292 \\
7.079 \\
6.825 \\
6.954 \\
7.513 \\
7.731 \\
7.224 \\
7.510 \\
7.725 \\
8.037 \\
7.967\end{array}$ & $\begin{array}{l}4.391 \\
3.996 \\
3.805 \\
4.181 \\
4.206 \\
4.070 \\
3.448 \\
3.035 \\
2.634 \\
2.449 \\
2.498 \\
2.535 \\
2.522 \\
2.555 \\
2.587 \\
2.600 \\
2.525 \\
2.173 \\
2.154 \\
2.126 \\
2.140 \\
2.094\end{array}$ & $\begin{array}{r}12.270 \\
11.771 \\
11.595 \\
12.250 \\
11.873 \\
11.908 \\
11.525 \\
10.721 \\
10.043 \\
10.063 \\
9.715 \\
10.036 \\
9.777 \\
9.556 \\
9.703 \\
10.280 \\
10.402 \\
9.553 \\
9.805 \\
9.993 \\
10.320 \\
10.200\end{array}$ & $\begin{array}{l}3.495 \\
3.475 \\
3.604 \\
3.747 \\
3.955 \\
4.116 \\
4.184 \\
4.355 \\
4.497 \\
4.566 \\
4.680 \\
4.928 \\
5.061 \\
5.235 \\
5.443 \\
5.724 \\
5.859 \\
6.015 \\
6.180 \\
6.096 \\
6.416 \\
6.560\end{array}$ & $\begin{array}{l}15.766 \\
15.246 \\
15.200 \\
15.997 \\
15.828 \\
16.023 \\
15.709 \\
15.075 \\
14.541 \\
14.629 \\
14.395 \\
14.964 \\
14.839 \\
14.791 \\
15.146 \\
16.004 \\
16.261 \\
15.568 \\
15.985 \\
16.089 \\
16.736 \\
16.760\end{array}$ & $\begin{array}{r}8.377 \\
8.480 \\
8.700 \\
9.021 \\
9.556 \\
10.061 \\
10.100 \\
10.580 \\
10.700 \\
11.000 \\
11.232 \\
11.510 \\
11.865 \\
12.061 \\
12.477 \\
12.920 \\
13.143 \\
13.218 \\
13.439 \\
13.010 \\
13.497 \\
13.673\end{array}$ & $\begin{array}{l}24.143 \\
23.725 \\
23.899 \\
25.018 \\
25.384 \\
26.084 \\
25.808 \\
25.655 \\
25.241 \\
25.629 \\
25.627 \\
26.474 \\
26.704 \\
26.852 \\
27.623 \\
28.925 \\
29.404 \\
28.786 \\
29.424 \\
29.099 \\
30.233 \\
30.433\end{array}$ \\
\hline 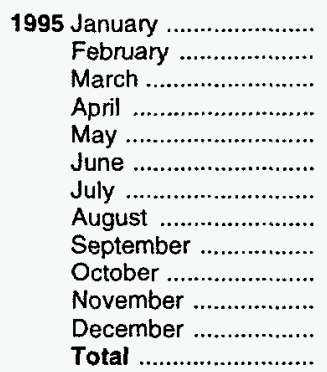 & $\begin{array}{l}.015 \\
.013 \\
.010 \\
.010 \\
.007 \\
.007 \\
.009 \\
.009 \\
.006 \\
.008 \\
.017 \\
.024 \\
.135\end{array}$ & $\begin{array}{l}1.276 \\
1.197 \\
.968 \\
.691 \\
.457 \\
.300 \\
.270 \\
.252 \\
.271 \\
.398 \\
.807 \\
1.209 \\
8.094\end{array}$ & $\begin{array}{l}.235 \\
.218 \\
.196 \\
.154 \\
.155 \\
.162 \\
.134 \\
.143 \\
.161 \\
.164 \\
.176 \\
.221 \\
2.120\end{array}$ & $\begin{array}{r}1.526 \\
1.428 \\
1.174 \\
.855 \\
.618 \\
.469 \\
.414 \\
.404 \\
.438 \\
.570 \\
.999 \\
1.454 \\
10.349\end{array}$ & $\begin{array}{l}.591 \\
.544 \\
.523 \\
.477 \\
.492 \\
.570 \\
.664 \\
.711 \\
.613 \\
.528 \\
.520 \\
.580 \\
6.813\end{array}$ & $\begin{array}{r}2.117 \\
1.973 \\
1.697 \\
1.332 \\
1.110 \\
1.039 \\
1.077 \\
1.115 \\
1.051 \\
1.098 \\
1.519 \\
2.034 \\
17.162\end{array}$ & 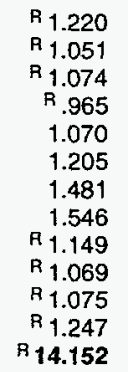 & $\begin{array}{r}R_{3.337} \\
R_{3.023} \\
R_{2.771} \\
R_{2.297} \\
2.180 \\
R_{2.243} \\
2.559 \\
R_{2.660} \\
2.201 \\
R_{2.167} \\
{ }_{2} 2.594 \\
{ }^{2} 3.281 \\
R_{31.314}\end{array}$ \\
\hline 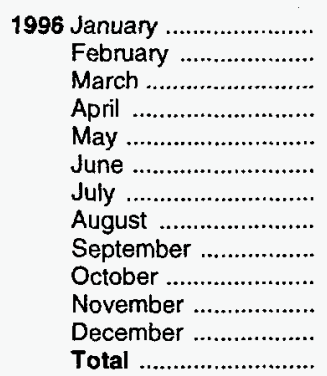 & $\begin{array}{l}.016 \\
.013 \\
.012 \\
.011 \\
.009 \\
.007 \\
.010 \\
.010 \\
.007 \\
.008 \\
.015 \\
.018 \\
.135\end{array}$ & $\begin{array}{l}1.452 \\
1.308 \\
1.122 \\
.778 \\
.467 \\
.304 \\
.257 \\
.248 \\
.269 \\
.426 \\
.819 \\
1.178 \\
8.626\end{array}$ & $\begin{array}{l}.250 \\
.233 \\
.208 \\
.162 \\
.164 \\
.155 \\
.151 \\
.153 \\
.156 \\
.177 \\
.191 \\
.221 \\
.2221\end{array}$ & $\begin{array}{r}1.718 \\
1.555 \\
1.342 \\
.951 \\
.639 \\
.466 \\
.417 \\
.410 \\
.433 \\
.611 \\
1.025 \\
1.417 \\
10.982\end{array}$ & $\begin{array}{r}.645 \\
R .591 \\
R .559 \\
R .504 \\
R .521 \\
R .604 \\
R .672 \\
R .678 \\
R .612 \\
R .533 \\
R .530 \\
R .591 \\
R 7.041\end{array}$ & $\begin{array}{r}2.363 \\
2.146 \\
R_{1} 1.901 \\
R_{1} 1.455 \\
R_{1} 1.160 \\
R_{1} 1.070 \\
R_{1} 1.089 \\
R_{1} 1.088 \\
R_{1} 1.044 \\
R_{1} 1.144 \\
R_{1} 1.555 \\
2.008 \\
R_{18.023}\end{array}$ & $\begin{array}{r}R_{1} 1.322 \\
R_{1.158} \\
R_{1} 1.153 \\
R_{1} 1.020 \\
R_{1} 1.168 \\
R_{1} 1.290 \\
R_{1} 1.454 \\
R_{1} 1.438 \\
R_{1} \\
R_{1.169} \\
R_{1.115} \\
R_{1} 1.246 \\
R_{14.615}\end{array}$ & $\begin{array}{r}R_{3.685} \\
R_{3.303} \\
R_{3.054} \\
R_{2.475} \\
R_{2.328} \\
R_{2} 2.360 \\
R_{2} .543 \\
R_{2} 2.526 \\
R_{2} 2.213 \\
R_{2.226} \\
R_{2} .670 \\
R_{3.253} \\
R_{32.638}\end{array}$ \\
\hline 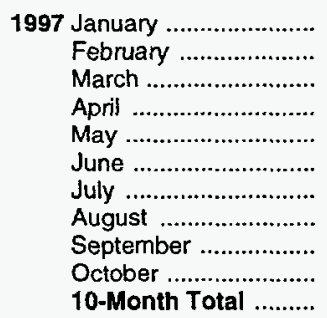 & $\begin{array}{r}.019 \\
.014 \\
.012 \\
.013 \\
.009 \\
.008 \\
.011 \\
.009 \\
.008 \\
\mathrm{E} .013 \\
\mathrm{E} .116\end{array}$ & $\begin{array}{r}R_{1} .426 \\
R 1.226 \\
R .989 \\
.720 \\
R .505 \\
.316 \\
.270 \\
.260 \\
R .279 \\
F .446 \\
E_{6.436}\end{array}$ & $\begin{array}{l}.261 \\
.212 \\
.202 \\
.173 \\
.163 \\
.154 \\
.153 \\
.149 \\
.159 \\
.179 \\
1.807\end{array}$ & $\begin{array}{r}R 1.706 \\
R \quad 1.452 \\
R \quad 1.203 \\
.907 \\
R .676 \\
.478 \\
R .434 \\
R .419 \\
R .445 \\
.638 \\
8.360\end{array}$ & $\begin{array}{l}.644 \\
.569 \\
.539 \\
.506 \\
.506 \\
.580 \\
.700 \\
.683 \\
.635 \\
.585 \\
\mathbf{5 . 9 4 7}\end{array}$ & $\begin{array}{r}R_{2} .350 \\
R_{2} .021 \\
R_{1.743} \\
1.413 \\
R_{1.182} \\
1.058 \\
R_{1.134} \\
R_{1.102} \\
R_{1.080} \\
1.223 \\
14.307\end{array}$ & $\begin{array}{r}1.349 \\
1.083 \\
1.146 \\
1.034 \\
1.110 \\
1.271 \\
1.565 \\
1.471 \\
1.259 \\
1.163 \\
12.450\end{array}$ & $\begin{array}{r}R_{3} 3.699 \\
R_{3.105} \\
R_{2.888} \\
2.447 \\
R_{2} .292 \\
R_{2} .330 \\
R_{2} .699 \\
\text { R }_{2.573} \\
\text { R }_{2.339} \\
2.386 \\
26.757\end{array}$ \\
\hline $\begin{array}{l}1996 \text { 10-Month Total .......... } \\
1995 \text { 10-Month Total ......... }\end{array}$ & $\begin{array}{l}.102 \\
.094\end{array}$ & $\begin{array}{l}6.630 \\
6.078\end{array}$ & $\begin{array}{l}1.809 \\
1.724\end{array}$ & $\begin{array}{l}8.540 \\
7.896\end{array}$ & $\begin{array}{l}5.920 \\
5.712\end{array}$ & $\begin{array}{l}14.460 \\
13.608\end{array}$ & $\begin{array}{l}12.254 \\
11.830\end{array}$ & $\begin{array}{l}26.714 \\
25.438\end{array}$ \\
\hline
\end{tabular}

a Includes supplemental gaseous fuels.

b Products obtained from the processing of crude oil (including lease condensate), natural gas, and other hydrocarton compounds.

$R=$ Revised data. $E=$ Estimate. $F=$ Forecast.
Notes: - Totals may not equal sum of components due to independent rounding. - Geographic coverage is the 50 States and the District of Columbia.

Additional Notes and Sources: See end of section.

Please Read: Due to a lack of consistent monthly historical data, some renewable energy sources are not included in this table. In 1996, for example, an estimated 0.7 quadrillion Btu of renewable energy used by the residential and commercial sectors (primarily the residential sector) is not included. See Note 12 at the end of section for details. 
Figure 2.3 Industrial Energy Consumption

(Quadrillion Btu)

By Major Sources, 1973-1996

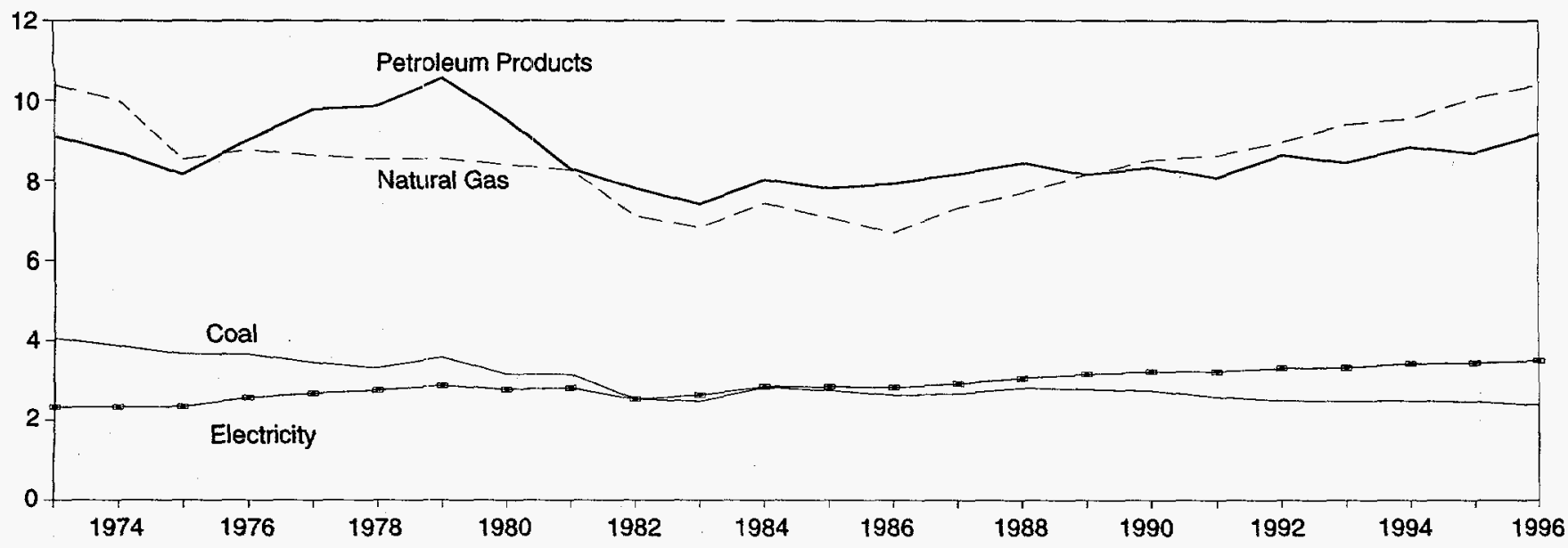

By Major Sources, Monthly

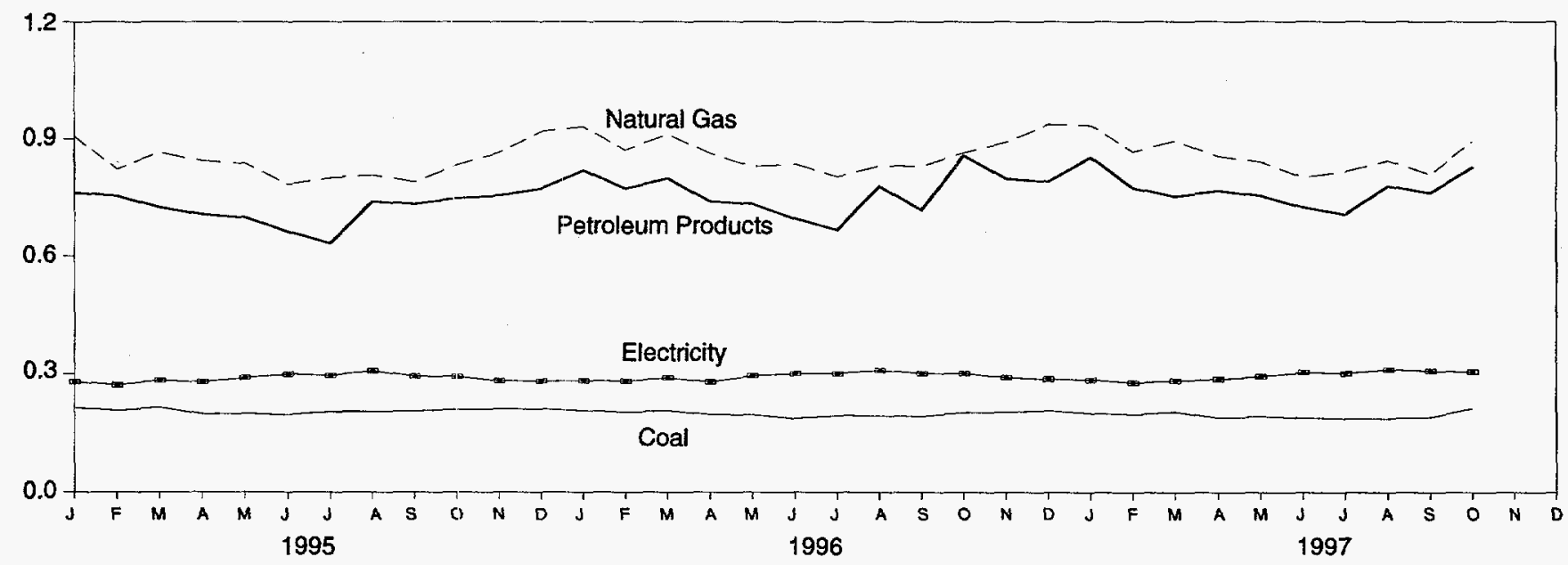

Total, January-October

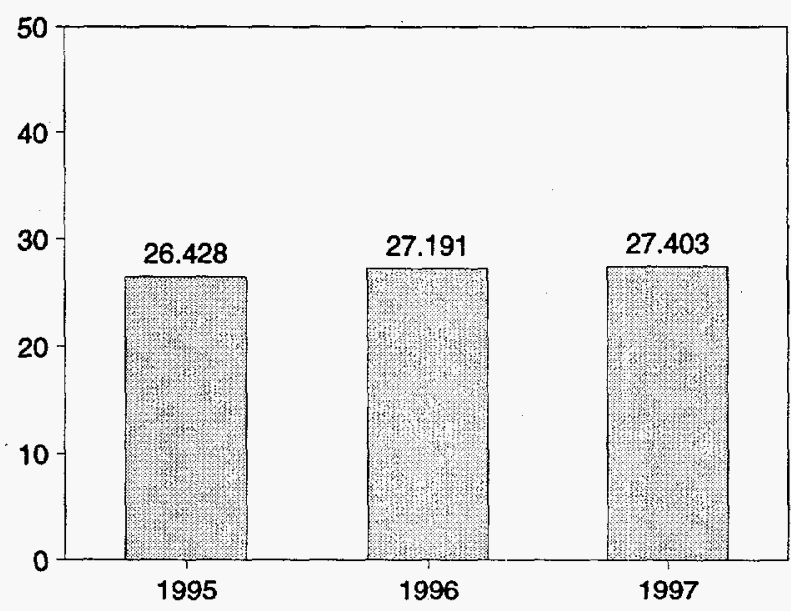

By Major Sources, October 1997

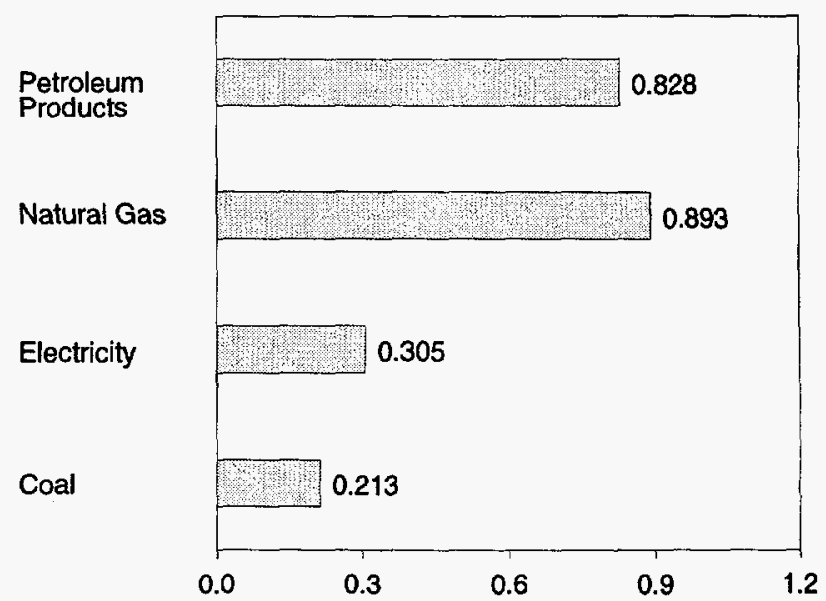

Note: Because vertical scales differ, graphs should not be compared. Source: Table 2.4. 
Table 2.4 Industrial Energy Consumption

(Quadrillion Btu)

\begin{tabular}{|c|c|c|c|c|c|c|c|c|c|c|}
\hline & Coal & $\begin{array}{c}\text { Natural } \\
\text { Gas }^{2}\end{array}$ & $\begin{array}{l}\text { Petroleum } \\
\text { Products }^{b}\end{array}$ & $\begin{array}{l}\text { Hydro- } \\
\text { electric } \\
\text { Power }\end{array}$ & $\begin{array}{c}\text { Net } \\
\text { Imports } \\
\text { of Coal } \\
\text { Coke }\end{array}$ & $\begin{array}{c}\text { Primary } \\
\text { Consumption }\end{array}$ & Electricity & $\begin{array}{c}\text { Net } \\
\text { Consumption }\end{array}$ & \begin{tabular}{|c|} 
Electrical \\
System \\
Energy \\
Losses
\end{tabular} & $\begin{array}{c}\text { Total } \\
\text { Consumption }\end{array}$ \\
\hline 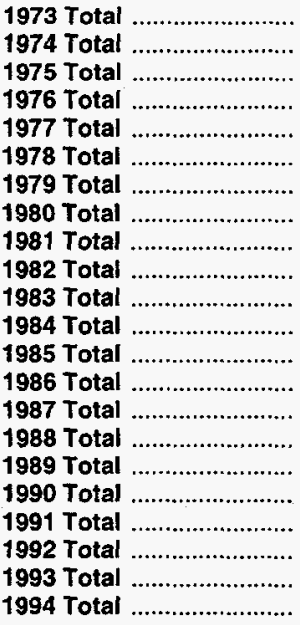 & $\begin{array}{l}4.057 \\
3.870 \\
3.667 \\
3.661 \\
3.454 \\
3.314 \\
3.593 \\
3.155 \\
3.157 \\
2.552 \\
2.490 \\
2.842 \\
2.760 \\
2.640 \\
2.673 \\
2.828 \\
2.787 \\
2.756 \\
2.601 \\
2.515 \\
2.496 \\
2.510\end{array}$ & $\begin{array}{r}10.388 \\
10.004 \\
8.532 \\
8.762 \\
8.635 \\
8.539 \\
8.549 \\
8.395 \\
8.257 \\
7.121 \\
6.826 \\
7.448 \\
7.080 \\
6.690 \\
7.323 \\
7.696 \\
8.131 \\
8.502 \\
8.619 \\
8.967 \\
9.410 \\
9.560\end{array}$ & $\begin{array}{r}9.104 \\
8.694 \\
8.146 \\
9.010 \\
9.774 \\
9.867 \\
10.568 \\
9.525 \\
8.285 \\
7.794 \\
7.420 \\
8.014 \\
7.805 \\
7.920 \\
8.150 \\
8.430 \\
8.133 \\
8.319 \\
8.057 \\
8.638 \\
8.449 \\
8.849\end{array}$ & $\begin{array}{l}0.035 \\
.033 \\
.032 \\
.033 \\
.033 \\
.032 \\
.034 \\
.033 \\
.033 \\
.033 \\
.033 \\
.033 \\
.033 \\
.033 \\
.033 \\
.033 \\
.033 \\
.033 \\
.033 \\
.033 \\
.032 \\
.032\end{array}$ & $\begin{array}{r}-0.007 \\
.056 \\
.014 \\
(\mathrm{~s}) \\
.015 \\
.125 \\
.063 \\
-.035 \\
-.016 \\
-.022 \\
-.016 \\
-.011 \\
-.013 \\
-.017 \\
.009 \\
.040 \\
.030 \\
.005 \\
.009 \\
.027 \\
.017 \\
.024\end{array}$ & $\begin{array}{l}23.576 \\
22.657 \\
20.391 \\
21.465 \\
21.911 \\
21.876 \\
22.807 \\
21.073 \\
19.715 \\
17.479 \\
16.753 \\
18.325 \\
17.665 \\
17.267 \\
18.188 \\
19.026 \\
19.113 \\
19.615 \\
19.319 \\
20.180 \\
20.405 \\
20.975\end{array}$ & $\begin{array}{l}2.341 \\
2.337 \\
2.346 \\
2.573 \\
2.682 \\
2.761 \\
2.873 \\
2.781 \\
2.817 \\
2.542 \\
2.648 \\
2.859 \\
2.855 \\
2.834 \\
2.928 \\
3.059 \\
3.158 \\
3.226 \\
3.230 \\
3.319 \\
3.334 \\
3.439\end{array}$ & $\begin{array}{l}25.917 \\
24.994 \\
22.737 \\
24.038 \\
24.593 \\
24.637 \\
25.679 \\
23.854 \\
22.533 \\
20.020 \\
19.401 \\
21.184 \\
20.520 \\
20.101 \\
21.116 \\
22.085 \\
22.272 \\
22.841 \\
22.549 \\
23.498 \\
23.739 \\
24.414\end{array}$ & $\begin{array}{l}5.611 \\
5.700 \\
5.665 \\
6.198 \\
6.484 \\
6.755 \\
6.936 \\
6.752 \\
6.707 \\
6.125 \\
6.359 \\
6.683 \\
6.694 \\
6.529 \\
6.710 \\
6.901 \\
7.082 \\
7.095 \\
7.021 \\
7.079 \\
7.010 \\
7.167\end{array}$ & $\begin{array}{l}31.528 \\
30.694 \\
28.402 \\
30.236 \\
31.077 \\
31.392 \\
32.616 \\
30.606 \\
29.240 \\
26.145 \\
25.759 \\
27.867 \\
27.214 \\
26.630 \\
27.826 \\
28.986 \\
29.353 \\
29.936 \\
29.570 \\
30.577 \\
30.749 \\
31.581\end{array}$ \\
\hline 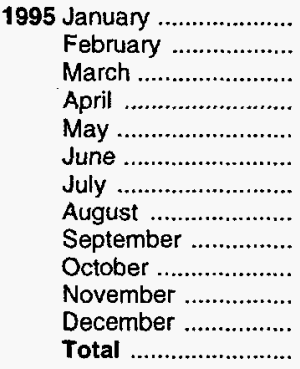 & $\begin{array}{l}.214 \\
.207 \\
.215 \\
.199 \\
.200 \\
.197 \\
.205 \\
.205 \\
.207 \\
.211 \\
.212 \\
.212 \\
2.483\end{array}$ & $\begin{array}{r}.906 \\
.822 \\
.865 \\
.843 \\
.836 \\
.783 \\
.800 \\
.807 \\
.790 \\
.833 \\
.864 \\
.919 \\
10.064\end{array}$ & $\begin{array}{l}.762 \\
.754 \\
.724 \\
.706 \\
.698 \\
.662 \\
.633 \\
.739 \\
.733 \\
.748 \\
.755 \\
.773 \\
8.688\end{array}$ & $\begin{array}{l}.003 \\
.003 \\
.003 \\
.003 \\
.003 \\
.003 \\
.003 \\
.002 \\
.002 \\
.002 \\
.002 \\
.002 \\
.033\end{array}$ & $\begin{array}{l}.004 \\
.002 \\
.003 \\
.001 \\
.004 \\
.001 \\
.002 \\
.001 \\
.002 \\
.003 \\
.002 \\
.002 \\
.026\end{array}$ & $\begin{array}{r}1.889 \\
1.788 \\
1.809 \\
1.752 \\
1.743 \\
1.645 \\
1.642 \\
1.755 \\
1.734 \\
1.796 \\
1.835 \\
1.908 \\
21.294\end{array}$ & $\begin{array}{l}.279 \\
.271 \\
.283 \\
.279 \\
.290 \\
.299 \\
.296 \\
.308 \\
.294 \\
.293 \\
.282 \\
.281 \\
.3 .455\end{array}$ & $\begin{array}{r}2.168 \\
2.059 \\
2.092 \\
2.031 \\
2.033 \\
1.944 \\
1.938 \\
2.063 \\
2.027 \\
2.089 \\
2.117 \\
2.189 \\
24.749\end{array}$ & $\begin{array}{r}R .576 \\
.522 \\
.581 \\
.566 \\
.631 \\
.632 \\
.660 \\
.670 \\
.551 \\
.593 \\
.583 \\
R .605 \\
R 7.170\end{array}$ & $\begin{array}{r}R_{2} .744 \\
R_{2} .581 \\
R_{2} .674 \\
2.597 \\
2.665 \\
2.576 \\
2.598 \\
R_{2} .733 \\
2.578 \\
R_{2} .683 \\
R_{2} .700 \\
2.794 \\
R_{31.920}\end{array}$ \\
\hline 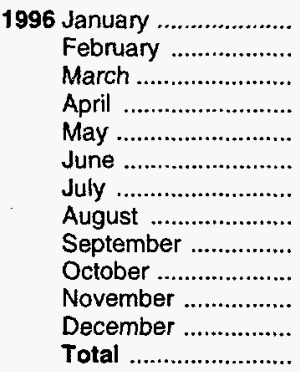 & $\begin{array}{l}.208 \\
.204 \\
.208 \\
.198 \\
.197 \\
.188 \\
.195 \\
.194 \\
.193 \\
.203 \\
.204 \\
.208 \\
2.399\end{array}$ & $\begin{array}{r}.931 \\
.871 \\
.912 \\
.863 \\
.829 \\
.835 \\
.804 \\
.831 \\
.830 \\
.864 \\
.891 \\
.937 \\
10.394\end{array}$ & $\begin{array}{l}.819 \\
.773 \\
.799 \\
.740 \\
.734 \\
.696 \\
.668 \\
.779 \\
.718 \\
.857 \\
.798 \\
.791 \\
9.172\end{array}$ & $\begin{array}{l}.003 \\
.003 \\
.003 \\
.003 \\
.003 \\
.003 \\
.003 \\
.002 \\
.002 \\
.002 \\
.002 \\
.002 \\
.033\end{array}$ & $\begin{array}{r}.001 \\
.003 \\
.003 \\
-.001 \\
-.001 \\
-.002 \\
(\mathrm{~s}) \\
-.003 \\
(\mathrm{~s}) \\
(\mathrm{s}) \\
(\mathrm{s}) \\
-.001 \\
(\mathrm{~s})\end{array}$ & $\begin{array}{r}1.963 \\
1.853 \\
1.925 \\
1.802 \\
1.763 \\
1.719 \\
1.669 \\
1.804 \\
1.744 \\
1.926 \\
1.895 \\
1.937 \\
21.997\end{array}$ & $\begin{array}{r}\text { R. } .282 \\
\text { R.281 } \\
\text { R.289 } \\
\text { R.279 } \\
\text { R.295 } \\
\text { R.301 } \\
\text { R.301 } \\
\text { R. } .309 \\
\text { R. } .301 \\
\text { R. } .301 \\
\text { R.290 } \\
\text { R.287 } \\
\text { R. } 3.516\end{array}$ & $\begin{array}{r}R_{2} .245 \\
R_{2} 2.133 \\
R_{2} 214 \\
R_{2} 2.082 \\
R_{2} 2.057 \\
R_{2} .020 \\
R_{1} .971 \\
R_{2} 2.112 \\
R_{2} .044 \\
R_{2} .228 \\
R_{2} 2.185 \\
R_{2.225} \\
R_{25} 5.513\end{array}$ & $\begin{array}{r}\text { R. } .578 \\
\text { R. } .549 \\
\text { R. } .596 \\
\text { R. } .565 \\
\text { R. } .661 \\
\text { R. } .643 \\
\text { R. } .652 \\
\text { R. } .655 \\
\text { R. } .574 \\
\text { R. } .612 \\
\text { R } .609 \\
\text { R. } .606 \\
\text { R } 7.300\end{array}$ & $\begin{array}{r}R_{2.823} \\
R_{2.683} \\
R_{2.810} \\
A_{2.647} \\
A_{2.718} \\
R_{2} .663 \\
R_{2.622} \\
R_{2} 2.767 \\
R_{2} .619 \\
R_{2.840} \\
R_{2.794} \\
R_{2.830} \\
R_{32.812}\end{array}$ \\
\hline 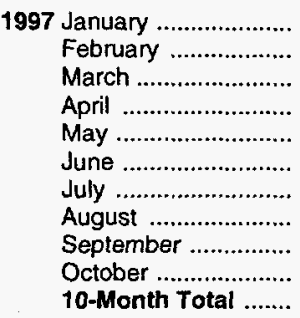 & $\begin{array}{r}.201 \\
.198 \\
.205 \\
.189 \\
.193 \\
.189 \\
R .187 \\
R .187 \\
R .190 \\
\text { E.213 } \\
\text { E } 1.952\end{array}$ & $\begin{array}{r}\text { R. } .935 \\
\text { R. } .866 \\
.894 \\
\text { R. } .855 \\
.841 \\
\text { R. } .803 \\
\text { R } .818 \\
\text { R. } .845 \\
\text { R. } .810 \\
\text { F } .893 \\
\text { E } 8.560\end{array}$ & $\begin{array}{l}.852 \\
.774 \\
.752 \\
.767 \\
.755 \\
.726 \\
.708 \\
.780 \\
.762 \\
.828 \\
7.704\end{array}$ & $\begin{array}{l}.003 \\
.003 \\
.003 \\
.003 \\
.003 \\
.003 \\
.003 \\
.002 \\
.002 \\
.002 \\
.028\end{array}$ & $\begin{array}{r}.002 \\
.002 \\
.002 \\
(s) \\
.002 \\
.001 \\
.002 \\
.007 \\
-.003 \\
.002 \\
.016\end{array}$ & $\begin{array}{r}R 1.992 \\
1.843 \\
1.856 \\
R_{1.814} \\
1.794 \\
1.722 \\
R 1.718 \\
R 1.822 \\
R \quad 1.761 \\
1.937 \\
18.259\end{array}$ & $\begin{array}{l}.285 \\
.278 \\
.283 \\
.287 \\
.294 \\
.304 \\
.302 \\
.311 \\
.307 \\
.305 \\
.2 .957\end{array}$ & $\begin{array}{r}R_{2} .277 \\
2.120 \\
R_{2} .140 \\
R_{2} .101 \\
R_{2} .089 \\
2.026 \\
R_{2} .020 \\
R_{2} .133 \\
R_{2} .068 \\
2.242 \\
21.216\end{array}$ & $\begin{array}{l}.597 \\
.528 \\
.602 \\
.586 \\
.646 \\
.667 \\
.675 \\
.670 \\
.609 \\
.606 \\
6.187\end{array}$ & $\begin{array}{r}R_{2.875} \\
2.648 \\
R_{2.742} \\
R_{2.687} \\
2.735 \\
R_{2.693} \\
R_{2.695} \\
R_{2} .804 \\
R_{2.677} \\
2.848 \\
27.403\end{array}$ \\
\hline $\begin{array}{l}1996 \text { 10-Month Total ....... } \\
1995 \text { 10-Month Total ....... }\end{array}$ & $\begin{array}{l}1.988 \\
2.059\end{array}$ & $\begin{array}{l}8.569 \\
8.284\end{array}$ & $\begin{array}{l}7.583 \\
7.161\end{array}$ & $\begin{array}{l}.028 \\
.028\end{array}$ & $\begin{array}{r}(s) \\
.022\end{array}$ & $\begin{array}{l}18.168 \\
17.554\end{array}$ & $\begin{array}{l}2.939 \\
2.892\end{array}$ & $\begin{array}{l}21.106 \\
20.446\end{array}$ & $\begin{array}{l}6.085 \\
5.982\end{array}$ & $\begin{array}{l}27.191 \\
26.428\end{array}$ \\
\hline
\end{tabular}

a Includes supplemental gaseous fuels.

Notes: - Totals may not equal sum of components due to independent

b Products obtained from the processing of crude oil (including lease condensate), natural gas, and other hydrocarbon compounds.

$R=$ Revised data. $E=E s t i m a t e . ~ F=F o r e c a s t . ~(s)=L e s s$ than +0.5 trillion Btu

rounding. - Geographic coverage is the 50 States and the District of Columbia.

Additional Notes and Sources: See end of section. and greater than -0.5 trillion Btu.

Please Read: Due to a lack of consistent monthly historical data, some renewable energy sources are not included in this table. In 1996, for example, an estimated 2.7 quadrillion Btu of renewable energy used by the industrial sector (primarily the pulp and paper industry) is not included. See Note 12 at the end of section for details. 
Figure 2.4 Transportation Energy Consumption

(Quadrillion Biu)

By Major Sources, 1973-1996

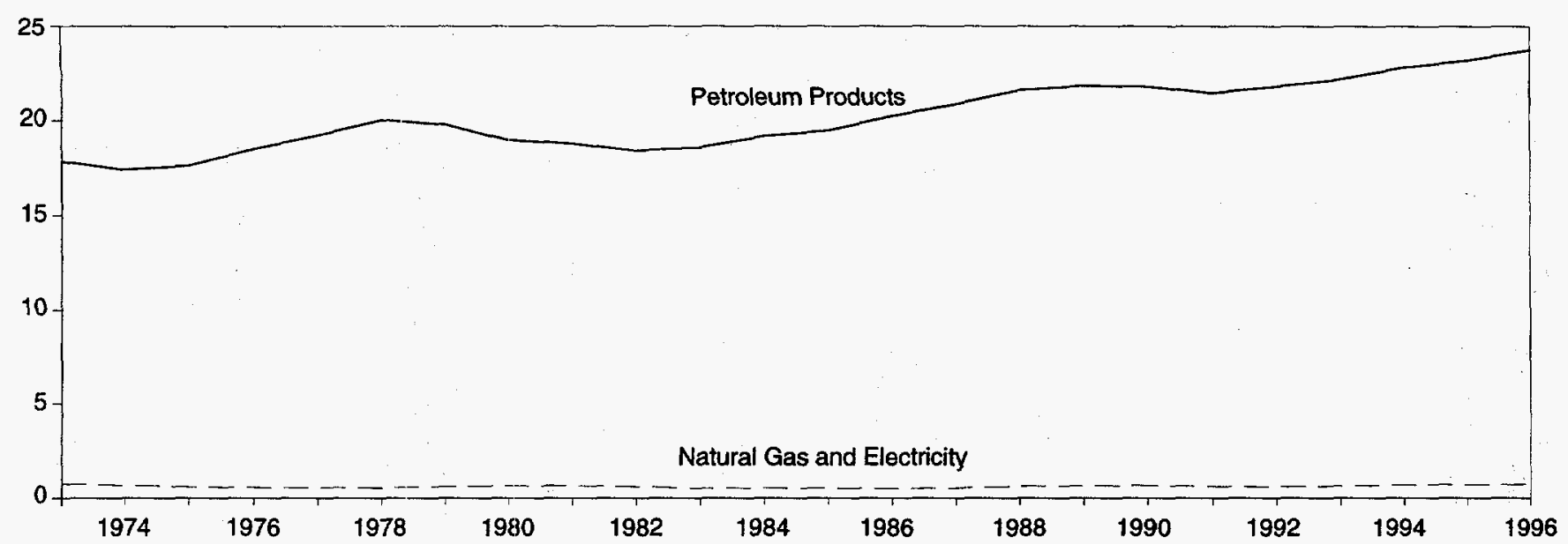

By Major Sources, Monthly

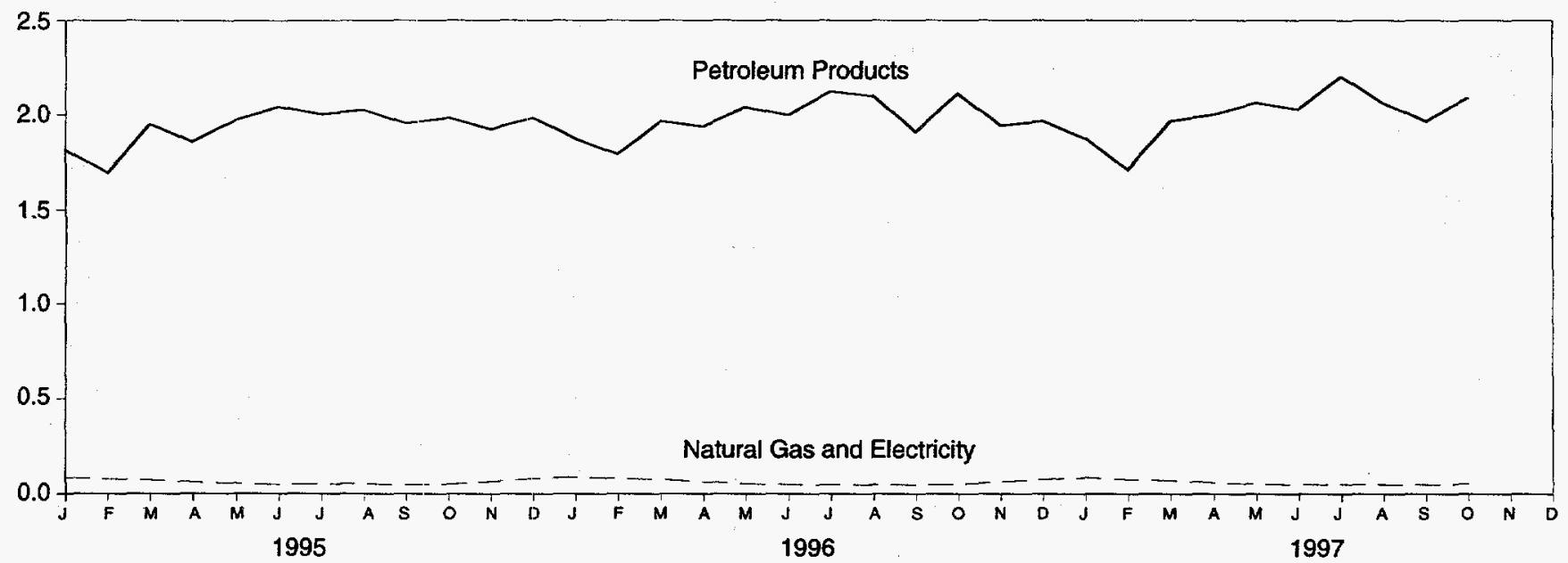

Total, January-October

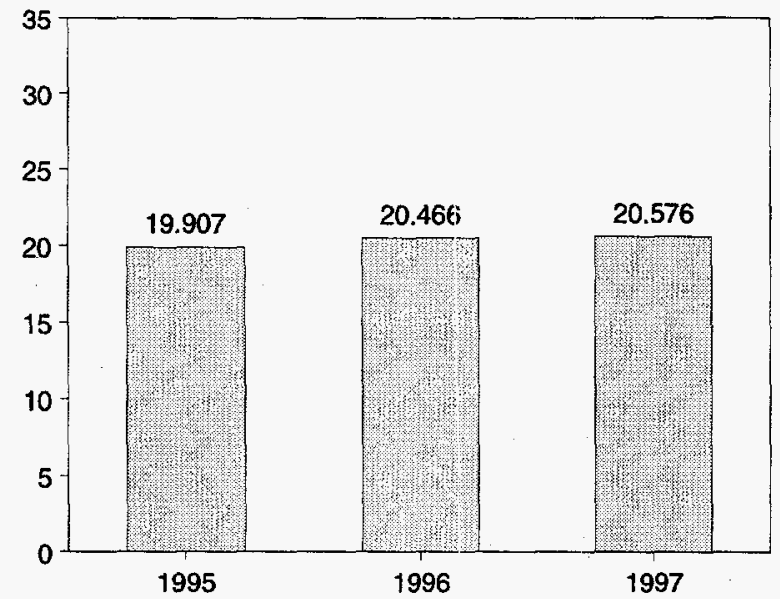

Total, Monthly

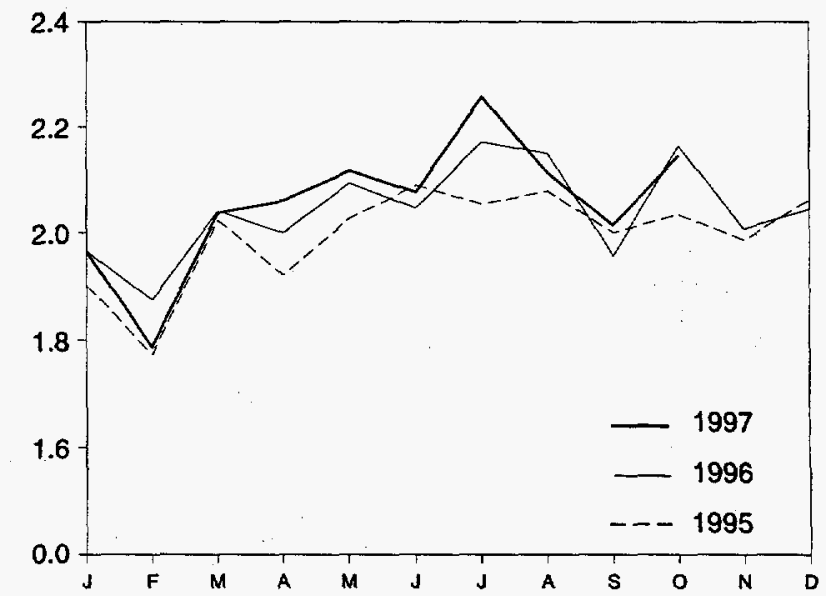

Note: Because vertical scales differ, graphs should not be compared. Source: Table 2.5. 


\begin{tabular}{|c|c|c|c|c|c|c|c|c|}
\hline & Coal & $\begin{array}{c}\text { Natural } \\
\text { Gas }^{a}\end{array}$ & $\begin{array}{l}\text { Petroleum } \\
\text { Products }{ }^{b, c}\end{array}$ & $\begin{array}{c}\text { Primary } \\
\text { Consumption }\end{array}$ & Electricity & $\begin{array}{c}\text { Net } \\
\text { Consumption }\end{array}$ & $\begin{array}{l}\text { Electrical } \\
\text { System } \\
\text { Energy } \\
\text { Losses }\end{array}$ & $\begin{array}{c}\text { Total } \\
\text { Consumption }\end{array}$ \\
\hline 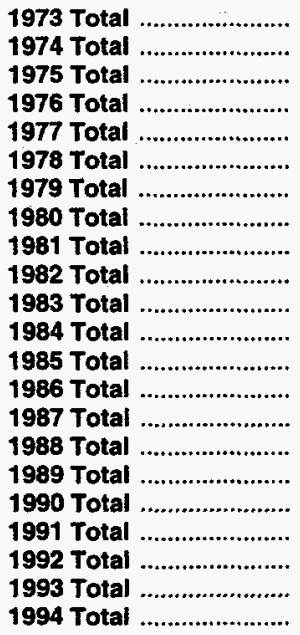 & $\begin{array}{l}0.003 \\
.002 \\
.001 \\
(s) \\
(s) \\
(d) \\
\text { (d) } \\
(d) \\
\text { dd) } \\
\text { (d) } \\
\text { (d) } \\
\text { (d) } \\
\text { (d) } \\
\text { (d) } \\
\text { (d) } \\
\text { (d) } \\
\text { (d) } \\
\text { (d) } \\
\text { (d) } \\
\text { (d) } \\
\text { (d) } \\
\text { (d) }\end{array}$ & $\begin{array}{r}0.743 \\
.685 \\
.595 \\
.559 \\
.543 \\
.539 \\
.612 \\
.650 \\
.658 \\
.612 \\
.505 \\
.545 \\
.519 \\
.499 \\
.535 \\
.632 \\
.649 \\
.680 \\
.620 \\
.606 \\
.643 \\
.707\end{array}$ & $\begin{array}{l}17.831 \\
17.399 \\
17.614 \\
18.506 \\
19.241 \\
20.041 \\
19.825 \\
19.008 \\
18.811 \\
18.420 \\
18.593 \\
19.216 \\
19.504 \\
20.269 \\
20.871 \\
21.629 \\
21.868 \\
21.810 \\
21.456 \\
21.812 \\
22.201 \\
22.824\end{array}$ & $\begin{array}{l}18.576 \\
18.086 \\
18.209 \\
19.065 \\
19.784 \\
20.580 \\
20.436 \\
19.658 \\
19.469 \\
19.032 \\
19.098 \\
19.761 \\
20.024 \\
20.768 \\
21.406 \\
22.260 \\
22.517 \\
22.490 \\
22.077 \\
22.419 \\
22.843 \\
23.531\end{array}$ & $\begin{array}{l}0.008 \\
.009 \\
.010 \\
.010 \\
.010 \\
.009 \\
.010 \\
.011 \\
.011 \\
.011 \\
.011 \\
.012 \\
.013 \\
.013 \\
.013 \\
.014 \\
.014 \\
.014 \\
.014 \\
.014 \\
.013 \\
.014\end{array}$ & $\begin{array}{l}18.584 \\
18.095 \\
18.219 \\
19.076 \\
19.794 \\
20.589 \\
20.447 \\
19.669 \\
19.480 \\
19.043 \\
19.109 \\
19.773 \\
20.036 \\
20.781 \\
21.419 \\
22.274 \\
22.530 \\
22.504 \\
22.091 \\
22.432 \\
22.857 \\
23.544\end{array}$ & $\begin{array}{l}0.020 \\
.022 \\
.025 \\
.025 \\
.025 \\
.022 \\
.025 \\
.026 \\
.026 \\
.026 \\
.026 \\
.028 \\
.030 \\
.031 \\
.029 \\
.031 \\
.031 \\
.031 \\
.030 \\
.029 \\
.028 \\
.028\end{array}$ & $\begin{array}{l}18.605 \\
18.117 \\
18.244 \\
19.101 \\
19.819 \\
20.611 \\
20.472 \\
19.695 \\
19.507 \\
19.069 \\
19.135 \\
19.801 \\
20.067 \\
20.812 \\
21.448 \\
22.305 \\
22.561 \\
22.535 \\
22.121 \\
22.462 \\
22.884 \\
23.573\end{array}$ \\
\hline 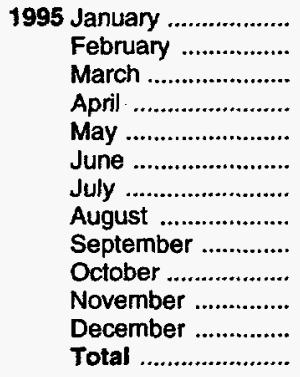 & $\begin{array}{l}\left(\begin{array}{l}d \\
d\end{array}\right) \\
\left(\begin{array}{l}d \\
d\end{array}\right) \\
\left(\begin{array}{l}d \\
d \\
d\end{array}\right) \\
\left(\begin{array}{l}d \\
d\end{array}\right) \\
\left(\begin{array}{l}d \\
d\end{array}\right) \\
\left(\begin{array}{l}d \\
d\end{array}\right) \\
\left(\begin{array}{l}d \\
d\end{array}\right) \\
\left(\begin{array}{l}d \\
\text { d }\end{array}\right.\end{array}$ & $\begin{array}{l}.081 \\
.075 \\
.070 \\
.059 \\
.052 \\
.046 \\
.049 \\
.051 \\
.046 \\
.049 \\
.063 \\
.078 \\
.722\end{array}$ & $\begin{array}{r}1.817 \\
1.695 \\
1.950 \\
1.859 \\
1.972 \\
2.041 \\
2.002 \\
2.024 \\
1.952 \\
1.982 \\
1.921 \\
1.982 \\
23.197\end{array}$ & $\begin{array}{r}1.898 \\
1.770 \\
2.021 \\
1.919 \\
2.024 \\
2.087 \\
2.051 \\
2.075 \\
1.998 \\
2.031 \\
1.984 \\
2.060 \\
23.919\end{array}$ & $\begin{array}{l}.001 \\
.001 \\
.001 \\
.001 \\
.001 \\
.001 \\
.001 \\
.001 \\
.001 \\
.001 \\
.001 \\
.001 \\
.013\end{array}$ & $\begin{array}{r}1.899 \\
1.771 \\
2.022 \\
1.920 \\
2.025 \\
2.088 \\
2.052 \\
2.076 \\
1.999 \\
2.032 \\
1.985 \\
2.061 \\
23.933\end{array}$ & $\begin{array}{l}.002 \\
.002 \\
.002 \\
.002 \\
.002 \\
.002 \\
.003 \\
.003 \\
.002 \\
.002 \\
.002 \\
.002 \\
.027\end{array}$ & $\begin{array}{r}1.902 \\
1.773 \\
2.024 \\
1.922 \\
2.027 \\
2.090 \\
2.055 \\
2.079 \\
2.001 \\
2.035 \\
1.987 \\
2.063 \\
23.960\end{array}$ \\
\hline 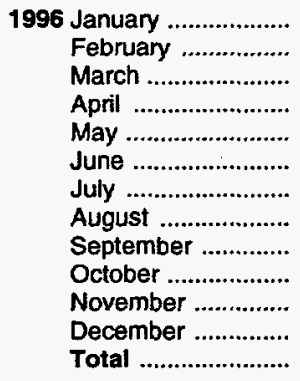 & 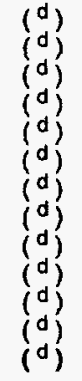 & $\begin{array}{l}.087 \\
.079 \\
.074 \\
.061 \\
.052 \\
.048 \\
.047 \\
.048 \\
.046 \\
.050 \\
.063 \\
.076 \\
.734\end{array}$ & $\begin{array}{r}1.875 \\
1.793 \\
1.963 \\
1.936 \\
2.039 \\
1.996 \\
2.121 \\
2.098 \\
1.909 \\
2.110 \\
1.941 \\
1.965 \\
23.746\end{array}$ & $\begin{array}{r}1.962 \\
1.872 \\
2.037 \\
1.997 \\
2.091 \\
2.044 \\
2.168 \\
2.146 \\
1.954 \\
2.160 \\
2.004 \\
2.042 \\
24.480\end{array}$ & $\begin{array}{l}.001 \\
.001 \\
.001 \\
.001 \\
.001 \\
.001 \\
.001 \\
.001 \\
.001 \\
.001 \\
.001 \\
.001 \\
\mathrm{R} .013\end{array}$ & $\begin{array}{r}1.963 \\
1.873 \\
2.039 \\
1.998 \\
2.092 \\
2.045 \\
2.169 \\
2.147 \\
1.956 \\
2.161 \\
2.005 \\
2.043 \\
R_{24.493}\end{array}$ & $\begin{array}{r}.002 \\
.002 \\
.002 \\
.002 \\
\mathrm{R} .002 \\
.002 \\
\mathrm{R} .002 \\
.003 \\
.002 \\
.002 \\
.002 \\
.002 \\
\mathrm{R} .028\end{array}$ & $\begin{array}{r}1.966 \\
1.875 \\
2.041 \\
2.000 \\
2.094 \\
2.047 \\
2.172 \\
2.150 \\
1.958 \\
2.164 \\
2.008 \\
2.045 \\
\text { ค }_{24.521}\end{array}$ \\
\hline 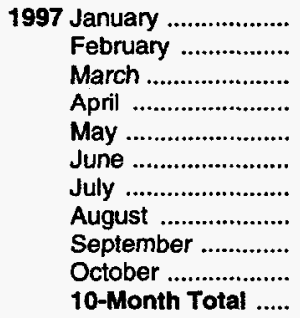 & $\begin{array}{l}\left(\begin{array}{l}d \\
d \\
d\end{array}\right) \\
\left(\begin{array}{l}d \\
d\end{array}\right) \\
\left(\begin{array}{l}d \\
d\end{array}\right) \\
\left(\begin{array}{l}d \\
d\end{array}\right) \\
\left(\begin{array}{l}d \\
d\end{array}\right) \\
\left(\begin{array}{l}d \\
d\end{array}\right) \\
\left(\begin{array}{l}d\end{array}\right)\end{array}$ & $\begin{array}{r}.084 \\
.075 \\
\mathrm{R} .070 \\
.059 \\
.053 \\
.048 \\
.051 \\
.050 \\
\mathrm{R} .048 \\
\mathrm{~F} .054 \\
\mathrm{E} .592\end{array}$ & $\begin{array}{r}1.877 \\
1.709 \\
1.965 \\
1.998 \\
2.061 \\
2.026 \\
2.202 \\
2.059 \\
1.964 \\
2.088 \\
19.949\end{array}$ & $\begin{array}{r}1.961 \\
1.784 \\
2.034 \\
2.058 \\
R 2.113 \\
2.074 \\
2.253 \\
2.109 \\
R 2.012 \\
2.143 \\
20.541\end{array}$ & $\begin{array}{l}.001 \\
.001 \\
.001 \\
.001 \\
.001 \\
.001 \\
.001 \\
.001 \\
.001 \\
.001 \\
.011\end{array}$ & $\begin{array}{r}1.962 \\
1.785 \\
2.035 \\
2.059 \\
2.115 \\
2.075 \\
2.254 \\
2.111 \\
R 2.013 \\
2.144 \\
20.553\end{array}$ & $\begin{array}{l}.002 \\
.002 \\
.002 \\
.002 \\
.002 \\
.002 \\
.003 \\
.003 \\
.002 \\
.002 \\
.024\end{array}$ & $\begin{array}{r}1.965 \\
1.787 \\
2.037 \\
2.061 \\
2.117 \\
2.077 \\
2.257 \\
2.113 \\
R 2.015 \\
2.146 \\
20.576\end{array}$ \\
\hline $\begin{array}{l}1996 \text { 10-Month Total ..... } \\
1995 \text { 10-Month Total ..... }\end{array}$ & $\left(\begin{array}{l}d \\
d\end{array}\right)$ & $\begin{array}{l}.591 \\
.578\end{array}$ & $\begin{array}{l}19.840 \\
19.295\end{array}$ & $\begin{array}{l}20.431 \\
19.873\end{array}$ & $\begin{array}{l}.011 \\
.011\end{array}$ & $\begin{array}{l}20.442 \\
19.884\end{array}$ & $\begin{array}{l}.023 \\
.023\end{array}$ & $\begin{array}{l}20.466 \\
19.907\end{array}$ \\
\hline
\end{tabular}

a Natural gas consumed in the operation of pipelines (primarily in compressors) and small amounts consumed as vehicle fuel. See Table 4.4

b Products obtained from the processing of crude oil (including lease condensate), natural gas, and other hydrocarbon compounds.

c Includes small quantities (about 0.1 quadrillion Btu per year since 1990) of renewable energy in the form of ethanol blended into motor gasoline. See Note 12 at end of section. d Since 1978, the small amounts of coal consumed for transportation are reported as industrial sector consumption.

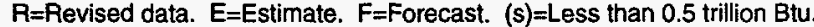

Notes: - Totals may not equal sum of components due to independent rounding. - Geographic coverage is the 50 States and the District of Columbia.

Additional Notes and Sources: See end of section. 
Figure 2.5 Energy Input at Electric Utilities

(Quadrillion Btu)

Total, 1973-1996

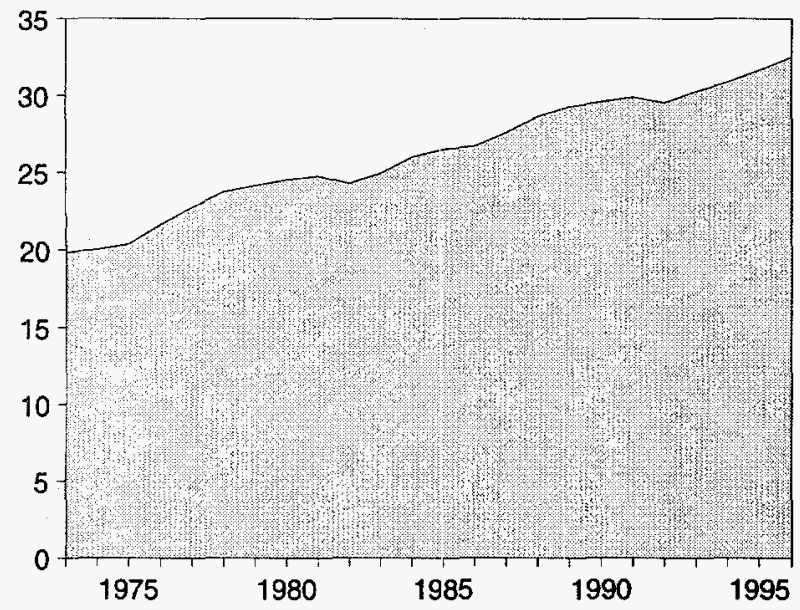

By Major Sources, 1973-1996

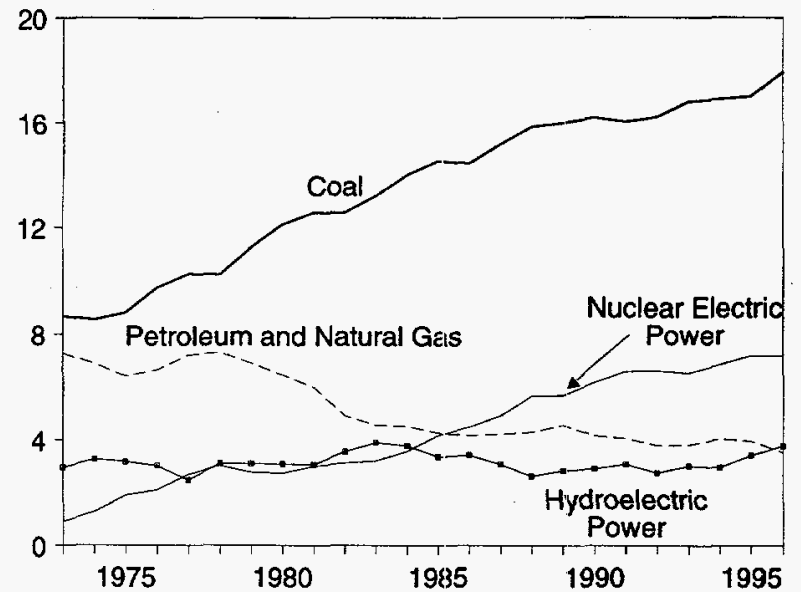

Total, January-October

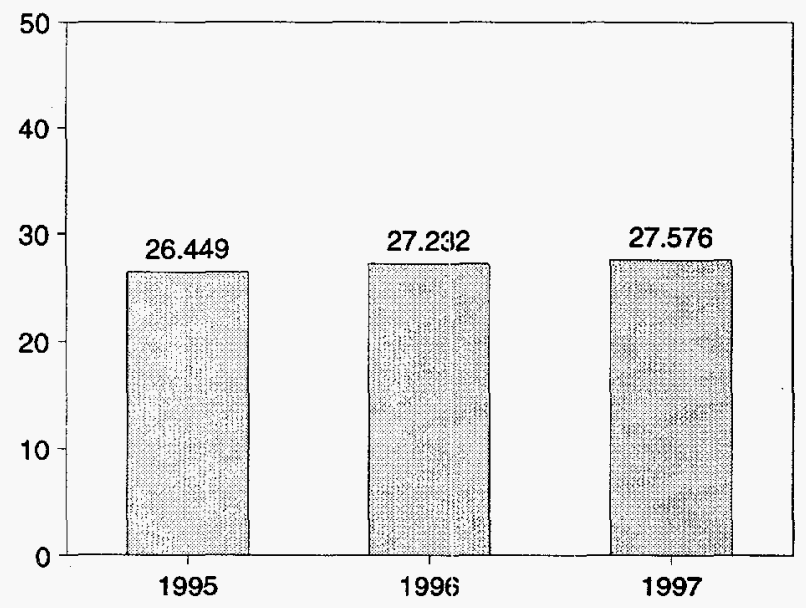

Total, Monthly

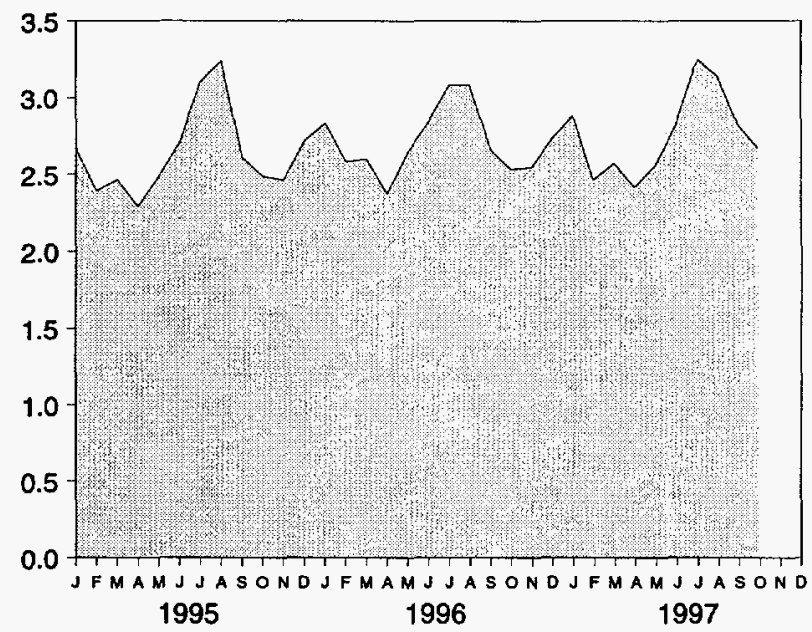

By Major Sources, Monthly

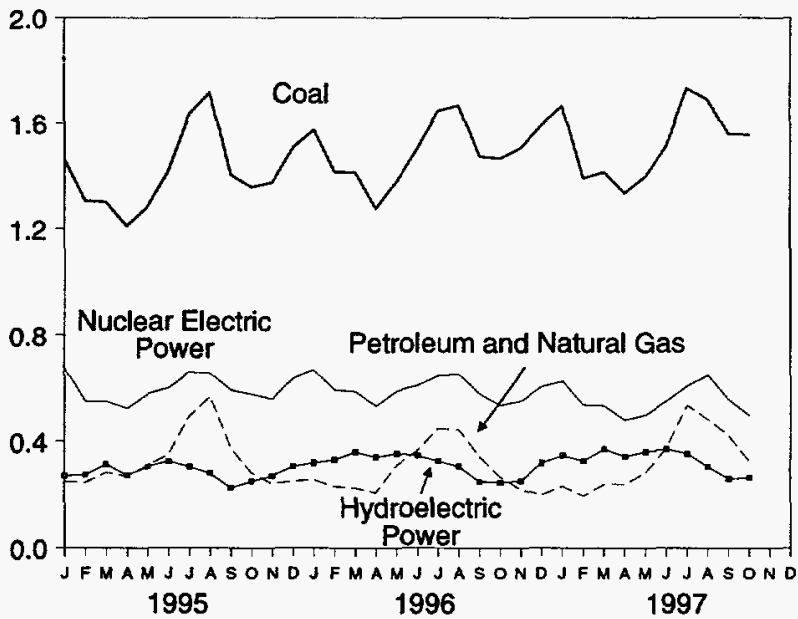

By Major Sources, October 1997

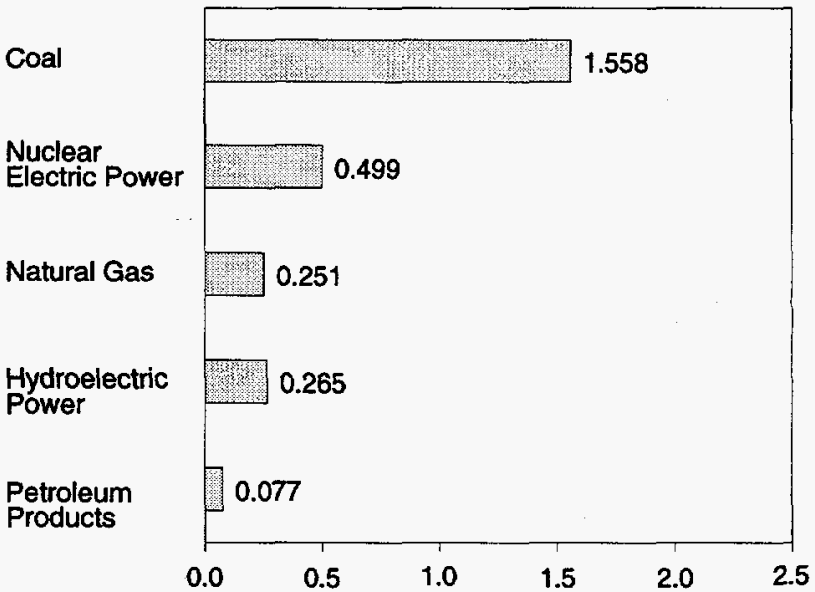

Note: Because vertical scales differ, graphs; should not be compared. Source: Table 2.6 . 
Table 2.6 Energy Input at Electric Utilities (Quadrillion Btu)

\begin{tabular}{|c|c|c|c|c|c|c|c|c|}
\hline & Coal & $\begin{array}{l}\text { Natural } \\
\text { Gas }^{a}\end{array}$ & $\begin{array}{l}\text { Petroleum } \\
\text { Products }^{b}\end{array}$ & $\begin{array}{c}\text { Nuclear } \\
\text { Electric } \\
\text { Power }\end{array}$ & $\begin{array}{l}\text { Hydro- } \\
\text { electric } \\
\text { Powerc }\end{array}$ & $\begin{array}{l}\text { Geothermal } \\
\text { Energy }\end{array}$ & Otherd & Total \\
\hline 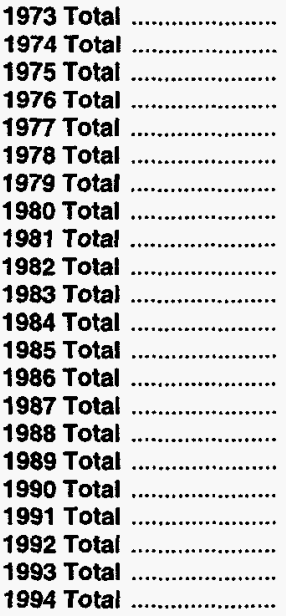 & $\begin{array}{r}8.658 \\
8.534 \\
8.786 \\
9.720 \\
10.262 \\
10.238 \\
11.260 \\
12.123 \\
12.583 \\
12.582 \\
13.213 \\
14.020 \\
14.542 \\
14.444 \\
15.173 \\
15.850 \\
15.988 \\
16.189 \\
16.028 \\
16.211 \\
16.790 \\
16.895\end{array}$ & $\begin{array}{l}3.748 \\
3.519 \\
3.240 \\
3.152 \\
3.284 \\
3.297 \\
3.613 \\
3.810 \\
3.768 \\
3.342 \\
2.998 \\
3.220 \\
3.160 \\
2.691 \\
2.935 \\
2.709 \\
2.871 \\
2.882 \\
2.856 \\
2.826 \\
2.741 \\
3.053\end{array}$ & $\begin{array}{r}3.515 \\
3.365 \\
3.166 \\
3.477 \\
3.901 \\
3.987 \\
3.283 \\
2.634 \\
2.202 \\
1.568 \\
1.544 \\
1.286 \\
1.090 \\
1.452 \\
1.257 \\
1.563 \\
1.685 \\
1.250 \\
1.178 \\
.951 \\
1.052 \\
.968\end{array}$ & $\begin{array}{l}0.910 \\
1.272 \\
1.900 \\
2.111 \\
2.702 \\
3.024 \\
2.776 \\
2.739 \\
3.008 \\
3.131 \\
3.203 \\
3.553 \\
4.149 \\
4.471 \\
4.906 \\
5.661 \\
5.677 \\
6.161 \\
6.579 \\
6.607 \\
6.519 \\
6.837\end{array}$ & $\begin{array}{l}2.975 \\
3.276 \\
3.187 \\
3.032 \\
2.482 \\
3.110 \\
3.107 \\
3.085 \\
3.072 \\
3.539 \\
3.866 \\
3.767 \\
3.365 \\
3.413 \\
3.084 \\
2.630 \\
2.848 \\
2.914 \\
3.083 \\
2.760 \\
3.017 \\
2.962\end{array}$ & $\begin{array}{r}0.043 \\
.053 \\
.070 \\
.078 \\
.077 \\
.064 \\
.084 \\
.110 \\
.123 \\
.105 \\
.129 \\
.165 \\
.198 \\
.219 \\
.229 \\
.217 \\
.197 \\
.181 \\
.170 \\
.169 \\
.158 \\
.145\end{array}$ & $\begin{array}{r}0.003 \\
.003 \\
.002 \\
.003 \\
.005 \\
.003 \\
.005 \\
.005 \\
.004 \\
.003 \\
.004 \\
.009 \\
.015 \\
.012 \\
.016 \\
.017 \\
.020 \\
.021 \\
.021 \\
.022 \\
.021 \\
.020\end{array}$ & $\begin{array}{l}19.852 \\
20.022 \\
20.350 \\
21.574 \\
22.713 \\
23.724 \\
24.128 \\
24.505 \\
24.760 \\
24.270 \\
24.956 \\
26.020 \\
26.519 \\
26.703 \\
27.600 \\
28.648 \\
29.286 \\
29.599 \\
29.915 \\
29.547 \\
30.299 \\
30.881\end{array}$ \\
\hline 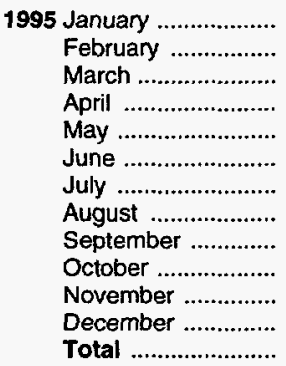 & $\begin{array}{r}1.464 \\
1.307 \\
1.303 \\
1.211 \\
1.284 \\
1.421 \\
1.633 \\
1.716 \\
1.406 \\
1.359 \\
1.377 \\
1.508 \\
16.990\end{array}$ & $\begin{array}{l}.204 \\
.172 \\
.251 \\
.235 \\
.264 \\
.304 \\
.417 \\
.480 \\
.324 \\
.246 \\
.203 \\
.177 \\
3.276\end{array}$ & $\begin{array}{l}.046 \\
.075 \\
.034 \\
.036 \\
.047 \\
.048 \\
.079 \\
.091 \\
.051 \\
.038 \\
.039 \\
.075 \\
.658\end{array}$ & $\begin{array}{l}.675 \\
.553 \\
.553 \\
.526 \\
.580 \\
.601 \\
.661 \\
.657 \\
.594 \\
.579 \\
.562 \\
.638 \\
7.177\end{array}$ & $\begin{array}{r}\text { R. } .271 \\
\text { R. } .276 \\
R .316 \\
\text { R. } 275 \\
.306 \\
.327 \\
.306 \\
\text { R. } 282 \\
.226 \\
R .251 \\
.271 \\
R .307 \\
\text { R. } 3.412\end{array}$ & $\begin{array}{l}.009 \\
.006 \\
.007 \\
.006 \\
.005 \\
.006 \\
.006 \\
.011 \\
.008 \\
.013 \\
.012 \\
.011 \\
.099\end{array}$ & $\begin{array}{l}.001 \\
.001 \\
.001 \\
.002 \\
.001 \\
.001 \\
.002 \\
.002 \\
.002 \\
.002 \\
.002 \\
.001 \\
.017\end{array}$ & $\begin{array}{r}R_{2} .669 \\
R_{2} .391 \\
R_{2} .464 \\
R_{2} .290 \\
2.487 \\
2.709 \\
3.105 \\
R_{3.238} \\
2.610 \\
R_{2} .487 \\
R_{2} .464 \\
R_{2} .717 \\
R_{31.631}\end{array}$ \\
\hline 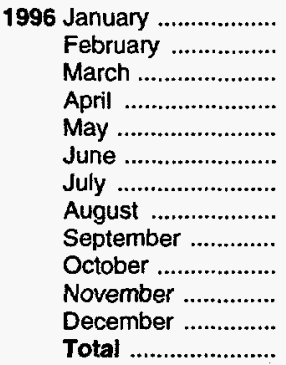 & $\begin{array}{l}1.575 \\
1.416 \\
1.415 \\
1.278 \\
1.381 \\
1.506 \\
1.646 \\
1.667 \\
1.474 \\
1.467 \\
1.507 \\
1.594 \\
17.927\end{array}$ & $\begin{array}{l}.172 \\
.140 \\
.160 \\
.174 \\
.271 \\
.307 \\
.366 \\
.376 \\
.292 \\
.232 \\
.174 \\
.136 \\
2.798\end{array}$ & $\begin{array}{l}.085 \\
.091 \\
.066 \\
.034 \\
.042 \\
.060 \\
.082 \\
.066 \\
.052 \\
.036 \\
.046 \\
.064 \\
.725\end{array}$ & $\begin{array}{l}.669 \\
.594 \\
.589 \\
.535 \\
.591 \\
.611 \\
.648 \\
.653 \\
.580 \\
.538 \\
.554 \\
.607 \\
7.168\end{array}$ & $\begin{array}{l}.321 \\
.332 \\
.361 \\
.343 \\
.356 \\
.348 \\
.328 \\
.307 \\
.250 \\
.247 \\
.253 \\
.321 \\
3.765\end{array}$ & $\begin{array}{l}.007 \\
.008 \\
.007 \\
.008 \\
.005 \\
.008 \\
.012 \\
.012 \\
.010 \\
.011 \\
.011 \\
.010 \\
.110\end{array}$ & $\begin{array}{l}.002 \\
.001 \\
.002 \\
.001 \\
.001 \\
.002 \\
.002 \\
.002 \\
.002 \\
.002 \\
.002 \\
.002 \\
.020\end{array}$ & $\begin{array}{r}2.830 \\
2.582 \\
2.600 \\
2.372 \\
2.648 \\
2.841 \\
3.083 \\
3.084 \\
2.659 \\
2.533 \\
2.547 \\
2.733 \\
32.512\end{array}$ \\
\hline 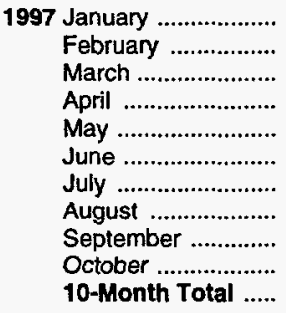 & $\begin{array}{r}1.664 \\
1.392 \\
1.416 \\
1.336 \\
1.400 \\
1.514 \\
1.732 \\
1.691 \\
1.559 \\
1.558 \\
\mathbf{1 5 . 2 6 1}\end{array}$ & $\begin{array}{l}.143 \\
.147 \\
.194 \\
.197 \\
.236 \\
.302 \\
.437 \\
.400 \\
.340 \\
.251 \\
2.648\end{array}$ & $\begin{array}{l}.089 \\
.048 \\
.046 \\
.043 \\
.049 \\
.074 \\
.100 \\
.082 \\
.082 \\
.077 \\
.690\end{array}$ & $\begin{array}{l}.626 \\
.538 \\
.536 \\
.481 \\
.500 \\
.553 \\
.609 \\
.649 \\
.559 \\
.499 \\
\mathbf{5 . 5 5 0}\end{array}$ & $\begin{array}{l}.348 \\
.328 \\
.372 \\
.346 \\
.363 \\
.372 \\
.355 \\
.306 \\
.261 \\
.265 \\
\mathbf{3 . 3 1 6}\end{array}$ & $\begin{array}{l}.009 \\
.006 \\
.009 \\
.010 \\
.010 \\
.008 \\
.011 \\
.011 \\
.010 \\
.010 \\
.094\end{array}$ & $\begin{array}{l}.002 \\
.002 \\
.002 \\
.002 \\
.002 \\
.002 \\
.002 \\
.002 \\
.002 \\
.002 \\
.017\end{array}$ & $\begin{array}{r}2.879 \\
2.461 \\
2.574 \\
2.416 \\
2.559 \\
2.825 \\
3.246 \\
3.140 \\
2.813 \\
2.662 \\
27.576\end{array}$ \\
\hline $\begin{array}{l}1996 \text { 10-Month Total ..... } \\
199510 \text {-Month Total ..... }\end{array}$ & $\begin{array}{l}14.826 \\
14.106\end{array}$ & $\begin{array}{l}2.488 \\
2.897\end{array}$ & $\begin{array}{l}.615 \\
.544\end{array}$ & $\begin{array}{l}6.007 \\
5.978\end{array}$ & $\begin{array}{l}3.191 \\
2.835\end{array}$ & $\begin{array}{l}.089 \\
.077\end{array}$ & $\begin{array}{l}.017 \\
.014\end{array}$ & $\begin{array}{l}27.232 \\
26.449\end{array}$ \\
\hline
\end{tabular}

a Includes supplemental gaseous fuels.

b Includes residual and distillate fuel oils, petroleum coke, and small amounts of kerosene and jet fuel.

c includes net imports of electricity.

d "Other" is electricity generated for distribution from wood, waste, wind, photovoltaic, and solar thermal energy.
$R=$ Revised data.

Notes: - Totals may not equal sum of components due to independent rounding. - Geographic coverage is the 50 States and the District of Columbia.

Additional Notes and Sources: See end of section.

Please Read: This table reports energy input at electric utilities and does not include data on nonutility power producers (NUPP). NUPP data are collected by EIA on an annual basis starting in 1989. See EIA's Electric Power Annual 1995, Volume II, the "Nonutility Power Producers" chapter for additional information. 


\section{Energy Consumption Notes and Sources}

The data in this section of the Monthly Energy Review $(M E R)$ are obtained initially from a group of energy-related surveys, typically called "supply surveys," conducted by the Energy Information Administration (EIA). Supply surveys are those surveys directed to suppliers and marketers of specific energy sources. They measure the quantities of specific energy sources produced, or the quantities supplied to the market, or both. The data obtained from the EIA's supply surveys are integrated to yield the summary consumption statistics published in this section (and in Section 1) of the $M E R$. Users of the EIA's energy consumption statistics should be aware of a second group of energy-related surveys, typically called "consumption surveys." Consumption surveys gather information on the types of energy consumed by end users of energy, along with the characteristics of those end users that can be associated with energy use. For example, the Manufacturing Energy Consumption Survey belongs to the consumption survey group because it collects information directly from end users (the manufacturing establishments). There are important differences between the supply and consumption surveys that need to be taken into account in any analysis that uses both data sources. For information on those differences, see Energy Consumption by End-Use Sector, A Comparison of Measures by Consumption and Supply Surveys, DOE/EIA-0533, Energy Information Administration, Washington, DC, April 6, 1990. The numbered notes that follow elaborate on essential information in Section 2.

1. Total Energy Consumed: Total energy consumed includes coal, natural gas (including supplernental gaseous fuels), petroleum products supplied, electric utility and industrial generation of hydroelectric power, net imports of electricity generated from hydroelectric power, and electricity generated from nuclear power. Total energy consumed also includes electricity generated from geothermal, wood, waste, wind, photovoltaic, and solar thermal energy but excludes other energy obtained from those sources because consistent historical data are not available.

2. Economic Sectors: Energy use is assigned to the major economic sectors according to the following guidelines as closely as possible:

- Residential-All private residences, whether occupied or vacant, owned or rented, including single-family homes, multifamily housing units, and mobile homes. Secondary homes, such as summer homes, are also included. Institutional housing, such as school dormitories, hospitals, and military barracks, generally are not included in the residential sector; they are included in the commercial sector.

- Commercial-Business establishments that are not engaged in transportation or in manufacturing or other types of industrial activity (agriculture, mining, or construction). Commercial establishments include hotels, motels, restaurants, wholesale businesses, retail stores, laundries, and other service enterprises; religious and nonprofit organizations; health, social, and educational institutions; and Federal, State, and local governments. Street lights, pumps, bridges, and public services are also included if the establishment operating them is considered commercial.

- Industrial-Manufacturing industries, which make up the largest part of the sector, along with mining, construction, agriculture, fisheries, and forestry. Establishments in this sector range from steel mills to small farms to companies assembling electronic components.

- Transportation-Private and public vehicles that move people and commodities. Included are automobiles, trucks, buses, motorcycles, railroads and railways (including streetcars), aircraft, ships, barges, and natural gas pipelines.

- Electric Utility-Privately and publicly owned establishments that generate, transmit, distribute, and sell electricity primarily for use by the public and meet the definition of an electric utility. Nonutility power producers are not included in the electric utility sector.

Although the end-use allocations are made according to these aggregations as closely as possible, some data are collected by using different classifications. For example, data on agricultural use of natural gas are collected and reported in the commercial sector, rather than in the industrial sector. Since agricultural use of natural gas cannot be identified separately, it is included in the commercial sector in this report. Another example is master-metered condominiums and apartments, and buildings with a combination of residential and commercial units. In many cases, the metering and billing practices cause residential energy usage of electricity, natural gas, or fuel oil to be included in the commercial sector. No adjustments for these discrepancies were made.

3. Conversion Factors: See the conversion factors listed in Appendix A.

4. Coal: Coal is anthracite, bituminous coal (including subbituminous coal), and lignite. Sources:

- 1973-October 1977: U.S. Department of the Interior (DOI), Bureau of Mines (BOM), Minerals Yearbook and Minerals Industry Surveys.

- Electric Utilities-October 1977 forward: Energy Information Administration (EIA), Form EIA-759 (formerly Federal Power Commission (FPC) Form FPC-4), "Monthly Power Plant Report."

- Other Industrial-October 1977-December 1979: EIA, Form EIA-3, "Monthly Coal Consumption Report -Manufacturing Plants"; January 1980 forward: EIA, Form EIA-3, "Quarterly Coal Consumption Report - Manufacturing Plants," and Form EIA-6, "Coal Distribution Report," quarterly. 
- Coke Plants-October 1977-December 1980: EIA, Form EIA-5/5A, "Coke and Coal Chemicals - Monthly/Annual"; January 1981-December 1984: EIA, Form EIA-5/5A, "Coke Plant Report - Quarterly/Annual Supplement"; January 1985 forward: EIA, Form EIA-5/5A, "Coke Plant Report - Quarterly."

- Residential and Commercial-October 1977-December 1979: EIA, Form EIA-2, "Monthly Coal Report, Retail Dealers - Upper Lake Docks"; January 1980 forward: EIA, Form EIA-6, "Coal Distribution Report," quarterly.

5. Natural Gas: Natural gas consumption by end use is based on data presented in Table 4.4 of this report. For Section 2 calculations, lease and plant fuel consumption are added to industrial deliveries, and pipeline fuel represents transportation use of natural gas. Values in Btu are derived by using the conversion factors provided in Appendix A. Sources:

- 1973-1975: DOI, BOM, Minerals Yearbook, "Natural Gas" chapter.

- 1976-1978: EIA, Energy Data Reports, "Natural Gas, Annual."

- 1979: EIA, Natural Gas Production and Consumption 1979.

- 1980-1995: EIA, Natural Gas Annual.

- 1996 and 1997: EIA, Natural Gas Monthly.

- Electric Utilities-1973-1976: Form FPC-4, "Monthly Power Plant Report"; 1977-1981: Federal Energy Regulatory Commission (FERC), Form FPC-4, "Monthly Power Plant Report"; 1982 forward: EIA, Form EIA-759, "Monthly Power Plant Report."

- American Gas Association, "Monthly Gas Utility Statistical Report," residential and commercial monthly sales data for 1973-1979, which are used to estimate monthly consumption values from EIA annual consumption values.

6. Petroleum: Petroleum consumption by end use is the sum of all individual petroleum products estimated to be consumed in each end-use sector. First, total consumption by product is determined. Petroleum consumption in this section of the Monthly Energy Review $(M E R)$ is the series called "petroleum products supplied" in Section 3. Sources for petroleum products supplied by individual products are:

- 1973-1975: DOI, BOM, Mineral Industry Surveys, "Petroleum Statement, Annual."

- 1976-1980: EIA, Energy Data Reports, "Petroleum Statement, Annual."

- 1981-1996: EIA, Petroleum Supply Annual.

- 1997: EIA, Petroleum Supply Monthly.

Specific petroleum products' end-use allocation procedures follow:
- Aviation Gasoline-All product supplied is assigned to the transportation sector.

- Asphalt-All product supplied is assigned to the industrial sector.

- Distillate Fuel-Product supplied is assigned to electric utilities and non-electric utilities as follows:

\section{Electric Utilities, All Periods.}

For 1973-1979, consumption of distillate fuel is assumed to be the amount of petroleum (minus small amounts of kerosene and kerosene-type jet fuel deliveries) consumed in gas turbine and internal combustion plants. For 1980 forward, consumption of distillate fuel is assumed to be the amount of light oil (minus small amounts of kerosene deliveries through 1982) consumed at electric utilities. (See Table 7.3)

Sources: 1973-September 1977: FPC, Form FPC4, "Monthly Power Plant Report"; October 1977. 1981: FERC, Form FPC-4, "Monthly Power Plant Report"; 1982 forward: EIA, Form EIA-759, "Monthly Power Plant Report."

Sectors Other Than Electric Utilities, Annual Estimates Through 1994.

The aggregate non-electric utility use of distillate fuel is total distillate fuel supplied minus the electric utility consumption. The non-electric utility annual consumption totals are allocated to the individual non-electric utility sectors (residential, commercial, industrial, and transportation) in proportion to the share of "adjusted sales" of each end-use sector, as reported in EIA's Fuel Oil and Kerosene Sales report series (DOE/EIA-0535), which is based primarily on data collected by Form EIA-821, previously Form EIA-172. "Adjusted sales" are sales that have been adjusted at the PAD district level to equal EIA volume estimates of petroleum products supplied in the U.S. market. Following are notes on the individual sector groupings:

- Since 1979, the residential sector adjusted sales total is directly from the Sales reports. Prior to 1979 , each year's sales subtotal of the heating plus industrial category is split into residential, commercial, and industrial (including farm) in proportion to the 1979 shares.

- Since 1979, the commercial sector adjusted sales total is directly from the Sales reports. Prior to 1979 , each year's sales subtotal of the heating plus industrial category is split into residential, commercial, and industrial (including farm) in proportion to the 1979 shares. 
- Since 1979, the industrial sector adjusted sales total is the sum of the adjusted sales for industrial, farm, oil company, off-highway, diesel, and all other uses. Prior to 1979 , each year's sales subtotal of the heating plus industrial category is split into residential, commercial, and industrial (including farm) in proportion to the 1979 shares, and this estimated industrial portion is added to oil company, off-highway diesel, and all other uses.

- The transportation sector adjusted sales total is the sum of the adjusted sales for railroad, vessel bunkering, on-highway diesel, and military uses for all years.

\section{Sectors Other Than Electric Utilities, Monthly Es- timates Through 1994.}

- Residential and commercial monthly consumption is estimated by allocating the annual estimates, which are described above, into the months in proportion to each month's share of the year's sales of No. 2 heating oil. The years' sales totals are from the following sources: for 1973-1980, the Ethyl Corporation, Monthly Report of Heating Oil Sales; for 1981 and 1982, the American Petroleum Institute, Monthly Report of Heating Oil Sales; and for 1983-1992, EIA, Form EIA-782A, "Refiners'/Gas Plant Operators' Monthly Petroleum Product Sales Report," No. 2 Fuel Oil Sales to End Users and for Resale.

- The transportation highway use portion is allocated into the months in proportion to each month's share of the year's total sales for highway use as reported by the Federal Highway Administration's Table MF-25, "Private and Commercial Highway Use of Special Fuels by Months.” After 1993, the sales-for-highway-use data are no longer available as a monthly series; the 1993 data are used for allocating succeeding year's totals into months. The remaining transportation use of distillate fuel (i.e., for railroads, vessel bunkering, and military use) is evenly distributed over the months, adjusted for the number of days per month.

- Industrial monthly estimates are made by subtracting the residential and commercial, transportation, and electric utility sector estimates from each month's total distillate fuel supplied.

\section{Sectors Other Than Electric Utilities, 1995-1997.}

Each month's non-electric utility consumption subtotal is disaggregated into the major end-use sectors in proportion to the shares each sector held of the non-electric utility subtotal in the same month in 1994.

- Jet Fuel-Through 1982, small amourts of kerosene-type jet fuel were consumed by electric utilities. Kerosene-type jet fuel deliveries to electric utilities as reported on the Form FERC-423 (formerly Form FPC-423) were used as estimates of this consumption. All remaining jet fuel (kerosene-type and naphtha-type) is consumed by the transportation sector.

- Kerosene-Total product supplied monthly is allocated to the major end-use sectors in proportion to annual sales grouped into end-use sectors from EIA's Fuel Oil and Kerosene Sales reports (based primarily on data collected by Form EIA-821, previously Form EIA-172), as follows:

- Residential deliveries are taken directly from the Sales reports for 1979-1994. Sales for 1994 are used as estimates for succeeding periods. Prior to 1979 , each year's sales category called "heating" is split into residential, commercial, and industrial in proportion to the 1979 shares.

- Commercial sales are directly from the Sales reports for 1979-1994. Sales for 1994 are used as estimates for succeeding periods. Prior to 1979 , each year's sales category called "heating" is split into residential, commercial, and industrial in proportion to the 1979 shares.

- Industrial sales are directly from the Sales reports for 1979-1994. Sales for 1994 are used as estimates for succeeding periods. Prior to 1979 , each year's sales category called "heating" is split into residential, commercial and industrial in proportion to the 1979 shares, and this estimated industrial (including farm) portion is added to all other uses.

- Liquefied Petroleum Gases (LPG)-The annual shares of LPG's total consumption that are estimated to be consumed by each end-use sector are applied to each month's total LPG consumption (i.e., product supplied) to create monthly end-use consumption estimates. The annual end-use shares are calculated in the following manner:

- Sales of LPG to the residential and commercial sector are converted from thousand gallons per year to thousand barrels per year and are assumed to be the annual consumption of LPG by the sector.

- The quantity of LPG sold each year for consumption in internal combustion engines is allocated between the transportation and industrial sectors on the basis of data for special fuels used on highways published by the U.S. Department of Transportation, Federal Highway Administration, in Highway Statistics. The allocations of LPG sold for internal combustion engine use to the transportation sector range from a low of 37 percent in 1987 to a high of 73 percent in 1994.

- LPG consumed annually by the industrial sector is estimated as the difference between LPG total supplied and the estimated consumption of LPG by the sum of the residential and commercial sector and the transportation sector. The industrial sector includes LPG used by chemical plants as raw ma- 
terials or solvents and used in the production of synthetic rubber; refinery fuel use; use as synthetic natural gas feedstock and use in secondary recovery projects; all farm use; LPG sold to gas utility companies for distribution through the mains; and a portion of the use of LPG as an internal combustion engine fuel.

The sources of the annual sales data for creating annual end-use shares are:

- 1973-1982: EIA's "Sales of Liquefied Petroleum Gases and Ethane" reports, based primarily on data collected by Form EIA-174.

- 1983: End-use consumption estimates for 1983 are based on 1982 end-use consumption because the collection of data under Form EIA-174 was discontinued after data year 1982 .

- 1984-1994: American Petroleum Institute (API), "Sales of Natural Gas Liquids and Liquefied Refinery Gases," which is based on an LPG sales survey jointly sponsored by API, the Gas Processors Association, and the National Liquefied Petroleum Gas Association.

- 1995-1997: The 1994 source is used to estimate succeeding periods.

- Lubricants-Total product supplied is allocated to the industrial and transportation sectors for all months according to proportions developed from annual sales of lubricants to the two sectors from U.S. Department of Commerce, Bureau of the Census, Current Industrial Reports, "Sales of Lubricating and Industrial Oils and Greases." The 1973 shares are applied to 1973 and 1974; the 1975 shares are applied to 1975 and 1976; and the 1977 shares are applied to 1977 forward.

- Motor Gasoline-Total product supplied monthly is allocated to the major end-use sectors in proportion to aggregations of annual sales categories created on the basis of the U.S. Department of Transportation, Federal Highway Administration, Highway Statistics, Tables MF-21, MF-24, and MF-25, as follows:

- Commercial sales are the sum of sales for public non-highway use and miscellaneous and unclassified uses.

- Industrial sales are the sum of sales for agriculture, construction, and industrial and commercial use as classified in the Highway Statistics.

- Transportation sales are the sum of sales for highway use (minus the sales of special fuels, which are primarily diesel fuel and are accounted for in the transportation sector of distillate fuel) and sales for marine use.
- Petroleum Coke--The portion consumed by electric utilities is from Form EIA-759, "Monthly Power Plant Report" (formerly Form FPC-4). The remaining petroleum coke is assigned to the industrial sector.

- Residual Fuel-Product supplied is assigned to electric utilities and non-electric utilities as follows:

\section{Electric Utilities, All Periods.}

For 1973-1979, consumption of residual fuel is assumed to be the amount of petroleum consumed in steam-electric power plants. For 1980 forward, consumption of residual fuel is assumed to be the amount of heavy oil consumed at electric utilities. (See Table 7.3)

Sources: 1973-September 1977: Form FPC-4, "Monthly Power Plant Report"; October 1977. 1981: FERC, Form FPC-4, "Monthly Power Plant Report"; 1982 forward: EIA, Form EIA-759, "Monthly Power Plant Report."

\section{Sectors Other Than Electric Utilities, Annual Es- timates Through 1994.}

The aggregate non-electric utility use of residual fuel is total residual fuel supplied minus the electric utility consumption. The non-electric utility annual totals are allocated into the individual non-electric utility sectors in proportion to the amount of residual fuel sold to end users, grouped into sectors from EIA's Fuel Oil and Kerosene Sales reports (based primarily on data collected by Form EIA-821, previously Form EIA-172), as follows:

- Since 1979, commercial sales data are directly from the Sales reports. Prior to 1979, each year's sales subtotal of the heating plus industrial category is split into commercial and industrial in proportion to the 1979 shares.

- Since 1979, industrial sales data are the sum of sales for industrial, oil company, and all other uses. Prior to 1979 , each year's sales subtotal of the heating plus industrial category is split into commercial and industrial in proportion to the 1979 shares, and this estimated industrial portion is added to oil company and all other uses.

- Transportation sales are the sum of sales for railroad, vessel bunkering, and military uses for all years.

Sectors Other Than Electric Utilities, Monthly Es. timates Through 1994.

- Commercial monthly consumption is estimated by allocating the annual estimates, which are described above, into the months in proportion to each 
month's share of the year's sales of No. 2 heating oil. The years' sales totals are from the following sources: for 1973-1980, the Ethyl Corporation, Monthly Report of Heating Oil Sales; for 1981 and 1982, the American Petroleum Institute, Monthly Report of Heating Oil Sales; and for 1983-1992, EIA, Form EIA-782A, "Refiners'/Gas Plant Operators' Monthly Petroleum Product Sales Report," No. 2 Fuel Oil Sales to End Users and for Resale.

- Transportation monthly estimates are made by evenly distributing the annual sector estimate over the months, adjusting for the number of days per month.

- Industrial monthly estimates are made by subtracting the commercial, transportation, and electric utility sector estimates from each month's total residual fuel supplied.

\section{Sectors Other Than Electric Utilities, 1995-1997.}

Each month's non-electric utility consumption subtotal is disaggregated into the major end-use sectors in proportion to the shares each sector held of the non-electric utility subtotal in the same month in 1994.

- Road Oil-All product supplied is assigned to the industrial sector.

- All Other Petroleum Products-The product supplied of all remaining petroleum products is assigned to the industrial sector.

7. Nuclear Electric Power, Geothermal, and Wood, Waste, Wind, Photovoltaic, and Solar Thermal Energy Sources Connected to Electric Utility Distribution Systems: Sources:

- 1973-1976: FPC, Form FPC-4, "Monthly Power Plant Report."

- 1977-1981: FERC, Form FPC-4, "Monthly Power Plant Report."

- 1982 forward: EIA, Form EIA-759, "Monthly Power Plant Report."

8. Hydroelectric Power: Includes electricity generated by hydroelectric power at electric utilities, small amounts in the industrial sector, and net imports of electricity, which are assumed to be generated by hydroelectric power and are included in the electric utilities sector.

Sources for electric utilities sector:

- 1973-1976: FPC, Form FPC-4, "Monthly Power Plant Report."

- 1977-1981: FERC, Form FPC-4, “Monthly Power Plant Report."

- 1982 forward: EIA, Form EIA-759, "Monthly Power Plant Report."

Sources for industrial sector:
- 1973-1978: FPC, Form FPC-4, "Monthly Power Plant Report," for plants with generating capacity exceeding 10 megawatts, and FPC, Form FPC12C, "Industrial Electric Generating Capacity," for all other plants.

- 1979: FPC, Form FPC-4, "Monthly Power Plant Report," for plants with generating capacity exceeding 10 megawatts and EIA estimates for all other plants.

- 1980 forward: Annual generation estimated by EIA as the average generation over the 6-year period of 1974-1979; monthly generation estimated to be in proportion to each month's hydroelectricity generation in the electric utility industry in 1980.

Sources for imports and exports of electricity:

- 1973-September 1977: Power Commission data.

- October 1977-1980: Unpublished Economic Regulatory Administration (ERA) data.

- 1981: DOE, Office of Energy Emergency Operations, "Report on Electric Energy Exchanges with Canada and Mexico for Calendar Year 1981," April 1982 (revised June 1982).

- 1982 and 1983: DOE, ERA, Electricity Exchanges Across International Borders.

- 1984-1986: DOE, ERA, Electricity Transactions Across International Borders.

- 1987 and 1988: DOE, ERA, Form ERA-781R, "Annual Report of International Electrical Export/Import Data."

- 1989-1993: DOE, Assistant Secretary for Fossil Energy, Form FE-781-R, "Annual Report of International Electrical Export/Import Data."

- 1994 forward: EIA estimates based on preliminary data from the National Energy Board of Canada and DOE, Assistant Secretary for Fossil Energy.

9. Net Imports of Coal Coke: Net imports means imports minus exports, and a minus sign indicates that exports are greater than imports. Sources:

- 1973-1975: DOI, BOM, Minerals Yearbook, "Coke and Coal Chemicals" chapter.

- 1976-1980: EIA, Energy Data Report, "Coke and Coal Chemicals" annual.

- 1981: EIA, Energy Data Report, "Coke Plant Report," quarterly.

- 1982 forward: EIA, Quarterly Coal Report.

10. Electricity: End-use consumption of electricity is based on Table 7.2 sales data. "Other," which is primarily for use in government buildings, is added to the commercial sector, except for approximately 4 percent used by railroads and railways and attributed to the 
transportation sector. Kilowathours are converted to Btu at the rate of 3,412 Btu per kilowatthour. See Table 7.2 for sources of the electricity sales data.

11. Electrical System Energy Losses: Electrical system energy losses are calculated as the difference between total energy input at electric utilities and the total energy content of electricity sold to end-use consumers. Most of those losses occur at steam-electric power plants (conventional and nuclear) in the conversion of heat energy into mechanical energy to turn electric generators. The loss is a thermodynamically necessary feature of the steam-electric cycle. Part of the energy input-to-output losses is a result of imputing fossil energy equivalent inputs for hydroelectric and other energy sources, since there is no generally accepted practice for measuring those thermal conversion rates. In addition to conversion losses, other losses include power plant use of electricity, transmission and distribution of electricity from power plants to end-use consumers (also called "line losses"), and unaccounted for electricity. Total losses are allocated to the end-use sectors in proportion to each sector's share of total electricity sales. Overall, approximately 67 percent of total energy input is lost in conversion; of electricity generated, approximately 5 percent is lost in plant use and 9 percent is lost in transmission and distribution. Calculated electrical system energy losses may be less than actual losses, because primary consumption does not include the energy equivalent of utility purchases of electricity from non-electric utilities and from Canada and Mexico, although they are included in electricity sales.

12. Renewable Energy: Monthly Energy Review (MER) consumption and production totals currently capture about half of estimated total renewable energy resources. Coverage is complete for the electric utilities as reported under "Hydroelectric Power," "Geothermal Energy," and "Other" on Table 2.6. Small amounts of hydroelectric power (about 0.03 quadrillion Btu in 1996) included on Table 2.6 are used at pumped storage facilities and are not considered renewable. Small quantities of ethanol (about 0.07 quadrillion Btu in 1996) are blended into motor gasoline, which are accounted for under "Petroleum Products" on Table 2.5 for the transportation sector.

Renewable energy used by residential, commercial, and industrial consumers is not currently included in the $M E R$ data series because consistent monthly series are not available. On an annual basis, the estimated quantities in quadrillion Btu are:

\begin{tabular}{|c|c|c|c|c|c|c|c|c|c|}
\hline \multirow[b]{2}{*}{ Year } & \multicolumn{3}{|c|}{ Residential and Commercial } & \multicolumn{6}{|c|}{ Industrial } \\
\hline & Biofuels & $\begin{array}{l}\text { Solar } \\
\text { Energy }\end{array}$ & Total & Biofuels & $\begin{array}{l}\text { Geothermal } \\
\text { Energy }\end{array}$ & $\begin{array}{l}\text { Conventional } \\
\text { Hydroelectric } \\
\text { Power }\end{array}$ & $\begin{array}{l}\text { Solar } \\
\text { Energy }\end{array}$ & $\begin{array}{l}\text { Wind } \\
\text { Energy }\end{array}$ & Total \\
\hline 1990 & 0.581 & 0.060 & 0.641 & 1.948 & 0.153 & 0.084 & 0.007 & 0.023 & 2.215 \\
\hline 1991 & 0.613 & 0.060 & 0.673 & 1.943 & 0.168 & 0.085 & 0.008 & 0.027 & 2.231 \\
\hline 1992 & 0.645 & 0.060 & 0.705 & 2.042 & 0.179 & 0.097 & 0.008 & 0.030 & 2.357 \\
\hline 1993 & 0.592 & 0.060 & 0.652 & 2.084 & 0.204 & 0.118 & 0.009 & 0.031 & 2.446 \\
\hline 1994 & 0.582 & 0.060 & 0.642 & 2.138 & 0.212 & 0.136 & 0.008 & 0.036 & 2.530 \\
\hline 1995 & 0.641 & 0.064 & 0.705 & 2.184 & 0.207 & 0.152 & 0.008 & 0.033 & 2.584 \\
\hline $1996^{\mathrm{E}}$ & 0.644 & 0.065 & 0.709 & 2.279 & 0.231 & 0.172 & 0.009 & 0.036 & 2.727 \\
\hline
\end{tabular}

$\mathrm{E}=$ Estimate

Source: Energy Information Administration, Annual Energy Review 1996 (July 1997), Table 10.2.

Note: More information about renewable energy is available in EIA's Renewable Energy Annual 1996, which was released in March 1997. See the inside front cover of the Monthly Energy Review for information about ordering EIA reports, or, for direct access to several reports on the subject of renewable energy, go to our Web site at http:/www.eia.doe.gov and tap "Alternative/Renewables" under "Fuel Groups." 


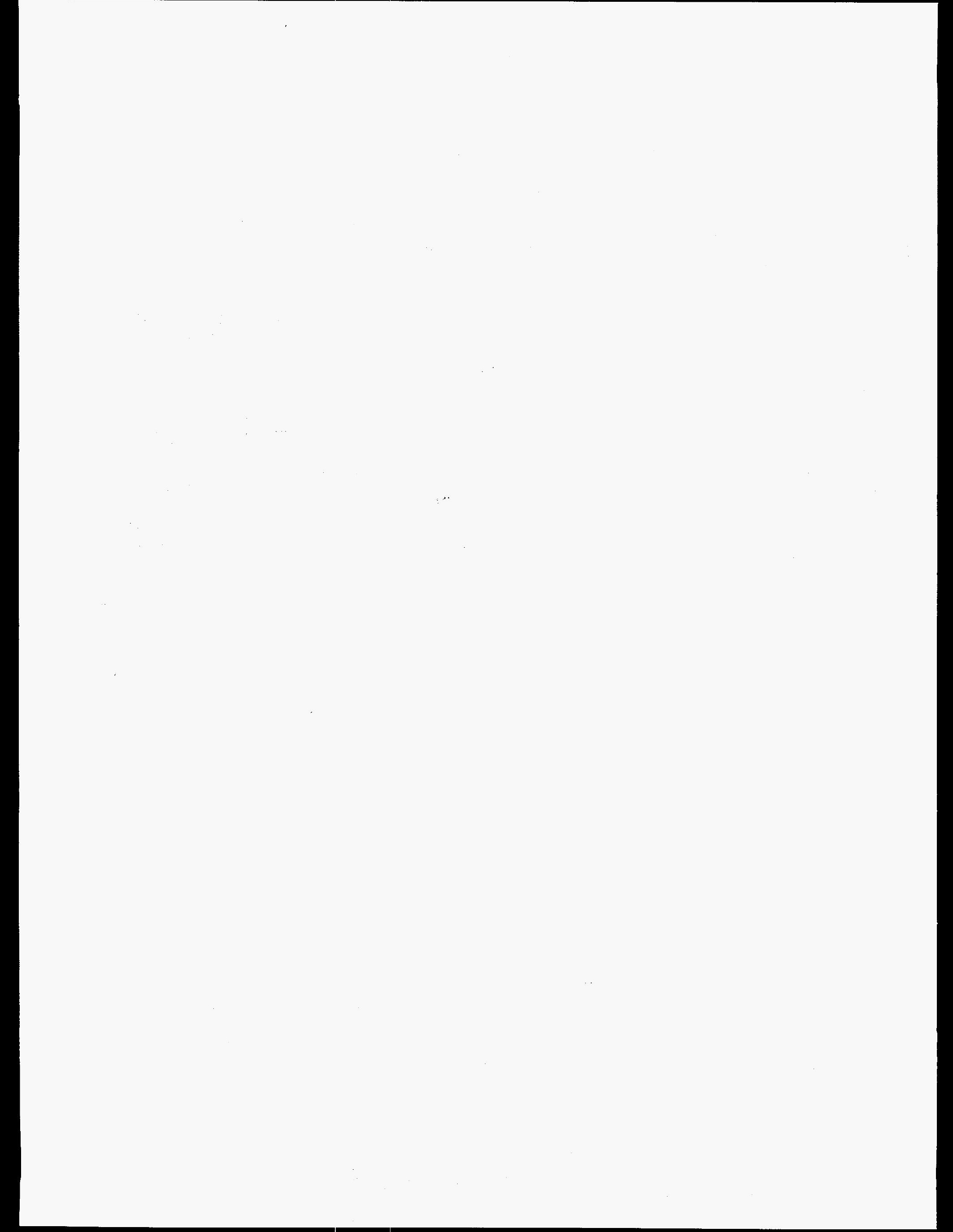




\section{Section 3. Petroleum}

Total petroleum imports ${ }^{1}$ averaged 9.1 million barrels per day in December 1997, 6 percent lower than the previous month's rate and 3 percent lower than the December 1996 rate.

In December 1997, 19.0 million barrels per day of petroleum products were supplied for domestic use, 4 percent higher than the December 1996 rate. Motor gasoline accounted for 43 percent of the total; distillate fuel oil, 19 percent; and kerosene-type jet fuel, 9 percent.

Motor gasoline supplied during December 1997 averaged 8.1 million barrels per day, 2 percent higher than the previous month's rate and 4 percent higher than the December 1996 rate. Total motor gasoline stocks were 209 million barrels at the end of December 1997, 7 million barrels above the stock level in the previous month and 14 million barrels above the level 1 year earlier.
Distillate fuel oil supplied during December 1997 averaged 3.6 million barrels per day, 6 percent higher than both the previous month's rate and the December 1996 rate. Distillate fuel oil ending stocks for December 1997 were 136 million barrels, 4 million barrels below the stock level in the previous month but 9 million barrels above the level 1 year earlier.

Kerosene-type jet fuel supplied in December 1997 averaged 1.7 million barrels per day, 5 percent higher than the previous month's rate and 8 percent higher than the December 1996 rate. Kerosene-type jet fuel stocks measured 44 million barrels at the end of December 1997, 2 million barrels below the stock level in the previous month but 4 million barrels higher than the stock level 1 year earlier.

Estimates (except of crude production) for the most current month are based on Energy Information Administration (EIA) weekly data and will be revised to conform with data from the EIA Petroleum Reporting System as available. For the most recent month, crude production is an EIA estimate based on historical and provisional data through September 1997.

\footnotetext{
${ }^{1}$ Total import data include imports into the Strategic Petroleum Reserve.
} 
Table 3.1a Petroleum Overview: Field Production, Stock Change, Petroleum Products Supplied, and Ending Stocks

\begin{tabular}{|c|c|c|c|c|c|c|c|}
\hline & \multicolumn{3}{|c|}{ Field Production } & \multicolumn{2}{|c|}{ Stock Change a } & \multirow[b]{2}{*}{$\begin{array}{l}\text { Petroleum } \\
\text { Products } \\
\text { Supplied }\end{array}$} & \multirow{2}{*}{$\begin{array}{c}\text { Ending Stocks }{ }^{b} \\
\text { Crude Oild and } \\
\text { Petroleum } \\
\text { Products }\end{array}$} \\
\hline & $\begin{array}{c}\text { Total } \\
\text { Dornestic }\end{array}$ & $\begin{array}{c}\text { Crude } \\
\text { Oil }\end{array}$ & $\begin{array}{l}\text { Natural } \\
\text { Gas Plant } \\
\text { Liquids }\end{array}$ & $\begin{array}{c}\text { Crude } \\
\text { Oild }^{d}\end{array}$ & $\begin{array}{l}\text { Petroleum } \\
\text { Products }\end{array}$ & & \\
\hline & \multicolumn{6}{|c|}{ Thousand Barrels per Day } & Million Barrels \\
\hline $\begin{array}{l}1973 \text { Average } \\
1974 \text { Average } \\
1975 \text { Average } \\
1976 \text { Average } \\
1977 \text { Average } \\
1978 \text { Average } \\
1979 \text { Average } \\
1980 \text { Average } \\
1981 \text { Average } \\
1982 \text { Average } \\
1983 \text { Average } \\
1984 \text { Average } \\
1985 \text { Average } \\
1986 \text { Average } \\
1987 \text { Average } \\
1988 \text { Average } \\
1989 \text { Average } \\
1990 \text { Average } \\
1991 \text { Average }\end{array}$ & $\begin{array}{r}10,975 \\
10,498 \\
10,045 \\
9,774 \\
9,913 \\
10,328 \\
10,179 \\
10,214 \\
10,230 \\
10,252 \\
10,299 \\
10,554 \\
10,636 \\
10,289 \\
10,008 \\
9,818 \\
9,219 \\
8,994 \\
9,168 \\
8,996 \\
98,836 \\
8,645\end{array}$ & $\begin{array}{l}9,208 \\
8,774 \\
8,375 \\
8,132 \\
8,245 \\
8,707 \\
8,552 \\
8,597 \\
8,572 \\
8,649 \\
8,688 \\
8,879 \\
8,971 \\
8,680 \\
8,349 \\
8,140 \\
7,613 \\
7,355 \\
7,417 \\
7,171 \\
6,847 \\
6,662\end{array}$ & $\begin{array}{r}1,738 \\
1,688 \\
1,633 \\
+1,604 \\
1,618 \\
1,567 \\
1,584 \\
1,573 \\
1,609 \\
1,550 \\
1,559 \\
1,630 \\
1,609 \\
1,551 \\
1,595 \\
1,625 \\
1,546 \\
1,559 \\
1,659 \\
1,697 \\
1,736 \\
1,727\end{array}$ & $\begin{array}{r}-11 \\
62 \\
e 17 \\
39 \\
170 \\
78 \\
148 \\
98 \\
e_{290} \\
136 \\
\text { e214 } \\
199 \\
50 \\
78 \\
128 \\
1 \\
86 \\
-35 \\
-42 \\
-1 \\
81 \\
18\end{array}$ & $\begin{array}{r}146 \\
117 \\
e_{15} \\
-96 \\
378 \\
-172 \\
25 \\
42 \\
e_{-}-130 \\
-283 \\
e_{-234} \\
81 \\
-153 \\
124 \\
-87 \\
-29 \\
-129 \\
142 \\
32 \\
-68 \\
e 70 \\
-2\end{array}$ & $\begin{array}{l}17,308 \\
16,653 \\
16,322 \\
17,461 \\
18,431 \\
18,847 \\
18,513 \\
17,056 \\
16,058 \\
15,296 \\
15,231 \\
15,726 \\
15,726 \\
16,281 \\
16,665 \\
17,283 \\
17,325 \\
16,988 \\
16,714 \\
17,033 \\
17,237 \\
17,718\end{array}$ & $\begin{array}{r}1,008 \\
e_{1,074} \\
1,133 \\
1,112 \\
1,312 \\
1,278 \\
1,341 \\
e_{1,392} \\
1,484 \\
e_{1,430} \\
1,454 \\
1,556 \\
1,519 \\
1,593 \\
1,607 \\
1,597 \\
1,581 \\
1,621 \\
1,617 \\
e_{1,592} \\
e_{1,647} \\
1,653\end{array}$ \\
\hline 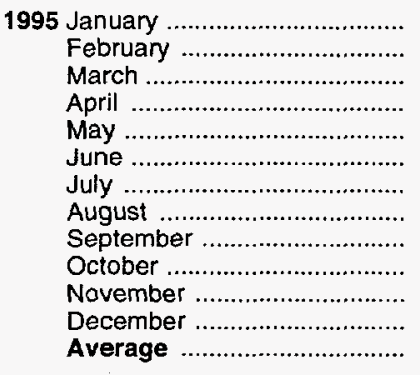 & $\begin{array}{l}8,764 \\
8,935 \\
8,619 \\
8,720 \\
8,729 \\
8,607 \\
8,500 \\
8,498 \\
8,467 \\
8,501 \\
8,662 \\
8,533 \\
8,626\end{array}$ & $\begin{array}{l}6,682 \\
6,794 \\
6,600 \\
6,604 \\
6,629 \\
6,579 \\
6,449 \\
6,447 \\
6,416 \\
6,421 \\
6,585 \\
6,530 \\
6,560\end{array}$ & $\begin{array}{l}1,787 \\
1,780 \\
1,776 \\
1,794 \\
1,790 \\
1,740 \\
1,751 \\
1,730 \\
1,757 \\
1,757 \\
1,797 \\
1,691 \\
1,762\end{array}$ & $\begin{array}{r}-219 \\
-49 \\
336 \\
-101 \\
-132 \\
-148 \\
-397 \\
-253 \\
-64 \\
168 \\
263 \\
-505 \\
-93\end{array}$ & $\begin{array}{r}-84 \\
-1,225 \\
-552 \\
114 \\
464 \\
57 \\
897 \\
-73 \\
243 \\
-589 \\
-352 \\
-822 \\
-153\end{array}$ & $\begin{array}{l}17,219 \\
18,279 \\
17,484 \\
17,142 \\
17,293 \\
18,131 \\
17,147 \\
18,044 \\
18,026 \\
17,651 \\
17,979 \\
18,366 \\
17,725\end{array}$ & $\begin{array}{l}1,643 \\
1,608 \\
1,601 \\
1,601 \\
1,612 \\
1,609 \\
1,624 \\
1,614 \\
1,620 \\
1,607 \\
1,604 \\
1,563 \\
1,563\end{array}$ \\
\hline 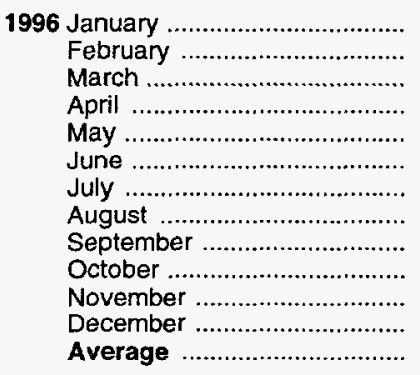 & $\begin{array}{l}8,564 \\
8,558 \\
8,718 \\
8,597 \\
8,502 \\
8,550 \\
8,486 \\
8,535 \\
8,623 \\
8,685 \\
8,730 \\
8,738 \\
8,1507\end{array}$ & $\begin{array}{l}6,495 \\
6,577 \\
6,571 \\
6,444 \\
6,394 \\
6,458 \\
6,338 \\
6,360 \\
6,482 \\
6,481 \\
6,476 \\
6,506 \\
6,465\end{array}$ & $\begin{array}{l}1,716 \\
1,680 \\
1,814 \\
1,845 \\
1,806 \\
1,833 \\
1,829 \\
1,858 \\
1,872 \\
1,912 \\
1,915 \\
1,876 \\
1,830\end{array}$ & $\begin{array}{r}-8 \\
-63 \\
-132 \\
29 \\
2 \\
305 \\
-244 \\
-19 \\
-499 \\
186 \\
-414 \\
-627 \\
-124\end{array}$ & $\begin{array}{r}-592 \\
-1,454 \\
-464 \\
633 \\
576 \\
593 \\
358 \\
-130 \\
701 \\
-630 \\
-117 \\
165 \\
-28\end{array}$ & $\begin{array}{l}18,261 \\
18,620 \\
18,301 \\
17,885 \\
17,957 \\
18,107 \\
18,211 \\
18,658 \\
17,655 \\
19,171 \\
18,535 \\
18,334 \\
18,309\end{array}$ & $\begin{array}{l}1,544 \\
1,500 \\
1,482 \\
1,502 \\
1,520 \\
1,546 \\
1,550 \\
1,545 \\
1,551 \\
1,538 \\
1,522 \\
1,507 \\
1,507\end{array}$ \\
\hline 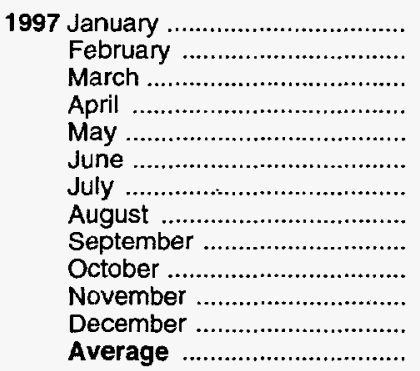 & $\begin{array}{r}E_{8,187} \\
E_{8,739} \\
E_{8,690} \\
E_{8,172} \\
E_{8,559} \\
E_{8,546} \\
E_{8,553} \\
E_{8,480} \\
E_{8,617} \\
E_{8,621} \\
\text { E }_{8,580} \\
E_{8,685} \\
E_{8,601}\end{array}$ & $\begin{array}{l}E_{6,387} \\
E_{6,514} \\
E_{6,470} \\
E_{6,483} \\
E_{6,401} \\
E_{6,341} \\
E_{6,316} \\
E_{6,282} \\
E_{6,388} \\
E_{6,435} \\
\text { RE }_{6,450} \\
P_{6,455} \\
P_{6,409}\end{array}$ & $\begin{array}{r}1,815 \\
1,900 \\
1,907 \\
1,849 \\
1,832 \\
1,842 \\
1,850 \\
1,850 \\
1,871 \\
1,840 \\
1,753 \\
\text { E } 1,872 \\
\text { E } 1,848\end{array}$ & $\begin{array}{r}497 \\
-167 \\
529 \\
208 \\
212 \\
-172 \\
-399 \\
-278 \\
78 \\
412 \\
\text { R } 252 \\
\text { E }-417 \\
\text { E } 65\end{array}$ & $\begin{array}{r}-717 \\
-569 \\
447 \\
10 \\
1,172 \\
676 \\
-191 \\
634 \\
720 \\
-279 \\
R-199 \\
E-224 \\
E 127\end{array}$ & $\begin{array}{r}18,560 \\
18,308 \\
17,869 \\
18,572 \\
18,244 \\
18,563 \\
19,065 \\
18,506 \\
18,480 \\
19,121 \\
R 18,491 \\
\text { E } 19,025 \\
\text { E } 18,570\end{array}$ & $\begin{array}{r}1,503 \\
1,482 \\
1,512 \\
1,519 \\
1,562 \\
1,577 \\
1,559 \\
1,570 \\
1,594 \\
1,598 \\
\text { R }_{1,599} \\
\text { E } 1,564^{-1,564}\end{array}$ \\
\hline
\end{tabular}

a A negative number indicates a decrease in stocks and a positive number indicates an increase.

b Stocks are totals as of end of period.

c Includes crude oil, natural gas plant liquids, and other liquids.

d Includes stocks located in the Strategic Petroleum Reserve.

See Note 4 at end of section.

$f$ See Note 6 at end of section.

9 Beginning in 1993, includes fuel ethanol blended into finished motor gasoline and oxygenate production from merchant MTBE (methyl tertiary butyl ether) plants.

$P E=$ Preliminary estimate. $R=$ Revised data. $E=E s t i m a t e$.

Notes: - Crude oil includes lease condensate. - Geographic coverage is the 50 States and the District of Columbia.

Sources: - 1973-1980: Energy Information Administration (EIA), Petroleum Supply Monthly, February 1993, Table S1. • 1981 forward: EIA, Petroleum Supply Monthly, January 1998, Table S1. 
Table 3.1b Petroleum Overview: Imports, Exports, and Net Imports

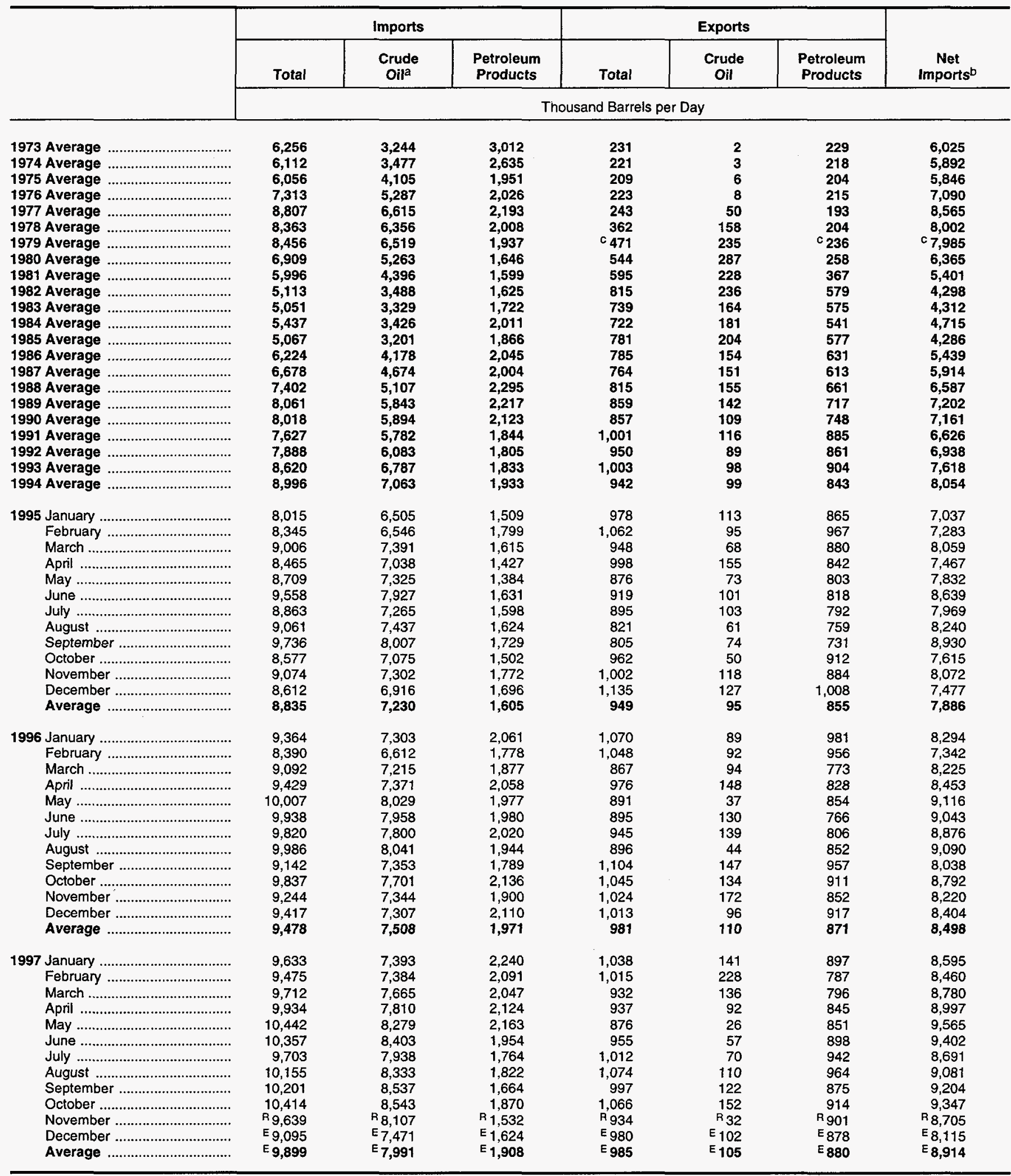

a Includes crude oil for storage in the Strategic Petroleum Reserve.

b Net imports equals imports minus exports.

c See Note 6 at end of section.

$R=R e v i s e d$ data. $E=E s t i m a t e$.

Notes: - Crude oil includes lease condensate. - Totals may not equal sum of components due to independent rounding. - Geographic coverage is the 50 States and the District of Columbia.

Sources: - 1973-1980: Energy Information Administration (EIA), Petroleum Supply Monthly, February 1993, Table St. 1981 forward: EIA, Petroleum Supply Monthly, January 1998, Table S1. 
Figure 3.1 Petroleum Overview

(Million Barrels per Day)

Overview, January-December

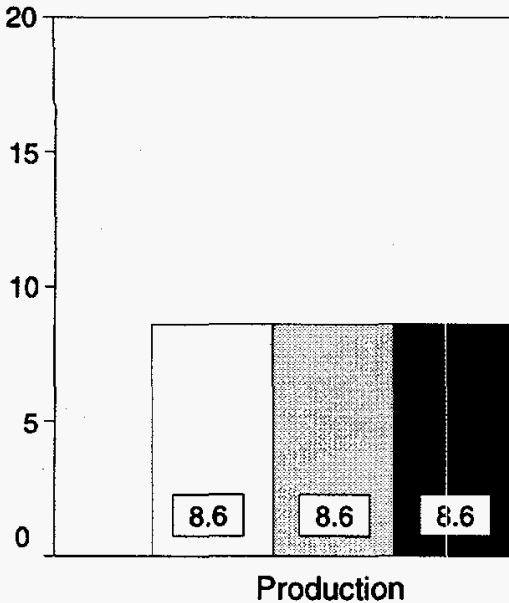

Oveniew, 1973-1997

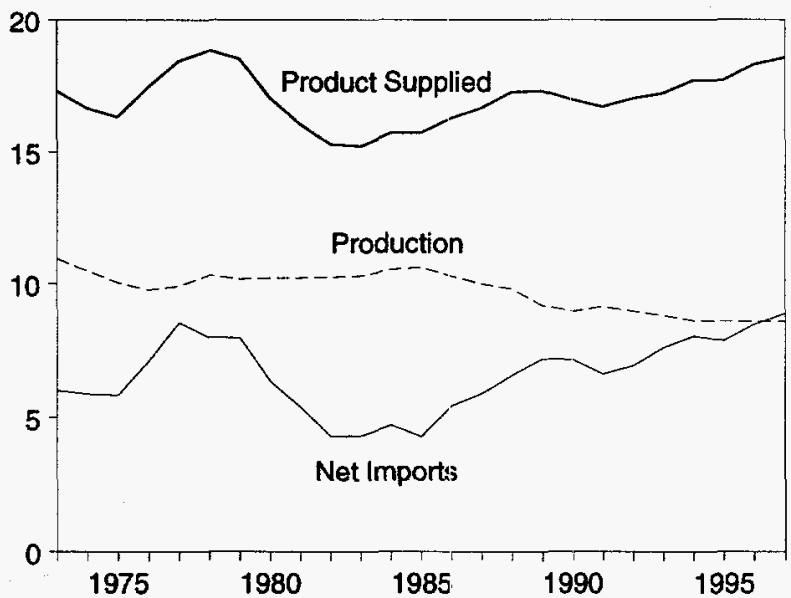

Crude Oil Production, 1973-1997

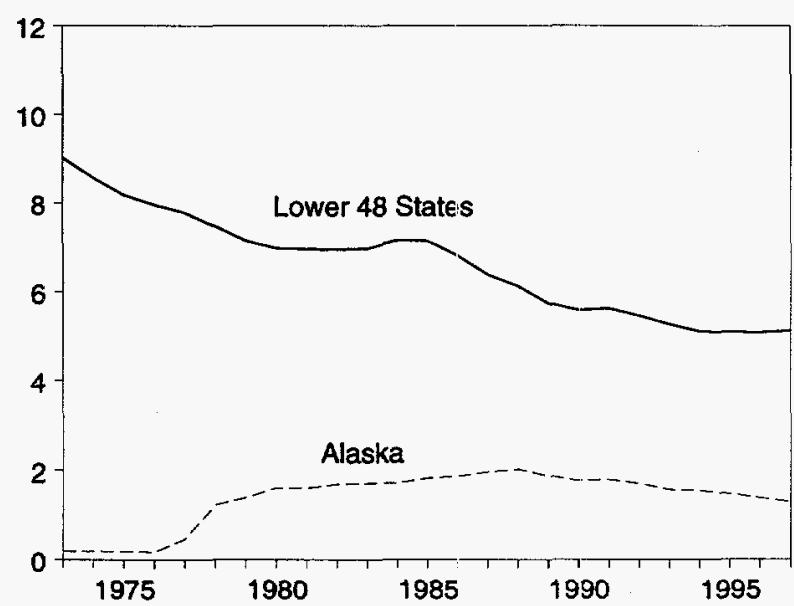

Production, 1973-1997

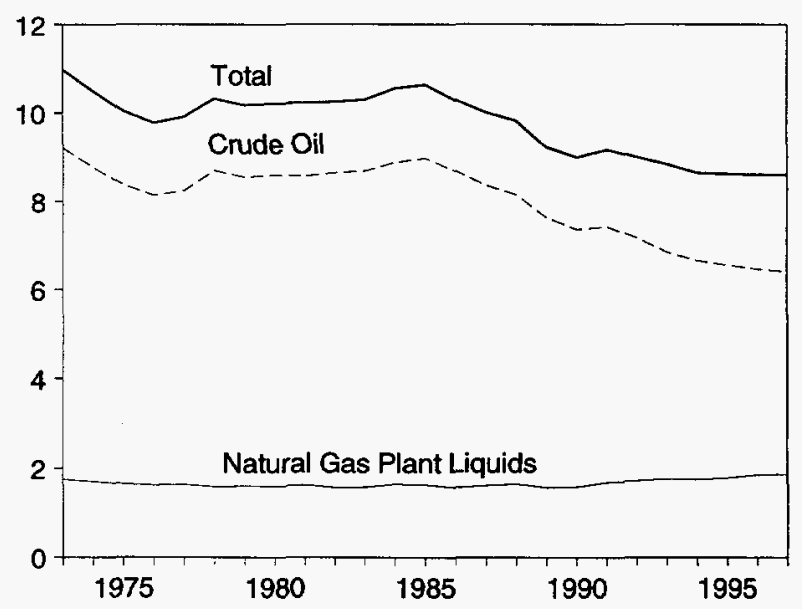

Total Production, Monthly

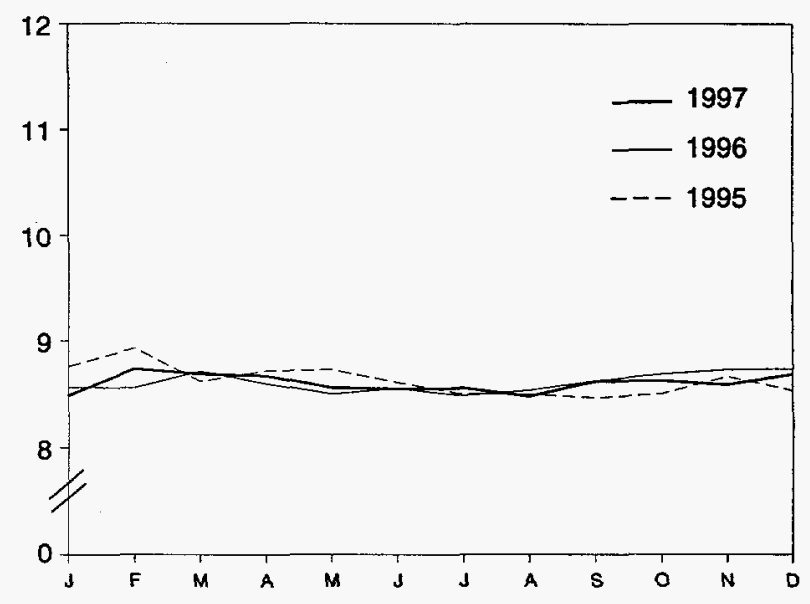

Note: Because vertical scales differ, graphs should not be compared.

Sources: Tables 3.1a, 3.1b, and 3.2a. 
Figure 3.1 Petroleum Overview (Continued)

(Million Barrels per Day, Except as Noted)

Product Supplied, 1973-1997

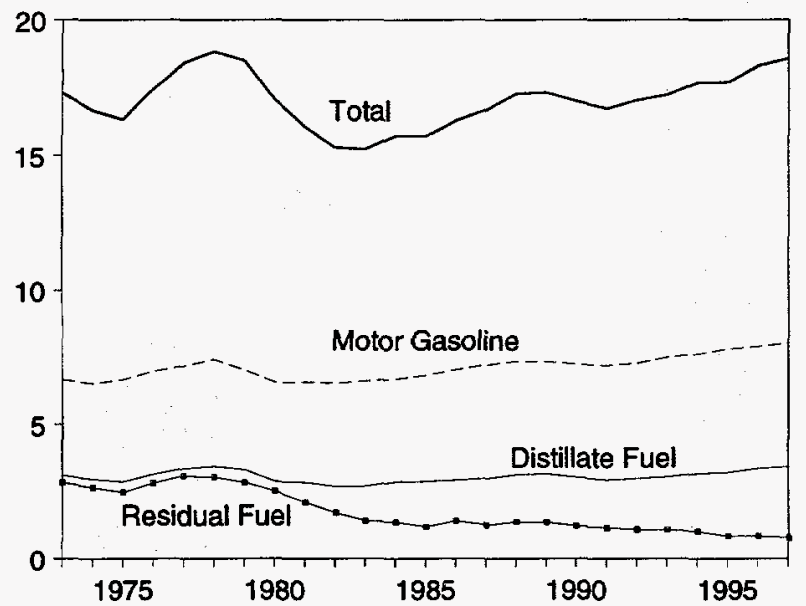

Imports from Selected Countries, November 1997

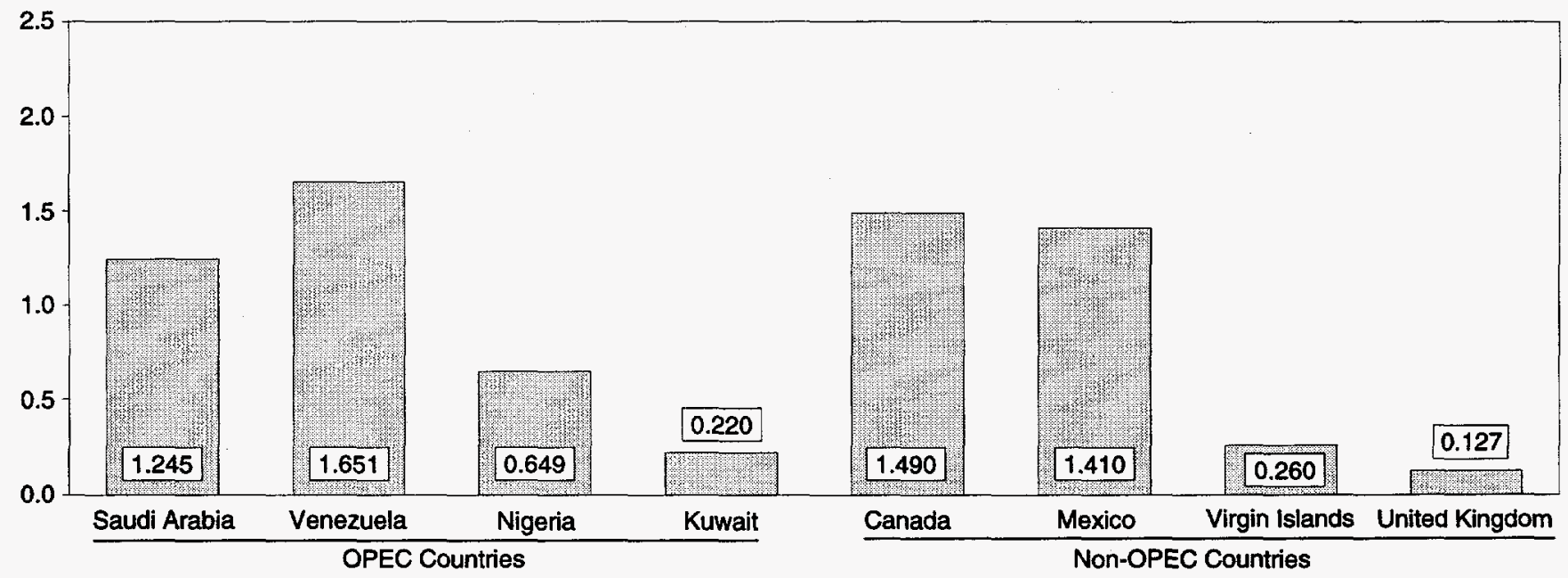

Stocks, End of Year, 1973-1997

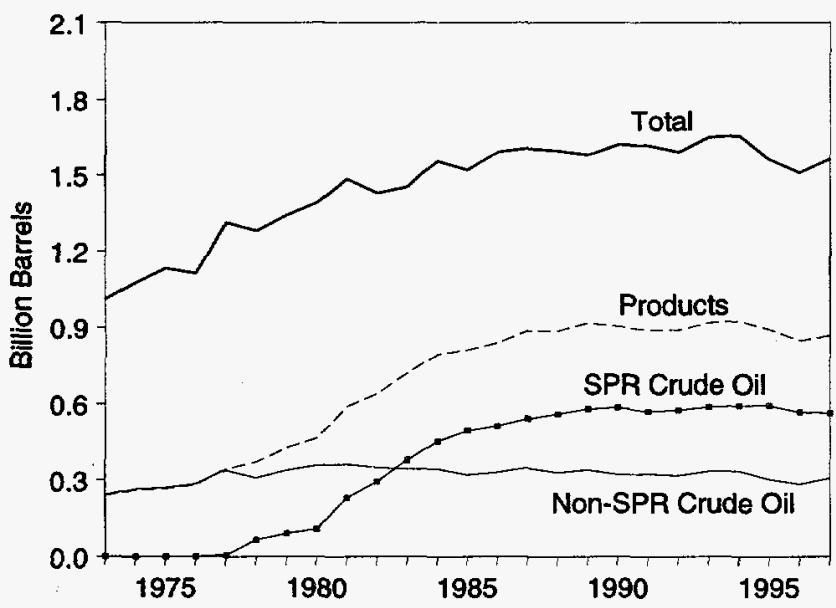

Notes: - OPEC = Organization of Petroleum Exporting Countries. - SPR = Strategic Petroleum Reserve. - Because vertical scales differ, graphs should not be compared.
Product Supplied, Monthly

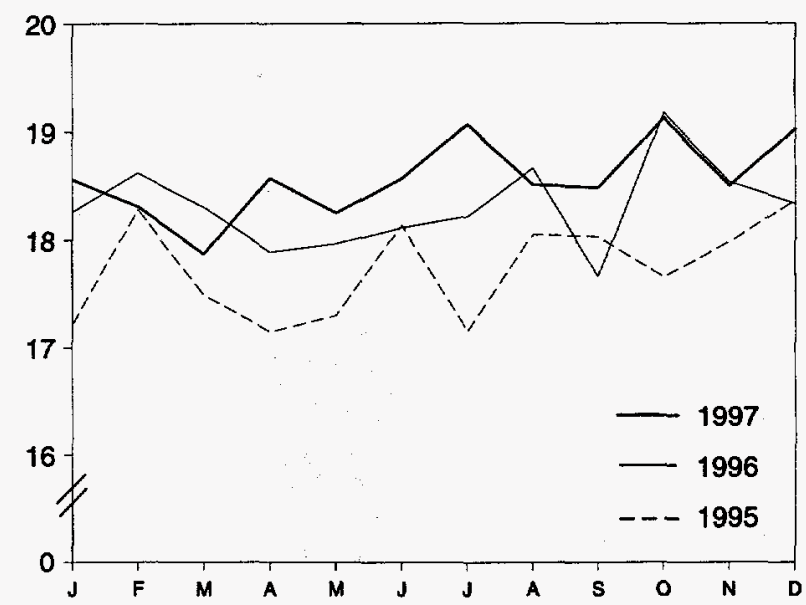

Total Stocks, End of Month

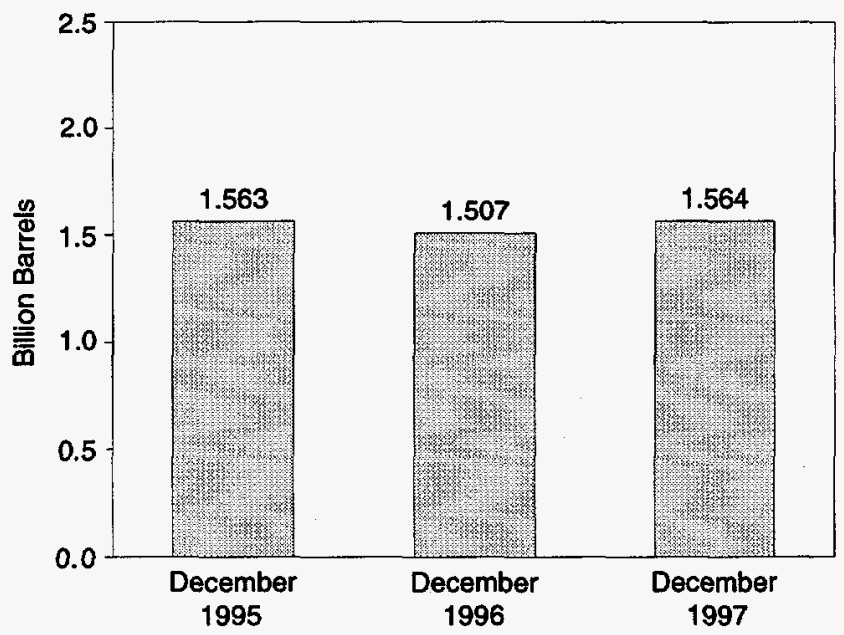

Sources: Tables 3.1a, 3.2b, 3.3a, 3.3b, 3.3d-3.3h, 3.4, 3.5, and 3.6. 
Table 3.2a Crude Oil Supply and Disposition: Supply

\begin{tabular}{|c|c|c|c|c|c|c|c|}
\hline & \multicolumn{7}{|c|}{ Supply } \\
\hline & \multicolumn{2}{|c|}{ Fisld Production } & \multicolumn{3}{|c|}{ Imports } & \multirow{2}{*}{$\begin{array}{c}\text { Unaccounted- } \\
\text { for Crude } \\
\text { Oif }\end{array}$} & \multirow{2}{*}{$\begin{array}{c}\text { Crude Oil } \\
\text { Used } \\
\text { Directlyc }\end{array}$} \\
\hline & $\begin{array}{c}\text { Total } \\
\text { Domestic }\end{array}$ & Alaskan & Total & SPR $^{a}$ & Other & & \\
\hline & \multicolumn{7}{|c|}{ Thousand Barrels per Day } \\
\hline $\begin{array}{l}1973 \text { Average } \\
1974 \text { Average } \\
1975 \text { Average } \\
1976 \text { Average } \\
1977 \text { Average } \\
1978 \text { Average } \\
1979 \ldots \ldots \ldots \ldots \\
1980 \text { Average } \\
1981 \text { Average } \\
1982 \text { Average }\end{array}$ & $\begin{array}{l}9,208 \\
8,774 \\
8,375 \\
8,132 \\
8,245 \\
8,707 \\
8,552 \\
8,597 \\
8,572 \\
8,649 \\
8,688 \\
8,879 \\
8,971 \\
8,680 \\
8,349 \\
8,140 \\
7,613 \\
7,355 \\
7,417 \\
7,171 \\
6,847 \\
6,662\end{array}$ & $\begin{array}{r}198 \\
193 \\
191 \\
173 \\
464 \\
1,229 \\
1,401 \\
1,617 \\
1,609 \\
1,696 \\
1,714 \\
1,722 \\
1,825 \\
1,867 \\
1,962 \\
2,017 \\
1,874 \\
1,773 \\
1,798 \\
1,714 \\
1,582 \\
1,559\end{array}$ & $\begin{array}{l}3,244 \\
3,477 \\
4,105 \\
5,287 \\
6,615 \\
6,356 \\
6,519 \\
5,263 \\
4,396 \\
3,488 \\
3,329 \\
3,426 \\
3,201 \\
4,178 \\
4,674 \\
5,107 \\
5,843 \\
5,894 \\
5,782 \\
6,083 \\
6,787 \\
7,063\end{array}$ & $\begin{array}{r}- \\
- \\
- \\
- \\
21 \\
d 161 \\
67 \\
44 \\
256 \\
165 \\
234 \\
197 \\
118 \\
48 \\
73 \\
51 \\
56 \\
27 \\
0 \\
10 \\
15 \\
12\end{array}$ & $\begin{array}{l}3,244 \\
3,477 \\
4,105 \\
5,287 \\
6,594 \\
6,195 \\
6,452 \\
5,219 \\
4,141 \\
3,323 \\
3,096 \\
3,229 \\
3,083 \\
4,130 \\
4,601 \\
5,055 \\
5,787 \\
5,867 \\
5,782 \\
6,073 \\
6,772 \\
7,051\end{array}$ & $\begin{array}{r}3 \\
-25 \\
17 \\
77 \\
-6 \\
-57 \\
-11 \\
34 \\
83 \\
71 \\
114 \\
185 \\
145 \\
139 \\
145 \\
196 \\
200 \\
258 \\
195 \\
258 \\
168 \\
266\end{array}$ & $\begin{array}{r}-19 \\
-15 \\
-17 \\
d-19 \\
-14 \\
d-15 \\
d-14 \\
d-14 \\
-58 \\
-59 \\
- \\
- \\
- \\
- \\
- \\
- \\
- \\
- \\
- \\
- \\
- \\
-\end{array}$ \\
\hline 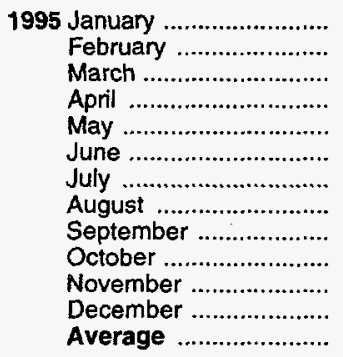 & $\begin{array}{l}6,682 \\
6,794 \\
6,600 \\
6,604 \\
6,629 \\
6,579 \\
6,449 \\
6,447 \\
6,416 \\
6,421 \\
6,585 \\
6,530 \\
6,560\end{array}$ & $\begin{array}{l}1,575 \\
1,578 \\
1,525 \\
1,511 \\
1,518 \\
1,484 \\
1,401 \\
1,432 \\
1,377 \\
1,475 \\
1,472 \\
1,466 \\
1,484\end{array}$ & $\begin{array}{l}6,505 \\
6,546 \\
7,391 \\
7,038 \\
7,325 \\
7,927 \\
7,265 \\
7,437 \\
8,007 \\
7,075 \\
7,302 \\
6,916 \\
7,230\end{array}$ & $\begin{array}{l}0 \\
0 \\
0 \\
0 \\
0 \\
0 \\
0 \\
0 \\
0 \\
0 \\
0 \\
0 \\
0\end{array}$ & $\begin{array}{l}6,505 \\
6,546 \\
7,391 \\
7,038 \\
7,325 \\
7,927 \\
7,265 \\
7,437 \\
8,007 \\
7,075 \\
7,302 \\
6,916 \\
7,230\end{array}$ & $\begin{array}{r}318 \\
78 \\
-101 \\
237 \\
296 \\
6 \\
402 \\
207 \\
-5 \\
328 \\
334 \\
193 \\
193\end{array}$ & $\begin{array}{l}- \\
\overline{-} \\
\overline{-} \\
\bar{z} \\
- \\
- \\
- \\
- \\
-\end{array}$ \\
\hline 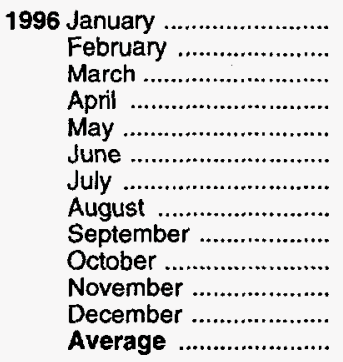 & $\begin{array}{l}6,495 \\
6,577 \\
6,571 \\
6,444 \\
6,394 \\
6,458 \\
6,338 \\
6,360 \\
6,482 \\
6,481 \\
6,476 \\
6,506 \\
6,465\end{array}$ & $\begin{array}{l}1,444 \\
1,482 \\
1,454 \\
1,367 \\
1,341 \\
1,419 \\
1,317 \\
1,327 \\
1,401 \\
1,379 \\
1,403 \\
1,392 \\
1,393\end{array}$ & $\begin{array}{l}7,303 \\
6,612 \\
7,215 \\
7,371 \\
8,029 \\
7,958 \\
7,800 \\
8,041 \\
7,353 \\
7,701 \\
7,344 \\
7,307 \\
7,508\end{array}$ & $\begin{array}{l}0 \\
0 \\
0 \\
0 \\
0 \\
0 \\
0 \\
0 \\
0 \\
0 \\
0 \\
0 \\
0\end{array}$ & $\begin{array}{l}7,303 \\
6,612 \\
7,215 \\
7,371 \\
8,029 \\
7,958 \\
7,800 \\
8,041 \\
7,353 \\
7,701 \\
7,344 \\
7,307 \\
7,508\end{array}$ & $\begin{array}{r}20 \\
413 \\
-25 \\
665 \\
61 \\
594 \\
121 \\
54 \\
303 \\
420 \\
148 \\
-153 \\
215\end{array}$ & $\begin{array}{l}- \\
- \\
- \\
- \\
- \\
- \\
- \\
- \\
- \\
- \\
-\end{array}$ \\
\hline 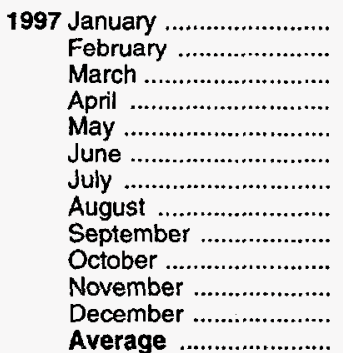 & $\begin{array}{r}E_{6,387} \\
E_{6,514} \\
E_{6,470} \\
E_{6,483} \\
E_{6,401} \\
E_{6,341} \\
E_{6,316} \\
E_{6,282} \\
E_{6,388} \\
E_{6,435} \\
\text { RE } 6,450^{6,45} \\
P E_{6,455} \\
P E_{6,409}\end{array}$ & $\begin{array}{r}E_{1}, 380 \\
E_{1}, 384 \\
E_{1}, 331 \\
E_{1,330} \\
E_{1}, 303 \\
E_{1,260} \\
E_{1}, 238 \\
E_{1}, 200 \\
E_{1}, 276 \\
E_{1}, 286 \\
P_{1}, 278 \\
P E_{1,277} \\
P E_{1,295}\end{array}$ & $\begin{array}{r}7,393 \\
7,384 \\
7,665 \\
7,810 \\
8,279 \\
8,403 \\
7,938 \\
8,333 \\
8,537 \\
8,543 \\
R 8,107 \\
E 7,471 \\
\text { E } 7,991\end{array}$ & $\begin{array}{l}0 \\
0 \\
0 \\
0 \\
0 \\
0 \\
0 \\
0 \\
0 \\
0 \\
0 \\
\mathrm{E}_{0} \\
\mathrm{E}\end{array}$ & $\begin{array}{r}7,393 \\
7,384 \\
7,665 \\
7,810 \\
8,279 \\
8,403 \\
7,938 \\
8,333 \\
8,537 \\
8,543 \\
\text { R } 8,107 \\
\text { E } 7,471 \\
\text { E } 7,991\end{array}$ & $\begin{array}{r}496 \\
-407 \\
582 \\
293 \\
646 \\
282 \\
377 \\
434 \\
572 \\
376 \\
\text { R } 382 \\
\text { E } 717^{2} \\
\text { E }_{403}\end{array}$ & $\begin{array}{l}- \\
- \\
- \\
- \\
- \\
- \\
- \\
- \\
- \\
- \\
-\end{array}$ \\
\hline
\end{tabular}

a Strategic Petroleum Reserve.

b A balancing item.

c Beginning in January 1983, crude oil used directly as fuel is shown as product supplied.

$d$ See Note 6 at end of section.

$\mathrm{PE}=$ Preliminary estimate. $\mathrm{R}=$ Revised data. $-=$ Not applicable. $E=$ Estimate.
Notes: - Crude oil includes lease condensate. - Totals may not equal sum of components due to independent rounding. - Geographic coverage is the 50 States and the District of Columbia.

Sources: - 1973-1980: Energy Information Administration (EIA), Petroleum Supply Monthly, February 1993, Table S2. • 1981 forward: EIA, Petroleum Supply Monthly, January 1998, Table S2. 
Table 3.2b Crude Oil Supply and Disposition: Disposition and Ending Stocks

\begin{tabular}{|c|c|c|c|c|c|c|c|c|c|}
\hline & \multicolumn{6}{|c|}{ Disposition } & \multicolumn{3}{|c|}{ Ending Stocks ${ }^{a}$} \\
\hline & \multirow{2}{*}{$\begin{array}{c}\text { Crude } \\
\text { Losses }\end{array}$} & \multicolumn{2}{|c|}{ Stock Changeb } & \multirow{2}{*}{$\begin{array}{l}\text { Refinery } \\
\text { Inputs }\end{array}$} & \multirow[b]{2}{*}{ Exports } & \multirow{2}{*}{$\begin{array}{l}\text { Product } \\
\text { Supplied }\end{array}$} & \multirow[b]{2}{*}{ Total } & \multirow[b]{2}{*}{ SPRC } & \multirow{2}{*}{$\begin{array}{c}\text { Other } \\
\text { Primary }\end{array}$} \\
\hline & & SPR ${ }^{C}$ & Other & & & & & & \\
\hline & \multicolumn{6}{|c|}{ Thousand Barrels per Day } & \multicolumn{3}{|c|}{ Million Barrels } \\
\hline $\begin{array}{l}1973 \text { Average } \\
1974 \text { Average } \\
1975 \text { Average } \\
1976 \text { Average } \\
1977 \text { Average } \\
1978 \text { Average } \\
1979 \text { Average } \\
1980 \text { Average } \\
1981 \text { Average } \\
1982 \text { Average } \\
1983 \text { Average } \\
1984 \text { Average } \\
1985 \text { Average } \\
1986 \text { Average } \\
1987 \text { Average } \\
1988 \text { Average } \\
1989 \text { Average } \\
1990 \text { Average } \\
1991 \text { Average } \\
1992 \text { Average } \\
1993 \text { Average } \\
1994 \text { Average }\end{array}$ & $\begin{array}{r}13 \\
13 \\
13 \\
14 \\
16 \\
16 \\
16 \\
14 \\
5 \\
3 \\
2 \\
2 \\
1 \\
(s) \\
(s) \\
(s) \\
(s) \\
(s) \\
(s) \\
(s) \\
(s) \\
(s)\end{array}$ & $\begin{array}{r}- \\
- \\
- \\
- \\
20 \\
163 \\
67 \\
45 \\
336 \\
174 \\
234 \\
195 \\
117 \\
50 \\
80 \\
52 \\
56 \\
16 \\
-47 \\
17 \\
34 \\
13\end{array}$ & $\begin{array}{r}-11 \\
62 \\
17 \\
39 \\
150 \\
-84 \\
81 \\
52 \\
r-46 \\
-38 \\
9-20 \\
4 \\
-67 \\
28 \\
49 \\
-51 \\
30 \\
-51 \\
5 \\
-18 \\
47 \\
5\end{array}$ & $\begin{array}{l}12,431 \\
12,133 \\
12,442 \\
13,416 \\
14,602 \\
14,739 \\
14,648 \\
13,481 \\
12,470 \\
11,774 \\
11,685 \\
12,044 \\
12,002 \\
12,716 \\
12,854 \\
13,246 \\
13,401 \\
13,409 \\
13,301 \\
13,411 \\
13,613 \\
13,866\end{array}$ & $\begin{array}{r}2 \\
3 \\
6 \\
8 \\
50 \\
158 \\
235 \\
287 \\
228 \\
236 \\
164 \\
181 \\
204 \\
154 \\
151 \\
155 \\
142 \\
109 \\
116 \\
89 \\
98 \\
99\end{array}$ & $\begin{array}{l}- \\
- \\
- \\
- \\
- \\
- \\
- \\
- \\
- \\
- \\
66 \\
64 \\
60 \\
49 \\
34 \\
40 \\
28 \\
24 \\
18 \\
13 \\
10 \\
9\end{array}$ & $\begin{array}{r}242 \\
265 \\
271 \\
285 \\
348 \\
376 \\
430 \\
+466 \\
594 \\
9644 \\
723 \\
796 \\
814 \\
843 \\
890 \\
890 \\
921 \\
908 \\
893 \\
893 \\
922 \\
929\end{array}$ & $\begin{array}{r}- \\
- \\
- \\
- \\
7 \\
67 \\
91 \\
108 \\
230 \\
294 \\
379 \\
451 \\
493 \\
512 \\
541 \\
560 \\
580 \\
586 \\
569 \\
575 \\
587 \\
592\end{array}$ & $\begin{array}{r}242 \\
265 \\
271 \\
285 \\
340 \\
309 \\
339 \\
+358 \\
363 \\
9350 \\
344 \\
345 \\
321 \\
331 \\
349 \\
330 \\
341 \\
323 \\
325 \\
318 \\
335 \\
337\end{array}$ \\
\hline 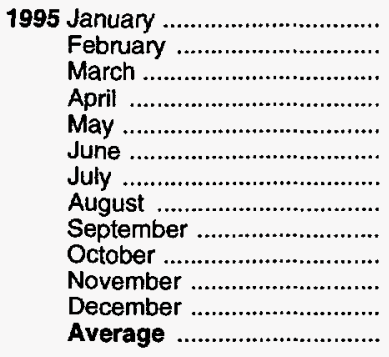 & $\begin{array}{c}(s) \\
0 \\
(s) \\
0 \\
0 \\
0 \\
0 \\
(s) \\
0 \\
(s) \\
0 \\
0 \\
(s)\end{array}$ & $\begin{array}{l}\text { (s) } \\
(s) \\
(s) \\
(s) \\
(s) \\
(s) \\
(s) \\
(s) \\
(s) \\
(s) \\
-1 \\
(s) \\
\text { (s) }\end{array}$ & $\begin{array}{r}-219 \\
-49 \\
336 \\
-101 \\
-132 \\
-148 \\
-397 \\
-253 \\
-63 \\
169 \\
264 \\
-505 \\
-93\end{array}$ & $\begin{array}{l}13,604 \\
13,365 \\
13,480 \\
13,817 \\
14,303 \\
14,553 \\
14,403 \\
14,276 \\
14,402 \\
13,598 \\
13,833 \\
14,011 \\
13,973\end{array}$ & $\begin{array}{r}113 \\
95 \\
68 \\
155 \\
73 \\
101 \\
103 \\
61 \\
74 \\
50 \\
118 \\
127 \\
95\end{array}$ & $\begin{array}{l}7 \\
8 \\
7 \\
7 \\
7 \\
5 \\
7 \\
6 \\
6 \\
8 \\
7 \\
6 \\
7\end{array}$ & $\begin{array}{l}922 \\
921 \\
931 \\
928 \\
924 \\
920 \\
907 \\
899 \\
898 \\
903 \\
911 \\
895 \\
895\end{array}$ & $\begin{array}{l}592 \\
592 \\
592 \\
592 \\
592 \\
592 \\
592 \\
592 \\
592 \\
592 \\
592 \\
592 \\
592\end{array}$ & $\begin{array}{l}330 \\
329 \\
339 \\
336 \\
332 \\
328 \\
316 \\
308 \\
306 \\
311 \\
319 \\
303 \\
303\end{array}$ \\
\hline 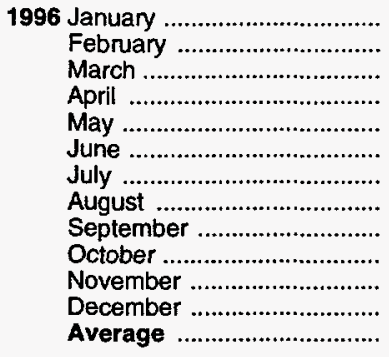 & $\begin{array}{c}0 \\
0 \\
0 \\
(s) \\
0 \\
0 \\
(s) \\
0 \\
0 \\
0 \\
0 \\
0 \\
\text { (s) }\end{array}$ & $\begin{array}{r}(s) \\
(s) \\
-80 \\
-88 \\
-22 \\
-45 \\
-50 \\
-172 \\
-130 \\
-1 \\
-127 \\
-129 \\
-71\end{array}$ & $\begin{array}{r}-8 \\
-62 \\
-52 \\
117 \\
24 \\
350 \\
-194 \\
153 \\
-368 \\
187 \\
-288 \\
-498 \\
-53\end{array}$ & $\begin{array}{l}13,728 \\
13,564 \\
13,793 \\
14,295 \\
14,439 \\
14,569 \\
14,359 \\
14,424 \\
14,484 \\
14,277 \\
14,204 \\
14,185 \\
14,195\end{array}$ & $\begin{array}{r}89 \\
92 \\
94 \\
148 \\
37 \\
130 \\
139 \\
44 \\
147 \\
134 \\
172 \\
96 \\
110\end{array}$ & $\begin{array}{r}11 \\
8 \\
7 \\
6 \\
7 \\
6 \\
5 \\
6 \\
6 \\
5 \\
5 \\
6 \\
6\end{array}$ & $\begin{array}{l}895 \\
893 \\
889 \\
890 \\
890 \\
899 \\
891 \\
891 \\
876 \\
882 \\
869 \\
850 \\
850\end{array}$ & $\begin{array}{l}592 \\
592 \\
589 \\
586 \\
586 \\
584 \\
583 \\
578 \\
574 \\
574 \\
570 \\
566 \\
566\end{array}$ & $\begin{array}{l}303 \\
301 \\
300 \\
303 \\
304 \\
314 \\
308 \\
313 \\
302 \\
308 \\
299 \\
284 \\
284\end{array}$ \\
\hline 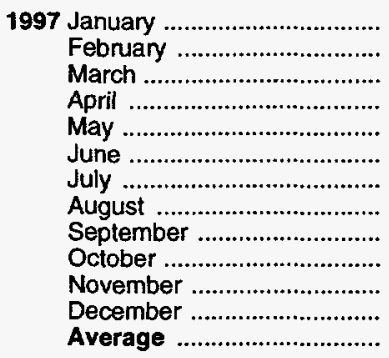 & $\begin{array}{l}0 \\
0 \\
0 \\
0 \\
0 \\
0 \\
0 \\
0 \\
0 \\
0 \\
0 \\
\mathrm{E}_{0} \\
\mathrm{E}_{0}\end{array}$ & $\begin{array}{r}-75 \\
(s) \\
(s) \\
(s) \\
(s) \\
(s) \\
(s) \\
(s) \\
(s) \\
(s) \\
(s) \\
E(s) \\
E-7\end{array}$ & $\begin{array}{r}572 \\
-167 \\
529 \\
208 \\
212 \\
-171 \\
-399 \\
-278 \\
78 \\
412 \\
4253 \\
E_{-417} \\
E 71\end{array}$ & $\begin{array}{r}13,632 \\
13,425 \\
14,047 \\
14,283 \\
15,083 \\
15,139 \\
14,958 \\
15,217 \\
15,297 \\
14,790 \\
R 14,654 \\
E_{14,957} \\
E_{14,631}\end{array}$ & $\begin{array}{r}141 \\
228 \\
136 \\
92 \\
26 \\
57 \\
70 \\
110 \\
122 \\
152 \\
R_{32} \\
E_{102} \\
E_{105}\end{array}$ & $\begin{array}{r}5 \\
6 \\
5 \\
3 \\
4 \\
2 \\
2 \\
(s) \\
(s) \\
0 \\
0 \\
E_{0} \\
E_{2}\end{array}$ & $\begin{array}{r}866 \\
861 \\
878 \\
884 \\
890 \\
885 \\
873 \\
864 \\
867 \\
879 \\
\text { R } 887 \\
\text { E } 871 \\
\text { E }_{\mathbf{8 7 1}}\end{array}$ & $\begin{array}{r}563 \\
563 \\
563 \\
563 \\
563 \\
563 \\
563 \\
563 \\
563 \\
563 \\
563 \\
\text { E563 } \\
\text { E563 }\end{array}$ & $\begin{array}{r}302 \\
298 \\
314 \\
320 \\
327 \\
322 \\
309 \\
301 \\
303 \\
316 \\
R_{324} \\
E_{307} \\
E_{307}\end{array}$ \\
\hline
\end{tabular}

a Stocks are totals as of end of period.

b A negative number indicates a decrease in stocks and a positive number indicates an increase.

c Strategic Petroleum Reserve.

d Beginning in January 1983 , crude oil used directly as fuel is shown as product supplied.

See Note 6 at end of section.

f Stocks of Alaskan crude oil in transit are included from January 1981 forward. See Note 5 at end of section.
9 See Note 4 at end of section.

$\mathrm{R}=$ Revised data. - =Not applicable. E=Estimate. (s)=Less than +500 barrels per day and greater than -500 barrels per day.

Notes: - Crude oil includes lease condensate. - Totals may not equal sum of components due to independent rounding. - Geographic coverage is the 50 States and the District of Columbia.

Sources: - 1973-1980: Energy Information Administration (EIA), Petroleum Supply Monthly, February 1993, Table S2. • 1981 forward: EIA, Petroleum Supply Monthly, January 1998, Table S2. 
Table 3.3a Petroleum Imports: Bahrain, Iran, Iraq, and Kuwait

(Thousand Barrels per Day)

\begin{tabular}{|c|c|c|c|c|c|c|c|c|}
\hline & \multicolumn{8}{|c|}{ Persian Gulfa } \\
\hline & \multicolumn{2}{|c|}{ Eahrain } & \multicolumn{2}{|c|}{ Iran } & \multicolumn{2}{|c|}{ Iraq } & \multicolumn{2}{|c|}{ Kuwait ${ }^{b}$} \\
\hline & Total & Crude Oil & Total & Crude Oil & Total : & Crude Oil & Total & Crude Oil \\
\hline 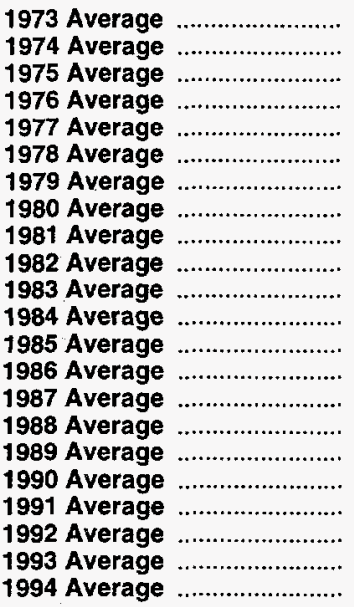 & $\begin{array}{r}11 \\
12 \\
16 \\
3 \\
10 \\
3 \\
1 \\
(s) \\
1 \\
1 \\
2 \\
1 \\
4 \\
2 \\
0 \\
2 \\
0 \\
1 \\
2 \\
0 \\
1 \\
1\end{array}$ & $\begin{array}{l}0 \\
0 \\
0 \\
0 \\
0 \\
0 \\
0 \\
0 \\
0 \\
0 \\
0 \\
0 \\
0 \\
0 \\
0 \\
0 \\
0 \\
0 \\
0 \\
0 \\
0 \\
0\end{array}$ & $\begin{array}{r}223 \\
469 \\
280 \\
298 \\
535 \\
555 \\
304 \\
9 \\
0 \\
35 \\
48 \\
10 \\
27 \\
19 \\
98 \\
\text { cs) } \\
0 \\
0 \\
32 \\
0 \\
0 \\
0\end{array}$ & $\begin{array}{r}216 \\
463 \\
278 \\
298 \\
530 \\
554 \\
297 \\
8 \\
0 \\
35 \\
48 \\
10 \\
27 \\
19 \\
98 \\
\mathbf{( s )} \\
0 \\
0 \\
32 \\
0 \\
0 \\
0\end{array}$ & $\begin{array}{r}4 \\
0 \\
2 \\
26 \\
74 \\
62 \\
88 \\
28 \\
(5) \\
3 \\
10 \\
12 \\
46 \\
81 \\
83 \\
345 \\
449 \\
518 \\
0 \\
0 \\
0 \\
0\end{array}$ & $\begin{array}{r}4 \\
0 \\
2 \\
26 \\
74 \\
62 \\
88 \\
28 \\
0 \\
3 \\
10 \\
12 \\
46 \\
81 \\
82 \\
343 \\
441 \\
514 \\
0 \\
0 \\
0 \\
0\end{array}$ & $\begin{array}{r}47 \\
5 \\
16 \\
5 \\
48 \\
6 \\
8 \\
27 \\
0 \\
5 \\
14 \\
36 \\
21 \\
68 \\
84 \\
92 \\
157 \\
86 \\
6 \\
51 \\
353 \\
312\end{array}$ & $\begin{array}{r}42 \\
5 \\
4 \\
1 \\
42 \\
5 \\
5 \\
27 \\
0 \\
2 \\
7 \\
24 \\
4 \\
28 \\
70 \\
80 \\
155 \\
79 \\
6 \\
39 \\
344 \\
307\end{array}$ \\
\hline 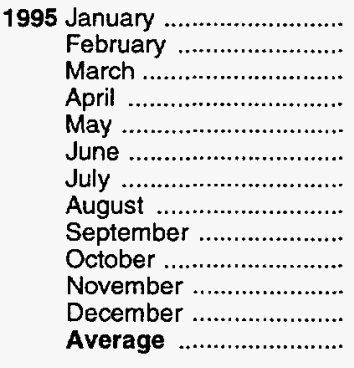 & $\begin{array}{r}0 \\
11 \\
0 \\
0 \\
0 \\
0 \\
0 \\
0 \\
0 \\
0 \\
0 \\
0 \\
1\end{array}$ & $\begin{array}{l}0 \\
0 \\
0 \\
0 \\
0 \\
0 \\
0 \\
0 \\
0 \\
0 \\
0 \\
0 \\
0\end{array}$ & $\begin{array}{l}0 \\
0 \\
0 \\
0 \\
0 \\
0 \\
0 \\
0 \\
0 \\
0 \\
0 \\
0 \\
0\end{array}$ & $\begin{array}{l}0 \\
0 \\
0 \\
0 \\
0 \\
0 \\
0 \\
0 \\
0 \\
0 \\
0 \\
0 \\
0\end{array}$ & $\begin{array}{l}0 \\
0 \\
0 \\
0 \\
0 \\
0 \\
0 \\
0 \\
0 \\
0 \\
0 \\
0 \\
0\end{array}$ & $\begin{array}{l}0 \\
0 \\
0 \\
0 \\
0 \\
0 \\
0 \\
0 \\
0 \\
0 \\
0 \\
0 \\
0\end{array}$ & $\begin{array}{l}130 \\
346 \\
252 \\
171 \\
208 \\
260 \\
195 \\
180 \\
187 \\
250 \\
238 \\
215 \\
218\end{array}$ & $\begin{array}{l}120 \\
324 \\
252 \\
164 \\
204 \\
259 \\
195 \\
175 \\
182 \\
244 \\
238 \\
215 \\
213\end{array}$ \\
\hline 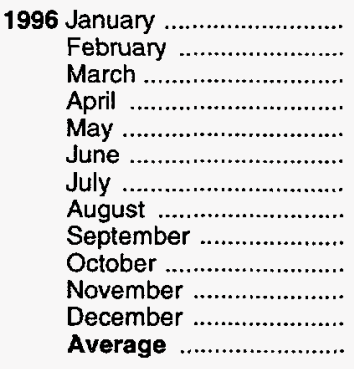 & $\begin{array}{r}0 \\
0 \\
0 \\
17 \\
0 \\
0 \\
0 \\
0 \\
0 \\
0 \\
0 \\
0 \\
1\end{array}$ & $\begin{array}{l}0 \\
0 \\
0 \\
0 \\
0 \\
0 \\
0 \\
0 \\
0 \\
0 \\
0 \\
0 \\
0\end{array}$ & $\begin{array}{l}0 \\
0 \\
0 \\
0 \\
0 \\
0 \\
0 \\
0 \\
0 \\
0 \\
0 \\
0 \\
0\end{array}$ & $\begin{array}{l}0 \\
0 \\
0 \\
0 \\
0 \\
0 \\
0 \\
0 \\
0 \\
0 \\
0 \\
0 \\
0\end{array}$ & $\begin{array}{r}0 \\
0 \\
0 \\
0 \\
0 \\
0 \\
0 \\
0 \\
0 \\
0 \\
0 \\
14 \\
1\end{array}$ & $\begin{array}{r}0 \\
0 \\
0 \\
0 \\
0 \\
0 \\
0 \\
0 \\
0 \\
0 \\
0 \\
14 \\
1\end{array}$ & $\begin{array}{l}148 \\
216 \\
127 \\
201 \\
230 \\
388 \\
266 \\
271 \\
236 \\
260 \\
228 \\
262 \\
236\end{array}$ & $\begin{array}{l}145 \\
216 \\
127 \\
201 \\
230 \\
388 \\
266 \\
266 \\
236 \\
260 \\
228 \\
262 \\
235\end{array}$ \\
\hline 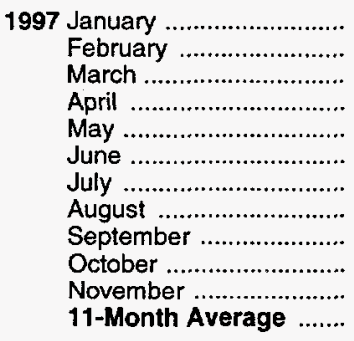 & $\begin{array}{l}0 \\
0 \\
0 \\
0 \\
0 \\
0 \\
0 \\
0 \\
0 \\
0 \\
0 \\
0\end{array}$ & $\begin{array}{l}0 \\
0 \\
0 \\
0 \\
0 \\
0 \\
0 \\
0 \\
0 \\
0 \\
0 \\
0\end{array}$ & $\begin{array}{l}0 \\
0 \\
0 \\
0 \\
0 \\
0 \\
0 \\
0 \\
0 \\
0 \\
0 \\
0\end{array}$ & $\begin{array}{l}0 \\
0 \\
0 \\
0 \\
0 \\
0 \\
0 \\
0 \\
0 \\
0 \\
0 \\
0\end{array}$ & $\begin{array}{r}0 \\
0 \\
35 \\
69 \\
102 \\
115 \\
88 \\
(s) \\
0 \\
177 \\
220 \\
74\end{array}$ & $\begin{array}{r}0 \\
0 \\
35 \\
69 \\
102 \\
115 \\
88 \\
(s) \\
0 \\
177 \\
220 \\
74\end{array}$ & $\begin{array}{l}209 \\
172 \\
315 \\
204 \\
128 \\
361 \\
331 \\
229 \\
322 \\
349 \\
220 \\
259\end{array}$ & $\begin{array}{l}209 \\
172 \\
315 \\
204 \\
128 \\
361 \\
331 \\
229 \\
322 \\
349 \\
220 \\
259\end{array}$ \\
\hline $\begin{array}{l}1996 \text { 11-Month Average ....... } \\
199511-\text { Month Average ....... }\end{array}$ & $\begin{array}{l}1 \\
1\end{array}$ & $\begin{array}{l}0 \\
0\end{array}$ & $\begin{array}{l}0 \\
0\end{array}$ & $\begin{array}{l}0 \\
0\end{array}$ & $\begin{array}{l}0 \\
0\end{array}$ & $\begin{array}{l}0 \\
0\end{array}$ & $\begin{array}{l}234 \\
219\end{array}$ & $\begin{array}{l}233 \\
213\end{array}$ \\
\hline
\end{tabular}

a Excludes petroleum imported into the United States indirectly from members of the Organization of Petroleum Exporting Countries (OPEC), primarily from Caribbean and West European areas, as petroleum products that were refined from crude oil produced by OPEC.

$b$ Imports from the Neutral Zone between Kuwait and Saudi Arabia ara included in Saudi Arabia.

$C$ A small amount of Iranian crude oil entered the United States in January 1988 from the Virgin Islands. The oil originated in Iran and was exported to the Virgin islands prior to the signing of Executive Order 12613 on October
$29,1987$.

(s)=Less than 500 barrels per day.

Notes: - Beginning in October 1977, Strategic Petroleum Reserve imports are included. - U.S. geographic coverage is the $\mathbf{5 0}$ States and the District of Columbia.

Sources: - Bahrain: Energy Information Administration (EIA), Form ElA-814, "Monthly Imports Report." - All Other Data: 1973-1980-EIA. Petroleum Supply Monthly, February 1993, Table S3. 1981 forward-EIA, Petroleum Supply Monthly, January 1998, Table S3. 
Table 3.3b Petroleum Imports: Qatar, Saudi Arabia, U.A.E., and Total Persian Gulf (Thousand Barrels per Day)

\begin{tabular}{|c|c|c|c|c|c|c|c|c|}
\hline & \multicolumn{8}{|c|}{ Persian Gulfa } \\
\hline & \multicolumn{2}{|c|}{ Qatar } & \multicolumn{2}{|c|}{ Saudi Arabiab } & \multicolumn{2}{|c|}{ United Arab Emirates } & \multicolumn{2}{|c|}{ Totala } \\
\hline & Total & Crude Oil & Total & Crude Oil & Total & Crude Oil & Total & Crude Oil \\
\hline $\begin{array}{l}1973 \text { Average } \\
1974 \text { Average } \\
1975 \text { Average } \\
1976 \text { Average } \\
1977 \text { Average } \\
1978 \text { Average } \\
1979 \text { Average } \\
1980 \text { Average } \\
1981 \text { Average } \\
1982 \text { Average } \\
1983 \text { Average } \\
1984 \text { Average } \\
1985 \text { Average } \\
1986 \text { Average } \\
1987 \text { Average } \\
1988 \text { Average } \\
1989 \text { Average } \\
1990 \text { Average } \\
1991 \text { Average }\end{array}$ & $\begin{array}{r}7 \\
17 \\
18 \\
24 \\
67 \\
64 \\
31 \\
22 \\
7 \\
7 \\
(s) \\
5 \\
(s) \\
13 \\
0 \\
0 \\
2 \\
4 \\
0 \\
1 \\
1 \\
0\end{array}$ & $\begin{array}{r}7 \\
17 \\
18 \\
24 \\
67 \\
64 \\
31 \\
22 \\
7 \\
7 \\
0 \\
4 \\
0 \\
12 \\
0 \\
0 \\
2 \\
4 \\
0 \\
0 \\
0 \\
0\end{array}$ & $\begin{array}{r}486 \\
461 \\
715 \\
1,230 \\
1,380 \\
1,144 \\
1,356 \\
1,261 \\
1,129 \\
552 \\
337 \\
325 \\
168 \\
685 \\
751 \\
1,073 \\
1,224 \\
1,339 \\
1,802 \\
1,720 \\
1,414 \\
1,402\end{array}$ & $\begin{array}{r}462 \\
438 \\
701 \\
1,222 \\
1,373 \\
1,142 \\
1,347 \\
1,250 \\
1,112 \\
530 \\
321 \\
309 \\
132 \\
618 \\
642 \\
911 \\
1,116 \\
1,195 \\
1,703 \\
1,597 \\
1,282 \\
1,297\end{array}$ & $\begin{array}{r}71 \\
74 \\
117 \\
254 \\
335 \\
385 \\
281 \\
172 \\
81 \\
92 \\
30 \\
117 \\
45 \\
44 \\
61 \\
29 \\
28 \\
17 \\
3 \\
6 \\
14 \\
13\end{array}$ & $\begin{array}{r}71 \\
69 \\
117 \\
254 \\
333 \\
385 \\
281 \\
172 \\
77 \\
81 \\
18 \\
90 \\
35 \\
38 \\
56 \\
23 \\
21 \\
9 \\
2 \\
0 \\
12 \\
11\end{array}$ & $\begin{array}{r}848 \\
1,039 \\
1,165 \\
1,840 \\
2,448 \\
2,219 \\
2,069 \\
1,519 \\
1,219 \\
696 \\
442 \\
506 \\
311 \\
912 \\
1,077 \\
1,541 \\
1,861 \\
1,966 \\
1,845 \\
1,778 \\
1,782 \\
1,728\end{array}$ & $\begin{array}{r}802 \\
992 \\
1,121 \\
1,825 \\
2,418 \\
2,212 \\
2,049 \\
1,508 \\
1,196 \\
659 \\
405 \\
450 \\
244 \\
796 \\
949 \\
1,357 \\
1,734 \\
1,801 \\
1,743 \\
1,636 \\
1,637 \\
1,615\end{array}$ \\
\hline 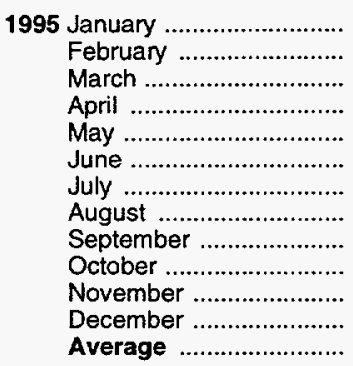 & $\begin{array}{l}0 \\
0 \\
0 \\
0 \\
0 \\
0 \\
0 \\
0 \\
0 \\
0 \\
0 \\
0 \\
0\end{array}$ & $\begin{array}{l}0 \\
0 \\
0 \\
0 \\
0 \\
0 \\
0 \\
0 \\
0 \\
0 \\
0 \\
0 \\
0\end{array}$ & $\begin{array}{l}1,309 \\
1,181 \\
1,535 \\
1,375 \\
1,281 \\
1,287 \\
1,265 \\
1,340 \\
1,474 \\
1,260 \\
1,429 \\
1,378 \\
1,344\end{array}$ & $\begin{array}{l}1,251 \\
1,134 \\
1,410 \\
1,321 \\
1,237 \\
1,221 \\
1,165 \\
1,245 \\
1,357 \\
1,181 \\
1,326 \\
1,263 \\
1,260\end{array}$ & $\begin{array}{r}20 \\
13 \\
0 \\
0 \\
0 \\
12 \\
0 \\
20 \\
29 \\
14 \\
10 \\
0 \\
10\end{array}$ & $\begin{array}{r}20 \\
13 \\
0 \\
0 \\
0 \\
1 \\
0 \\
20 \\
0 \\
0 \\
10 \\
0 \\
5\end{array}$ & $\begin{array}{l}1,459 \\
1,550 \\
1,788 \\
1,547 \\
1,490 \\
1,558 \\
1,460 \\
1,541 \\
1,691 \\
1,524 \\
1,677 \\
1,593 \\
1,573\end{array}$ & $\begin{array}{l}1,391 \\
1,471 \\
1,662 \\
1,485 \\
1,441 \\
1,481 \\
1,360 \\
1,440 \\
1,539 \\
1,426 \\
1,574 \\
1,478 \\
1,479\end{array}$ \\
\hline 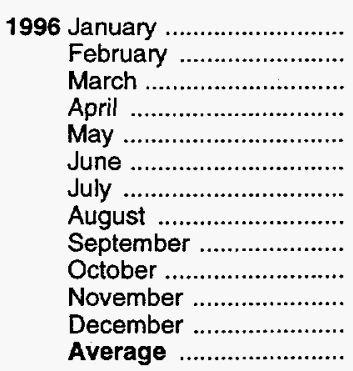 & $\begin{array}{l}0 \\
0 \\
0 \\
0 \\
0 \\
0 \\
0 \\
0 \\
0 \\
0 \\
0 \\
0 \\
0\end{array}$ & $\begin{array}{l}0 \\
0 \\
0 \\
0 \\
0 \\
0 \\
0 \\
0 \\
0 \\
0 \\
0 \\
0 \\
0\end{array}$ & $\begin{array}{l}1,398 \\
1,128 \\
1,422 \\
1,288 \\
1,518 \\
1,138 \\
1,548 \\
1,477 \\
1,355 \\
1,357 \\
1,297 \\
1,400 \\
1,363\end{array}$ & $\begin{array}{l}1,334 \\
1,053 \\
1,318 \\
1,200 \\
1,414 \\
1,035 \\
1,371 \\
1,333 \\
1,255 \\
1,209 \\
1,201 \\
1,236 \\
1,248\end{array}$ & $\begin{array}{r}0 \\
0 \\
0 \\
0 \\
0 \\
11 \\
4 \\
0 \\
0 \\
17 \\
0 \\
0 \\
3\end{array}$ & $\begin{array}{r}0 \\
0 \\
0 \\
0 \\
0 \\
11 \\
4 \\
0 \\
0 \\
17 \\
0 \\
0 \\
3\end{array}$ & $\begin{array}{l}1,546 \\
1,344 \\
1,549 \\
1,506 \\
1,748 \\
1,537 \\
1,819 \\
1,747 \\
1,591 \\
1,635 \\
1,525 \\
1,675 \\
1,604\end{array}$ & $\begin{array}{l}1,479 \\
1,268 \\
1,446 \\
1,401 \\
1,643 \\
1,433 \\
1,642 \\
1,599 \\
1,491 \\
1,486 \\
1,429 \\
1,511 \\
1,488\end{array}$ \\
\hline 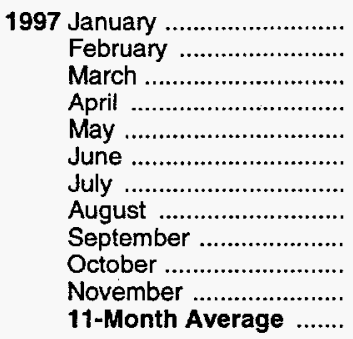 & $\begin{array}{r}0 \\
0 \\
0 \\
15 \\
0 \\
0 \\
0 \\
0 \\
0 \\
16 \\
0 \\
3\end{array}$ & $\begin{array}{l}0 \\
0 \\
0 \\
0 \\
0 \\
0 \\
0 \\
0 \\
0 \\
0 \\
0 \\
0\end{array}$ & $\begin{array}{l}1,344 \\
1,361 \\
1,292 \\
1,573 \\
1,475 \\
1,303 \\
1,285 \\
1,621 \\
1,551 \\
1,340 \\
1,245 \\
1,399\end{array}$ & $\begin{array}{l}1,253 \\
1,250 \\
1,157 \\
1,408 \\
1,333 \\
1,180 \\
1,188 \\
1,501 \\
1,463 \\
1,245 \\
1,195 \\
1,289\end{array}$ & $\begin{array}{r}0 \\
0 \\
0 \\
0 \\
0 \\
6 \\
14 \\
0 \\
0 \\
0 \\
0 \\
2\end{array}$ & $\begin{array}{l}0 \\
0 \\
0 \\
0 \\
0 \\
0 \\
0 \\
0 \\
0 \\
0 \\
0 \\
0\end{array}$ & $\begin{array}{l}1,553 \\
1,533 \\
1,641 \\
1,862 \\
1,706 \\
1,785 \\
1,719 \\
1,850 \\
1,873 \\
1,882 \\
1,686 \\
1,736\end{array}$ & $\begin{array}{l}1,462 \\
1,421 \\
1,506 \\
1,682 \\
1,564 \\
1,656 \\
1,607 \\
1,730 \\
1,785 \\
1,771 \\
1,635 \\
1,621\end{array}$ \\
\hline $\begin{array}{l}1996 \text { 11-Month Average ....... } \\
1995 \text { 11-Month Average ....... }\end{array}$ & $\begin{array}{l}0 \\
0\end{array}$ & $\begin{array}{l}0 \\
0\end{array}$ & $\begin{array}{l}1,359 \\
1,341\end{array}$ & $\begin{array}{l}1,250 \\
1,259\end{array}$ & $\begin{array}{r}3 \\
11\end{array}$ & $\begin{array}{l}3 \\
6\end{array}$ & $\begin{array}{l}1,597 \\
1,571\end{array}$ & $\begin{array}{l}1,485 \\
1,479\end{array}$ \\
\hline
\end{tabular}

a Excludes petroleum imported into the United States indirectly from members of the Organization of Petroleum Exporting Countries (OPEC), primarily from Caribbean and West European areas, as petroleum products that were refined from crude oil produced by OPEC.

b Imports from the Neutral Zone between Kuwait and Saudi Arabia are included in Saudi Arabia.

(s)=Less than 500 barrels per day.

Notes: - Beginning in October 1977, Strategic Petroleum Reserve imports are included. - Totals may not equal sum of components due to independent rounding. - U.S. geographic coverage is the 50 States and the District of Columbia.

Sources: - 1973-1980: Energy Information Administration (EIA) Petroleum Supply Monthly, February 1993, Table S3. • 1981 forward: EIA, Petroleum Supply Monthly, January 1998, Table S3. 
Table 3.3c Petroleum Innports: Algeria, Ecuador, Gabon, Indonesia, and Libya (Thousand Barrels per Day)

\begin{tabular}{|c|c|c|c|c|c|c|c|c|c|c|}
\hline & \multicolumn{10}{|c|}{ Other OPECa } \\
\hline & \multicolumn{2}{|c|}{ Algeria } & \multicolumn{2}{|c|}{ Ecuador ${ }^{b}$} & \multicolumn{2}{|c|}{ Gabonc } & \multicolumn{2}{|c|}{ Indonesia } & \multicolumn{2}{|c|}{ Libya } \\
\hline & Total & Crude Oil & Total & Crude Oil & Total & Crude Oil & Total & Crude Oil & Total & Crude Oil \\
\hline $\begin{array}{l}1973 \text { Average } \\
1974 \text { Average } \\
1975 \text { Average } \\
1976 \text { Average } \\
1977 \text { Average } \\
1978 \text { Average } \\
1979 \text { Average } \\
1980 \text { Average } \\
1981 \text { Average } \\
1982 \text { Average } \\
1983 \text { Average } \\
1984 \text { Average } \\
1985 \text { Average } \\
1986 \text { Average } \\
1987 \text { Average } \\
1988 \text { Average } \\
1989 \text { Average } \\
1990 \text { Average } \\
1991 \text { Average }\end{array}$ & 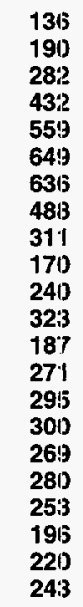 & $\begin{array}{r}1: 20 \\
1: 30 \\
2154 \\
408 \\
544 \\
634 \\
608 \\
456 \\
261 \\
90 \\
90 \\
176 \\
194 \\
34 \\
78 \\
115 \\
58 \\
100 \\
163 \\
44 \\
24 \\
24 \\
21\end{array}$ & $\begin{array}{r}48 \\
42 \\
57 \\
51 \\
57 \\
54 \\
42 \\
27 \\
48 \\
42 \\
61 \\
55 \\
67 \\
77 \\
29 \\
47 \\
89 \\
49 \\
63 \\
65 \\
\text { (b) } \\
\text { b) }\end{array}$ & $\begin{array}{r}47 \\
42 \\
57 \\
51 \\
55 \\
38 \\
30 \\
17 \\
38 \\
32 \\
56 \\
47 \\
56 \\
64 \\
23 \\
33 \\
80 \\
\mathbf{3 8} \\
53 \\
\mathbf{6 2} \\
\text { (b) } \\
\text { (b) }\end{array}$ & $\begin{array}{r}0 \\
23 \\
27 \\
28 \\
42 \\
41 \\
42 \\
26 \\
35 \\
40 \\
59 \\
58 \\
52 \\
26 \\
35 \\
16 \\
50 \\
64 \\
84 \\
124 \\
152 \\
194\end{array}$ & $\begin{array}{r}0 \\
23 \\
27 \\
26 \\
35 \\
38 \\
42 \\
25 \\
35 \\
40 \\
59 \\
57 \\
51 \\
25 \\
35 \\
15 \\
49 \\
64 \\
84 \\
123 \\
151 \\
194\end{array}$ & $\begin{array}{r}213 \\
300 \\
390 \\
539 \\
541 \\
573 \\
420 \\
348 \\
366 \\
248 \\
338 \\
343 \\
314 \\
318 \\
285 \\
205 \\
183 \\
114 \\
111 \\
78 \\
81 \\
111\end{array}$ & $\begin{array}{r}200 \\
284 \\
379 \\
537 \\
507 \\
533 \\
380 \\
314 \\
318 \\
226 \\
315 \\
304 \\
292 \\
297 \\
262 \\
186 \\
158 \\
98 \\
102 \\
70 \\
65 \\
92\end{array}$ & $\begin{array}{r}164 \\
4 \\
232 \\
453 \\
723 \\
654 \\
658 \\
554 \\
319 \\
26 \\
0 \\
1 \\
4 \\
0 \\
0 \\
0 \\
0 \\
0 \\
0 \\
0 \\
0 \\
0\end{array}$ & $\begin{array}{r}133 \\
4 \\
223 \\
444 \\
704 \\
638 \\
642 \\
548 \\
317 \\
23 \\
0 \\
0 \\
0 \\
0 \\
0 \\
0 \\
0 \\
0 \\
0 \\
0 \\
0 \\
0\end{array}$ \\
\hline 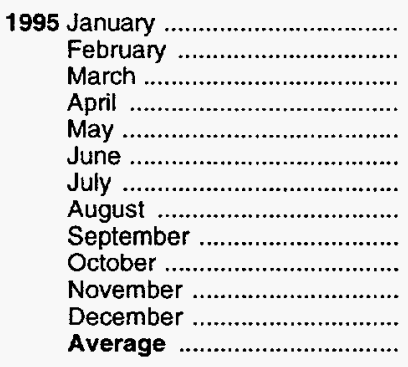 & $\begin{array}{l}15: 3 \\
3513 \\
1915 \\
251 \\
16: 3 \\
277 \\
257 \\
29: 3 \\
2510 \\
229 \\
241 \\
15: 2 \\
234\end{array}$ & $\begin{array}{r}0 \\
64 \\
19 \\
31 \\
36 \\
39 \\
11 \\
65 \\
20 \\
39 \\
0 \\
0 \\
27\end{array}$ & $\begin{array}{l}\left(\begin{array}{l}b \\
b\end{array}\right) \\
(b) \\
b \\
(b) \\
b \\
\left(\begin{array}{l}b \\
b\end{array}\right) \\
\left(\begin{array}{l}b \\
b\end{array}\right) \\
\left(\begin{array}{l}b \\
b\end{array}\right) \\
\left(\begin{array}{l}b \\
b\end{array}\right)\end{array}$ & $\begin{array}{l}\left(\begin{array}{l}b \\
b\end{array}\right) \\
(b) \\
b \\
(b) \\
b \\
\left(\begin{array}{l}b \\
b\end{array}\right) \\
\left(\begin{array}{l}b \\
b\end{array}\right) \\
\left(\begin{array}{l}b \\
b\end{array}\right) \\
(b)\end{array}$ & $\begin{array}{l}\left(\begin{array}{l}c \\
c\end{array}\right) \\
(c) \\
(c) \\
(c) \\
(c) \\
\left(\begin{array}{l}c \\
c\end{array}\right) \\
\left(\begin{array}{l}c \\
c\end{array}\right) \\
\left(\begin{array}{l}c \\
c\end{array}\right) \\
(c)\end{array}$ & 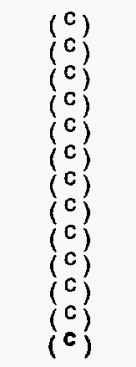 & $\begin{array}{r}38 \\
129 \\
51 \\
95 \\
65 \\
96 \\
104 \\
122 \\
94 \\
87 \\
107 \\
72 \\
88\end{array}$ & $\begin{array}{l}38 \\
87 \\
29 \\
87 \\
36 \\
51 \\
96 \\
95 \\
66 \\
68 \\
73 \\
41 \\
64\end{array}$ & $\begin{array}{l}0 \\
0 \\
0 \\
0 \\
0 \\
0 \\
0 \\
0 \\
0 \\
0 \\
0 \\
0 \\
0\end{array}$ & $\begin{array}{l}0 \\
0 \\
0 \\
0 \\
0 \\
0 \\
0 \\
0 \\
0 \\
0 \\
0 \\
0 \\
0\end{array}$ \\
\hline 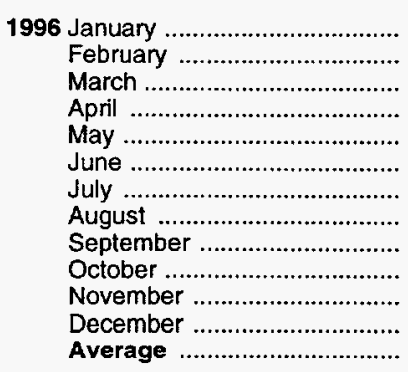 & $\begin{array}{l}31: 3 \\
2010 \\
241 \\
211 \\
341 \\
31: 3 \\
30: 5 \\
32: 3 \\
1815 \\
209 \\
21.4 \\
21.4 \\
2516\end{array}$ & $\begin{array}{r}38 \\
16 \\
38 \\
2 \\
0 \\
0 \\
0 \\
0 \\
0 \\
0 \\
3 \\
0 \\
8\end{array}$ & $\begin{array}{l}\left(\begin{array}{l}b \\
b\end{array}\right) \\
\left(\begin{array}{l}b \\
b\end{array}\right) \\
\left(\begin{array}{l}b \\
b\end{array}\right) \\
\left(\begin{array}{l}b \\
b\end{array}\right) \\
\left(\begin{array}{l}b \\
b\end{array}\right) \\
\left(\begin{array}{l}b \\
b\end{array}\right) \\
\left(\begin{array}{l}b \\
b\end{array}\right) \\
\left(\begin{array}{l}b \\
b\end{array}\right)\end{array}$ & $\begin{array}{l}\left(\begin{array}{l}b \\
b\end{array}\right) \\
(b) \\
(b) \\
(b) \\
(b) \\
(b) \\
(b) \\
(b) \\
(b) \\
(b) \\
\left(\begin{array}{c}b \\
b\end{array}\right)\end{array}$ & $\begin{array}{l}\left(\begin{array}{l}c \\
c\end{array}\right) \\
\left(\begin{array}{l}c \\
c\end{array}\right) \\
(c) \\
(c) \\
(c) \\
(c) \\
(c) \\
(c) \\
(c) \\
(c) \\
(c) \\
(c)\end{array}$ & $\begin{array}{l}\left(\begin{array}{l}c \\
c\end{array}\right) \\
\left(\begin{array}{l}c \\
c\end{array}\right) \\
(c) \\
(c) \\
(c) \\
(c) \\
(c) \\
(c) \\
(c) \\
(c) \\
(c) \\
(c)\end{array}$ & $\begin{array}{r}52 \\
44 \\
58 \\
57 \\
49 \\
72 \\
56 \\
53 \\
26 \\
125 \\
36 \\
81 \\
59\end{array}$ & $\begin{array}{l}43 \\
43 \\
55 \\
57 \\
15 \\
65 \\
48 \\
49 \\
26 \\
82 \\
12 \\
32 \\
44\end{array}$ & $\begin{array}{l}0 \\
0 \\
0 \\
0 \\
0 \\
0 \\
0 \\
0 \\
0 \\
0 \\
0 \\
0 \\
0\end{array}$ & $\begin{array}{l}0 \\
0 \\
0 \\
0 \\
0 \\
0 \\
0 \\
0 \\
0 \\
0 \\
0 \\
0 \\
0\end{array}$ \\
\hline 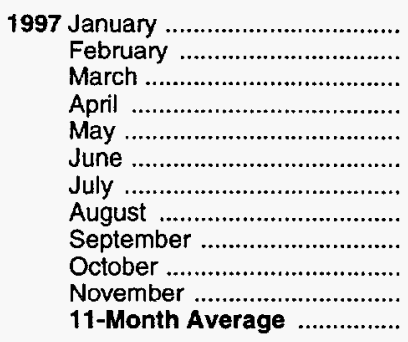 & $\begin{array}{l}282 \\
319 \\
309 \\
329 \\
290 \\
349 \\
291 \\
261 \\
259 \\
272 \\
267 \\
292\end{array}$ & $\begin{array}{r}0 \\
0 \\
0 \\
23 \\
0 \\
0 \\
0 \\
4 \\
6 \\
3 \\
7 \\
4\end{array}$ & $\begin{array}{l}\left(\begin{array}{l}b \\
b\end{array}\right) \\
\left(\begin{array}{l}b \\
b\end{array}\right) \\
\left(\begin{array}{l}b \\
b\end{array}\right) \\
\left(\begin{array}{l}b \\
b\end{array}\right) \\
\left(\begin{array}{l}b \\
b\end{array}\right) \\
\left(\begin{array}{l}b \\
b\end{array}\right) \\
\left(\begin{array}{l}b \\
b\end{array}\right)\end{array}$ & 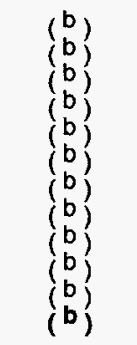 & $\begin{array}{l}\left(\begin{array}{l}c \\
c\end{array}\right) \\
(c) \\
(c) \\
c \\
(c) \\
(c) \\
(c) \\
(c) \\
(c) \\
(c) \\
(c) \\
(c) \\
(c)\end{array}$ & $\begin{array}{l}\left(\begin{array}{l}c \\
c\end{array}\right. \\
\left(\begin{array}{l}c \\
c\end{array}\right. \\
\left(\begin{array}{l}c \\
c\end{array}\right. \\
(c) \\
(c) \\
(c) \\
(c) \\
(c) \\
(c) \\
(c)\end{array}$ & $\begin{array}{l}73 \\
51 \\
18 \\
40 \\
86 \\
57 \\
73 \\
14 \\
82 \\
42 \\
79 \\
56\end{array}$ & $\begin{array}{l}38 \\
39 \\
15 \\
32 \\
86 \\
50 \\
66 \\
11 \\
75 \\
42 \\
74 \\
48\end{array}$ & $\begin{array}{l}0 \\
0 \\
0 \\
0 \\
0 \\
0 \\
0 \\
0 \\
0 \\
0 \\
0 \\
0\end{array}$ & $\begin{array}{l}0 \\
0 \\
0 \\
0 \\
0 \\
0 \\
0 \\
0 \\
0 \\
0 \\
0 \\
0\end{array}$ \\
\hline $\begin{array}{l}1996 \text { 11-Month Average } . . . . . . . . . . . . . \\
1995 \text { 11-Month Average .............. }\end{array}$ & $\begin{array}{l}260 \\
242\end{array}$ & $\begin{array}{r}9 \\
29\end{array}$ & $\left(\begin{array}{l}b \\
b\end{array}\right)$ & $\left.\begin{array}{l}b \\
b\end{array}\right)$ & $\left(\begin{array}{l}c \\
c\end{array}\right)$ & $\left(\begin{array}{l}c \\
c\end{array}\right)$ & $\begin{array}{l}57 \\
89\end{array}$ & $\begin{array}{l}45 \\
66\end{array}$ & $\begin{array}{l}0 \\
0\end{array}$ & $\begin{array}{l}0 \\
0\end{array}$ \\
\hline
\end{tabular}

a Excludes petroleum imported into the United States indirectly from members of the Organization of Petroleum Exporting Countries (OPEC), primarily from Caribbean and West European areas, as petroleum products that were refined from crude oil produced by OPEC.

$b$ Ecuador withdrew from OPEC on December 31, 1992. As of January 1993, imports from Ecuador appear on Table 3.3f under "Non-OPEC."

C Gabon withdrew from OPEC on December 31, 1994. As of January
1995, imports from Gabon appear on Table 3.3f under "Non-OPEC."

Notes: - Beginning in October 1977, Strategic Petroleum Resenve imports are included. - U.S. geographic coverage is the 50 States and the District of Columbia.

Sources: - 1973-1980: Energy Information Administration (EIA) Petroleum Supply Monthly, February 1993, Table S3. • 1981 forward: EIA, Petroleum Supply Monthly, January 1998, Table S3. 
Table 3.3d Petroleum Imports: Nigeria, Venezuela, Total Other OPEC, and Total OPEC

(Thousand Barrels per Day)

\begin{tabular}{|c|c|c|c|c|c|c|c|c|}
\hline & \multicolumn{6}{|c|}{ Other OPECa } & & \\
\hline & \multicolumn{2}{|c|}{ Nigeria } & \multicolumn{2}{|c|}{ Venezuela } & \multicolumn{2}{|c|}{ Total } & \multicolumn{2}{|c|}{$\begin{array}{c}\text { Total } \\
\text { OPEC }\end{array}$} \\
\hline & Total & Crude Oil & Total & Crude Oil & Total & Crude Oil & Total & Crude Oit \\
\hline $\begin{array}{l}1973 \text { Average } \\
1974 \text { Average } \\
1975 \text { Average } \\
1976 \text { Average } \\
1977 \text { Average } \\
1978 \text { Average } \\
1979 \text { Average } \\
1980 \text { Average } \\
1981 \text { Average } \\
1982 \text { Average } \\
1983 \text { Average } \\
1984 \text { Average } \\
1985 \text { Average } \\
1986 \text { Average } \\
1987 \text { Average } \\
1988 \text { Average } \\
1989 \text { Average } \\
1990 \text { Average } \\
1991 \text { Average }\end{array}$ & $\begin{array}{r}459 \\
713 \\
762 \\
1,025 \\
1,143 \\
919 \\
1,080 \\
857 \\
620 \\
514 \\
302 \\
216 \\
293 \\
440 \\
535 \\
618 \\
815 \\
800 \\
703 \\
681 \\
740 \\
637\end{array}$ & $\begin{array}{r}448 \\
697 \\
746 \\
1,014 \\
1,130 \\
910 \\
1,069 \\
841 \\
611 \\
510 \\
301 \\
207 \\
280 \\
437 \\
529 \\
607 \\
800 \\
784 \\
683 \\
665 \\
722 \\
624\end{array}$ & $\begin{array}{r}1,135 \\
979 \\
702 \\
700 \\
690 \\
646 \\
690 \\
481 \\
406 \\
412 \\
422 \\
548 \\
605 \\
793 \\
804 \\
794 \\
873 \\
1,025 \\
1,035 \\
1,170 \\
1,300 \\
1,334\end{array}$ & $\begin{array}{r}344 \\
319 \\
395 \\
241 \\
250 \\
181 \\
293 \\
156 \\
147 \\
155 \\
164 \\
253 \\
306 \\
416 \\
488 \\
439 \\
495 \\
666 \\
668 \\
826 \\
1,010 \\
1,034\end{array}$ & $\begin{array}{l}2,156 \\
2,253 \\
2,452 \\
3,229 \\
3,754 \\
3,536 \\
3,569 \\
2,781 \\
2,106 \\
1,451 \\
1,422 \\
1,544 \\
1,522 \\
1,926 \\
1,983 \\
1,981 \\
2,279 \\
2,332 \\
2,249 \\
2,313 \\
2,493 \\
2,520\end{array}$ & $\begin{array}{l}1,293 \\
1,549 \\
2,091 \\
2,721 \\
3,225 \\
2,972 \\
3,063 \\
2,356 \\
1,726 \\
1,075 \\
1,072 \\
1,062 \\
1,069 \\
1,317 \\
1,451 \\
1,339 \\
1,642 \\
1,713 \\
1,634 \\
1,770 \\
1,972 \\
1,965\end{array}$ & $\begin{array}{l}2,993 \\
3,280 \\
3,601 \\
5,066 \\
6,193 \\
5,751 \\
5,637 \\
4,300 \\
3,323 \\
2,146 \\
1,862 \\
2,049 \\
1,830 \\
2,837 \\
3,060 \\
3,520 \\
4,140 \\
4,296 \\
4,092 \\
4,092 \\
4,273 \\
4,247\end{array}$ & $\begin{array}{l}2,095 \\
2,540 \\
3,211 \\
4,545 \\
5,643 \\
5,184 \\
5,112 \\
3,864 \\
2,922 \\
1,734 \\
1,477 \\
1,512 \\
1,312 \\
2,113 \\
2,400 \\
2,696 \\
3,376 \\
3,514 \\
3,377 \\
3,406 \\
3,609 \\
3,580\end{array}$ \\
\hline 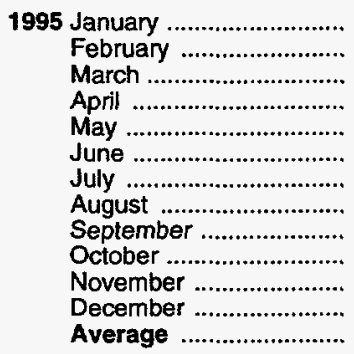 & $\begin{array}{l}625 \\
463 \\
687 \\
467 \\
603 \\
696 \\
696 \\
482 \\
851 \\
649 \\
646 \\
652 \\
627\end{array}$ & $\begin{array}{l}617 \\
463 \\
676 \\
458 \\
592 \\
696 \\
696 \\
463 \\
841 \\
649 \\
637 \\
652 \\
621\end{array}$ & $\begin{array}{l}1,442 \\
1,439 \\
1,499 \\
1,365 \\
1,480 \\
1,479 \\
1,536 \\
1,449 \\
1,655 \\
1,453 \\
1,507 \\
1,459 \\
1,480\end{array}$ & $\begin{array}{l}1,061 \\
1,083 \\
1,208 \\
1,083 \\
1,176 \\
1,209 \\
1,162 \\
1,162 \\
1,288 \\
1,159 \\
1,140 \\
1,074 \\
1,151\end{array}$ & $\begin{array}{l}2,258 \\
2,389 \\
2,432 \\
2,177 \\
2,311 \\
2,548 \\
2,592 \\
2,352 \\
2,851 \\
2,418 \\
2,501 \\
2,334 \\
2,430\end{array}$ & $\begin{array}{l}1,717 \\
1,697 \\
1,933 \\
1,659 \\
1,840 \\
1,995 \\
1,965 \\
1,784 \\
2,214 \\
1,914 \\
1,851 \\
1,767 \\
1,862\end{array}$ & $\begin{array}{l}3,718 \\
3,929 \\
4,220 \\
3,724 \\
3,801 \\
4,106 \\
4,052 \\
3,892 \\
4,541 \\
3,942 \\
4,178 \\
3,927 \\
4,002\end{array}$ & $\begin{array}{l}3,108 \\
3,168 \\
3,595 \\
3,144 \\
3,281 \\
3,476 \\
3,325 \\
3,225 \\
3,753 \\
3,340 \\
3,424 \\
3,245 \\
3,341\end{array}$ \\
\hline 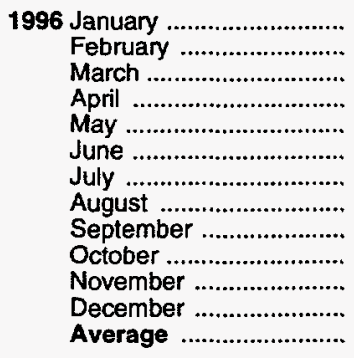 & $\begin{array}{l}690 \\
647 \\
594 \\
518 \\
705 \\
711 \\
750 \\
793 \\
694 \\
521 \\
465 \\
320 \\
617\end{array}$ & $\begin{array}{l}663 \\
639 \\
548 \\
497 \\
705 \\
697 \\
696 \\
785 \\
677 \\
488 \\
453 \\
298 \\
595\end{array}$ & $\begin{array}{l}1,518 \\
1,495 \\
1,719 \\
1,732 \\
1,700 \\
1,642 \\
1,690 \\
1,749 \\
1,708 \\
1,781 \\
1,728 \\
1,641 \\
1,676\end{array}$ & $\begin{array}{l}1,148 \\
1,166 \\
1,341 \\
1,288 \\
1,333 \\
1,236 \\
1,332 \\
1,431 \\
1,269 \\
1,448 \\
1,303 \\
1,324 \\
1,303\end{array}$ & $\begin{array}{l}2,574 \\
2,385 \\
2,611 \\
2,519 \\
2,794 \\
2,738 \\
2,800 \\
2,918 \\
2,613 \\
2,636 \\
2,443 \\
2,256 \\
2,609\end{array}$ & $\begin{array}{l}1,892 \\
1,865 \\
1,981 \\
1,844 \\
2,054 \\
1,999 \\
2,076 \\
2,265 \\
1,972 \\
2,019 \\
1,770 \\
1,654 \\
1,950\end{array}$ & $\begin{array}{l}4,120 \\
3,730 \\
4,161 \\
4,007 \\
4,541 \\
4,275 \\
4,619 \\
4,665 \\
4,204 \\
4,271 \\
3,967 \\
3,931 \\
4,211\end{array}$ & $\begin{array}{l}3,371 \\
3,133 \\
3,427 \\
3,245 \\
3,697 \\
3,432 \\
3,718 \\
3,865 \\
3,463 \\
3,504 \\
3,199 \\
3,166 \\
3,438\end{array}$ \\
\hline 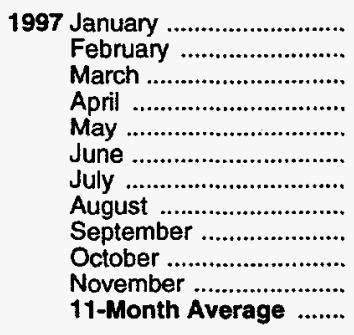 & $\begin{array}{l}531 \\
625 \\
558 \\
705 \\
961 \\
768 \\
580 \\
882 \\
765 \\
688 \\
649 \\
702\end{array}$ & $\begin{array}{l}505 \\
620 \\
557 \\
696 \\
944 \\
768 \\
571 \\
866 \\
765 \\
675 \\
649 \\
693\end{array}$ & $\begin{array}{l}1,637 \\
1,595 \\
1,753 \\
1,640 \\
1,872 \\
1,852 \\
1,628 \\
1,703 \\
1,771 \\
1,948 \\
1,651 \\
1,733\end{array}$ & $\begin{array}{l}1,212 \\
1,255 \\
1,324 \\
1,254 \\
1,384 \\
1,475 \\
1,312 \\
1,310 \\
1,443 \\
1,562 \\
1,391 \\
1,357\end{array}$ & $\begin{array}{l}2,523 \\
2,591 \\
2,638 \\
2,706 \\
3,209 \\
3,026 \\
2,573 \\
2,860 \\
2,878 \\
2,950 \\
2,645 \\
2,783\end{array}$ & $\begin{array}{l}1,755 \\
1,913 \\
1,895 \\
2,005 \\
2,414 \\
2,293 \\
1,949 \\
2,191 \\
2,289 \\
2,283 \\
2,120 \\
2,101\end{array}$ & $\begin{array}{l}4,077 \\
4,123 \\
4,279 \\
4,567 \\
4,915 \\
4,811 \\
4,291 \\
4,710 \\
4,750 \\
4,833 \\
4,331 \\
4,519\end{array}$ & $\begin{array}{l}3,217 \\
3,335 \\
3,402 \\
3,687 \\
3,977 \\
3,949 \\
3,556 \\
3,921 \\
4,074 \\
4,054 \\
3,755 \\
3,722\end{array}$ \\
\hline $\begin{array}{l}1996 \text { 11-Month Average ....... } \\
1995 \text { 11-Month Average ....... }\end{array}$ & $\begin{array}{l}645 \\
625\end{array}$ & $\begin{array}{l}623 \\
618\end{array}$ & $\begin{array}{l}1,679 \\
1,482\end{array}$ & $\begin{array}{l}1,301 \\
1,158\end{array}$ & $\begin{array}{l}2,641 \\
2,439\end{array}$ & $\begin{array}{l}1,978 \\
1,871\end{array}$ & $\begin{array}{l}4,237 \\
4,009\end{array}$ & $\begin{array}{l}3,463 \\
3,349\end{array}$ \\
\hline
\end{tabular}

a Excludes petroleum imported into the United States indirectly from members of the Organization of Petroleum Exporting Countries (OPEC), primarily from Caribbean and West European areas, as petroleum products that were refined from crude oil produced by OPEC.

$b$ OPEC includes the Persian Gulf nations that are displayed on Tables $3.3 a$ and $3.3 b$ except Bahrain, which is not a member of OPEC, and the nations displayed under "Other OPEC" on Tables $3.3 \mathrm{c}$ and $3.3 \mathrm{~d}$. Ecuador withdrew from OPEC on December 31, 1992; as of January 1993, imports from Ecuador appear on Table 3.3 f under "Non-OPEC." Gabon withdrew on December 31, 1994; as of January 1995, imports from Gabon appear on
Table 3.3t under "Non-OPEC." Imports from Bahrain are accounted for under "Other Non-OPEC" on Table $3.3 \mathrm{~h}$.

Notes: - Beginning in October 1977, Strategic Petroleum Reserve imports are included. - Totals may not equal sum of components due to independent rounding. - U.S. geographic coverage is the 50 States and the District of Columbia.

Sources: - 1973-1980: Energy Information Administration (EIA), Petroleum Supply Monthiy, February 1993, Table S3. • 1981 forward: EIA, Petroleum Supply Monthly, January 1998, Table S3. 
Table 3.3e Petroleum Imports: Angola, Australia, Bahama Islands, Brazil, Canada, and China

(Thousand Barrels per Day)

\begin{tabular}{|c|c|c|c|c|c|c|c|c|c|c|c|c|}
\hline & \multicolumn{12}{|c|}{ Non-OPEC ${ }^{\mathbf{a}}$} \\
\hline $\begin{array}{l}1973 \text { Average } \\
1974 \text { Average } \\
1975 \text { Average ................ } \\
1976 \text { Average . . } \\
1977 \text { Average } \\
1978 \text { Average }\end{array}$ & $\begin{array}{r}49 \\
49 \\
75 \\
12 \\
24 \\
20 \\
43 \\
43 \\
42 \\
49 \\
44 \\
78 \\
90 \\
110 \\
112 \\
192 \\
212 \\
284 \\
237 \\
254 \\
336 \\
336 \\
331\end{array}$ & $\begin{array}{r}49 \\
48 \\
71 \\
7 \\
17 \\
6 \\
39 \\
37 \\
45 \\
42 \\
71 \\
85 \\
104 \\
102 \\
180 \\
203 \\
279 \\
236 \\
254 \\
336 \\
336 \\
322 \\
\end{array}$ & $\begin{array}{l}2 \\
1 \\
5 \\
2 \\
3 \\
5 \\
6 \\
1 \\
5 \\
5 \\
4 \\
38 \\
37 \\
41 \\
58 \\
64 \\
36 \\
53 \\
26 \\
19 \\
19 \\
17\end{array}$ & $\begin{array}{r}0 \\
0 \\
0 \\
0 \\
0 \\
0 \\
0 \\
0 \\
0 \\
(\mathbf{s}) \\
0 \\
25 \\
21 \\
30 \\
49 \\
59 \\
31 \\
47 \\
21 \\
17 \\
18 \\
16\end{array}$ & $\begin{array}{r}174 \\
164 \\
152 \\
118 \\
171 \\
160 \\
147 \\
78 \\
74 \\
65 \\
125 \\
88 \\
40 \\
37 \\
37 \\
32 \\
34 \\
37 \\
35 \\
36 \\
28 \\
29\end{array}$ & $\begin{array}{l}0 \\
0 \\
0 \\
0 \\
0 \\
0 \\
0 \\
0 \\
0 \\
0 \\
0 \\
0 \\
0 \\
0 \\
0 \\
0 \\
0 \\
0 \\
0 \\
0 \\
0 \\
0\end{array}$ & $\begin{array}{l}9 \\
2 \\
5 \\
0 \\
0 \\
0 \\
1 \\
3 \\
23 \\
47 \\
41 \\
60 \\
61 \\
50 \\
84 \\
98 \\
82 \\
49 \\
22 \\
20 \\
33 \\
31\end{array}$ & $\begin{array}{r}0 \\
0 \\
0 \\
0 \\
0 \\
0 \\
0 \\
1 \\
14 \\
19 \\
2 \\
(s) \\
0 \\
0 \\
0 \\
0 \\
0 \\
0 \\
0 \\
0 \\
0 \\
1\end{array}$ & $\begin{array}{r}1,325 \\
1,070 \\
846 \\
599 \\
517 \\
467 \\
538 \\
455 \\
447 \\
482 \\
547 \\
630 \\
770 \\
807 \\
848 \\
999 \\
931 \\
934 \\
1,033 \\
1,069 \\
1,181 \\
1,272\end{array}$ & $\begin{array}{r}1,001 \\
791 \\
600 \\
371 \\
279 \\
248 \\
271 \\
199 \\
164 \\
214 \\
274 \\
341 \\
468 \\
570 \\
608 \\
681 \\
630 \\
643 \\
743 \\
797 \\
900 \\
983\end{array}$ & $\begin{array}{c}\text { (s) } \\
0 \\
0 \\
0 \\
0 \\
0 \\
13 \\
(s) \\
18 \\
40 \\
34 \\
46 \\
59 \\
90 \\
82 \\
88 \\
80 \\
80 \\
91 \\
90 \\
51 \\
65\end{array}$ & $\begin{array}{r}0 \\
0 \\
0 \\
0 \\
0 \\
0 \\
13 \\
0 \\
0 \\
8 \\
6 \\
15 \\
36 \\
68 \\
63 \\
82 \\
76 \\
77 \\
87 \\
84 \\
50 \\
64\end{array}$ \\
\hline 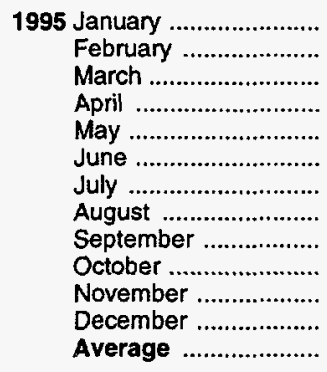 & $\begin{array}{l}273 \\
348 \\
427 \\
412 \\
419 \\
371 \\
295 \\
367 \\
444 \\
366 \\
318 \\
366 \\
367\end{array}$ & $\begin{array}{l}262 \\
335 \\
416 \\
402 \\
407 \\
358 \\
287 \\
355 \\
444 \\
366 \\
318 \\
366 \\
360\end{array}$ & $\begin{array}{r}21 \\
22 \\
0 \\
33 \\
21 \\
10 \\
42 \\
0 \\
0 \\
15 \\
(\mathrm{~s}) \\
23 \\
16\end{array}$ & $\begin{array}{r}21 \\
22 \\
0 \\
33 \\
21 \\
10 \\
42 \\
0 \\
0 \\
15 \\
0 \\
23 \\
16\end{array}$ & $\begin{array}{l}6 \\
8 \\
7 \\
0 \\
0 \\
0 \\
0 \\
0 \\
8 \\
0 \\
0 \\
0 \\
2\end{array}$ & $\begin{array}{l}0 \\
0 \\
0 \\
0 \\
0 \\
0 \\
0 \\
0 \\
0 \\
0 \\
0 \\
0 \\
0\end{array}$ & $\begin{array}{r}1 \\
0 \\
0 \\
0 \\
0 \\
0 \\
8 \\
9 \\
43 \\
9 \\
12 \\
12 \\
8\end{array}$ & $\begin{array}{l}0 \\
0 \\
0 \\
0 \\
0 \\
0 \\
0 \\
0 \\
0 \\
0 \\
0 \\
0 \\
0\end{array}$ & $\begin{array}{l}1,345 \\
1,311 \\
1,208 \\
1,243 \\
1,406 \\
1,420 \\
1,279 \\
1,345 \\
1,252 \\
1,300 \\
1,403 \\
1,471 \\
1,332\end{array}$ & $\begin{array}{r}1,011 \\
965 \\
891 \\
999 \\
1,167 \\
1,169 \\
1,028 \\
1,058 \\
959 \\
1,057 \\
1,069 \\
1,099 \\
1,040\end{array}$ & $\begin{array}{l}64 \\
21 \\
54 \\
65 \\
35 \\
26 \\
80 \\
40 \\
73 \\
40 \\
66 \\
73 \\
53\end{array}$ & $\begin{array}{l}62 \\
21 \\
54 \\
65 \\
35 \\
26 \\
80 \\
40 \\
73 \\
40 \\
66 \\
73 \\
53\end{array}$ \\
\hline 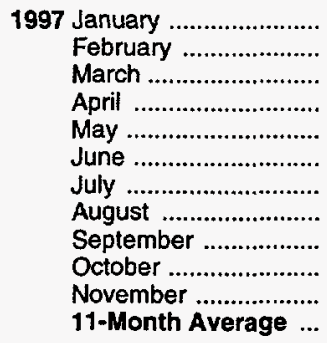 & $\begin{array}{l}485 \\
422 \\
467 \\
435 \\
312 \\
418 \\
416 \\
270 \\
399 \\
475 \\
437 \\
412\end{array}$ & $\begin{array}{l}485 \\
422 \\
461 \\
422 \\
307 \\
418 \\
416 \\
270 \\
399 \\
457 \\
437 \\
408\end{array}$ & $\begin{array}{r}21 \\
0 \\
37 \\
22 \\
61 \\
23 \\
77 \\
91 \\
53 \\
92 \\
23 \\
46\end{array}$ & $\begin{array}{r}21 \\
0 \\
37 \\
22 \\
44 \\
23 \\
48 \\
60 \\
12 \\
53 \\
23 \\
31\end{array}$ & $\begin{array}{r}0 \\
13 \\
0 \\
0 \\
0 \\
0 \\
0 \\
0 \\
0 \\
0 \\
0 \\
1\end{array}$ & $\begin{array}{l}0 \\
0 \\
0 \\
0 \\
0 \\
0 \\
0 \\
0 \\
0 \\
0 \\
0 \\
0\end{array}$ & $\begin{array}{r}1 \\
0 \\
4 \\
0 \\
0 \\
20 \\
21 \\
4 \\
3 \\
6 \\
2 \\
6\end{array}$ & $\begin{array}{l}0 \\
0 \\
0 \\
0 \\
0 \\
0 \\
0 \\
0 \\
0 \\
0 \\
0 \\
0\end{array}$ & $\begin{array}{l}1,508 \\
1,548 \\
1,412 \\
1,448 \\
1,423 \\
1,406 \\
1,403 \\
1,499 \\
1,503 \\
1,370 \\
1,490 \\
1,455\end{array}$ & $\begin{array}{l}1,137 \\
1,127 \\
1,103 \\
1,071 \\
1,068 \\
1,057 \\
1,085 \\
1,158 \\
1,185 \\
1,059 \\
1,176 \\
1,111\end{array}$ & $\begin{array}{r}84 \\
50 \\
120 \\
46 \\
21 \\
44 \\
0 \\
42 \\
26 \\
48 \\
0 \\
44\end{array}$ & $\begin{array}{r}84 \\
50 \\
120 \\
46 \\
21 \\
44 \\
0 \\
42 \\
20 \\
47 \\
0 \\
43\end{array}$ \\
\hline $\begin{array}{l}1996 \text { 11-Month Average ... } \\
1995 \text { 11-Month Average ... }\end{array}$ & $\begin{array}{l}345 \\
367\end{array}$ & $\begin{array}{l}338 \\
359\end{array}$ & $\begin{array}{l}31 \\
15\end{array}$ & $\begin{array}{l}25 \\
15\end{array}$ & $\begin{array}{l}1 \\
3\end{array}$ & $\begin{array}{l}0 \\
0\end{array}$ & $\begin{array}{r}10 \\
7\end{array}$ & $\begin{array}{l}0 \\
0\end{array}$ & $\begin{array}{l}1,400 \\
1,319\end{array}$ & $\begin{array}{l}1,061 \\
1,034\end{array}$ & $\begin{array}{l}56 \\
52\end{array}$ & $\begin{array}{l}56 \\
51\end{array}$ \\
\hline
\end{tabular}

a Includes petroleum imported into the United States indirectly from members of the Organization of Petroleum Exporting Countries (OPEC), primarily from Caribbean and West European areas, as petroleum products that were refined from crude oil produced by OPEC.

(s)=Less than 500 barrels per day.

Notes: - Beginning in October 1977, Strategic Petroleum Reserve imports are included. - U.S. geographic coverage is the 50 States and the District of Columbia.

Sources: - 1973-1980: Energy Information Administration (EIA), Petroleum Supply Monthly, February 1993, Table S3. - 1981 forward: EIA, Petroleum Supply Monthly, January 1998, Table S3. 
Table 3.3f Petroleum Imports: Colombia, Ecuador, Gabon, Italy, Malaysia, and Mexico

(Thousand Barrels per Day)

\begin{tabular}{|c|c|c|c|c|c|c|c|c|c|c|c|c|}
\hline & \multicolumn{12}{|c|}{ Non-OPECa } \\
\hline & \multicolumn{2}{|c|}{ Colombia } & \multicolumn{2}{|c|}{ Ecuadorb } & \multicolumn{2}{|c|}{ Gabonc } & \multicolumn{2}{|c|}{ Italy } & \multicolumn{2}{|c|}{ Malaysia } & \multicolumn{2}{|c|}{ Mexico } \\
\hline . & Total & Crude OIJ & Total & Crude Oil & Total & Crude Oil & Total & Crude Oil & Total & Crude Oil & Total & Crude Oil \\
\hline 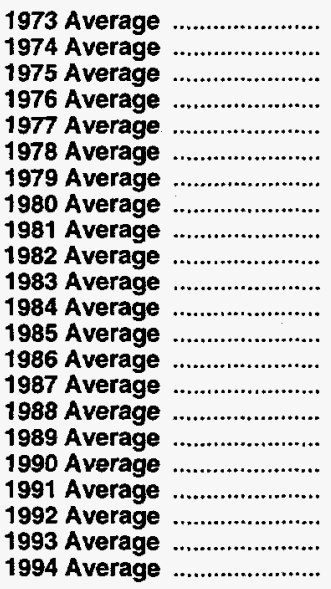 & $\begin{array}{r}9 \\
5 \\
9 \\
21 \\
17 \\
20 \\
18 \\
4 \\
1 \\
5 \\
10 \\
8 \\
23 \\
87 \\
148 \\
134 \\
172 \\
182 \\
163 \\
126 \\
171 \\
161\end{array}$ & $\begin{array}{r}2 \\
0 \\
0 \\
6 \\
0 \\
0 \\
0 \\
0 \\
0 \\
0 \\
0 \\
0 \\
0 \\
57 \\
115 \\
106 \\
136 \\
140 \\
123 \\
102 \\
141 \\
146\end{array}$ & 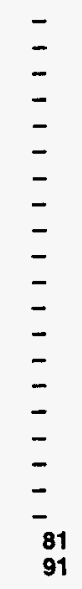 & $\begin{array}{l}- \\
- \\
- \\
- \\
- \\
- \\
- \\
- \\
- \\
- \\
- \\
- \\
- \\
- \\
- \\
- \\
- \\
-78\end{array}$ & $\begin{array}{l}- \\
- \\
- \\
- \\
- \\
- \\
- \\
- \\
- \\
- \\
- \\
- \\
- \\
- \\
- \\
- \\
- \\
- \\
- \\
-\end{array}$ & $\begin{array}{l}- \\
- \\
- \\
- \\
- \\
- \\
- \\
- \\
- \\
- \\
- \\
- \\
- \\
- \\
- \\
- \\
- \\
-\end{array}$ & $\begin{array}{r}125 \\
74 \\
27 \\
39 \\
51 \\
38 \\
30 \\
4 \\
11 \\
18 \\
18 \\
45 \\
60 \\
76 \\
54 \\
65 \\
34 \\
58 \\
47 \\
55 \\
31 \\
22\end{array}$ & $\begin{array}{c}0 \\
0 \\
0 \\
0 \\
0 \\
0 \\
0 \\
0 \\
0 \\
(s) \\
(s) \\
(s) \\
(s) \\
0 \\
1 \\
5 \\
3 \\
2 \\
3 \\
0 \\
0 \\
0\end{array}$ & $\begin{array}{r}12 \\
12 \\
8 \\
18 \\
66 \\
42 \\
66 \\
70 \\
36 \\
20 \\
4 \\
1 \\
3 \\
12 \\
13 \\
19 \\
39 \\
41 \\
24 \\
10 \\
11 \\
10\end{array}$ & $\begin{array}{r}1 \\
1 \\
5 \\
16 \\
55 \\
37 \\
52 \\
61 \\
33 \\
18 \\
3 \\
0 \\
1 \\
11 \\
12 \\
19 \\
39 \\
40 \\
24 \\
10 \\
10 \\
6\end{array}$ & $\begin{array}{r}16 \\
8 \\
71 \\
87 \\
179 \\
318 \\
439 \\
533 \\
522 \\
685 \\
826 \\
748 \\
816 \\
699 \\
655 \\
747 \\
767 \\
755 \\
807 \\
830 \\
919 \\
984\end{array}$ & $\begin{array}{r}1 \\
2 \\
70 \\
87 \\
177 \\
316 \\
437 \\
507 \\
469 \\
645 \\
766 \\
659 \\
715 \\
621 \\
602 \\
674 \\
716 \\
689 \\
759 \\
787 \\
863 \\
939\end{array}$ \\
\hline 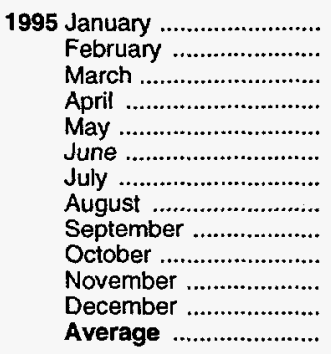 & $\begin{array}{l}223 \\
139 \\
239 \\
175 \\
171 \\
225 \\
223 \\
330 \\
252 \\
199 \\
240 \\
200 \\
219\end{array}$ & $\begin{array}{l}214 \\
129 \\
221 \\
175 \\
153 \\
202 \\
223 \\
311 \\
236 \\
190 \\
229 \\
190 \\
207\end{array}$ & $\begin{array}{r}130 \\
107 \\
104 \\
146 \\
116 \\
137 \\
87 \\
116 \\
61 \\
12 \\
102 \\
51 \\
97\end{array}$ & $\begin{array}{r}130 \\
107 \\
104 \\
146 \\
116 \\
137 \\
87 \\
104 \\
61 \\
12 \\
102 \\
51 \\
96\end{array}$ & $\begin{array}{l}193 \\
186 \\
159 \\
163 \\
206 \\
357 \\
311 \\
246 \\
216 \\
270 \\
271 \\
171 \\
229\end{array}$ & $\begin{array}{l}193 \\
186 \\
159 \\
163 \\
206 \\
357 \\
311 \\
246 \\
216 \\
270 \\
271 \\
171 \\
229\end{array}$ & $\begin{array}{r}4 \\
1 \\
8 \\
13 \\
0 \\
13 \\
4 \\
0 \\
0 \\
11 \\
4 \\
3 \\
5\end{array}$ & $\begin{array}{l}0 \\
0 \\
0 \\
0 \\
0 \\
0 \\
0 \\
0 \\
0 \\
0 \\
0 \\
0 \\
0\end{array}$ & $\begin{array}{r}21 \\
0 \\
0 \\
7 \\
0 \\
7 \\
0 \\
0 \\
14 \\
13 \\
16 \\
17 \\
8\end{array}$ & $\begin{array}{r}21 \\
0 \\
0 \\
0 \\
0 \\
0 \\
0 \\
0 \\
14 \\
5 \\
16 \\
11 \\
6\end{array}$ & $\begin{array}{r}925 \\
922 \\
1,006 \\
993 \\
1,118 \\
1,138 \\
1,188 \\
1,201 \\
1,311 \\
894 \\
1,114 \\
996 \\
1,068\end{array}$ & $\begin{array}{r}892 \\
890 \\
961 \\
963 \\
1,063 \\
1,076 \\
1,166 \\
1,172 \\
1,238 \\
854 \\
1,060 \\
978 \\
1,027\end{array}$ \\
\hline 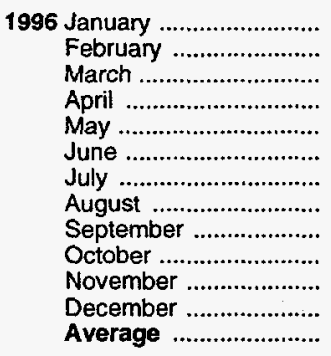 & $\begin{array}{l}186 \\
149 \\
262 \\
280 \\
263 \\
250 \\
204 \\
221 \\
213 \\
265 \\
267 \\
246 \\
234\end{array}$ & $\begin{array}{l}183 \\
139 \\
250 \\
280 \\
249 \\
247 \\
198 \\
217 \\
213 \\
252 \\
267 \\
218 \\
226\end{array}$ & $\begin{array}{r}126 \\
81 \\
131 \\
158 \\
100 \\
138 \\
113 \\
83 \\
48 \\
66 \\
111 \\
89 \\
104\end{array}$ & $\begin{array}{r}120 \\
81 \\
125 \\
143 \\
95 \\
133 \\
96 \\
71 \\
48 \\
60 \\
111 \\
72 \\
96\end{array}$ & $\begin{array}{l}171 \\
191 \\
154 \\
212 \\
154 \\
218 \\
191 \\
156 \\
104 \\
226 \\
253 \\
184 \\
184\end{array}$ & $\begin{array}{l}171 \\
191 \\
154 \\
212 \\
154 \\
218 \\
191 \\
156 \\
104 \\
226 \\
253 \\
184 \\
184\end{array}$ & $\begin{array}{r}2 \\
0 \\
13 \\
(s) \\
0 \\
16 \\
19 \\
8 \\
15 \\
4 \\
13 \\
8 \\
8\end{array}$ & $\begin{array}{l}0 \\
0 \\
0 \\
0 \\
0 \\
0 \\
0 \\
0 \\
0 \\
0 \\
0 \\
0 \\
0\end{array}$ & $\begin{array}{r}0 \\
24 \\
4 \\
0 \\
47 \\
19 \\
0 \\
5 \\
0 \\
31 \\
7 \\
0 \\
11\end{array}$ & $\begin{array}{r}0 \\
17 \\
0 \\
0 \\
40 \\
11 \\
0 \\
0 \\
0 \\
0 \\
0 \\
0 \\
6\end{array}$ & $\begin{array}{l}1,281 \\
1,083 \\
1,176 \\
1,303 \\
1,288 \\
1,351 \\
1,216 \\
1,157 \\
1,355 \\
1,213 \\
1,157 \\
1,346 \\
1,244\end{array}$ & $\begin{array}{l}1,245 \\
1,062 \\
1,165 \\
1,273 \\
1,222 \\
1,274 \\
1,186 \\
1,142 \\
1,306 \\
1,189 \\
1,110 \\
1,301 \\
1,207\end{array}$ \\
\hline 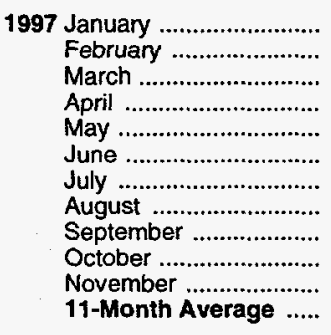 & $\begin{array}{l}227 \\
248 \\
260 \\
236 \\
288 \\
228 \\
251 \\
303 \\
271 \\
286 \\
304 \\
264\end{array}$ & $\begin{array}{l}226 \\
248 \\
257 \\
236 \\
282 \\
228 \\
241 \\
303 \\
271 \\
286 \\
304 \\
262\end{array}$ & $\begin{array}{r}112 \\
110 \\
148 \\
73 \\
109 \\
121 \\
122 \\
128 \\
143 \\
143 \\
91 \\
118\end{array}$ & $\begin{array}{r}107 \\
110 \\
148 \\
73 \\
104 \\
121 \\
122 \\
128 \\
143 \\
143 \\
91 \\
117\end{array}$ & $\begin{array}{r}62 \\
262 \\
217 \\
203 \\
178 \\
226 \\
264 \\
203 \\
271 \\
235 \\
256 \\
215\end{array}$ & $\begin{array}{r}62 \\
262 \\
217 \\
203 \\
178 \\
226 \\
264 \\
203 \\
271 \\
235 \\
256 \\
215\end{array}$ & $\begin{array}{r}8 \\
27 \\
5 \\
26 \\
9 \\
0 \\
0 \\
2 \\
0 \\
8 \\
0 \\
8\end{array}$ & $\begin{array}{l}0 \\
0 \\
0 \\
0 \\
0 \\
0 \\
0 \\
0 \\
0 \\
0 \\
0 \\
0\end{array}$ & $\begin{array}{r}32 \\
7 \\
33 \\
33 \\
9 \\
32 \\
28 \\
14 \\
37 \\
19 \\
8 \\
23\end{array}$ & $\begin{array}{r}0 \\
7 \\
0 \\
0 \\
0 \\
24 \\
0 \\
6 \\
29 \\
19 \\
0 \\
8\end{array}$ & $\begin{array}{l}1,307 \\
1,277 \\
1,310 \\
1,448 \\
1,429 \\
1,401 \\
1,366 \\
1,425 \\
1,386 \\
1,463 \\
1,410 \\
1,384\end{array}$ & $\begin{array}{l}1,264 \\
1,241 \\
1,249 \\
1,416 \\
1,408 \\
1,382 \\
1,347 \\
1,421 \\
1,371 \\
1,437 \\
1,403 \\
1,359\end{array}$ \\
\hline $\begin{array}{l}1996 \text { 11-Month Average ..... } \\
1995 \text { 11-Month Average ..... }\end{array}$ & $\begin{array}{l}233 \\
220\end{array}$ & $\begin{array}{l}227 \\
208\end{array}$ & $\begin{array}{l}105 \\
101\end{array}$ & $\begin{array}{r}98 \\
100\end{array}$ & $\begin{array}{l}184 \\
235\end{array}$ & $\begin{array}{l}184 \\
235\end{array}$ & $\begin{array}{l}8 \\
5\end{array}$ & $\begin{array}{l}0 \\
0\end{array}$ & $\begin{array}{r}13 \\
7\end{array}$ & $\begin{array}{l}\mathbf{6} \\
\mathbf{5}\end{array}$ & $\begin{array}{l}1,235 \\
1,074\end{array}$ & $\begin{array}{l}1,198 \\
1,031\end{array}$ \\
\hline
\end{tabular}

a Includes petroleum imported into the United States indirectly from members of the Organization of Petroleum Exporting Countries (OPEC) primarily from Caribbean and West European areas, as petroleum products that were refined from crude oil produced by OPEC.

b Through 1992, Ecuador was a member of OPEC. See Table 3.3c. 3.3c.

c Through December 1994, Gabon was a member of OPEC. See Table
$-=$ Not applicable. $(s)=$ Less than 500 barrels per day.

Notes: - Beginning in October 1977, Strategic Petroleum Resenve imports are included. - U.S. geographic coverage is the 50 States and the District of Columbia.

Sources: - 1973-1980: Energy Information Administration (EIA), Petroleum Supply Monthly, February 1993, Table S3. - 1981 forward: EIA, Petroleum Supply Monthly, January 1998, Table S3. 
Table 3.3g Petroleum Imports: Netherlands, Netherlands Antilles, Norway, Puerto Rico, Russia, and Spain

(Thousand Barrels per Day)

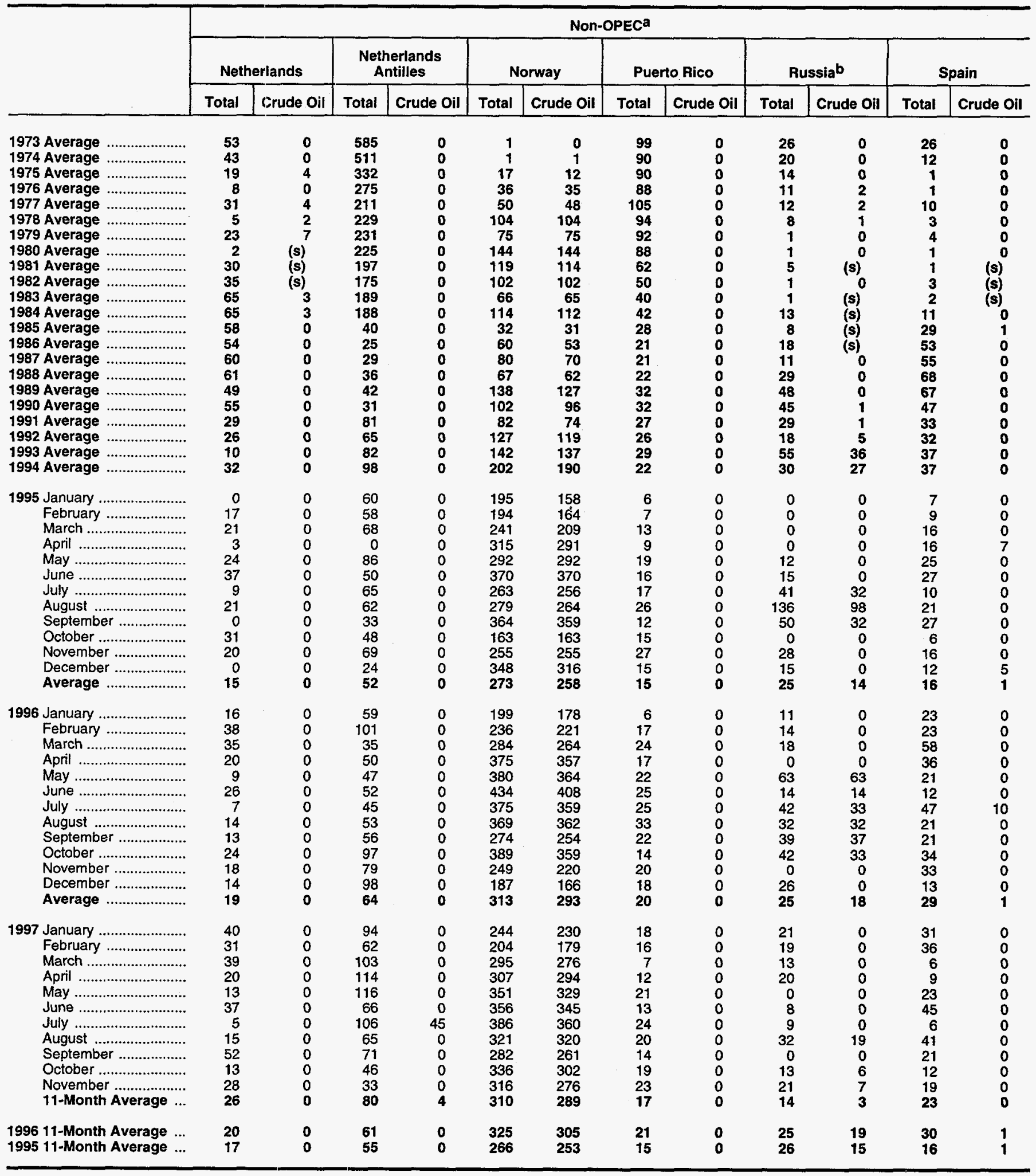

a Includes petroleum imported into the United States indirectly from members of the Organization of Petroleum Exporting Countries (OPEC), primarily from Caribbean and West Europiean areas, as petroleum products that were refined from crude oil produced by OPEC.

b Imports from other States in the former U.S.S.R. may be included in imports from Russia for the years 1973 through 1992.

(s)=Less than 500 barrels per day.
Notes: - Beginning in October 1977, Strategic Petroleum Reserve imports are included. - U.S. geographic coverage is the 50 States and the District of Columbia.

Sources: - 1973-1980: Energy Information Administration (EIA), Petroleum Supply Monthly, February 1993, Table S3. - 1981 forward: EIA, Petroleum Supply Monthly, January 1998, Table S3. 
Table 3.3h Petroleum Imports: Trinidad and Tobago, United Kingdom, Virgin Islands, Other Non-OPEC, Total Non-OPEC, and Total Imports

(Thousand Barrels per Day)

\begin{tabular}{|c|c|c|c|c|c|c|c|c|c|c|c|c|}
\hline & \multicolumn{10}{|c|}{ Non-OPECa } & & \\
\hline & \multicolumn{2}{|c|}{$\begin{array}{l}\text { Trinidad } \\
\text { and Tobago }\end{array}$} & \multicolumn{2}{|c|}{$\begin{array}{l}\text { United } \\
\text { Kingdom }\end{array}$} & \multicolumn{2}{|c|}{ Virgin islands } & \multicolumn{2}{|c|}{$\begin{array}{l}\text { Other } \\
\text { Non-OPEC }\end{array}$} & \multicolumn{2}{|c|}{ Totalb,c } & \multicolumn{2}{|c|}{$\begin{array}{l}\text { Total } \\
\text { Imports }\end{array}$} \\
\hline & Total & Crude Oil & Total & Crude Oil & Total & Crude Oil & Total & Crude Oil & Total & Crude Oil & Total & Crude Oil \\
\hline $\begin{array}{l}1973 \text { Average } \\
1974 \text { Average } \\
1975 \text { Average } \\
1976 \text { Average } \\
197 \ldots \ldots \ldots \ldots \ldots \ldots \\
1977 \text { Average } \\
1978 \text { Average }\end{array}$ & $\begin{array}{r}255 \\
251 \\
242 \\
274 \\
289 \\
253 \\
190 \\
176 \\
133 \\
112 \\
96 \\
94 \\
113 \\
125 \\
106 \\
97 \\
94 \\
96 \\
88 \\
95 \\
74 \\
77\end{array}$ & $\begin{array}{r}60 \\
63 \\
115 \\
104 \\
134 \\
142 \\
123 \\
115 \\
102 \\
92 \\
83 \\
87 \\
98 \\
93 \\
75 \\
71 \\
73 \\
76 \\
72 \\
70 \\
55 \\
62\end{array}$ & $\begin{array}{r}15 \\
8 \\
14 \\
14 \\
31 \\
126 \\
180 \\
202 \\
176 \\
375 \\
456 \\
382 \\
402 \\
310 \\
350 \\
352 \\
315 \\
215 \\
189 \\
138 \\
230 \\
350 \\
458\end{array}$ & $\begin{array}{r}0 \\
0 \\
(s) \\
13 \\
97 \\
169 \\
197 \\
173 \\
369 \\
441 \\
365 \\
378 \\
278 \\
317 \\
304 \\
254 \\
160 \\
155 \\
106 \\
200 \\
312 \\
396\end{array}$ & $\begin{array}{l}329 \\
391 \\
406 \\
422 \\
466 \\
428 \\
431 \\
388 \\
327 \\
316 \\
282 \\
294 \\
247 \\
244 \\
272 \\
242 \\
321 \\
282 \\
243 \\
249 \\
254 \\
328\end{array}$ & $\begin{array}{l}0 \\
0 \\
0 \\
0 \\
0 \\
0 \\
0 \\
0 \\
0 \\
0 \\
0 \\
0 \\
0 \\
0 \\
0 \\
0 \\
0 \\
0 \\
0 \\
0 \\
0 \\
0\end{array}$ & $\begin{array}{l}153 \\
122 \\
120 \\
203 \\
287 \\
239 \\
269 \\
219 \\
236 \\
306 \\
378 \\
411 \\
394 \\
426 \\
459 \\
487 \\
457 \\
417 \\
282 \\
335 \\
452 \\
450\end{array}$ & $\begin{array}{r}36 \\
30 \\
14 \\
101 \\
157 \\
146 \\
192 \\
162 \\
163 \\
174 \\
215 \\
210 \\
137 \\
144 \\
196 \\
196 \\
197 \\
180 \\
137 \\
149 \\
240 \\
239\end{array}$ & $\begin{array}{r}3,263 \\
2,832 \\
2,454 \\
2,247 \\
2,614 \\
2,612 \\
2,819 \\
2,609 \\
2,672 \\
2,968 \\
3,189 \\
3,388 \\
3,237 \\
3,387 \\
3,617 \\
3,882 \\
3,921 \\
3,721 \\
3,535 \\
3,796 \\
c 4,347 \\
4,749\end{array}$ & $\begin{array}{r}1,149 \\
937 \\
893 \\
742 \\
971 \\
1,172 \\
1,407 \\
1,399 \\
1,474 \\
1,754 \\
1,853 \\
1,914 \\
1,888 \\
2,065 \\
2,274 \\
2,411 \\
2,467 \\
2,381 \\
2,405 \\
2,676 \\
63,178 \\
3,483\end{array}$ & $\begin{array}{l}6,256 \\
6,112 \\
6,056 \\
7,313 \\
8,807 \\
8,363 \\
8,456 \\
6,909 \\
5,996 \\
5,113 \\
5,051 \\
5,437 \\
5,067 \\
6,224 \\
6,678 \\
7,402 \\
8,061 \\
8,018 \\
7,627 \\
7,888 \\
8,620 \\
8,996\end{array}$ & $\begin{array}{l}3,244 \\
3,477 \\
4,105 \\
5,287 \\
6,615 \\
6,356 \\
6,519 \\
5,263 \\
4,396 \\
3,488 \\
3,329 \\
3,426 \\
3,201 \\
4,178 \\
4,674 \\
5,107 \\
5,843 \\
5,894 \\
5,782 \\
6,083 \\
6,787 \\
7,063\end{array}$ \\
\hline 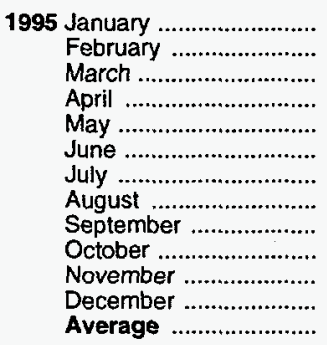 & $\begin{array}{l}91 \\
58 \\
70 \\
55 \\
61 \\
78 \\
73 \\
74 \\
73 \\
86 \\
61 \\
53 \\
70\end{array}$ & $\begin{array}{l}91 \\
58 \\
70 \\
55 \\
53 \\
74 \\
54 \\
53 \\
55 \\
70 \\
53 \\
53 \\
62\end{array}$ & $\begin{array}{l}240 \\
382 \\
663 \\
491 \\
405 \\
520 \\
137 \\
288 \\
427 \\
528 \\
284 \\
238 \\
383\end{array}$ & $\begin{array}{r}213 \\
359 \\
621 \\
450 \\
366 \\
418 \\
97 \\
249 \\
386 \\
479 \\
284 \\
177 \\
341\end{array}$ & $\begin{array}{l}283 \\
322 \\
298 \\
284 \\
203 \\
268 \\
240 \\
264 \\
223 \\
299 \\
317 \\
334 \\
278\end{array}$ & $\begin{array}{l}0 \\
0 \\
0 \\
0 \\
0 \\
0 \\
0 \\
0 \\
0 \\
0 \\
0 \\
0 \\
0\end{array}$ & $\begin{array}{l}209 \\
304 \\
183 \\
317 \\
286 \\
368 \\
441 \\
343 \\
312 \\
331 \\
273 \\
262 \\
302\end{array}$ & $\begin{array}{r}131 \\
143 \\
91 \\
143 \\
165 \\
253 \\
277 \\
261 \\
180 \\
214 \\
155 \\
156 \\
181\end{array}$ & $\begin{array}{l}4,297 \\
4,416 \\
4,787 \\
4,741 \\
4,907 \\
5,453 \\
4,812 \\
5,168 \\
5,194 \\
4,635 \\
4,896 \\
4,684 \\
4,833\end{array}$ & $\begin{array}{l}3,397 \\
3,378 \\
3,797 \\
3,894 \\
4,044 \\
4,451 \\
3,940 \\
4,212 \\
4,254 \\
3,735 \\
3,878 \\
3,671 \\
3,889\end{array}$ & $\begin{array}{l}8,015 \\
8,345 \\
9,006 \\
8,465 \\
8,709 \\
9,558 \\
8,863 \\
9,061 \\
9,736 \\
8,577 \\
9,074 \\
8,612 \\
8,835\end{array}$ & $\begin{array}{l}6,505 \\
6,546 \\
7,391 \\
7,038 \\
7,325 \\
7,927 \\
7,265 \\
7,437 \\
8,007 \\
7,075 \\
7,302 \\
6,916 \\
7,230\end{array}$ \\
\hline 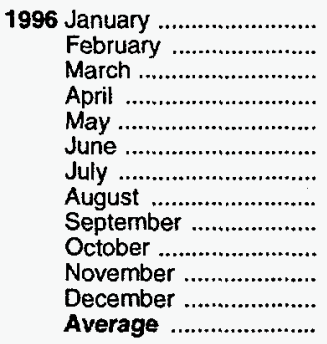 & $\begin{array}{l}92 \\
56 \\
63 \\
87 \\
97 \\
86 \\
70 \\
81 \\
51 \\
70 \\
96 \\
58 \\
76\end{array}$ & $\begin{array}{l}71 \\
56 \\
52 \\
55 \\
71 \\
54 \\
58 \\
59 \\
37 \\
55 \\
75 \\
54 \\
58\end{array}$ & $\begin{array}{l}364 \\
374 \\
346 \\
481 \\
421 \\
312 \\
244 \\
274 \\
165 \\
264 \\
199 \\
253 \\
308\end{array}$ & $\begin{array}{r}238 \\
280 \\
252 \\
347 \\
316 \\
234 \\
195 \\
177 \\
90 \\
136 \\
160 \\
167 \\
216\end{array}$ & $\begin{array}{l}390 \\
343 \\
311 \\
359 \\
298 \\
292 \\
344 \\
279 \\
268 \\
325 \\
253 \\
294 \\
313\end{array}$ & $\begin{array}{l}0 \\
0 \\
0 \\
0 \\
0 \\
0 \\
0 \\
0 \\
0 \\
0 \\
0 \\
0 \\
0\end{array}$ & $\begin{array}{l}406 \\
275 \\
373 \\
333 \\
429 \\
561 \\
456 \\
508 \\
502 \\
477 \\
513 \\
438 \\
440\end{array}$ & $\begin{array}{l}188 \\
169 \\
215 \\
157 \\
282 \\
402 \\
292 \\
348 \\
318 \\
240 \\
318 \\
245 \\
265\end{array}$ & $\begin{array}{l}5,244 \\
4,660 \\
4,932 \\
5,421 \\
5,465 \\
5,663 \\
5,201 \\
5,321 \\
4,938 \\
5,566 \\
5,277 \\
5,487 \\
\mathbf{5 , 2 6 7}\end{array}$ & $\begin{array}{l}3,932 \\
3,479 \\
3,788 \\
4,125 \\
4,332 \\
4,526 \\
4,082 \\
4,177 \\
3,891 \\
4,196 \\
4,145 \\
4,142 \\
4,070\end{array}$ & $\begin{array}{r}9,364 \\
8,390 \\
9,092 \\
9,429 \\
10,007 \\
9,938 \\
9,820 \\
9,986 \\
9,142 \\
9,837 \\
9,244 \\
9,417 \\
9,478\end{array}$ & $\begin{array}{l}7,303 \\
6,612 \\
7,215 \\
7,371 \\
8,029 \\
7,958 \\
7,800 \\
8,041 \\
7,353 \\
7,701 \\
7,344 \\
7,307 \\
7,508\end{array}$ \\
\hline 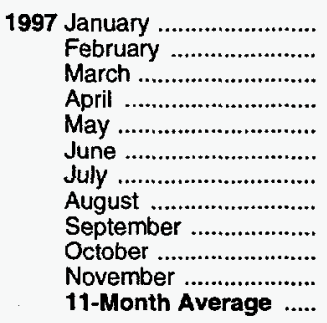 & $\begin{array}{l}62 \\
69 \\
56 \\
69 \\
70 \\
55 \\
62 \\
41 \\
66 \\
58 \\
57 \\
60\end{array}$ & $\begin{array}{l}55 \\
61 \\
55 \\
62 \\
66 \\
55 \\
54 \\
37 \\
58 \\
55 \\
57 \\
56\end{array}$ & $\begin{array}{l}400 \\
239 \\
236 \\
124 \\
261 \\
372 \\
198 \\
268 \\
167 \\
154 \\
127 \\
232\end{array}$ & $\begin{array}{r}333 \\
172 \\
161 \\
35 \\
181 \\
311 \\
165 \\
220 \\
110 \\
119 \\
87 \\
172\end{array}$ & $\begin{array}{l}335 \\
331 \\
254 \\
321 \\
300 \\
300 \\
310 \\
319 \\
248 \\
301 \\
260 \\
298\end{array}$ & $\begin{array}{l}0 \\
0 \\
0 \\
0 \\
0 \\
0 \\
0 \\
0 \\
0 \\
0 \\
0 \\
0\end{array}$ & $\begin{array}{l}464 \\
380 \\
411 \\
401 \\
531 \\
375 \\
357 \\
343 \\
439 \\
484 \\
403 \\
417\end{array}$ & $\begin{array}{l}173 \\
170 \\
180 \\
242 \\
314 \\
220 \\
237 \\
225 \\
334 \\
271 \\
236 \\
237\end{array}$ & $\begin{array}{l}\mathbf{5}, 557 \\
5,352 \\
5,433 \\
5,366 \\
5,527 \\
5,546 \\
5,411 \\
5,445 \\
5,451 \\
5,581 \\
\mathbf{5 , 3 0 8} \\
\mathbf{5 , 4 5 4}\end{array}$ & $\begin{array}{l}4,176 \\
4,049 \\
4,263 \\
4,123 \\
4,301 \\
4,453 \\
4,382 \\
4,411 \\
4,463 \\
4,490 \\
4,352 \\
4,317\end{array}$ & $\begin{array}{r}9,633 \\
9,475 \\
9,712 \\
9,934 \\
10,442 \\
10,357 \\
9,703 \\
10,155 \\
10,201 \\
10,414 \\
9,639 \\
9,973\end{array}$ & $\begin{array}{l}7,393 \\
7,384 \\
7,665 \\
7,810 \\
8,279 \\
8,403 \\
7,938 \\
8,333 \\
8,537 \\
8,543 \\
8,107 \\
8,039\end{array}$ \\
\hline $\begin{array}{l}1996 \text { 11-Month Average ..... } \\
1995 \text { 11-Month Average ..... }\end{array}$ & $\begin{array}{l}77 \\
71\end{array}$ & $\begin{array}{l}58 \\
63\end{array}$ & $\begin{array}{l}313 \\
397\end{array}$ & $\begin{array}{l}220 \\
356\end{array}$ & $\begin{array}{l}315 \\
272\end{array}$ & $\begin{array}{l}0 \\
0\end{array}$ & $\begin{array}{l}440 \\
306\end{array}$ & $\begin{array}{l}266 \\
183\end{array}$ & $\begin{array}{l}5,247 \\
4,847\end{array}$ & $\begin{array}{l}4,063 \\
3,909\end{array}$ & $\begin{array}{l}9,484 \\
8,856\end{array}$ & $\begin{array}{l}7,526 \\
7,259\end{array}$ \\
\hline
\end{tabular}

a Includes petroleum imported into the United States indirectly from members of the Organization of Petroleum Exporting Countries (OPEC), primarily from Caribbean and West European areas, as petroleum products that were refined from crude oil produced by OPEC.

Includes Bahrain, which is shown on Table 3.3a.

c As of January 1993, includes petroleum imported from Ecuador, which withdrew from OPEC on December 31, 1992. As of January 1995, includes petroleum imported from Gabon, which withdrew from OPEC on December 31 , 1994.
(s)=Less than 500 barrels per day. are included. - Totals may not equal sum of components due to independent rounding. - U.S. geographic coverage is the 50 States and the District of Columbia.

Sources: - 1973-1980: Energy Information Administration (EIA), Petroleum Supply Monthly, February 1993, Table S3. - 1981 forward: ElA, Petroleum Supply Monthly, January 1998, Table S3. 
Figure 3.2 Finished Motor Gasoline

(Million Barrels per Day, Except as Noted)

Overview, 1973-1997

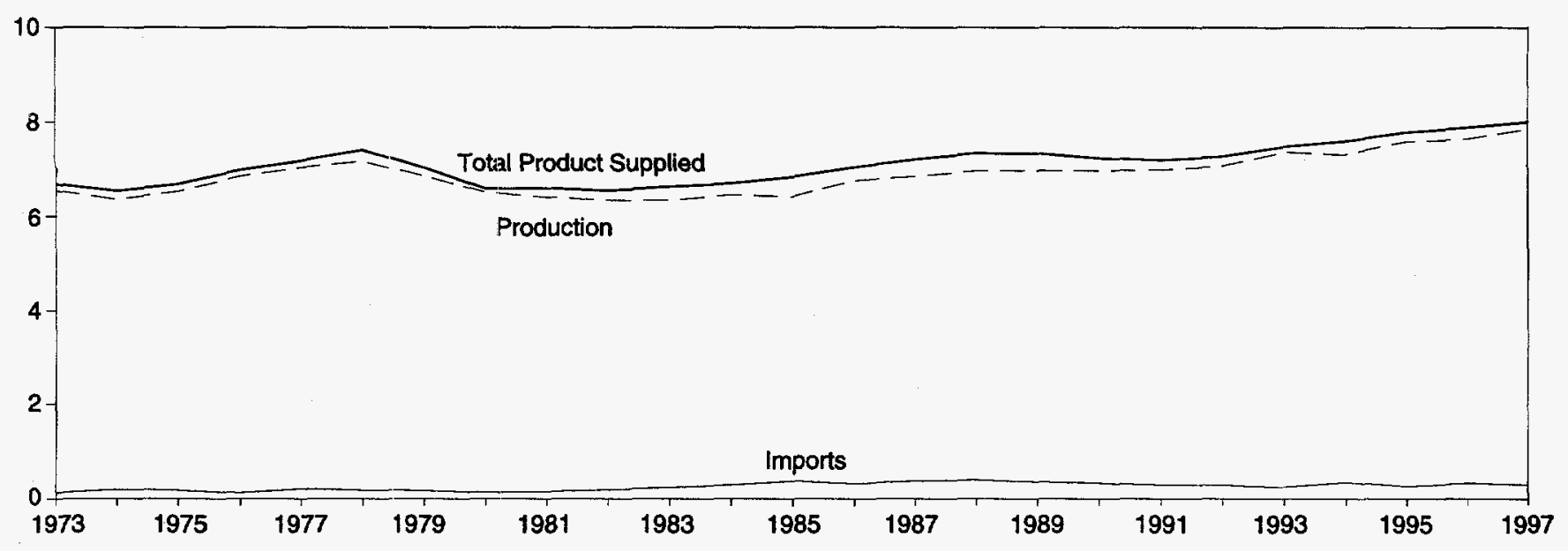

\section{Overview, Monthly}

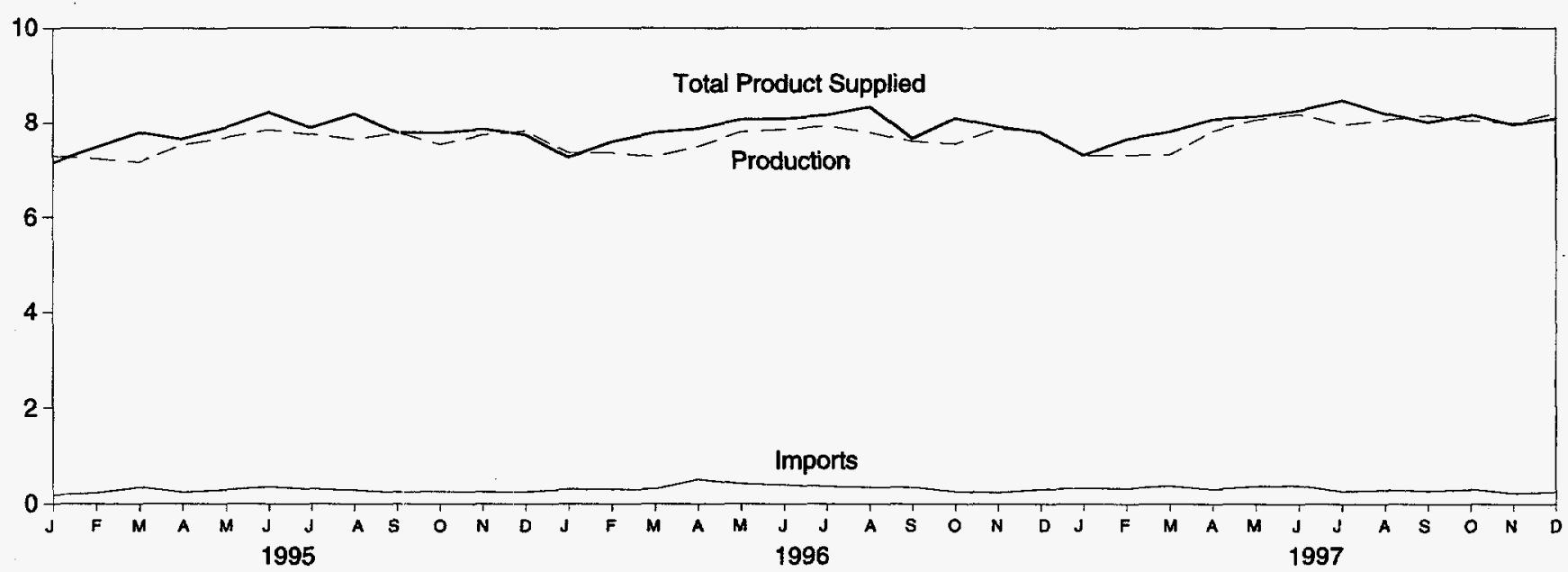

\section{Product Supplied, January-December}

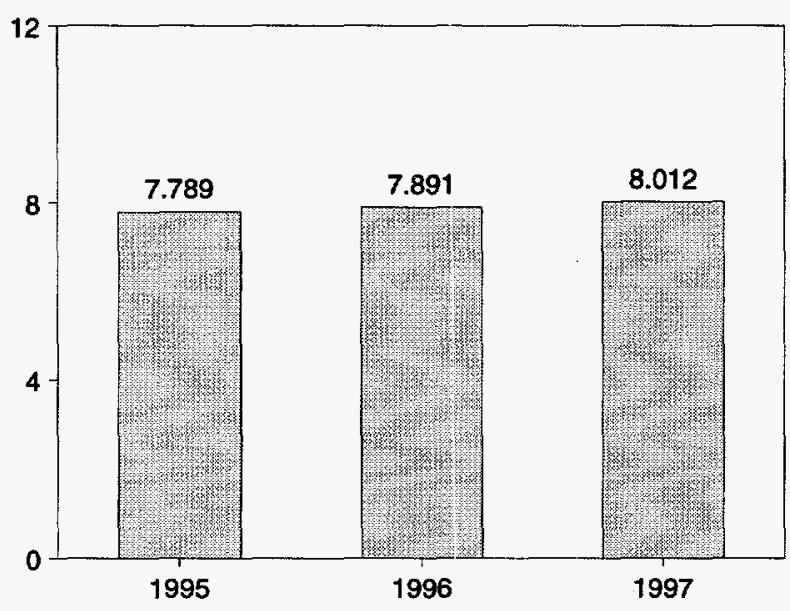

Stocks, End of Month

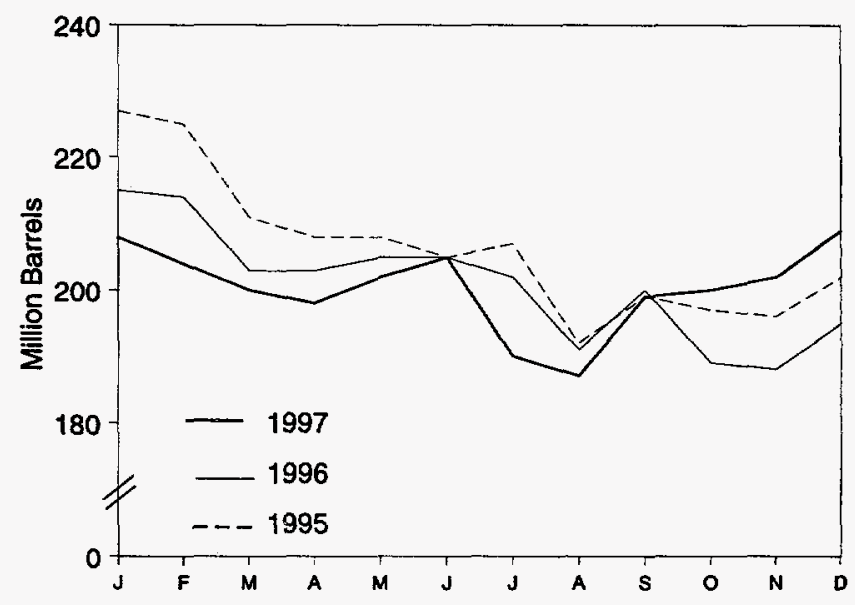

Note: Because vertical scales differ, graphs should not be compared. Source: Table 3.4. 
Table 3.4 Finished Motor Gasoline Supply and Disposition

\begin{tabular}{|c|c|c|c|c|c|c|c|c|}
\hline & \multicolumn{2}{|c|}{ Supply } & \multicolumn{3}{|c|}{ Disposition } & \multicolumn{2}{|c|}{$\begin{array}{l}\text { Motor Gasoline } \\
\text { Ending Stocks }\end{array}$} & \multirow{2}{*}{$\begin{array}{c}\text { Oxygenates } \\
\text { Ending } \\
\text { Stocks }\end{array}$} \\
\hline & $\begin{array}{c}\text { Total } \\
\text { Production }\end{array}$ & Imports $^{b}$ & $\begin{array}{c}\text { Stock } \\
\text { Changeb,c }\end{array}$ & Exports & $\begin{array}{l}\text { Product } \\
\text { Supplied }\end{array}$ & Totald & Finished & \\
\hline & \multicolumn{5}{|c|}{ Thousand Barrels per Day } & \multicolumn{3}{|c|}{ Million Barrels } \\
\hline $\begin{array}{l}1973 \text { Average } \\
1974 \text { Average } \\
1975 \text { Average } \\
1976 \text { Average }\end{array}$ & $\begin{array}{r}6,535 \\
6,360 \\
6,520 \\
6,841 \\
7,033 \\
7,169 \\
6,852 \\
6,506 \\
6,405 \\
6,338 \\
6,340 \\
6,453 \\
6,419 \\
6,752 \\
6,841 \\
6,956 \\
6,963 \\
6,959 \\
6,975 \\
7,058 \\
97,360 \\
7,312\end{array}$ & $\begin{array}{l}134 \\
204 \\
184 \\
131 \\
217 \\
190 \\
181 \\
140 \\
157 \\
197 \\
247 \\
299 \\
381 \\
326 \\
384 \\
405 \\
369 \\
342 \\
297 \\
294 \\
247 \\
356\end{array}$ & $\begin{array}{r}-9 \\
24 \\
e 28 \\
-10 \\
72 \\
-54 \\
-2 \\
66 \\
e-28 \\
-25 \\
--45 \\
54 \\
-41 \\
11 \\
-15 \\
3 \\
-35 \\
10 \\
3 \\
-11 \\
26 \\
-31\end{array}$ & $\begin{array}{r}4 \\
2 \\
2 \\
3 \\
2 \\
1 \\
(\mathrm{~s}) \\
1 \\
2 \\
20 \\
10 \\
6 \\
10 \\
33 \\
35 \\
22 \\
39 \\
55 \\
82 \\
96 \\
105 \\
97\end{array}$ & $\begin{array}{r}\mathbf{6 , 6 7 4} \\
\mathbf{6 , 5 3 7} \\
6,675 \\
\mathbf{6 , 9 7 8} \\
\mathbf{7 , 1 7 7} \\
\mathbf{7 , 4 1 2} \\
\mathbf{7 , 0 3 4} \\
\mathbf{6 , 5 7 9} \\
\mathbf{6 , 5 8 8} \\
\mathbf{6 , 5 3 9} \\
\mathbf{6 , 6 2 2} \\
\mathbf{6 , 6 9 3} \\
\mathbf{6 , 8 3 1} \\
\mathbf{7 , 0 3 4} \\
\mathbf{7 , 2 0 6} \\
\mathbf{7 , 3 3 6} \\
\mathbf{7 , 3 2 8} \\
\mathbf{7 , 2 3 5} \\
\mathbf{7 , 1 8 8} \\
\mathbf{7 , 2 6 8} \\
9 \mathbf{7 , 4 7 6} \\
\mathbf{7 , 6 0 1}\end{array}$ & $\begin{array}{r}209 \\
\text { e } 218 \\
235 \\
231 \\
258 \\
238 \\
237 \\
\text { e } 261 \\
253 \\
e^{235} \\
222 \\
243 \\
223 \\
233 \\
226 \\
228 \\
213 \\
220 \\
219 \\
216 \\
226 \\
215\end{array}$ & $\begin{array}{l}\text { NA } \\
\text { NA } \\
\text { NA } \\
\text { NA } \\
\text { NA } \\
\text { NA } \\
\text { NA } \\
\text { NA } \\
203 \\
e_{194} \\
186 \\
205 \\
190 \\
194 \\
189 \\
190 \\
177 \\
181 \\
182 \\
178 \\
187 \\
176\end{array}$ & $\begin{array}{l}\text { NA } \\
\text { NA } \\
\text { NA } \\
\text { NA } \\
\text { NA } \\
\text { NA } \\
\text { NA } \\
\text { NA } \\
\text { NA } \\
\text { NA } \\
\text { NA } \\
\text { NA } \\
\text { NA } \\
\text { NA } \\
\text { NA } \\
\text { NA } \\
\text { NA } \\
\text { NA } \\
\text { NA } \\
\text { NA } \\
\text { h13 } \\
17\end{array}$ \\
\hline 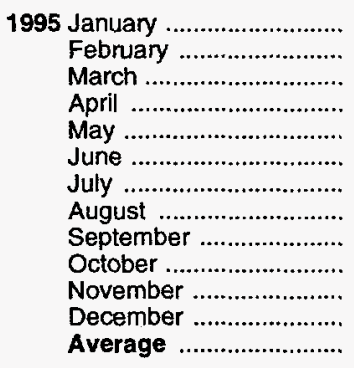 & $\begin{array}{l}7,303 \\
7,243 \\
7,168 \\
7,529 \\
7,678 \\
7,843 \\
7,747 \\
7,642 \\
7,785 \\
7,544 \\
7,739 \\
7,821 \\
7,588\end{array}$ & $\begin{array}{l}182 \\
223 \\
336 \\
235 \\
286 \\
347 \\
306 \\
280 \\
238 \\
253 \\
246 \\
244 \\
265\end{array}$ & $\begin{array}{r}221 \\
-99 \\
-391 \\
-26 \\
3 \\
-122 \\
80 \\
-367 \\
143 \\
-106 \\
1 \\
182 \\
-40\end{array}$ & $\begin{array}{r}100 \\
84 \\
107 \\
139 \\
67 \\
91 \\
86 \\
103 \\
94 \\
121 \\
118 \\
141 \\
104\end{array}$ & $\begin{array}{l}7,163 \\
7,481 \\
7,788 \\
7,651 \\
7,894 \\
8,220 \\
7,888 \\
8,187 \\
7,786 \\
7,781 \\
7,866 \\
7,742 \\
7,789\end{array}$ & $\begin{array}{l}227 \\
225 \\
211 \\
208 \\
208 \\
205 \\
207 \\
192 \\
199 \\
197 \\
196 \\
202 \\
202\end{array}$ & $\begin{array}{l}183 \\
180 \\
168 \\
167 \\
167 \\
163 \\
166 \\
155 \\
159 \\
156 \\
156 \\
161 \\
161\end{array}$ & $\begin{array}{l}16 \\
16 \\
15 \\
15 \\
15 \\
14 \\
15 \\
16 \\
15 \\
14 \\
11 \\
12 \\
12\end{array}$ \\
\hline 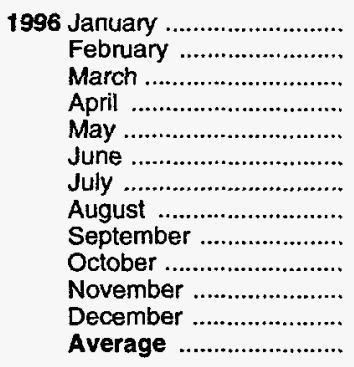 & $\begin{array}{l}7,370 \\
7,369 \\
7,289 \\
7,497 \\
7,804 \\
7,858 \\
7,924 \\
7,796 \\
7,606 \\
7,557 \\
7,864 \\
7,815 \\
7,647\end{array}$ & $\begin{array}{l}303 \\
293 \\
303 \\
501 \\
414 \\
393 \\
359 \\
346 \\
339 \\
253 \\
234 \\
298 \\
336\end{array}$ & $\begin{array}{r}240 \\
-10 \\
-327 \\
49 \\
66 \\
68 \\
-5 \\
-284 \\
215 \\
-396 \\
55 \\
202 \\
-12\end{array}$ & $\begin{array}{r}163 \\
72 \\
128 \\
77 \\
81 \\
95 \\
123 \\
82 \\
68 \\
113 \\
128 \\
117 \\
104\end{array}$ & $\begin{array}{l}7,271 \\
7,599 \\
7,792 \\
7,873 \\
8,071 \\
8,088 \\
8,165 \\
8,343 \\
7,662 \\
8,093 \\
7,915 \\
7,794 \\
7,891\end{array}$ & $\begin{array}{l}215 \\
214 \\
203 \\
203 \\
205 \\
205 \\
202 \\
191 \\
200 \\
189 \\
188 \\
195 \\
195\end{array}$ & $\begin{array}{c}169 \\
168 \\
158 \\
160 \\
162 \\
164 \\
164 \\
155 \\
161 \\
149 \\
151 \\
157 \\
157\end{array}$ & $\begin{array}{l}12 \\
12 \\
13 \\
13 \\
12 \\
11 \\
11 \\
12 \\
11 \\
11 \\
12 \\
13 \\
13\end{array}$ \\
\hline 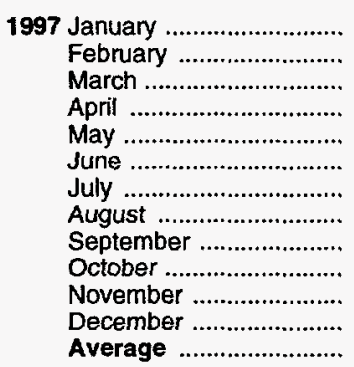 & $\begin{array}{r}7,308 \\
7,315 \\
7,322 \\
7,822 \\
8,056 \\
8,180 \\
7,947 \\
8,048 \\
8,147 \\
8,039 \\
R_{7,984} \\
E_{8,208} \\
E_{7,867}\end{array}$ & $\begin{array}{l}320 \\
317 \\
370 \\
300 \\
362 \\
377 \\
259 \\
292 \\
269 \\
309 \\
\text { R } 225 \\
\text { E } 260 \\
\text { E } 305\end{array}$ & $\begin{array}{r}240 \\
-130 \\
-240 \\
-62 \\
189 \\
202 \\
-429 \\
-30 \\
282 \\
-4 \\
R_{103} \\
E_{257} \\
E_{32}\end{array}$ & $\begin{array}{r}75 \\
111 \\
123 \\
117 \\
101 \\
96 \\
164 \\
175 \\
130 \\
186 \\
R 151 \\
E_{118} \\
E_{129}\end{array}$ & $\begin{array}{r}7,312 \\
7,651 \\
7,808 \\
8,067 \\
8,128 \\
8,260 \\
8,471 \\
8,195 \\
8,004 \\
8,166 \\
\text { R } 7,955 \\
\text { E } 8,093 \\
\text { E } 8,012\end{array}$ & $\begin{array}{r}208 \\
204 \\
200 \\
198 \\
202 \\
205 \\
190 \\
187 \\
199 \\
200 \\
R 202 \\
E_{209} \\
E_{209}\end{array}$ & $\begin{array}{l}165 \\
161 \\
154 \\
152 \\
158 \\
164 \\
151 \\
150 \\
158 \\
158 \\
\text { R 161 } \\
\text { E } 166 \\
\text { E 166 }\end{array}$ & $\begin{array}{l}13 \\
13 \\
13 \\
13 \\
13 \\
12 \\
13 \\
13 \\
13 \\
12 \\
12 \\
\text { NA } \\
\text { NA }\end{array}$ \\
\hline
\end{tabular}

a Stocks are totals as of end of period.

b From 1981 forward, blending components are excluded.

c A negative number indicates a decrease in stocks and a positive number indicates an increase.

d Includes motor gasoline blending components and gasohol, but excludes oxygenates, which are reported separately.

See Note 4 at end of section.

See Note 2 at end of section.

9 Beginning in 1993, motor gasoline production and product supplied include blending of fuel ethanol and an adjustment to correct for the

imbalance of motor gasoline blending components. See Nole 2 at end of section.

$h$ See Note 1 at end of section.

$R=$ Revised data. NA=Not available. E=Estimate. (s)=Less than 500 barrels per day.

Note: Geographic coverage is the 50 States and the District of Columbia.

Sources: - 1973-1980: Energy Information Administration (EIA),

Petroleum Supply Monthly, February 1993, Table S4. • 1981 forward: EIA Petroleum Supply Monthly, January 1998, Table S4. 
Figure 3.3 Distillate Fuel

(Million Barrels per Day, Except as Noted)

Oveniew, 1973-1997

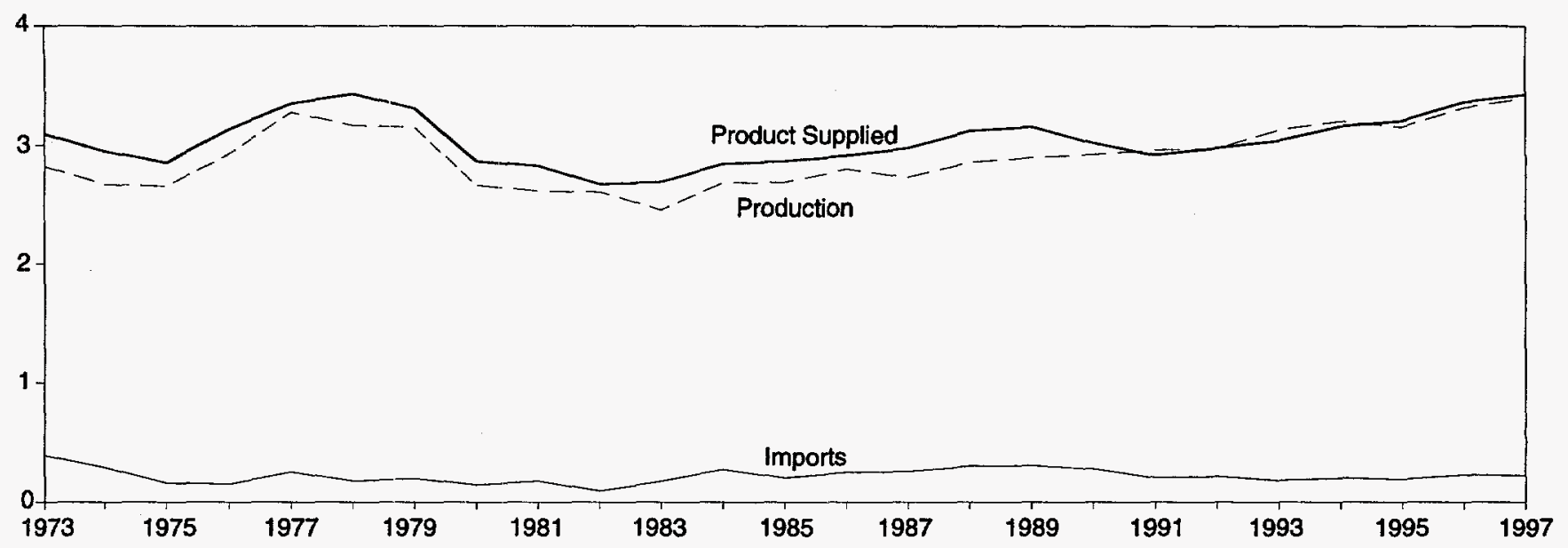

Overview, Monthly

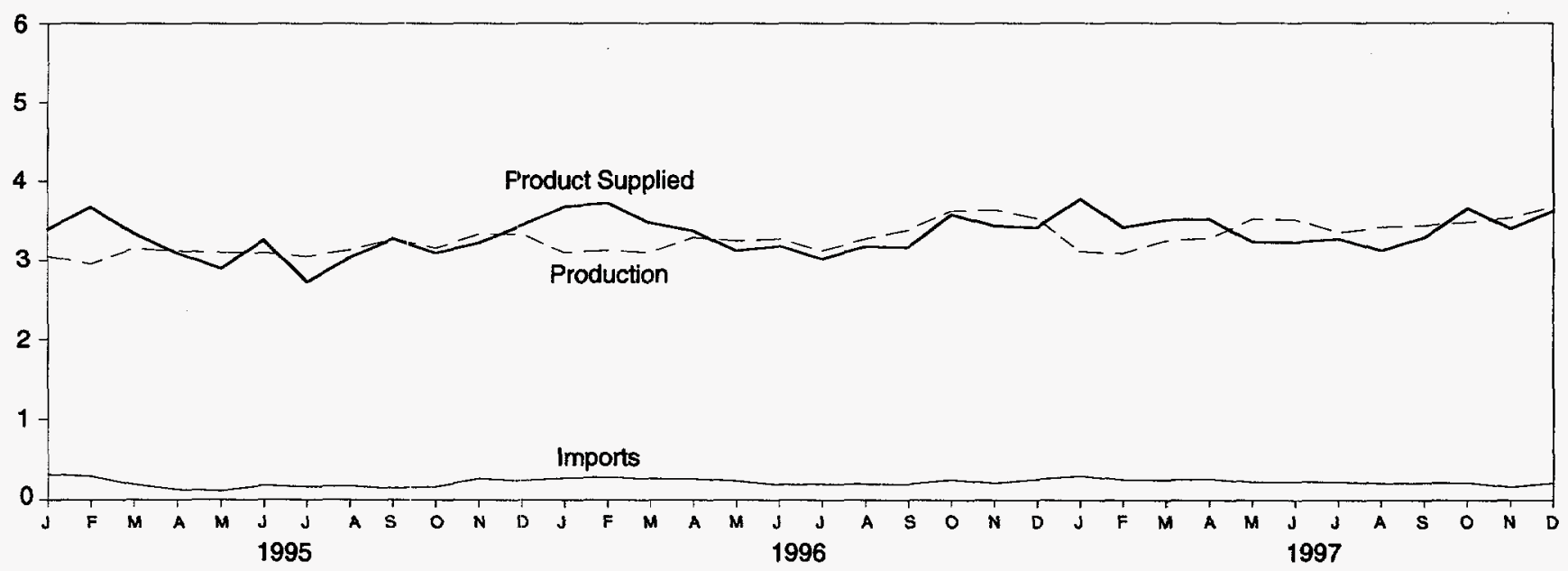

Product Supplied, January-December

Stocks, End of Month
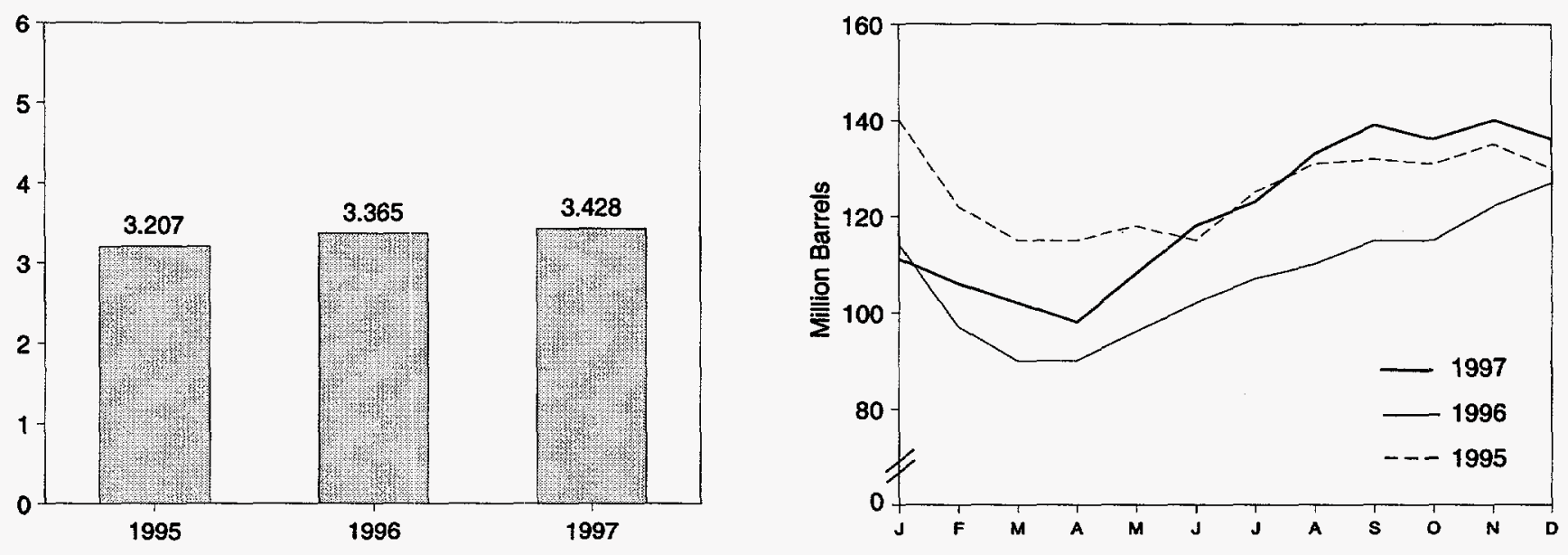

Source: Table $\mathbf{3 . 5}$. 
Table 3.5 Distillate Fuel Oil Supply and Disposition

\begin{tabular}{|c|c|c|c|c|c|c|c|c|c|}
\hline & \multicolumn{3}{|c|}{ Supply } & \multicolumn{3}{|c|}{ Disposition } & \multicolumn{3}{|c|}{ Ending Stocks ${ }^{a}$} \\
\hline & \multirow[b]{2}{*}{$\begin{array}{c}\text { Total } \\
\text { Production }\end{array}$} & \multirow[b]{2}{*}{ Imports } & \multirow[b]{2}{*}{$\begin{array}{c}\text { Crude Oif } \\
\text { Used } \\
\text { Directlyb }\end{array}$} & \multirow[b]{2}{*}{$\begin{array}{c}\text { Stock } \\
\text { Changec }\end{array}$} & \multirow[b]{2}{*}{ Exports } & \multirow[b]{2}{*}{$\begin{array}{l}\text { Product } \\
\text { Supplied }\end{array}$} & \multirow[b]{2}{*}{ Total } & \multicolumn{2}{|c|}{ Sulfur Content } \\
\hline & & & & & & & & $\begin{array}{l}0.05 \text { Percent } \\
\text { or Less }{ }^{d}\end{array}$ & $\begin{array}{l}\text { Greater Than } \\
0.05 \text { Percent }\end{array}$ \\
\hline & \multicolumn{6}{|c|}{ Thousand Barreis per Day } & \multicolumn{3}{|c|}{ Million Barrels } \\
\hline 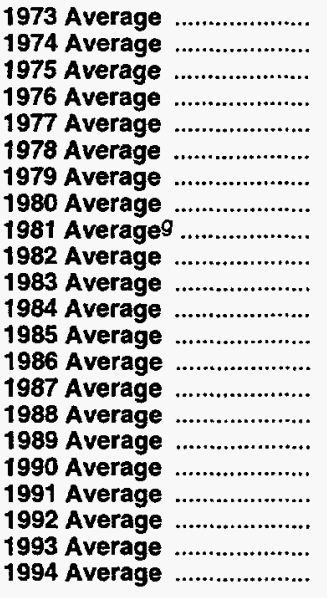 & $\begin{array}{l}2,822 \\
2,669 \\
2,654 \\
2,924 \\
3,278 \\
3,167 \\
3,153 \\
2,662 \\
2,613 \\
2,606 \\
2,456 \\
2,681 \\
2,687 \\
2,798 \\
2,731 \\
2,859 \\
2,899 \\
2,925 \\
2,962 \\
2,974 \\
3,132 \\
3,205\end{array}$ & $\begin{array}{r}392 \\
289 \\
155 \\
146 \\
250 \\
173 \\
193 \\
142 \\
173 \\
93 \\
974 \\
174 \\
272 \\
200 \\
247 \\
255 \\
302 \\
306 \\
278 \\
205 \\
216 \\
184 \\
203\end{array}$ & $\begin{array}{r}2 \\
2 \\
2 \\
1 \\
1 \\
1 \\
1 \\
1 \\
10 \\
10 \\
- \\
- \\
- \\
- \\
- \\
- \\
- \\
- \\
- \\
-\end{array}$ & $\begin{array}{r}115 \\
010 \\
e, 1-41 \\
-62 \\
176 \\
-93 \\
34 \\
-64 \\
1-38 \\
-35 \\
-124 \\
57 \\
-48 \\
31 \\
-56 \\
-30 \\
-49 \\
73 \\
31 \\
-8 \\
1 \\
12\end{array}$ & $\begin{array}{r}9 \\
2 \\
1 \\
1 \\
1 \\
3 \\
3 \\
3 \\
5 \\
74 \\
64 \\
51 \\
67 \\
100 \\
66 \\
69 \\
97 \\
109 \\
215 \\
219 \\
274 \\
234\end{array}$ & $\begin{array}{l}3,092 \\
2,948 \\
2,851 \\
3,133 \\
3,352 \\
3,432 \\
3,311 \\
2,866 \\
2,829 \\
2,671 \\
2,690 \\
2,845 \\
2,868 \\
2,914 \\
2,976 \\
3,122 \\
3,157 \\
3,021 \\
2,921 \\
2,979 \\
3,041 \\
3,162\end{array}$ & $\begin{array}{r}196 \\
1200 \\
209 \\
186 \\
250 \\
216 \\
229 \\
1205 \\
192 \\
1179 \\
140 \\
161 \\
144 \\
155 \\
134 \\
124 \\
106 \\
132 \\
144 \\
141 \\
141 \\
145\end{array}$ & $\begin{array}{c}\text { NA } \\
\text { NA } \\
\text { NA } \\
\text { NA } \\
\text { NA } \\
\text { NA } \\
\text { NA } \\
\text { NA } \\
\text { NA } \\
\text { NA } \\
\text { NA } \\
\text { NA } \\
\text { NA } \\
\text { NA } \\
\text { NA } \\
\text { NA } \\
\text { NA } \\
\text { NA } \\
\text { NA } \\
\text { NA } \\
\text { 964 } \\
73\end{array}$ & $\begin{array}{c}\text { NA } \\
\text { NA } \\
\text { NA } \\
\text { NA } \\
\text { NA } \\
\text { NA } \\
\text { NA } \\
\text { NA } \\
\text { NA } \\
\text { NA } \\
\text { NA } \\
\text { NA } \\
\text { NA } \\
\text { NA } \\
\text { NA } \\
\text { NA } \\
\text { NA } \\
\text { NA } \\
\text { NA } \\
\text { NA } \\
977 \\
73\end{array}$ \\
\hline 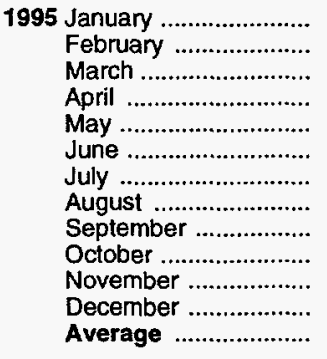 & $\begin{array}{l}3,054 \\
2,954 \\
3,157 \\
3,126 \\
3,111 \\
3,109 \\
3,056 \\
3,145 \\
3,287 \\
3,169 \\
3,341 \\
3,344 \\
3,155\end{array}$ & $\begin{array}{l}313 \\
289 \\
188 \\
125 \\
109 \\
176 \\
157 \\
171 \\
142 \\
162 \\
262 \\
235 \\
193\end{array}$ & $\begin{array}{l}- \\
- \\
- \\
z \\
z \\
z \\
- \\
- \\
-\end{array}$ & $\begin{array}{r}-163 \\
-645 \\
-216 \\
-27 \\
119 \\
-119 \\
333 \\
189 \\
28 \\
-11 \\
135 \\
-168 \\
-41\end{array}$ & $\begin{array}{r}141 \\
212 \\
216 \\
172 \\
202 \\
137 \\
148 \\
84 \\
116 \\
238 \\
236 \\
298 \\
183\end{array}$ & $\begin{array}{l}3,389 \\
3,675 \\
3,344 \\
3,106 \\
2,899 \\
3,267 \\
2,732 \\
3,044 \\
3,285 \\
3,104 \\
3,233 \\
3,449 \\
3,207\end{array}$ & $\begin{array}{l}140 \\
122 \\
115 \\
115 \\
118 \\
115 \\
125 \\
131 \\
132 \\
131 \\
135 \\
130 \\
130\end{array}$ & $\begin{array}{l}70 \\
63 \\
59 \\
62 \\
62 \\
60 \\
62 \\
62 \\
64 \\
61 \\
65 \\
67 \\
67\end{array}$ & $\begin{array}{l}70 \\
59 \\
56 \\
53 \\
56 \\
55 \\
63 \\
69 \\
68 \\
70 \\
70 \\
63 \\
63\end{array}$ \\
\hline 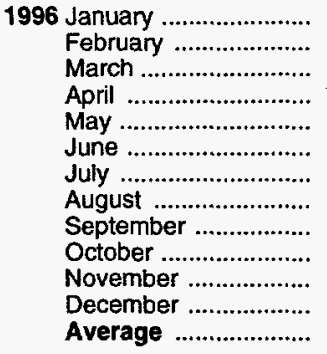 & $\begin{array}{l}3,105 \\
3,133 \\
3,107 \\
3,300 \\
3,256 \\
3,283 \\
3,127 \\
3,280 \\
3,392 \\
3,627 \\
3,641 \\
3,536 \\
3,316\end{array}$ & $\begin{array}{l}267 \\
279 \\
256 \\
258 \\
231 \\
185 \\
194 \\
195 \\
193 \\
246 \\
205 \\
253 \\
230\end{array}$ & $\begin{array}{l}- \\
- \\
- \\
- \\
- \\
- \\
- \\
- \\
-\end{array}$ & $\begin{array}{r}-528 \\
-570 \\
-247 \\
13 \\
182 \\
198 \\
166 \\
112 \\
157 \\
-8 \\
234 \\
160 \\
-10\end{array}$ & $\begin{array}{r}216 \\
256 \\
139 \\
166 \\
176 \\
81 \\
134 \\
182 \\
256 \\
300 \\
171 \\
206 \\
190\end{array}$ & $\begin{array}{l}3,684 \\
3,727 \\
3,471 \\
3,379 \\
3,128 \\
3,189 \\
3,021 \\
3,180 \\
3,172 \\
3,581 \\
3,442 \\
3,422 \\
3,365\end{array}$ & $\begin{array}{r}114 \\
97 \\
90 \\
90 \\
96 \\
102 \\
107 \\
110 \\
115 \\
115 \\
122 \\
127 \\
127\end{array}$ & $\begin{array}{l}58 \\
53 \\
49 \\
52 \\
57 \\
60 \\
62 \\
62 \\
64 \\
60 \\
65 \\
68 \\
68\end{array}$ & $\begin{array}{l}55 \\
44 \\
40 \\
38 \\
39 \\
41 \\
45 \\
49 \\
51 \\
54 \\
57 \\
58 \\
58\end{array}$ \\
\hline 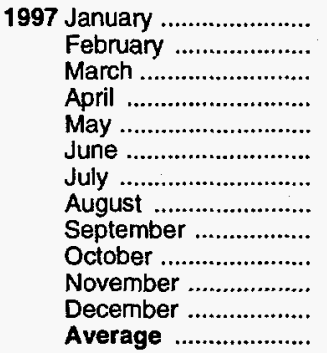 & $\begin{array}{r}3,119 \\
3,089 \\
3,258 \\
3,291 \\
3,525 \\
3,517 \\
3,362 \\
3,427 \\
3,452 \\
3,488 \\
R_{3,543} \\
\text { E } 3,684 \\
\text { E }_{3,398}\end{array}$ & $\begin{array}{r}293 \\
246 \\
245 \\
256 \\
220 \\
219 \\
223 \\
202 \\
210 \\
213 \\
R_{161} \\
\text { E } 214_{2} \\
E_{225}\end{array}$ & $\begin{array}{l}- \\
- \\
- \\
- \\
- \\
- \\
- \\
- \\
- \\
- \\
-\end{array}$ & $\begin{array}{r}-502 \\
-193 \\
-133 \\
-142 \\
352 \\
327 \\
154 \\
320 \\
201 \\
-90 \\
\mathrm{R}_{144} \\
\mathrm{E}_{63} \\
\mathrm{E}_{43}\end{array}$ & $\begin{array}{l}133 \\
107 \\
120 \\
166 \\
153 \\
174 \\
151 \\
185 \\
160 \\
133 \\
R 149 \\
\Sigma_{204} \\
\Sigma_{153}\end{array}$ & $\begin{array}{r}3,780 \\
3,422 \\
3,515 \\
3,523 \\
3,240 \\
3,235 \\
3,279 \\
3,124 \\
3,302 \\
3,659 \\
\text { R } 3,411 \\
\text { E } 3,632 \\
\text { E } 3,428\end{array}$ & $\begin{array}{r}111 \\
106 \\
102 \\
98 \\
108 \\
118 \\
123 \\
133 \\
139 \\
136 \\
R_{140} \\
E_{136} \\
E_{136}\end{array}$ & $\begin{array}{r}60 \\
57 \\
59 \\
59 \\
63 \\
65 \\
65 \\
69 \\
70 \\
64 \\
R_{68} \\
E_{67} \\
E_{67}\end{array}$ & $\begin{array}{r}51 \\
49 \\
43 \\
39 \\
45 \\
53 \\
58 \\
64 \\
69 \\
73 \\
\text { R } 73 \\
E_{70} \\
E_{70}\end{array}$ \\
\hline
\end{tabular}

a Stocks are totals as of end of period.

b Beginning in January 1983, crude oil used directly as distillate fuel oil is reported as crude oil product supplied on Table $3.2 \mathrm{~b}$ rather than as distillate fuel oil product supplied.

c A negative number indicates a decrease in stocks and a positive number indicates an increase.

d By weight.

See Note 6 at end of section.

$f$ See Note 4 at end of section.
9 See Note 3 at end of section.

$R=$ Revised data. NA=Not avaitable. $-=$ Not applicable. E=Estimate.

Notes: - Totals may not equal sum of components due to independent rounding. - Geographic coverage is the 50 States and the District of Columbia.

Sources: - 1973-1980: Energy Information Administration (EIA), Petroleum Supply Monthly, February 1993, Table S5. - 1981 forward: EIA, Petroleum Supply Monthly, January 1998, Table S5. 
Figure 3.4 Residual Fuel

(Million Barrels per Day, Except as Noted)

Ovenview, 1973-1997

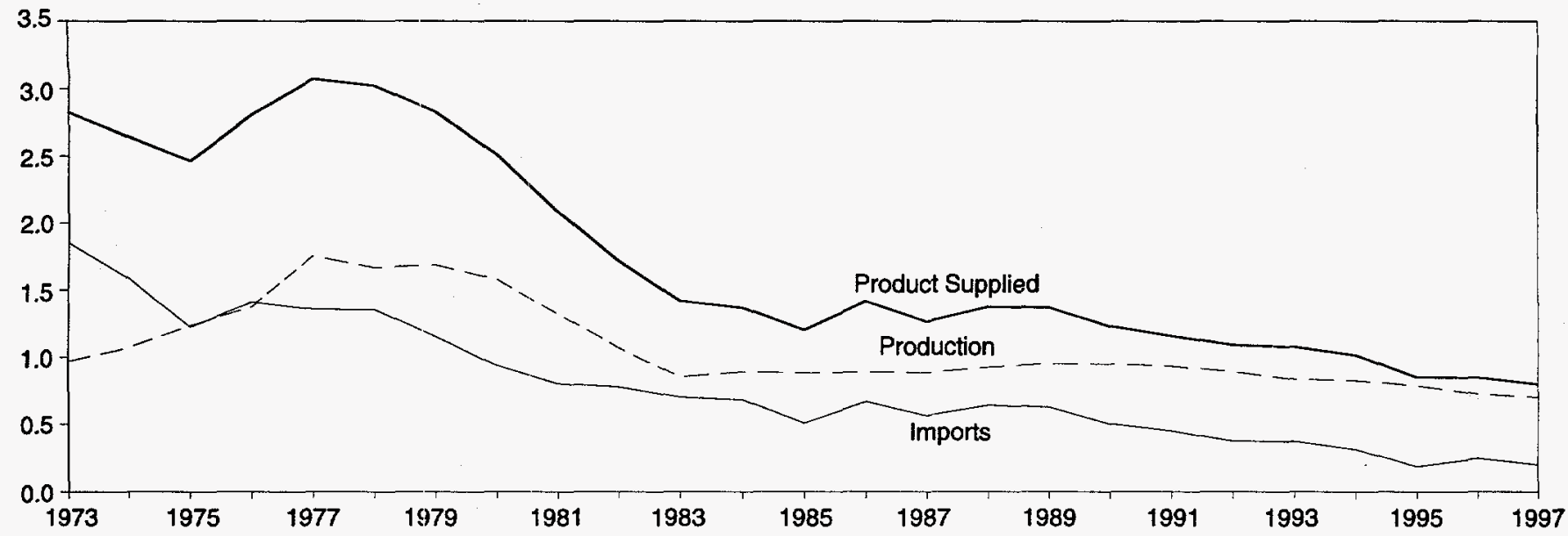

\section{Overview, Monthly}

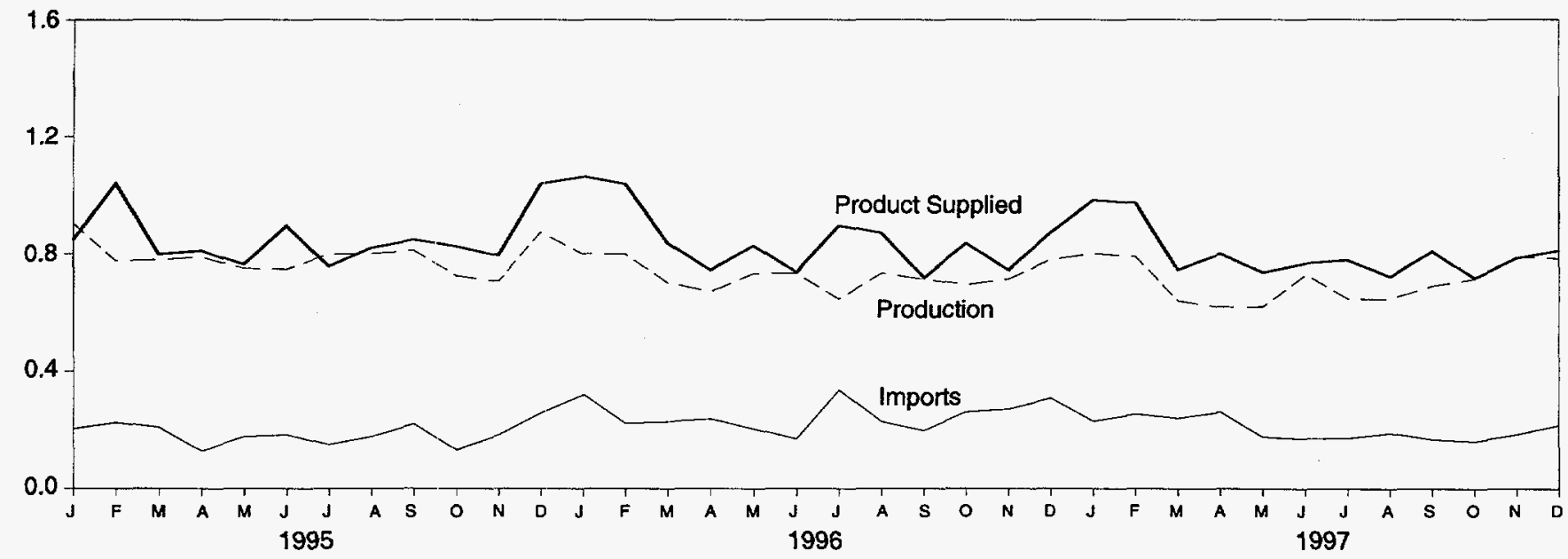

Product Supplied, January-December

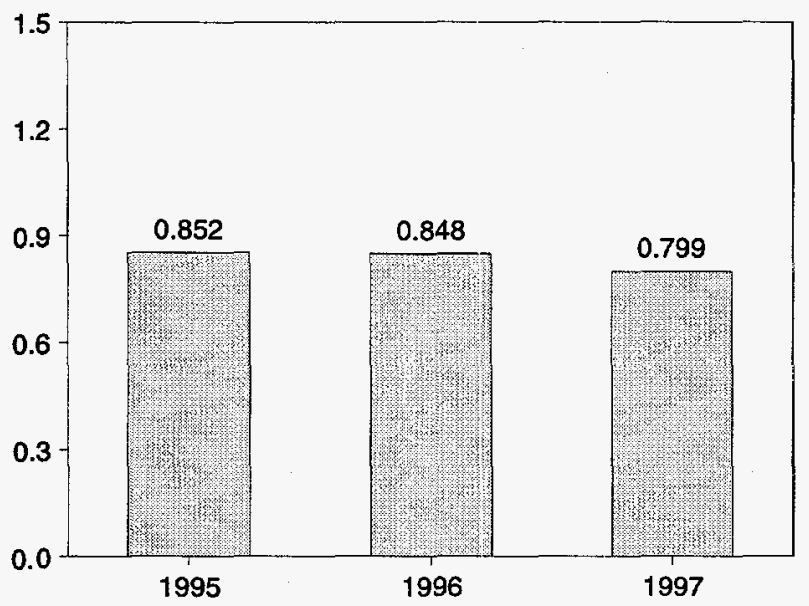

Stocks, End of Month

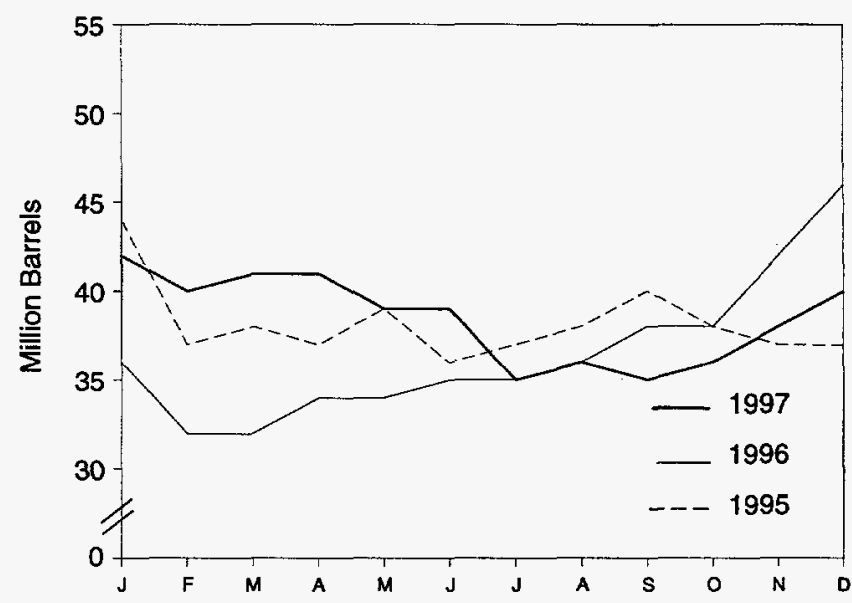

Note: Because vertical scales differ, graphs should not be compared. Source: Table 3.6. 
Table 3.6 Residual Fuel Oil Supply and Disposition

\begin{tabular}{|c|c|c|c|c|c|c|c|}
\hline & \multicolumn{3}{|c|}{ Supply } & \multicolumn{3}{|c|}{ Disposition } & \multirow[b]{2}{*}{$\begin{array}{l}\text { Ending } \\
\text { Stocks }\end{array}$} \\
\hline & $\begin{array}{c}\text { Total } \\
\text { Production }\end{array}$ & Imports & $\begin{array}{c}\text { Crude Oil } \\
\text { Used } \\
\text { Directlya }\end{array}$ & $\begin{array}{c}\text { Stock } \\
\text { Change }\end{array}$ & Exports & $\begin{array}{l}\text { Product } \\
\text { Supplieda }\end{array}$ & \\
\hline & \multicolumn{6}{|c|}{ Thousand Barrels per Day } & Million Barrels \\
\hline $\begin{array}{l}1973 \text { Average } \\
1974 \text { Average } \\
1975 \text { Average } . . . \ldots \ldots \ldots \ldots \ldots \ldots \\
1976 \text { Average } \\
1977 \text { Average } \\
1978 \text { Average } \\
1979 \text { Average } \\
1980 \text { Average }\end{array}$ & $\begin{array}{r}971 \\
1,070 \\
1,235 \\
1,377 \\
1,754 \\
1,667 \\
1,687 \\
1,580 \\
1,321 \\
1,070 \\
852 \\
891 \\
882 \\
889 \\
885 \\
926 \\
954 \\
950 \\
934 \\
892 \\
835 \\
826\end{array}$ & $\begin{array}{r}1,853 \\
1,587 \\
1,223 \\
1,413 \\
1,359 \\
1,355 \\
1,151 \\
939 \\
800 \\
776 \\
699 \\
681 \\
510 \\
669 \\
565 \\
644 \\
629 \\
504 \\
453 \\
375 \\
373 \\
314\end{array}$ & $\begin{array}{l}17 \\
13 \\
15 \\
17 \\
13 \\
13 \\
12 \\
12 \\
48 \\
48 \\
- \\
- \\
- \\
- \\
- \\
- \\
- \\
- \\
- \\
-\end{array}$ & $\begin{array}{r}-5 \\
17 \\
d-2 \\
-5 \\
48 \\
1 \\
15 \\
-10 \\
d-37 \\
-32 \\
d-55 \\
12 \\
-7 \\
-8 \\
(s) \\
-8 \\
-2 \\
13 \\
4 \\
-20 \\
4 \\
-6\end{array}$ & $\begin{array}{r}23 \\
14 \\
15 \\
12 \\
6 \\
13 \\
9 \\
33 \\
118 \\
209 \\
185 \\
190 \\
197 \\
147 \\
186 \\
200 \\
215 \\
211 \\
226 \\
193 \\
123 \\
125\end{array}$ & $\begin{array}{l}2,822 \\
2,639 \\
2,462 \\
2,801 \\
3,071 \\
3,023 \\
2,826 \\
2,508 \\
2,088 \\
1,716 \\
1,421 \\
1,369 \\
1,202 \\
1,418 \\
1,264 \\
1,378 \\
1,370 \\
1,229 \\
1,158 \\
1,094 \\
1,080 \\
1,021\end{array}$ & $\begin{array}{r}53 \\
d 60 \\
74 \\
72 \\
90 \\
90 \\
96 \\
d 92 \\
98 \\
d 66 \\
49 \\
53 \\
50 \\
47 \\
47 \\
45 \\
44 \\
49 \\
50 \\
43 \\
44 \\
42\end{array}$ \\
\hline 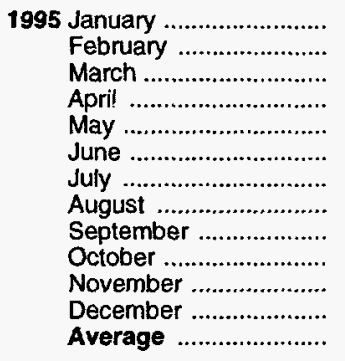 & $\begin{array}{l}903 \\
776 \\
778 \\
789 \\
748 \\
746 \\
797 \\
801 \\
811 \\
724 \\
705 \\
874 \\
788\end{array}$ & $\begin{array}{l}204 \\
225 \\
209 \\
128 \\
177 \\
184 \\
149 \\
177 \\
220 \\
131 \\
182 \\
257 \\
187\end{array}$ & $\begin{array}{l}- \\
- \\
- \\
- \\
- \\
- \\
- \\
- \\
- \\
- \\
- \\
- \\
-\end{array}$ & $\begin{array}{r}56 \\
-246 \\
35 \\
-22 \\
48 \\
-87 \\
27 \\
36 \\
58 \\
-55 \\
-17 \\
-8 \\
-13\end{array}$ & $\begin{array}{r}203 \\
208 \\
154 \\
129 \\
115 \\
120 \\
164 \\
122 \\
124 \\
84 \\
111 \\
98 \\
136\end{array}$ & $\begin{array}{r}848 \\
1,040 \\
798 \\
810 \\
762 \\
896 \\
755 \\
820 \\
848 \\
825 \\
793 \\
1,040 \\
852\end{array}$ & $\begin{array}{l}44 \\
37 \\
38 \\
37 \\
39 \\
36 \\
37 \\
38 \\
40 \\
38 \\
37 \\
37 \\
37\end{array}$ \\
\hline 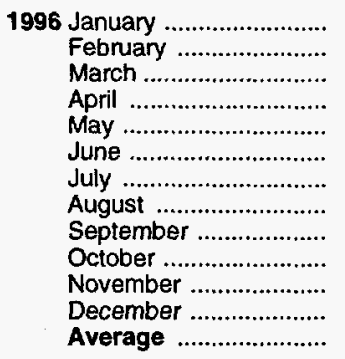 & $\begin{array}{l}799 \\
798 \\
700 \\
671 \\
732 \\
731 \\
646 \\
732 \\
713 \\
694 \\
714 \\
778 \\
726\end{array}$ & $\begin{array}{l}320 \\
222 \\
227 \\
237 \\
203 \\
168 \\
335 \\
227 \\
197 \\
260 \\
270 \\
307 \\
248\end{array}$ & $\begin{array}{l}- \\
- \\
- \\
- \\
- \\
- \\
- \\
- \\
- \\
-\end{array}$ & $\begin{array}{r}-54 \\
-132 \\
-4 \\
69 \\
18 \\
21 \\
-3 \\
32 \\
68 \\
16 \\
139 \\
112 \\
24\end{array}$ & $\begin{array}{r}108 \\
114 \\
95 \\
96 \\
89 \\
144 \\
88 \\
56 \\
125 \\
104 \\
101 \\
102 \\
102\end{array}$ & $\begin{array}{r}1,064 \\
1,038 \\
836 \\
743 \\
827 \\
735 \\
896 \\
871 \\
717 \\
835 \\
744 \\
872 \\
848\end{array}$ & $\begin{array}{l}36 \\
32 \\
32 \\
34 \\
34 \\
35 \\
35 \\
36 \\
38 \\
38 \\
42 \\
46 \\
46\end{array}$ \\
\hline 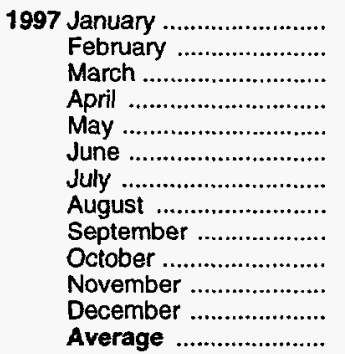 & $\begin{array}{l}800 \\
789 \\
639 \\
617 \\
618 \\
727 \\
645 \\
643 \\
688 \\
711 \\
\text { R } 786 \\
\text { E } 781 \\
\text { E } 703\end{array}$ & $\begin{array}{r}229 \\
253 \\
239 \\
260 \\
175 \\
168 \\
170 \\
187 \\
165 \\
158 \\
R_{182} \\
E_{214} \\
E_{200}\end{array}$ & $\begin{array}{l}- \\
- \\
- \\
- \\
- \\
- \\
- \\
- \\
-\end{array}$ & $\begin{array}{r}-124 \\
-68 \\
45 \\
-27 \\
-44 \\
-1 \\
-119 \\
31 \\
-42 \\
22 \\
R 64 \\
\text { E } 74 \\
\text { E-16 }\end{array}$ & $\begin{array}{r}171 \\
137 \\
89 \\
105 \\
102 \\
130 \\
159 \\
80 \\
91 \\
133 \\
1122 \\
E_{113} \\
E_{119}\end{array}$ & $\begin{array}{r}983 \\
972 \\
744 \\
798 \\
734 \\
765 \\
776 \\
719 \\
804 \\
714 \\
\text { R } 782 \\
\text { E } 808 \\
\text { E } 799\end{array}$ & $\begin{array}{r}42 \\
40 \\
41 \\
41 \\
39 \\
39 \\
35 \\
36 \\
35 \\
36 \\
38 \\
E_{40} \\
E_{40}\end{array}$ \\
\hline
\end{tabular}

a Beginning in January 1983, crude oil used directly as residual fuel oil is reported as crude oil product supplied on Table $3.2 \mathrm{~b}$ rather than as residual fuel oil product supplied.

$b$ A negative number indicates a decrease in stocks and a positive number indicates an increase.

c Stocks are totals as of end of period.

d See Note 4 at end of section.
- See Note 3 at end of section.

$R=$ Revised data. $\quad=$ Not applicable. E=Estimate. (s)=Less than +500 barrels per day and greater than -500 barrels per day.

Note: Geographic coverage is the 50 States and the District of Columbia.

Sources: - 1973-1980: Energy Information Administration (EIA) Petroleum Supply Monthly, February 1993, Table S6. - 1981 forward: EIA, Petroleum Supply Monthly, January 1998, Table S6. 
Figure 3.5 Jet Fuel

(Million Barrels per Day, Except as Noted)

Overview, 1973-1997

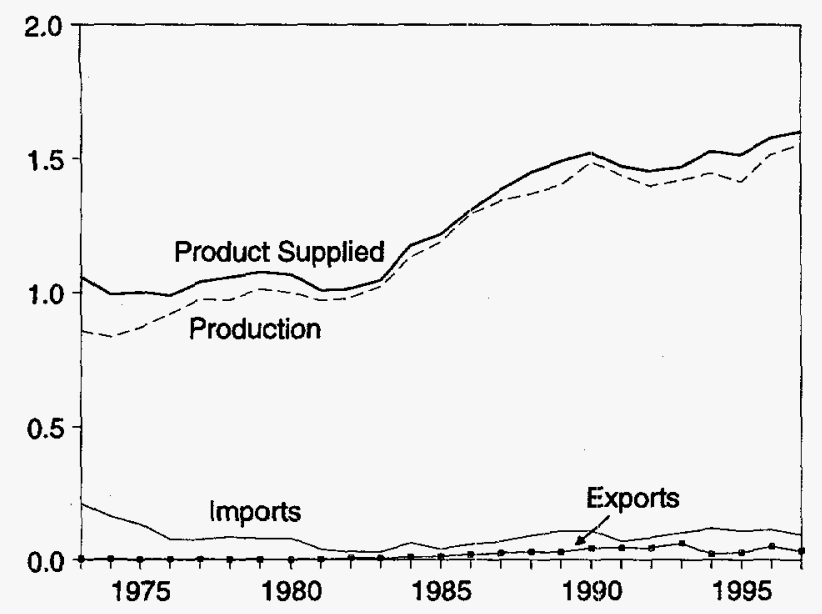

Overview, Monthly

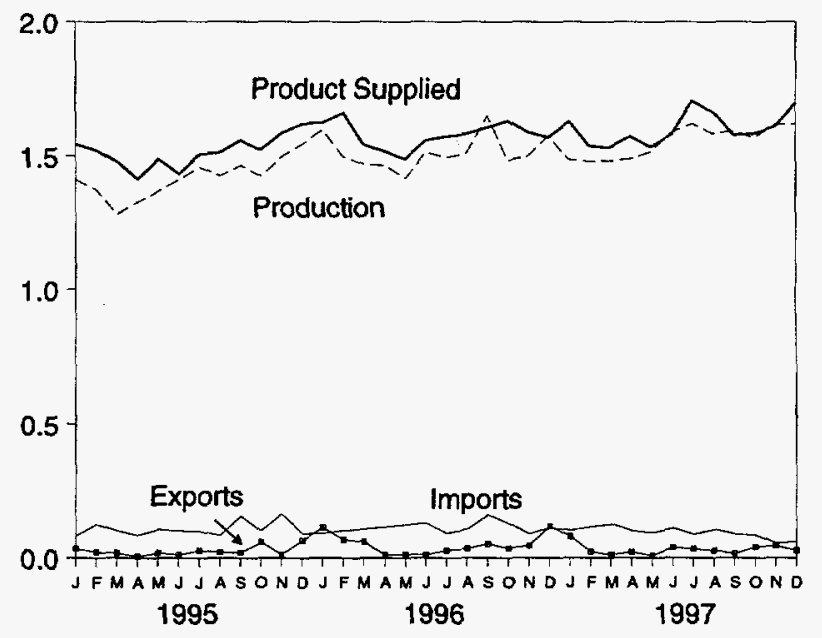

Product Supplied by Type, 1973-1997

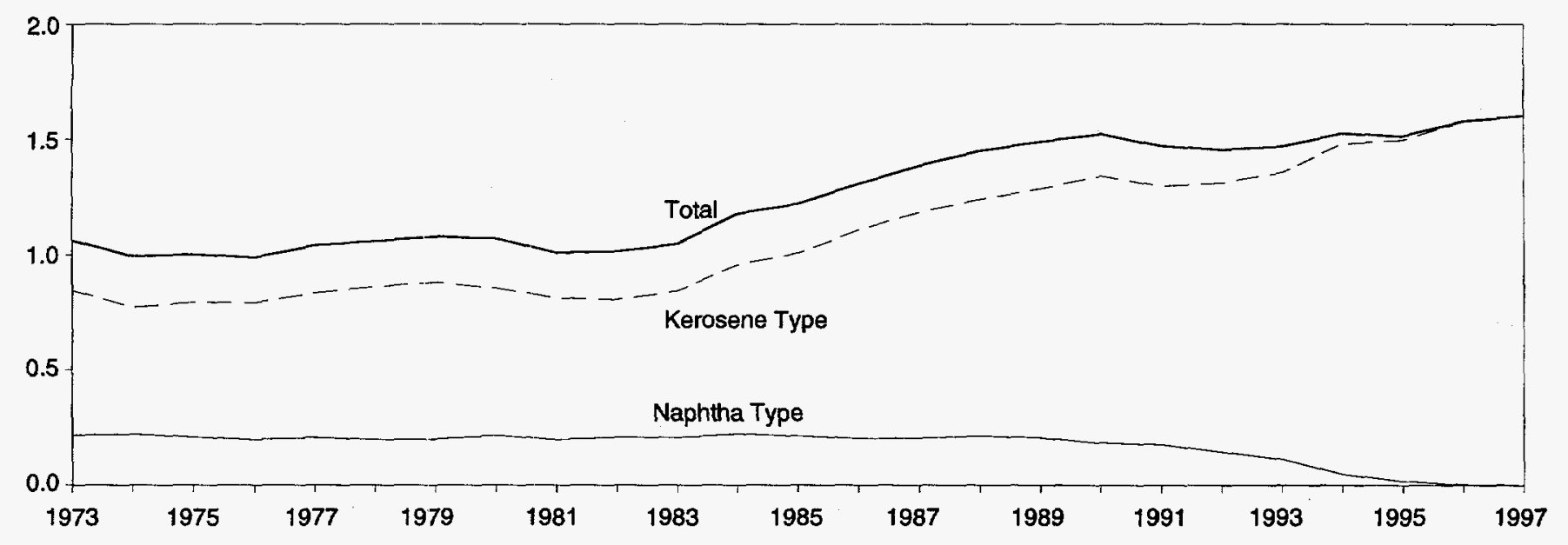

Product Supplied, January-December

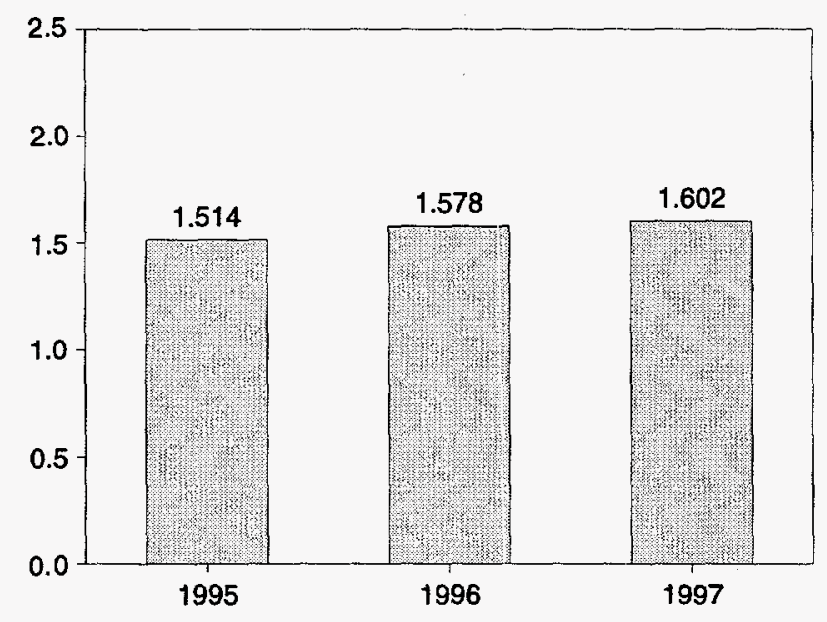

Stocks, End of Month

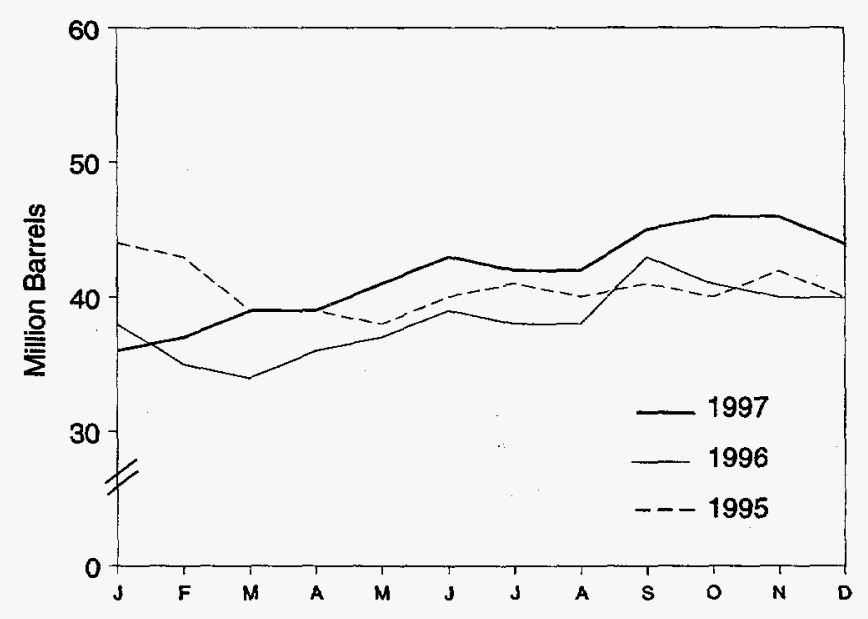

Source: Table 3.7 . 
Table 3.7 Jet Fuel Supply and Disposition

\begin{tabular}{|c|c|c|c|c|c|c|c|c|c|}
\hline & \multicolumn{3}{|c|}{ Supply } & \multicolumn{4}{|c|}{ Disposition } & \multirow{2}{*}{\multicolumn{2}{|c|}{ Ending Stocks ${ }^{a}$}} \\
\hline & \multicolumn{2}{|c|}{ Production } & \multirow[b]{2}{*}{ Imports } & \multirow{2}{*}{$\begin{array}{c}\text { Stock } \\
\text { Changeb }\end{array}$} & \multirow[b]{2}{*}{ Exports } & \multicolumn{2}{|c|}{ Product Supplied } & & \\
\hline & Total & Kerosene Type & & & & Total & Kerosene Type & Total & Kerosene Type \\
\hline & & & Thous & and Barrels & er Day & & & & on Barrels \\
\hline $\begin{array}{l}1973 \text { Average } \\
1974 \text { Average } \\
1975 \text { Average } \\
1976 \text { Average } \\
1977 \text { Average } \\
1978 \text { Average } \\
1979 \text { Average } \\
1980 \text { Average } \\
1981 \text { Average } \\
1982 \text { Average } \\
1983 \text { Average } \\
1984 \text { Average } \\
1985 \text { Average } \\
1986 \text { Average } \\
1987 \text { Average } \\
1988 \text { Average } \\
1989 \text { Average } \\
1990 \text { Average } \\
1991 \text { Average }\end{array}$ & $\begin{array}{r}859 \\
836 \\
871 \\
918 \\
973 \\
970 \\
1,012 \\
999 \\
968 \\
978 \\
1,022 \\
1,132 \\
1,189 \\
1,293 \\
1,343 \\
1,370 \\
1,403 \\
1,488 \\
1,438 \\
1,399 \\
1,422 \\
1,448\end{array}$ & $\begin{array}{r}679 \\
641 \\
691 \\
731 \\
787 \\
791 \\
835 \\
811 \\
775 \\
778 \\
817 \\
919 \\
983 \\
1,097 \\
1,138 \\
1,164 \\
1,197 \\
1,311 \\
1,274 \\
1,254 \\
1,309 \\
1,410\end{array}$ & $\begin{array}{r}212 \\
163 \\
133 \\
76 \\
75 \\
86 \\
78 \\
80 \\
38 \\
29 \\
29 \\
62 \\
39 \\
57 \\
67 \\
90 \\
106 \\
108 \\
67 \\
82 \\
100 \\
117\end{array}$ & $\begin{array}{r}8 \\
2 \\
c 2 \\
5 \\
7 \\
-2 \\
13 \\
10 \\
c^{-4} \\
-12 \\
c(s) \\
9 \\
-4 \\
25 \\
(s) \\
-17 \\
-8 \\
31 \\
-9 \\
-16 \\
-7 \\
18\end{array}$ & $\begin{array}{r}4 \\
3 \\
2 \\
2 \\
2 \\
1 \\
1 \\
1 \\
2 \\
6 \\
6 \\
9 \\
13 \\
18 \\
24 \\
28 \\
27 \\
43 \\
43 \\
43 \\
59 \\
20\end{array}$ & $\begin{array}{r}1,059 \\
993 \\
1,001 \\
987 \\
1,039 \\
1,057 \\
1,076 \\
1,068 \\
1,007 \\
1,013 \\
1,046 \\
1,175 \\
1,218 \\
1,307 \\
1,385 \\
1,449 \\
1,489 \\
1,522 \\
1,471 \\
1,454 \\
1,469 \\
1,527\end{array}$ & $\begin{array}{r}842 \\
771 \\
791 \\
789 \\
831 \\
858 \\
876 \\
851 \\
809 \\
804 \\
839 \\
953 \\
1,005 \\
1,105 \\
1,181 \\
1,236 \\
1,284 \\
1,340 \\
1,296 \\
1,310 \\
1,357 \\
1,480\end{array}$ & $\begin{array}{r}29 \\
c 29 \\
30 \\
32 \\
35 \\
34 \\
39 \\
c 42 \\
c^{2} \\
41 \\
c^{37} \\
39 \\
42 \\
40 \\
50 \\
50 \\
44 \\
41 \\
52 \\
49 \\
43 \\
40 \\
47\end{array}$ & $\begin{array}{r}23 \\
c 24 \\
25 \\
26 \\
28 \\
28 \\
33 \\
c^{\circ} 36 \\
34 \\
c^{3} \\
31 \\
32 \\
35 \\
34 \\
43 \\
42 \\
38 \\
34 \\
46 \\
44 \\
39 \\
38 \\
46\end{array}$ \\
\hline 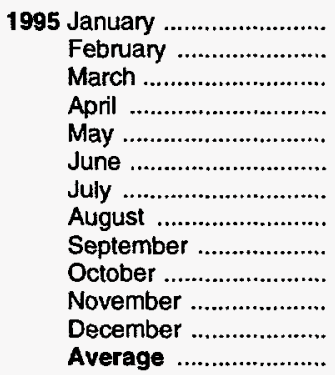 & $\begin{array}{l}1,412 \\
1,375 \\
1,281 \\
1,326 \\
1,367 \\
1,412 \\
1,458 \\
1,427 \\
1,465 \\
1,426 \\
1,496 \\
1,542 \\
1,416\end{array}$ & $\begin{array}{l}1,402 \\
1,366 \\
1,272 \\
1,317 \\
1,354 \\
1,398 \\
1,444 \\
1,418 \\
1,459 \\
1,422 \\
1,493 \\
1,538 \\
1,407\end{array}$ & $\begin{array}{r}79 \\
123 \\
99 \\
82 \\
104 \\
99 \\
97 \\
82 \\
155 \\
99 \\
164 \\
89 \\
106\end{array}$ & $\begin{array}{r}-84 \\
-43 \\
-115 \\
-12 \\
-35 \\
67 \\
23 \\
-23 \\
44 \\
-54 \\
64 \\
-51 \\
-19\end{array}$ & $\begin{array}{r}33 \\
21 \\
17 \\
5 \\
18 \\
11 \\
27 \\
21 \\
20 \\
57 \\
13 \\
63 \\
26\end{array}$ & $\begin{array}{l}1,542 \\
1,520 \\
1,478 \\
1,414 \\
1,487 \\
1,433 \\
1,505 \\
1,511 \\
1,557 \\
1,521 \\
1,584 \\
1,619 \\
1,514\end{array}$ & $\begin{array}{l}1,525 \\
1,514 \\
1,464 \\
1,402 \\
1,478 \\
1,393 \\
1,469 \\
1,505 \\
1,500 \\
1,518 \\
1,578 \\
1,618 \\
1,497\end{array}$ & $\begin{array}{l}44 \\
43 \\
39 \\
39 \\
38 \\
40 \\
41 \\
40 \\
41 \\
40 \\
42 \\
40 \\
40\end{array}$ & $\begin{array}{l}43 \\
42 \\
39 \\
38 \\
37 \\
39 \\
40 \\
39 \\
41 \\
39 \\
41 \\
39 \\
39\end{array}$ \\
\hline 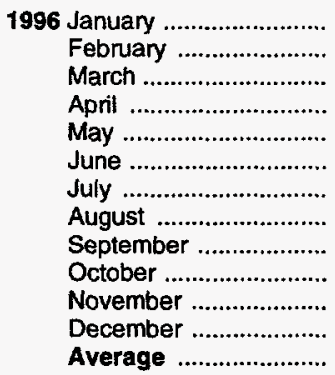 & $\begin{array}{l}1,596 \\
1,499 \\
1,470 \\
1,466 \\
1,419 \\
1,514 \\
1,496 \\
1,510 \\
1,650 \\
1,485 \\
1,501 \\
1,575 \\
1,515\end{array}$ & $\begin{array}{l}1,593 \\
1,495 \\
1,468 \\
1,464 \\
1,418 \\
1,512 \\
1,493 \\
1,507 \\
1,647 \\
1,484 \\
1,500 \\
1,574 \\
1,513\end{array}$ & $\begin{array}{r}89 \\
100 \\
105 \\
113 \\
122 \\
127 \\
89 \\
104 \\
159 \\
126 \\
87 \\
110 \\
111\end{array}$ & $\begin{array}{r}-49 \\
-129 \\
-24 \\
51 \\
39 \\
71 \\
-14 \\
-2 \\
152 \\
-55 \\
-45 \\
(\mathrm{~s}) \\
(\mathrm{s})\end{array}$ & $\begin{array}{r}111 \\
67 \\
59 \\
11 \\
13 \\
11 \\
27 \\
34 \\
51 \\
35 \\
45 \\
115 \\
48\end{array}$ & $\begin{array}{l}1,624 \\
1,661 \\
1,541 \\
1,517 \\
1,489 \\
1,558 \\
1,572 \\
1,582 \\
1,606 \\
1,631 \\
1,588 \\
1,570 \\
1,578\end{array}$ & $\begin{array}{l}1,607 \\
1,658 \\
1,547 \\
1,515 \\
1,467 \\
1,556 \\
1,569 \\
1,580 \\
1,604 \\
1,636 \\
1,588 \\
1,573 \\
1,575\end{array}$ & $\begin{array}{l}38 \\
35 \\
34 \\
36 \\
37 \\
39 \\
38 \\
38 \\
43 \\
41 \\
40 \\
40 \\
40\end{array}$ & $\begin{array}{l}38 \\
35 \\
34 \\
35 \\
37 \\
39 \\
38 \\
38 \\
43 \\
41 \\
40 \\
40 \\
40\end{array}$ \\
\hline 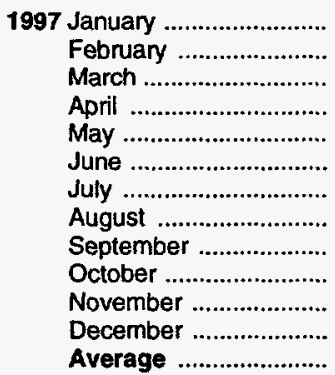 & $\begin{array}{r}1,489 \\
1,482 \\
1,484 \\
1,491 \\
1,516 \\
1,588 \\
1,620 \\
1,583 \\
1,592 \\
1,567 \\
A_{1,617} \\
E_{1,619} \\
E_{1,554}\end{array}$ & $\begin{array}{r}1,488 \\
1,482 \\
1,483 \\
1,490 \\
1,515 \\
1,588 \\
1,619 \\
1,583 \\
1,591 \\
1,566 \\
R_{1,616} \\
E_{1,619} \\
E_{1,554}\end{array}$ & $\begin{array}{r}100 \\
113 \\
123 \\
98 \\
91 \\
108 \\
86 \\
103 \\
87 \\
83 \\
R_{55} \\
\mathrm{E}_{60} \\
E_{92}\end{array}$ & $\begin{array}{r}-117 \\
35 \\
63 \\
-5 \\
65 \\
78 \\
-34 \\
-5 \\
85 \\
26 \\
\mathrm{~A}_{19} \\
\mathrm{E}_{-45} \\
\mathrm{E}_{13}\end{array}$ & $\begin{array}{r}78 \\
23 \\
11 \\
21 \\
9 \\
38 \\
33 \\
27 \\
16 \\
40 \\
\mathrm{R}_{44} \\
\mathrm{E}_{28} \\
\mathrm{E}_{31}\end{array}$ & $\begin{array}{r}1,629 \\
1,537 \\
1,532 \\
1,573 \\
1,533 \\
1,580 \\
1,707 \\
1,664 \\
1,577 \\
1,583 \\
R_{1,609} \\
E_{1,697} \\
E_{1,602}\end{array}$ & $\begin{array}{r}1,625 \\
1,530 \\
1,531 \\
1,572 \\
1,533 \\
1,579 \\
1,706 \\
1,663 \\
1,576 \\
1,584 \\
R_{1}, 609 \\
E_{1}, 696 \\
E_{1,601}\end{array}$ & $\begin{array}{r}36 \\
37 \\
39 \\
39 \\
41 \\
43 \\
42 \\
42 \\
45 \\
46 \\
R_{46} \\
E_{44} \\
E_{44}\end{array}$ & $\begin{array}{r}36 \\
37 \\
39 \\
39 \\
41 \\
43 \\
42 \\
42 \\
45 \\
46 \\
R_{46} \\
E_{44} \\
E_{44}\end{array}$ \\
\hline
\end{tabular}

a Stocks are totals as of end of period.

b A negative number indicates a decrease in stocks and a positive number indicates an increase.

c See Note 4 at end of section.

$R=$ Revised data. $E=$ Estimate. (s)=Less than +500 barrels per day and greater than -500 barrels per day.

Note: Geographic coverage is the 50 States and the District of Columbia.

Sources: - 1973-1980: Energy Information Administration (EIA), Petroleum Supply Monthly, February 1993, Table S7. • 1981 torward: EIA Petroleum Supply Monthly, January 1998, Table S7. 
Figure 3.6 Liquefied Petroleum Gases

(Million Barrels per Day, Except as Noted)

\section{Overview, 1973-1996}

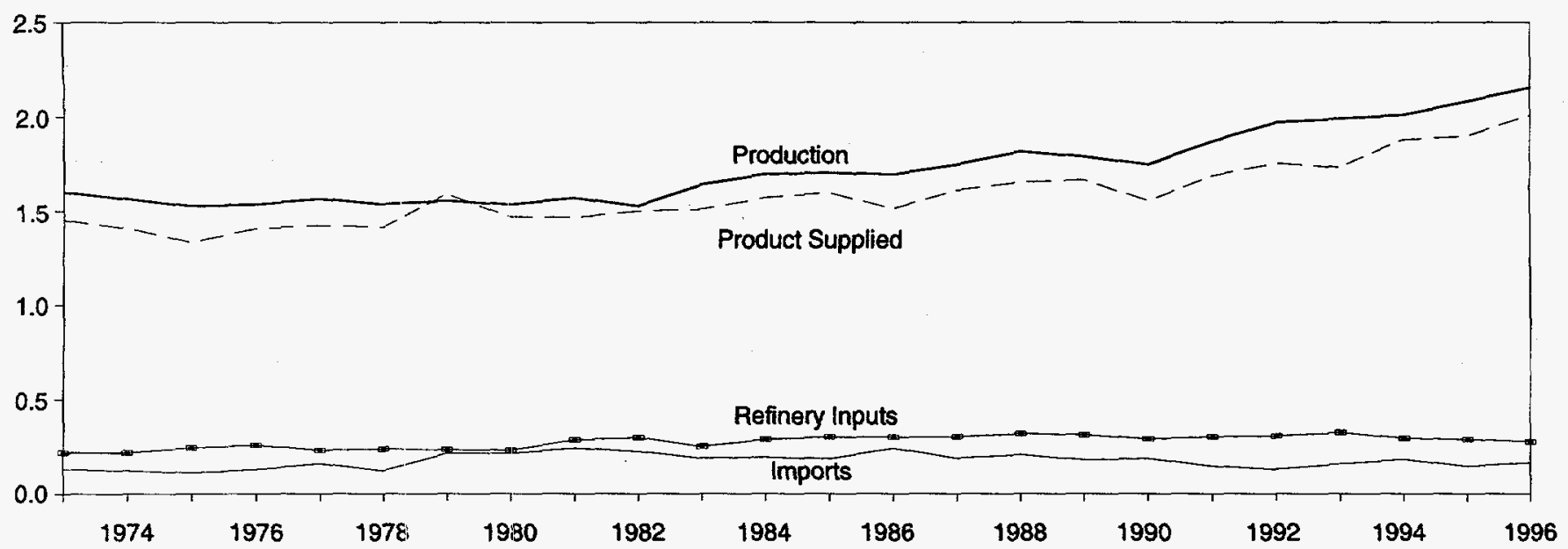

\section{Overview, Monthly}

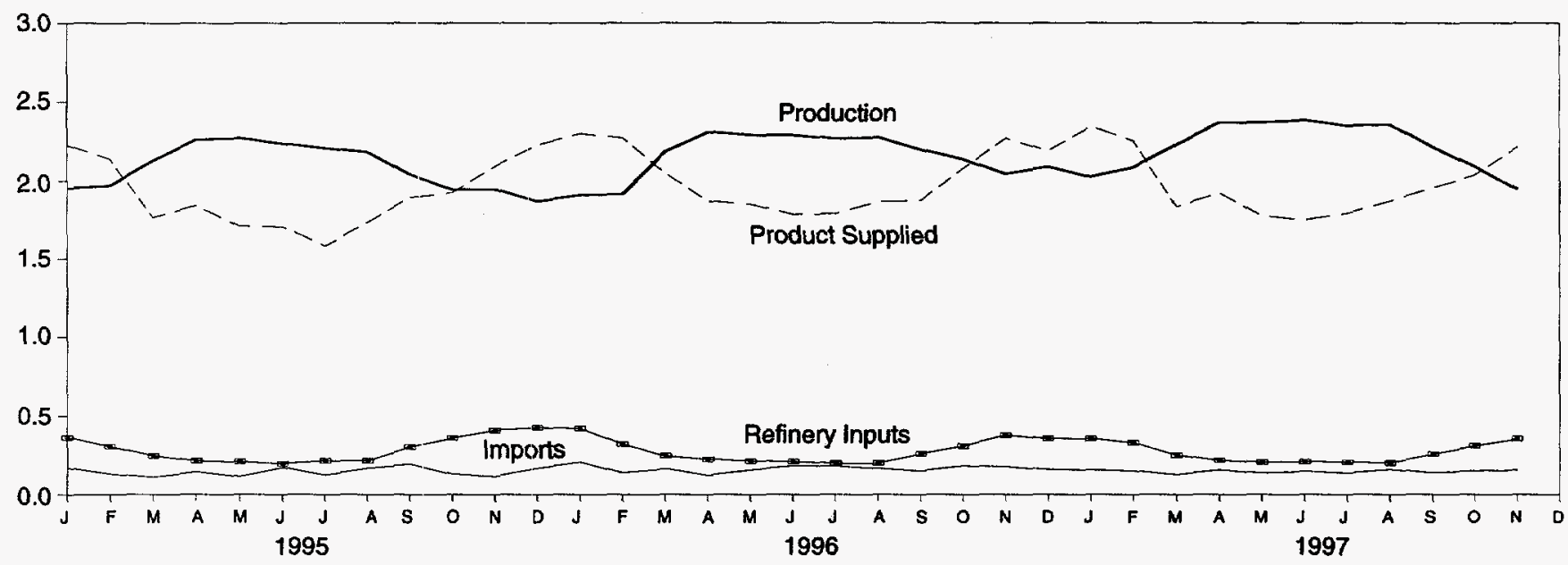

Product Supplied, January-Novernber

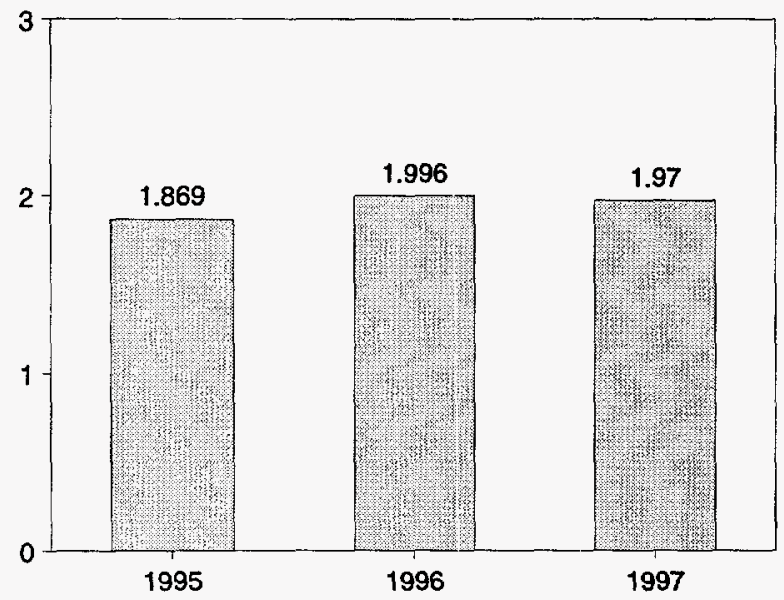

Stocks, End of Month

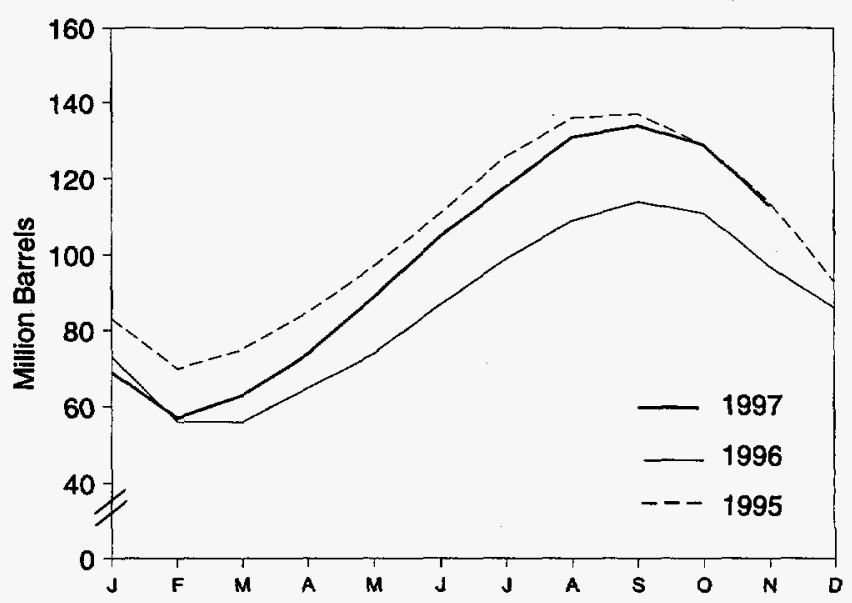

Note: Because vertical scales differ, graphs should not be compared.

Source: Table 3.8 . 
Table 3.8 Liquefied Petroleum Gases Supply and Disposition

\begin{tabular}{|c|c|c|c|c|c|c|c|}
\hline & \multicolumn{2}{|c|}{ Supply } & \multicolumn{4}{|c|}{ Disposition } & \multirow{3}{*}{$\begin{array}{c}\begin{array}{c}\text { Ending } \\
\text { Stocks }^{\mathrm{b}}\end{array} \\
\text { Million Barrels }\end{array}$} \\
\hline & $\begin{array}{c}\text { Total } \\
\text { Production }\end{array}$ & Imports & $\begin{array}{l}\text { Stock } \\
\text { Change }^{a}\end{array}$ & $\begin{array}{l}\text { Refinery } \\
\text { Inputs }\end{array}$ & Exports & $\begin{array}{l}\text { Product } \\
\text { Supplied }\end{array}$ & \\
\hline & \multicolumn{6}{|c|}{ Thousand Barrels per Day } & \\
\hline 1973 Average & 1,600 & 132 & 35 & 220 & 27 & 1,449 & 99 \\
\hline 1974 Average & 1,565 & 123 & 38 & 220 & 25 & 1,406 & $c_{113}$ \\
\hline 1975 Average & $\mathbf{1 , 5 2 7}$ & 112 & $c_{35}$ & 246 & 26 & 1,333 & 125 \\
\hline 1976 Average & 1,535 & 130 & -24 & 260 & 25 & 1,404 & 116 \\
\hline 1977 Average ............................. & 1,566 & 161 & 55 & 233 & 18 & $\mathbf{1 , 4 2 2}$ & 136 \\
\hline 1978 Average & 1,537 & 123 & -12 & 239 & 20 & $1, \mathbf{4 1 3}$ & $c^{6} 132$ \\
\hline 1979 Average & 1,556 & 217 & $c_{-70}$ & 236 & 15 & 1,592 & 111 \\
\hline 1980 Average & 1,535 & 216 & 27 & 233 & 21 & 1,469 & $c_{120}$ \\
\hline 1981 Average & 1,571 & 244 & $\mathbf{1 8}$ & 289 & 42 & 1,466 & 135 \\
\hline 1982 Average & d 1,527 & 226 & -111 & 300 & 65 & 1,499 & 94 \\
\hline 1983 Average ............................. & 1,642 & 190 & $c_{-4}$ & 253 & 73 & 1,509 & $c_{101}$ \\
\hline 1984 Average & 1,697 & 195 & $c_{-19}$ & 291 & 48 & 1,572 & 101 \\
\hline 1985 Average ........................... & 1,704 & 187 & -75 & 304 & 62 & 1,599 & 74 \\
\hline 1986 Average ............................ & 1,695 & 242 & 80 & 302 & 42 & 1,512 & 103 \\
\hline 1987 Average ......................... & 1,748 & 190 & -15 & 304 & 38 & 1,612 & 97 \\
\hline 1988 Average & 1,817 & 209 & 1 & 321 & 49 & 1,656 & 97 \\
\hline 1989 Average .......................... & 1,791 & 181 & -47 & 315 & 35 & 1,668 & 80 \\
\hline 1990 Average ............................ & 1,749 & 188 & 48 & 293 & 40 & 1,556 & 98 \\
\hline 1991 Average & 1,871 & 147 & -15 & 304 & 41 & 1,689 & 92 \\
\hline 1992 Average & 1,972 & 131 & -10 & 309 & 49 & 1,755 & 89 \\
\hline 1993 Average ............................. & 1,993 & 160 & 49 & 327 & 43 & 1,734 & 106 \\
\hline 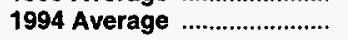 & 2,012 & 183 & -19 & 296 & 38 & 1,880 & 99 \\
\hline 1995 January ............................. & 1,952 & 172 & -527 & 363 & 64 & 2,225 & 83 \\
\hline February & 1,969 & 134 & -463 & 306 & 122 & 2,138 & 70 \\
\hline 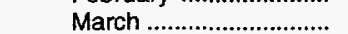 & 2,126 & 111 & 170 & 247 & 57 & 1,763 & 75 \\
\hline April & 2,259 & 147 & 307 & 216 & 43 & 1,841 & 85 \\
\hline May ................................... & 2,269 & 115 & 403 & 211 & 62 & 1,709 & 97 \\
\hline June ................................ & 2,233 & 174 & 448 & 198 & 55 & 1,705 & 111 \\
\hline 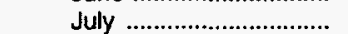 & 2,203 & 124 & 488 & 217 & 41 & 1,581 & 126 \\
\hline 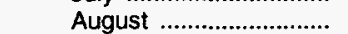 & 2,178 & 169 & 343 & 217 & 57 & 1,730 & 136 \\
\hline September ......................... & 2,038 & 195 & 14 & 300 & 29 & 1,890 & 137 \\
\hline 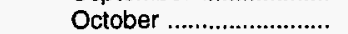 & 1,940 & 130 & -245 & 358 & 35 & 1,921 & 129 \\
\hline November .......................... & 1,943 & 115 & -500 & 407 & 63 & 2,087 & 114 \\
\hline December ............................ & 1,865 & 169 & -680 & 424 & 67 & 2,223 & 93 \\
\hline Average & 2,082 & 146 & -17 & 289 & 58 & 1,899 & 93 \\
\hline 1996 January ............................ & 1,906 & 208 & -649 & 419 & 49 & 2,295 & 73 \\
\hline February .......................... & 1,912 & 138 & -596 & 320 & 60 & 2,267 & 56 \\
\hline 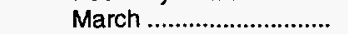 & 2,181 & 165 & 15 & 246 & 38 & 2,047 & 56 \\
\hline April ....1...1. & 2,305 & 122 & 279 & 226 & 56 & 1,867 & 65 \\
\hline 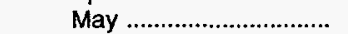 & 2,287 & 156 & 315 & 215 & 67 & 1,846 & 74 \\
\hline 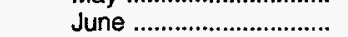 & 2,285 & 184 & 439 & 211 & 36 & 1,783 & 87 \\
\hline 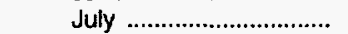 & 2,264 & 182 & 385 & 201 & 72 & 1,787 & 99 \\
\hline August & 2,271 & 166 & 321 & 201 & 50 & 1,864 & 109 \\
\hline September ............................ & 2,194 & 150 & 165 & 260 & 47 & 1,871 & 114 \\
\hline 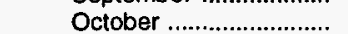 & 2,133 & 183 & -103 & 309 & 37 & 2,073 & 111 \\
\hline November .......................... & 2,041 & 177 & -466 & 377 & 41 & 2,265 & 97 \\
\hline December ............................ & 2,086 & 159 & -352 & 355 & 56 & 2,186 & 86 \\
\hline Average & 2,156 & 166 & -19 & 278 & 51 & 2,012 & 86 \\
\hline 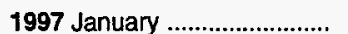 & 2,022 & 156 & .555 & 356 & 36 & 2,341 & 69 \\
\hline February .......................... & 2,082 & 150 & -424 & 330 & 78 & 2,249 & 57 \\
\hline March .................................. & 2,225 & 126 & 206 & 252 & 62 & 1,831 & 63 \\
\hline April & 2,366 & 157 & 345 & 218 & 41 & 1,918 & 74 \\
\hline 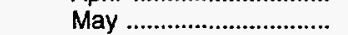 & 2,367 & 136 & 485 & 207 & 40 & 1,773 & 89 \\
\hline 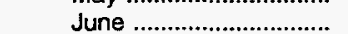 & 2,382 & 148 & 531 & 210 & 43 & 1,746 & 105 \\
\hline 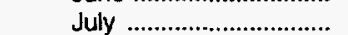 & 2,346 & 136 & 430 & 206 & 56 & 1,789 & 118 \\
\hline August .............................. & 2,352 & 159 & 407 & 201 & 37 & 1,866 & 131 \\
\hline September ......................... & 2,209 & 138 & 110 & 258 & 29 & 1,950 & 134 \\
\hline 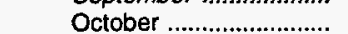 & 2,088 & 151 & -147 & 312 & 42 & 2,032 & 129 \\
\hline November ........................... & 1,934 & 155 & -534 & 355 & 66 & 2,203 & 113 \\
\hline 11-Month Average ..... & 2,217 & 146 & 82 & 264 & 48 & 1,970 & 113 \\
\hline $\begin{array}{l}1996 \text { 11-Month Average ..... } \\
1995 \text { 11-Month Average ..... }\end{array}$ & $\begin{array}{l}2,163 \\
2,102\end{array}$ & $\begin{array}{l}167 \\
144\end{array}$ & $\begin{array}{l}12 \\
44\end{array}$ & $\begin{array}{l}271 \\
276\end{array}$ & $\begin{array}{l}50 \\
57\end{array}$ & $\begin{array}{l}1,996 \\
1,869\end{array}$ & $\begin{array}{r}97 \\
114\end{array}$ \\
\hline
\end{tabular}

a A negative number indicates a decrease in stocks and a positive number indicates an increase.

$b$ Stocks are totals as of end of period.

c See Nole 4 at end of section.

d See Note 6 at end of section.

Notes: - Liquefied petroleum gases include ethane, ethylene, propane,

propylene, normal butane butylene, isobutane and isobutylene. - Geographic coverage is the 50 States and the District of Columbia.

Sources: - 1973-1980: Energy Information Administration (EIA), Petroleum Supply Monthly, February 1993, Table S8. - 1981 forward: EIA Petroleum Supply Monthly, January 1998, Table S9. 
Figure 3.7 Propane and Propylene

(Million Barrels per Day, Except as Noted)

Overview, 1973-1996

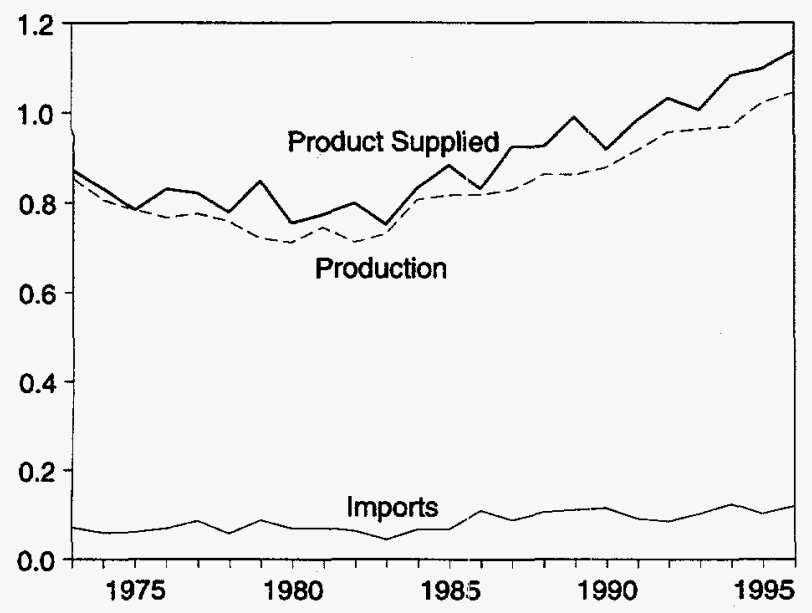

Product Supplied, Monthly

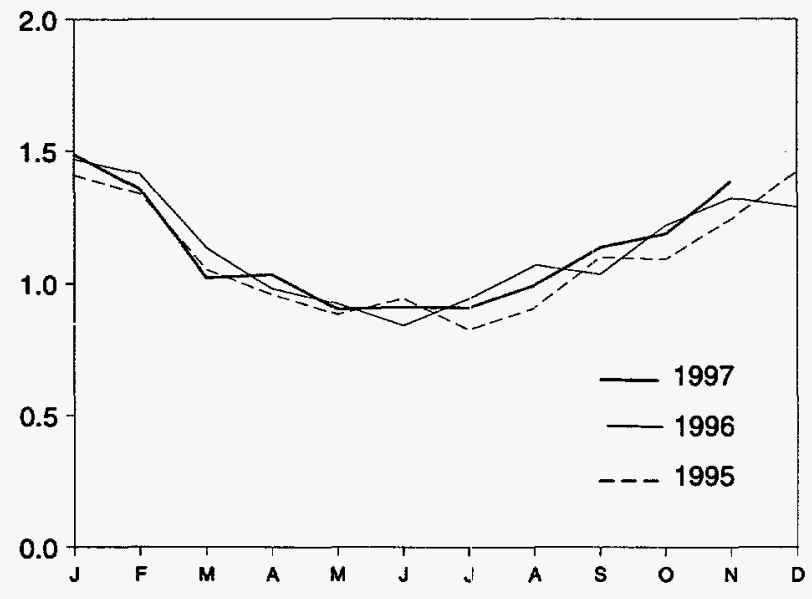

Stocks, End of Month

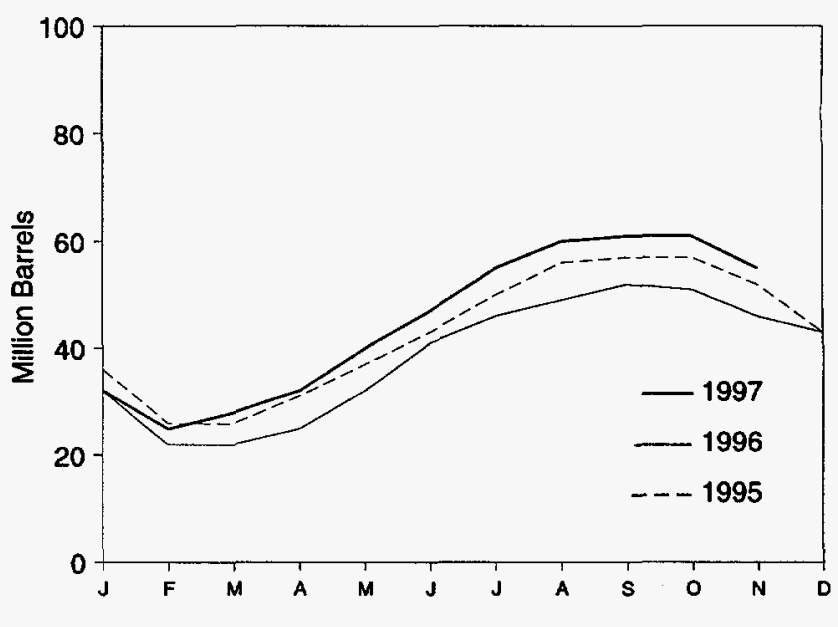

Product Supplied, January-November

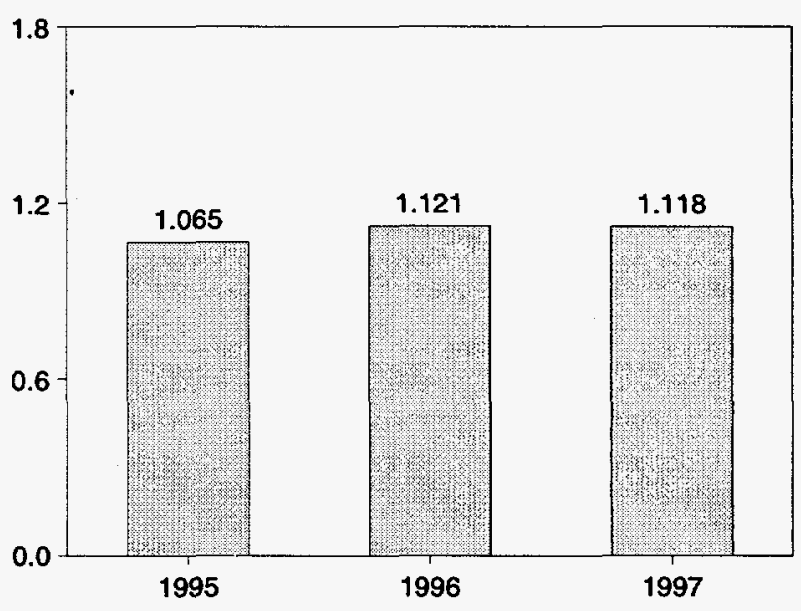

\section{Share of Liquefied Petroleum Gases, November}

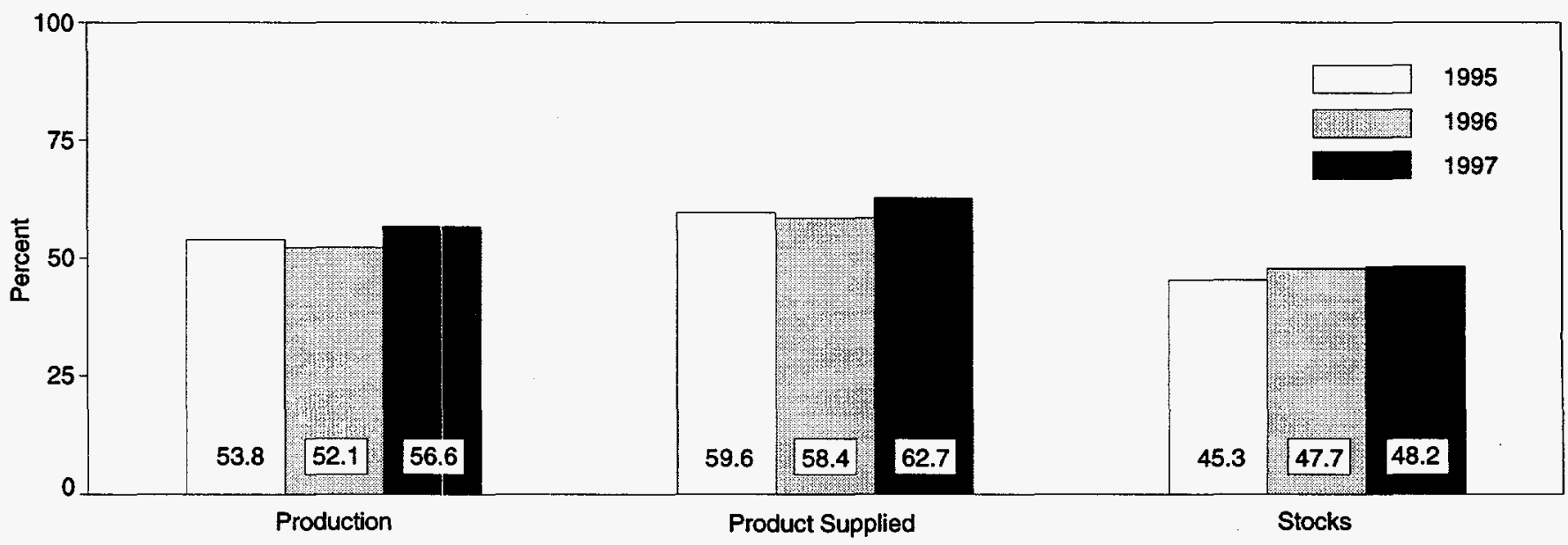

Note: Because vertical scales differ, graphs should not be compared.

Sources: Table 3.9 and, for calculation of shares, data prior to rounding for publication in Tables 3.8 and 3.9 . 
Table 3.9 Propane and Propylene Supply and Disposition (A Subset of Table 3.8)

\begin{tabular}{|c|c|c|c|c|c|c|c|}
\hline & \multicolumn{2}{|c|}{ Supply } & \multicolumn{4}{|c|}{ Disposition } & \multirow[b]{2}{*}{$\begin{array}{l}\text { Ending } \\
\text { Stocks }\end{array}$} \\
\hline & $\begin{array}{c}\text { Total } \\
\text { Production }\end{array}$ & Imports & $\begin{array}{c}\text { Stock } \\
\text { Changea }\end{array}$ & $\begin{array}{l}\text { Refinery } \\
\text { Inputs }\end{array}$ & Exports & $\begin{array}{l}\text { Product } \\
\text { Supplied }\end{array}$ & \\
\hline & \multicolumn{6}{|c|}{ Thousand Barrels per Day } & Million Barrels \\
\hline $\begin{array}{l}1973 \text { Average } \\
1974 \text { Average } \\
1975 \text { Average } \\
1976 \text { Average } \\
1977 \text { Average } \\
197 \ldots \ldots \ldots \ldots \ldots \ldots \ldots \\
1978 \text { Average }\end{array}$ & $\begin{array}{l}854 \\
805 \\
783 \\
766 \\
775 \\
758 \\
721 \\
711 \\
745 \\
711 \\
730 \\
806 \\
816 \\
817 \\
828 \\
863 \\
862 \\
878 \\
915 \\
956 \\
963 \\
969\end{array}$ & $\begin{array}{r}71 \\
59 \\
60 \\
68 \\
86 \\
57 \\
88 \\
69 \\
70 \\
63 \\
44 \\
67 \\
67 \\
110 \\
88 \\
106 \\
111 \\
115 \\
91 \\
85 \\
103 \\
124\end{array}$ & $\begin{array}{r}30 \\
11 \\
36 \\
-22 \\
21 \\
15 \\
c-61 \\
4 \\
c 18 \\
-59 \\
c-24 \\
c 7 \\
-50 \\
64 \\
-41 \\
7 \\
-52 \\
48 \\
-3 \\
-24 \\
34 \\
-13\end{array}$ & $\begin{array}{r}8 \\
9 \\
11 \\
12 \\
10 \\
13 \\
14 \\
12 \\
5 \\
4 \\
4 \\
4 \\
3 \\
4 \\
8 \\
8 \\
11 \\
(\mathrm{~s}) \\
\text { (s) } \\
\text { (s) } \\
\text { (s) } \\
0\end{array}$ & $\begin{array}{r}15 \\
14 \\
13 \\
13 \\
10 \\
9 \\
8 \\
10 \\
18 \\
31 \\
43 \\
30 \\
48 \\
28 \\
24 \\
31 \\
24 \\
28 \\
28 \\
33 \\
26 \\
24\end{array}$ & $\begin{array}{r}872 \\
830 \\
783 \\
830 \\
821 \\
778 \\
849 \\
754 \\
773 \\
798 \\
751 \\
833 \\
883 \\
831 \\
924 \\
923 \\
990 \\
917 \\
982 \\
1,032 \\
1,006 \\
1,082\end{array}$ & $\begin{array}{r}65 \\
69 \\
82 \\
74 \\
81 \\
\times 87 \\
644 \\
665 \\
66 \\
\mathrm{c} 54 \\
\mathrm{c} 48 \\
58 \\
39 \\
63 \\
48 \\
50 \\
32 \\
49 \\
48 \\
39 \\
51 \\
46\end{array}$ \\
\hline 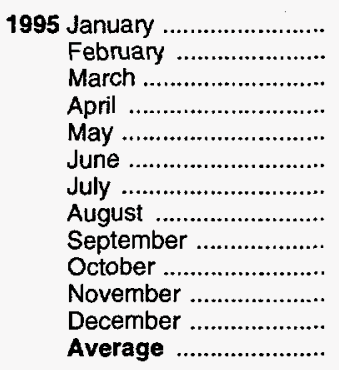 & $\begin{array}{r}1,007 \\
985 \\
1,017 \\
1,040 \\
1,046 \\
1,042 \\
1,011 \\
1,008 \\
1,022 \\
999 \\
1,045 \\
1,033 \\
1,021\end{array}$ & $\begin{array}{r}108 \\
94 \\
90 \\
107 \\
73 \\
114 \\
75 \\
107 \\
146 \\
98 \\
76 \\
135 \\
102\end{array}$ & $\begin{array}{r}-349 \\
-362 \\
14 \\
157 \\
209 \\
188 \\
236 \\
187 \\
45 \\
-22 \\
-160 \\
-285 \\
-10\end{array}$ & $\begin{array}{l}0 \\
0 \\
0 \\
0 \\
0 \\
0 \\
0 \\
0 \\
0 \\
0 \\
0 \\
0 \\
0\end{array}$ & $\begin{array}{r}55 \\
100 \\
39 \\
31 \\
29 \\
27 \\
27 \\
24 \\
25 \\
30 \\
37 \\
31 \\
38\end{array}$ & $\begin{array}{r}1,409 \\
1,341 \\
1,055 \\
958 \\
882 \\
941 \\
823 \\
905 \\
1,098 \\
1,090 \\
1,243 \\
1,422 \\
1,096\end{array}$ & $\begin{array}{l}36 \\
26 \\
26 \\
31 \\
37 \\
43 \\
50 \\
56 \\
57 \\
57 \\
52 \\
43 \\
43\end{array}$ \\
\hline 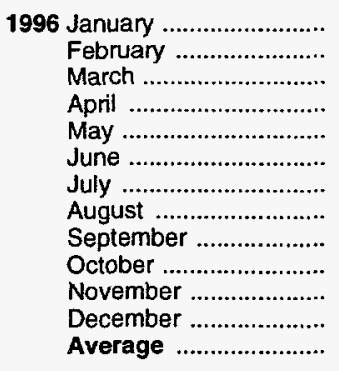 & $\begin{array}{r}995 \\
1,001 \\
1,043 \\
1,047 \\
1,048 \\
1,031 \\
1,043 \\
1,051 \\
1,057 \\
1,058 \\
1,063 \\
1,093 \\
1,044\end{array}$ & $\begin{array}{r}151 \\
106 \\
116 \\
78 \\
104 \\
122 \\
114 \\
126 \\
95 \\
151 \\
147 \\
122 \\
119\end{array}$ & $\begin{array}{r}-353 \\
-347 \\
-1 \\
114 \\
209 \\
293 \\
188 \\
83 \\
97 \\
-37 \\
-148 \\
-106 \\
(s)\end{array}$ & $\begin{array}{l}0 \\
0 \\
0 \\
0 \\
0 \\
0 \\
0 \\
0 \\
0 \\
0 \\
0 \\
0 \\
0\end{array}$ & $\begin{array}{l}30 \\
39 \\
25 \\
31 \\
21 \\
21 \\
29 \\
24 \\
21 \\
29 \\
34 \\
31 \\
28\end{array}$ & $\begin{array}{r}1,468 \\
1,415 \\
1,135 \\
981 \\
922 \\
839 \\
940 \\
1,069 \\
1,034 \\
1,218 \\
1,324 \\
1,289 \\
1,136\end{array}$ & $\begin{array}{l}32 \\
22 \\
22 \\
25 \\
32 \\
41 \\
46 \\
49 \\
52 \\
51 \\
46 \\
43 \\
43\end{array}$ \\
\hline 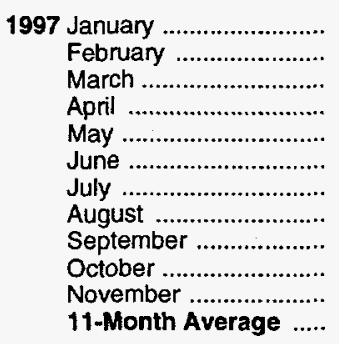 & $\begin{array}{l}1,042 \\
1,043 \\
1,065 \\
1,114 \\
1,113 \\
1,111 \\
1,085 \\
1,092 \\
1,111 \\
1,111 \\
1,099 \\
1,090\end{array}$ & $\begin{array}{r}121 \\
105 \\
84 \\
99 \\
69 \\
79 \\
76 \\
97 \\
78 \\
111 \\
113 \\
94\end{array}$ & $\begin{array}{r}-352 \\
-252 \\
86 \\
146 \\
258 \\
250 \\
231 \\
172 \\
39 \\
7 \\
-222 \\
35\end{array}$ & $\begin{array}{l}0 \\
0 \\
0 \\
0 \\
0 \\
0 \\
0 \\
0 \\
0 \\
0 \\
0 \\
0\end{array}$ & $\begin{array}{l}28 \\
42 \\
40 \\
32 \\
23 \\
31 \\
24 \\
24 \\
16 \\
29 \\
48 \\
31\end{array}$ & $\begin{array}{r}1,486 \\
1,358 \\
1,023 \\
1,035 \\
901 \\
909 \\
906 \\
993 \\
1,134 \\
1,185 \\
1,386 \\
1,118\end{array}$ & $\begin{array}{l}32 \\
25 \\
28 \\
32 \\
40 \\
47 \\
55 \\
60 \\
61 \\
61 \\
55 \\
55\end{array}$ \\
\hline $\begin{array}{l}1996 \text { 11-Month Average ..... } \\
1995 \text { 11-Month Average ..... }\end{array}$ & $\begin{array}{l}1,040 \\
1,020\end{array}$ & $\begin{array}{r}119 \\
99\end{array}$ & $\begin{array}{l}10 \\
16\end{array}$ & $\begin{array}{l}0 \\
0\end{array}$ & $\begin{array}{l}28 \\
38\end{array}$ & $\begin{array}{l}1,121 \\
1,065\end{array}$ & $\begin{array}{l}46 \\
52\end{array}$ \\
\hline
\end{tabular}

a A negative number indicates a decrease in stocks and a positive number indicates an increase.

b Stocks are totals as of end period.

c See Note 4 at end of section.

(s)=Less than 500 barrels per day.

Note: Geographic coverage is the 50 States and the District of Columbia.
Sources: - 1973 through 1975: U.S. Department of the Interior, Bureau of Mines, Mineral Industry Surveys, "Petroleum Statement, Annual." - 1976 through 1980: Energy Information Administration (EIA), Energy Data Reports, Petroleum Statement, Annual." 1981 forward: EIA, Petroleum Supply Monthly, January 1998, Table S8. 
Table 3.10 Other Petroleum Products Supply and Disposition

\begin{tabular}{|c|c|c|c|c|c|c|c|}
\hline & \multicolumn{2}{|c|}{ Supply } & \multicolumn{4}{|c|}{ Disposition } & \multirow{3}{*}{$\begin{array}{c}\begin{array}{c}\text { Ending } \\
\text { Stocks }\end{array} \\
\text { Million Barrels }\end{array}$} \\
\hline & $\begin{array}{c}\text { Total } \\
\text { Production }\end{array}$ & Innports & $\begin{array}{c}\text { Stock } \\
\text { Change }^{a}\end{array}$ & $\begin{array}{l}\text { Refinery } \\
\text { Inputs }\end{array}$ & Exports & $\begin{array}{l}\text { Products } \\
\text { Supplied }\end{array}$ & \\
\hline & \multicolumn{6}{|c|}{ Thousand Barrels per Day } & \\
\hline 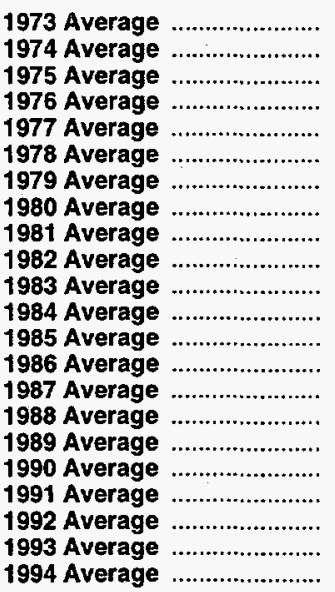 & $\begin{array}{r}2,833 \\
2,722 \\
2,547 \\
2,725 \\
2,939 \\
3,076 \\
3,141 \\
2,957 \\
2,771 \\
2,475 \\
2,437 \\
2,500 \\
2,532 \\
2,704 \\
2,737 \\
2,773 \\
2,771 \\
2,842 \\
2,826 \\
2,928 \\
e 3,035 \\
2,973\end{array}$ & $\begin{array}{r}290 \\
269 \\
144 \\
129 \\
130 \\
80 \\
116 \\
130 \\
188 \\
305 \\
382 \\
503 \\
550 \\
504 \\
543 \\
645 \\
627 \\
705 \\
675 \\
707 \\
770 \\
761\end{array}$ & $\begin{array}{r}1 \\
25 \\
c-6 \\
(s) \\
20 \\
-12 \\
24 \\
15 \\
c-42 \\
-68 \\
c-6 \\
c-32 \\
22 \\
-15 \\
-1 \\
22 \\
12 \\
-32 \\
18 \\
-3 \\
c-2 \\
24\end{array}$ & $\begin{array}{r}750 \\
665 \\
537 \\
524 \\
514 \\
492 \\
352 \\
310 \\
723 \\
787 \\
712 \\
791 \\
886 \\
888 \\
829 \\
799 \\
797 \\
\mathbf{8 8 7} \\
\mathbf{9 3 6} \\
\mathbf{9 0 6} \\
\mathbf{1 , 0 8 1} \\
\mathbf{8 6 1}\end{array}$ & $\begin{array}{l}162 \\
172 \\
158 \\
172 \\
164 \\
165 \\
208 \\
197 \\
197 \\
205 \\
236 \\
236 \\
227 \\
291 \\
264 \\
294 \\
305 \\
289 \\
277 \\
263 \\
e 300 \\
329\end{array}$ & $\begin{array}{r}2,211 \\
2,129 \\
2,001 \\
2,158 \\
2,371 \\
2,511 \\
2,673 \\
2,566 \\
2,081 \\
d 1,857 \\
1,877 \\
2,007 \\
1,947 \\
2,045 \\
2,187 \\
2,303 \\
2,285 \\
2,402 \\
2,269 \\
2,470 \\
e, 426 \\
2,518\end{array}$ & $\begin{array}{r}179 \\
c 188 \\
188 \\
188 \\
195 \\
191 \\
200 \\
c 205 \\
241 \\
c 216 \\
c 217 \\
198 \\
206 \\
201 \\
200 \\
208 \\
213 \\
201 \\
208 \\
c 207 \\
206 \\
215\end{array}$ \\
\hline 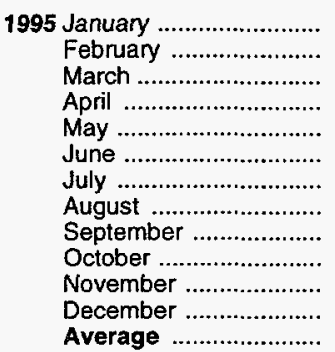 & $\begin{array}{l}2,879 \\
2,960 \\
2,842 \\
2,916 \\
3,009 \\
3,142 \\
3,312 \\
3,246 \\
3,256 \\
2,939 \\
2,918 \\
2,953 \\
3,031\end{array}$ & $\begin{array}{l}559 \\
806 \\
672 \\
711 \\
593 \\
651 \\
765 \\
745 \\
779 \\
727 \\
803 \\
701 \\
708\end{array}$ & $\begin{array}{r}413 \\
271 \\
-35 \\
-106 \\
-74 \\
-130 \\
-54 \\
-250 \\
-44 \\
-120 \\
-35 \\
-97 \\
-23\end{array}$ & $\begin{array}{r}657 \\
758 \\
914 \\
1,064 \\
801 \\
917 \\
1,126 \\
1,123 \\
1,077 \\
919 \\
1,003 \\
1,125 \\
958\end{array}$ & $\begin{array}{l}324 \\
320 \\
329 \\
355 \\
339 \\
403 \\
326 \\
372 \\
348 \\
376 \\
343 \\
341 \\
348\end{array}$ & $\begin{array}{l}2,044 \\
2,417 \\
2,306 \\
2,313 \\
2,535 \\
2,604 \\
2,679 \\
2,746 \\
2,654 \\
2,491 \\
2,409 \\
2,286 \\
2,457\end{array}$ & $\begin{array}{l}227 \\
235 \\
234 \\
231 \\
229 \\
225 \\
223 \\
215 \\
214 \\
210 \\
209 \\
206 \\
206\end{array}$ \\
\hline 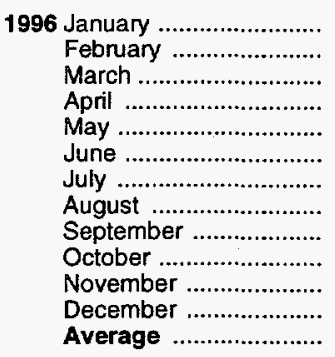 & $\begin{array}{l}2,833 \\
2,817 \\
2,983 \\
3,108 \\
3,128 \\
3,227 \\
3,223 \\
3,332 \\
3,306 \\
3,146 \\
3,093 \\
3,088 \\
3,108\end{array}$ & $\begin{array}{r}873 \\
745 \\
820 \\
828 \\
852 \\
923 \\
862 \\
907 \\
751 \\
1,068 \\
928 \\
982 \\
\mathbf{8 7 9}\end{array}$ & $\begin{array}{r}448 \\
-18 \\
122 \\
174 \\
-45 \\
-203 \\
-170 \\
-311 \\
-56 \\
-84 \\
-34 \\
42 \\
-11\end{array}$ & $\begin{array}{r}613 \\
872 \\
759 \\
841 \\
1,010 \\
1,207 \\
1,131 \\
1,289 \\
1,083 \\
1,023 \\
1,113 \\
1,224 \\
1,014\end{array}$ & $\begin{array}{l}335 \\
388 \\
315 \\
421 \\
427 \\
399 \\
361 \\
448 \\
410 \\
323 \\
366 \\
321 \\
376\end{array}$ & $\begin{array}{l}2,311 \\
2,320 \\
2,607 \\
2,500 \\
2,588 \\
2,748 \\
2,764 \\
2,812 \\
2,620 \\
2,952 \\
2,576 \\
2,485 \\
2,608\end{array}$ & $\begin{array}{l}220 \\
219 \\
223 \\
228 \\
227 \\
221 \\
216 \\
206 \\
204 \\
202 \\
201 \\
202 \\
202\end{array}$ \\
\hline 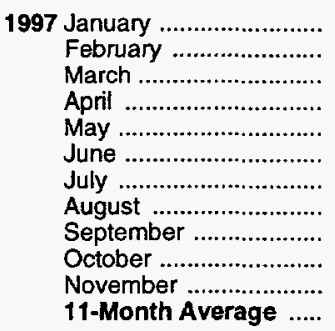 & $\begin{array}{l}2,963 \\
2,990 \\
3,103 \\
3,172 \\
3,343 \\
3,391 \\
3,451 \\
3,446 \\
3,434 \\
3,235 \\
3,092 \\
3,240\end{array}$ & $\begin{array}{r}1,142 \\
1,012 \\
945 \\
1,053 \\
1,178 \\
934 \\
892 \\
880 \\
796 \\
957 \\
754 \\
959\end{array}$ & $\begin{array}{r}341 \\
213 \\
505 \\
-99 \\
125 \\
-461 \\
-193 \\
-89 \\
83 \\
-86 \\
7 \\
31\end{array}$ & $\begin{array}{r}850 \\
988 \\
718 \\
1,240 \\
1,119 \\
1,395 \\
1,114 \\
1,017 \\
853 \\
930 \\
941 \\
1,014\end{array}$ & $\begin{array}{l}403 \\
332 \\
391 \\
395 \\
446 \\
417 \\
380 \\
460 \\
450 \\
381 \\
369 \\
403\end{array}$ & $\begin{array}{l}2,511 \\
2,470 \\
2,434 \\
2,689 \\
2,831 \\
2,976 \\
3,041 \\
2,937 \\
2,843 \\
2,966 \\
2,530 \\
2,750\end{array}$ & $\begin{array}{l}214 \\
219 \\
235 \\
232 \\
236 \\
222 \\
216 \\
213 \\
216 \\
213 \\
213 \\
213\end{array}$ \\
\hline $\begin{array}{l}1996 \text { 11-Month Average ..... } \\
199511 \text {-Month Average ..... }\end{array}$ & $\begin{array}{l}3,110 \\
3,038\end{array}$ & $\begin{array}{l}870 \\
709\end{array}$ & $\begin{array}{l}-16 \\
-17\end{array}$ & $\begin{array}{l}995 \\
943\end{array}$ & $\begin{array}{l}381 \\
349\end{array}$ & $\begin{array}{l}2,620 \\
2,473\end{array}$ & $\begin{array}{l}201 \\
209\end{array}$ \\
\hline
\end{tabular}

a A negative number indicates a decrease in stocks and a positive number indicates an increase.

Stocks are totals as of end of period.

c See Note 4 at end of section.

d See Note 6 at end of section.

- Beginning in 1993, other petroleum products production, exports, and products supplied include an adjustment to oxygenates and motor gasoline blending components.

(s) =Less than +500 barrels per day and greater than -500 barrels per day.

Notes: - Other petroleum products include pentanes plus, other hydrocarbons and alcohol, unfinished oils, gasoline blending components, and all finished petroleum products except finished motor gasoline, distillate fuel oil, residual fuel oil, jet fuel, liquefied petroleum gases, and crude oil that is used as fuel. - Geographic coverage is the 50 States and the District of Columbia.

Sources: - 1973-1980: Energy Information Administration (EIA), Petroleum Supply Monthly, February 1993, Table S9. • 1981 forward: EIA Petroleum Supply Monthly, January 1998, Table S10. 


\section{Petroleum Notes}

1. The Energy Information Administration (EIA) uses a number of sources and methods to maintain the survey respondent lists. On a regular basis, survey managers review such industry publications as the Oil and Gas Journal and Oil Daily for information on facilities or companies starting up or closing down operations. Those sources are augmented by articles in newspapers, letters from respondents indicating changes in status, and information received from survey systems.

To supplement routine frames maintenance and to provide more thorough coverage, a comprehensive frames investigation is conducted every 3 years. This investigation results in the reassessment and recompilation of the complete frame for each survey. The effort also includes the evaluation of the impact of potential frame changes on the historical time series of data from these respondents. The results of this frame study are usually implemented in January to provide a full year under the same frame.

In 1991, the EIA conducted a frame identifier survey of companies that produce, blend, store, or import oxygenates. A summary of the results from the identification survey was published in the Weekly Petroleum Status Report dated February 12, 1992, and in the February 1992 issue of the Petroleum Supply Monthly. In order to continue to provide relevant information about U.S. and regional gasoline supply, the EIA conducted a second frame identifier survey of those companies during 1992. As a result, numerous respondents were added to the monthly surveys effective in January 1993. See Explanatory Note 7 in the Petroleum Supply Monthly.

2. Motor Gasoline: Beginning in January 1981, the EIA expanded its universe to include non-refinery blenders and separated blending components from finished motor gasoline as a reporting category. Also, survey forms were modified to describe refinery operations more accurately.

Beginning with the reporting of January 1993 data, the EIA made adjustments to the product supplied series for finished motor gasoline. It was recognized that motor gasoline statistics published by the EIA through 1992 were underreported because the reporting system was (1) not collecting all fuel ethanol blending, and (2) there was a misreporting of motor gasoline blending components that were blended into finished gasoline. The adjustments are incorporated into EIA's data beginning in January 1993 . To facilitate data analysis across the 1992-1993 period, EIA has prepared a table of 1992 data adjusted according to the 1993 basis. See Petroleum Supply Monthly, March 1993, Table H3.

3. Distillate and Residual Fuel Oils: The requirement to report crude oil in pipelines or burned on leases as either distillate or residual fuel oil has been eliminated. Prior to January 1981, the refinery input of unfinished oils typically exceeded the available supply of unfinished oils. That discrepancy was assumed to be due to the redesignation of distillate and residual fuel oils received as such but used as unfinished oil inputs by the receiving refinery. The imbalance between supply and disposition of unfinished oils would then be subtracted from the production of distillate and residual fuel oils. Two-thirds of that difference was subtracted from distillate and onethird from residual. Beginning in January 1981, the EIA modified its survey forms to account for redesignated product and discontinued the above-mentioned adjustment.

Beginning in January 1993, the end-of-month stocks of distillate fuel oil are split into two sulfur categories $(0.05$ percent sulfur or less and greater than 0.05 percent sulfur) to meet Environmental Protection Agency requirements effective in October 1992. For further details, see the EIA, Petroleum Supply Monthly.

4. New Stock Basis: In January $1975,1979,1981$, and 1983, numerous respondents were added to bulk terminal and pipeline surveys, affecting subsequent stocks reported and stock change calculations. Using the expanded coverage (new basis), the end-of-year stocks, in million barrels, would have been:

- Crude Oil: 1982-645 (Total) and 351 (Other Primary).

- Crude Oil and Petroleum Products: 1974-1,121; 1980-1,425; and 1982-1,461.

- Motor Gasoline: 1974-225; 1980-263 (Total) and 214 (Finished); 1982-244 (Total) and 202 (Finished).

- Distillate Fuel Oil: 1974-224; 1980-205; and 1982-186.

- Residual Fuel Oil: 1974-75; 1980-91; and 1982-69.

- Jet Fuel: 1974-30 (Total) and 24 (Kerosene Type); $1980-42$ (Total) and 36 (Kerosene Type); and 1982-39 (Total) and 32 (Kerosene Type).

- Liquefied Petroleum Gases: 1974-113; 1978 -136; 1980-128; and 1982-102.

- Propane and Propylene: 1978-86; 1980—69; and 1982-57.

- Other Petroleum Products: 1974-190; 1980 -207; and 1982-219.

Stock change calculations beginning in 1975, 1979, 1981, and 1983 were made by using new basis stock levels.

In January 1984, changes were made in the reporting of natural gas liquids. As a result, unfractionated stream, which was formerly included in the "Other Petroleum Products Supply and Disposition" table, is now reported on a component basis (ethane, propane, normal butane, isobutane, and pentanes plus). Most of these stocks now appear in the "Liquefied Petroleum Gases Supply and 
Disposition" table. This change affects stocks reported and stock change calculations in each table. Under the new basis, end-of-year 1983 stocks, in million barrels, would have been:

- Liquefied Petroleum Gases: 1983-108.

- Propane and Propylene: 1983-55.

- Other Petroleum Products: 1983-210.

In January 1993, changes were made in the monthly surveys to begin collecting bulk terminal and pipeline stocks of oxygenates. This change affected stocks reported and stock change calculations. However, a new basis stock level was not calculated for 1992 end-of-year stocks.
5. Stocks of Alaskan Crude Oil: Stocks of Alaskan Crude oil in transit were included for the first time in January 1981. The major impact of this change is on the reporting of stock change calculations. Using the expanded coverage (new basis), 1980 end-of-year stocks, in million barrels, would have been 488 (Total) and 380 (Other Primary).

6. Data Discrepancies: Due to differences internal to EIA data processing systems, some small discrepancies exist between data in the Monthly Energy Review (MER) and the Petroleum Supply Annual (PSA) and Petroleum Supply Monthly (PSM). The data that have discrepancies are footnoted in Section 3 tables and summarized here.

\begin{tabular}{llcrr} 
Table & \multicolumn{1}{c}{ Data Series } & Year & MER & PSA and $P S M$ \\
Data & Data \\
\hline $3.1 \mathrm{a}$ & Natural Gas Plant Production & 1976 & 1,604 & 1,603 \\
$3.1 \mathrm{~b}$ & Exports, Total & 1979 & 471 & 472 \\
$3.1 \mathrm{~b}$ & Exports, Petroleum Products & 1979 & 236 & 237 \\
$3.1 \mathrm{~b}$ & Net Imports & 1979 & 7,985 & 7,984 \\
$3.2 \mathrm{a}$ & Crude Used Directly & 1976 & -19 & -18 \\
$3.2 \mathrm{a}$ & Imports, SPR & 1978 & 161 & 162 \\
$3.2 \mathrm{a}$ & Crude Used Directly & 1978 & -15 & -14 \\
$3.2 \mathrm{a}$ & Crude Used Directly & 1979 & -14 & -13 \\
$3.2 \mathrm{a}$ & Crude Used Directly & 1980 & -14 & -13 \\
$3.2 \mathrm{~b}$ & Crude Losses & 1976 & 14 & 15 \\
$3.2 \mathrm{~b}$ & Crude Losses & 1980 & 14 & 15 \\
3.5 & Stock Change & 1974 & 10 & 9 \\
3.5 & Stock Change & 1975 & -41 & -40 \\
3.8 & Total Production & 1982 & 1,527 & 1,525 \\
3.10 & Products Supplied & 1982 & 1,857 & 1,856 \\
\hline
\end{tabular}




\section{Section 4. Natural Gas}

Total dry natural gas production in the United States during December 1997 was forecast as 1.6 trillion cubic feet, 4 percent higher than production during the previous December. Dry natural gas production during 1997 was an estimated 19.0 trillion cubic feet, 1 percent higher than production during 1996.

Consumption of natural and supplemental gas in December 1997 was forecast as 2.4 trillion cubic feet, 6 percent above the level in December 1996. Consumption of natural gas and supplemental gas during 1997 was an estimated 22.1 trillion cubic feet, 1 percent higher than consumption during 1996.

Deliveries to residential consumers in December 1997 were forecast as 756 billion cubic feet, 2 percent above the previous December's deliveries. During 1997, deliveries to residential consumers were an estimated 5.0 trillion cubic feet, 4 percent lower than residential deliveries 1 year earlier. Total deliveries to industrial consumers during December 1997 were forecast as 827 billion cubic feet, 2 percent higher than the previous December's level. During 1997, deliveries to industrial consumers were an estimated 8.9 trillion cubic feet, slightly higher than industrial deliveries during 1996.

Net imports of natural gas in December 1997 were estimated as 265 billion cubic feet, 2 percent higher than net imports in the previous December. During 1977 , net imports of natural gas were estimated at 2.8 trillion cubic feet, 2 percent higher than net imports during 1996.

Stocks of working gas ${ }^{1}$ in underground natural gas storage reservoirs at the end of December 1997 were forecast as 2.3 trillion cubic feet, 5 percent above the level of stocks available 1 year earlier. Net injections to storage during December 1997 were forecast as 410 billion cubic feet, 10 percent higher than the amount of net injections during the previous December.

\footnotetext{
${ }^{1}$ Gas available for withdrawal.
} 
Figure 4.1 Natural Gas

(Trillion Cubic Feet)

Overview, 1973-1997

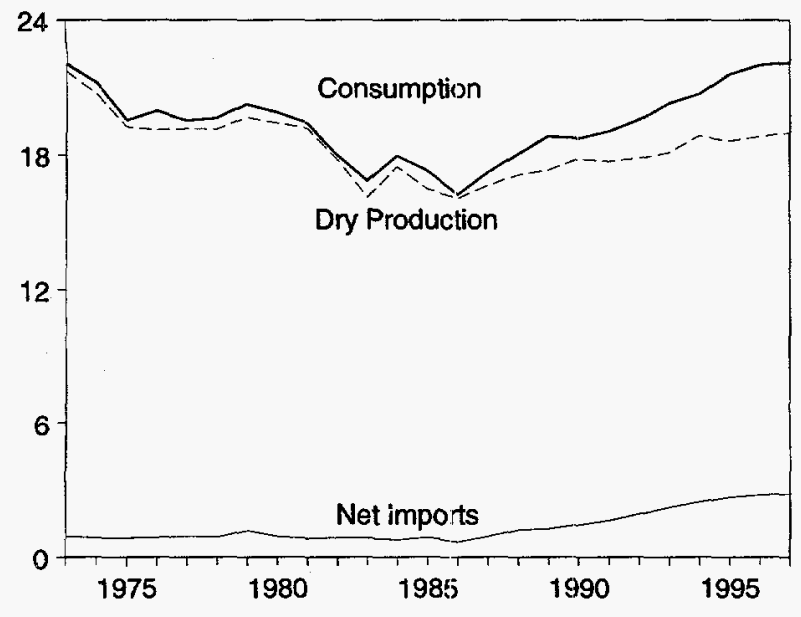

Consumption by Sector, 1973-1997

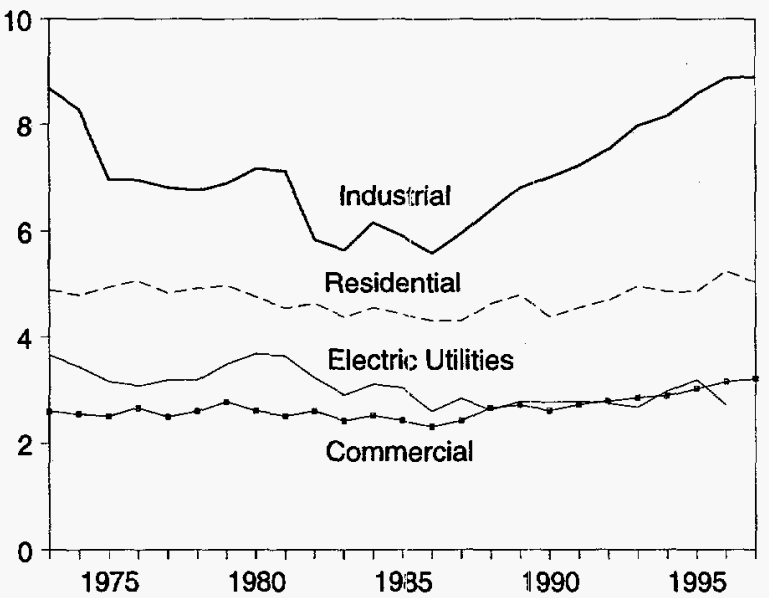

Underground Storage, End of Year, 1973-1997

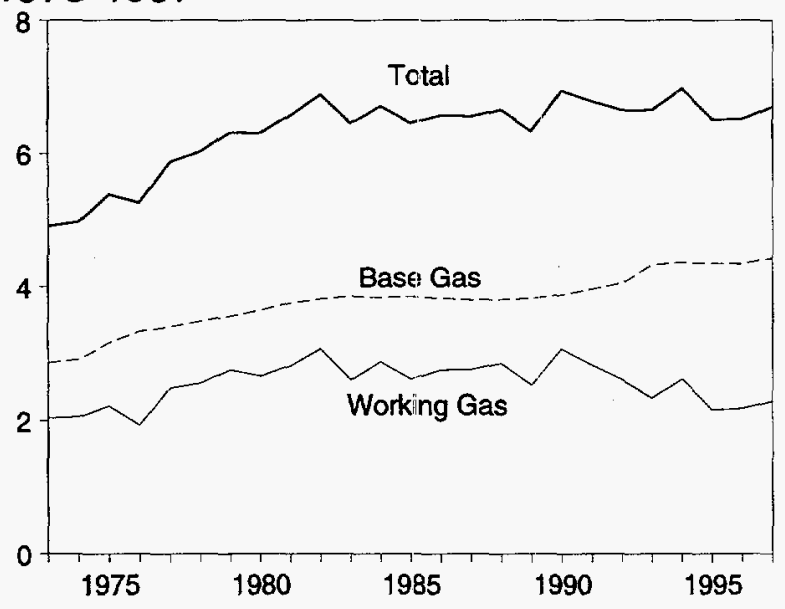

Overview, Monthly

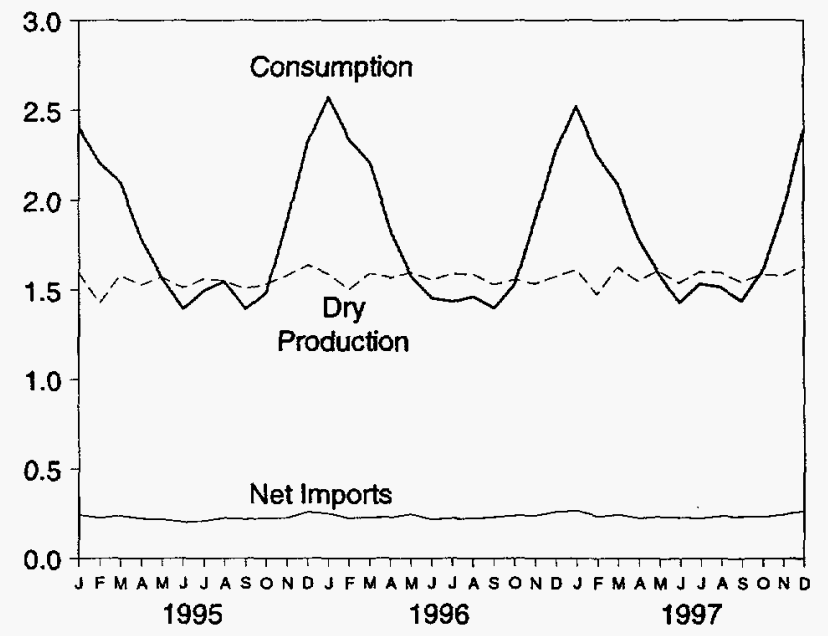

Consumption by Sector, Monthly

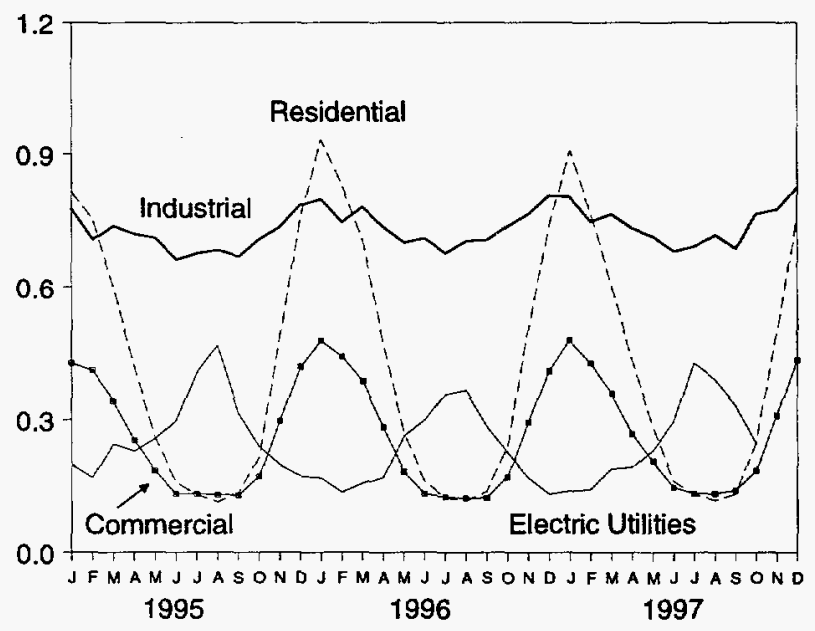

Underground Storage, End of Month

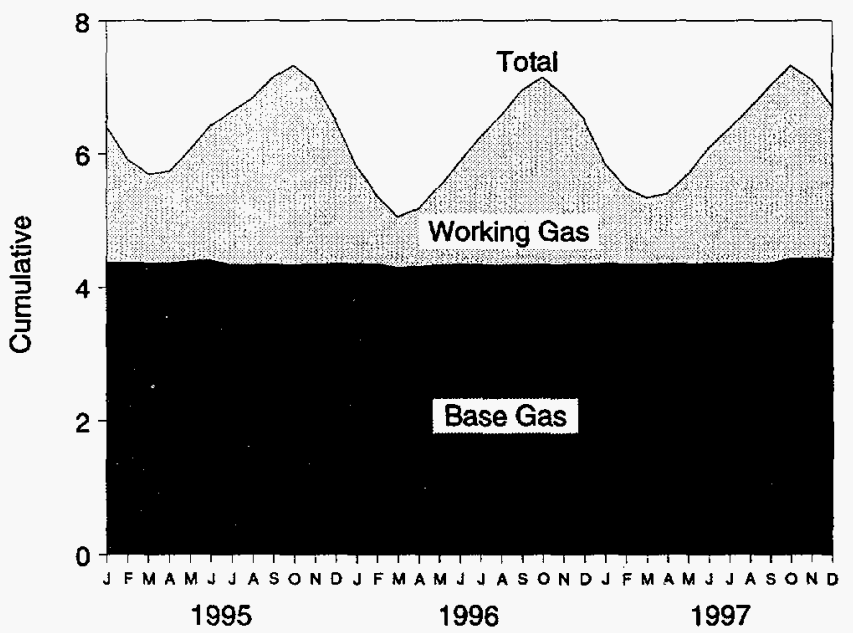

Note: Because vertical scales differ, graphs should not be compared. Sources: Tables $4.1,4.3,4.4$, and 4.5 . 
Table 4.1 Natural Gas Overview

(Billion Cubic Feet)

\begin{tabular}{|c|c|c|c|c|c|c|}
\hline & $\begin{array}{c}\text { Dry Gas } \\
\text { Productiona }\end{array}$ & $\begin{array}{c}\text { Supplemental } \\
\text { Gaseous } \\
\text { Fuels }^{b}\end{array}$ & $\begin{array}{c}\text { Net } \\
\text { Imports }\end{array}$ & $\begin{array}{c}\text { Net } \\
\text { Withdrawals } \\
\text { From } \\
\text { Storaged }\end{array}$ & $\begin{array}{c}\text { Balancing } \\
\text { Item }^{e}\end{array}$ & Consumption ${ }^{f}$ \\
\hline 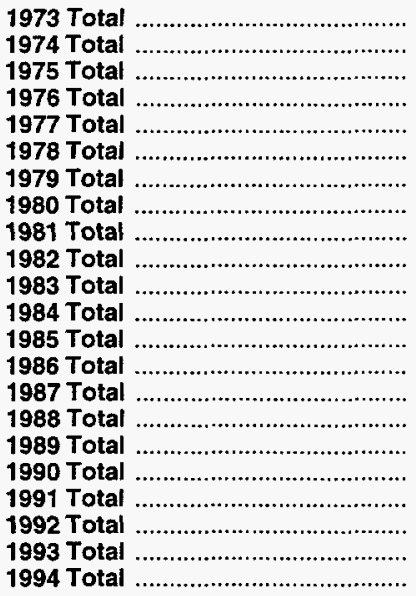 & $\begin{array}{r}921,731 \\
920,713 \\
919,236 \\
919,098 \\
919,163 \\
919,122 \\
919,663 \\
19,403 \\
19,181 \\
17,820 \\
16,094 \\
17,466 \\
16,454 \\
16,059 \\
16,621 \\
17,103 \\
17,311 \\
17,810 \\
17,698 \\
17,840 \\
18,095 \\
18,821\end{array}$ & $\begin{array}{l}\text { NA } \\
\text { NA } \\
\text { NA } \\
\text { NA } \\
\text { NA } \\
\text { NA } \\
\text { NA } \\
155 \\
176 \\
145 \\
132 \\
110 \\
126 \\
113 \\
101 \\
101 \\
107 \\
123 \\
113 \\
118 \\
119 \\
111\end{array}$ & $\begin{array}{r}956 \\
882 \\
880 \\
899 \\
955 \\
913 \\
1,198 \\
936 \\
845 \\
882 \\
864 \\
788 \\
894 \\
689 \\
939 \\
1,220 \\
1,275 \\
1,447 \\
1,644 \\
1,921 \\
2,210 \\
2,462\end{array}$ & $\begin{array}{r}-442 \\
-84 \\
-344 \\
165 \\
-557 \\
-120 \\
-248 \\
23 \\
-297 \\
-308 \\
447 \\
-197 \\
235 \\
-147 \\
-6 \\
59 \\
326 \\
-513 \\
80 \\
173 \\
-36 \\
-286\end{array}$ & $\begin{array}{r}-196 \\
-289 \\
-235 \\
-216 \\
-41 \\
-287 \\
-372 \\
-640 \\
-500 \\
9-537 \\
9-703 \\
-217 \\
-428 \\
-493 \\
-444 \\
-453 \\
-218 \\
-149 \\
-500 \\
-508 \\
-110 \\
-400\end{array}$ & $\begin{array}{l}22,049 \\
21,223 \\
19,538 \\
19,946 \\
19,521 \\
19,627 \\
20,241 \\
19,877 \\
19,404 \\
18,001 \\
16,835 \\
17,951 \\
17,281 \\
16,221 \\
17,211 \\
18,030 \\
18,801 \\
18,716 \\
19,035 \\
19,544 \\
20,279 \\
20,708\end{array}$ \\
\hline 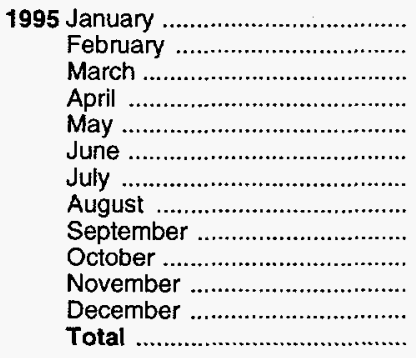 & $\begin{array}{r}1,599 \\
1,426 \\
1,582 \\
1,530 \\
1,572 \\
1,513 \\
1,563 \\
1,552 \\
1,507 \\
1,535 \\
1,580 \\
1,639 \\
18,599\end{array}$ & $\begin{array}{r}12 \\
10 \\
10 \\
7 \\
8 \\
8 \\
8 \\
8 \\
7 \\
9 \\
10 \\
12 \\
110\end{array}$ & $\begin{array}{r}240 \\
223 \\
236 \\
220 \\
216 \\
202 \\
208 \\
223 \\
216 \\
224 \\
224 \\
256 \\
2,687\end{array}$ & $\begin{array}{r}613 \\
531 \\
228 \\
-51 \\
-343 \\
-380 \\
-313 \\
-212 \\
-321 \\
-210 \\
278 \\
595 \\
415\end{array}$ & $\begin{array}{r}-60 \\
17 \\
42 \\
74 \\
115 \\
52 \\
30 \\
-24 \\
-17 \\
-72 \\
-206 \\
-181 \\
-230\end{array}$ & $\begin{array}{r}2,403 \\
2,207 \\
2,098 \\
1,780 \\
1,567 \\
1,395 \\
1,497 \\
1,548 \\
1,393 \\
1,486 \\
1,886 \\
2,321 \\
21,581\end{array}$ \\
\hline 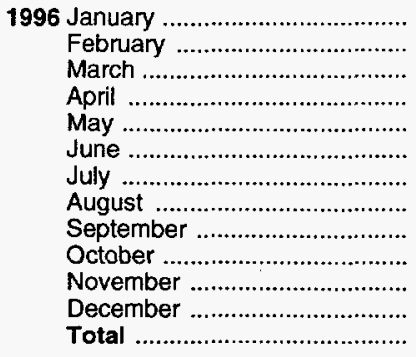 & $\begin{array}{r}1,591 \\
1,504 \\
1,592 \\
1,570 \\
1,598 \\
1,555 \\
1,591 \\
1,590 \\
1,531 \\
1,558 \\
1,537 \\
1,576 \\
18,793\end{array}$ & $\begin{array}{r}12 \\
11 \\
11 \\
9 \\
6 \\
8 \\
8 \\
8 \\
8 \\
9 \\
10 \\
10 \\
109\end{array}$ & $\begin{array}{r}249 \\
221 \\
226 \\
227 \\
244 \\
214 \\
222 \\
221 \\
227 \\
236 \\
238 \\
259 \\
2,784\end{array}$ & $\begin{array}{r}723 \\
462 \\
333 \\
-119 \\
-339 \\
-388 \\
-382 \\
-358 \\
-379 \\
-210 \\
272 \\
387 \\
2\end{array}$ & $\begin{array}{r}-2 \\
138 \\
46 \\
139 \\
67 \\
65 \\
-3 \\
4 \\
12 \\
-62 \\
-161 \\
34 \\
279\end{array}$ & $\begin{array}{r}2,574 \\
2,335 \\
2,209 \\
1,826 \\
1,576 \\
1,454 \\
1,436 \\
1,465 \\
1,399 \\
1,531 \\
1,896 \\
2,266 \\
21,967\end{array}$ \\
\hline 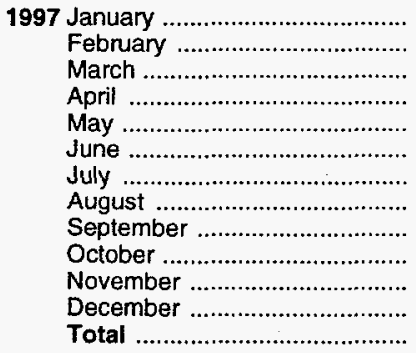 & $\begin{array}{r}1,614 \\
1,476 \\
1,629 \\
1,549 \\
1,609 \\
1,541 \\
R_{1,603} \\
R_{1}, 599 \\
E_{1}, 545 \\
R_{1} \mathbf{1 , 5 8 8} \\
F_{1,578} \\
F_{1,633} \\
E_{18,963}\end{array}$ & $\begin{array}{r}12 \\
11 \\
10 \\
9 \\
9 \\
7 \\
8 \\
9 \\
E_{7} \\
R E_{9} \\
F_{11} \\
F_{12} \\
E_{116}\end{array}$ & $\begin{array}{r}E_{264} \\
E_{231} \\
E_{243} \\
E_{221} \\
E_{229} \\
E_{226} \\
E_{222} \\
R E_{233} \\
R_{229} \\
R E_{229} \\
F_{241} \\
F_{265} \\
E_{2,834}\end{array}$ & $\begin{array}{r}683 \\
358 \\
156 \\
-59 \\
-322 \\
-366 \\
-274 \\
-323 \\
-330 \\
\mathrm{R}_{-212} \\
\mathrm{RF}_{221} \\
\mathrm{~F}_{410} \\
\mathrm{E}_{\mathbf{- 5}} \mathbf{5 8}\end{array}$ & $\begin{array}{r}-55 \\
R 177 \\
R 53 \\
R 63 \\
R 66 \\
22 \\
R-23 \\
R_{-1} \\
R-12 \\
R E-12 \\
R F-120 \\
R_{74} \\
E_{234}\end{array}$ & 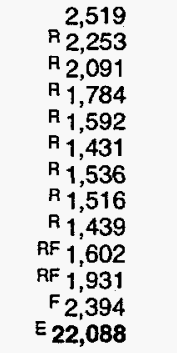 \\
\hline
\end{tabular}

a "Marketed Production (Wet)" minus "Extraction Loss." See Table 4.2.

b See Note 4 at end of section.

c "Imports" minus "Exports." See Table 4.3.

d "Withdrawals". minus "Injections." Data for 1980-1996 cover underground storage and liquefied natural gas storage. All other time periods cover underground storage only. See also Note 8 at end of section.

See Note 7 at end of section. Since 1980, excludes transit shipments that cross the U.S.-Canada border (i.e., natural gas delivered to its destination via the other country).

See Note 6 at end of section.

$g$ May include unknown quantities of nonhydrocarbon gases.

R=Revised data. $N A=$ Not available. $E=$ Estimate. $F=$ Forecast.

Notes: - Totals may not equal sum of components due to independent rounding. - Geographic coverage is the 50 States and the District of Columbia.

Sources: - 1973-1990: Energy Information Administration (EIA), Natural Gas Annual 1996, Table 100. - 1991 torward: EIA, Natural Gas Monthly December 1997, Table 2, except for Balancing Item and Consumption, which incorporate the most current electric utilities data from Table 4.4 of this report. Forecast values are derived from ElA's Short-Term Integrated Forecasting System. See Note 9 at end of section. 
Table 4.2 Natural Gas Production

(Billion Cubic Feet)

\begin{tabular}{|c|c|c|c|c|c|c|c|}
\hline & $\begin{array}{c}\text { Gross } \\
\text { Withdravalsa }\end{array}$ & Repressuringb & $\begin{array}{l}\text { Nonhydro- } \\
\text { carbon Gases } \\
\text { Removed" }\end{array}$ & $\begin{array}{l}\text { Vented } \\
\text { and } \\
\text { Flaredd }\end{array}$ & $\begin{array}{c}\text { Marketed } \\
\text { Production } \\
\text { (Wet)e }\end{array}$ & $\begin{array}{c}\text { Extraction } \\
\text { Loss }^{\dagger}\end{array}$ & $\begin{array}{c}\text { Total } \\
\text { Dry Gas } \\
\text { Productiong }\end{array}$ \\
\hline 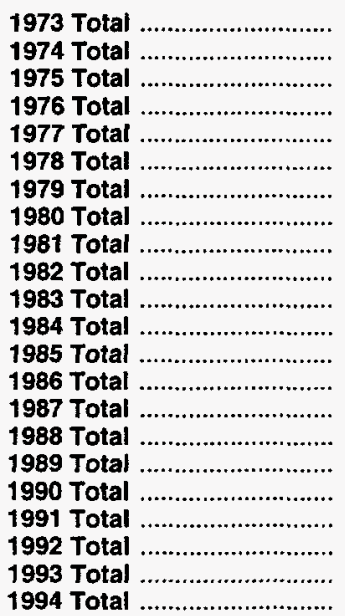 & $\begin{array}{l}24,067 \\
22,850 \\
21,104 \\
20,944 \\
21,097 \\
21,309 \\
21,88: 3 \\
21,870 \\
21,587 \\
20,27: 2 \\
18,659 \\
20,267 \\
19,607 \\
19,131 \\
20,140 \\
20,999 \\
21,074 \\
21,523 \\
21,750 \\
22,132 \\
22,726 \\
23,581\end{array}$ & $\begin{array}{r}1,171 \\
1,080 \\
861 \\
859 \\
935 \\
1,181 \\
1,245 \\
1,365 \\
1,312 \\
1,388 \\
1,458 \\
1,630 \\
1,915 \\
1,838 \\
2,208 \\
2,478 \\
2,475 \\
2,489 \\
2,772 \\
2,973 \\
3,103 \\
3,231\end{array}$ & $\begin{array}{l}\text { NA } \\
\text { NA } \\
\text { NA } \\
\text { NA } \\
\text { NA } \\
\text { NA } \\
\text { NA } \\
199 \\
222 \\
208 \\
222 \\
224 \\
326 \\
337 \\
376 \\
460 \\
362 \\
289 \\
276 \\
280 \\
414 \\
412\end{array}$ & $\begin{array}{r}248 \\
169 \\
134 \\
132 \\
137 \\
153 \\
167 \\
125 \\
98 \\
93 \\
95 \\
108 \\
95 \\
98 \\
124 \\
143 \\
142 \\
150 \\
170 \\
168 \\
227 \\
228\end{array}$ & $\begin{array}{r}h_{22,648} \\
h_{21,601} \\
h_{20,109} \\
h_{19,952} \\
h_{20,025} \\
h_{19,974} \\
h_{20,471} \\
20,180 \\
19,956 \\
18,582 \\
16,884 \\
18,304 \\
17,270 \\
16,859 \\
17,433 \\
17,918 \\
18,095 \\
18,594 \\
18,532 \\
18,712 \\
18,982 \\
19,710\end{array}$ & $\begin{array}{l}917 \\
887 \\
872 \\
854 \\
863 \\
852 \\
808 \\
777 \\
775 \\
762 \\
790 \\
838 \\
816 \\
800 \\
812 \\
816 \\
785 \\
784 \\
835 \\
872 \\
886 \\
889\end{array}$ & $\begin{array}{r}h_{21,731} \\
h_{20,713} \\
h_{19,236} \\
h_{19,098} \\
h_{19,163} \\
h_{19,122} \\
h_{19,663} \\
19,403 \\
19,181 \\
17,820 \\
16,094 \\
17,466 \\
16,454 \\
16,059 \\
16,621 \\
17,103 \\
17,311 \\
17,810 \\
17,698 \\
17,840 \\
18,095 \\
18,821\end{array}$ \\
\hline 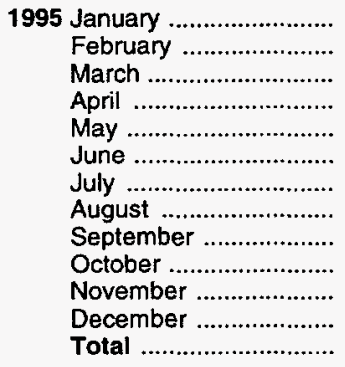 & $\begin{array}{r}2,043 \\
1,822 \\
2,026 \\
1,945 \\
1,997 \\
1,910 \\
1,960 \\
1,965 \\
1,914 \\
1,988 \\
2,045 \\
2,128 \\
23,744\end{array}$ & $\begin{array}{r}311 \\
276 \\
314 \\
287 \\
291 \\
264 \\
264 \\
284 \\
276 \\
319 \\
331 \\
348 \\
3,565\end{array}$ & $\begin{array}{r}34 \\
30 \\
32 \\
32 \\
33 \\
31 \\
31 \\
30 \\
33 \\
34 \\
33 \\
35 \\
388\end{array}$ & $\begin{array}{l}21 \\
20 \\
20 \\
21 \\
24 \\
28 \\
26 \\
22 \\
25 \\
25 \\
24 \\
26 \\
284\end{array}$ & $\begin{array}{r}1,677 \\
1,495 \\
1,660 \\
1,604 \\
1,649 \\
1,587 \\
1,639 \\
1,628 \\
1,581 \\
1,610 \\
1,657 \\
1,719 \\
19,506\end{array}$ & $\begin{array}{l}78 \\
70 \\
77 \\
75 \\
77 \\
74 \\
76 \\
76 \\
74 \\
75 \\
77 \\
80 \\
908\end{array}$ & $\begin{array}{r}1,599 \\
1,426 \\
1,582 \\
1,530 \\
1,572 \\
1,513 \\
1,563 \\
1,552 \\
1,507 \\
1,535 \\
1,580 \\
1,639 \\
18,599\end{array}$ \\
\hline 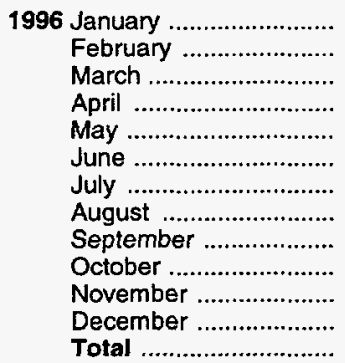 & $\begin{array}{r}2,0512 \\
1,941 \\
2,05 i 4 \\
2,0013 \\
2,02.5 \\
1,9622 \\
2,0018 \\
2,02.1 \\
1,958 \\
2,011 \\
1,984 \\
2,032 \\
24,052\end{array}$ & $\begin{array}{l}310 \\
294 \\
313 \\
289 \\
281 \\
276 \\
271 \\
281 \\
283 \\
306 \\
299 \\
307 \\
\mathbf{3 , 5 1 0}\end{array}$ & $\begin{array}{r}44 \\
41 \\
45 \\
42 \\
42 \\
36 \\
42 \\
45 \\
44 \\
44 \\
47 \\
46 \\
518\end{array}$ & $\begin{array}{l}26 \\
24 \\
23 \\
22 \\
23 \\
16 \\
24 \\
24 \\
22 \\
23 \\
23 \\
23 \\
272\end{array}$ & $\begin{array}{r}1,673 \\
1,580 \\
1,674 \\
1,650 \\
1,679 \\
1,634 \\
1,672 \\
1,671 \\
1,609 \\
1,638 \\
1,615 \\
1,656 \\
19,751\end{array}$ & $\begin{array}{r}81 \\
77 \\
81 \\
80 \\
81 \\
79 \\
81 \\
81 \\
78 \\
79 \\
78 \\
80 \\
958\end{array}$ & $\begin{array}{r}1,591 \\
1,504 \\
1,592 \\
1,570 \\
1,598 \\
1,555 \\
1,591 \\
1,590 \\
1,531 \\
1,558 \\
1,537 \\
1,576 \\
18,793\end{array}$ \\
\hline 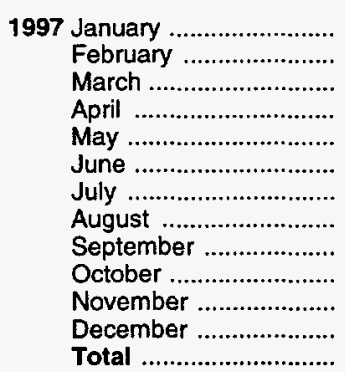 & $\begin{array}{r}E_{2,032} \\
E_{1,905} \\
E_{2,036} \\
E_{1}, 974 \\
E_{2,055} \\
E_{1}, 962 \\
R E_{2,031} \\
R E_{2,015} \\
R E_{1}, 957 \\
R E_{2,009} \\
\text { NAA } \\
\text { NA } \\
\text { NAA }\end{array}$ & $\begin{array}{r}E_{327} \\
E_{301} \\
E_{321} \\
E_{296} \\
E_{313} \\
E_{294} \\
E_{295} \\
R E_{283} \\
R E_{284} \\
R E_{288} \\
N A \\
N A \\
\text { NA }\end{array}$ & $\begin{array}{r}41 \\
38 \\
34 \\
33 \\
\text { E } 33 \\
31 \\
34 \\
\text { RE } 34 \\
\text { E } 32 \\
\text { RE } 33 \\
\text { NA } \\
\text { NA } \\
\text { NA }\end{array}$ & $\begin{array}{r}E_{21} \\
E_{19} \\
E_{22} \\
E_{21} \\
E_{21} \\
E_{20} \\
R_{22} \\
R_{22} \\
E_{21} \\
\text { RE } 21_{21} \\
\text { NA } \\
\text { NA } \\
\text { NA }\end{array}$ & $\begin{array}{r}E_{1,693} \\
E_{1,548} \\
E_{1,708} \\
E_{1,625} \\
E_{1,688} \\
E_{1,616} \\
R_{1,681} \\
R_{1,677} \\
E_{1,620} \\
E_{1,666} \\
F_{1,658} \\
E_{1,19,897}\end{array}$ & $\begin{array}{r}79 \\
72 \\
80 \\
76 \\
79 \\
75 \\
R 78 \\
78 \\
\text { E } 75 \\
\text { RE } 78 \\
\text { F } 80 \\
\text { F } 84 \\
\text { E } 934\end{array}$ & $\begin{array}{r}1,614 \\
1,476 \\
1,629 \\
1,549 \\
1,609 \\
1,541 \\
R_{1,603} \\
R_{1,599} \\
E_{1,545} \\
R_{1} 1,588 \\
F_{1,578} \\
F_{1}, 633 \\
E_{18,963}\end{array}$ \\
\hline
\end{tabular}

a Gas withdrawn from gas and oil wells.

b The injection of natural gas into cil and gas formations for pressure maintenance and cycling purposes.

$c$ See Note 1 at end of section.

d Vented: Natural gas released into the air on the base site or at processing plants. Flared: Natural gas bumed in flares on the base site or at gas processing plants.

e "Gross Withdrawals" minus "Repressuring," "Nonhydrocarbon Gases Removed," and "Vented and Flared." See Note 2 at end of section.

1 See Note 3 at end of section.

g "Marketed Production (Wet)" minus "Extraction Loss."

h May include unknown quantities of nonhydrocarbon gases.

$\mathrm{R}=$ Revised data. $\mathrm{NA}=$ Not available. $\mathrm{E}=$ Estimate. F=Forecast.

Notes: - Totals may not equal sum of components due to independent rounding. - Geographic coverage is the 50 States and the District of Columbia.

Sources: - 1973-1990: Energy Information Administration (E|A), Natural Gas Annual 1996, Table 99. - 1991 forward: ElA, Natural Gas Monthly, December 1997, Table 1. Forecast values are derived from EIA's Short-Term Integrated Forecasting System. See Note 9 at end of section. 


\section{Table 4.3 Natural Gas Trade by Country}

(Billion Cubic Feet)

\begin{tabular}{|c|c|c|c|c|c|c|c|c|c|}
\hline & \multicolumn{5}{|c|}{ Imports } & \multicolumn{4}{|c|}{ Exports } \\
\hline & Canada ${ }^{a}$ & Algeriab & Mexico ${ }^{a}$ & $\begin{array}{l}\text { United Arab } \\
\text { Emirates }^{b}\end{array}$ & Total & Canada $^{a}$ & Mexicoa & Japanb & Total \\
\hline 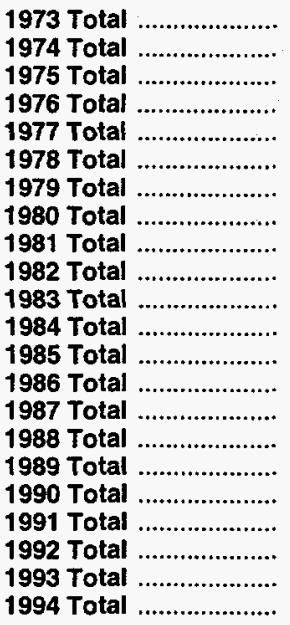 & $\begin{array}{r}1,028 \\
959 \\
948 \\
954 \\
997 \\
881 \\
1,001 \\
797 \\
762 \\
783 \\
712 \\
755 \\
926 \\
749 \\
993 \\
1,276 \\
1,339 \\
1,448 \\
1,710 \\
2,094 \\
2,267 \\
2,566\end{array}$ & $\begin{array}{r}3 \\
0 \\
5 \\
10 \\
11 \\
84 \\
253 \\
86 \\
37 \\
55 \\
131 \\
36 \\
24 \\
0 \\
0 \\
17 \\
42 \\
84 \\
64 \\
43 \\
82 \\
51\end{array}$ & $\begin{array}{r}2 \\
(s) \\
0 \\
0 \\
2 \\
0 \\
0 \\
102 \\
105 \\
95 \\
75 \\
52 \\
0 \\
0 \\
0 \\
0 \\
0 \\
0 \\
0 \\
0 \\
2 \\
7\end{array}$ & $\begin{array}{l}0 \\
0 \\
0 \\
0 \\
0 \\
0 \\
0 \\
0 \\
0 \\
0 \\
0 \\
0 \\
0 \\
0 \\
0 \\
0 \\
0 \\
0 \\
0 \\
0 \\
0 \\
0\end{array}$ & $\begin{array}{r}1,033 \\
959 \\
953 \\
964 \\
1,011 \\
966 \\
1,253 \\
985 \\
904 \\
933 \\
918 \\
843 \\
950 \\
6750 \\
993 \\
1,294 \\
1,382 \\
1,532 \\
1,773 \\
2,138 \\
2,350 \\
2,624\end{array}$ & $\begin{array}{r}15 \\
13 \\
10 \\
8 \\
(s) \\
(s) \\
(s) \\
(s) \\
(s) \\
(s) \\
(s) \\
(s) \\
(s) \\
9 \\
3 \\
20 \\
38 \\
17 \\
15 \\
68 \\
45 \\
53\end{array}$ & $\begin{array}{r}14 \\
13 \\
9 \\
7 \\
4 \\
4 \\
4 \\
4 \\
3 \\
2 \\
2 \\
2 \\
2 \\
2 \\
2 \\
2 \\
17 \\
16 \\
60 \\
96 \\
40 \\
47\end{array}$ & $\begin{array}{l}48 \\
50 \\
53 \\
50 \\
\mathbf{5 2} \\
\mathbf{4 8} \\
\mathbf{5 1} \\
\mathbf{4 5} \\
\mathbf{5 6} \\
\mathbf{5 0} \\
\mathbf{5 3} \\
\mathbf{5 3} \\
\mathbf{5 3} \\
\mathbf{5 0} \\
\mathbf{4 9} \\
\mathbf{5 2} \\
\mathbf{5 1} \\
\mathbf{5 3} \\
\mathbf{5 4} \\
\mathbf{5 3} \\
\mathbf{5 6} \\
\mathbf{6 3}\end{array}$ & $\begin{array}{r}77 \\
77 \\
73 \\
65 \\
56 \\
53 \\
56 \\
49 \\
59 \\
52 \\
55 \\
55 \\
55 \\
61 \\
54 \\
74 \\
107 \\
86 \\
129 \\
216 \\
140 \\
162\end{array}$ \\
\hline 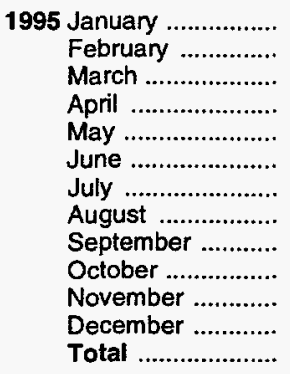 & $\begin{array}{r}251 \\
233 \\
248 \\
232 \\
226 \\
217 \\
223 \\
233 \\
224 \\
234 \\
234 \\
262 \\
2,816\end{array}$ & $\begin{array}{r}3 \\
3 \\
3 \\
0 \\
3 \\
0 \\
0 \\
3 \\
0 \\
0 \\
2 \\
3 \\
18\end{array}$ & $\begin{array}{r}(\mathrm{s}) \\
0 \\
(\mathrm{~s}) \\
0 \\
0 \\
0 \\
0 \\
1 \\
4 \\
2 \\
0 \\
0 \\
7\end{array}$ & $\begin{array}{l}0 \\
0 \\
0 \\
0 \\
0 \\
0 \\
0 \\
0 \\
0 \\
0 \\
0 \\
0 \\
0\end{array}$ & $\begin{array}{r}253 \\
236 \\
250 \\
232 \\
228 \\
217 \\
223 \\
237 \\
228 \\
236 \\
236 \\
264 \\
2,841\end{array}$ & $\begin{array}{r}3 \\
2 \\
2 \\
2 \\
2 \\
2 \\
2 \\
3 \\
3 \\
3 \\
2 \\
1 \\
28\end{array}$ & $\begin{array}{l}6 \\
6 \\
7 \\
6 \\
7 \\
8 \\
7 \\
3 \\
2 \\
6 \\
4 \\
1 \\
61\end{array}$ & $\begin{array}{l}6 \\
6 \\
6 \\
4 \\
4 \\
6 \\
6 \\
8 \\
6 \\
4 \\
8 \\
6 \\
65\end{array}$ & $\begin{array}{r}14 \\
13 \\
15 \\
12 \\
12 \\
16 \\
15 \\
14 \\
11 \\
12 \\
13 \\
8 \\
154\end{array}$ \\
\hline 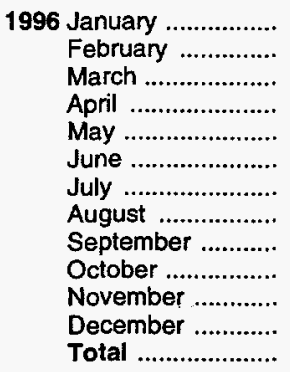 & $\begin{array}{r}260 \\
231 \\
238 \\
231 \\
246 \\
226 \\
233 \\
235 \\
234 \\
241 \\
246 \\
264 \\
2,883\end{array}$ & $\begin{array}{l}2 \\
3 \\
3 \\
5 \\
3 \\
0 \\
3 \\
3 \\
0 \\
5 \\
5 \\
5 \\
35\end{array}$ & $\begin{array}{r}1 \\
1 \\
1 \\
1 \\
4 \\
1 \\
1 \\
(\mathrm{~s}) \\
1 \\
1 \\
1 \\
(\mathrm{~s}) \\
14\end{array}$ & $\begin{array}{l}0 \\
0 \\
0 \\
0 \\
0 \\
0 \\
0 \\
0 \\
3 \\
0 \\
0 \\
2 \\
5\end{array}$ & $\begin{array}{r}264 \\
234 \\
242 \\
237 \\
252 \\
227 \\
237 \\
238 \\
238 \\
248 \\
252 \\
271 \\
2,937\end{array}$ & $\begin{array}{r}7 \\
5 \\
7 \\
2 \\
3 \\
3 \\
4 \\
2 \\
3 \\
4 \\
7 \\
5 \\
52\end{array}$ & $\begin{array}{r}2 \\
2 \\
3 \\
2 \\
2 \\
3 \\
3 \\
9 \\
2 \\
2 \\
2 \\
2 \\
34\end{array}$ & $\begin{array}{l}6 \\
6 \\
6 \\
6 \\
4 \\
6 \\
8 \\
6 \\
6 \\
6 \\
6 \\
6 \\
68\end{array}$ & $\begin{array}{r}14 \\
13 \\
15 \\
10 \\
8 \\
12 \\
14 \\
17 \\
11 \\
12 \\
14 \\
13 \\
153\end{array}$ \\
\hline 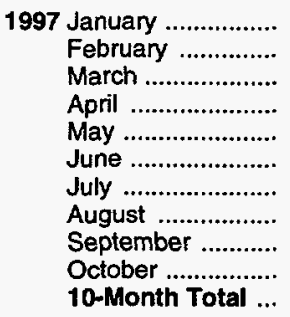 & $\begin{array}{r}265 \\
234 \\
254 \\
232 \\
232 \\
229 \\
226 \\
R_{241} \\
R_{2} 233 \\
\text { E }_{235} \\
\text { E } 2,381_{2,38}\end{array}$ & $\begin{array}{r}8 \\
8 \\
3 \\
3 \\
3 \\
5 \\
5 \\
8 \\
5 \\
5 \\
51\end{array}$ & $\begin{array}{r}1 \\
2 \\
3 \\
(s) \\
2 \\
2 \\
E_{1} \\
E_{0} \\
E_{1}(s) \\
E_{1} \\
E_{12}\end{array}$ & $\begin{array}{l}2 \\
0 \\
0 \\
0 \\
0 \\
0 \\
0 \\
0 \\
0 \\
0 \\
2\end{array}$ & $\begin{array}{r}276 \\
243 \\
260 \\
235 \\
\mathrm{~d}_{239} \\
235 \\
\mathrm{E} 231 \\
\mathrm{RE} 249 \\
\mathrm{~d} 240 \\
\mathrm{E} 241 \\
2, \mathbf{4 5 1}\end{array}$ & $\begin{array}{r}4 \\
5 \\
9 \\
5 \\
4 \\
3 \\
\mathrm{E}_{3} \\
\mathrm{E}_{3} \\
\mathrm{E}_{3} \\
\mathrm{E}_{3} \\
\mathrm{E}_{\mathbf{4 1}}\end{array}$ & $\begin{array}{r}2 \\
2 \\
1 \\
3 \\
2 \\
3 \\
E_{3} \\
E_{6} \\
E_{5} \\
E_{4} \\
E_{31}\end{array}$ & $\begin{array}{r}6 \\
6 \\
6 \\
6 \\
4 \\
4 \\
4 \\
8 \\
4 \\
6 \\
51\end{array}$ & $\begin{array}{r}12 \\
12 \\
16 \\
14 \\
10 \\
10 \\
E_{9} \\
E_{16} \\
E_{12} \\
E_{12} \\
E_{123}\end{array}$ \\
\hline $\begin{array}{l}1996 \text { 10-Month Total ... } \\
1995 \text { 10-Month Total ... }\end{array}$ & $\begin{array}{l}2,374 \\
2,321\end{array}$ & $\begin{array}{l}25 \\
13\end{array}$ & $\begin{array}{r}13 \\
7\end{array}$ & $\begin{array}{l}3 \\
0\end{array}$ & $\begin{array}{l}2,414 \\
2,340\end{array}$ & $\begin{array}{l}40 \\
25\end{array}$ & $\begin{array}{l}30 \\
56\end{array}$ & $\begin{array}{l}56 \\
52\end{array}$ & $\begin{array}{l}127 \\
133\end{array}$ \\
\hline
\end{tabular}

a By pipeline, except for very small amounts of liquefied natural gas imported from Canada in 1973, 1977 and 1981. See Note 5 at end of section.

b As liquefied natural gas.

c Includes 2 billion cubic feet of liquefied natural gas from Indonesia.

Includes 2 billion cubic feet of liquefied natural gas from Australia.

$R=$ Revised data. $E=E s t i m a t e$. (s)=Less than 500 million cubic feet.
Notes: - See Note 5 at end of section. - Totals may not equal sum of components due to independent rounding. - U.S. geographic coverage is the 50 States and the District of Columbia.

Sources: - 1973-1990: Energy Information Administration (EIA), Form FPC-14, "Annual Report for Importers and Exporters of Natural Gas." - 1991 forward: EIA, Natural Gas Monthly, December 1997, Tables 5 and 6. 
Table 4.4 Natural Gas Consumption by End-Use Sector

(Billion Cubic Feet)

\begin{tabular}{|c|c|c|c|c|c|c|c|c|c|}
\hline & \multirow[b]{2}{*}{$\begin{array}{l}\text { Lease and } \\
\text { Plant Fuel }\end{array}$} & \multirow[b]{2}{*}{$\begin{array}{c}\text { Pipeline } \\
\text { Fuela }\end{array}$} & \multicolumn{6}{|c|}{ Delivered to Consumers } & \multirow[b]{2}{*}{$\begin{array}{c}\text { Total } \\
\text { Consumption }\end{array}$} \\
\hline & & & Residential & Commercial & Industrial & Vehicles & $\begin{array}{l}\text { Electric } \\
\text { Utilities }\end{array}$ & Total & \\
\hline 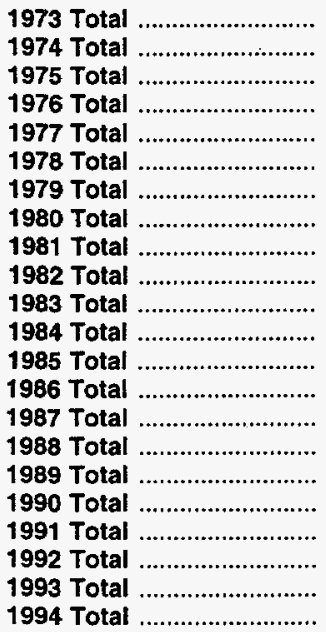 & $\begin{array}{r}1,496 \\
1,477 \\
1,396 \\
1,634 \\
1,659 \\
1,648 \\
1,499 \\
1,026 \\
928 \\
1,109 \\
978 \\
1,077 \\
966 \\
923 \\
1,149 \\
1,096 \\
1,070 \\
1,236 \\
1,129 \\
1,171 \\
1,172 \\
1,124\end{array}$ & $\begin{array}{l}7: 28 \\
6159 \\
5133 \\
548 \\
533 \\
530 \\
6101 \\
6: 35 \\
6412 \\
5196 \\
4 ! 90 \\
5: 29 \\
5134 \\
4135 \\
5: 19 \\
614 \\
6: 29 \\
6150 \\
6101 \\
5: 88 \\
6: 24 \\
6: 35\end{array}$ & $\begin{array}{l}4,879 \\
4,786 \\
4,924 \\
5,051 \\
4,821 \\
4,903 \\
4,965 \\
4,752 \\
4,546 \\
4,633 \\
4,381 \\
4,555 \\
4,433 \\
4,314 \\
4,315 \\
4,630 \\
4,781 \\
4,391 \\
4,556 \\
4,690 \\
4,956 \\
4,848\end{array}$ & $\begin{array}{l}2,597 \\
2,556 \\
2,508 \\
2,668 \\
2,501 \\
2,601 \\
2,786 \\
2,611 \\
2,520 \\
2,606 \\
2,433 \\
2,524 \\
2,432 \\
2,318 \\
2,430 \\
2,670 \\
2,718 \\
2,623 \\
2,729 \\
2,803 \\
2,862 \\
2,895\end{array}$ & $\begin{array}{l}8,689 \\
8,292 \\
6,968 \\
6,964 \\
6,815 \\
6,757 \\
6,899 \\
7,172 \\
7,128 \\
5,831 \\
5,643 \\
6,154 \\
5,901 \\
5,579 \\
5,953 \\
6,383 \\
6,816 \\
7,018 \\
7,231 \\
7,527 \\
7,981 \\
8,167\end{array}$ & $\begin{array}{l}\text { NA } \\
\text { NA } \\
\text { NA } \\
\text { NA } \\
\text { NA } \\
\text { NA } \\
\text { NA } \\
\text { NA } \\
\text { NA } \\
\text { NA } \\
\text { NA } \\
\text { NA } \\
\text { NA } \\
\text { NA } \\
\text { NA } \\
\text { NA } \\
\text { NA } \\
\text { (s) } \\
\text { (s) } \\
1 \\
1 \\
2\end{array}$ & $\begin{array}{l}3,660 \\
3,443 \\
3,158 \\
3,081 \\
3,191 \\
3,188 \\
3,491 \\
3,682 \\
3,640 \\
3,226 \\
2,911 \\
3,111 \\
3,044 \\
2,602 \\
2,844 \\
2,636 \\
2,787 \\
2,787 \\
2,789 \\
2,766 \\
2,682 \\
2,987\end{array}$ & $\begin{array}{l}19,825 \\
19,077 \\
17,558 \\
17,764 \\
17,329 \\
17,449 \\
18,141 \\
18,216 \\
17,834 \\
16,295 \\
15,367 \\
16,345 \\
15,811 \\
14,814 \\
15,542 \\
16,320 \\
17,102 \\
16,820 \\
17,305 \\
17,786 \\
18,483 \\
18,899\end{array}$ & $\begin{array}{l}22,049 \\
21,223 \\
19,538 \\
19,946 \\
19,521 \\
19,627 \\
20,241 \\
19,877 \\
19,404 \\
18,001 \\
16,835 \\
17,951 \\
17,281 \\
16,221 \\
17,211 \\
18,030 \\
18,801 \\
18,716 \\
19,035 \\
19,544 \\
20,279 \\
20,708\end{array}$ \\
\hline 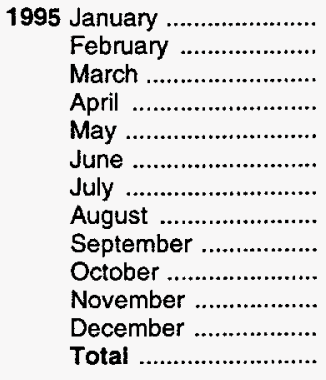 & $\begin{array}{r}105 \\
94 \\
104 \\
100 \\
103 \\
99 \\
101 \\
101 \\
99 \\
102 \\
105 \\
109 \\
1,220\end{array}$ & $\begin{array}{r}79 \\
73 \\
69 \\
58 \\
50 \\
45 \\
48 \\
50 \\
45 \\
48 \\
61 \\
76 \\
700\end{array}$ & $\begin{array}{r}816 \\
754 \\
600 \\
419 \\
260 \\
159 \\
131 \\
114 \\
134 \\
216 \\
489 \\
758 \\
4,850\end{array}$ & $\begin{array}{r}427 \\
411 \\
342 \\
254 \\
184 \\
133 \\
133 \\
130 \\
130 \\
171 \\
297 \\
420 \\
3,031\end{array}$ & $\begin{array}{r}777 \\
707 \\
738 \\
720 \\
711 \\
663 \\
677 \\
684 \\
670 \\
709 \\
736 \\
786 \\
8,580\end{array}$ & $\begin{array}{r}\text { NA } \\
\text { NA } \\
\text { NA } \\
\text { NA } \\
\text { NA } \\
\text { NA } \\
\text { NA } \\
\text { NA } \\
\text { NA } \\
\text { NA } \\
\text { NA } \\
\text { NA } \\
3\end{array}$ & $\begin{array}{r}199 \\
168 \\
245 \\
229 \\
258 \\
297 \\
407 \\
468 \\
316 \\
240 \\
198 \\
172 \\
3,197\end{array}$ & $\begin{array}{r}2,218 \\
2,040 \\
1,926 \\
1,622 \\
1,414 \\
1,252 \\
1,347 \\
1,397 \\
1,250 \\
1,336 \\
1,720 \\
2,136 \\
19,660\end{array}$ & $\begin{array}{r}2,403 \\
2,207 \\
2,098 \\
1,780 \\
1,567 \\
1,395 \\
1,497 \\
1,548 \\
1,393 \\
1,486 \\
1,886 \\
2,321 \\
21,581\end{array}$ \\
\hline 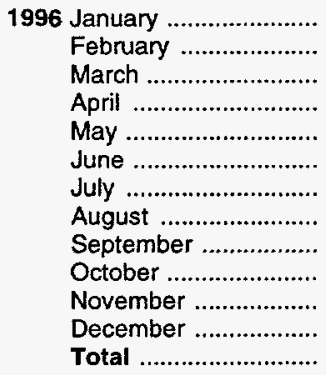 & $\begin{array}{r}106 \\
101 \\
106 \\
104 \\
106 \\
102 \\
105 \\
105 \\
102 \\
104 \\
103 \\
105 \\
1,250\end{array}$ & $\begin{array}{r}85 \\
77 \\
72 \\
59 \\
50 \\
46 \\
46 \\
47 \\
45 \\
49 \\
62 \\
74 \\
711\end{array}$ & $\begin{array}{r}934 \\
831 \\
705 \\
474 \\
271 \\
162 \\
124 \\
118 \\
138 \\
243 \\
503 \\
738 \\
5,241\end{array}$ & $\begin{array}{r}480 \\
443 \\
387 \\
284 \\
183 \\
133 \\
126 \\
123 \\
124 \\
171 \\
295 \\
409 \\
3,158\end{array}$ & $\begin{array}{r}800 \\
747 \\
781 \\
736 \\
701 \\
710 \\
677 \\
704 \\
706 \\
737 \\
764 \\
807 \\
\mathbf{8 , 8 7 0}\end{array}$ & $\begin{array}{l}\text { NA } \\
\text { NA } \\
\text { NA } \\
\text { NA } \\
\text { NA } \\
\text { NA } \\
\text { NA } \\
\text { NA } \\
\text { NA } \\
\text { NA } \\
\text { NA } \\
\text { NA } \\
3\end{array}$ & $\begin{array}{r}168 \\
137 \\
156 \\
170 \\
264 \\
299 \\
358 \\
367 \\
285 \\
226 \\
170 \\
132 \\
2,732\end{array}$ & $\begin{array}{r}2,382 \\
2,157 \\
2,030 \\
1,663 \\
1,420 \\
1,305 \\
1,285 \\
1,312 \\
1,253 \\
1,378 \\
1,732 \\
2,086 \\
20,005\end{array}$ & $\begin{array}{r}2,574 \\
2,335 \\
2,209 \\
1,826 \\
1,576 \\
1,454 \\
1,436 \\
1,465 \\
1,399 \\
1,531 \\
1,896 \\
2,266 \\
21,967\end{array}$ \\
\hline 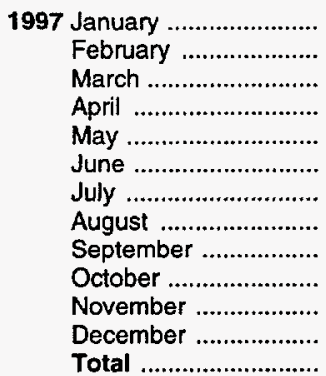 & $\begin{array}{r}106 \\
97 \\
107 \\
102 \\
106 \\
101 \\
R 105 \\
105 \\
R 101 \\
F 104 \\
F 103 \\
F 113 \\
E_{1,250}\end{array}$ & $\begin{array}{r}82 \\
73 \\
68 \\
58 \\
52 \\
46 \\
50 \\
49 \\
\text { R } 47 \\
\text { F } 53 \\
\text { F } 63 \\
\text { F } 77 \\
\text { E } 717\end{array}$ & $\begin{array}{r}R 908 \\
R 766 \\
604 \\
434 \\
R_{285} \\
R_{161} \\
131 \\
119 \\
R_{132} \\
F_{250} \\
R_{495} \\
F_{756} \\
E_{5} \mathbf{5 , 0 4 1}\end{array}$ & $\begin{array}{r}R_{480} \\
427 \\
359 \\
R_{267} \\
R_{206} \\
147 \\
R_{133} \\
134 \\
R_{140} \\
F_{184} \\
F_{309} \\
F_{434} \\
\text { E }_{3,220}\end{array}$ & $\begin{array}{r}\text { R } 804 \\
747 \\
\text { R } 764 \\
\text { R } 731 \\
713 \\
680 \\
691 \\
\text { R } 718 \\
\text { R } 687 \\
\text { F } 765 \\
F 775 \\
\text { F } 827 \\
\text { E } 8,903\end{array}$ & $\begin{array}{l}\text { NA } \\
\text { NA } \\
\text { NA } \\
\text { NA } \\
\text { NA } \\
\text { NA } \\
\text { NA } \\
\text { NA } \\
\text { NA } \\
\text { NA } \\
\text { NA } \\
\text { NA } \\
\text { NA }\end{array}$ & $\begin{array}{c}139 \\
143 \\
189 \\
193 \\
231 \\
295 \\
427 \\
390 \\
332 \\
R 246 \\
\text { NA } \\
\text { NA } \\
\text { NA }\end{array}$ & $\begin{array}{r}2,331 \\
R_{2,083} \\
R_{1,917} \\
R_{1,625} \\
R_{1,435} \\
1,283 \\
R_{1,381} \\
R_{1,362} \\
R_{1,291} \\
F_{1,445} \\
R_{1,765} \\
F_{2,204} \\
E_{20,121}\end{array}$ & $\begin{array}{r}2,519 \\
R_{2,253} \\
R_{2,091} \\
R_{1,784} \\
R_{1,592} \\
R_{1,431} \\
R_{1,536} \\
R_{1,516} \\
R_{1,439} \\
R_{1} F_{1,602} \\
R_{1,931} F_{1,934} \\
F_{2,394} \\
E_{22,088}\end{array}$ \\
\hline
\end{tabular}

a Natural gas consumed in the operation of pipelines, primarily in compressors.

$R=$ Revised data. $N A=N o t$ available. E=Estimate. F=Forecast. $(s)=$ Less than $\mathbf{5 0 0}$ million cubic feet.

Notes: - Natural gas includes supplemental gaseous fuels. - Totals may not equal sum of components due to independent rounding. - Geographic coverage is the $\mathbf{5 0}$ States and the District of Columbia.
Sources: - 1973-1990: Energy Information Administration (ElA), Natural Gas Annual 1996, Table 101. - 1991 forward: ElA, Natural Gas Monthly, December 1997, Table 3, except for the electric utilities values, which come from Table 7.3 of this report, and columns 8 and 9 , which incorporate the values from column 7. Forecast values are derived from ElA's Short-Term Integrated Forecasting System. 
Table 4.5 Natural Gas in Underground Storage (Volumes in Billion Cubic Feet)

\begin{tabular}{|c|c|c|c|c|c|c|c|c|}
\hline & \multicolumn{3}{|c|}{$\begin{array}{l}\text { Natural Gas in } \\
\text { Underground Storage, } \\
\text { End of Period }\end{array}$} & \multicolumn{2}{|c|}{$\begin{array}{c}\text { Change in Working Gas } \\
\text { from Same Period } \\
\text { Previous Year }\end{array}$} & \multicolumn{3}{|c|}{ Storage Activity } \\
\hline & Base Gas & Working Gas & Totala & Volume & Percent & Withdrawals & Injections & $\operatorname{Net}^{b, c}$ \\
\hline 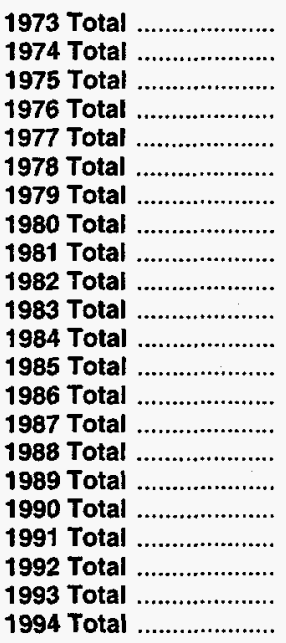 & $\begin{array}{l}2,864 \\
2,912 \\
3,162 \\
3,323 \\
3,391 \\
3,473 \\
3,553 \\
3,642 \\
3,752 \\
3,808 \\
3,847 \\
3,830 \\
3,842 \\
3,819 \\
3,792 \\
3,800 \\
3,812 \\
3,868 \\
3,954 \\
4,044 \\
4,327 \\
4,360\end{array}$ & $\begin{array}{l}2,034 \\
2,050 \\
2,212 \\
1,926 \\
2,475 \\
2,547 \\
2,753 \\
2,655 \\
2,817 \\
3,071 \\
2,595 \\
2,876 \\
2,607 \\
2,749 \\
2,756 \\
2,850 \\
2,513 \\
3,068 \\
2,824 \\
2,597 \\
2,322 \\
2,606\end{array}$ & $\begin{array}{l}4,898 \\
4,962 \\
5,374 \\
5,250 \\
5,866 \\
6,020 \\
6,306 \\
6,297 \\
6,569 \\
6,879 \\
6,442 \\
6,706 \\
6,448 \\
6,567 \\
6,548 \\
6,650 \\
6,325 \\
6,936 \\
6,778 \\
6,641 \\
6,649 \\
6,966\end{array}$ & $\begin{array}{r}305 \\
16 \\
162 \\
-286 \\
549 \\
72 \\
207 \\
-99 \\
162 \\
255 \\
-476 \\
281 \\
-270 \\
142 \\
7 \\
94 \\
-337 \\
555 \\
-244 \\
-227 \\
-275 \\
284\end{array}$ & $\begin{array}{r}17.6 \\
.8 \\
7.9 \\
-12.9 \\
28.5 \\
2.9 \\
8.1 \\
-3.6 \\
6.1 \\
9.0 \\
-15.5 \\
10.8 \\
-9.4 \\
5.5 \\
.3 \\
3.4 \\
-11.8 \\
22.1 \\
-8.0 \\
-8.0 \\
-10.6 \\
12.2\end{array}$ & $\begin{array}{l}1,533 \\
1,701 \\
1,760 \\
1,921 \\
1,750 \\
2,158 \\
2,047 \\
1,910 \\
1,887 \\
2,094 \\
2,142 \\
2,064 \\
2,359 \\
1,812 \\
1,881 \\
2,244 \\
2,804 \\
1,934 \\
2,689 \\
2,724 \\
2,717 \\
2,508\end{array}$ & $\begin{array}{l}1,974 \\
1,784 \\
2,104 \\
1,756 \\
2,307 \\
2,278 \\
2,295 \\
1,896 \\
2,180 \\
2,399 \\
1,700 \\
2,252 \\
2,128 \\
1,952 \\
1,887 \\
2,174 \\
2,491 \\
2,433 \\
2,608 \\
2,555 \\
2,760 \\
2,796\end{array}$ & $\begin{array}{r}-442 \\
-84 \\
-344 \\
165 \\
-557 \\
-120 \\
-248 \\
14 \\
-293 \\
-306 \\
442 \\
-188 \\
231 \\
-140 \\
-6 \\
69 \\
313 \\
-499 \\
80 \\
168 \\
-43 \\
-288\end{array}$ \\
\hline 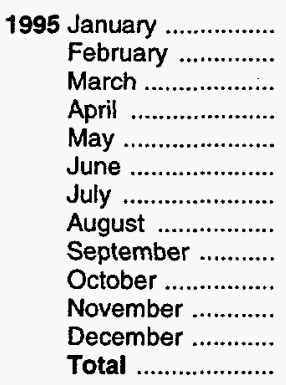 & $\begin{array}{l}4,365 \\
4,368 \\
4,362 \\
4,360 \\
4,393 \\
4,406 \\
4,340 \\
4,339 \\
4,341 \\
4,338 \\
4,342 \\
4,349 \\
4,349\end{array}$ & $\begin{array}{l}2,045 \\
1,542 \\
1,332 \\
1,379 \\
1,668 \\
2,014 \\
2,301 \\
2,495 \\
2,802 \\
2,996 \\
2,728 \\
2,153 \\
2,153\end{array}$ & $\begin{array}{l}6,410 \\
5,910 \\
5,694 \\
5,740 \\
6,061 \\
6,420 \\
6,641 \\
6,834 \\
7,143 \\
7,334 \\
7,070 \\
6,503 \\
6,503\end{array}$ & $\begin{array}{r}466 \\
451 \\
374 \\
207 \\
114 \\
118 \\
28 \\
-112 \\
-110 \\
-79 \\
-249 \\
-453 \\
-453\end{array}$ & $\begin{array}{r}29.5 \\
41.4 \\
39.0 \\
17.7 \\
7.3 \\
6.2 \\
1.2 \\
-4.3 \\
-3.8 \\
-2.6 \\
-8.4 \\
-17.4 \\
-17.4\end{array}$ & $\begin{array}{r}644 \\
564 \\
327 \\
127 \\
34 \\
40 \\
54 \\
86 \\
29 \\
68 \\
367 \\
635 \\
2,974\end{array}$ & $\begin{array}{r}45 \\
44 \\
104 \\
177 \\
369 \\
410 \\
359 \\
293 \\
343 \\
274 \\
96 \\
53 \\
2,566\end{array}$ & $\begin{array}{r}599 \\
519 \\
223 \\
-49 \\
-335 \\
-371 \\
-306 \\
-207 \\
-313 \\
-205 \\
272 \\
582 \\
408\end{array}$ \\
\hline 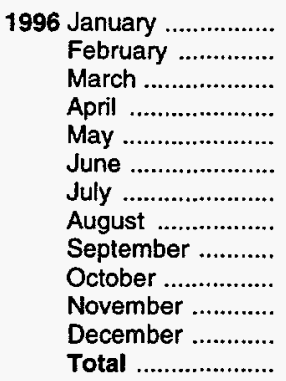 & $\begin{array}{l}4,354 \\
4,349 \\
4,290 \\
4,312 \\
4,332 \\
4,341 \\
4,336 \\
4,332 \\
4,338 \\
4,335 \\
4,339 \\
4,341 \\
4,341\end{array}$ & $\begin{array}{r}1,462 \\
1,021 \\
758 \\
854 \\
1,161 \\
1,529 \\
1,898 \\
2,245 \\
2,605 \\
2,810 \\
2,549 \\
2,173 \\
2,173\end{array}$ & $\begin{array}{l}5,817 \\
5,369 \\
5,048 \\
5,166 \\
5,493 \\
5,870 \\
6,234 \\
6,577 \\
6,943 \\
7,145 \\
6,889 \\
6,513 \\
6,513\end{array}$ & $\begin{array}{r}-583 \\
-521 \\
-574 \\
-525 \\
-507 \\
-485 \\
-404 \\
-250 \\
-197 \\
-186 \\
-179 \\
19 \\
19\end{array}$ & $\begin{array}{r}-28.5 \\
-33.8 \\
-43.1 \\
-38.1 \\
-30.4 \\
-24.1 \\
-17.5 \\
-10.0 \\
-7.0 \\
-6.2 \\
-6.6 \\
.9 \\
.9\end{array}$ & $\begin{array}{r}749 \\
544 \\
403 \\
112 \\
45 \\
35 \\
49 \\
54 \\
32 \\
73 \\
354 \\
461 \\
2,911\end{array}$ & $\begin{array}{r}49 \\
97 \\
80 \\
227 \\
373 \\
410 \\
418 \\
400 \\
398 \\
276 \\
90 \\
86 \\
2,906\end{array}$ & $\begin{array}{r}700 \\
447 \\
323 \\
-115 \\
-328 \\
-375 \\
-370 \\
-346 \\
-366 \\
-203 \\
264 \\
374 \\
6\end{array}$ \\
\hline 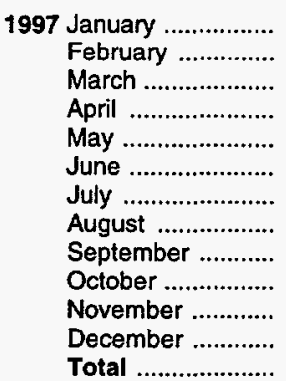 & $\begin{array}{r}4,347 \\
4,341 \\
4,344 \\
4,340 \\
4,342 \\
4,355 \\
4,354 \\
4,355 \\
4,357 \\
\text { R } 4,424 \\
\text { RF } 4,424 \\
F_{4,424} \\
\text { E } 4,424\end{array}$ & $\begin{array}{r}1,496 \\
1,140 \\
990 \\
1,049 \\
1,360 \\
1,731 \\
2,018 \\
2,334 \\
2,667 \\
R_{2,905} \\
R_{2} 2,684 \\
F_{2,274} \\
E_{2,274}\end{array}$ & $\begin{array}{r}5,843 \\
5,481 \\
5,334 \\
5,390 \\
5,701 \\
6,087 \\
6,372 \\
6,689 \\
7,024 \\
R_{7,329} \\
\text { RF }_{7,108} \\
F_{6,698} \\
\text { E }_{\mathbf{6 , 6 9 8}}\end{array}$ & $\begin{array}{r}34 \\
119 \\
232 \\
195 \\
199 \\
202 \\
120 \\
90 \\
62 \\
R 94 \\
R F 134 \\
F_{101} \\
E_{101}\end{array}$ & $\begin{array}{r}2.3 \\
11.7 \\
30.6 \\
22.9 \\
17.1 \\
13.2 \\
6.3 \\
4.0 \\
2.4 \\
R_{3.3} \\
F_{5} 5.3 \\
F_{4.7} \\
E_{4} 4.7\end{array}$ & $\begin{array}{r}749 \\
411 \\
281 \\
143 \\
38 \\
39 \\
81 \\
52 \\
43 \\
84 \\
\text { NA } \\
\text { NA } \\
\text { NA }\end{array}$ & $\begin{array}{r}66 \\
53 \\
126 \\
202 \\
360 \\
405 \\
355 \\
376 \\
373 \\
296 \\
\text { NA } \\
\text { NA } \\
\text { NA }\end{array}$ & $\begin{array}{r}683 \\
358 \\
156 \\
-59 \\
-322 \\
-366 \\
-274 \\
-323 \\
-330 \\
R_{-212} \\
R_{2} 221 \\
F_{410} \\
E_{-58}\end{array}$ \\
\hline
\end{tabular}

a For total underground storage capacity at the end of each calendar year, see Note 8 at end of section.

$\mathrm{b}$ For 1980-1996, data differ from those shown on Table 4.1, which includes liquefied natural gas storage for that period.

c Positive numbers indicate that withdrawals are greater than injections. Negative numbers indicate that injections are greater than withdrawals. Net withdrawals or injections may not equal the difference between applicable ending stocks. See Note 8 at end of section. $\mathrm{R}=$ Revised data. $F=$ Forecast.

Notes: - Totals may not equal sum of components due to independent rounding. - Geographic coverage is the 50 States and the District of Columbia.

Sources: See end of section. 


\section{Natural Gas Notes}

1. Nonhydrocarbon Gases Rernoved: Annual data on nonhydrocarbon gases removed from marketed production-carbon dioxide, helium, hydrogen sulfide, and nitrogen-are from the Energy Information Administration (EIA) Natural Gas Annual (NGA) 1992. Data are not available prior to 1980 . Monthly data are reported by three States and computed for six States. Monthly data are preliminary until after publication of the EIA $N G A$. Differences between annual data published in the EIA $N G A$ and the sum of the preliminary monthly data (January-December) are allocated proportionally to the months to create final monthly data. For further information on methods of estimating prelimainary monthly data, see the EIA Natural Gas Monthly (NGM).

\section{Production.}

- Annual data: Final annual data are from the EIA $N G A$.

- Estimated monthly data: Data for the two most recent months presented are estimated. Some of the data for earlier months are also estimated or computed. For a discussion of computation and estimation procedures, see the EIA $N G M$.

- Preliminary monthly data: Monthly data are considered preliminary until after publication of the EIA $N G A$. Preliminary monthly data are gathered from reports to the Interstate Oil Compact Commission and the U.S. Minerals Management Service. Volumetric data are converted, as necessary, to a standard 14.73 psi pressure base. Unless there are major changes, data are not revised until after publication of the ElA $N G A$.

- Final monthly data: Differences between annual data in the EIA NGA and the sum of preliminary monthly data (January-December) are allocated proportionally to the months to create final monthly data.

3. Extraction Loss: Extraction loss is the reduction in volume of natural gas resulting from the removal of natural gas liquid constituents at natural gas processing plants.

Annual data are from the EIA $N G A$, where they are estimated on the basis of the type and quantity of liquid products extracted from the gas stream and the calculated volume of such products at standard conditions. For a detailed explanation of the calculations used to derive estimated extraction losses, see the EIA $N G A$.

Preliminary monthly data are estimated on the basis of extraction loss as an annual percentage of marketed production. This percentage is applied to each month's marketed production to estimate monthly extraction loss.

Monthly data are revised and considered final after the publication of the EIA NGA. Final monthly data are estimated by allocating annual extraction loss data to the months on the basis of total natural gas marketed production data from the EIA $N G A$.
4. Supplemental Gaseous Fuels: Any gaseous substance that, introduced into or commingled with natural gas, increases the volume available for disposition. Such substances include, but are not limited to, propane-air, refinery gas, coke oven gas, still gas, manufactured gas, biomass gas, or air or inert gases added for Btu stabilization.

Annual data beginning with 1980 are from the EIA NGA. Unknown quantities of supplemental gaseous fuels are included in consumption data for 1979 and earlier years.

Monthly data are considered preliminary until after the publication of the EIA NGA. Monthly estimates are based on the annual ratio of supplemental gaseous fuels to the sum of dry gas production, net imports, and net withdrawals from storage. The ratio is applied to the monthly sum of the three elements to compute a monthly supplemental gaseous fuels figure.

5. Imports and Exports: The United States imports natural gas via pipeline from Canada and Mexico. Liquefied natural gas (LNG) arrives via tanker from Algeria and United Arab Emirates. One shipment of LNG was received from Indonesia in December 1986. Very small amounts of LNG arrived from Canada in 1973 (667 million cubic feet), 1977 (572 million cubic feet), and 1981 ( 6 million cubic feet). The United States exports natural gas via pipeline to Canada and Mexico and LNG via tanker to Japan.

Annual and final monthly data are from the annual EIA Form FPC-14, "Annual Report for Importers and Exporters of Natural Gas," which requires data to be reported by month for the calendar year.

Preliminary monthly data are EIA estimates. For a discussion of estimation procedures, see the EIA NGM. Preliminary data are revised after the publication of the EIA U.S. Imports and Exports of Natural Gas.

6. Consumption: Consumption includes pipeline fuel use, lease and plant fuel use, and deliveries to consuming sectors.

Final data are from the EIA NGA. Monthly data are considered preliminary until after publication of the EIA $N G A$. For more detailed information on the methods of estimating preliminary and final monthly data, see the EIA NGM.

7. Balancing Item: The balancing item for natural gas represents the difference between the sum of the components of natural gas supply and the sum of components of natural gas disposition. The differences may be due to quantities lost or to the effects of data reporting problems. Reporting problems include differences due to the net result of conversions of flow data metered at varying temperature and pressure bases and converted to a standard temperature and pressure base; the effect of variations in company accounting and billing practices; differences between billing cycle and calendar period time frames; and imbalances resulting from the merger of data reporting systems which vary in scope, format, definitions, and type of respondents.

The increase of 0.2 trillion cubic feet (Tcf) in the "Balancing Item" category in 1983, followed by a decline of 0.5 Tcf in 1984, reflected unusually large differences resulting 
from the use of the annual billing cycle (essentially December 15 through the following December 14) consumption data in conjunction with calendar year supply data. Record cold temperatures during the last half of December 1983 resulted in a reported $0.3 \mathrm{Tcf}$ increase in net withdrawals from underground storage for peak shaving as compared with the same period in 1982 , but the effect of this cold weather was reflected primarily in 1984 consumption data. For underground storage data, see Table F2 in the May 1985 NGM, which was published in July 1985.

8. Natural Gas Storage: Gas in storage at the end of a reporting period may not equal the quantity derived by adding or subtracting net injections or withdrawals from the quantity in storage at the end of the previous period. The difference is due to changes in the quantity of native gas included in the base gas and/or losses in base gas due to migration from storage reservoirs.

Monthly underground storage data are collected from the Federal Energy Regulatory Commission (FERC) Forms FERC-8 (interstate data) and EIA-191 (intrastate data). Beginning in January 1991, all data are collected on the revised Form EIA-191. Injection and withdrawal data from the FERC-8/EIA-191 survey are adjusted to correspond to data from Form EIA-176 following publication of the EIA NGA.

The final monthly and annual storage and withdrawal data for 1980-1996 include both underground and liquefied natural gas (LNG) storage. Annual data on LNG additions and withdrawals are from Form EIA-176. Monthly data are estimated by computing the ratio of each month's underground storage additions and withdrawals to annual underground storage additions and withdrawals and applying the ratio to the annual LNG data.

Total underground storage capacity at the end of each calendar year since 1975 (first year data were available), in billion cubic feet, was:

$\begin{array}{llll}1975 & 6,280 & 1986 & 8145 \\ 1976 & 6,544 & 1987 & 8,124 \\ 1977 & 6,678 & 1988 & 8,124 \\ 1978 & 6,890 & 1989 & 8,124 \\ 1979 & 6,929 & 1990 & 8,125 \\ 1980 & 7,434 & 1991 & 7,993 \\ 1981 & 7,805 & 1992 & 7,932 \\ 1982 & 7,915 & 1993 & 7,989 \\ 1983 & 7,985 & 1994 & 8,043 \\ 1984 & 8,043 & 1995 & 7,953 \\ 1985 & 8,087 & 1996 & 7,980\end{array}$

Current capacity is 7,980 billion cubic feet.

9. Forecast Values: Data values preceded by "F" in this section are forecast values. They are derived from EIA's Short-Term Integrated Forecasting System
(STIFS). The model is driven primarily by data and assumptions about key macroeconomic variables, the world oil price, and weather. The natural gas forecast relies on other variables as well, such as gas wellhead prices, electric power generation by other sources, and U.S. gas import capacity. Each month, EIA staff review the model output and make adjustments, if appropriate, based on their knowledge of developments in the natural gas industry.

The STIFS model results are published quarterly in EIA's Short-Term Energy Outlook, which is available from the National Energy Information Center (202-5868800 ) and accessible on the world wide web at http://www.eia.doe.gov. Documentation for the model and instructions for downloading and operating it on a personal computer are provided.

\section{Sources for Table 4.5}

\section{Storage Activity}

1973-1975 : Energy Information Administration (EIA) Natural Gas Annual 1994, Volume 2, Table 9.

1976-1979: EIA, Natural Gas Production and Consumption 1979, Table 1.

1980-1990: EIA, Historical Natural Gas Annual 1930 Through 1995, Table 11.

1991 forward: EIA, Natural Gas Monthly, December 1997, Table 9. Forecast values are derived from EIA's Short-Term Integrated Forecasting System. See Note 9 on this page.

\section{Other Data}

1973 and 1974: American Gas Association (AGA), Gas Facts, 1972 Data, Table 57, Gas Facts, 1973 Data, Table 57, and Gas Facts, 1974 Data, Table 40.

1975 and 1976: Federal Energy Administration (FEA), Form FEA-G318-M-O, "Underground Gas Storage Report," and Federal Power Commission (FPC), Form FPC-8, "Underground Gas Storage Report."

1977 and 1978: EIA, Form FEA-G-318-M-O, "Underground Gas Storage Report," and Federal Energy Regulatory Commission (FERC), Form FERC-8, "Underground Gas Storage Report.

1979-1990: EIA, Form EIA-191, "Underground Gas Storage Report," and FERC, Form FERC-8, "Underground Gas Storage Report."

1991 forward: EIA, Natural Gas Monthly, December 1997, Table 9. Forecast values are derived from EIA's Short-Term Integrated Forecasting System. See Note 9 on this page. 


\section{Section 5. Oil and Gas Resource Development}

The December 1997 rotary rig count of 1,013 was 3 percent higher than the count in November and 19 percent higher than the count in December 1996. Of the total number of rigs in operation in December 1997, 884 were onshore and 129 were offshore. The number of onshore rigs was up 20 percent and the number of offshore rigs rose 11 percent from their December 1996 values.
There were 3.4 thousand well servicing units active in December 1997, 4 percent higher than in December 1996.

Oil and gas wells drilled and total footage drilled data updates were not available.

Figure 5.1 Oil and Gas Resource Development Indicators

Active Well Servicing Units

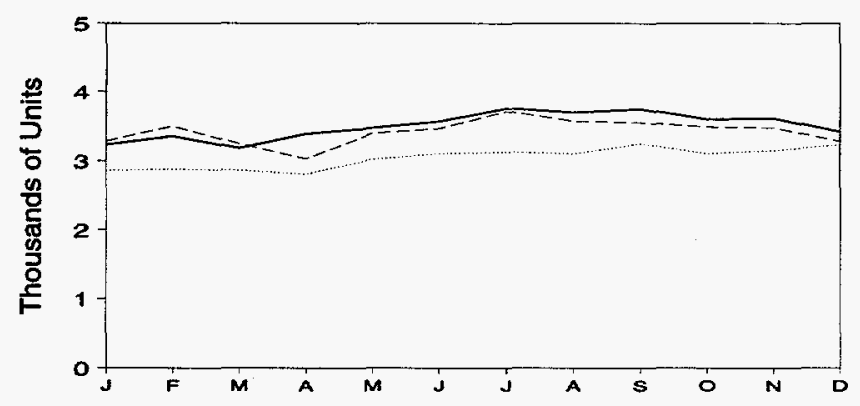

Wells Drilled

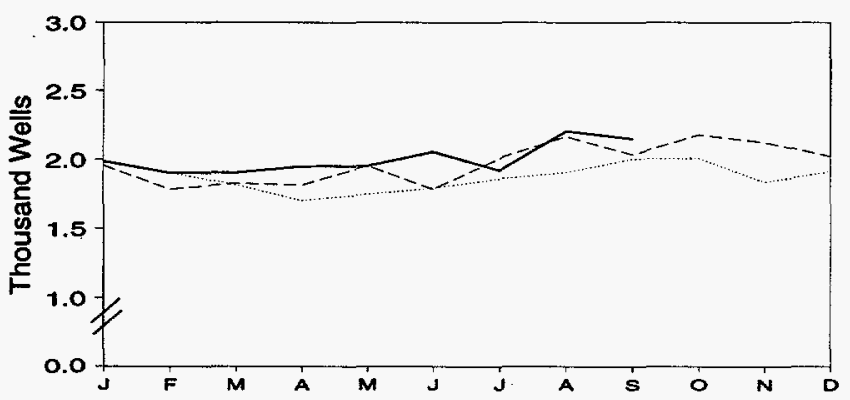

Rotary Rigs in Operation

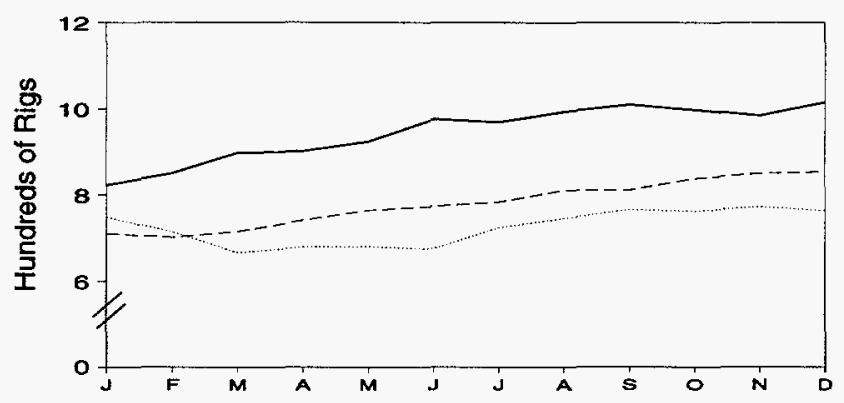

Footage Drilled

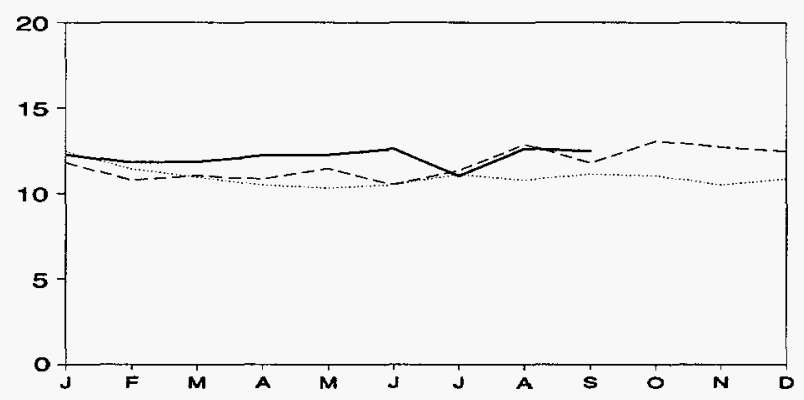


Table 5.1 Oil and Gas Drilling Activity Measurements

\begin{tabular}{|c|c|c|c|c|c|c|c|c|c|c|}
\hline & \multicolumn{3}{|c|}{$\begin{array}{l}\text { Crews Engaged in } \\
\text { Seismic Exploration }\end{array}$} & \multicolumn{5}{|c|}{ Rotary Rigs in Operation ${ }^{a}$} & \multirow{4}{*}{$\begin{array}{c}\begin{array}{c}\text { Total } \\
\text { Footage } \\
\text { Drilledc }\end{array} \\
\text { housand Feet }\end{array}$} & \multirow{4}{*}{$\begin{array}{c}\begin{array}{c}\text { Active } \\
\text { Well Servicing } \\
\text { Units }\end{array} \\
\text { Number }\end{array}$} \\
\hline & \multirow[b]{2}{*}{ Offshore } & \multirow[b]{2}{*}{ Dnshore } & \multirow{3}{*}{ Total } & \multicolumn{2}{|c|}{ By Site } & \multicolumn{2}{|c|}{ By Type } & \multirow[b]{2}{*}{ Total ${ }^{\mathrm{b}}$} & & \\
\hline & & & & Oftshore & Onshore & Oil & Gas & & & \\
\hline & \multicolumn{2}{|c|}{ Monthly Average } & & \multicolumn{5}{|c|}{ Weekly Average } & & \\
\hline 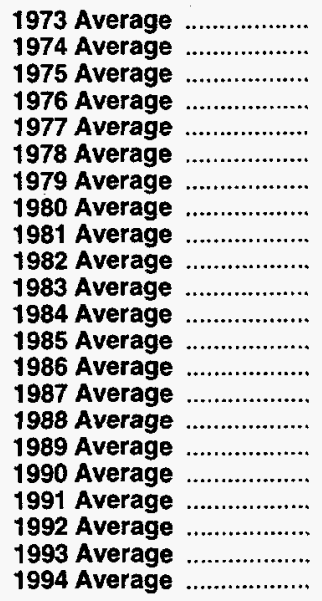 & $\begin{array}{l}23 \\
31 \\
30 \\
25 \\
27 \\
25 \\
30 \\
37 \\
44 \\
57 \\
47 \\
49 \\
45 \\
24 \\
24 \\
29 \\
23 \\
23 \\
19 \\
12 \\
16 \\
\text { NA }\end{array}$ & $\begin{array}{r}227 \\
274 \\
254 \\
237 \\
281 \\
327 \\
370 \\
493 \\
637 \\
531 \\
426 \\
445 \\
333 \\
176 \\
153 \\
153 \\
109 \\
102 \\
85 \\
64 \\
63 \\
\text { NA }\end{array}$ & $\begin{array}{r}250 \\
305 \\
284 \\
262 \\
308 \\
352 \\
400 \\
530 \\
681 \\
588 \\
473 \\
494 \\
378 \\
200 \\
177 \\
182 \\
132 \\
125 \\
104 \\
76 \\
79 \\
\text { NA }\end{array}$ & $\begin{array}{r}84 \\
94 \\
106 \\
129 \\
167 \\
185 \\
207 \\
231 \\
256 \\
243 \\
199 \\
213 \\
206 \\
99 \\
95 \\
123 \\
105 \\
108 \\
81 \\
52 \\
82 \\
102\end{array}$ & $\begin{array}{r}1,110 \\
1,378 \\
1,554 \\
1,529 \\
1,834 \\
2,074 \\
1,970 \\
2,678 \\
3,714 \\
2,862 \\
2,033 \\
2,215 \\
1,774 \\
865 \\
841 \\
813 \\
764 \\
902 \\
779 \\
669 \\
672 \\
673\end{array}$ & $\begin{array}{l}\text { NA } \\
\text { NA } \\
\text { NA } \\
\text { NA } \\
\text { NA } \\
\text { NA } \\
\text { NA } \\
\text { NA } \\
\text { NA } \\
\text { NA } \\
\text { NA } \\
\text { NA } \\
\text { NA } \\
\text { NA } \\
\text { NA } \\
\mathbf{5 5 4} \\
\mathbf{4 5 3} \\
\mathbf{5 3 2} \\
\mathbf{4 8 2} \\
\mathbf{3 7 3} \\
\mathbf{3 7 3} \\
\mathbf{3 3 5}\end{array}$ & $\begin{array}{l}\text { NA } \\
\text { NA } \\
\text { NA } \\
\text { NA } \\
\text { NA } \\
\text { NA } \\
\text { NA } \\
\text { NA } \\
\text { NA } \\
\text { NA } \\
\text { NA } \\
\text { NA } \\
\text { NA } \\
\text { NA } \\
\text { NA } \\
354 \\
401 \\
464 \\
351 \\
331 \\
364 \\
427\end{array}$ & $\begin{array}{r}1,194 \\
1,472 \\
1,660 \\
1,658 \\
2,001 \\
2,259 \\
2,177 \\
2,909 \\
3,970 \\
3,105 \\
2,232 \\
2,428 \\
1,980 \\
964 \\
936 \\
936 \\
869 \\
1,010 \\
860 \\
721 \\
754 \\
775\end{array}$ & $\begin{array}{l}139,427 \\
153,791 \\
181,046 \\
187,291 \\
215,696 \\
238,388 \\
243,686 \\
312,303 \\
408,842 \\
378,437 \\
318,585 \\
370,730 \\
312,569 \\
177,486 \\
161,226 \\
153,340 \\
133,383 \\
154,632 \\
146,383 \\
124,879 \\
140,330 \\
127,934\end{array}$ & $\begin{array}{r}\text { NA } \\
\text { NA } \\
\text { NA } \\
2,601 \\
2,828 \\
2,988 \\
3,399 \\
4,089 \\
4,850 \\
4,248 \\
3,732 \\
4,663 \\
4,716 \\
3,036 \\
3,060 \\
3,341 \\
3,391 \\
3,658 \\
3,331 \\
2,732 \\
3,158 \\
2,961\end{array}$ \\
\hline 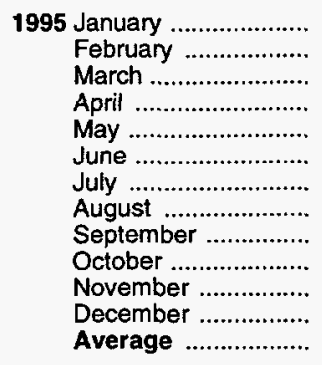 & $\begin{array}{l}\text { NA } \\
\text { NA } \\
\text { NA } \\
\text { NA } \\
\text { NA } \\
\text { NA } \\
\text { NA } \\
\text { NA } \\
\text { NA } \\
\text { NA } \\
\text { NA } \\
\text { NA } \\
\text { NA }\end{array}$ & $\begin{array}{l}\text { NA } \\
\text { NA } \\
\text { NA } \\
\text { NA } \\
\text { NA } \\
\text { NA } \\
\text { NA } \\
\text { NA } \\
\text { NA } \\
\text { NA } \\
\text { NA } \\
\text { NA } \\
\text { NA }\end{array}$ & $\begin{array}{l}\text { NA } \\
\text { NA } \\
\text { NA } \\
\text { NA } \\
\text { NA } \\
\text { NA } \\
\text { NA } \\
\text { NA } \\
\text { NA } \\
\text { NA } \\
\text { NA } \\
\text { NA } \\
\text { NA }\end{array}$ & $\begin{array}{r}106 \\
100 \\
90 \\
91 \\
100 \\
96 \\
104 \\
103 \\
103 \\
105 \\
104 \\
109 \\
101\end{array}$ & $\begin{array}{l}642 \\
613 \\
575 \\
587 \\
579 \\
578 \\
619 \\
642 \\
662 \\
656 \\
668 \\
654 \\
622\end{array}$ & $\begin{array}{l}325 \\
326 \\
322 \\
328 \\
325 \\
301 \\
301 \\
327 \\
333 \\
332 \\
330 \\
325 \\
323\end{array}$ & $\begin{array}{l}411 \\
375 \\
331 \\
336 \\
335 \\
352 \\
399 \\
399 \\
413 \\
414 \\
430 \\
427 \\
385\end{array}$ & $\begin{array}{l}748 \\
713 \\
665 \\
678 \\
679 \\
674 \\
723 \\
745 \\
765 \\
761 \\
772 \\
763 \\
723\end{array}$ & $\begin{array}{r}12,457 \\
11,423 \\
10,956 \\
10,487 \\
10,319 \\
10,525 \\
11,115 \\
10,798 \\
11,138 \\
11,054 \\
10,503 \\
10,854 \\
131,629\end{array}$ & $\begin{array}{l}2,855 \\
2,877 \\
2,862 \\
2,806 \\
3,020 \\
3,107 \\
3,133 \\
3,103 \\
3,255 \\
3,105 \\
3,157 \\
3,239 \\
3,043\end{array}$ \\
\hline 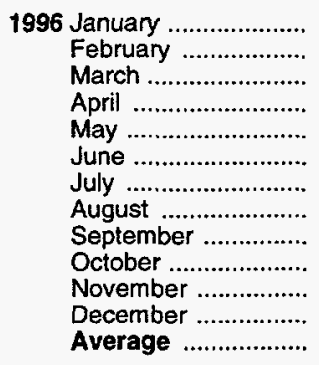 & $\begin{array}{l}\text { NA } \\
\text { NA } \\
\text { NA } \\
\text { NA } \\
\text { NA } \\
\text { NA } \\
\text { NA } \\
\text { NA } \\
\text { NA } \\
\text { NA } \\
\text { NA } \\
\text { NA } \\
\text { NA }\end{array}$ & $\begin{array}{l}\text { NA } \\
\text { NA } \\
\text { NA } \\
\text { NA } \\
\text { NA } \\
\text { NA } \\
\text { NA } \\
\text { NA } \\
\text { NA } \\
\text { NA } \\
\text { NA } \\
\text { NA } \\
\text { NA }\end{array}$ & $\begin{array}{l}\text { NA } \\
\text { NA } \\
\text { NA } \\
\text { NA } \\
\text { NA } \\
\text { NA } \\
\text { NA } \\
\text { NA } \\
\text { NA } \\
\text { NA } \\
\text { NA } \\
\text { NA } \\
\text { NA }\end{array}$ & $\begin{array}{r}111 \\
102 \\
96 \\
113 \\
116 \\
112 \\
107 \\
108 \\
109 \\
108 \\
107 \\
116 \\
108\end{array}$ & $\begin{array}{l}598 \\
598 \\
618 \\
628 \\
648 \\
662 \\
677 \\
703 \\
702 \\
728 \\
741 \\
736 \\
671\end{array}$ & $\begin{array}{l}295 \\
283 \\
286 \\
286 \\
288 \\
298 \\
290 \\
297 \\
301 \\
328 \\
363 \\
361 \\
306\end{array}$ & $\begin{array}{l}406 \\
411 \\
421 \\
446 \\
467 \\
471 \\
488 \\
488 \\
505 \\
499 \\
482 \\
489 \\
464\end{array}$ & $\begin{array}{l}709 \\
700 \\
714 \\
741 \\
764 \\
774 \\
784 \\
811 \\
811 \\
836 \\
848 \\
852 \\
779\end{array}$ & $\begin{array}{r}11,807 \\
10,768 \\
11,045 \\
10,835 \\
11,456 \\
10,518 \\
11,344 \\
12,867 \\
11,797 \\
13,062 \\
12,697 \\
12,460 \\
140,656\end{array}$ & $\begin{array}{l}3,290 \\
3,509 \\
3,253 \\
3,031 \\
3,405 \\
3,473 \\
3,723 \\
3,582 \\
3,560 \\
3,498 \\
3,489 \\
3,287 \\
3,425\end{array}$ \\
\hline 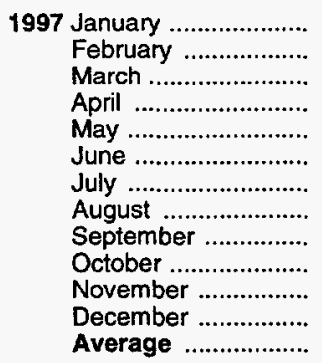 & $\begin{array}{l}\text { NA } \\
\text { NA } \\
\text { NA } \\
\text { NA } \\
\text { NA } \\
\text { NA } \\
\text { NA } \\
\text { NA } \\
\text { NA } \\
\text { NA } \\
\text { NA } \\
\text { NA } \\
\text { NA }\end{array}$ & $\begin{array}{l}\text { NA } \\
\text { NA } \\
\text { NA } \\
\text { NA } \\
\text { NA } \\
\text { NA } \\
\text { NA } \\
\text { NA } \\
\text { NA } \\
\text { NA } \\
\text { NA } \\
\text { NA } \\
\text { NA }\end{array}$ & $\begin{array}{l}\text { NA } \\
\text { NA } \\
\text { NA } \\
\text { NA } \\
\text { NA } \\
\text { NA } \\
\text { NA } \\
\text { NA } \\
\text { NA } \\
\text { NA } \\
\text { NA } \\
\text { NA } \\
\text { NA }\end{array}$ & $\begin{array}{l}110 \\
107 \\
127 \\
126 \\
120 \\
121 \\
125 \\
125 \\
128 \\
121 \\
126 \\
129 \\
122\end{array}$ & $\begin{array}{l}712 \\
742 \\
770 \\
775 \\
804 \\
855 \\
844 \\
868 \\
881 \\
875 \\
857 \\
884 \\
822\end{array}$ & $\begin{array}{l}342 \\
356 \\
377 \\
373 \\
379 \\
396 \\
382 \\
409 \\
392 \\
390 \\
354 \\
361 \\
376\end{array}$ & $\begin{array}{l}478 \\
492 \\
518 \\
526 \\
541 \\
577 \\
584 \\
581 \\
614 \\
602 \\
625 \\
648 \\
564\end{array}$ & $\begin{array}{r}822 \\
849 \\
897 \\
901 \\
924 \\
976 \\
969 \\
993 \\
1,009 \\
996 \\
983 \\
1,013 \\
943\end{array}$ & $\begin{array}{r}12,268 \\
11,809 \\
11,855 \\
12,215 \\
12,260 \\
12,600 \\
11,026 \\
12,630 \\
12,473 \\
\text { NA } \\
\text { NA } \\
\text { NA } \\
\text { NA }\end{array}$ & $\begin{array}{r}3,237 \\
3,364 \\
3,189 \\
3,398 \\
3,483 \\
3,575 \\
3,766 \\
3,705 \\
3,755 \\
3,607 \\
\text { R } 3,622 \\
3,429 \\
\mathbf{3 , 5 1 0}\end{array}$ \\
\hline
\end{tabular}

a Rotary rigs in operation are reported weekly. Monthly data are averages of 4- or 5- week reporting periods, not calendar months. Multi-month data are averages of the reported data over the covered months, not averages of the weekly data. Annual data are averages over 52- or 53- weeks, not calendar years. Published data are rounded to the nearest whole number.

Sum of oil, gas, and miscellaneous other rigs, which is not shown.

c Values shown are totals.

d See Glossary.

$R=$ Revised data. NA=Not available.

Note: Geographic coverage is the 50 States and the District of Columbia.
Sources: - Crews Engaged in Seismic Exploration: Society of Exploration Geophysicists, Tulsa, Oklahoma, Monthly Seismic Crew Count - Rotary Rigs in Operation: By Site-Baker Hughes, Inc., Houston, Texas, Rotary Rigs Running--by State. By Type - Baker Hughes, Inc., Houston, Texas, weekly phone recording. - Total Footage Drilled: Energy Information Administration computations, which are based on well reports submitted to the American Petroleum Institute by the Petroleum Information Corporation Denver, Colorado. Active Well Servicing Units: Association of Energy Service Companies, Dallas, Texas, Field Reports. 
Table 5.2 Oil and Gas Wells Drilled

(Number of Wells)

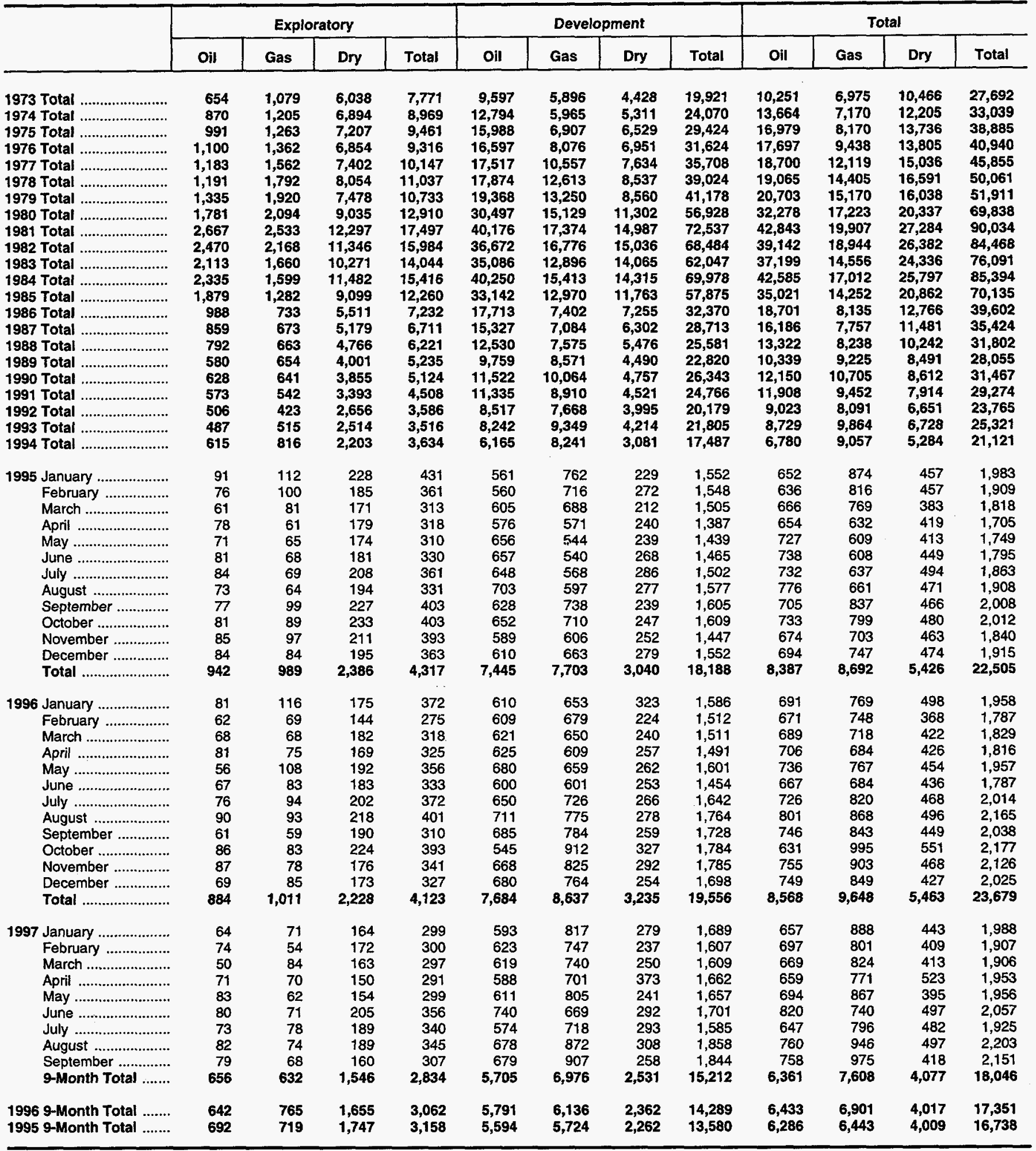

Notes: - Service wells, stratigraphic tests, and core tests are excluded.

- Due to the method of estimation, data shown on this page are frequently revised. See end of section. - Geographic coverage is the 50 States and the District of Columbia.
Sources: Energy Information Administration computations, which are based on well reports submitted by the Petroleum Information Corporation Denver, Colorado.

An update to Table 5.2 was not available in time for inclusion this month. 


\section{Oil and Gas Resource Develop- ment Notes}

Three well types are considered in the Monthly Energy Review (MER) drilling statistics: "completed for oil," "completed for gas," and "dry hole." Wells that productively encounter both crude oil and natural gas are categorized as "completed for oil." Both development wells and exploratory wells (new field wildcats, new pool tests, and extension tests) are included in the statistics. All other classes of wells drilled in connection with the search for producible hydrocarbons are excluded.

Prior to the March $1985 M E R$, drilling statistics consisted of completion data for the above types and classes of wells as reported to the American Petroleum Institute (API) during a given month. Due to time lags between the date of well completion and the date of completion reporting to the API, as-reported well completions proved to be an inaccurate indicator of drilling activity. During 1982, for example, as-reported well completions rose, while the number of actual completions fell. Consequently, the drilling statistics published since the March 1985 MER are Energy Information Administration-generated (EIA) estimates produced by statistically imputing well counts and footage based on the partial data available from the API.

Estimates for a given month are first published in the $M E R$ for that month. Revisions of the "oil," "gas," and "dry" components are made in the 6th, 12th, and 24 th subsequent months, as newly reported data allow refinement of the estimates. Unscheduled revisions may also occur when the latest estimate differs by more than 15 percent during the first 5 months, more than 10 percent during the next 6 months, or more than 2 percent thereafter through 5 years: After 5 years, the reported API data are published in lieu of EIA-generated estimates. A comprehensive, one-time reestimation of Total Footage Drilled (Table 5.1) and Oil and Gas Wells Drilled (Table 5.2) from 1990 through March 1995 was published in the June 1995 MER.

Since 1985 when EIA began to produce estimates from the partial data, changes in the industry and in data collection systems have introduced greater uncertainty in to the estimation results. Consequently, EIA has a project underway to enhance the estimation system, and an adjustment to the system is anticipated at the end of 1997. Meanwhile, readers should be aware that estimates published for the most recent months may not be as reliable as comparable estimates in the past.

Additional information about the EIA estimation methodology may be found in "Estimating Well Completions," the feature article published in the March 1985 MER.

A significant input data shortfall has been corrected involving data in 1995 and 1996. 


\section{Section 6. Coal}

Coal production in December 1997 totaled 98 million short tons, 12 percent higher than the 87 million short tons produced in December 1996. During 1997, coal production totaled 1,095 million short tons, 3 percent higher than the 1,064 million short tons produced during 1996.

Electric utility coal consumption in October 1997 totaled 76 million short tons, 6 percent higher than the consumption level in October 1996.
Electric utility coal stocks were 104 million short tons at the end of October 1997, 16 percent below the 123 million short tons at the end of October 1996.

Coal exports in October 1997 totaled 7 million short tons, 8 percent lower than exports in October 1996. Coal imports in October 1997 totaled 564 thousand short tons, 12 percent lower than imports in October 1996. 
Figure 6.1 Coal

(Million Short Tons)

Overview, 1973-1997

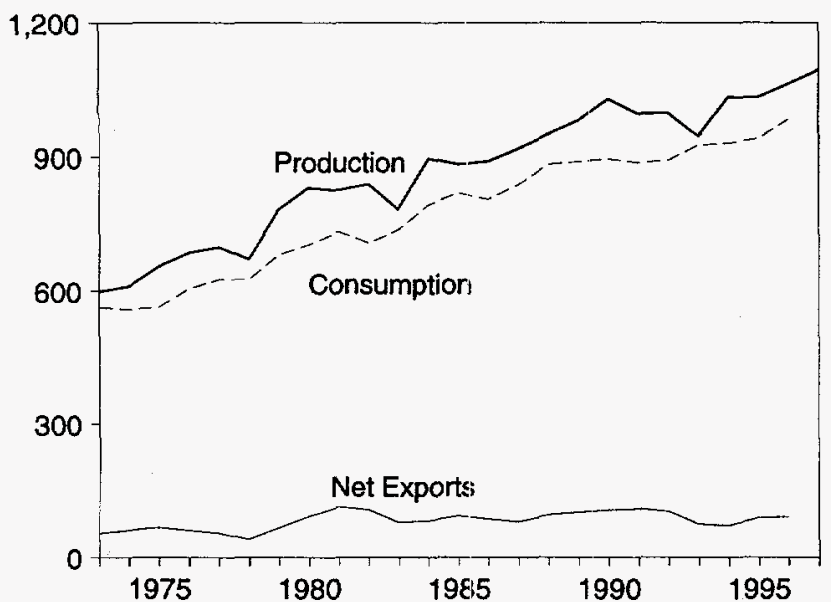

Consumption by Sector, 1973-1996

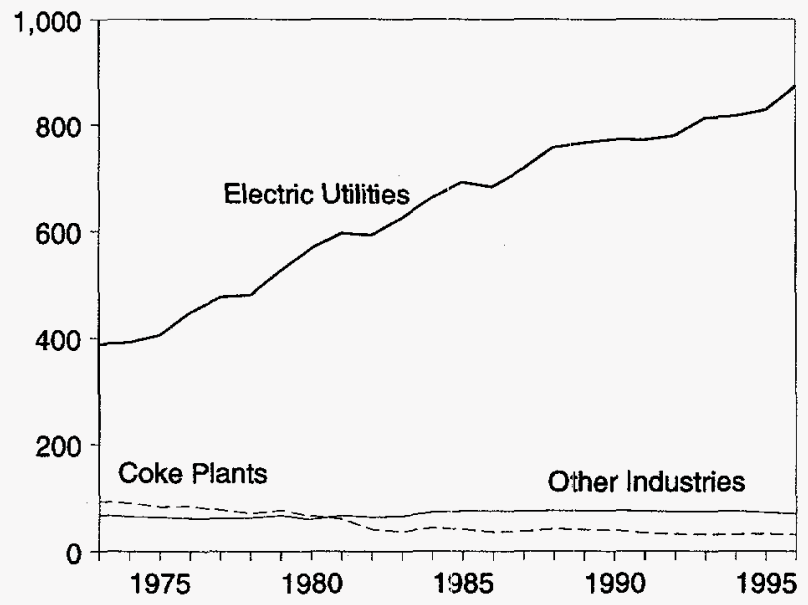

Stocks, End of Year, 1973-1996

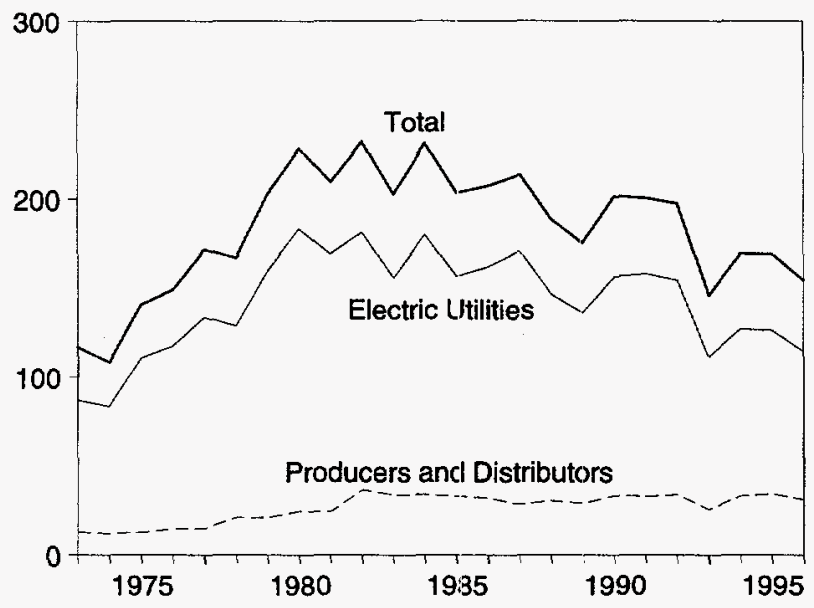

Overview, Monthly

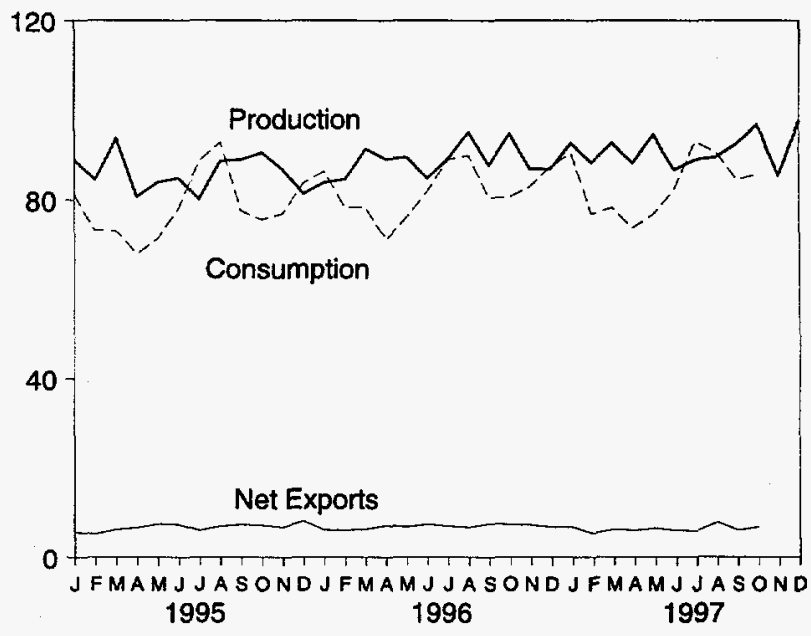

Consumption by Electric Utilities, Monthly

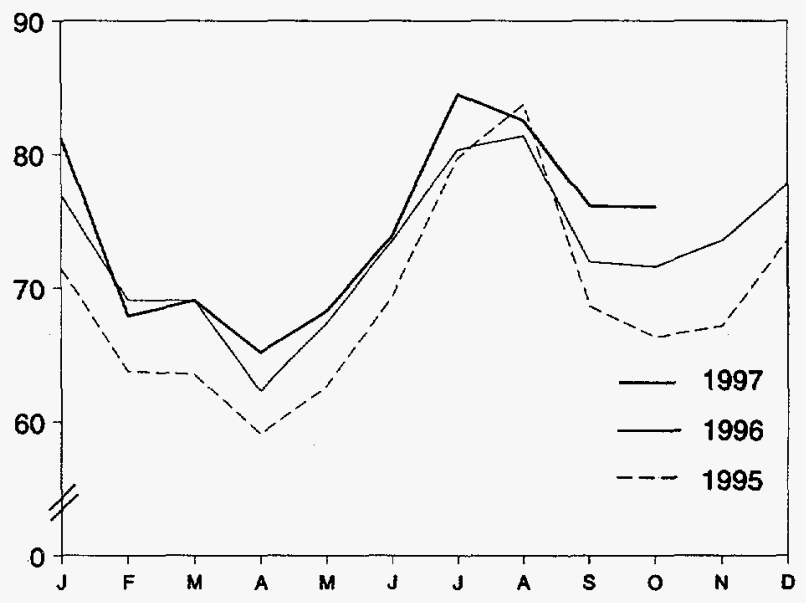

Stocks at Electric Utilities, End of Month

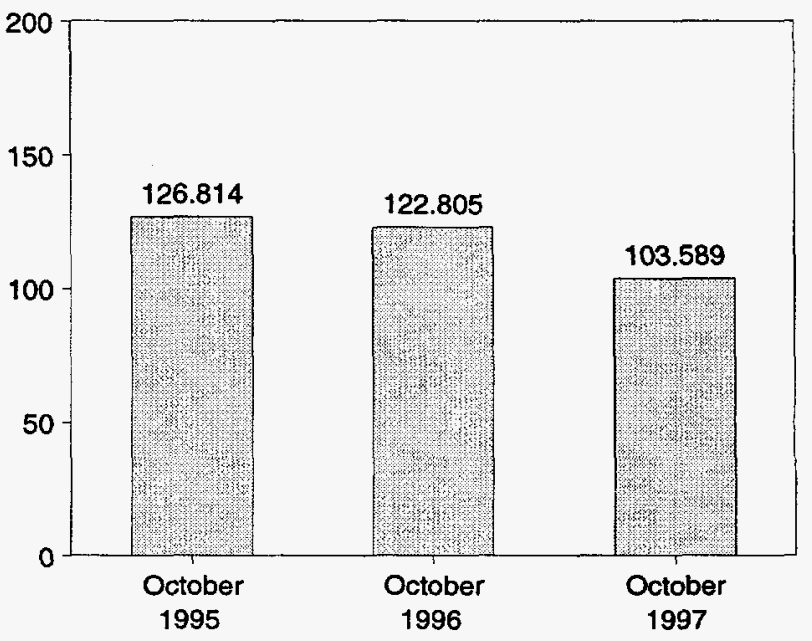

Note: Because vertical scales differ, graphs should not be compared. Sources: Tables 6.1, 6.2, and 6.3. 
Table 6.1 Coal Overview

(Thousand Short Tons)

\begin{tabular}{|c|c|c|c|c|c|}
\hline & Production & Consumption & Imports ${ }^{a}$ & Exports & Stocks ${ }^{b}$ \\
\hline 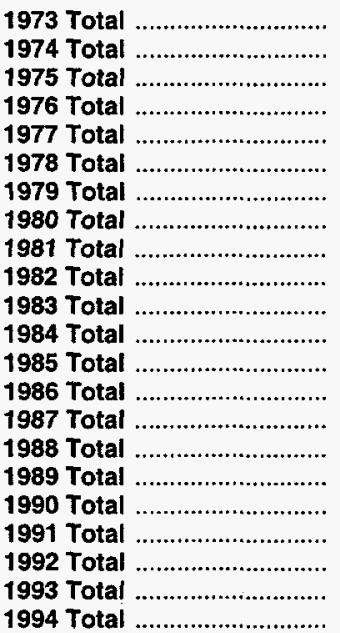 & $\begin{array}{r}598,568 \\
610,023 \\
654,641 \\
684,913 \\
697,205 \\
670,164 \\
781,134 \\
829,700 \\
823,775 \\
838,112 \\
782,091 \\
895,921 \\
883,638 \\
890,315 \\
918,762 \\
950,265 \\
980,729 \\
1,029,076 \\
995,984 \\
997,545 \\
945,424 \\
1,033,504\end{array}$ & $\begin{array}{l}562,584 \\
558,402 \\
562,640 \\
603,790 \\
625,291 \\
625,225 \\
680,524 \\
702,730 \\
732,627 \\
706,911 \\
736,672 \\
791,296 \\
818,049 \\
804,231 \\
836,941 \\
883,642 \\
889,699 \\
895,480 \\
887,621 \\
892,421 \\
925,944 \\
930,201\end{array}$ & $\begin{array}{r}127 \\
2,080 \\
940 \\
1,203 \\
1,647 \\
2,953 \\
2,059 \\
1,194 \\
1,043 \\
742 \\
1,271 \\
1,286 \\
1,952 \\
2,212 \\
1,747 \\
2,134 \\
2,851 \\
2,699 \\
3,390 \\
3,803 \\
7,309 \\
7,584\end{array}$ & $\begin{array}{r}53,587 \\
60,661 \\
66,309 \\
60,021 \\
54,312 \\
40,714 \\
66,042 \\
91,742 \\
112,541 \\
106,277 \\
77,772 \\
81,483 \\
92,680 \\
85,518 \\
79,607 \\
95,023 \\
100,815 \\
105,804 \\
108,969 \\
102,516 \\
74,519 \\
71,359\end{array}$ & $\begin{array}{l}117,155 \\
108,237 \\
140,391 \\
148,899 \\
171,543 \\
166,606 \\
202,812 \\
228,407 \\
209,423 \\
232,038 \\
202,584 \\
231,300 \\
203,367 \\
207,319 \\
213,780 \\
188,831 \\
175,087 \\
201,629 \\
200,682 \\
197,685 \\
145,742 \\
169,358\end{array}$ \\
\hline 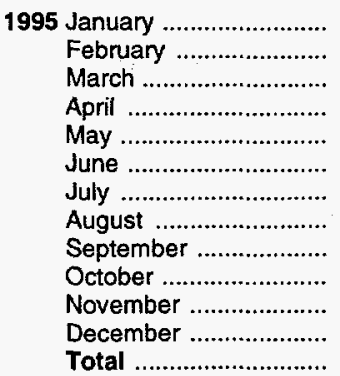 & $\begin{array}{r}88,953 \\
84,472 \\
93,696 \\
80,660 \\
83,874 \\
84,818 \\
80,093 \\
88,712 \\
89,052 \\
90,573 \\
86,779 \\
81,292 \\
1,032,974\end{array}$ & $\begin{array}{r}81,201 \\
73,236 \\
73,167 \\
67,990 \\
71,456 \\
77,993 \\
88,801 \\
92,860 \\
77,692 \\
75,664 \\
76,947 \\
83,632 \\
940,638\end{array}$ & $\begin{array}{r}530 \\
486 \\
780 \\
525 \\
517 \\
567 \\
566 \\
547 \\
613 \\
613 \\
721 \\
738 \\
7,201\end{array}$ & $\begin{array}{r}6,184 \\
5,774 \\
7,029 \\
7,212 \\
8,036 \\
7,935 \\
6,632 \\
7,530 \\
8,012 \\
7,823 \\
7,494 \\
8,883 \\
88,547\end{array}$ & $\begin{array}{l}171,339 \\
177,689 \\
186,463 \\
192,948 \\
198,349 \\
193,761 \\
178,797 \\
167,780 \\
167,932 \\
170,876 \\
173,096 \\
169,083 \\
169,083\end{array}$ \\
\hline 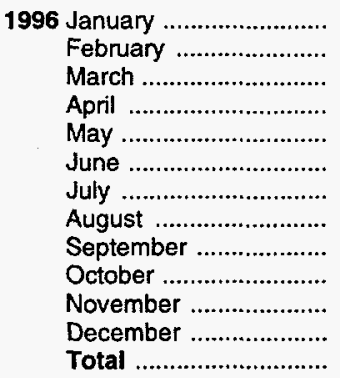 & $\begin{array}{r}83,814 \\
84,533 \\
91,409 \\
89,124 \\
89,525 \\
84,748 \\
89,262 \\
95,083 \\
87,773 \\
94,752 \\
86,905 \\
86,928 \\
1,063,856\end{array}$ & $\begin{array}{r}86,376 \\
78,384 \\
78,461 \\
71,198 \\
76,124 \\
81,819 \\
89,055 \\
90,004 \\
80,469 \\
80,604 \\
82,893 \\
87,419 \\
982,805\end{array}$ & $\begin{array}{r}524 \\
715 \\
474 \\
172 \\
790 \\
591 \\
802 \\
620 \\
649 \\
642 \\
668 \\
479 \\
7,126\end{array}$ & $\begin{array}{r}6,743 \\
6,892 \\
6,880 \\
7,330 \\
7,663 \\
8,046 \\
7,877 \\
7,412 \\
8,214 \\
8,077 \\
7,976 \\
7,361 \\
90,473\end{array}$ & $\begin{array}{l}159,779 \\
159,203 \\
161,611 \\
170,131 \\
175,001 \\
171,611 \\
163,929 \\
160,657 \\
161,374 \\
163,876 \\
160,811 \\
154,089 \\
154,089\end{array}$ \\
\hline 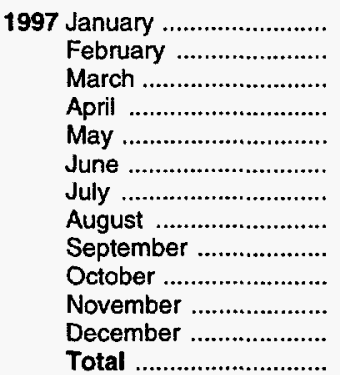 & $\begin{array}{r}92,776 \\
88,394 \\
92,757 \\
88,283 \\
94,647 \\
86,772 \\
\text { R } 89,073 \\
\text { R } 89,625 \\
\text { R } 92,593 \\
96,794 \\
85,491 \\
97,633 \\
1,094,837\end{array}$ & $\begin{array}{c}90,598 \\
77,008 \\
78,361 \\
73,854 \\
76,893 \\
82,300 \\
\text { R } 92,933 \\
\text { R } 90,880 \\
\text { R } 84,515 \\
\text { E } 85,674 \\
\text { NA } \\
\text { NA } \\
\text { NA }\end{array}$ & $\begin{array}{l}409 \\
338 \\
585 \\
528 \\
580 \\
599 \\
781 \\
620 \\
820 \\
564 \\
\text { NA } \\
\text { NA } \\
\text { NA }\end{array}$ & $\begin{array}{l}7,298 \\
5,778 \\
6,936 \\
6,657 \\
7,195 \\
6,751 \\
6,807 \\
8,551 \\
6,997 \\
7,446 \\
\text { NA } \\
\text { NA } \\
\text { NA }\end{array}$ & $\begin{array}{r}146,225 \\
150,543 \\
157,390 \\
164,402 \\
171,500 \\
170,616 \\
R_{158,505} \\
R_{151,970} \\
R_{149,330} \\
E_{143,805} \\
\text { NA } \\
\text { NA } \\
\text { NA }\end{array}$ \\
\hline
\end{tabular}

a Includes Puerto Rico.

b Stocks held by electric utilities, coke plants, general industry, and coal producers and distributors at end of period. Excludes stocks held at retail dealers for consumption by the residential and commercial sector.

$\mathrm{R}=$ Revised data. NA=Not available. E=Estimate.

Notes: - Data through 1995 are final. Subsequent data are preliminary.
- For methodology used to calculate production, consumption, and stocks, see Notes 1, 2, and 3 at end of section. - Totals may not equal sum of components due to independent rounding. - Geographic coverage is the 50 States and the District of Columbia.

Sources: See end of section. 
Table 6.2 Coal Consunnption by End-Use Sector

(Thousand Short Tons)

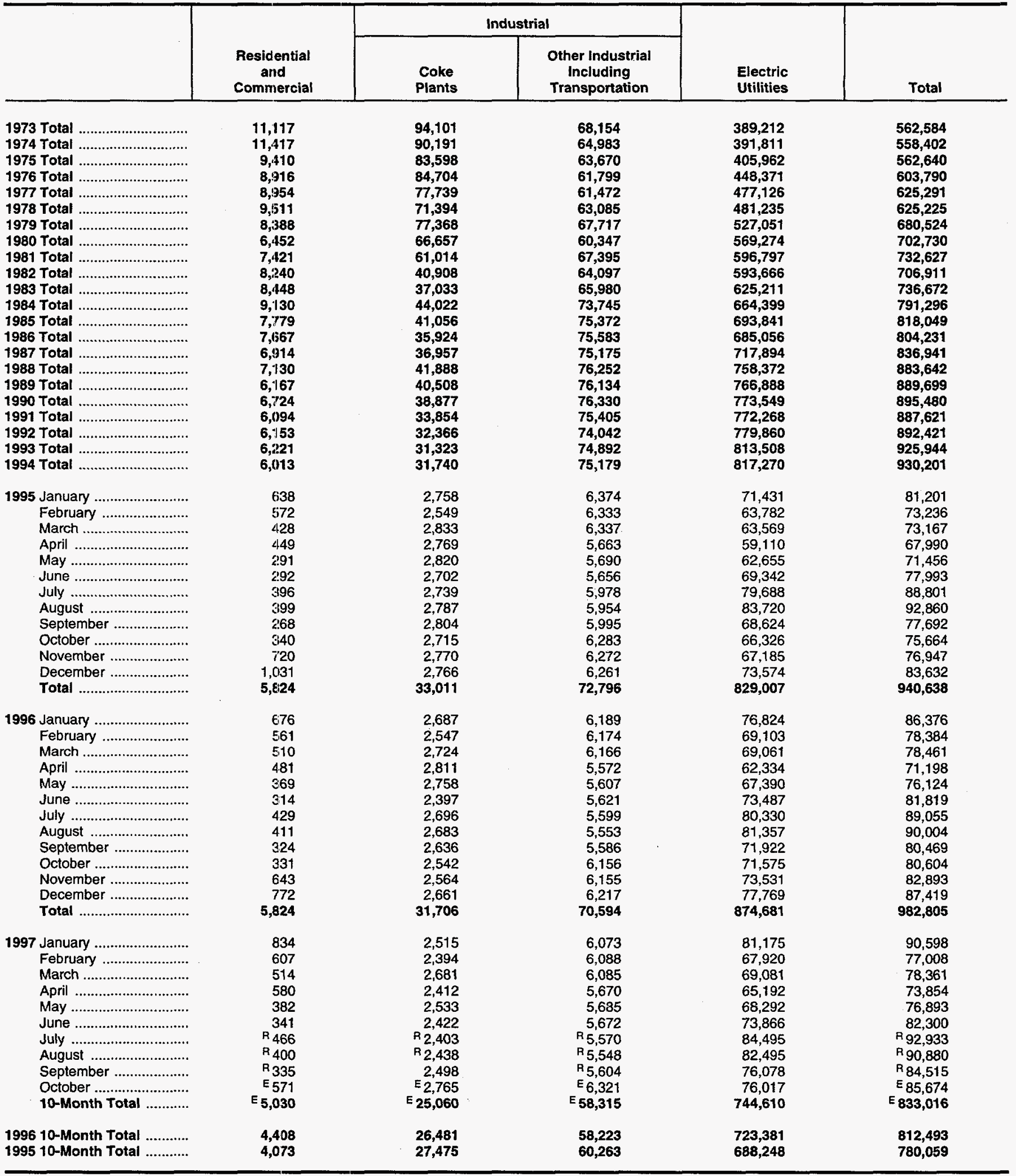

$R=$ Revised data. E=Estimate.

Notes: - For sector-specific reporting and estimating information, see Note 2 at end of section. - Data through 1994 are final. Subsequent data are preliminary. - Totals may not equal sum of componerits due to independent rounding. - Geographic coverage is the 50 States and the District of Columbia.

Sources: See end of section. 


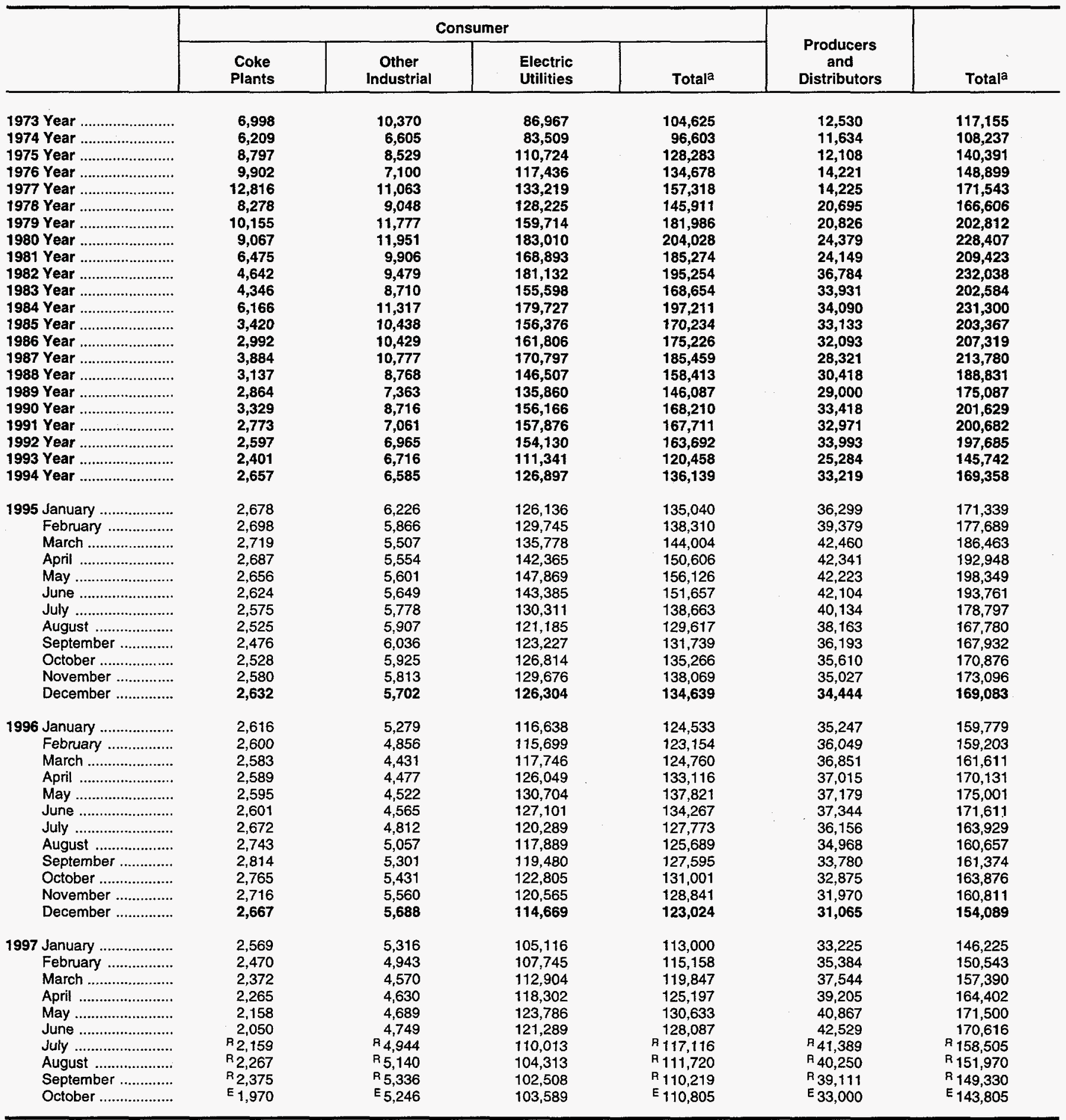

a Includes stocks held at retail dealers for consumption by the residential and commercial sector in thousand short tons: $1973290 ; 1974280 ; 1975$ 233; 1976 240; $1977220 ; 1978360$; and 1979340.

$\mathrm{R}=$ Revised data. $\mathrm{E}=$ Estimate.

Notes: - For sector-specific reporting and estimating information, see Note 3 at end of section. - Data through 1994 are final. Subsequent data are preliminary. - Totals may not equal sum of components due to independent rounding. - Geographic coverage is the 50 States and the District of Columbia.

Sources: See end of section. 


\section{Coal Notes}

1. Production: Preliminary monthly estimates of national coal production are the sum of weekly estimates developed by the Energy Information Administration (EIA) and published in the Weekly Coal Production report. When a week extends into a new month, production is allocated on a daily basis and added to the appropriate month. Weekly estimates are based on Association of American Railroads data showing the number of railcars loaded with coal during the week by Class I and certain other railroads. This number is converted into tons of coal by EIA by using the average number of tons of coal per railcar loaded reported in the most recent "Quarterly Freight Commodity Statistics" from the Surface Transportation Board. If an average coal tonnage per railcar loaded is not available for a specific railroad, the national average is used. To derive the estimate of total weekly production, the total rail tonnage for the week is divided by the ratio of quarterly production shipped by rail and total quarterly production. Data for the corresponding quarter of previous years are used to derive this ratio. This method ensures that the seasonal variations are preserved in the production estimates.

When preliminary quarterly data become available, the monthly and weekly estimates are adjusted to conform to the quarterly figure. The adjustment procedure uses State-level production data and is explained in EIA's Quarterly Coal Report. Initial estimates of annual production published in January of the following year are based on preliminary production data covering the first 9 months (three quarters) and weekly/monthly estimates for the fourth quarter. The fourth quarter estimates may or may not be revised when preliminary data become available in March of the following year, depending on the magnitude of the difference between the estimates and the preliminary data. In any event, all quarterly, monthly, and weekly production figures are adjusted to conform to the final annual production data published in the Monthly Energy Review in the fall of the following year.

2. Consumption: Coal consumption data are reported by major end-use sector. Estimated data for the most recent months (designated by an "E") are derived from forecasted values shown in the EIA Short-Term Energy Outlook (DOE/EIA-0202) table titled "Supply and Disposition of Coal: Mid World Oil Price Case." The monthly estimates are one-third of the quarterly values shown in the then current issue of the publication, regularly released in February, May, November, and November. The estimates are revised quarterly as collected data become available from the data sources. Sector-specific information follows.

- Residential and Commercial-Prior to 1980, monthly consumption estimates for the residential and commercial sector were derived by using reported data to modify baseline figures developed by the Bureau of Mines. From 1980-1987, monthly estimates were derived by proportioning reported quarterly data by using the ratios of monthly-to-quarterly consumption data in 1979 , the last year in which monthly data were reported on Form EIA-2. During 1981 and 1982, the estimates were also modified to reflect air temperature degree-days. Quarterly consumption data were taken directly from reported data and were defined as distribution to the residential and commercial sector as reported by coal producers and distributors on Form EIA-6. Beginning in January 1988 , monthly residential and commercial consumption estimates are derived from reported quarterly data by using monthly national average population weighted heating/cooling degree-days obtained from the National Oceanic and Atmospheric Administration. The monthly ratios are the monthly national sum of heating and cooling degree-days as a proportion of the quarterly national sum. Quarterly consumption data are taken directly from reported data.

- Coke Plants-Prior to 1980 , monthly coke plant consumption data were taken directly from reported data. From 1980-1987, coke plant consumption estimates were derived by proportioning reported quarterly data by using the ratios of monthly-to-quarterly consumption data in 1979 , the last year in which monthly data were reported. Beginning in January 1988 , monthly coke plant consumption estimates are derived from the reported quarterly data by using monthly ratios of raw steel production data from the American Iron and Steel Institute. The ratios are the monthly raw steel production from open hearth and basic oxygen process furnaces as a proportion of the quarterly production from those kinds of furnaces.

- Other Industrial-Prior to 1978 , monthly consumption data for the other industrial sector (all industrial users minus coke plants) were derived by using reported data to modify baseline consumption figures from the most recent Bureau of the Census Annual Survey of Manufactures or Census of Manufactures. For 1978 and 1979, monthly estimates were derived from data reported on Forms EIA-3 and EIA-6. From 1980-1987, monthly figures were estimated by proportioning quarterly data by using the ratios of monthly-to-quarterly consumption data in 1979, the last year in which monthly data were reported on Form EIA-3. Quarterly consumption data were derived by adding beginning stocks at manufacturing plants to current receipts and subtracting ending stocks at manufacturing plants. In this calculation, current receipts were the greater of either reported receipts from manufacturing plants (Form EIA-3) or reported shipments to the other industrial sector (Form EIA-6), thereby ensuring that agriculture, forestry, fishing, mining, and construction consumption data were included where appropriate. Starting in January 1988, monthly consumption for the other industrial sector is estimated from reported quarterly data by using ratios derived from industrial production indices published by the Board of Governors of the Federal Reserve System. Indices for six major industry groups are used as the basis 
for calculating the ratios: foods, Standard Industrial Classification (SIC) 20; paper and products, SIC 26; chemicals and products, SIC 28; petroleum products, SIC 29; clay, glass, and stone products, SIC 32; and primary metals, SIC 33. The monthly ratios are computed as the monthly sum of the weighted indices as a proportion of the quarterly sum of the weighted indices by using the 1977 proportion as the weights

- Electric Utilities-Monthly consumption data for electric utility plants are taken directly from reported data.

3. Stocks: Coal stocks data are reported by major enduse sector. Estimated data for the most recent months (designated by an "E") are derived from forecasted values shown in the EIA Short-Term Energy Outlook (DOE/EIA-0202) table titled "Supply and Disposition of Coal: Mid World Oil Price Case." The monthly estimates are one-third of the quarterly values shown in the then current issue of the publication, regularly released in February, May, October, and November. The estimates are revised quarterly as collected data become available from the data sources. Sector-specific information follows.

- Coke Plants--Prior to 1980, monthly stocks at coke plants were taken directly from reported data. From 1980 forward, coke plant stocks are estimated by using one-third of the current quarterly change to indicate the monthly change in stocks. Quarterly stocks are taken directly from data reported on Form EIA-5.

- Other Industrial-Prior to 1978 , stocks for the other industrial sector were derived by using reported data to modify baseline figures from a one-time Bureau of Mines survey of consumers. For 1978-1982, monthly estimates were derived by judgmentally proportioning reported quarterly data based on representative seasonal patterns of supply and demand. From 1983 forward, other industrial coal stocks are estimated as indicated above for coke plants. Quarterly stocks are taken directly from data reported on Form EIA-3 and therefore include only manufacturing industries; data for agriculture, forestry, fishing, mining, and construction stocks are not available.

- Electric Utilities-Monthly stocks data at electric utility plants are taken directly from reported data.

- Producers and Distributors-Quarterly stocks at producers and distributors are taken directly from reported data. Monthly data are estimated by using one-third of the current quarterly change to indicate the monthly change in stocks.

4. Imports and Exports: All coal import and export figures are taken directly from data reported monthly by the Bureau of the Census.

5. Additional Information: EIA's Quarterly Coal Report provides additional information about coal data and estimation procedures.

\section{Sources for Table 6.1}

\section{Production}

1973-September 1977-U.S. Department of the Interior, Bureau of Mines, Minerals Yearbook and Minerals Industry Surveys.

October 1977 forward-Energy Information Administration, Weekly Coal Production.

\section{Consumption}

Table 6.2.

\section{Imports and Exports}

U.S. Department of Commerce, Bureau of the Census, Monthly Reports IM-145 (Imports) and EM-545 (Exports).

\section{Stocks}

Table 6.3.

\section{Sources for Table 6.2}

\section{Residential and Commercial}

1973-1976-U.S. Department of the Interior (DOI), Bureau of Mines (BOM), Minerals Yearbook.

January-September 1977-DOI, BOM, Form 6-1400, "Monthly Coal Report, Retail Dealers-Upper Lake Docks."

October 1977-1979-Energy Information Administration (EIA), Form EIA-2, "Monthly Coal Report, Retail Dealers-Upper Lake Docks."

1980 forward-EIA, Form EIA-6, "Coal Distribution Report," quarterly.

\section{Coke Plants}

1973-September 1977-DOI, BOM, Minerals Yearbook and Minerals Industry Surveys

October 1977-1980-EIA, Form EIA-5/5A, "Coke and Coal Chemicals-Monthly/Annual Supplement."

1981-1984_EIA, Form EIA-5/5A, "Coke Plant ReportQuarterly/Annual Supplement."

1985 forward-EIA, Form EIA-5, "Coke Plant ReportQuarterly."

\section{Other Industrial}

1973-September 1977-DOI, BOM, Minerals Yearbook and Minerals Industry Surveys. 
October 1977-1979-EIA, Form EIA-3, "Monthly Coal Consumption Report-Manufacturing Plants."

1980 forward-EIA, Form EIA-3, "Quarterly Coal Consumption Report-Manufacturing Plants," and Form EIA-6, "Coal Distribution Report," quarterly.

\section{Electric Utilities}

1973-September 1977-DOI, BOM, Minerals Yearbook and Minerals Industry Surveys.

October 1977 forward-EIA, Form EIA-759 (formerly

Form FPC-4), "Monthly Power Plant Report."

\section{Sources for Table 6.3}

\section{Coke Plants}

1973-September 1977-U.S. Department of the Interior (DOI), Bureau of Mines (BOM), Minerals Yearbook and Minerals Industry Surveys.

October 1977-1980-Energy Information Administration (EIA), Form EIA-5/5A, "Coke and Coal Chemicals-Monthly/Annual."

1981-1984—EIA, Form EIA 5/5A, "Coke Plant ReportQuarterly/Annual Supplement."
1985 forward-EIA, Form EIA-5, "Coke Plant ReportQuarterly."

\section{Other Industrial}

1973-September 1977-DOI, BOM, Minerals Yearbook and Minerals Industry Surveys.

October 1977-1979-EIA, Form EIA-3, "Monthly Coal Consumption Report-Manufacturing Plants."

1980 forward-EIA, Form EIA-3, "Quarterly Coal Consumption Report-Manufacturing Plants," and Form EIA-6, "Coal Distribution Report," quarterly.

\section{Electric Utilities}

1973-September 1977-DOI, BOM, Minerals Yearbook and Minerals Industry Surveys.

October 1977 forward-EIA, Form EI-A759 (formerly Form FPC-4), "Monthly Power Plant Report."

\section{Producers and Distributors}

EIA, Form EIA-6, "Coal Distribution Report," quarterly. 


\section{Section 7. Electricity}

Electric Power Industry. Electricity is produced by electric utilities and nonutility power producers. Electric utilities are the traditional, highly regulated part of the industry, and nonutility power producers are the unregulated, competitive part of the industry. In general, the electric power industry is moving away from regulated entities, and the nonutility power producers are expanding rapidly.

The Energy Information Administration maintains comprehensive data about electric utilities, which still account for most electric power in the country. Less information is available about nonutility power production, but some data are beginning to become available that provide perspective on the overall industry.

While little monthly data are available on the activities of nonutility power producers, some annual data can be provided. Monthly Energy Review Tables 7.1, 7.5, and 7.6 now provide annual data about nonutility power net generation and fossil fuel consumption.

In 1996, the total electric power industry net generation was 3.5 trillion kilowatthours of electricity. Of that sum, 3.1 trillion kilowatthours, or 88 percent, was produced by electric utilities and 0.4 trillion kilowatthours, or 12 percent, from nonutility power producers. While electric utilities relied most heavily on coal for producing power, nonutilities derived most of their power from natural gas.

Electric Utility Net Generation. During October 1997, electric utilities generated 253 billion kilowatthours of electricity, 5 percent more than in October 1996. Coalfired generation totaled 152 billion kilowathours, 6 percent higher than the October 1996 level. Nuclear generation totaled 47 billion kilowatthours, 7 percent lower than the level 1 year earlier. Natural gas-fired generation was 23 billion kilowatthours, 8 percent higher than the October 1996 level. Hydroelectric generation totaled 23 billion kilowatthours, 10 percent more than the October 1996 level. Petroleum-fired generation totaled 7 billion kilowatthours, 116 percent above the level 1 year earlier.

Electric Utility Sales. Electric utility sales of electricity to all ultimate consumers in the United States in October 1997 were 261 billion kilowatthours, 7 percent higher than sales during October 1996. Sales to industrial consumers totaled 89 billion kilowatthours in October 1997, 1 percent above the level of sales during the previous year. Residential sales totaled 84 billion kilowatthours in October 1997, 11 percent above the level 1 year earlier. Commercial sales totaled 79 billion kilowatthours, 8 percent higher than the level of sales during the previous year. In October 1997, other sales totaled 9 billion kilowatthours, 7 percent higher than the October 1996 level.

Electric Utililty Consumption of Fossil Fuels. Electric utility consumption of coal during October 1997 was 76 million short tons, 6 percent higher than consumption in October 1996. Petroleum consumption (excluding petroleum coke) during October 1997 was 12 million barrels, 109 percent above the level of consumption in October 1996. During October 1997, electric utilities consumed 246 billion cubic feet of natural gas, 8 percent above the October 1996 consumption level.

Electric Utility Stocks of Coal and Petroleum. On October 31,1997 , electric utility stocks of all types of coal totaled 104 million short tons, 16 percent lower than the level on October 31, 1996. Stocks of petroleum (excluding petroleum coke) on October 31,1997 , totaled 45 million barrels, 5 percent below the level on October 31, 1996. 
Figure 7.1 Electric Power Industry Net Generation

(Billion Kilowatthours)

Electric Power Industry, 1996

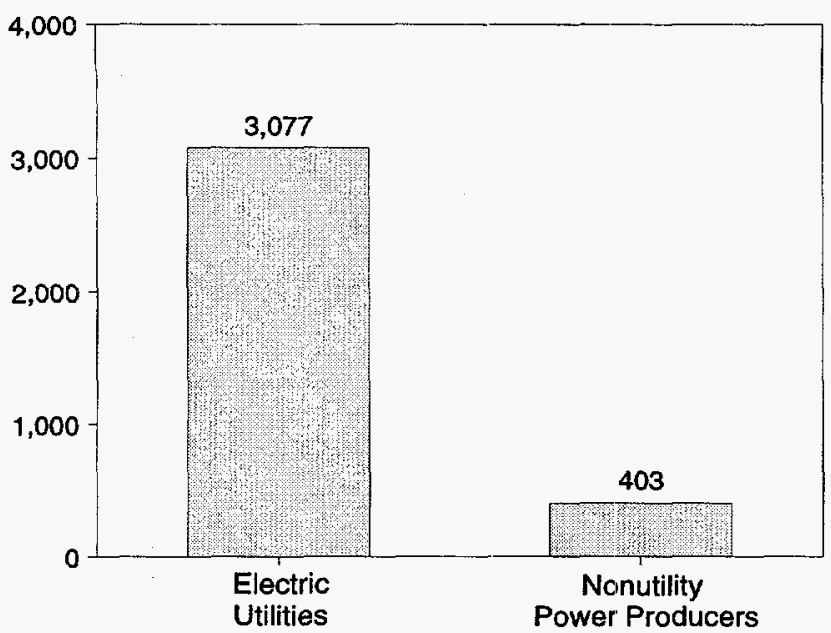

Electric Power Industry, 1989-1996

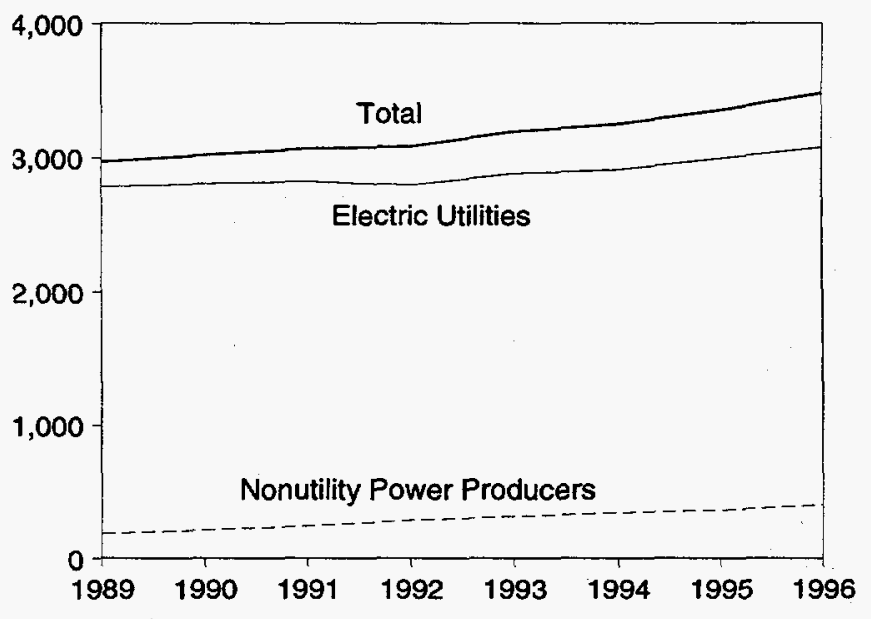

Electric Utilities by Source, 1973-1996

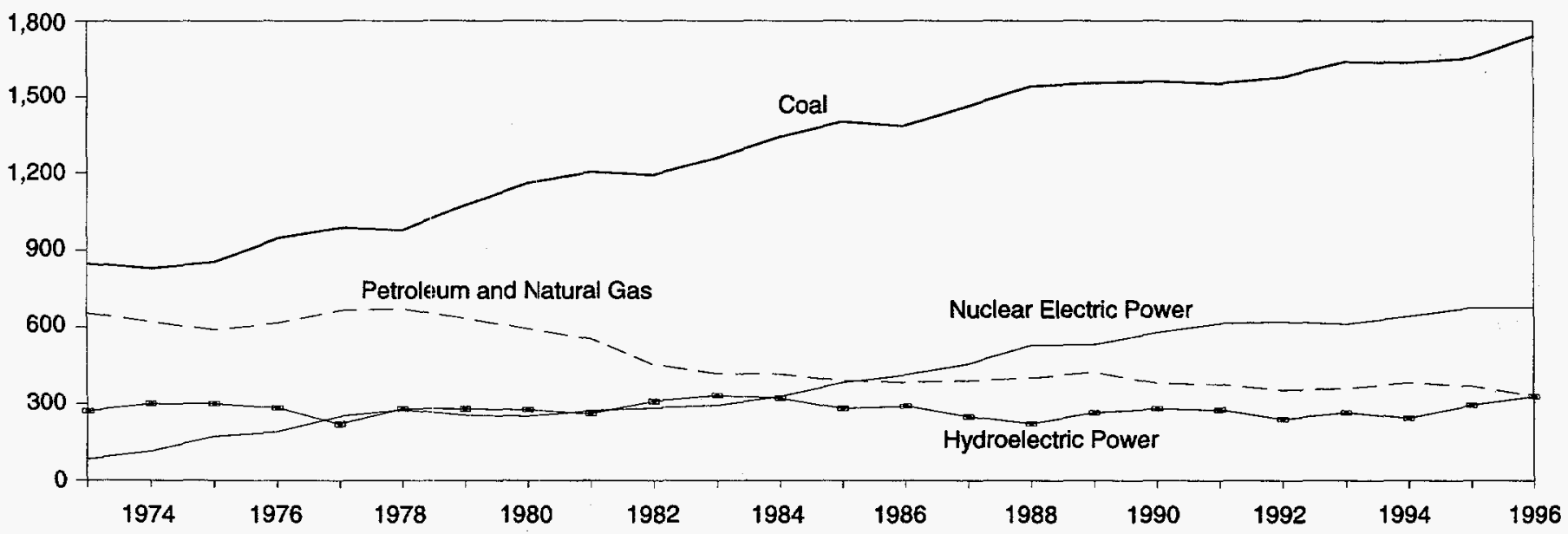

Electric Utilities Total, Janulary-October

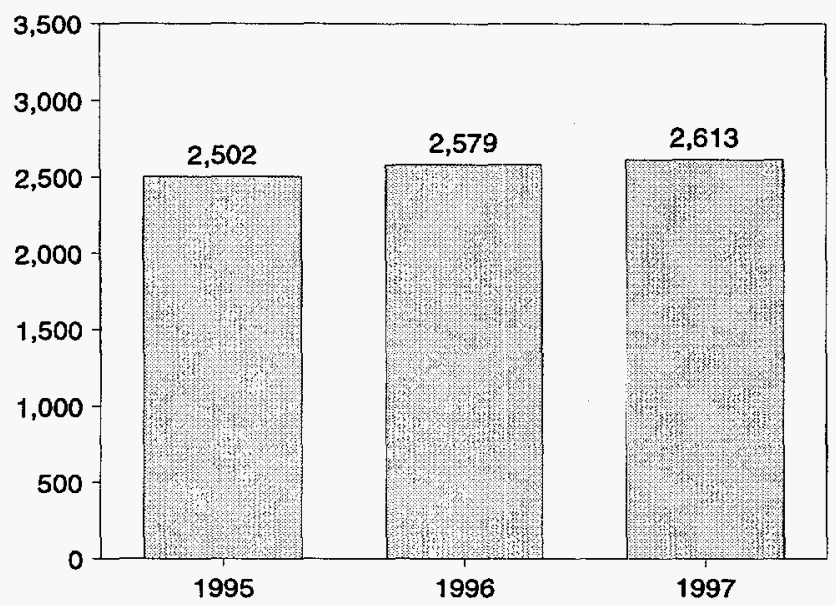

Electric Utilities Total, October 1997

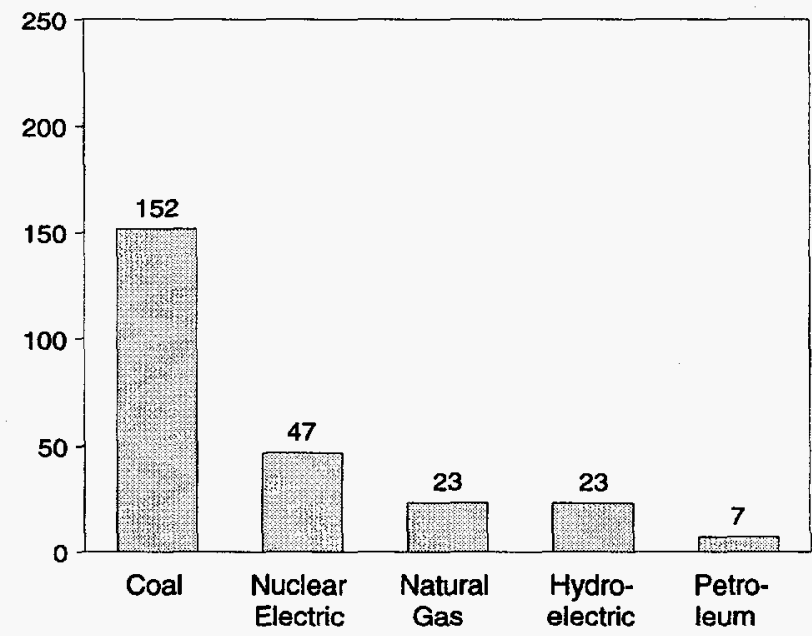

Note: Because vertical scales differ, graphs should not be compared. Source: Table 7.1 . 
Table 7.1 Electric Power Industry Net Generation

(Million Kilowatthours)

\begin{tabular}{|c|c|c|c|c|c|c|c|c|c|c|c|}
\hline & \multicolumn{9}{|c|}{ Electric Utilities } & \multirow[b]{2}{*}{$\begin{array}{l}\text { Nonutility } \\
\text { Power } \\
\text { Producers }\end{array}$} & \multirow[b]{2}{*}{$\begin{array}{c}\text { Total } \\
\text { Electric } \\
\text { Power } \\
\text { Industr }\end{array}$} \\
\hline & Coal & $\begin{array}{c}\text { Natural } \\
\text { Gas }^{a}\end{array}$ & Petroleumb & $\begin{array}{l}\text { Nuclear } \\
\text { Electric } \\
\text { Power }\end{array}$ & $\begin{array}{l}\text { Hydro- } \\
\text { electric } \\
\text { Power }\end{array}$ & $\begin{array}{c}\text { Geo- } \\
\text { thermal } \\
\text { Energy }\end{array}$ & $\begin{array}{l}\text { Wood } \\
\text { and } \\
\text { Waste }\end{array}$ & Otherc & Total & & \\
\hline $\begin{array}{l}1973 \text { Total } \\
1974 \text { Total } \\
1975 \text { Total } \\
1976 \text { Total } \\
1977 \text { Total } \\
1978 \text { Total } \\
1979 \text { Total } \\
1980 \text { Total } \\
1981 \text { Total } \\
1982 \text { Total } \\
1983 \text { Total } \\
1984 \text { Total } \\
1985 \text { Total } \\
1986 \text { Total } \\
1987 \text { Total } \\
1988 \text { Total } \\
1989 \text { Total } \\
1990 \text { Total } \\
1991 \text { Total } \\
1992 \text { Total } \\
1993 \text { Total } \\
1994 \text { Total }\end{array}$ & $\begin{array}{r}\mathbf{8 4 7 , 6 5 1} \\
\mathbf{8 2 8 , 4 3 3} \\
\mathbf{8 5 2 , 7 8 6} \\
\mathbf{9 4 4 , 3 9 1} \\
\mathbf{9 8 5 , 2 1 9} \\
\mathbf{9 7 5 , 7 4 2} \\
\mathbf{1 , 0 7 5 , 0 3 7} \\
\mathbf{1 , 1 6 1 , 5 6 2} \\
\mathbf{1 , 2 0 3 , 2 0 3} \\
\mathbf{1 , 1 9 2 , 0 0 4} \\
\mathbf{1 , 2 5 9 , 4 2 4} \\
\mathbf{1 , 3 4 1 , 6 8 1} \\
\mathbf{1 , 4 0 2 , 1 2 8} \\
\mathbf{1 , 3 8 5 , 8 3 1} \\
\mathbf{1 , 4 6 3 , 7 8 1} \\
\mathbf{1 , 5 4 0 , 6 5 3} \\
\mathbf{1 , 5 5 3 , 6 6 1} \\
\mathbf{1 , 5 5 9 , 6 0 6} \\
\mathbf{1 , 5 5 1 , 1 6 7} \\
\mathbf{1 , 5 7 5 , 8 9 5} \\
\mathbf{1 , 6 3 9 , 1 5 1} \\
\mathbf{1 , 6 3 5 , 4 9 3}\end{array}$ & $\begin{array}{l}340,858 \\
320,065 \\
299,778 \\
294,624 \\
305,505 \\
305,391 \\
329,485 \\
346,240 \\
345,777 \\
305,260 \\
274,098 \\
297,394 \\
291,946 \\
248,508 \\
272,621 \\
252,801 \\
266,598 \\
264,089 \\
264,172 \\
263,872 \\
258,915 \\
291,115\end{array}$ & $\begin{array}{r}314,343 \\
300,931 \\
289,095 \\
319,988 \\
358,179 \\
365,060 \\
303,525 \\
245,994 \\
206,421 \\
146,797 \\
144,499 \\
119,808 \\
100,202 \\
136,585 \\
118,493 \\
148,900 \\
158,318 \\
117,017 \\
111,463 \\
88,916 \\
99,539 \\
91,039\end{array}$ & $\begin{array}{r}83,479 \\
113,976 \\
172,505 \\
191,104 \\
250,883 \\
276,403 \\
255,155 \\
251,116 \\
272,674 \\
282,773 \\
293,677 \\
327,634 \\
383,691 \\
414,038 \\
455,270 \\
526,973 \\
529,355 \\
576,362 \\
612,565 \\
618,776 \\
610,291 \\
640,440\end{array}$ & $\begin{array}{l}272,083 \\
301,032 \\
300,047 \\
283,707 \\
220,475 \\
280,419 \\
279,783 \\
276,021 \\
260,684 \\
309,213 \\
332,130 \\
321,150 \\
281,149 \\
290,844 \\
249,695 \\
222,940 \\
265,063 \\
279,926 \\
275,519 \\
239,559 \\
265,063 \\
243,693\end{array}$ & \begin{tabular}{r|}
$\mathbf{1}, 966$ \\
$\mathbf{2 , 4 5 3}$ \\
$\mathbf{3 , 2 4 6}$ \\
$\mathbf{3 , 6 1 6}$ \\
$\mathbf{3 , 5 8 2}$ \\
$\mathbf{2 , 9 7 8}$ \\
$\mathbf{3 , 8 8 9}$ \\
$\mathbf{5 , 0 7 3}$ \\
$\mathbf{5 , 6 8 6}$ \\
$\mathbf{4 , 8 4 3}$ \\
$\mathbf{6 , 0 7 5}$ \\
$\mathbf{7 , 7 4 1}$ \\
$\mathbf{9 , 3 2 5}$ \\
10,308 \\
10,775 \\
10,300 \\
$\mathbf{9 , 3 4 2}$ \\
$\mathbf{8 , 5 8 1}$ \\
$\mathbf{8 , 0 8 7}$ \\
$\mathbf{8 , 1 0 4}$ \\
$\mathbf{7 , 5 7 1}$ \\
$\mathbf{6 , 9 4 1}$
\end{tabular} & $\begin{array}{r}328 \\
251 \\
191 \\
266 \\
481 \\
338 \\
498 \\
433 \\
368 \\
321 \\
379 \\
886 \\
1,383 \\
1,177 \\
1,477 \\
1,674 \\
1,965 \\
2,067 \\
2,046 \\
2,093 \\
1,990 \\
1,988\end{array}$ & $\begin{array}{r}0 \\
0 \\
0 \\
0 \\
0 \\
0 \\
0 \\
0 \\
0 \\
0 \\
3 \\
12 \\
16 \\
18 \\
14 \\
10 \\
3 \\
3 \\
4 \\
3 \\
4 \\
4\end{array}$ & $\begin{array}{l}1,860,710 \\
1,867,140 \\
1,917,649 \\
2,037,696 \\
2,124,323 \\
2,206,331 \\
2,247,372 \\
2,286,439 \\
2,294,812 \\
2,241,211 \\
2,310,285 \\
2,416,304 \\
2,469,841 \\
2,487,310 \\
2,572,127 \\
2,704,250 \\
2,784,304 \\
2,808,151 \\
2,825,023 \\
2,797,219 \\
2,882,525 \\
2,910,712\end{array}$ & $\begin{array}{l}\text { NA } \\
\text { NA } \\
\text { NA } \\
\text { NA } \\
\text { NA } \\
\text { NA } \\
\text { NA } \\
\text { NA } \\
\text { NA } \\
\text { NA } \\
\text { NA } \\
\text { NA } \\
\text { NA } \\
\text { NA } \\
\text { NA } \\
\text { NA } \\
183,728 \\
212,779 \\
243,006 \\
286,148 \\
314,399 \\
343,087\end{array}$ & $\begin{array}{c}\text { NA } \\
\text { NA } \\
\text { NA } \\
\text { NA } \\
\text { NA } \\
\text { NA } \\
\text { NA } \\
\text { NA } \\
\text { NA } \\
\text { NA } \\
\text { NA } \\
\text { NA } \\
\text { NA } \\
\text { NA } \\
\text { NA } \\
\text { NA } \\
2,968,032 \\
3,020,930 \\
3,068,029 \\
3,083,367 \\
3,196,924 \\
3,253,799\end{array}$ \\
\hline 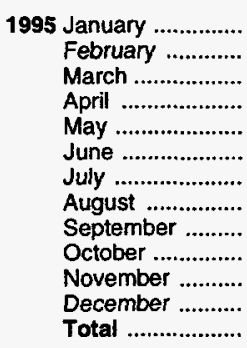 & $\begin{array}{r}142,412 \\
128,447 \\
126,970 \\
118,786 \\
126,013 \\
138,089 \\
158,378 \\
166,700 \\
135,241 \\
131,318 \\
133,899 \\
146,662 \\
1,652,914\end{array}$ & $\begin{array}{r}19,339 \\
16,422 \\
23,844 \\
22,062 \\
24,662 \\
28,394 \\
38,756 \\
44,402 \\
30,479 \\
23,076 \\
19,261 \\
16,609 \\
307,306\end{array}$ & \begin{tabular}{r|}
4,159 \\
7,042 \\
3,080 \\
3,315 \\
4,390 \\
4,422 \\
7,252 \\
8,257 \\
4,850 \\
3,500 \\
3,521 \\
7,056 \\
60,844
\end{tabular} & $\begin{array}{r}63,342 \\
51,858 \\
51,880 \\
49,321 \\
54,387 \\
56,381 \\
62,037 \\
61,661 \\
55,690 \\
54,293 \\
52,708 \\
59,844 \\
673,402\end{array}$ & $\begin{array}{r}23,291 \\
23,956 \\
27,458 \\
23,464 \\
26,570 \\
28,387 \\
25,942 \\
22,999 \\
18,798 \\
21,440 \\
24,019 \\
27,329 \\
293,653\end{array}$ & $\begin{array}{r}408 \\
296 \\
326 \\
282 \\
255 \\
281 \\
305 \\
524 \\
367 \\
619 \\
554 \\
528 \\
4,745\end{array}$ & $\begin{array}{r}126 \\
105 \\
116 \\
150 \\
102 \\
127 \\
154 \\
162 \\
147 \\
162 \\
154 \\
143 \\
1,649\end{array}$ & $\begin{array}{r}(s) \\
(s) \\
(s) \\
(s) \\
2 \\
2 \\
3 \\
2 \\
2 \\
1 \\
1 \\
(s) \\
15\end{array}$ & $\begin{array}{r}253,077 \\
228,127 \\
233,675 \\
217,381 \\
236,381 \\
256,083 \\
292,827 \\
304,709 \\
245,574 \\
234,409 \\
234,117 \\
258,170 \\
2,994,529\end{array}$ & $\begin{array}{l}\text { NA } \\
\text { NA } \\
\text { NA } \\
\text { NA } \\
\text { NA } \\
\text { NA } \\
\text { NA } \\
\text { NA } \\
\text { NA } \\
\text { NA } \\
\text { NA } \\
\text { NA } \\
361,889\end{array}$ & $\begin{array}{c}\text { NA } \\
\text { NA } \\
\text { NA } \\
\text { NA } \\
\text { NA } \\
\text { NA } \\
\text { NA } \\
\text { NA } \\
\text { NA } \\
\text { NA } \\
\text { NA } \\
\text { NA } \\
\mathbf{3 , 3 5 6 , 4 1 8}\end{array}$ \\
\hline 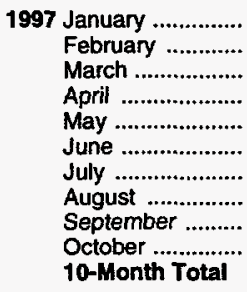 & $\begin{array}{r}161,276 \\
135,218 \\
137,554 \\
131,720 \\
136,185 \\
146,072 \\
166,893 \\
162,363 \\
151,238 \\
151,840 \\
1,480,359\end{array}$ & $\begin{array}{r}13,927 \\
13,455 \\
18,170 \\
18,783 \\
22,098 \\
28,265 \\
40,143 \\
37,186 \\
32,245 \\
23,454 \\
247,728\end{array}$ & $\begin{array}{r}8,392 \\
4,644 \\
4,525 \\
4,094 \\
4,489 \\
6,789 \\
9,204 \\
7,580 \\
7,865 \\
7,242 \\
64,822 \\
64,822\end{array}$ & $\begin{array}{r}58,914 \\
50,658 \\
50,414 \\
45,313 \\
47,032 \\
52,095 \\
57,352 \\
61,084 \\
52,586 \\
46,981 \\
522,428\end{array}$ & $\begin{array}{r}31,090 \\
29,882 \\
33,313 \\
30,483 \\
32,753 \\
32,801 \\
30,070 \\
25,494 \\
22,121 \\
23,241 \\
291,248\end{array}$ & $\begin{array}{r}414 \\
310 \\
438 \\
484 \\
471 \\
385 \\
512 \\
505 \\
482 \\
477 \\
4,478\end{array}$ & $\begin{array}{r}162 \\
147 \\
155 \\
169 \\
177 \\
158 \\
168 \\
173 \\
153 \\
193 \\
1,654\end{array}$ & $\begin{array}{r}(\mathrm{s}) \\
(\mathrm{s}) \\
1 \\
1 \\
1 \\
1 \\
1 \\
1 \\
1 \\
1 \\
9\end{array}$ & $\begin{array}{r}274,177 \\
234,315 \\
244,569 \\
231,045 \\
243,206 \\
266,565 \\
304,344 \\
294,386 \\
266,690 \\
253,430 \\
2,612,726\end{array}$ & $\begin{array}{l}\text { NA } \\
\text { NA } \\
\text { NA } \\
\text { NA } \\
\text { NA } \\
\text { NA } \\
\text { NA } \\
\text { NA } \\
\text { NA } \\
\text { NA } \\
\text { NA }\end{array}$ & $\begin{array}{l}\text { NA } \\
\text { NA } \\
\text { NA } \\
\text { NA } \\
\text { NA } \\
\text { NA } \\
\text { NA } \\
\text { NA } \\
\text { NA } \\
\text { NA } \\
\text { NA }\end{array}$ \\
\hline $\begin{array}{l}1996 \text { 10-Month Total } \\
1995 \text { 10-Month Total }\end{array}$ & $\begin{array}{l}1,439,263 \\
1,372,354\end{array}$ & $\begin{array}{l}233,791 \\
271,436\end{array}$ & $\begin{array}{l}57,118 \\
50,267\end{array}$ & $\begin{array}{l}565,438 \\
560,850\end{array}$ & $\begin{array}{l}277,216 \\
242,305\end{array}$ & $\begin{array}{l}\mathbf{4 , 2 4 0} \\
\mathbf{3 , 6 6 3}\end{array}$ & $\begin{array}{l}1,603 \\
1,352\end{array}$ & $\begin{array}{l}12 \\
14\end{array}$ & $\begin{array}{l}2,578,682 \\
2,502,241\end{array}$ & $\begin{array}{l}\text { NA } \\
\text { NA }\end{array}$ & $\begin{array}{l}\text { NA } \\
\text { NA }\end{array}$ \\
\hline
\end{tabular}

a Includes supplemental gaseous fuel. coke.

fuel oil nos. $1,2,4,5$, and 6 , crude oil, kerosene, and petroleum

$c$ "Other" is electricity produced from wind, photovoltaic, and solar thermal energy sources connected to electric utility distribution systems.
$N A=$ Not available. (s)=Less than 500 thousand kilowatthours.

Notes: - Totals may not equal sum of components due to independent rounding. - Geographic coverage is the 50 States and the District of Columbia.

Sources: See end of section.

See Table 7.5 for nonutility power producers' annual net generation of electricity by source. 
Figure 7.2 Electric Utility Retail Sales of Electricity

(Billion Kilowatthours)

Total, January-October

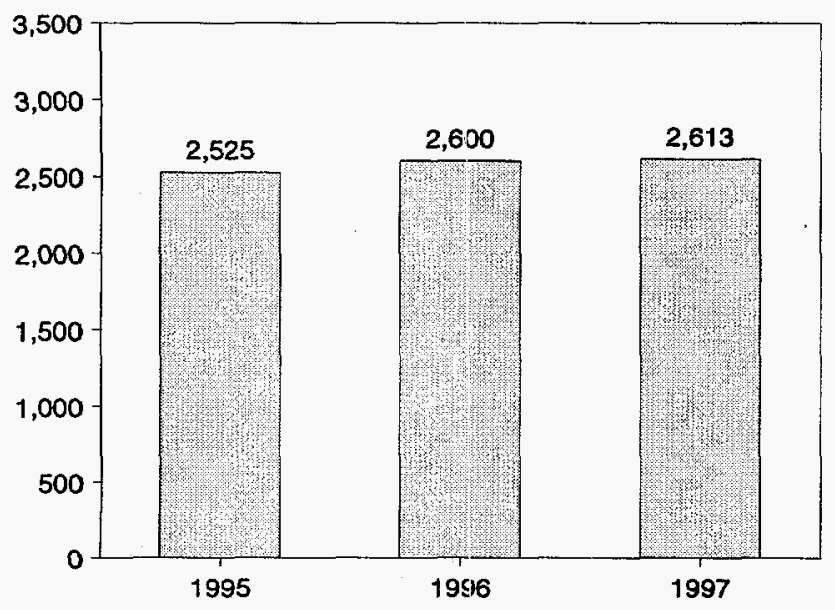

Total, Monthly

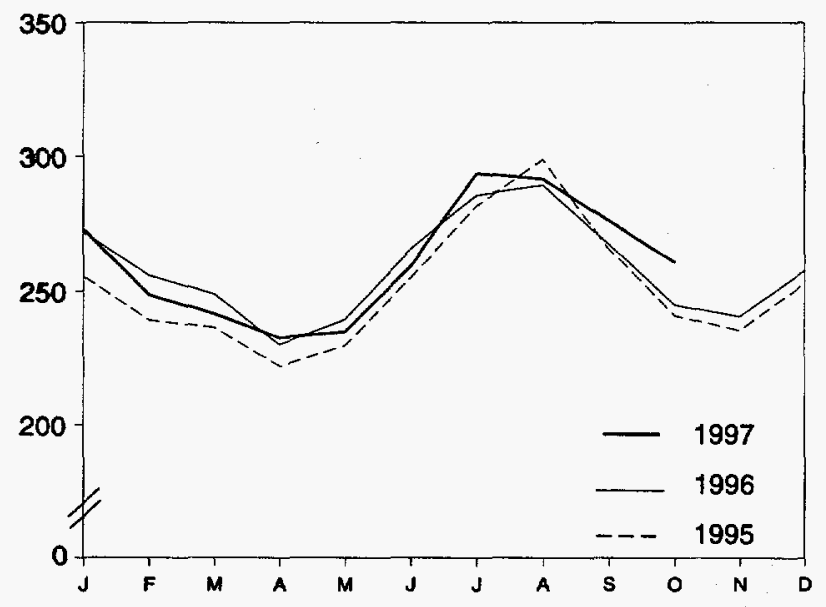

\section{By Sector, Monthly}

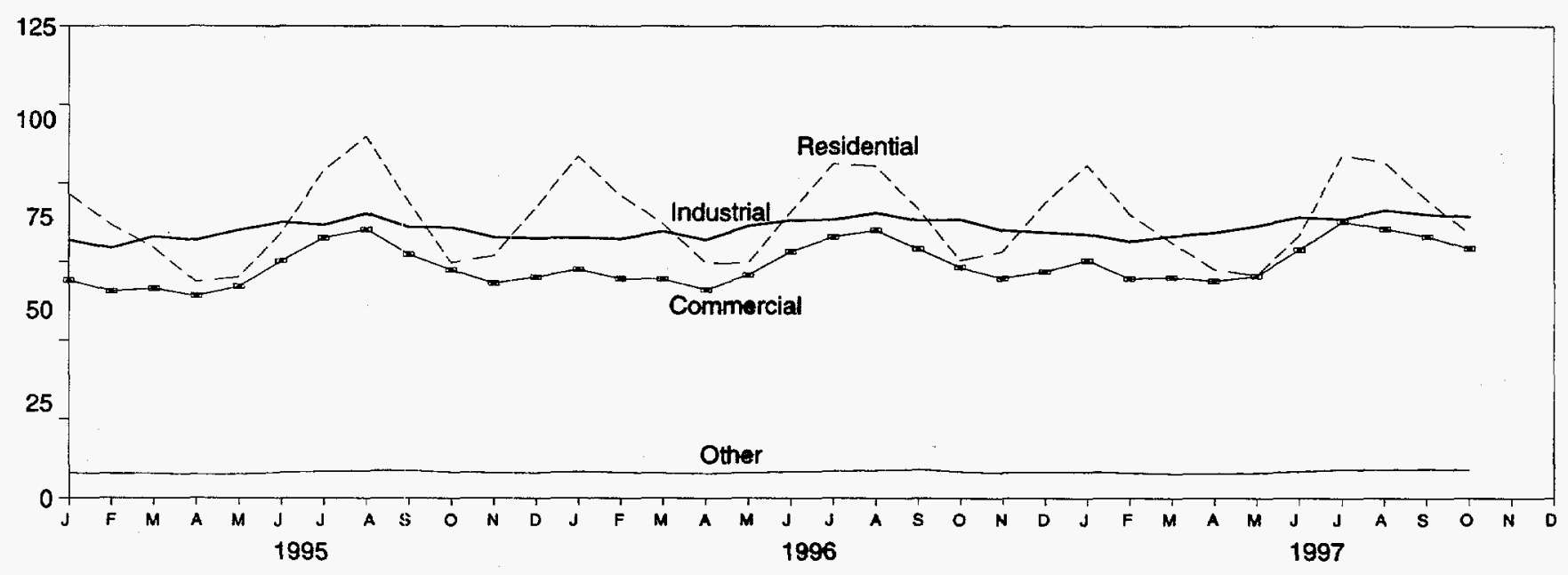

By Sector, 1973-1996

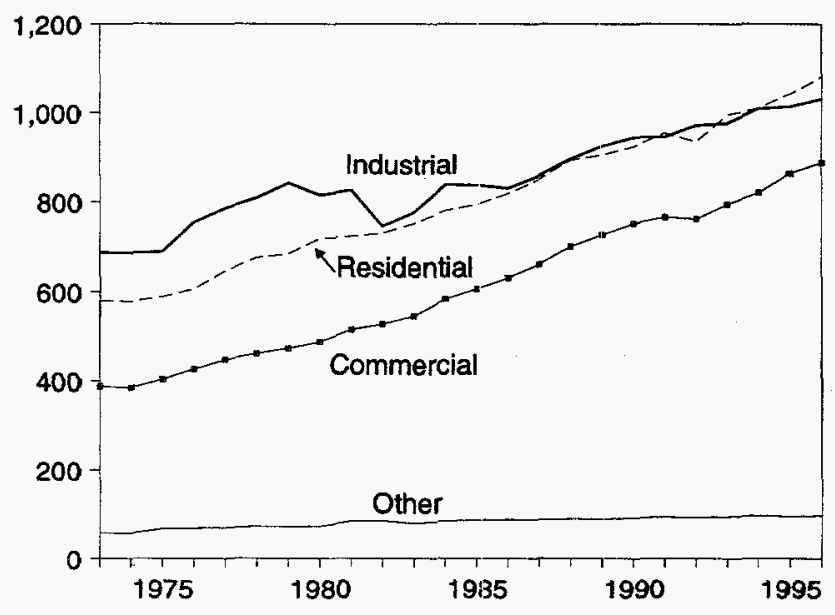

By Sector, October 1997

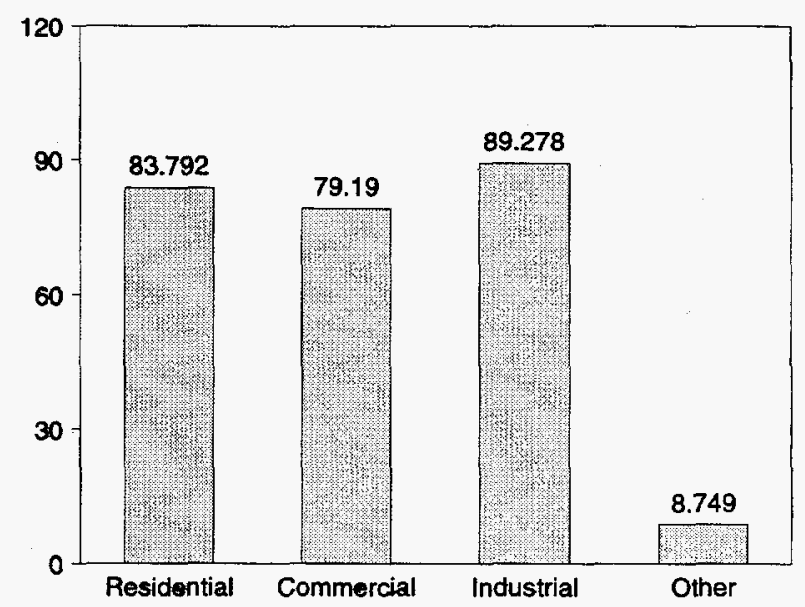

Note: Because vertical scales differ, graphs should not be compared. Source: Table 7.2, Monthly Series. 
Table 7.2 Electric Utility Retail Sales of Electricity by End-Use Sector

(Million Kilowatthours)

\begin{tabular}{|c|c|c|c|c|c|}
\hline & Residential & Commercial & Industrial & Othera & Total \\
\hline 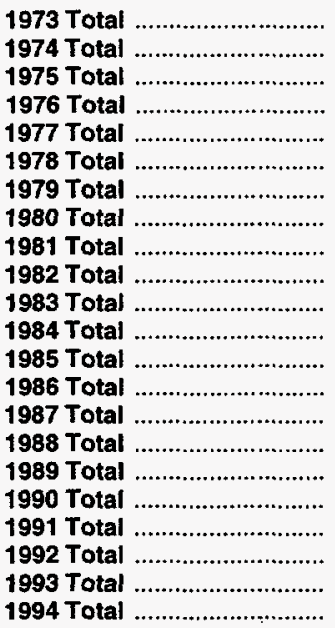 & $\begin{array}{r}579,231 \\
578,184 \\
588,140 \\
606,452 \\
645,239 \\
674,466 \\
682,819 \\
717,495 \\
722,265 \\
729,520 \\
750,948 \\
780,092 \\
793,934 \\
819,088 \\
850,410 \\
892,866 \\
905,525 \\
924,019 \\
955,417 \\
935,939 \\
994,781 \\
1,008,482\end{array}$ & $\begin{array}{l}388,266 \\
384,826 \\
403,049 \\
425,094 \\
446,514 \\
461,163 \\
473,307 \\
488,155 \\
514,338 \\
526,397 \\
543,788 \\
582,621 \\
605,989 \\
630,520 \\
660,433 \\
699,100 \\
725,861 \\
751,027 \\
765,664 \\
761,271 \\
794,573 \\
820,269\end{array}$ & $\begin{array}{r}686,085 \\
684,875 \\
687,680 \\
754,069 \\
786,037 \\
809,078 \\
841,903 \\
815,067 \\
825,743 \\
744,949 \\
775,999 \\
837,836 \\
836,772 \\
830,531 \\
858,233 \\
896,498 \\
925,659 \\
945,522 \\
946,583 \\
972,714 \\
977,164 \\
1,007,981\end{array}$ & $\begin{array}{l}59,326 \\
58,039 \\
68,222 \\
69,631 \\
70,571 \\
73,215 \\
73,070 \\
73,732 \\
84,756 \\
85,575 \\
80,219 \\
85,248 \\
87,279 \\
88,615 \\
88,196 \\
89,598 \\
89,765 \\
91,988 \\
94,339 \\
93,442 \\
94,944 \\
97,830\end{array}$ & $\begin{array}{l}1,712,909 \\
1,705,924 \\
1,747,091 \\
1,855,246 \\
1,948,361 \\
2,017,922 \\
2,071,099 \\
2,094,449 \\
2,147,103 \\
2,086,441 \\
2,150,955 \\
2,285,796 \\
2,323,974 \\
2,368,753 \\
2,457,272 \\
2,578,062 \\
2,646,809 \\
2,712,555 \\
2,762,003 \\
2,763,365 \\
2,861,462 \\
2,934,563\end{array}$ \\
\hline 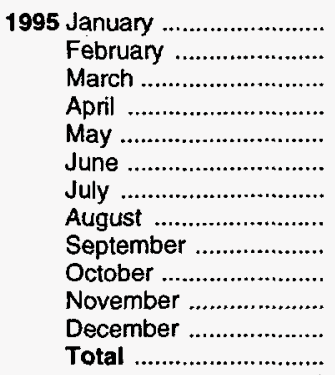 & $\begin{array}{r}96,573 \\
86,711 \\
79,475 \\
68,574 \\
70,082 \\
84,218 \\
104,021 \\
114,903 \\
93,900 \\
74,704 \\
76,927 \\
92,414 \\
1,042,501\end{array}$ & $\begin{array}{r}68,986 \\
65,468 \\
66,368 \\
64,069 \\
66,973 \\
75,189 \\
82,537 \\
85,203 \\
77,380 \\
72,376 \\
68,025 \\
70,110 \\
862,685\end{array}$ & $\begin{array}{r}81,785 \\
79,305 \\
82,942 \\
81,866 \\
85,087 \\
87,603 \\
86,676 \\
90,320 \\
86,026 \\
85,901 \\
82,701 \\
82,482 \\
1,012,693\end{array}$ & $\begin{array}{r}7,936 \\
7,655 \\
7,680 \\
7,350 \\
7,447 \\
8,000 \\
8,312 \\
8,574 \\
8,680 \\
8,071 \\
7,826 \\
7,876 \\
95,407\end{array}$ & $\begin{array}{r}255,281 \\
239,139 \\
236,465 \\
221,859 \\
229,589 \\
255,010 \\
281,546 \\
299,000 \\
265,986 \\
241,053 \\
235,479 \\
252,882 \\
3,013,287\end{array}$ \\
\hline 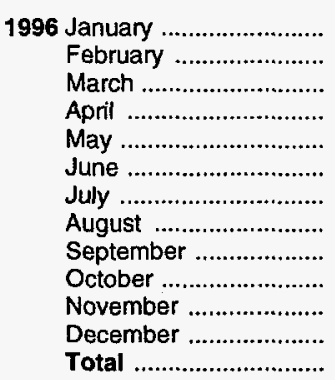 & $\begin{array}{r}R_{108,619} \\
R_{96,116} \\
R_{87,038} \\
R_{74,613} \\
R_{74,537} \\
R_{90,945} \\
R_{106,124} \\
R_{105,556} \\
R_{91,584} \\
R_{75,377} \\
R_{78,253} \\
R_{93,729} \\
R_{1,082,491}\end{array}$ & $\begin{array}{l}R_{72,499} \\
R_{69,524} \\
R_{69,328} \\
R_{65,961} \\
R_{70,619} \\
R_{78,244} \\
R_{82,882} \\
R_{84,927} \\
R_{79,093} \\
R_{73,076} \\
R_{69,526} \\
R_{71,746} \\
R_{887,425}\end{array}$ & 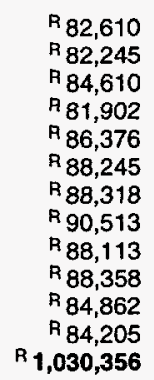 & $\begin{array}{l}R_{8,173} \\
R_{7,956} \\
R_{7,776} \\
R_{7,590} \\
R_{7,855} \\
R_{8,195} \\
R_{8,367} \\
R_{8,597} \\
R_{8,955} \\
R_{8,140} \\
R_{7,879} \\
R_{8,058} \\
R_{\mathbf{9 7 , 5 3 9}}\end{array}$ & $\begin{array}{l}R_{271,901} \\
R_{255,841} \\
R_{248,752} \\
R_{230,065} \\
R_{239,386} \\
R_{265,629} \\
R_{285,690} \\
R_{289,592} \\
R_{267,744} \\
R_{244,951} \\
R_{240,520} \\
R_{257,738} \\
R_{3,097,810}\end{array}$ \\
\hline 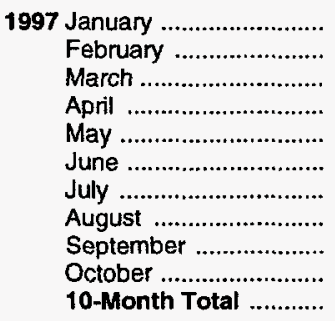 & $\begin{array}{r}105,774 \\
89,970 \\
81,030 \\
72,451 \\
70,492 \\
83,291 \\
108,916 \\
106,476 \\
94,413 \\
83,792 \\
896,605\end{array}$ & $\begin{array}{r}75,282 \\
69,439 \\
69,823 \\
68,635 \\
70,258 \\
78,745 \\
87,645 \\
85,349 \\
82,988 \\
79,190 \\
767,355\end{array}$ & $\begin{array}{r}83,643 \\
81,339 \\
83,029 \\
84,115 \\
86,298 \\
89,102 \\
88,487 \\
91,283 \\
89,996 \\
89,278 \\
866,570\end{array}$ & $\begin{array}{r}8,106 \\
7,803 \\
7,523 \\
7,511 \\
7,781 \\
8,260 \\
8,877 \\
8,792 \\
8,996 \\
8,749 \\
\mathbf{8 2 , 3 9 6}\end{array}$ & $\begin{array}{r}272,805 \\
248,552 \\
241,405 \\
232,711 \\
234,828 \\
259,398 \\
293,925 \\
291,900 \\
276,393 \\
261,009 \\
2,612,926\end{array}$ \\
\hline $\begin{array}{l}1996 \text { 10-Month Total ............ } \\
1995 \text { 10-Month Total ........... }\end{array}$ & $\begin{array}{l}910,508 \\
873,161\end{array}$ & $\begin{array}{l}746,153 \\
724,550\end{array}$ & $\begin{array}{l}861,290 \\
847,511\end{array}$ & $\begin{array}{l}81,601 \\
79,705\end{array}$ & $\begin{array}{l}2,599,552 \\
2,524,926\end{array}$ \\
\hline
\end{tabular}

a "Other" is public street and highway lighting, other sales to public authorities, sales to railroads and railways, and interdepartmental sales.

$R=$ Revised data.

Notes: - Totals may not equal sum of components due to independent rounding. - Geographic coverage is the 50 States and the District of Columbia.

Sources: See end of section.

Please Read: This table reports electric utility retail sales of electricity. Retail sales include electricity that the utilities purchased from nonutility power producers (NUPP) for resale to the end-use sectors. It does not include NUPP-produced electricity for their own use (141,480 million kilowatthours in 1996) or delivered directly to endusers (17,919 million kilowatthours in 1996). See EIA's Electric Power Annual 1995, Volume 11, the "Nonutility Power Producers" chapter for additional information. 
Figure 7.3 Electric Utility Consumption and Stocks of Fossil Fuels

Fuels Consumed, 1973-1996

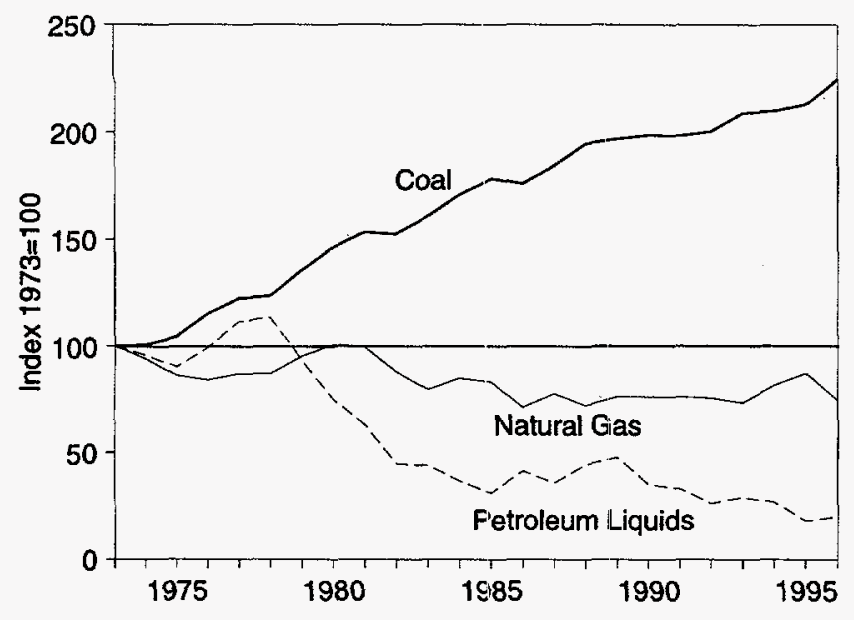

Petroleum Liquids Consumed, Monthly

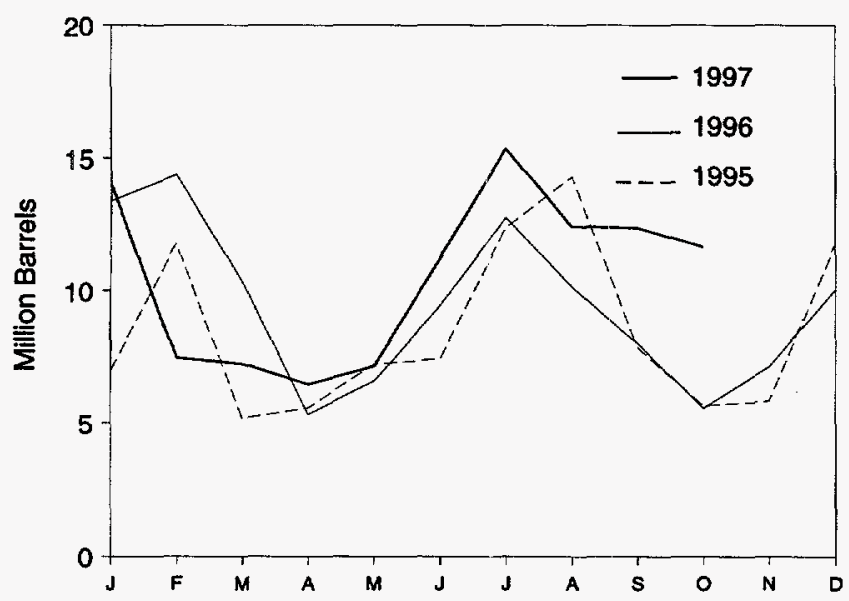

Coal Stocks, End of Month

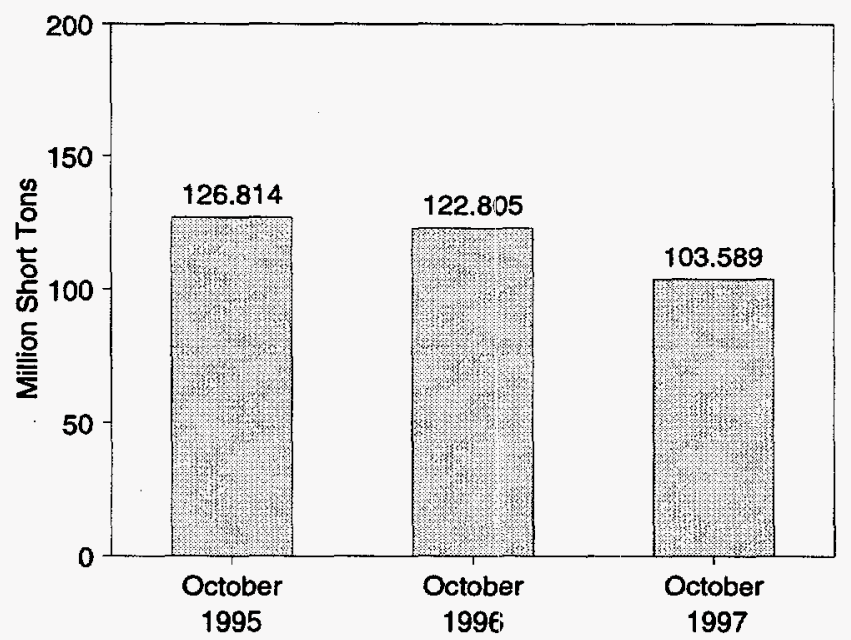

Coal Consumed, Monthly

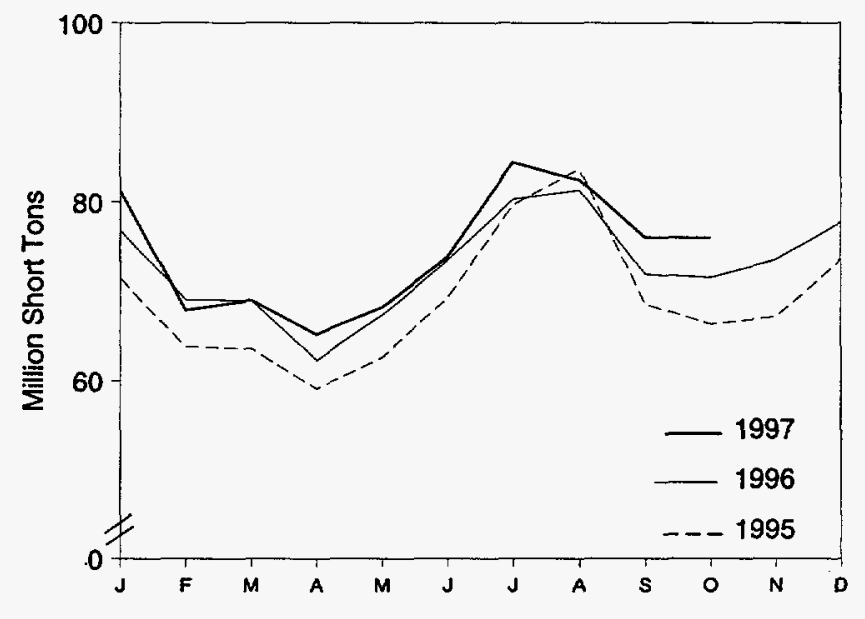

Natural Gas Consumed, Monthly

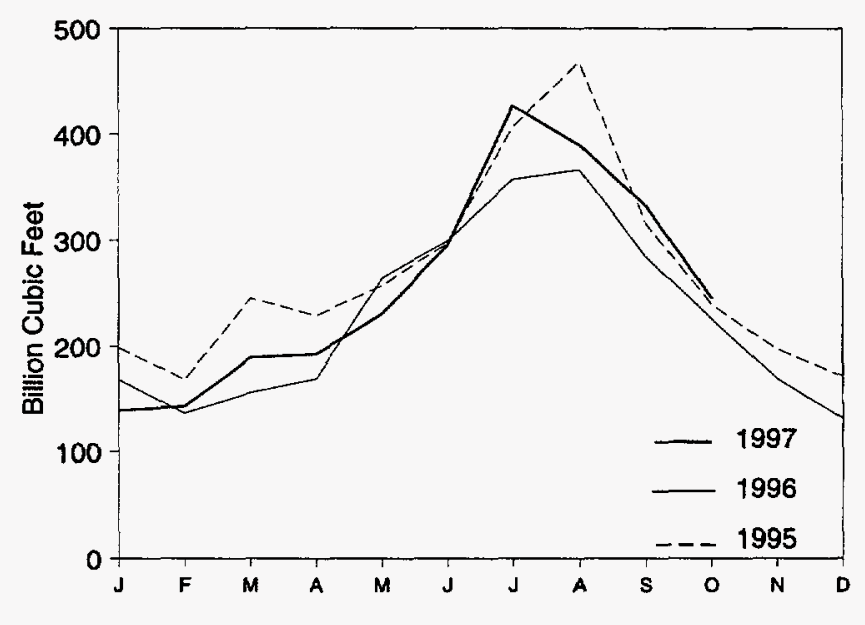

Petroleum Liquids Stocks, End of Month

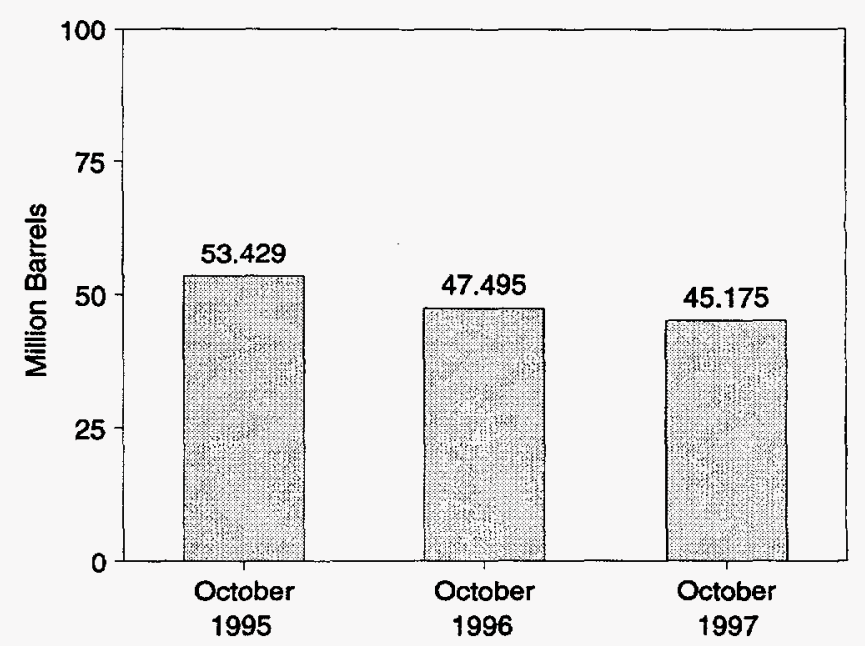

Note: Because vertical scales differ, graphs should not be compared. Sources: Tables 7.3 and 7.4 . 
Table 7.3 Electric Utility Consumption of Fossil Fuels To Generate Electricity

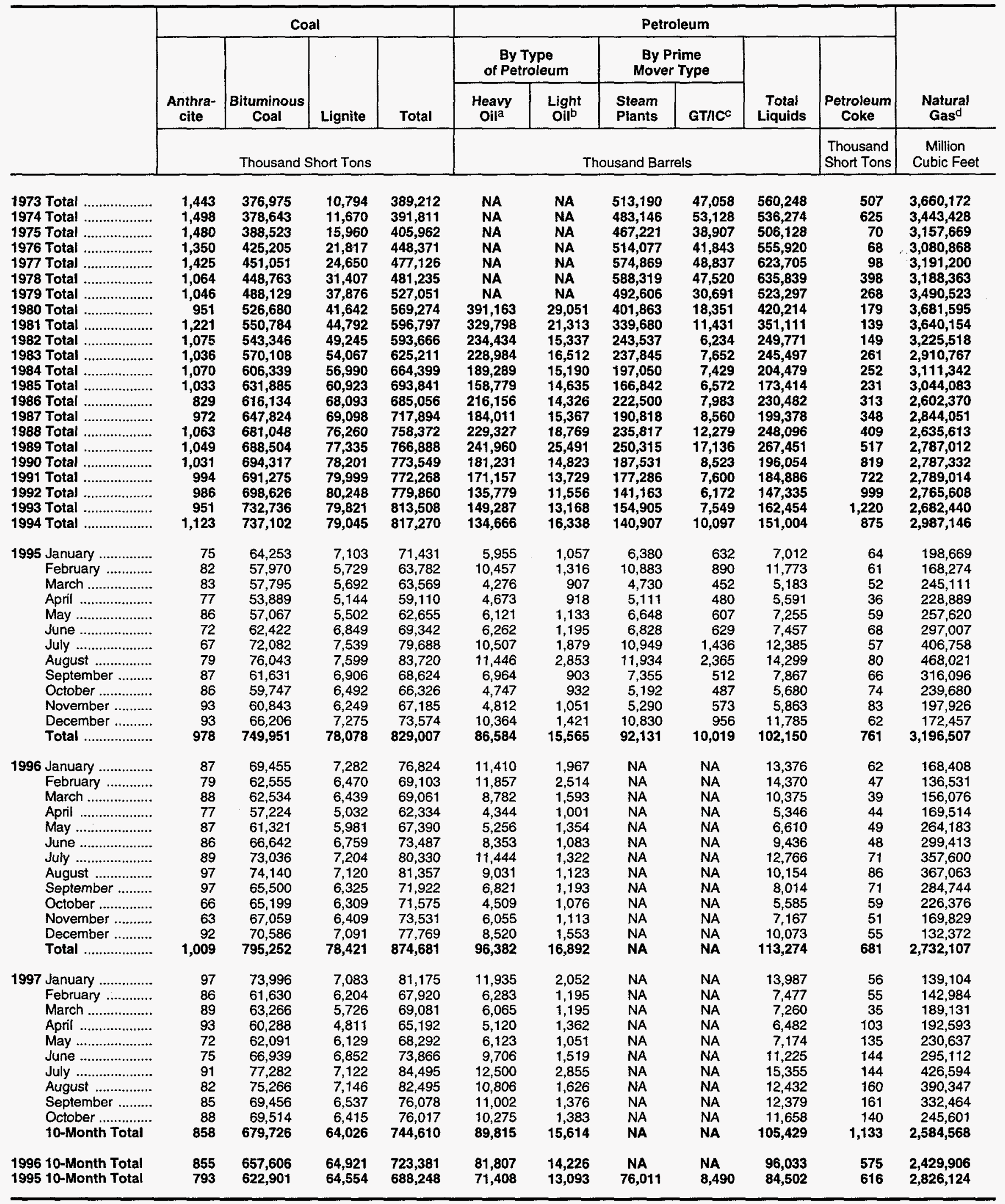

a Heavy oil includes fuel oil nos. 4, 5, and 6, and residual fuel oils.

b Light oil includes fuel oil nos. 1 and 2, kerosene, and jet fuel.

c $G T A C=$ Gas turbine and internal combustion plants.

d Includes supplemental gaseous fuels.

Notes: - Totals may not equal sum of components due to independent rounding. - Geographic coverage is the 50 States and the District of Columbia.

Sources: See end of section.

$\mathrm{NA}=$ Not available.

This table reports consumption of fossil fuels by electric utilities and does not include nonutility power producers. Please see Table 7.6 for annual consumption of fossil fuels by nonutility power producers. 
Table 7.4 Electric Utility Stocks of Coal and Petroleum, End of Period

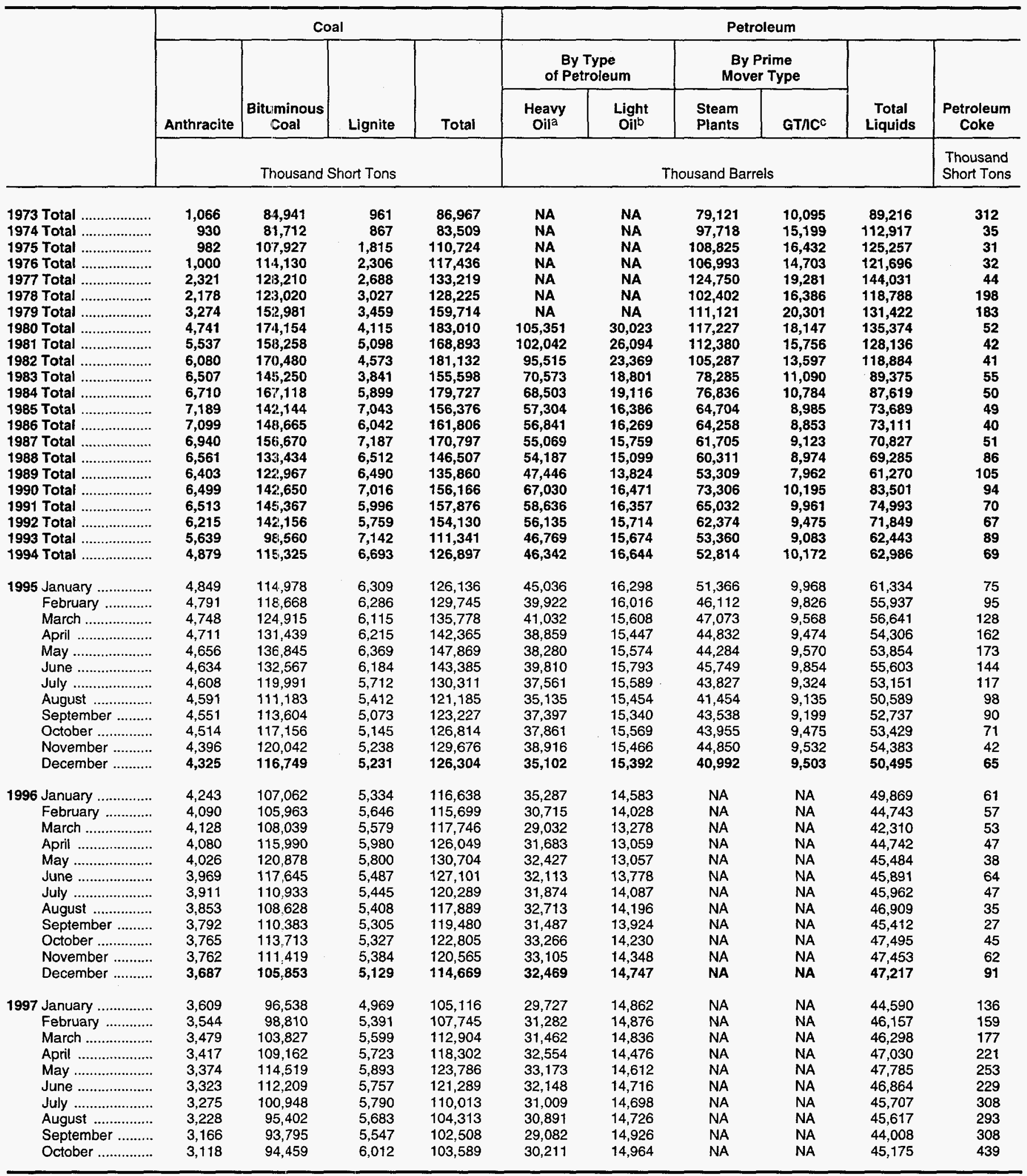

a Heavy oil includes fuel oil nos. 4,5 , and 6 , and residual fuel oils. b Light oil includes fuel oil nos. 1 and 2 , kerosene, and jet fuel. c GT/IC = Gas turbine and internal combustion plants.

$N A=$ Not available.
Notes: - Totals may not equal sum of components due to independent rounding. - Geographic coverage is the 50 States and the District of Columbia.

Sources: See end of section.

Please Read: This table reports stocks at electric utilities only and does not include stocks held by nonutility power producers, which are not collected by EIA. See EIA's Electric Power Annual 1995, Volume II, the "Nonutility Power Producers" chapter for additional information. 
Table 7.5 Nonutility Power Net Generation of Electricity (Million Kilowatthours)

\begin{tabular}{|c|c|c|c|c|c|c|c|c|c|}
\hline & Coala & $\begin{array}{c}\text { Natural } \\
\text { Gas }^{b}\end{array}$ & Petroleumc & $\begin{array}{l}\text { Nuclear } \\
\text { Electric } \\
\text { Powerd }\end{array}$ & $\begin{array}{l}\text { Hydro- } \\
\text { electric } \\
\text { Powere }\end{array}$ & $\begin{array}{c}\text { Geo- } \\
\text { thermal } \\
\text { Energy }\end{array}$ & $\begin{array}{c}\text { Wood } \\
\text { and } \\
\text { Wasteg }\end{array}$ & Otherh & Total \\
\hline $\begin{array}{l}1992 \text { Total ........ } \\
1993 \text { Total ........ } \\
1994 \text { Total ........ } \\
1995 \text { Total ........ } \\
1996 \text { Total ........ }\end{array}$ & $\begin{array}{l}45,189 \\
50,859 \\
56,197 \\
54,772 \\
60,794\end{array}$ & $\begin{array}{l}154,429 \\
169,502 \\
174,813 \\
191,069 \\
214,237\end{array}$ & $\begin{array}{l}10,508 \\
12,814 \\
14,464 \\
16,294 \\
19,605\end{array}$ & $\begin{array}{r}65 \\
76 \\
52 \\
0 \\
0\end{array}$ & $\begin{array}{r}9,352 \\
11,396 \\
13,095 \\
14,626 \\
16,545\end{array}$ & $\begin{array}{r}8,318 \\
9,454 \\
9,816 \\
9,614 \\
10,684\end{array}$ & $\begin{array}{l}\mathbf{5 1 , 2 6 4} \\
\mathbf{5 3 , 3 1 8} \\
\mathbf{5 4 , 8 9 8} \\
\mathbf{5 4 , 4 4 5} \\
\mathbf{5 9 , 3 3 3}\end{array}$ & $\begin{array}{r}7,023 \\
6,981 \\
19,752 \\
21,069 \\
22,292\end{array}$ & $\begin{array}{l}286,148 \\
314,399 \\
343,087 \\
361,889 \\
403,490\end{array}$ \\
\hline
\end{tabular}

a Coal, anthracite culm, and coal waste.

b Butane, ethane, propane, waste heat, and waste gases are included in "Natural Gas" for 1992 and 1993 but in "Other" for 1994-1996.

c Petroleum, petroleum coke, diesel, kerosene, and petroleum sludge and tar.

Nuclear reactor and generator at Argonne National Laboratory used primarily for research and development in testing reactor fuels as well as for training. Generation from the unit is for internal consumption.

e Conventional hydropower only; there are no pumped storage projects among the nonutility power producers.

Wood, wood waste, peat, wood liquors, railroad tjes, pitch, and wood sludge.

9 Municipal solid waste, agricultural waste, straw, tires, landfill gases, and other waste.

$h$ Wind, photovoltaic, and solar thermal energy; hydrogen, sulfur, batteries, chemicals, fish oil, and spent liquor, and, since 1994, butane, ethane, propane, waste heat, and waste gases.

Note: Total may not equal sum of components due to independent rounding.

Sources: Energy Information Administration, estimated from form EIA-867, "Annual Nonutility Power Producer Report."

\section{Table 7.6 Electric Power Industry Consumption of Fossil Fuels To Generate Electricity}

\begin{tabular}{|c|c|c|c|c|c|c|c|c|c|}
\hline & \multicolumn{3}{|c|}{ Coal } & \multicolumn{3}{|c|}{ Petroleum } & \multicolumn{3}{|c|}{ Natural Gas and Other Gas ${ }^{a}$} \\
\hline & $\begin{array}{l}\text { Electric } \\
\text { Utilities }\end{array}$ & $\begin{array}{c}\text { Nonutility } \\
\text { Power } \\
\text { Producers }{ }^{b}\end{array}$ & Total & $\begin{array}{c}\text { Electric } \\
\text { Utilities }^{c}\end{array}$ & $\begin{array}{c}\text { Nonutility } \\
\text { Power } \\
\text { Producers }\end{array}$ & Total & $\begin{array}{l}\text { Electric } \\
\text { Utility }^{\ominus}\end{array}$ & $\begin{array}{l}\text { Nonutility } \\
\text { Power } \\
\text { Producers }\end{array}$ & Total \\
\hline & \multicolumn{3}{|c|}{ Thousand Short Tons } & \multicolumn{3}{|c|}{ Thousand Barrels } & \multicolumn{3}{|c|}{ Million Cubic Feet } \\
\hline $\begin{array}{l}1992 \text { Total .... } \\
1993 \text { Total .... } \\
1994 \text { Total .... } \\
1995 \text { Total .... } \\
1996 \text { Total .... }\end{array}$ & $\begin{array}{l}779,860 \\
813,508 \\
817,270 \\
829,007 \\
874,681\end{array}$ & $\begin{array}{r}44,607 \\
48,343 \\
52,261 \\
47,849 \\
E_{49,130}\end{array}$ & $\begin{array}{l}824,467 \\
861,851 \\
869,531 \\
876,856 \\
923,811\end{array}$ & $\begin{array}{l}152,329 \\
168,556 \\
155,377 \\
105,956 \\
116,680\end{array}$ & $\begin{array}{r}31,539 \\
36,768 \\
40,460 \\
39,075 \\
\text { E } 42,096\end{array}$ & $\begin{array}{l}183,868 \\
205,324 \\
195,837 \\
145,031 \\
158,776\end{array}$ & $\begin{array}{l}2,765,608 \\
2,682,440 \\
2,987,146 \\
3,196,507 \\
2,732,107\end{array}$ & $\begin{array}{r}3,429,324 \\
3,691,954 \\
3,735,431 \\
3,915,614 \\
\varepsilon 4,104,490\end{array}$ & $\begin{array}{l}6,194,932 \\
6,374,394 \\
6,722,577 \\
7,112,121 \\
6,836,597\end{array}$ \\
\hline
\end{tabular}

a "Other Gas" is butane, ethane, propane, and other gases.

b Coal, anthracite culm, and coal waste.

- Includes petroleum coke (converted at 5 barrels per short ton).

d Petroleum, petroleum coke (converted at 5 barrels per short ton), diese, kerosene, petroleum sludge, and tar.

Natural gas only.

E=Estimate.
Note: Totals may not equal sum of components due to independent rounding.

Sources: - Electric Utilities: Energy Information Administration (E|A), Electric Power Monthly, December 1997, Table 14. - Nonutility Power Producers: EIA, estimated from Form EIA-867, "Annual Nonutility Power Producer Report" data. 


\section{Sources for Table 7.1}

\section{Electric Utilities}

1973-September 1977-Federal Power Commission Form FPC-4, "Monthly Power Plant Report."

October 1977-1979-Federal Energy Regulatory Commission (FERC), Form FPC-4, "Monthly Power Plant Report."

1980-Energy Information Administration (EIA), Electric Power Monthly, March 1991, Table 4, and (for geothermal energy and other) FERC, Form FPC-4, "Monthly Power Plant Report."

1981-EIA, Electric Power Monthly, March 1992, Table 4, and (for geothermal energy and other) FERC, Form FPC-4, "Monthly Power Plant Report."

1982-EIA, Electric Power Monthly, March 1993, Table 4, and (for geothermal energy and other) EIA, Form EIA-759, "Monthly Power Plant Report."

1983-1992-EIA, Eiectric Power Monthly, March 1994, Table 4, and (for geothermal energy and other) EIA, Form EIA-759, "Monthly Power Plant Report."

1993 and 1994 -EIA, Electric Power Monthly, May 1995, Tables 4 and 5.

1995 forward-EIA, Form EIA-759, "Monthly Power Plant Report."

\section{Nonutility Power Producers}

EIA, estimated from Form EIA-867, "Annual Nonutility Power Producer Report."

\section{Total Electric Power Industry}

Sum of Electric Utilities and Nonutility Power Producers.

\section{Sources for Table 7.2}

1973-September 1977-Federal Power Commission (FPC), Form FPC-5, "Monthly Statement of Electric Operating Revenue and Income."

October 1977-February 1980-Federal Energy Regulatory Commission (FERC), Form FPC-5, "Monthly Statement of Electric Operating Revenue and Income."

March 1980-1982_FERC, Form FPC-5, "Electric Utility Company Monthly Statement."

1983 Energy Information Administration (EIA), Form EIA-826, "Electric Utility Company Mlonthly Statement."

1984-1986_EIA, Form EIA-861, "Annual Electric Utility Report."
1987 forward-EIA, Electric Power Monthly, January 1998, Table 44.

\section{Sources for Table 7.3}

\section{Prime Mover Type Data}

1973-September 1977-Federal Power Commission (FPC), Form FPC-4, "Monthly Power Plant Report."

October 1977-1981-Federal Energy Regulatory Commission (FERC), Form FPC-4, "Monthly Power Plant Report." 1982 forward-Energy Information Administration (ELA), Form EIA-759, "Monthly Power Plant Report."

\section{All Other Data}

1973-September 1977-FPC, Form FPC-4, "Monthly Power Plant Report."

October 1977-1979_FERC, Form FPC-4, "Monthly Power Plant Report."

1980-1986-EIA, Electric Power Monthly, March issues. 1987-1995-EIA, Electric Power Monthly, January 1998, Table 14.

1996 forward-EIA, Form EIA-759, "Monthly Power Plant Report."

\section{Sources for Table 7.4}

\section{Prime Mover Type Data}

1973-September 1977-Federal Power Commission (FPC), Form FPC-4, "Monthly Power Plant Report." October 1977-1981-Federal Energy Regulatory Commission (FERC), Form FPC-4, "Monthly Power Plant Report." 1982 forward-Energy Information Administration (EIA), Form EIA-759, "Monthly Power Plant Report."

\section{All Other Data}

1973-September 1977-FPC, Form FPC-4, "Monthly Power Plant Report."

October 1977-1979_FERC, Form FPC-4 "Monthly Power Plant Report."

1980-1982-EIA, Electric Power Monthly, March issues. 1983 and 1993 monthly data-EIA, Electric Power Monthly, March 1994, Table 29.

1984-1986-EIA, Electric Power Monthly, March issues. 1987-1995 (except 1993 monthly data)-EIA, Electric Power Monthly, December 1997, Table 21.

1996 forward-EIA, Form EIA-759, "Monthly Power Plant Report." 


\section{Section 8. Nuclear Energy}

In October 1997, U.S. nuclear generating units produced a total of 47 net terawatthours (billion kilowatthours) of electricity, 7 percent lower than in October 1996. Nuclear units generated at an average capacity factor of 63.3 percent, 4.2 percentage points lower than in October 1996. Nuclear power supplied 18.5 percent of the total electric utility-generated electricity in October 1997, compared with 21.1 percent in October 1996.

No low-power or full-power licenses for nuclear power plants were issued by the Nuclear Regulatory Commission during October 1997.

On October 31, 1997, there were 109 operable nuclear generating units in the United States, with a collective net summer capability of 99.8 million kilowatts of electricity. Of the 109 operable units, 24 units generated at less than 25 percent of capacity because of maintenance, refueling, or repair outage, and 20 of the 24 units generated no electricity during the month. The aggregate net design capacity of the 109 operable units was 99.8 million kilowatts.

In addition, there were 3 other units with construction permits, although construction for all 3 units was canceled or halted. The design capacity of the 3 units with construction permits was 3.6 million kilowatts. The net design capacity of those units, when added to that of the 109 operable nuclear generating units, is 103.4 million kilowatts. 
Figure 8.1 Nuclear Power Plant Operations

Operable Units, End of Year, 1973-1996

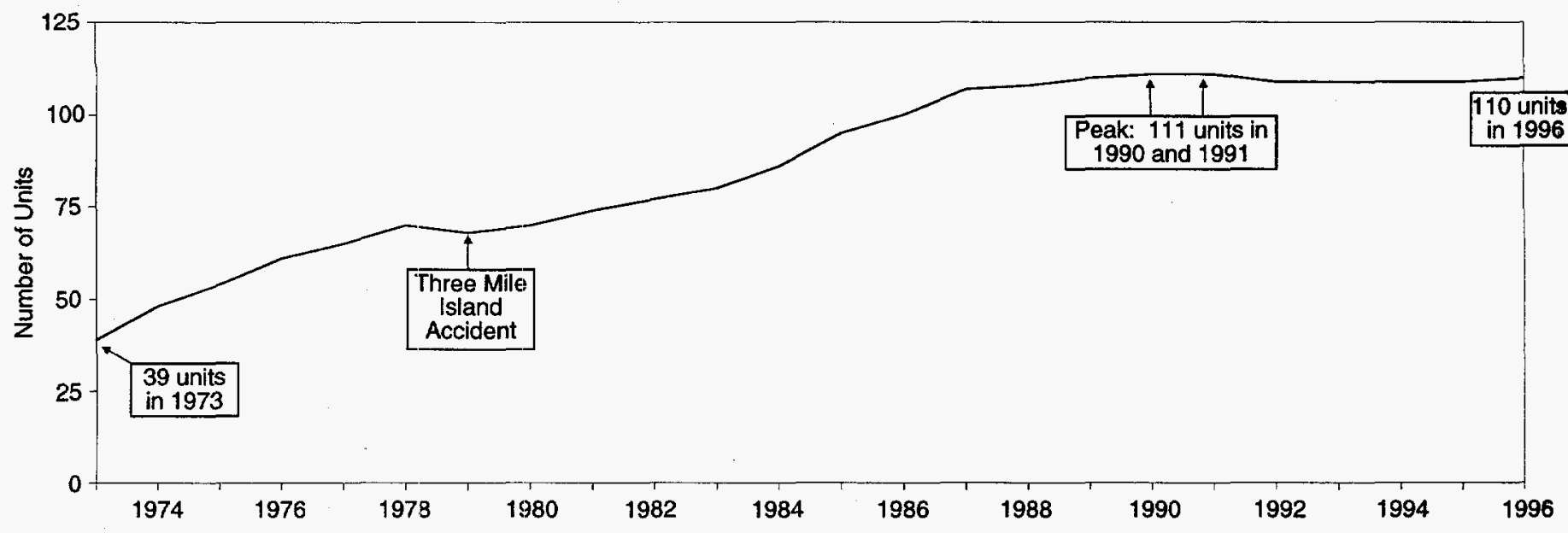

Net Generation of Electricity, 1973-1996

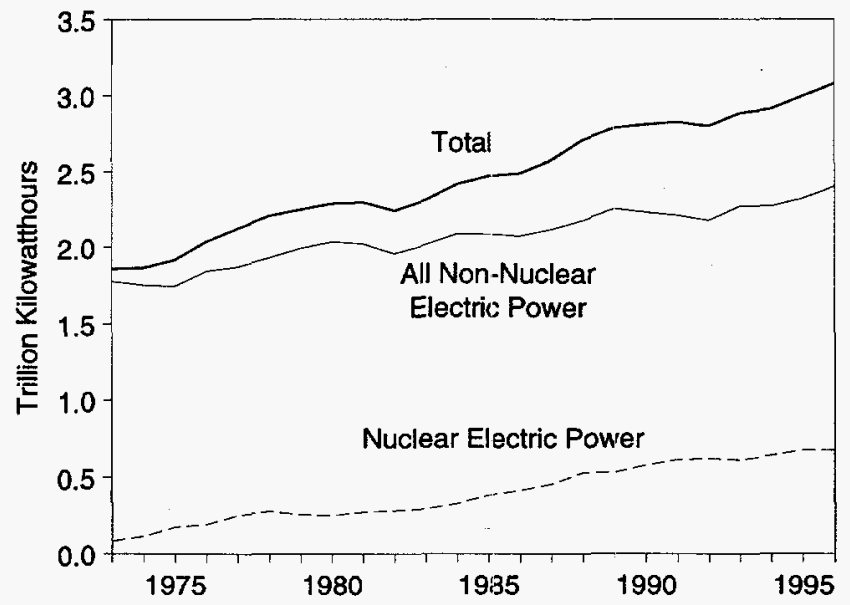

Nuclear Electricity Net Generation

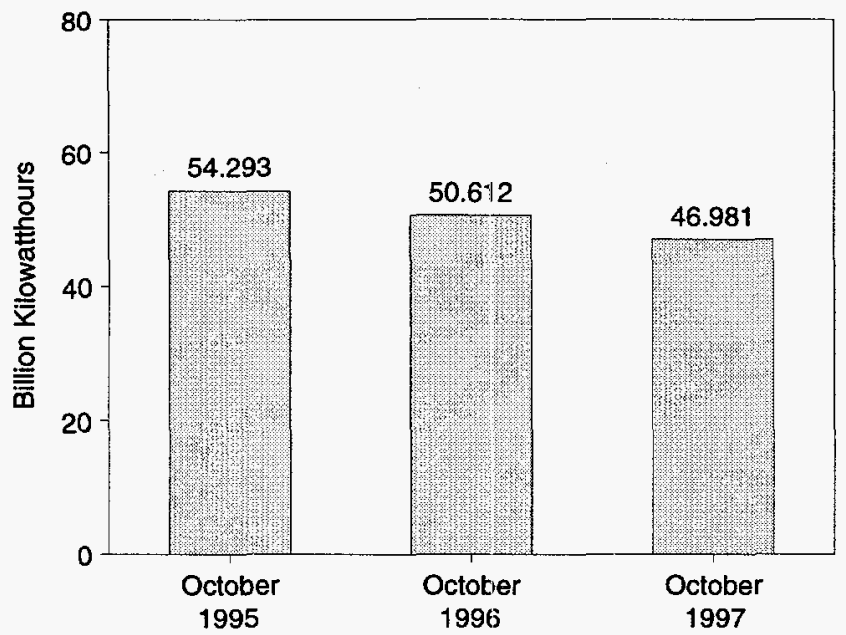

Nuclear Portion of Domestic Electricity

Net Generation

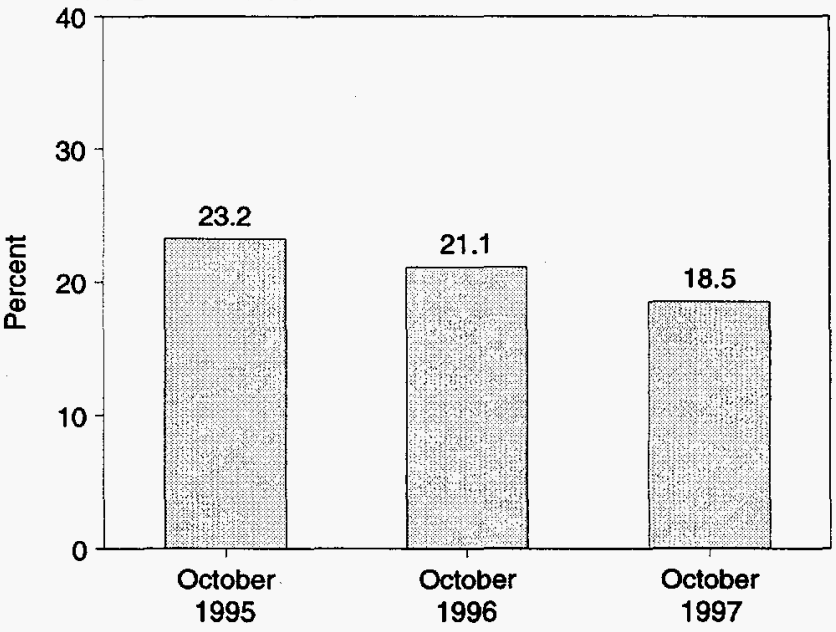

Capacity Factor, Monthly

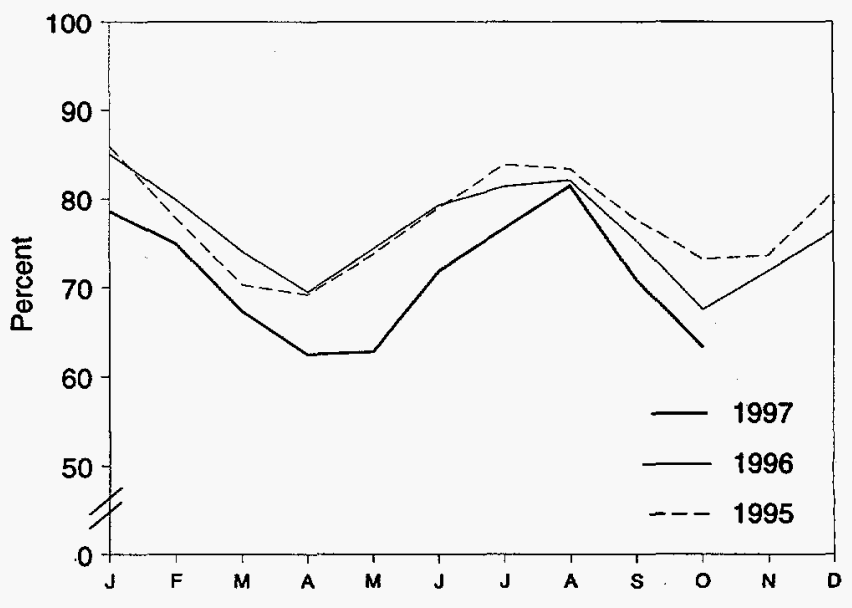

Note: Because vertical scales differ, graphs should not be compared. Sources: Tables 7.1 and 8.1 . 
Table 8.1 Nuclear Power Plant Operations

\begin{tabular}{|c|c|c|c|c|c|}
\hline & $\begin{array}{l}\text { Operable } \\
\text { Units } a, b\end{array}$ & $\begin{array}{c}\text { Nuclear } \\
\text { Electricity } \\
\text { Net } \\
\text { Generation }\end{array}$ & $\begin{array}{c}\text { Nuclear } \\
\text { Portion of } \\
\text { Domestic } \\
\text { Electricity } \\
\text { Net Generation }\end{array}$ & $\begin{array}{c}\text { Net } \\
\text { Summer } \\
\text { Capability } \\
\text { of Operable } \\
\text { Units }{ }^{\text {a,c }}\end{array}$ & $\begin{array}{l}\text { Capacity } \\
\text { Factord }\end{array}$ \\
\hline & Number & $\begin{array}{c}\text { Million } \\
\text { Kilowatthours }\end{array}$ & Percent & $\begin{array}{c}\text { Million } \\
\text { Kilowatts }\end{array}$ & Percent \\
\hline 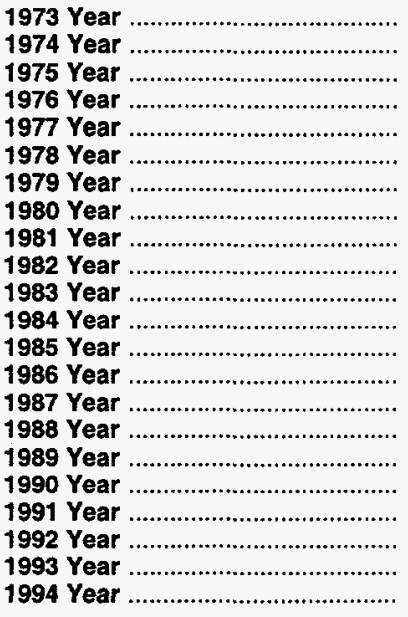 & $\begin{array}{r}39 \\
48 \\
54 \\
61 \\
65 \\
70 \\
68 \\
70 \\
74 \\
77 \\
80 \\
86 \\
95 \\
100 \\
107 \\
108 \\
110 \\
111 \\
111 \\
109 \\
109 \\
109\end{array}$ & $\begin{array}{r}83,479 \\
113,976 \\
172,505 \\
191,104 \\
250,883 \\
276,403 \\
255,155 \\
251,116 \\
272,674 \\
282,773 \\
293,677 \\
327,634 \\
383,691 \\
414,038 \\
455,270 \\
526,973 \\
529,355 \\
576,862 \\
612,565 \\
618,776 \\
610,291 \\
640,440\end{array}$ & $\begin{array}{r}4.5 \\
6.1 \\
9.0 \\
9.4 \\
11.8 \\
12.5 \\
11.4 \\
11.0 \\
11.9 \\
12.6 \\
12.7 \\
13.6 \\
15.5 \\
16.6 \\
17.7 \\
19.5 \\
19.0 \\
20.5 \\
21.7 \\
22.1 \\
21.2 \\
22.0\end{array}$ & $\begin{array}{l}22.683 \\
31.867 \\
37.267 \\
43.822 \\
46.303 \\
50.824 \\
49.747 \\
51.810 \\
56.042 \\
60.035 \\
63.009 \\
69.652 \\
79.397 \\
85.241 \\
93.583 \\
94.695 \\
98.161 \\
99.624 \\
99.589 \\
98.985 \\
99.041 \\
99.148\end{array}$ & $\begin{array}{l}53.5 \\
47.8 \\
55.9 \\
54.7 \\
63.3 \\
64.5 \\
58.4 \\
56.3 \\
58.2 \\
56.6 \\
54.4 \\
56.3 \\
58.0 \\
56.9 \\
57.4 \\
63.5 \\
62.2 \\
66.0 \\
70.2 \\
70.9 \\
70.5 \\
73.8\end{array}$ \\
\hline 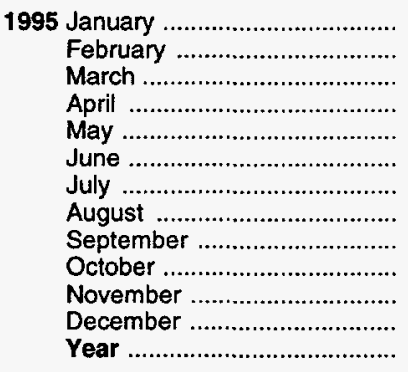 & $\begin{array}{l}109 \\
109 \\
109 \\
109 \\
109 \\
109 \\
109 \\
109 \\
109 \\
109 \\
109 \\
109 \\
109\end{array}$ & $\begin{array}{r}63,342 \\
51,858 \\
51,880 \\
49,321 \\
54,387 \\
56,381 \\
62,037 \\
61,661 \\
55,690 \\
54,293 \\
52,708 \\
59,844 \\
673,402\end{array}$ & $\begin{array}{l}25.0 \\
22.7 \\
22.2 \\
22.7 \\
23.0 \\
22.0 \\
21.2 \\
20.2 \\
22.7 \\
23.2 \\
22.5 \\
23.2 \\
22.5\end{array}$ & $\begin{array}{l}99.148 \\
99.148 \\
99.148 \\
99.148 \\
99.148 \\
99.148 \\
99.515 \\
99.515 \\
99.515 \\
99.515 \\
99.515 \\
99.515 \\
99.515\end{array}$ & $\begin{array}{l}85.9 \\
77.8 \\
70.3 \\
69.2 \\
73.7 \\
79.0 \\
83.8 \\
83.3 \\
77.7 \\
73.2 \\
73.6 \\
80.8 \\
77.4\end{array}$ \\
\hline 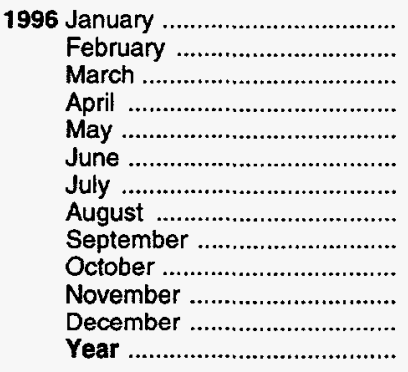 & $\begin{array}{l}109 \\
110 \\
110 \\
110 \\
110 \\
110 \\
110 \\
110 \\
110 \\
110 \\
110 \\
110 \\
110\end{array}$ & $\begin{array}{r}62,942 \\
55,928 \\
55,474 \\
50,325 \\
55,637 \\
57,498 \\
60,953 \\
61,477 \\
54,593 \\
50,612 \\
52,132 \\
57,159 \\
674,729\end{array}$ & $\begin{array}{l}23.4 \\
22.8 \\
22.4 \\
22.2 \\
22.1 \\
21.4 \\
21.1 \\
21.2 \\
21.8 \\
21.1 \\
21.6 \\
22.2 \\
21.9\end{array}$ & $\begin{array}{r}99.515 \\
100.685 \\
100.685 \\
100.685 \\
100.685 \\
100.685 \\
100.685 \\
100.685 \\
100.685 \\
100.685 \\
100.685 \\
100.685 \\
100.685\end{array}$ & $\begin{array}{l}85.0 \\
79.9 \\
74.1 \\
69.5 \\
74.3 \\
79.3 \\
81.4 \\
82.1 \\
75.3 \\
67.5 \\
71.9 \\
76.3 \\
76.4\end{array}$ \\
\hline 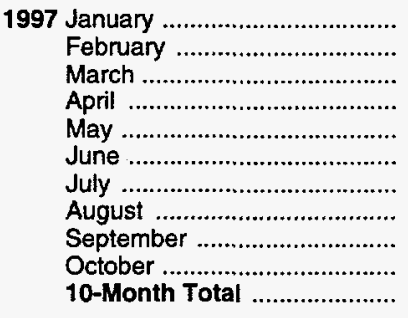 & $\begin{array}{l}110 \\
110 \\
110 \\
110 \\
110 \\
110 \\
110 \\
110 \\
109 \\
109 \\
109\end{array}$ & $\begin{array}{r}58,914 \\
50,658 \\
50,414 \\
45,313 \\
47,032 \\
52,095 \\
57,352 \\
61,084 \\
52,586 \\
46,981 \\
522,428\end{array}$ & $\begin{array}{l}21.5 \\
21.6 \\
20.6 \\
19.6 \\
19.3 \\
19.5 \\
18.8 \\
20.7 \\
19.7 \\
18.5 \\
20.0\end{array}$ & $\begin{array}{r}100.685 \\
100.685 \\
100.685 \\
100.685 \\
100.685 \\
100.685 \\
100.685 \\
100.685 \\
99.815 \\
99.815 \\
99.815\end{array}$ & $\begin{array}{l}78.6 \\
74.9 \\
67.3 \\
62.5 \\
62.8 \\
71.9 \\
76.6 \\
81.5 \\
70.8 \\
63.3 \\
71.0\end{array}$ \\
\hline 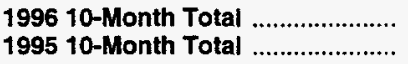 & $\begin{array}{l}110 \\
109\end{array}$ & $\begin{array}{l}565,438 \\
560,850\end{array}$ & $\begin{array}{l}21.9 \\
22.4\end{array}$ & $\begin{array}{r}100.685 \\
99.515\end{array}$ & $\begin{array}{l}76.8 \\
77.4\end{array}$ \\
\hline
\end{tabular}

a At end of period.

b See Note 1 at end of section.

c For the definition of "Net Summer Capability," see Note 3 at end of section.

d For an explanation of the method of calculating the capacity factor, see
Note 4 at end of section.

Notes: - Nuclear electricity net generation totals may not equal sum of components due to independent rounding. - Geographic coverage is the 50 States and the District of Columbia. 
Table 8.2 Nuclear Generating Units, End of Period

\begin{tabular}{|c|c|c|c|c|c|c|c|c|}
\hline & \multicolumn{2}{|c|}{$\begin{array}{c}\text { Licensed } \\
\text { for Operation }\end{array}$} & \multicolumn{2}{|c|}{$\begin{array}{c}\text { Construction } \\
\text { Permits }\end{array}$} & \multirow[b]{2}{*}{ On Order } & \multirow[b]{2}{*}{ Announced } & \multirow[b]{2}{*}{ Total } & \multirow{3}{*}{$\begin{array}{c}\begin{array}{c}\text { Total } \\
\text { Design } \\
\text { Capacityc }\end{array} \\
\begin{array}{c}\text { Million } \\
\text { Kilowatts }\end{array}\end{array}$} \\
\hline & Operable & In Startupb & Granted & Pending & & & & \\
\hline & \multicolumn{7}{|c|}{ Number of Units } & \\
\hline 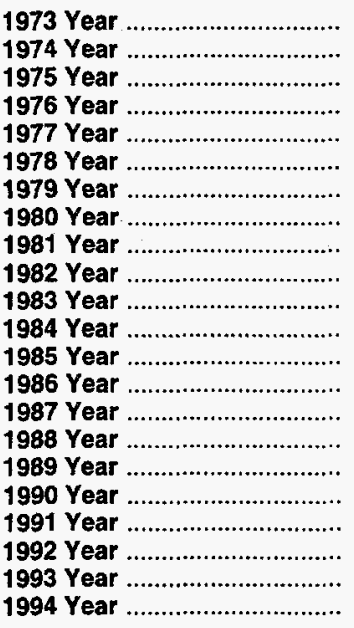 & $\begin{array}{r}39 \\
48 \\
54 \\
61 \\
65 \\
70 \\
68 \\
70 \\
74 \\
77 \\
80 \\
86 \\
95 \\
100 \\
107 \\
108 \\
110 \\
111 \\
111 \\
109 \\
109 \\
109\end{array}$ & $\begin{array}{l}2 \\
5 \\
2 \\
1 \\
2 \\
0 \\
0 \\
1 \\
0 \\
2 \\
3 \\
6 \\
3 \\
7 \\
4 \\
3 \\
1 \\
0 \\
0 \\
0 \\
0 \\
0\end{array}$ & $\begin{array}{r}57 \\
62 \\
69 \\
71 \\
78 \\
88 \\
90 \\
82 \\
76 \\
60 \\
53 \\
38 \\
30 \\
19 \\
14 \\
12 \\
10 \\
8 \\
8 \\
8 \\
7 \\
7\end{array}$ & $\begin{array}{l}52 \\
75 \\
69 \\
63 \\
49 \\
32 \\
24 \\
12 \\
11 \\
3 \\
0 \\
0 \\
0 \\
0 \\
0 \\
0 \\
0 \\
0 \\
0 \\
0 \\
0 \\
0\end{array}$ & $\begin{array}{r}49 \\
30 \\
14 \\
16 \\
13 \\
5 \\
3 \\
3 \\
2 \\
2 \\
2 \\
2 \\
2 \\
2 \\
2 \\
0 \\
0 \\
0 \\
0 \\
0 \\
0 \\
0\end{array}$ & $\begin{array}{l}9 \\
6 \\
5 \\
2 \\
2 \\
0 \\
0 \\
0 \\
0 \\
0 \\
0 \\
0 \\
0 \\
0 \\
0 \\
0 \\
0 \\
0 \\
0 \\
0 \\
0 \\
0\end{array}$ & $\begin{array}{l}208 \\
226 \\
213 \\
214 \\
209 \\
195 \\
185 \\
168 \\
163 \\
144 \\
138 \\
132 \\
130 \\
128 \\
127 \\
123 \\
121 \\
119 \\
119 \\
117 \\
116 \\
116\end{array}$ & $\begin{array}{l}198 \\
223 \\
212 \\
211 \\
203 \\
191 \\
180 \\
162 \\
157 \\
134 \\
129 \\
123 \\
121 \\
119 \\
119 \\
115 \\
113 \\
111 \\
111 \\
111 \\
110 \\
110\end{array}$ \\
\hline 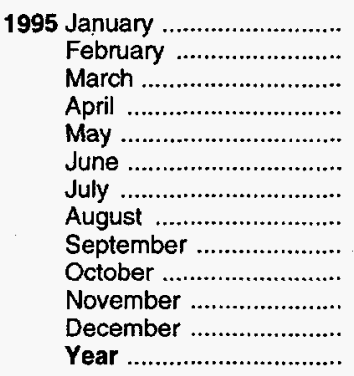 & $\begin{array}{l}109 \\
109 \\
109 \\
109 \\
109 \\
109 \\
109 \\
109 \\
109 \\
109 \\
109 \\
109 \\
109\end{array}$ & $\begin{array}{l}0 \\
0 \\
0 \\
0 \\
0 \\
0 \\
0 \\
0 \\
0 \\
0 \\
1 \\
1 \\
1\end{array}$ & $\begin{array}{l}6 \\
6 \\
6 \\
6 \\
6 \\
6 \\
6 \\
6 \\
6 \\
6 \\
5 \\
3 \\
3\end{array}$ & $\begin{array}{l}0 \\
0 \\
0 \\
0 \\
0 \\
0 \\
0 \\
0 \\
0 \\
0 \\
0 \\
0 \\
0\end{array}$ & $\begin{array}{l}0 \\
0 \\
0 \\
0 \\
0 \\
0 \\
0 \\
0 \\
0 \\
0 \\
0 \\
0 \\
0\end{array}$ & $\begin{array}{l}0 \\
0 \\
0 \\
0 \\
0 \\
0 \\
0 \\
0 \\
0 \\
0 \\
0 \\
0 \\
0\end{array}$ & $\begin{array}{l}115 \\
115 \\
115 \\
115 \\
115 \\
115 \\
115 \\
115 \\
115 \\
115 \\
115 \\
113 \\
113\end{array}$ & $\begin{array}{l}107 \\
107 \\
107 \\
107 \\
107 \\
107 \\
107 \\
107 \\
107 \\
107 \\
107 \\
104 \\
104\end{array}$ \\
\hline 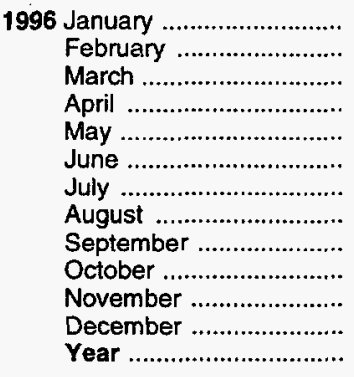 & $\begin{array}{l}109 \\
110 \\
110 \\
110 \\
110 \\
110 \\
110 \\
110 \\
110 \\
110 \\
110 \\
110 \\
110\end{array}$ & $\begin{array}{l}1 \\
0 \\
0 \\
0 \\
0 \\
0 \\
0 \\
0 \\
0 \\
0 \\
0 \\
0 \\
0\end{array}$ & $\begin{array}{l}3 \\
3 \\
3 \\
3 \\
3 \\
3 \\
3 \\
3 \\
3 \\
3 \\
3 \\
3 \\
3\end{array}$ & $\begin{array}{l}0 \\
0 \\
0 \\
0 \\
0 \\
0 \\
0 \\
0 \\
0 \\
0 \\
0 \\
0 \\
0\end{array}$ & $\begin{array}{l}0 \\
0 \\
0 \\
0 \\
0 \\
0 \\
0 \\
0 \\
0 \\
0 \\
0 \\
0 \\
0\end{array}$ & $\begin{array}{l}0 \\
0 \\
0 \\
0 \\
0 \\
0 \\
0 \\
0 \\
0 \\
0 \\
0 \\
0 \\
0\end{array}$ & $\begin{array}{l}113 \\
113 \\
113 \\
113 \\
113 \\
113 \\
113 \\
113 \\
113 \\
113 \\
113 \\
113 \\
113\end{array}$ & $\begin{array}{l}104 \\
104 \\
104 \\
104 \\
104 \\
104 \\
104 \\
104 \\
104 \\
104 \\
104 \\
104 \\
104\end{array}$ \\
\hline 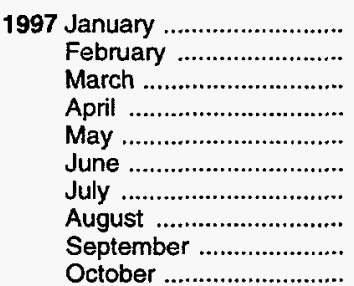 & $\begin{array}{l}110 \\
110 \\
110 \\
110 \\
110 \\
110 \\
110 \\
110 \\
109 \\
109\end{array}$ & $\begin{array}{l}0 \\
0 \\
0 \\
0 \\
0 \\
0 \\
0 \\
0 \\
0 \\
0\end{array}$ & $\begin{array}{l}3 \\
3 \\
3 \\
3 \\
3 \\
3 \\
3 \\
3 \\
3 \\
3\end{array}$ & $\begin{array}{l}0 \\
0 \\
0 \\
0 \\
0 \\
0 \\
0 \\
0 \\
0 \\
0\end{array}$ & $\begin{array}{l}0 \\
0 \\
0 \\
0 \\
0 \\
0 \\
0 \\
0 \\
0 \\
0\end{array}$ & $\begin{array}{l}0 \\
0 \\
0 \\
0 \\
0 \\
0 \\
0 \\
0 \\
0 \\
0\end{array}$ & $\begin{array}{l}113 \\
113 \\
113 \\
113 \\
113 \\
113 \\
113 \\
113 \\
112 \\
112\end{array}$ & $\begin{array}{l}104 \\
104 \\
104 \\
104 \\
104 \\
104 \\
104 \\
104 \\
100 \\
100\end{array}$ \\
\hline
\end{tabular}

a See Note 1 at end of section.

b See Note 2 at end of section.

c Net design electrical rating (DER) is used because many of the units were canceled prior to being assigned a nel summer capability. See Note 3 at end of section.

Note: Geographic coverage is the 50 States and the District of Columbia. Sources: See end of section. 


\section{Nuclear Energy Notes}

1. Operable Units: Nuclear generating units that have been issued a full-power license by the Nuclear Regulatory Commission (NRC).

Exceptions: The Shippingport (60 megawatts (MW)) and the Hanford-N ( $840 \mathrm{MW})$ nuclear units were included in the operable units until 1982 and 1988 , respectively. The Shippingport unit was excluded from the operable category during March 1974-November 1977 due to a major core modification outage. Hanford$\mathrm{N}$, an unlicensed unit used for defense materiel production, was included in the operable category because power was produced as by-product and sold commercially. Three Mile Island $2(880 \mathrm{MW})$ experienced a major accident in 1979 and, although that unit still retains its operating license and site cleanup continues, there is no plan to restart it. Therefore, it has not been included in the operable category since March 1979. Although Shoreham received a full-power license in April 1989, the unit is not currently scheduled to operate and, therefore, has not been included in the operable category. Rancho Seco ( $873 \mathrm{MW}$ ) was shut down by the Sacramento Municipal Utility District (SMUD) in June 1989 following a referendum on its continued operation. Because there are currently no plans to operate it as a nuclear unit, it is no longer included as an operable unit but is identified as a unit shut down for an extended period. As soon as SMUD and the NRC formalize the plant's official retirement, it will be noted as such in this report. The Department of Energy-operated Experimental Breeder Reactor 2 unit is not a commercial reactor and is therefore not included in the operable category.

In addition, nine units have been retired and therefore removed from the operable category. Those units are: Peach Bottom 1 (40 MW) and Indian Point 1 (265 MW), both retired in 1974; Humboldt Bay (65 MW), officially retired in 1976; Dresden 1 (200 MW), retired in October 1979; LaCrosse (51 MW), retired in May 1987; Fort Saint Vrain (217 MW), retired in October 1989; Yankee Rowe 1 (185 MW), retired in February 1992; San Onofre 1 (436 MW), retired in December 1992; and Trojan (1,104 MW), retired in February 1993.

2. In Startup: The period of time between a nuclear generating unit's initial fuel loading date and the issuance of its full-power license. During that period, the unit is undergoing low-power testing and the maximum level of operation is 5 percent of the unit's design thermal rating.

3. Capacity: Nuclear generating units may have more than one type of net capacity rating, including the following:

(a) Net Summer Capability-The steady hourly output that generating equipment is expected to supply to system load, exclusive of auxiliary power, as demonstrated by test at the time of summer peak demand. Auxiliary power of a typical nuclear power plant is about 5 percent of gross generation.

(b) Net Design Capacity or Net Design Electrical Rating (DER) - The nominal net electrical output of a unit, specified by the utility and used for plant design.

4. Monthly Capacity Factors: The monthly capacity factors are computed as the actual monthly generation divided by the maximum possible generation for that month. The maximum possible generation is the number of hours in the month multiplied by the net summer capability at the end of the month. That fraction is then multiplied by 100 to obtain a percentage. Annual capacity factors are averages of the monthly values for that year.

\section{Sources for Table 8.1 Operable Units}

1973-1982: U.S. Department of Energy (DOE), Office of Nuclear Programs, "U.S. Central Station Nuclear Electric Generating Units: Significant Milestones."

1983-January 1996: Nuclear Regulatory Commission (NRC), "Licensed Operating Reactors" (NUREG-0020).

February 1996 forward: EIA estimates.

\section{Nuclear Electricity Net Generation}

Table 7.1.

\section{Nuclear Portion of Domestic Electricity Net Gen- eration}

Calculated from data in Table 7.1.

\section{Net Summer Capability of Operable Units}

1973-1982: Compiled from various sources, primarily DOE, Office of Nuclear Reactor Programs, "U.S. Central Station Nuclear Electric Generating Units: Significant Milestones."

1983 forward: Energy Information Administration (EIA), Form EIA-860, "Annual Electric Generator Report," and monthly updates as appropriate.

\section{Capacity Factor}

EIA, Office of Coal, Nuclear, Electric and Alternate Fuels.

\section{Sources for Table $\mathbf{8 . 2}$ Licensed for Operation}

1973-1982: U.S. Department of Energy (DOE), Office of Nuclear Programs, "U.S. Central Station 
Nuclear Electric Generating Units: Significant Milestones."

1983 forward: Nuclear Regulatory Commission (NRC), “Licensed Operating Reactors" (NUREG-0020).

\section{Construction Permits, On Order, and Announced}

1973-1982: Compiled from various sources, primarily DOE, Office of Nuclear Reactor Programs, "U.S. Central Station Nuclear Electric Generating Units: Significant Milestones"; Energy Information Administration (EIA), Office of Coal, Nuclear, Electric and Alternate Fuels (CNEAF), "Nuclear Steam-Electric Units That Have Been in Operation as of 1957-1989"; EIA, CNEAF, "Nuclear Plant Cancellations: Causes, Costs, and Consequences"; and Utility Data Institute, Inc., "U.S. Nuclear Plant Statistics, 1987.

1983 forward: NRC, "Summary Information Report"
(NUREG-0871); NRC, "Licensed Operating Reactors" (NUREG-0020); and various journals.

\section{Total Design Capacity}

1973-1982: Compiled from various sources, primarily DOE, Office of Nuclear Reactor Programs, "U.S. Central Station Nuclear Electric Generating Units: Significant Milestones"; EIA, CNEAF, "Nuclear SteamElectric Units That Have Been in Operation as of 1957-1987"; EIA, CNEAF, "Monthly Report for Electric Utilities-Power Generation"; EIA, CNEAF, "Nuclear Plant Cancellations: Causes, Costs, and Consequences"; and Utility Data Institute, Inc., "U.S. Nuclear Plant Statistics, 1987."

1983 forward: NRC, "Summary Information Report" (NUREG-0871); NRC, "Licensed Operating Reactors" (NUREG-0020); and EIA, Form EIA-860, "Annual Electric Generator Report." 


\section{Section 9. Energy Prices}

Crude Oil. The average price of domestic crude oil purchased at the wellhead was $\$ 17.68$ per barrel in October 1997, 16 percent lower than the level in October 1996. The refiner acquisition cost of imported crude oil in October 1997 was $\$ 18.95$ per barrel, 18 percent lower than the October 1996 level. The average cost of domestic crude oil in October 1997 was $\$ 19.58,15$ percent lower than the October 1996 average.

Motor Gasoline. The national city average retail price of unleaded regular gasoline at all types of stations was $\$ 1.21$ per gallon in November 1997,3 percent lower than the price in November 1996. The price of unleaded premium gasoline averaged $\$ 1.40$ per gallon in November 1997, 2 percent lower than the price in November 1996.

Residual Fuel Oil. The average price, excluding taxes, of residual fuel oil sold to end users in October 1997 was 44 cents per gallon, 6 percent higher than the previous month's price but 10 percent lower than the October 1996 average. The average resale price, excluding taxes, of residual fuel oil in October 1997 was 42 cents per gallon, 8 percent higher than the previous month's average but 7 percent lower than the price 1 year earlier.

Aviation Fuel. The average price, excluding taxes, of aviation gasoline sold to end users in October 1997 was $\$ 1.14$ per gallon, 1 percent lower than both the previous month's price and the October 1996 price. The average price, excluding taxes, of kerosene-type jet fuel sold to end users in October 1997 was 62 cents per gallon, 6 percent higher than the previous month's price but 16 percent lower than the October 1996 average price.

No. 2 Distillate Fuel Oil. The October 1997 national average price, excluding taxes, of heating oil sold to residential customers was 92 cents per gallon, 4 percent higher than the previous month's price but 10 percent lower than the price 1 year earlier. The average price of No. 2 fuel oil sold to all end users was 63 cents per gallon in October 1997, 8 percent higher than the previous month's price but 16 percent lower than the October 1996 price.
Electricity. The average price of electricity sold to all ultimate consumers in the United States in October 1997 was 6.91 cents per kilowatthour, the same as the October 1996 mean price. The price of electricity sold to residential consumers in October 1997 averaged 8.62 cents per kilowatthour, 1 percent lower than the October 1996 price. The price of electricity sold to commercial consumers averaged 7.71 cents per kilowatthour in October 1997, 2 percent lower than the October 1996 price. The price of electricity sold to other consumers was 6.83 cents per kilowatthour, 4 percent lower than the price 1 year earlier. The price of electricity sold to industrial users in October 1997 averaged 4.62 cents per kilowatthour, slightly higher than the October 1996 price.

Beginning with January 1986 , new series of national average price estimates were based on a statistically derived sample of both publicly and privately owned electric utilities. Previously, average price estimates were derived from selected privately owned electric utilities and were not national averages.

Natural Gas. The estimated average wellhead price of natural gas for September 1997 was $\$ 2.44$ per thousand cubic feet, 32 percent higher than the September 1996 price.

The average price of natural gas delivered to electric utility plants was $\$ 2.54$ per thousand cubic feet in August 1997 (latest date for which data are available) 1 percent below the August 1996 price. The average price of natural gas used by residential consumers in September 1997 was $\$ 8.55$ per thousand cubic feet, 7 percent higher than the September 1996 price. The average price of natural gas used by commercial consumers in September 1997 was $\$ 5.62$ per thousand cubic feet, 3 percent more than the September 1996 price. The average price of natural gas used by industrial consumers in September 1997 was $\$ 3.23$ per thousand cubic feet, 17 percent above the September 1996 price. 
Figure 9.1 Petroleum Prices

Crude Oil Prices, 1973-1996

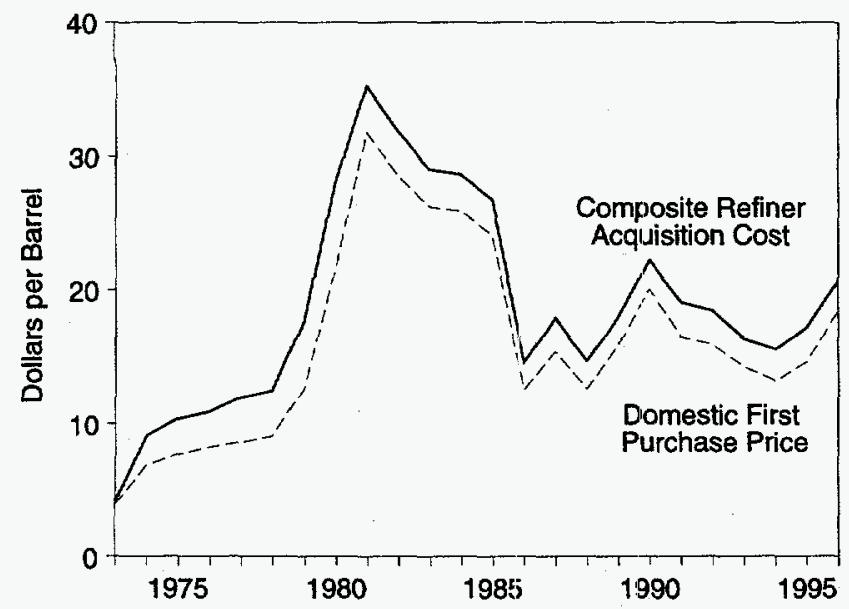

Composite Refiner Acquisition Cost, Monthly

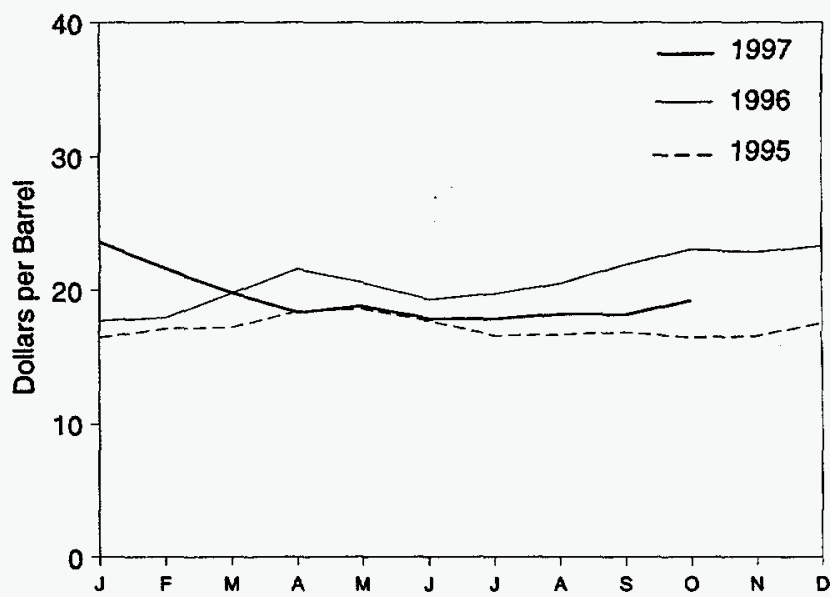

Refiner Prices to End Users: Motor Gasoline, Diesel Fuel, and Jet Fuel, Monthly

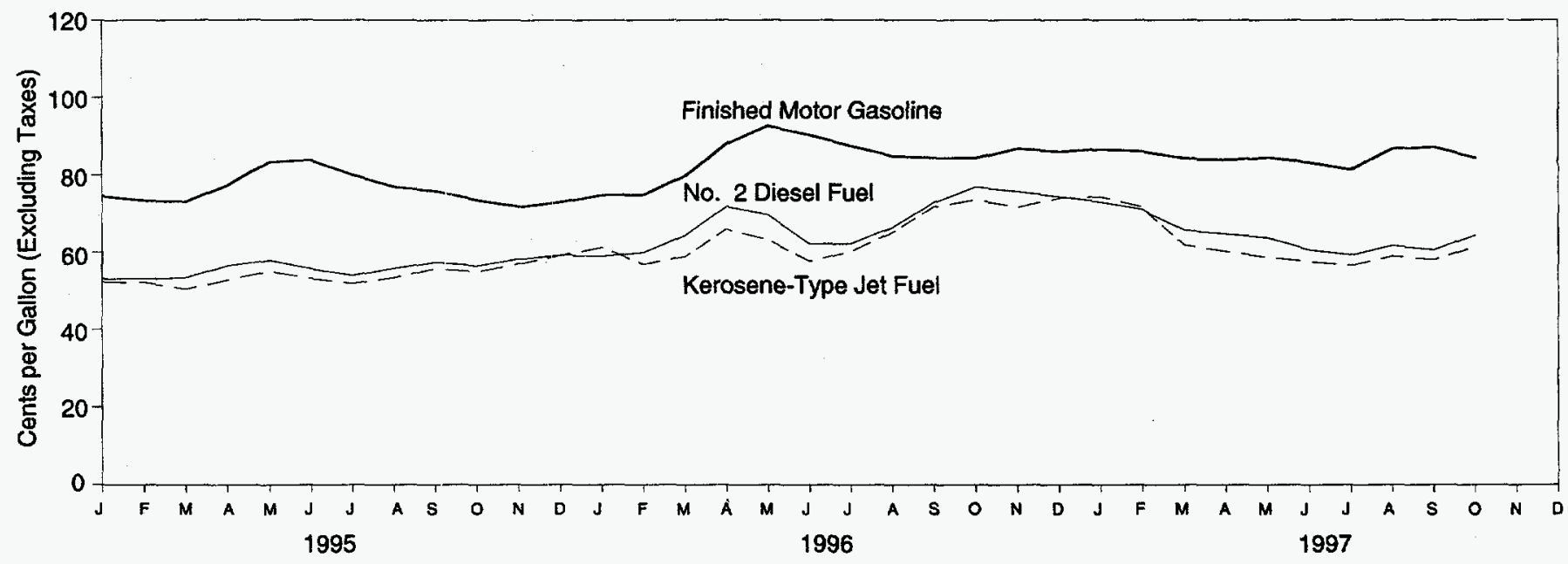

Refiner Prices to End Users: No. 2 Fuel Oil, Propane, and Residual Fuel, Monthly

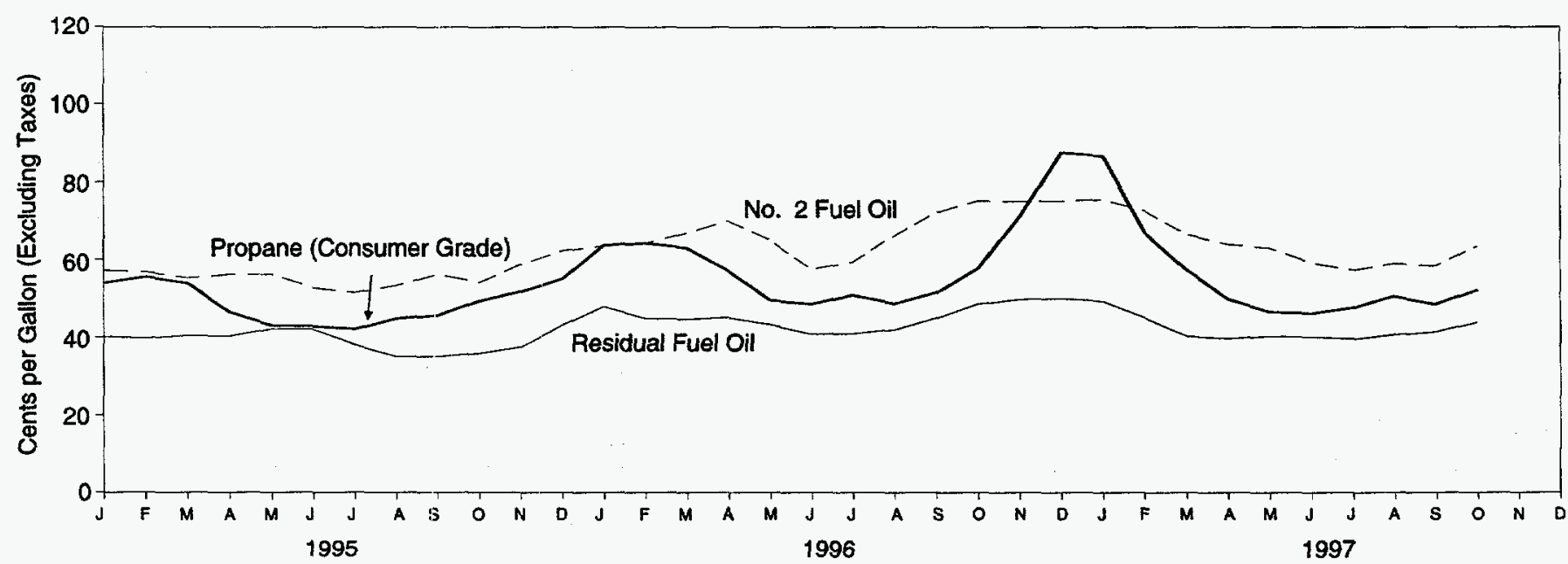

Sources: Tabies 9.1, 9.5, and 9.7. 
Table 9.1 Crude Oil Price Summary

(Dollars per Barrel)

\begin{tabular}{|c|c|c|c|c|c|c|}
\hline & \multirow[b]{2}{*}{$\begin{array}{l}\text { Domestic First } \\
\text { Purchase Priceb }\end{array}$} & \multirow[b]{2}{*}{$\begin{array}{l}\text { F.O.B. Cost } \\
\text { of Imports }\end{array}$} & \multirow[b]{2}{*}{$\begin{array}{l}\text { Landed Cost } \\
\text { of Imports }\end{array}$} & \multicolumn{3}{|c|}{ Refiner Acquisition Cost ${ }^{a}$} \\
\hline & & & & Domestic & Imported & Composite \\
\hline 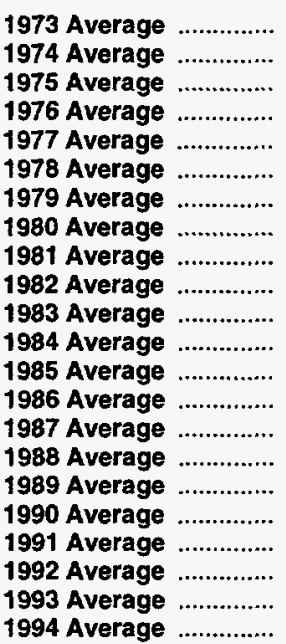 & $\begin{array}{r}3.89 \\
6.87 \\
7.67 \\
8.19 \\
8.57 \\
9.00 \\
12.64 \\
21.59 \\
31.77 \\
28.52 \\
26.19 \\
25.88 \\
24.09 \\
12.51 \\
15.40 \\
12.58 \\
15.86 \\
20.03 \\
16.54 \\
15.99 \\
14.25 \\
13.19\end{array}$ & $\begin{array}{l}\text { e } 5.21 \\
10.91 \\
11.18 \\
12.15 \\
13.24 \\
13.29 \\
20.07 \\
32.37 \\
35.15 \\
32.02 \\
27.81 \\
27.60 \\
25.84 \\
12.52 \\
16.69 \\
13.25 \\
16.89 \\
20.37 \\
16.89 \\
16.77 \\
14.71 \\
14.18\end{array}$ & $\begin{array}{l}\text { e } 6.41 \\
12.32 \\
12.70 \\
13.32 \\
14.36 \\
14.35 \\
21.45 \\
33.67 \\
36.47 \\
33.18 \\
28.93 \\
28.54 \\
26.67 \\
13.49 \\
17.65 \\
14.08 \\
17.68 \\
21.13 \\
18.02 \\
17.75 \\
15.72 \\
15.18\end{array}$ & $\begin{array}{r}E 4.17 \\
7.18 \\
8.39 \\
8.84 \\
9.55 \\
10.61 \\
14.27 \\
24.23 \\
34.33 \\
31.22 \\
28.87 \\
28.53 \\
26.66 \\
14.82 \\
17.76 \\
14.74 \\
17.87 \\
22.59 \\
19.33 \\
18.63 \\
16.67 \\
15.67\end{array}$ & $\begin{array}{l}\varepsilon_{4.08} \\
12.52 \\
13.93 \\
13.48 \\
14.53 \\
14.57 \\
21.67 \\
33.89 \\
37.05 \\
33.55 \\
29.30 \\
28.88 \\
26.99 \\
14.00 \\
18.13 \\
14.56 \\
18.08 \\
21.76 \\
18.70 \\
18.20 \\
16.14 \\
15.51\end{array}$ & $\begin{array}{r}E_{4.15} \\
9.07 \\
10.38 \\
10.89 \\
11.96 \\
12.46 \\
17.72 \\
28.07 \\
35.24 \\
31.87 \\
28.99 \\
28.63 \\
26.75 \\
14.55 \\
17.90 \\
14.67 \\
17.97 \\
22.22 \\
19.06 \\
18.43 \\
16.41 \\
15.59\end{array}$ \\
\hline 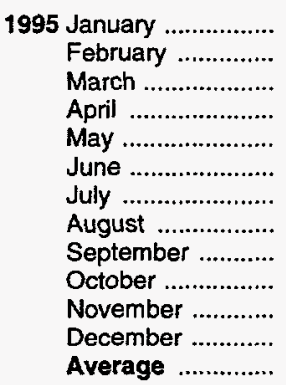 & $\begin{array}{l}14.00 \\
14.71 \\
14.68 \\
15.84 \\
15.85 \\
15.02 \\
14.01 \\
14.13 \\
14.49 \\
13.68 \\
14.03 \\
15.02 \\
14.62\end{array}$ & $\begin{array}{l}15.08 \\
15.65 \\
15.88 \\
17.28 \\
17.30 \\
15.91 \\
14.82 \\
15.05 \\
15.24 \\
14.68 \\
15.30 \\
16.06 \\
15.69\end{array}$ & $\begin{array}{l}16.23 \\
16.74 \\
17.04 \\
18.26 \\
18.18 \\
17.07 \\
15.96 \\
16.10 \\
16.38 \\
15.87 \\
16.30 \\
17.05 \\
16.78\end{array}$ & $\begin{array}{l}16.52 \\
17.16 \\
17.31 \\
18.20 \\
18.68 \\
17.94 \\
16.85 \\
16.96 \\
17.12 \\
16.82 \\
16.73 \\
17.55 \\
17.33\end{array}$ & $\begin{array}{l}16.56 \\
17.21 \\
17.21 \\
18.70 \\
18.56 \\
17.43 \\
16.50 \\
16.54 \\
16.71 \\
16.29 \\
16.52 \\
17.53 \\
17.14\end{array}$ & $\begin{array}{l}16.54 \\
17.18 \\
17.26 \\
18.43 \\
18.62 \\
17.69 \\
16.68 \\
16.75 \\
16.91 \\
16.55 \\
16.62 \\
17.54 \\
17.23\end{array}$ \\
\hline 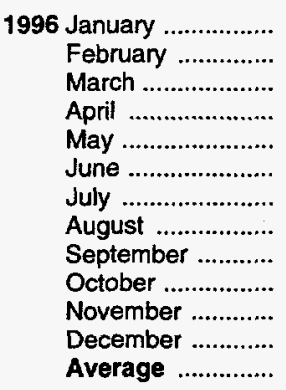 & $\begin{array}{l}15.43 \\
15.54 \\
17.63 \\
19.58 \\
17.94 \\
16.94 \\
17.63 \\
18.29 \\
19.93 \\
21.09 \\
20.20 \\
21.34 \\
18.46\end{array}$ & $\begin{array}{l}16.17 \\
16.86 \\
18.77 \\
19.56 \\
18.34 \\
17.61 \\
18.21 \\
19.27 \\
21.03 \\
22.23 \\
21.31 \\
21.56 \\
19.32\end{array}$ & $\begin{array}{l}17.31 \\
17.81 \\
19.61 \\
20.73 \\
19.61 \\
18.83 \\
19.35 \\
20.30 \\
21.95 \\
23.05 \\
22.24 \\
22.48 \\
20.31\end{array}$ & $\begin{array}{l}17.98 \\
18.10 \\
19.63 \\
21.88 \\
21.15 \\
19.30 \\
19.91 \\
20.55 \\
21.87 \\
22.93 \\
23.08 \\
23.38 \\
20.77\end{array}$ & $\begin{array}{l}17.48 \\
17.77 \\
19.90 \\
21.33 \\
20.12 \\
19.32 \\
19.60 \\
20.53 \\
22.04 \\
23.22 \\
22.66 \\
23.22 \\
20.64\end{array}$ & $\begin{array}{l}17.74 \\
17.95 \\
19.76 \\
21.63 \\
20.61 \\
19.31 \\
19.76 \\
20.54 \\
21.96 \\
23.08 \\
22.87 \\
23.30 \\
20.71\end{array}$ \\
\hline 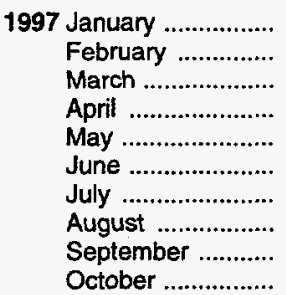 & $\begin{array}{l}21.76 \\
19.38 \\
17.85 \\
16.64 \\
17.24 \\
15.90 \\
15.91 \\
16.21 \\
16.44 \\
17.68\end{array}$ & $\begin{array}{r}21.31 \\
18.99 \\
17.11 \\
16.20 \\
16.81 \\
15.99 \\
16.38 \\
R 16.68 \\
R 16.73 \\
17.39\end{array}$ & $\begin{array}{r}22.31 \\
19.98 \\
18.45 \\
17.52 \\
17.87 \\
17.12 \\
17.28 \\
R 17.78 \\
A 17.82 \\
18.70\end{array}$ & $\begin{array}{r}24.29 \\
22.47 \\
20.57 \\
19.01 \\
19.20 \\
18.45 \\
18.35 \\
18.59 \\
R 18.49 \\
19.58\end{array}$ & $\begin{array}{l}23.05 \\
20.92 \\
19.16 \\
17.85 \\
18.54 \\
17.38 \\
17.48 \\
17.96 \\
17.96 \\
18.95\end{array}$ & $\begin{array}{r}23.62 \\
21.65 \\
19.82 \\
18.36 \\
18.84 \\
17.87 \\
17.88 \\
18.23 \\
\mathrm{R} \\
18.20 \\
19.23\end{array}$ \\
\hline
\end{tabular}

a See Note 4 at end of section.

b See Note 1 at end of section.

c See Note 2 at end of section.

d See Note 3 at end of section.

- Based on October, November, and December data only.

$R=$ Revised data. $E=$ Estimate.

Notes: - Values for Domestic First Purchase Price and Refiner Acquisition
Cost for the current month and for F.O.B. and Landed Costs of Imports for the current 2 months are preliminary. - F.O.B. and landed costs through 1980 reflect the period of reporting; prices since then reflect the period of loading. - Annual averages are the averages of the monthly prices, weighted by volume. - Geographic coverage is the 50 States, the District of Columbia, Puerto Rico, the Virgin Islands, and all U.S. Territories and Possessions.

Sources: See end of section. 


\begin{tabular}{|c|c|c|c|c|c|c|c|c|c|c|}
\hline & \multicolumn{7}{|c|}{ Selected Countries } & \multirow{2}{*}{$\begin{array}{c}\text { Persian } \\
\text { Gulf } \\
\text { Nations } \\
\end{array}$} & \multirow[b]{2}{*}{$\begin{array}{c}\text { Total } \\
\text { OPEC }\end{array}$} & \multirow[b]{2}{*}{$\begin{array}{c}\text { Total } \\
\text { Non-OPEC }\end{array}$} \\
\hline & Angola & Colombia & Mexico & Nigeria & $\begin{array}{l}\text { Saudi } \\
\text { Arabia }\end{array}$ & $\begin{array}{c}\text { United } \\
\text { Kingdom }\end{array}$ & Venezuela & & & \\
\hline 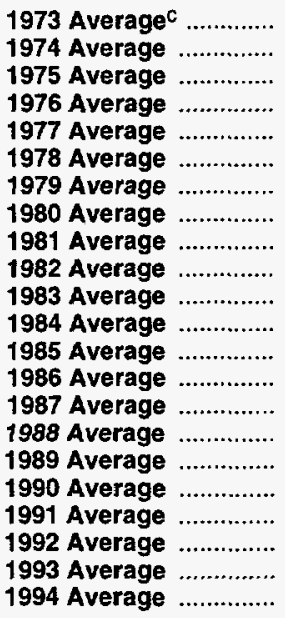 & $\begin{array}{c}W \\
11.87 \\
10.97 \\
12.02 \\
13.29 \\
13.32 \\
19.85 \\
33.45 \\
35.55 \\
31.86 \\
28.14 \\
27.46 \\
26.30 \\
13.30 \\
17.27 \\
13.70 \\
17.66 \\
20.23 \\
18.47 \\
18.41 \\
16.23 \\
15.40\end{array}$ & $\begin{array}{l}W \\
W \\
(d) \\
(d) \\
(d) \\
(d) \\
(d) \\
w \\
(d) \\
(d) \\
(d) \\
(d) \\
(d) \\
12.34 \\
17.84 \\
13.61 \\
17.89 \\
20.75 \\
18.49 \\
18.02 \\
15.87 \\
14.99\end{array}$ & $\begin{array}{c}\text { NA } \\
W V \\
11.44 \\
12.22 \\
13.42 \\
13.24 \\
20.27 \\
31.06 \\
33.01 \\
28.08 \\
25.20 \\
26.39 \\
25.33 \\
11.84 \\
16.36 \\
12.18 \\
15.96 \\
19.26 \\
15.37 \\
151.26 \\
131.74 \\
13.68\end{array}$ & $\begin{array}{r}7.81 \\
12.44 \\
11.82 \\
13.08 \\
14.44 \\
14.05 \\
21.69 \\
35.93 \\
38.31 \\
35.13 \\
29.81 \\
29.51 \\
28.04 \\
14.35 \\
18.47 \\
15.16 \\
18.31 \\
22.46 \\
20.29 \\
19.98 \\
17.79 \\
15.32\end{array}$ & $\begin{array}{r}3.25 \\
10.17 \\
10.87 \\
11.62 \\
12.38 \\
12.70 \\
17.28 \\
28.17 \\
32.60 \\
33.73 \\
27.53 \\
27.67 \\
22.04 \\
11.36 \\
15.12 \\
12.16 \\
16.29 \\
20.36 \\
14.62 \\
15.85 \\
13.77 \\
14.12\end{array}$ & $\begin{array}{c}\text { NA } \\
\text { NA } \\
\text { NA } \\
W \\
14.11 \\
13.82 \\
21.70 \\
34.36 \\
36.06 \\
33.42 \\
29.91 \\
28.87 \\
27.64 \\
13.84 \\
18.28 \\
14.80 \\
17.89 \\
23.43 \\
20.81 \\
19.61 \\
16.64 \\
15.66\end{array}$ & $\begin{array}{l}5.39 \\
10.71 \\
11.04 \\
11.39 \\
12.63 \\
12.38 \\
16.90 \\
24.81 \\
28.95 \\
23.74 \\
21.48 \\
24.23 \\
23.64 \\
10.92 \\
15.08 \\
12.96 \\
16.09 \\
19.55 \\
14.91 \\
14.39 \\
12.46 \\
12.21\end{array}$ & $\begin{array}{r}3.68 \\
10.60 \\
10.88 \\
11.65 \\
12.56 \\
12.77 \\
18.77 \\
28.92 \\
33.00 \\
33.55 \\
27.70 \\
27.48 \\
23.31 \\
11.35 \\
15.97 \\
12.38 \\
16.61 \\
18.54 \\
15.22 \\
16.35 \\
14.21 \\
13.97\end{array}$ & $\begin{array}{r}5.43 \\
11.33 \\
11.34 \\
12.23 \\
13.29 \\
13.31 \\
19.88 \\
32.21 \\
35.17 \\
33.48 \\
28.46 \\
27.79 \\
25.67 \\
12.21 \\
16.43 \\
13.43 \\
17.06 \\
20.40 \\
16.99 \\
16.87 \\
14.78 \\
14.00\end{array}$ & $\begin{array}{r}4.80 \\
9.59 \\
10.62 \\
11.70 \\
12.97 \\
13.23 \\
20.92 \\
32.85 \\
35.12 \\
30.58 \\
27.20 \\
27.45 \\
25.96 \\
12.87 \\
16.99 \\
13.05 \\
16.72 \\
20.32 \\
16.77 \\
16.66 \\
14.65 \\
14.34\end{array}$ \\
\hline 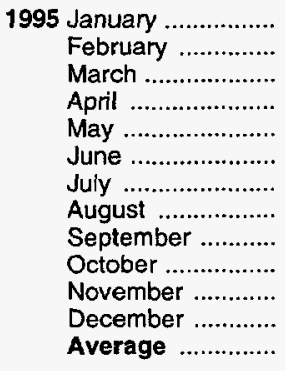 & $\begin{array}{l}15.63 \\
16.70 \\
16.68 \\
17.38 \\
18.25 \\
16.92 \\
15.63 \\
15.37 \\
16.44 \\
15.68 \\
16.39 \\
17.24 \\
16.58\end{array}$ & $\begin{array}{l}15.87 \\
W \\
16.77 \\
18.12 \\
18.27 \\
16.53 \\
15.85 \\
16.44 \\
16.79 \\
16.11 \\
16.65 \\
17.38 \\
16.13\end{array}$ & $\begin{array}{l}14.98 \\
15.79 \\
15.74 \\
17.16 \\
17.20 \\
16.07 \\
14.77 \\
14.54 \\
15.24 \\
15.02 \\
15.32 \\
16.41 \\
15.64\end{array}$ & $\begin{array}{l}17.13 \\
17.43 \\
17.19 \\
18.96 \\
18.66 \\
17.66 \\
15.97 \\
16.48 \\
16.91 \\
16.54 \\
17.28 \\
18.37 \\
17.40\end{array}$ & $\begin{array}{l}W \\
W \\
W \\
W \\
W \\
W \\
W \\
W \\
W \\
W \\
W \\
W \\
W\end{array}$ & $\begin{array}{c}W \\
16.84 \\
W \\
W \\
18.42 \\
W \\
W \\
16.23 \\
16.47 \\
16.41 \\
W \\
W \\
16.94\end{array}$ & $\begin{array}{l}12.61 \\
13.02 \\
14.23 \\
15.97 \\
15.76 \\
13.80 \\
13.33 \\
13.73 \\
13.30 \\
12.40 \\
13.38 \\
14.70 \\
13.86\end{array}$ & $\begin{array}{l}W \\
15.96 \\
W \\
W \\
W \\
W \\
W \\
W \\
W \\
W \\
W \\
W \\
W\end{array}$ & $\begin{array}{l}14.79 \\
15.14 \\
15.47 \\
17.20 \\
16.98 \\
15.48 \\
14.45 \\
14.89 \\
14.79 \\
14.26 \\
15.05 \\
15.74 \\
15.36\end{array}$ & $\begin{array}{l}15.37 \\
16.17 \\
16.28 \\
17.37 \\
17.69 \\
16.37 \\
15.15 \\
15.20 \\
15.67 \\
15.15 \\
15.50 \\
16.37 \\
16.02\end{array}$ \\
\hline 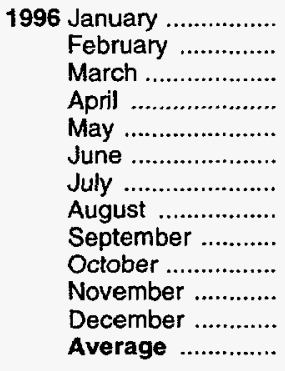 & $\begin{array}{l}16.95 \\
17.91 \\
19.78 \\
20.96 \\
19.72 \\
18.60 \\
19.72 \\
20.33 \\
22.23 \\
23.05 \\
22.38 \\
23.22 \\
20.70\end{array}$ & $\begin{array}{l}17.73 \\
18.09 \\
20.02 \\
22.65 \\
20.09 \\
19.49 \\
19.72 \\
20.79 \\
22.79 \\
23.57 \\
23.25 \\
24.56 \\
21.33\end{array}$ & $\begin{array}{l}16.52 \\
16.59 \\
18.39 \\
19.63 \\
17.93 \\
17.05 \\
17.85 \\
18.89 \\
20.96 \\
22.40 \\
20.96 \\
21.83 \\
19.14\end{array}$ & $\begin{array}{l}18.63 \\
18.53 \\
20.44 \\
21.49 \\
20.13 \\
19.25 \\
19.90 \\
21.13 \\
22.80 \\
24.71 \\
24.43 \\
24.39 \\
21.27\end{array}$ & $\begin{array}{c}W \\
W \\
18.29 \\
W \\
W \\
17.96 \\
18.48 \\
20.16 \\
20.60 \\
W \\
21.90 \\
19.24 \\
19.28\end{array}$ & $\begin{array}{c}W \\
W \\
19.42 \\
W \\
19.02 \\
W \\
W \\
18.82 \\
W \\
W \\
22.35 \\
W \\
19.43\end{array}$ & $\begin{array}{l}14.12 \\
15.22 \\
17.78 \\
17.99 \\
16.35 \\
16.08 \\
16.72 \\
17.35 \\
19.66 \\
20.29 \\
19.62 \\
20.41 \\
17.73\end{array}$ & $\begin{array}{c}W \\
W \\
18.62 \\
W \\
W \\
17.70 \\
18.45 \\
20.43 \\
21.01 \\
W \\
22.39 \\
19.99 \\
19.30\end{array}$ & $\begin{array}{l}15.86 \\
16.89 \\
18.77 \\
18.75 \\
17.72 \\
17.21 \\
17.78 \\
18.99 \\
20.57 \\
21.85 \\
21.04 \\
21.01 \\
18.94\end{array}$ & $\begin{array}{l}16.37 \\
16.81 \\
18.77 \\
20.20 \\
18.83 \\
17.94 \\
18.62 \\
19.59 \\
21.55 \\
22.59 \\
21.48 \\
22.04 \\
19.65\end{array}$ \\
\hline 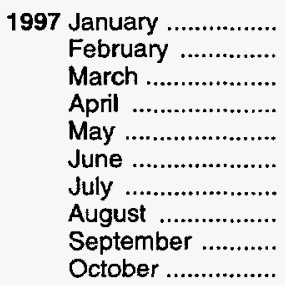 & $\begin{array}{r}23.20 \\
21.35 \\
18.66 \\
17.05 \\
18.25 \\
17.84 \\
17.72 \\
17.96 \\
\mathrm{R} 18.15 \\
19.53\end{array}$ & $\begin{array}{r}24.14 \\
21.12 \\
19.41 \\
17.87 \\
17.92 \\
16.87 \\
17.72 \\
\mathrm{R} 18.42 \\
\mathrm{R} 18.52 \\
19.52\end{array}$ & $\begin{array}{r}21.09 \\
18.57 \\
17.00 \\
15.94 \\
16.84 \\
15.70 \\
15.99 \\
R 16.29 \\
R 16.02 \\
17.53\end{array}$ & $\begin{array}{r}24.52 \\
21.53 \\
19.02 \\
17.97 \\
18.99 \\
18.22 \\
19.12 \\
R \quad 18.98 \\
R \quad 19.35 \\
19.80\end{array}$ & $\begin{array}{c}17.37 \\
W \\
W \\
15.82 \\
15.60 \\
15.26 \\
15.24 \\
16.89 \\
15.65 \\
W\end{array}$ & $\begin{array}{c}W \\
W \\
(d) \\
W \\
19.03 \\
18.09 \\
17.40 \\
18.17 \\
R 18.44 \\
W\end{array}$ & $\begin{array}{r}19.35 \\
16.68 \\
15.50 \\
14.81 \\
15.27 \\
14.66 \\
14.99 \\
\text { R } 15.33 \\
\text { R } 15.25 \\
16.26\end{array}$ & $\begin{array}{c}17.37 \\
W \\
W \\
15.95 \\
15.67 \\
15.11 \\
15.33 \\
16.47 \\
R 16.20 \\
W\end{array}$ & $\begin{array}{r}20.37 \\
17.96 \\
16.49 \\
15.92 \\
16.27 \\
15.61 \\
16.04 \\
R 16.37 \\
R 16.49 \\
16.62\end{array}$ & $\begin{array}{r}21.93 \\
19.71 \\
17.68 \\
16.44 \\
17.33 \\
16.36 \\
16.65 \\
R 16.96 \\
R 16.96 \\
18.03\end{array}$ \\
\hline
\end{tabular}

a Bahrain, Iran, Iraq, Kuwait, Qatar, Saudi Arabia, and United Arab Emirates.

b Current members are Algeria, Indonesia, Iran, Iraq, Kuwait, Libya, Nigeria, Qatar, Saudi Arabia, United Arab Emirates, and Venezuela. Ecuador withdrew at the end of 1992 and Gabon withdrew at the end of 1994

c Based on October, November, and December datal only.

d No data reported.

$R=$ Revised data. NA=Not available. $W=$ Value withheld to avoid disclosure of individual company data.

Notes: - The Free on Board (F.O.B.) cost at the country of origin excludes all costs related to insurance and transportation. See Note 2 at end of section. - Values for the current 2 months are preliminary. - Prices through 1980 reflect the period of reporting: prices since then reflect the period of loading. - Annual averages are averages of the monthly prices, including prices not published, weighted by volume. - Cargoes that are purchased on a "netback" basis, or under similar contractual arrangements whereby the actual purchase price is not established at the time the crude oil is acquired for importation into the United States, are not included in the published data until the actual prices have been determined and reported. - U.S. geographic coverage is the 50 States and the District of Columbia.

Sources: See end of section. 


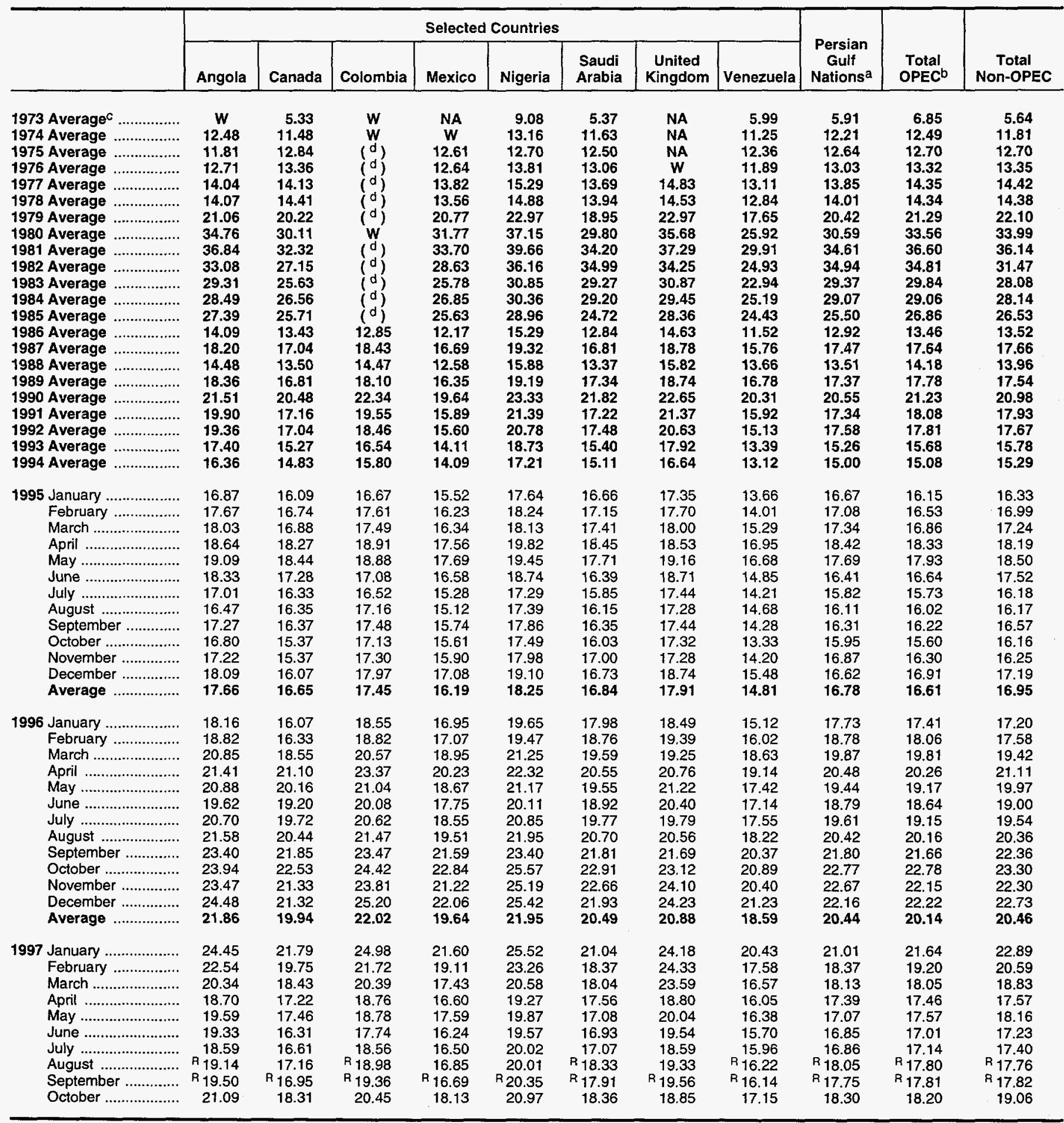

a Bahrain, Iran, Iraq, Kuwait, Qatar, Saudi Arabia, and United Arab Emirates.

b Current members are Algeria, Indonesia, Iran, Iraq, Kuwait, Libya, Nigeria, Qatar, Saudi Arabia, United Arab Emirates, and Venezuela. Ecuador withdrew at the end of 1992 and Gabon withdrew at the end of 1994.

c Based on October, November, and December data only.

I No data reported.

$R=$ Revised data. $N A=$ Not available. $W=$ Value withheld to avoid disclosure of individual company data.

Notes: - See Note 3 at end of section. - Values for the current 2 months are preliminary. - Prices through 1980 reflect the period of reporting; prices since then reflect the period of loading. - Annual averages are averages of the monthly prices, including prices not published, weighted by volume. - Cargoes that are purchased on a "netback" basis, or under similar contractual arrangements whereby the actual purchase price is not established at the time the crude oil is acquired for importation into the United States, are not included in the published data until the actual prices have been determined and reported. - U.S. geographic coverage is the 50 States and the District of Columbia.

Sources: - October 1973-September 1977: Federal Energy Administration, Form FEA-F701-M-O "Transfer Pricing Report." - October 1977-December 1977: Energy Information Administration (EIA), Form FEA-F701-M-0, "Transfer Pricing Report." - 1978 forward: EIA, Petroleum Marketing Monthly, January 1998, Table 25. 
Table 9.4 Motor Gasoline Retail Prices, U.S. City Average

(Cents per Gallon, Including Taxes)

\begin{tabular}{|c|c|c|c|c|}
\hline & $\begin{array}{l}\text { Leaded } \\
\text { Regular }\end{array}$ & $\begin{array}{c}\text { Unleaded } \\
\text { Regular }\end{array}$ & $\begin{array}{l}\text { Unleaded } \\
\text { Premium }\end{array}$ & All Types \\
\hline 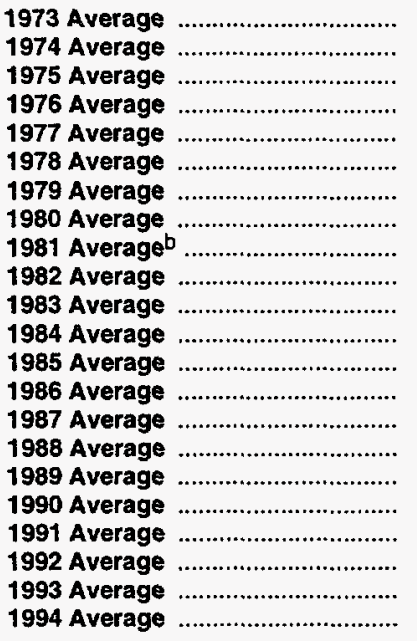 & $\begin{array}{r}38.8 \\
53.2 \\
56.7 \\
59.0 \\
62.2 \\
62.6 \\
85.7 \\
119.1 \\
131.1 \\
122.2 \\
115.7 \\
112.9 \\
111.5 \\
85.7 \\
89.7 \\
89.9 \\
99.8 \\
114.9 \\
\text { NA } \\
\text { NA } \\
\text { NA } \\
\text { NA }\end{array}$ & $\begin{array}{r}\text { NA } \\
\text { NA } \\
\text { NA } \\
61.4 \\
65.6 \\
67.0 \\
90.3 \\
124.5 \\
137.8 \\
129.6 \\
124.1 \\
121.2 \\
120.2 \\
92.7 \\
94.8 \\
94.6 \\
102.1 \\
116.4 \\
114.0 \\
112.7 \\
110.8 \\
111.2\end{array}$ & $\begin{array}{r}\text { NA } \\
\text { NA } \\
\text { NA } \\
\text { NA } \\
\text { NA } \\
\text { NA } \\
\text { NA } \\
\text { NA } \\
\text { c } 147.0 \\
141.5 \\
138.3 \\
136.6 \\
134.0 \\
108.5 \\
109.3 \\
110.7 \\
119.7 \\
134.9 \\
132.1 \\
131.6 \\
130.2 \\
130.5\end{array}$ & $\begin{array}{r}\text { NA } \\
\text { NA } \\
\text { NA } \\
\text { NA } \\
\text { NA } \\
65.2 \\
88.2 \\
122.1 \\
135.3 \\
128.1 \\
122.5 \\
119.8 \\
119.6 \\
93.1 \\
95.7 \\
96.3 \\
106.0 \\
121.7 \\
119.6 \\
119.0 \\
117.3 \\
117.4\end{array}$ \\
\hline 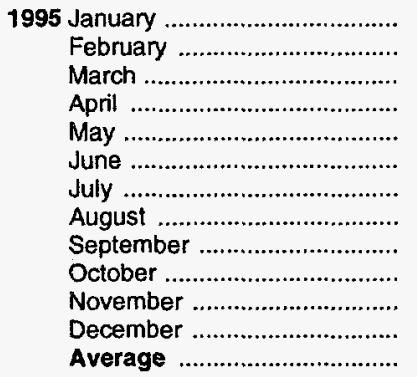 & $\begin{array}{l}\text { NA } \\
\text { NA } \\
\text { NA } \\
\text { NA } \\
\text { NA } \\
\text { NA } \\
\text { NA } \\
\text { NA } \\
\text { NA } \\
\text { NA } \\
\text { NA } \\
\text { NA } \\
\text { NA }\end{array}$ & $\begin{array}{l}112.9 \\
112.0 \\
111.5 \\
114.0 \\
120.0 \\
122.6 \\
119.5 \\
116.4 \\
114.8 \\
112.7 \\
110.1 \\
110.1 \\
114.7\end{array}$ & $\begin{array}{l}132.4 \\
131.6 \\
130.6 \\
132.5 \\
138.3 \\
141.1 \\
138.4 \\
135.2 \\
133.2 \\
131.5 \\
129.2 \\
129.0 \\
133.6\end{array}$ & $\begin{array}{l}119.0 \\
118.1 \\
117.3 \\
119.7 \\
125.6 \\
128.1 \\
125.2 \\
122.2 \\
120.6 \\
118.5 \\
116.1 \\
116.0 \\
120.5\end{array}$ \\
\hline 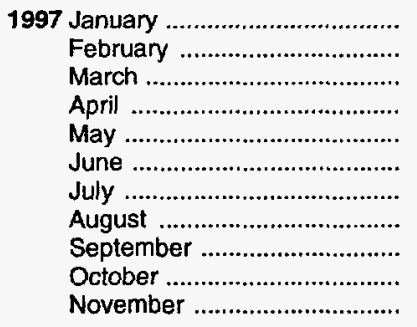 & $\begin{array}{l}\text { NA } \\
\text { NA } \\
\text { NA } \\
\text { NA } \\
\text { NA } \\
\text { NA } \\
\text { NA } \\
\text { NA } \\
\text { NA } \\
\text { NA } \\
\text { NA }\end{array}$ & $\begin{array}{l}126.1 \\
125.5 \\
123.5 \\
123.1 \\
122.6 \\
122.9 \\
120.5 \\
125.3 \\
127.7 \\
124.2 \\
121.3\end{array}$ & $\begin{array}{l}144.1 \\
143.4 \\
141.5 \\
141.3 \\
140.9 \\
141.1 \\
138.8 \\
143.3 \\
145.8 \\
142.6 \\
139.7\end{array}$ & $\begin{array}{l}131.8 \\
131.2 \\
129.3 \\
128.8 \\
128.4 \\
128.6 \\
126.3 \\
131.0 \\
133.4 \\
130.0 \\
127.1\end{array}$ \\
\hline
\end{tabular}

a Also includes types of motor gasoline not shown separately.

b In September 1981, the Bureau of Labor Statistics changed the weights used in the calculation of average motor gasoline prices. From September 1981 fonward, gasohol is included in the average for all types, and unleaded premium is weighted more heavily.

$c$ Based on September through December data only.

$\mathrm{NA}=$ Not available.

Notes: - See Note 5 at end of section. - Geographic coverage for

$1973-1977$ is 56 urban areas. Geographic coverage for 1978 fonward is 85 urban areas.

Sources: - Monthly Data: U.S. Department of Labor, Bureau of Labor Statistics, Consumer Prices: Energy. - Annual Data: 1973-Platt's Oil Price Handbook and Oilmanac, 1974, 51st Edition. 1974 forward-calculated by the Energy Information Administration as the simple averages of monthly data. 
Table 9.5 Refiner Prices of Residual Fuel Oil

(Cents per Gallon, Excluding Taxes)

\begin{tabular}{|c|c|c|c|c|c|c|}
\hline & \multicolumn{2}{|c|}{$\begin{array}{c}\text { Residual Fuel Oil } \\
\text { Sulfur Content Less } \\
\text { Than or Equal to } 1 \text { Percent }\end{array}$} & \multicolumn{2}{|c|}{$\begin{array}{c}\text { Residual Fuel Oil } \\
\text { Sulfur Content } \\
\text { Greater Than } 1 \text { Percent }\end{array}$} & \multicolumn{2}{|c|}{ Average } \\
\hline & $\begin{array}{l}\text { Sales for } \\
\text { Resale }\end{array}$ & $\begin{array}{l}\text { Sales to } \\
\text { End Users }\end{array}$ & $\begin{array}{l}\text { Sales for } \\
\text { Resale }\end{array}$ & $\begin{array}{l}\text { Sales to } \\
\text { End Users }\end{array}$ & $\begin{array}{l}\text { Sales for } \\
\text { Resale }\end{array}$ & $\begin{array}{l}\text { Sales to } \\
\text { End Users }\end{array}$ \\
\hline 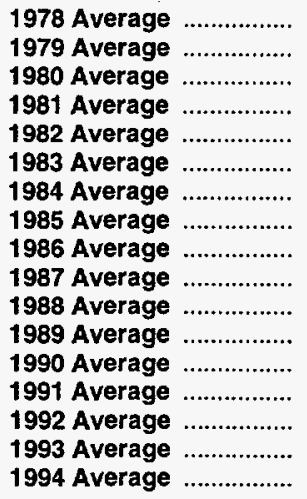 & $\begin{array}{l}29.3 \\
45.0 \\
60.8 \\
74.8 \\
69.5 \\
64.3 \\
68.5 \\
61.0 \\
32.8 \\
41.2 \\
33.3 \\
40.7 \\
47.2 \\
36.4 \\
35.1 \\
33.7 \\
34.5\end{array}$ & $\begin{array}{l}31.4 \\
46.8 \\
67.5 \\
82.9 \\
74.7 \\
69.5 \\
72.0 \\
64.4 \\
37.2 \\
44.7 \\
37.2 \\
43.6 \\
50.5 \\
40.2 \\
38.9 \\
39.7 \\
40.1\end{array}$ & $\begin{array}{l}24.5 \\
36.6 \\
47.9 \\
62.2 \\
57.2 \\
59.1 \\
63.9 \\
56.0 \\
28.9 \\
36.2 \\
27.1 \\
33.1 \\
37.2 \\
29.2 \\
28.6 \\
25.6 \\
28.7\end{array}$ & $\begin{array}{l}27.5 \\
38.9 \\
52.3 \\
67.3 \\
61.1 \\
61.1 \\
65.9 \\
58.2 \\
31.7 \\
39.6 \\
30.0 \\
34.4 \\
40.0 \\
30.6 \\
31.2 \\
30.3 \\
33.0\end{array}$ & $\begin{array}{l}26.3 \\
39.9 \\
52.8 \\
66.3 \\
61.2 \\
60.9 \\
65.4 \\
57.7 \\
30.5 \\
38.5 \\
30.0 \\
36.0 \\
41.3 \\
31.4 \\
30.8 \\
29.3 \\
31.7\end{array}$ & $\begin{array}{l}29.8 \\
43.6 \\
60.7 \\
75.6 \\
67.6 \\
65.1 \\
68.7 \\
61.0 \\
34.3 \\
42.3 \\
33.4 \\
38.5 \\
44.4 \\
34.0 \\
33.6 \\
33.7 \\
35.2\end{array}$ \\
\hline 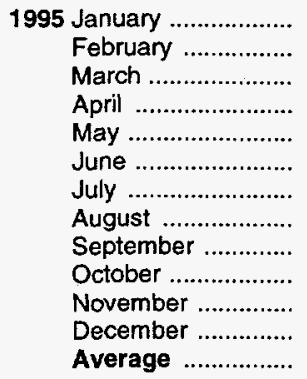 & $\begin{array}{l}39.1 \\
37.1 \\
38.3 \\
36.8 \\
40.4 \\
39.9 \\
36.8 \\
35.5 \\
36.4 \\
35.3 \\
36.6 \\
44.7 \\
38.3\end{array}$ & $\begin{array}{l}46.0 \\
43.7 \\
43.4 \\
42.6 \\
43.6 \\
45.1 \\
42.9 \\
39.1 \\
39.0 \\
41.7 \\
43.4 \\
49.2 \\
43.6\end{array}$ & $\begin{array}{l}33.3 \\
33.3 \\
35.2 \\
36.1 \\
37.3 \\
36.9 \\
32.5 \\
29.8 \\
30.4 \\
32.4 \\
31.8 \\
36.0 \\
33.8\end{array}$ & $\begin{array}{l}37.9 \\
38.2 \\
39.6 \\
39.6 \\
41.7 \\
41.3 \\
36.4 \\
33.7 \\
34.0 \\
34.5 \\
35.5 \\
40.5 \\
37.7\end{array}$ & $\begin{array}{l}36.6 \\
35.4 \\
37.0 \\
36.5 \\
38.8 \\
38.7 \\
35.3 \\
33.1 \\
33.8 \\
34.1 \\
34.4 \\
40.6 \\
36.3\end{array}$ & $\begin{array}{l}40.2 \\
39.8 \\
40.5 \\
40.3 \\
42.2 \\
42.1 \\
38.1 \\
35.1 \\
35.1 \\
35.9 \\
37.4 \\
43.2 \\
39.2\end{array}$ \\
\hline 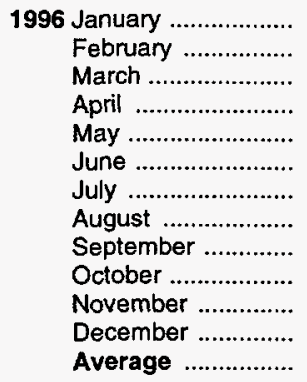 & $\begin{array}{l}49.9 \\
42.6 \\
47.1 \\
48.3 \\
45.0 \\
40.4 \\
41.4 \\
41.9 \\
42.6 \\
47.8 \\
49.2 \\
51.4 \\
45.6\end{array}$ & $\begin{array}{l}54.8 \\
53.2 \\
51.9 \\
51.1 \\
51.1 \\
47.3 \\
48.6 \\
49.8 \\
51.2 \\
54.7 \\
57.0 \\
58.6 \\
52.6\end{array}$ & $\begin{array}{l}38.0 \\
36.8 \\
36.0 \\
39.9 \\
36.9 \\
35.0 \\
37.3 \\
37.2 \\
40.3 \\
43.1 \\
44.5 \\
43.0 \\
38.9\end{array}$ & $\begin{array}{l}44.7 \\
41.7 \\
42.1 \\
43.0 \\
41.4 \\
38.4 \\
38.7 \\
39.5 \\
43.2 \\
47.1 \\
48.0 \\
47.5 \\
43.3\end{array}$ & $\begin{array}{l}45.2 \\
40.1 \\
42.0 \\
43.7 \\
41.0 \\
37.4 \\
38.9 \\
39.0 \\
41.2 \\
45.0 \\
46.3 \\
46.0 \\
42.0\end{array}$ & $\begin{array}{l}47.9 \\
44.9 \\
44.7 \\
45.1 \\
43.3 \\
40.8 \\
41.0 \\
42.0 \\
44.9 \\
48.5 \\
49.7 \\
49.9 \\
45.5\end{array}$ \\
\hline 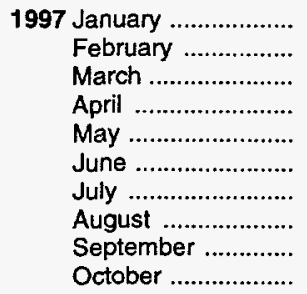 & $\begin{array}{r}46.2 \\
43.7 \\
39.6 \\
37.6 \\
36.6 \\
39.4 \\
38.5 \\
39.4 \\
\text { R } \\
44.6\end{array}$ & $\begin{array}{r}58.7 \\
54.6 \\
49.3 \\
46.4 \\
45.3 \\
44.5 \\
44.2 \\
44.6 \\
\text { R } \\
46.4 \\
48.3\end{array}$ & $\begin{array}{l}39.2 \\
35.4 \\
34.1 \\
35.2 \\
35.4 \\
34.8 \\
35.4 \\
37.6 \\
37.6 \\
39.8\end{array}$ & $\begin{array}{r}46.3 \\
41.8 \\
37.6 \\
37.5 \\
38.7 \\
38.7 \\
38.2 \\
39.5 \\
\text { R } \\
40.1 \\
42.8\end{array}$ & $\begin{array}{r}42.9 \\
39.4 \\
35.8 \\
36.1 \\
35.8 \\
36.7 \\
36.6 \\
38.3 \\
\text { R } 38.7 \\
41.9\end{array}$ & $\begin{array}{r}49.2 \\
45.0 \\
40.3 \\
39.7 \\
40.3 \\
40.1 \\
39.6 \\
40.7 \\
41.4 \\
43.8\end{array}$ \\
\hline
\end{tabular}

$\mathbf{R}=$ Revised data.

Notes: - Sales for resale are those made to purchasers other than ultimate consumers. Sales to end users are those made directly to ultimate consumers, including bulk consumers (such as agriculture, industry, and electric utilities) and commercial consumers. Values for the current month are preliminary. - Prices prior to 1983 are Energy Information Administration (EIA) estimates. See Note 6 at end of section. - Geographic coverage is the 50 States and the District of Columbia.

Source: EIA, Petroleum Marketing Monthly, January 1998, Table 19. 
Table 9.6 Refiner Prices of Petroleum Products for Resale (Cents per Gallon, Excluding Taxes)

\begin{tabular}{|c|c|c|c|c|c|c|c|}
\hline & $\begin{array}{l}\text { Finished } \\
\text { Motor } \\
\text { Gasolinea }\end{array}$ & $\begin{array}{l}\text { Finished } \\
\text { Aviation } \\
\text { Gasoline }\end{array}$ & $\begin{array}{c}\text { Kerosene- } \\
\text { Type } \\
\text { Jet Fuel }\end{array}$ & Kerosene & $\begin{array}{c}\text { No. } 2 \\
\text { Fuel } \\
\text { Oil }\end{array}$ & $\begin{array}{c}\text { No. } 2 \\
\text { Diesel } \\
\text { Fuel }\end{array}$ & $\begin{array}{c}\text { Propane } \\
\text { (Consumer } \\
\text { Grade) }\end{array}$ \\
\hline $\begin{array}{l}1978 \text { Average } \\
1979 \text { Average } \\
1980 \text { Average } \\
1981 \text { Average }\end{array}$ & $\begin{array}{r}43.4 \\
63.7 \\
94.1 \\
106.4 \\
97.3 \\
88.2 \\
83.2 \\
83.5 \\
53.1 \\
58.9 \\
57.7 \\
65.4 \\
78.6 \\
69.9 \\
67.7 \\
62.6 \\
59.9\end{array}$ & $\begin{array}{r}53.7 \\
72.1 \\
112.8 \\
125.0 \\
122.8 \\
117.8 \\
116.5 \\
113.0 \\
91.2 \\
85.9 \\
85.0 \\
95.0 \\
106.3 \\
100.1 \\
99.1 \\
96.5 \\
93.3\end{array}$ & $\begin{array}{r}38.6 \\
66.0 \\
86.8 \\
101.2 \\
95.3 \\
85.4 \\
83.0 \\
79.4 \\
49.5 \\
53.8 \\
49.5 \\
58.3 \\
77.3 \\
65.0 \\
60.5 \\
57.7 \\
53.4\end{array}$ & $\begin{array}{r}40.4 \\
62.4 \\
86.4 \\
106.6 \\
101.8 \\
89.2 \\
91.6 \\
87.4 \\
60.6 \\
59.2 \\
54.9 \\
66.9 \\
83.9 \\
72.2 \\
63.2 \\
60.4 \\
61.8\end{array}$ & $\begin{array}{l}36.9 \\
56.9 \\
80.3 \\
97.6 \\
91.4 \\
81.5 \\
82.1 \\
77.6 \\
48.6 \\
52.7 \\
47.3 \\
56.5 \\
69.7 \\
62.2 \\
57.9 \\
54.4 \\
50.6\end{array}$ & $\begin{array}{l}36.5 \\
57.4 \\
80.1 \\
97.2 \\
91.4 \\
80.8 \\
80.3 \\
77.2 \\
45.2 \\
53.4 \\
47.3 \\
56.7 \\
69.4 \\
61.5 \\
59.1 \\
57.0 \\
52.9\end{array}$ & $\begin{array}{l}23.7 \\
29.1 \\
41.5 \\
46.6 \\
42.7 \\
48.4 \\
45.0 \\
39.8 \\
29.0 \\
25.2 \\
24.0 \\
24.7 \\
38.6 \\
34.9 \\
32.8 \\
35.1 \\
32.4\end{array}$ \\
\hline 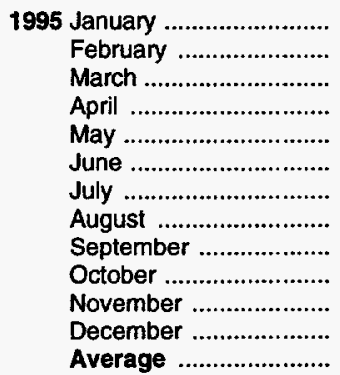 & $\begin{array}{l}60.0 \\
60.3 \\
60.0 \\
66.5 \\
71.8 \\
68.2 \\
62.9 \\
62.0 \\
62.3 \\
58.8 \\
58.0 \\
59.9 \\
62.6\end{array}$ & $\begin{array}{r}92.9 \\
93.2 \\
93.1 \\
96.6 \\
102.2 \\
101.6 \\
100.1 \\
98.9 \\
98.7 \\
96.3 \\
94.2 \\
95.3 \\
97.5\end{array}$ & $\begin{array}{l}52.2 \\
52.0 \\
50.1 \\
52.6 \\
54.7 \\
53.1 \\
51.3 \\
53.1 \\
55.2 \\
54.1 \\
56.3 \\
58.6 \\
53.9\end{array}$ & $\begin{array}{l}56.6 \\
55.2 \\
52.8 \\
56.0 \\
57.7 \\
53.2 \\
52.3 \\
54.9 \\
58.0 \\
57.0 \\
60.5 \\
64.0 \\
58.0\end{array}$ & $\begin{array}{l}49.4 \\
49.2 \\
48.1 \\
50.5 \\
52.4 \\
49.4 \\
48.1 \\
51.0 \\
52.0 \\
50.5 \\
53.4 \\
57.3 \\
51.1\end{array}$ & $\begin{array}{l}50.1 \\
50.6 \\
51.2 \\
54.7 \\
55.9 \\
52.6 \\
51.4 \\
54.2 \\
55.7 \\
54.6 \\
56.3 \\
57.6 \\
53.8\end{array}$ & $\begin{array}{l}35.6 \\
34.5 \\
34.3 \\
33.0 \\
33.1 \\
32.6 \\
32.1 \\
33.2 \\
33.8 \\
34.4 \\
34.7 \\
37.9 \\
34.4\end{array}$ \\
\hline 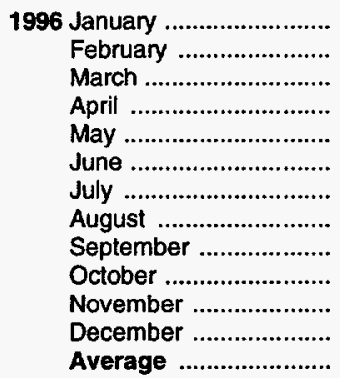 & $\begin{array}{l}61.0 \\
61.6 \\
67.9 \\
76.1 \\
78.0 \\
73.0 \\
72.3 \\
71.1 \\
71.6 \\
72.8 \\
74.5 \\
73.1 \\
71.3\end{array}$ & $\begin{array}{r}94.7 \\
96.5 \\
100.6 \\
107.5 \\
110.0 \\
107.0 \\
105.3 \\
107.1 \\
106.8 \\
107.1 \\
108.4 \\
107.1 \\
105.5\end{array}$ & $\begin{array}{l}60.3 \\
57.3 \\
59.6 \\
65.3 \\
62.2 \\
57.5 \\
59.6 \\
64.5 \\
71.6 \\
73.6 \\
72.2 \\
73.0 \\
64.6\end{array}$ & $\begin{array}{l}65.8 \\
65.7 \\
68.0 \\
75.1 \\
66.1 \\
59.8 \\
61.7 \\
66.6 \\
75.6 \\
80.7 \\
79.7 \\
79.0 \\
71.4\end{array}$ & $\begin{array}{l}56.8 \\
58.9 \\
62.8 \\
67.5 \\
61.1 \\
53.7 \\
57.1 \\
62.1 \\
68.7 \\
72.7 \\
71.4 \\
71.2 \\
63.9\end{array}$ & $\begin{array}{l}56.2 \\
57.9 \\
61.9 \\
70.1 \\
66.8 \\
59.1 \\
60.0 \\
64.9 \\
71.7 \\
75.4 \\
73.3 \\
71.0 \\
65.9\end{array}$ & $\begin{array}{l}41.6 \\
44.2 \\
41.1 \\
37.8 \\
36.2 \\
36.2 \\
36.9 \\
38.9 \\
45.2 \\
51.1 \\
57.9 \\
67.7 \\
46.1\end{array}$ \\
\hline 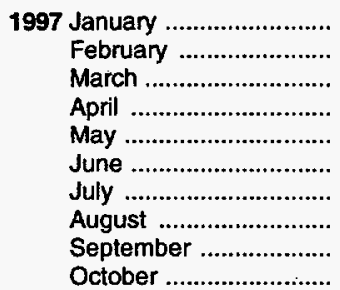 & $\begin{array}{l}74.8 \\
73.1 \\
71.5 \\
70.4 \\
71.1 \\
68.3 \\
67.5 \\
75.0 \\
72.3 \\
68.6\end{array}$ & $\begin{array}{l}109.0 \\
108.7 \\
107.9 \\
108.5 \\
108.2 \\
105.9 \\
104.9 \\
108.9 \\
108.9 \\
104.5\end{array}$ & $\begin{array}{l}73.5 \\
71.4 \\
61.8 \\
60.5 \\
59.4 \\
58.1 \\
56.8 \\
59.4 \\
58.8 \\
61.4\end{array}$ & $\begin{array}{r}77.7 \\
73.4 \\
63.2 \\
62.1 \\
61.1 \\
57.1 \\
56.2 \\
60.5 \\
\text { R } 60.1 \\
63.8\end{array}$ & $\begin{array}{l}69.8 \\
64.5 \\
57.7 \\
58.6 \\
58.8 \\
54.5 \\
53.8 \\
55.3 \\
54.3 \\
59.0\end{array}$ & $\begin{array}{l}69.9 \\
67.8 \\
62.5 \\
61.7 \\
60.7 \\
56.5 \\
55.8 \\
58.9 \\
57.8 \\
61.7\end{array}$ & $\begin{array}{l}59.9 \\
44.7 \\
41.3 \\
37.7 \\
36.9 \\
36.4 \\
35.9 \\
37.5 \\
39.5 \\
41.1\end{array}$ \\
\hline
\end{tabular}

a See Note 5 at end of section.

$R=$ Revised data.

Notes: - Sales for resale are those made to purchasers other than ultimate consumers. Sales to end users are shown in Table 9.7; they are sales made directly to ultimate consumers, iricluding bulk consumers (such as agriculture, industry, and electric utilities) and residential and commercial consumers. - Values for the current month are preliminary. Prices prior to 1983 are Energy Information Administration (EIA) estimates. See Note 6 at end of section. - Geographic coverage is the 50 States and the District of Columbia.

Source: EIA, Petroleum Marketing Monthly, January 1998, Table 4. 
Table 9.7 Refiner Prices of Petroleum Products to End Users (Cents per Gallon, Excluding Taxes)

\begin{tabular}{|c|c|c|c|c|c|c|c|}
\hline & $\begin{array}{l}\text { Finished } \\
\text { Motor } \\
\text { Gasoline }\end{array}$ & $\begin{array}{l}\text { Finished } \\
\text { Aviation } \\
\text { Gasoline }\end{array}$ & $\begin{array}{c}\text { Kerosene- } \\
\text { Type } \\
\text { Jet Fuel }\end{array}$ & Kerosene & $\begin{array}{c}\text { No. } 2 \\
\text { Fuel } \\
\text { Oil }\end{array}$ & $\begin{array}{c}\text { No. } 2 \\
\text { Diesel } \\
\text { Fuel }\end{array}$ & $\begin{array}{c}\text { Propane } \\
\text { (Consumer } \\
\text { Grade) }\end{array}$ \\
\hline $\begin{array}{l}1978 \text { Average } \\
1979 \text { Average } \\
1980 \text { Average } \ldots \ldots \ldots \ldots \ldots \ldots \ldots \\
1981 \text { Average } \ldots \ldots \ldots \ldots \ldots \ldots \\
1982 \text { Average } \ldots \ldots \ldots \ldots \ldots \ldots \\
1983 \text { Average } \\
1984 \text { Average } \\
1985 \text { Average } \\
1986 \text { Average } \\
1987 \text { Average }\end{array}$ & $\begin{array}{r}48.4 \\
71.3 \\
103.5 \\
114.7 \\
106.0 \\
95.4 \\
90.7 \\
91.2 \\
62.4 \\
66.9 \\
67.3 \\
75.6 \\
88.3 \\
79.7 \\
78.7 \\
75.9 \\
73.8\end{array}$ & $\begin{array}{r}51.6 \\
68.9 \\
108.4 \\
130.3 \\
131.2 \\
125.5 \\
123.4 \\
120.1 \\
101.1 \\
90.7 \\
89.1 \\
99.5 \\
112.0 \\
104.7 \\
102.7 \\
99.0 \\
95.7\end{array}$ & $\begin{array}{r}38.7 \\
54.7 \\
86.8 \\
102.4 \\
96.3 \\
87.8 \\
84.2 \\
79.6 \\
52.9 \\
54.3 \\
51.3 \\
59.2 \\
76.6 \\
65.2 \\
61.0 \\
58.0 \\
53.4\end{array}$ & $\begin{array}{r}42.1 \\
58.5 \\
90.2 \\
112.3 \\
108.9 \\
96.1 \\
103.6 \\
103.0 \\
79.0 \\
77.0 \\
73.8 \\
70.9 \\
92.3 \\
83.8 \\
78.8 \\
75.4 \\
66.0\end{array}$ & $\begin{array}{l}40.0 \\
51.6 \\
78.8 \\
91.4 \\
90.5 \\
91.6 \\
91.6 \\
84.9 \\
56.0 \\
58.1 \\
54.4 \\
58.7 \\
73.4 \\
66.5 \\
62.7 \\
60.2 \\
57.2\end{array}$ & $\begin{array}{l}37.7 \\
58.5 \\
81.8 \\
99.5 \\
94.2 \\
82.6 \\
82.3 \\
78.9 \\
47.8 \\
55.1 \\
50.0 \\
58.5 \\
72.5 \\
64.8 \\
61.9 \\
60.2 \\
55.4\end{array}$ & $\begin{array}{l}33.5 \\
35.7 \\
48.2 \\
56.5 \\
59.2 \\
70.9 \\
73.7 \\
71.7 \\
74.5 \\
70.1 \\
71.4 \\
61.5 \\
74.5 \\
73.0 \\
64.3 \\
67.3 \\
53.0\end{array}$ \\
\hline 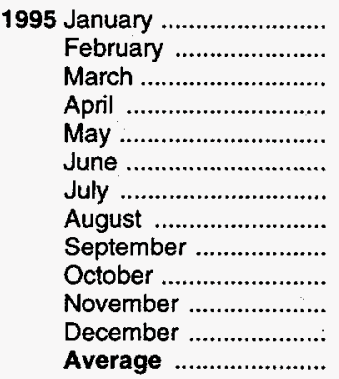 & $\begin{array}{l}74.5 \\
73.3 \\
73.1 \\
77.3 \\
83.4 \\
83.9 \\
80.0 \\
76.9 \\
75.8 \\
73.5 \\
71.8 \\
73.0 \\
76.5\end{array}$ & $\begin{array}{r}99.6 \\
99.8 \\
99.0 \\
101.3 \\
105.8 \\
106.4 \\
101.8 \\
99.2 \\
101.3 \\
96.8 \\
95.4 \\
96.0 \\
100.5\end{array}$ & $\begin{array}{l}52.3 \\
52.2 \\
50.5 \\
52.8 \\
55.0 \\
53.2 \\
51.9 \\
53.4 \\
55.7 \\
54.9 \\
57.0 \\
59.2 \\
54.0\end{array}$ & $\begin{array}{l}67.4 \\
62.8 \\
59.4 \\
56.1 \\
51.7 \\
54.9 \\
51.3 \\
53.3 \\
57.3 \\
56.5 \\
62.8 \\
70.0 \\
58.9\end{array}$ & $\begin{array}{l}57.3 \\
56.9 \\
55.3 \\
56.2 \\
56.2 \\
52.7 \\
51.5 \\
53.3 \\
56.2 \\
54.1 \\
58.7 \\
62.3 \\
\mathbf{5 6 . 2}\end{array}$ & $\begin{array}{l}53.2 \\
53.1 \\
53.4 \\
56.5 \\
57.9 \\
55.7 \\
54.0 \\
55.8 \\
57.4 \\
56.5 \\
58.2 \\
59.3 \\
\mathbf{5 6 . 0}\end{array}$ & $\begin{array}{l}54.0 \\
55.6 \\
53.9 \\
46.6 \\
43.1 \\
42.9 \\
42.2 \\
44.9 \\
45.7 \\
49.3 \\
51.7 \\
55.0 \\
49.2\end{array}$ \\
\hline 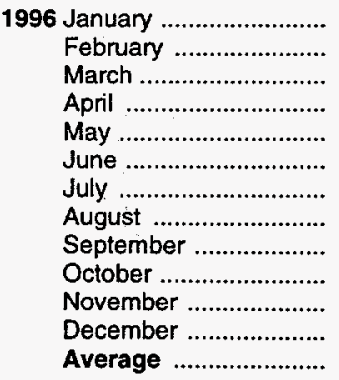 & $\begin{array}{l}74.8 \\
74.9 \\
79.8 \\
88.1 \\
92.7 \\
90.3 \\
87.5 \\
84.9 \\
84.4 \\
84.4 \\
86.8 \\
86.0 \\
84.7\end{array}$ & $\begin{array}{l}101.2 \\
100.6 \\
105.0 \\
111.4 \\
114.4 \\
113.5 \\
113.7 \\
114.4 \\
114.3 \\
115.0 \\
115.1 \\
115.3 \\
111.6\end{array}$ & $\begin{array}{l}61.3 \\
56.9 \\
59.0 \\
66.0 \\
63.3 \\
57.7 \\
60.3 \\
65.1 \\
71.8 \\
73.6 \\
71.7 \\
74.0 \\
65.1\end{array}$ & $\begin{array}{l}71.8 \\
73.4 \\
69.0 \\
80.5 \\
68.4 \\
58.5 \\
64.6 \\
69.5 \\
76.4 \\
87.1 \\
88.7 \\
90.7 \\
74.0\end{array}$ & $\begin{array}{l}63.5 \\
64.1 \\
66.8 \\
69.9 \\
64.9 \\
57.5 \\
59.4 \\
66.1 \\
72.1 \\
75.1 \\
75.0 \\
75.1 \\
67.3\end{array}$ & $\begin{array}{l}59.0 \\
60.0 \\
64.4 \\
71.9 \\
69.8 \\
62.2 \\
62.3 \\
66.4 \\
72.9 \\
76.9 \\
75.7 \\
74.4 \\
68.1\end{array}$ & $\begin{array}{l}63.7 \\
64.2 \\
63.0 \\
57.0 \\
49.5 \\
48.5 \\
50.8 \\
48.6 \\
51.4 \\
57.7 \\
71.1 \\
87.5 \\
60.5\end{array}$ \\
\hline 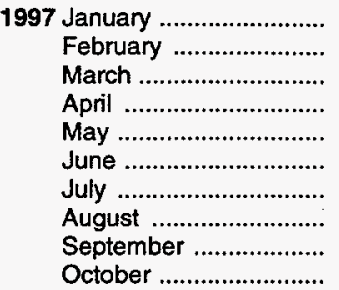 & $\begin{array}{l}86.6 \\
86.1 \\
84.3 \\
83.9 \\
84.5 \\
83.3 \\
81.5 \\
86.8 \\
87.2 \\
84.4\end{array}$ & $\begin{array}{l}113.7 \\
114.9 \\
113.8 \\
114.7 \\
115.7 \\
114.6 \\
112.5 \\
114.6 \\
115.6 \\
113.9\end{array}$ & $\begin{array}{l}74.4 \\
71.7 \\
61.9 \\
60.3 \\
58.8 \\
57.6 \\
56.7 \\
59.1 \\
58.2 \\
61.5\end{array}$ & $\begin{array}{l}88.7 \\
84.8 \\
N A \\
69.8 \\
68.4 \\
64.3 \\
63.1 \\
64.9 \\
63.4 \\
72.8\end{array}$ & $\begin{array}{r}75.5 \\
72.5 \\
66.4 \\
63.8 \\
62.9 \\
59.2 \\
57.3 \\
59.0 \\
\text { R } 58.4 \\
63.3\end{array}$ & $\begin{array}{r}73.0 \\
71.1 \\
65.8 \\
64.8 \\
63.8 \\
60.7 \\
59.4 \\
61.8 \\
60.7 \\
64.5\end{array}$ & $\begin{array}{l}86.6 \\
66.8 \\
57.3 \\
49.7 \\
46.5 \\
46.1 \\
47.5 \\
50.5 \\
48.4 \\
52.1\end{array}$ \\
\hline
\end{tabular}

a See Note 5 at end of section.

$R=$ Revised data. NA=Not available.

Notes: - Sales to end users are those made directly to ultimate consumers, including bulk consumers (such as agriculture, industry, and electric utilities) and residential and commercial consumers. Sales for resale are shown in Table 9.6; they are sales made to purchasers other than ultimate consumers. - Values for the current month are preliminary. - Prices prior to 1983 are Energy information Administration (EIA) estimates. See Note 6 at end of section. - Geographic coverage is the 50 States and the District of Columbia.

Source: EIA, Petroleum Marketing Monthly, January 1998, Table 2. 
Table 9.8a No. 2 Distillate Prices to Residences: Northeastern States (Cents per Gallon, Excluding Taxes)

\begin{tabular}{|c|c|c|c|c|c|c|c|c|c|}
\hline & Maine & $\begin{array}{c}\text { New } \\
\text { Hampshire }\end{array}$ & Vermont & Massachusetts & $\begin{array}{l}\text { Rhode } \\
\text { island }\end{array}$ & Connecticut & $\begin{array}{l}\text { New } \\
\text { York }\end{array}$ & $\begin{array}{c}\text { New } \\
\text { Jersey }\end{array}$ & Pennsylvania \\
\hline 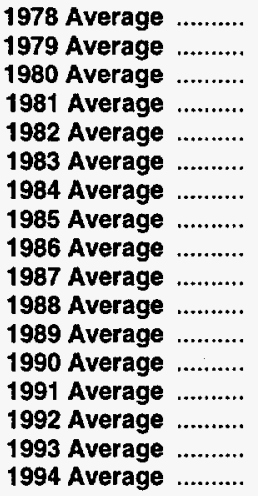 & $\begin{array}{r}48.6 \\
68.8 \\
96.3 \\
120.4 \\
115.5 \\
102.8 \\
103.9 \\
99.7 \\
74.4 \\
74.7 \\
77.7 \\
89.4 \\
98.9 \\
96.0 \\
87.1 \\
82.6 \\
81.8\end{array}$ & $\begin{array}{r}50.3 \\
72.5 \\
100.4 \\
123.7 \\
117.4 \\
104.1 \\
108.4 \\
102.4 \\
75.9 \\
76.5 \\
78.2 \\
89.3 \\
102.8 \\
91.6 \\
85.6 \\
82.8 \\
79.2\end{array}$ & $\begin{array}{r}50.8 \\
72.5 \\
101.5 \\
125.4 \\
120.1 \\
112.9 \\
111.9 \\
107.7 \\
86.6 \\
81.1 \\
82.6 \\
90.5 \\
107.0 \\
101.9 \\
92.1 \\
90.4 \\
87.6\end{array}$ & $\begin{array}{r}48.8 \\
70.9 \\
97.8 \\
121.3 \\
117.6 \\
109.1 \\
111.6 \\
107.0 \\
82.1 \\
80.6 \\
82.1 \\
92.6 \\
108.4 \\
103.0 \\
92.5 \\
89.7 \\
87.0\end{array}$ & $\begin{array}{r}50.7 \\
72.8 \\
101.1 \\
123.8 \\
120.1 \\
110.5 \\
111.4 \\
106.7 \\
82.8 \\
82.5 \\
83.6 \\
93.9 \\
108.6 \\
99.9 \\
91.2 \\
89.3 \\
88.5\end{array}$ & $\begin{array}{r}50.1 \\
72.0 \\
98.3 \\
121.7 \\
118.3 \\
109.1 \\
112.1 \\
108.0 \\
89.0 \\
83.4 \\
85.3 \\
92.9 \\
109.8 \\
106.2 \\
94.7 \\
91.9 \\
89.0\end{array}$ & $\begin{array}{r}50.1 \\
71.2 \\
98.2 \\
123.2 \\
120.5 \\
112.1 \\
115.5 \\
111.3 \\
91.1 \\
85.2 \\
86.3 \\
95.8 \\
112.5 \\
111.3 \\
102.8 \\
100.1 \\
96.6\end{array}$ & $\begin{array}{r}49.6 \\
71.0 \\
97.9 \\
121.5 \\
117.4 \\
107.9 \\
111.0 \\
105.9 \\
90.2 \\
84.3 \\
84.8 \\
91.8 \\
108.7 \\
104.0 \\
93.9 \\
92.4 \\
89.5\end{array}$ & $\begin{array}{r}48.8 \\
69.8 \\
96.4 \\
118.1 \\
113.7 \\
105.8 \\
107.9 \\
102.3 \\
81.4 \\
76.9 \\
77.8 \\
85.1 \\
102.6 \\
99.7 \\
89.0 \\
86.3 \\
85.7\end{array}$ \\
\hline 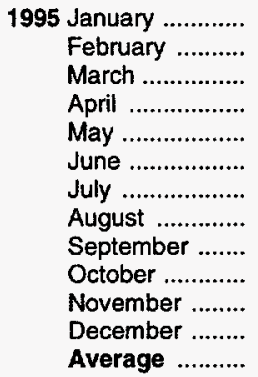 & $\begin{array}{l}77.8 \\
77.4 \\
76.3 \\
76.7 \\
78.7 \\
78.1 \\
76.9 \\
76.7 \\
76.2 \\
75.8 \\
79.1 \\
87.0 \\
78.7\end{array}$ & $\begin{array}{l}78.4 \\
78.5 \\
77.7 \\
76.6 \\
75.8 \\
74.5 \\
72.9 \\
73.0 \\
73.8 \\
73.9 \\
77.3 \\
83.8 \\
77.9\end{array}$ & $\begin{array}{l}85.7 \\
85.9 \\
85.6 \\
84.8 \\
84.5 \\
83.9 \\
81.7 \\
81.7 \\
82.5 \\
82.5 \\
84.5 \\
88.0 \\
85.3\end{array}$ & $\begin{array}{l}84.8 \\
84.9 \\
82.5 \\
81.9 \\
84.7 \\
82.5 \\
80.6 \\
80.9 \\
81.7 \\
82.3 \\
83.8 \\
88.9 \\
84.4\end{array}$ & $\begin{array}{l}87.3 \\
87.3 \\
87.0 \\
86.5 \\
86.1 \\
83.2 \\
81.7 \\
85.3 \\
84.9 \\
85.7 \\
87.4 \\
91.8 \\
87.4\end{array}$ & $\begin{array}{l}86.7 \\
87.8 \\
87.0 \\
85.2 \\
86.5 \\
84.2 \\
79.4 \\
77.4 \\
79.2 \\
83.1 \\
85.7 \\
90.5 \\
86.4\end{array}$ & $\begin{array}{l}95.2 \\
96.3 \\
95.9 \\
94.1 \\
95.9 \\
95.0 \\
92.3 \\
89.8 \\
90.5 \\
92.7 \\
94.3 \\
99.4 \\
95.5\end{array}$ & $\begin{array}{l}87.6 \\
89.0 \\
89.0 \\
87.1 \\
88.2 \\
87.7 \\
85.4 \\
82.2 \\
83.9 \\
85.2 \\
88.1 \\
94.3 \\
88.8\end{array}$ & $\begin{array}{l}83.1 \\
83.4 \\
82.3 \\
80.7 \\
81.1 \\
79.5 \\
75.8 \\
75.6 \\
77.2 \\
79.6 \\
81.9 \\
87.1 \\
\mathbf{8 2 . 6}\end{array}$ \\
\hline 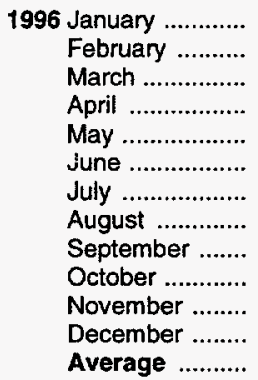 & $\begin{array}{r}93.0 \\
93.2 \\
96.7 \\
98.7 \\
95.4 \\
90.1 \\
87.5 \\
89.5 \\
96.4 \\
101.1 \\
103.4 \\
105.1 \\
97.2\end{array}$ & $\begin{array}{r}89.1 \\
90.8 \\
93.8 \\
96.5 \\
93.6 \\
87.2 \\
83.6 \\
85.1 \\
91.9 \\
99.1 \\
99.7 \\
101.6 \\
94.0\end{array}$ & $\begin{array}{r}92.6 \\
93.7 \\
97.3 \\
100.3 \\
98.8 \\
92.2 \\
88.5 \\
89.0 \\
94.4 \\
100.7 \\
101.9 \\
103.6 \\
96.9\end{array}$ & $\begin{array}{r}92.0 \\
93.8 \\
99.3 \\
101.5 \\
95.9 \\
87.9 \\
87.5 \\
89.0 \\
93.1 \\
103.0 \\
103.7 \\
105.9 \\
97.6\end{array}$ & $\begin{array}{r}94.9 \\
95.6 \\
99.7 \\
98.8 \\
94.9 \\
88.7 \\
87.7 \\
88.3 \\
96.6 \\
104.0 \\
104.5 \\
106.4 \\
98.6\end{array}$ & $\begin{array}{r}94.5 \\
96.2 \\
99.6 \\
102.1 \\
96.8 \\
88.8 \\
84.9 \\
84.0 \\
92.5 \\
103.0 \\
105.0 \\
108.1 \\
98.6\end{array}$ & $\begin{array}{r}102.9 \\
104.1 \\
106.6 \\
109.0 \\
105.2 \\
101.4 \\
97.2 \\
93.4 \\
99.1 \\
107.9 \\
111.6 \\
114.4 \\
106.3\end{array}$ & $\begin{array}{r}97.8 \\
100.5 \\
103.5 \\
105.4 \\
98.2 \\
91.8 \\
89.7 \\
90.6 \\
97.3 \\
105.7 \\
108.8 \\
111.1 \\
102.4\end{array}$ & $\begin{array}{r}92.3 \\
93.1 \\
95.9 \\
97.1 \\
92.9 \\
83.9 \\
79.4 \\
82.0 \\
88.9 \\
99.4 \\
102.2 \\
104.0 \\
95.3\end{array}$ \\
\hline $\begin{array}{l}1997 \text { January ............ } \\
\text { February .......... } \\
\text { March ................. } \\
\text { April ................. } \\
\text { May ................. } \\
\text { June ................. } \\
\text { July ................. } \\
\text { August ............. } \\
\text { September ........ } \\
\text { October ............. }\end{array}$ & $\begin{array}{r}105.2 \\
102.2 \\
94.3 \\
90.9 \\
90.6 \\
88.0 \\
86.7 \\
85.7 \\
\text { R } 87.1 \\
90.2\end{array}$ & $\begin{array}{r}102.2 \\
101.0 \\
98.6 \\
95.2 \\
91.9 \\
89.1 \\
85.6 \\
85.3 \\
R 86.3 \\
88.2\end{array}$ & $\begin{array}{r}104.4 \\
103.5 \\
103.1 \\
100.4 \\
97.7 \\
92.9 \\
91.1 \\
92.7 \\
91.7 \\
93.1\end{array}$ & $\begin{array}{r}106.4 \\
103.4 \\
97.7 \\
95.9 \\
93.0 \\
89.1 \\
87.5 \\
84.7 \\
\mathrm{R} 87.0 \\
89.4\end{array}$ & $\begin{array}{r}106.9 \\
104.5 \\
100.6 \\
99.6 \\
97.3 \\
94.1 \\
91.8 \\
91.0 \\
91.2 \\
94.3\end{array}$ & $\begin{array}{r}108.7 \\
105.2 \\
99.3 \\
97.6 \\
93.4 \\
89.9 \\
83.7 \\
84.5 \\
85.5 \\
89.1\end{array}$ & $\begin{array}{r}114.7 \\
112.0 \\
111.5 \\
109.7 \\
107.9 \\
103.9 \\
100.0 \\
92.9 \\
94.5 \\
100.7\end{array}$ & $\begin{array}{r}111.3 \\
108.4 \\
104.6 \\
102.5 \\
99.9 \\
96.9 \\
90.5 \\
89.6 \\
R 90.7 \\
95.0\end{array}$ & $\begin{array}{r}104.2 \\
102.2 \\
97.7 \\
95.0 \\
92.4 \\
87.8 \\
82.1 \\
80.7 \\
82.8 \\
85.8\end{array}$ \\
\hline
\end{tabular}

$R=$ Revised data.

Notes: - States are grouped in Tables $9.8 \mathrm{a}, 9.8 \mathrm{~b}$, and $9.8 \mathrm{c}$ by geographic region of the country. - Values for the current month are preliminary.
- Prices prior to 1983 are Energy Information Administration (EIA) estimates. See Note 6 at end of section.

Source: EIA, Petroleum Marketing Monthly, January 1998, Table 18. 
Table 9.8b No. 2 Distillate Prices to Residences: Selected South Atlantic and Midwestern States

(Cents per Gallon, Excluding Taxes)

\begin{tabular}{|c|c|c|c|c|c|c|c|c|c|c|c|}
\hline & Delaware & $\begin{array}{c}\text { District } \\
\text { of } \\
\text { Columbia }\end{array}$ & Maryland & Virginia & $\begin{array}{c}\text { West } \\
\text { Virginia }\end{array}$ & Ohio & Michigan & Indiana & Illinois & Wisconsin & Minnesota \\
\hline 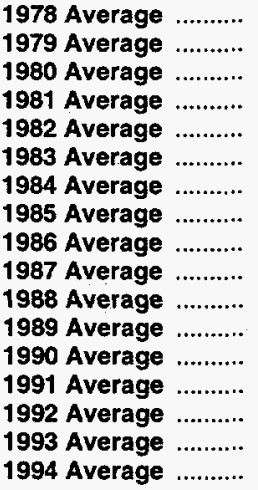 & $\begin{array}{r}47.8 \\
68.2 \\
95.4 \\
117.3 \\
111.3 \\
106.0 \\
109.6 \\
104.6 \\
85.0 \\
79.3 \\
80.1 \\
88.2 \\
105.8 \\
99.7 \\
92.3 \\
89.9 \\
89.4\end{array}$ & $\begin{array}{r}50.7 \\
74.2 \\
102.6 \\
127.4 \\
124.5 \\
117.0 \\
118.7 \\
114.3 \\
93.1 \\
91.8 \\
91.6 \\
98.6 \\
107.8 \\
112.2 \\
105.7 \\
104.5 \\
100.0\end{array}$ & $\begin{array}{r}49.2 \\
70.1 \\
97.9 \\
121.4 \\
117.1 \\
110.3 \\
113.5 \\
108.8 \\
91.4 \\
86.6 \\
87.0 \\
93.8 \\
111.9 \\
108.4 \\
100.0 \\
98.1 \\
95.0\end{array}$ & $\begin{array}{r}49.1 \\
70.4 \\
98.5 \\
120.5 \\
117.7 \\
108.7 \\
110.5 \\
106.3 \\
86.6 \\
79.5 \\
80.5 \\
87.0 \\
110.6 \\
101.1 \\
92.8 \\
89.3 \\
85.3\end{array}$ & $\begin{array}{r}46.2 \\
65.1 \\
92.2 \\
115.0 \\
109.3 \\
101.0 \\
102.1 \\
98.0 \\
74.6 \\
76.4 \\
74.2 \\
83.0 \\
99.1 \\
93.4 \\
86.4 \\
85.6 \\
80.9\end{array}$ & $\begin{array}{r}47.4 \\
68.6 \\
91.9 \\
113.2 \\
110.2 \\
101.3 \\
102.1 \\
99.7 \\
77.7 \\
74.7 \\
74.7 \\
81.6 \\
98.1 \\
91.0 \\
83.6 \\
84.0 \\
81.2\end{array}$ & $\begin{array}{r}47.9 \\
70.9 \\
97.8 \\
118.3 \\
113.9 \\
106.4 \\
105.0 \\
102.1 \\
81.0 \\
77.5 \\
77.5 \\
85.3 \\
100.9 \\
94.2 \\
87.2 \\
87.2 \\
86.3\end{array}$ & $\begin{array}{r}48.5 \\
72.7 \\
99.6 \\
118.5 \\
114.3 \\
100.7 \\
103.1 \\
99.1 \\
74.8 \\
75.4 \\
75.4 \\
83.2 \\
99.3 \\
91.8 \\
81.2 \\
81.0 \\
81.2\end{array}$ & $\begin{array}{r}46.5 \\
68.8 \\
95.8 \\
114.9 \\
110.9 \\
100.4 \\
100.1 \\
97.5 \\
\text { NA } \\
79.8 \\
77.6 \\
80.9 \\
96.1 \\
92.7 \\
87.7 \\
84.4 \\
78.4\end{array}$ & $\begin{array}{r}44.7 \\
67.3 \\
91.5 \\
109.1 \\
107.8 \\
101.2 \\
101.0 \\
98.3 \\
75.6 \\
75.1 \\
73.9 \\
81.1 \\
94.2 \\
89.5 \\
81.6 \\
82.3 \\
81.1\end{array}$ & $\begin{array}{r}47.8 \\
72.4 \\
99.9 \\
118.4 \\
115.1 \\
103.1 \\
104.1 \\
101.9 \\
79.2 \\
74.6 \\
73.5 \\
82.4 \\
101.4 \\
91.1 \\
82.6 \\
83.2 \\
80.6\end{array}$ \\
\hline 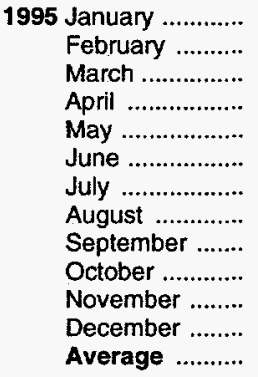 & $\begin{array}{l}88.4 \\
88.5 \\
87.6 \\
87.0 \\
85.2 \\
83.0 \\
80.0 \\
82.1 \\
82.4 \\
84.0 \\
84.5 \\
89.5 \\
87.0\end{array}$ & $\begin{array}{c}102.4 \\
103.4 \\
103.3 \\
100.0 \\
93.2 \\
\text { NA } \\
85.1 \\
W \\
86.1 \\
\text { NA } \\
100.2 \\
103.8 \\
101.0\end{array}$ & $\begin{array}{l}94.3 \\
95.1 \\
94.2 \\
91.3 \\
89.6 \\
86.8 \\
83.3 \\
82.6 \\
85.5 \\
89.5 \\
93.2 \\
98.5 \\
93.6\end{array}$ & $\begin{array}{l}85.0 \\
84.6 \\
84.0 \\
84.0 \\
83.0 \\
82.3 \\
81.2 \\
80.9 \\
81.6 \\
82.5 \\
83.8 \\
88.2 \\
84.4\end{array}$ & $\begin{array}{l}83.1 \\
82.1 \\
81.4 \\
80.3 \\
76.5 \\
77.7 \\
75.8 \\
74.1 \\
76.1 \\
77.4 \\
81.4 \\
89.4 \\
81.5\end{array}$ & $\begin{array}{l}81.2 \\
81.0 \\
80.1 \\
81.9 \\
80.8 \\
78.0 \\
76.6 \\
72.7 \\
77.5 \\
79.1 \\
81.8 \\
84.0 \\
80.8\end{array}$ & $\begin{array}{l}86.1 \\
85.5 \\
85.7 \\
86.2 \\
86.1 \\
83.6 \\
82.0 \\
82.1 \\
84.5 \\
83.9 \\
86.9 \\
88.8 \\
86.0\end{array}$ & $\begin{array}{l}81.6 \\
80.1 \\
82.3 \\
82.3 \\
83.6 \\
83.5 \\
81.9 \\
79.4 \\
80.9 \\
81.8 \\
79.2 \\
83.6 \\
81.6\end{array}$ & $\begin{array}{l}82.1 \\
80.8 \\
76.7 \\
78.7 \\
81.6 \\
77.0 \\
76.6 \\
72.9 \\
75.6 \\
74.6 \\
79.0 \\
82.9 \\
78.5\end{array}$ & $\begin{array}{l}81.1 \\
80.4 \\
80.5 \\
81.1 \\
81.5 \\
81.3 \\
81.0 \\
78.5 \\
80.7 \\
80.5 \\
81.6 \\
82.9 \\
81.2\end{array}$ & $\begin{array}{l}80.1 \\
79.0 \\
80.4 \\
80.4 \\
80.5 \\
77.3 \\
76.6 \\
77.3 \\
79.5 \\
80.1 \\
80.5 \\
81.8 \\
80.1\end{array}$ \\
\hline $\begin{array}{l}1996 \text { January ............ } \\
\text { February .......... } \\
\text { March ............... } \\
\text { April ................ } \\
\text { May .................. } \\
\text { June ................. } \\
\text { July ................ } \\
\text { August ............ } \\
\text { September ....... } \\
\text { October ............ } \\
\text { November ........ } \\
\text { December ........ } \\
\text { Average .......... }\end{array}$ & $\begin{array}{r}94.7 \\
94.4 \\
96.1 \\
100.7 \\
98.0 \\
91.9 \\
91.0 \\
91.0 \\
95.3 \\
103.1 \\
105.9 \\
106.7 \\
98.4\end{array}$ & $\begin{array}{r}111.7 \\
112.9 \\
117.7 \\
115.9 \\
109.7 \\
102.5 \\
97.3 \\
99.2 \\
106.2 \\
120.9 \\
125.7 \\
129.2 \\
117.8\end{array}$ & $\begin{array}{r}103.9 \\
104.1 \\
106.4 \\
105.8 \\
104.4 \\
97.3 \\
93.7 \\
93.7 \\
99.3 \\
108.1 \\
111.8 \\
114.9 \\
106.3\end{array}$ & $\begin{array}{r}91.3 \\
92.8 \\
93.6 \\
95.4 \\
91.7 \\
88.2 \\
88.5 \\
89.1 \\
92.6 \\
98.6 \\
102.2 \\
104.3 \\
95.2\end{array}$ & $\begin{array}{r}90.7 \\
93.8 \\
95.8 \\
97.0 \\
91.4 \\
89.9 \\
88.6 \\
88.9 \\
94.9 \\
101.1 \\
104.6 \\
104.3 \\
96.0\end{array}$ & $\begin{array}{r}85.5 \\
87.7 \\
91.6 \\
95.3 \\
91.3 \\
86.8 \\
86.5 \\
82.2 \\
92.8 \\
98.2 \\
100.8 \\
101.5 \\
92.1\end{array}$ & $\begin{array}{r}89.6 \\
91.2 \\
97.0 \\
101.0 \\
99.6 \\
94.6 \\
92.2 \\
92.5 \\
98.6 \\
102.6 \\
106.4 \\
106.4 \\
97.7\end{array}$ & $\begin{array}{r}85.6 \\
86.4 \\
90.7 \\
93.5 \\
93.0 \\
86.2 \\
85.6 \\
87.4 \\
92.8 \\
96.6 \\
102.4 \\
100.8 \\
91.2\end{array}$ & $\begin{array}{l}84.4 \\
85.8 \\
88.7 \\
90.4 \\
89.9 \\
80.6 \\
78.9 \\
83.0 \\
87.1 \\
92.4 \\
96.8 \\
98.1 \\
89.3\end{array}$ & $\begin{array}{l}83.3 \\
83.9 \\
87.1 \\
91.5 \\
92.2 \\
88.4 \\
88.6 \\
87.8 \\
91.1 \\
95.6 \\
98.7 \\
98.9 \\
89.9\end{array}$ & $\begin{array}{r}82.7 \\
83.7 \\
86.7 \\
91.4 \\
92.0 \\
85.5 \\
84.3 \\
86.2 \\
91.8 \\
97.8 \\
102.4 \\
100.4 \\
90.9\end{array}$ \\
\hline $\begin{array}{l}1997 \text { January ............. } \\
\text { February ........... } \\
\text { March ............... } \\
\text { April ................ } \\
\text { May ................... } \\
\text { June ................ } \\
\text { July ................. } \\
\text { August ............ } \\
\text { September ....... } \\
\text { October ............. }\end{array}$ & $\begin{array}{r}106.5 \\
104.2 \\
99.4 \\
99.1 \\
95.0 \\
89.8 \\
87.3 \\
87.8 \\
\text { R } 87.8 \\
91.2\end{array}$ & $\begin{array}{r}130.9 \\
127.0 \\
122.1 \\
W \\
108.6 \\
99.9 \\
99.9 \\
W \\
96.6 \\
W\end{array}$ & $\begin{array}{r}117.0 \\
115.0 \\
108.1 \\
105.6 \\
101.9 \\
98.0 \\
96.1 \\
93.8 \\
94.5 \\
97.8\end{array}$ & $\begin{array}{r}105.5 \\
102.6 \\
100.4 \\
96.7 \\
89.9 \\
87.8 \\
85.9 \\
85.2 \\
85.2 \\
88.5\end{array}$ & $\begin{array}{r}103.8 \\
101.2 \\
98.1 \\
95.7 \\
92.9 \\
90.6 \\
87.4 \\
85.0 \\
87.5 \\
88.3\end{array}$ & $\begin{array}{r}100.7 \\
98.4 \\
92.6 \\
92.4 \\
90.1 \\
86.8 \\
83.1 \\
81.7 \\
84.3 \\
88.1\end{array}$ & $\begin{array}{c}105.6 \\
104.4 \\
\text { NA } \\
91.7 \\
90.7 \\
88.3 \\
84.9 \\
87.4 \\
88.3 \\
88.9\end{array}$ & $\begin{array}{c}100.9 \\
97.0 \\
94.6 \\
\text { NA } \\
88.4 \\
84.0 \\
79.7 \\
83.6 \\
80.2 \\
84.0\end{array}$ & $\begin{array}{r}98.8 \\
93.3 \\
90.2 \\
83.4 \\
79.9 \\
79.7 \\
78.4 \\
81.2 \\
\text { R } 77.4 \\
82.3\end{array}$ & $\begin{array}{r}98.3 \\
96.8 \\
96.7 \\
92.9 \\
93.4 \\
90.8 \\
86.7 \\
86.5 \\
\text { R } 88.0 \\
89.5\end{array}$ & $\begin{array}{r}99.2 \\
96.9 \\
91.7 \\
89.7 \\
89.1 \\
87.4 \\
84.8 \\
86.0 \\
R 84.9 \\
87.4\end{array}$ \\
\hline
\end{tabular}

$R=$ Revised data. NA=Not available. $W=$ Value withneld to avoid disclosure of individual company data.

Notes: - States are grouped in Tables $9.8 \mathrm{a}, 9.8 \mathrm{~b}$, and $9.8 \mathrm{c}$ by geographic region of the country. - Values for the current month are preliminary.
- Prices prior to 1983 are Energy Information Administration (EIA) estimates. See Note 6 at end of section.

Source: EIA, Petroleum Marketing Monthly, January 1998, Table 18. 
Table 9.8c No. 2 Distillate Prices to Residences: Selected Western States and U.S. Average

(Cents per Gallon, Excluding Taxes)

\begin{tabular}{|c|c|c|c|c|c|}
\hline & Idaho & Washington & Oregon & Alaska & $\begin{array}{c}\text { U.S. } \\
\text { Average }\end{array}$ \\
\hline $\begin{array}{l}1978 \text { Average } \\
1979 \text { Average } \\
1980 \text { Average } \\
1981 \text { Average }\end{array}$ & $\begin{array}{r}43.15 \\
62.1 \\
91.15 \\
110.4 \\
110.4 \\
101.1 \\
98.15 \\
97.12 \\
73.13 \\
68.13 \\
68.13 \\
77.13 \\
97.4 \\
95.1 \\
85.7 \\
86.2 \\
78.1\end{array}$ & $\begin{array}{r}48.6 \\
69.7 \\
100.8 \\
116.5 \\
117.6 \\
109.0 \\
102.6 \\
101.1 \\
77.5 \\
79.5 \\
78.5 \\
87.4 \\
102.9 \\
101.6 \\
94.0 \\
99.9 \\
95.0\end{array}$ & $\begin{array}{r}45.8 \\
68.0 \\
97.3 \\
111.4 \\
111.6 \\
103.6 \\
99.3 \\
97.1 \\
70.4 \\
72.5 \\
70.9 \\
80.2 \\
97.0 \\
93.3 \\
87.6 \\
91.8 \\
88.7\end{array}$ & $\begin{array}{r}53.2 \\
68.2 \\
97.8 \\
118.0 \\
117.4 \\
108.8 \\
106.9 \\
108.3 \\
94.9 \\
86.5 \\
86.9 \\
96.4 \\
110.1 \\
105.0 \\
94.1 \\
96.1 \\
86.5\end{array}$ & $\begin{array}{r}49.0 \\
70.4 \\
97.4 \\
119.4 \\
116.0 \\
107.8 \\
109.1 \\
105.3 \\
83.6 \\
80.3 \\
81.3 \\
90.0 \\
106.3 \\
101.9 \\
93.4 \\
91.1 \\
88.4\end{array}$ \\
\hline 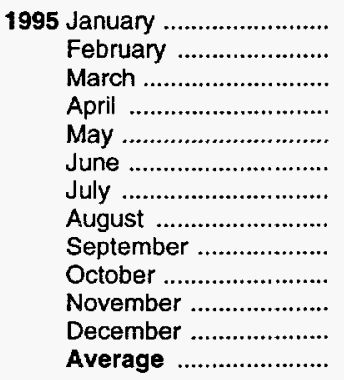 & $\begin{array}{l}80.4 \\
80.1 \\
80.6 \\
80.7 \\
82.7 \\
82.1 \\
82.5 \\
83.5 \\
86.4 \\
88.3 \\
88.15 \\
89.2 \\
83.9\end{array}$ & $\begin{array}{r}95.4 \\
94.5 \\
94.5 \\
96.7 \\
\text { NA } \\
95.2 \\
94.0 \\
91.2 \\
95.5 \\
97.8 \\
99.2 \\
100.7 \\
96.2\end{array}$ & $\begin{array}{l}88.4 \\
86.9 \\
88.7 \\
90.7 \\
91.6 \\
90.1 \\
\text { NA } \\
86.3 \\
87.1 \\
90.5 \\
92.2 \\
90.5 \\
89.4\end{array}$ & $\begin{array}{l}83.7 \\
84.0 \\
83.7 \\
82.6 \\
81.9 \\
82.7 \\
81.7 \\
81.9 \\
83.2 \\
83.4 \\
84.6 \\
84.2 \\
83.4\end{array}$ & $\begin{array}{l}86.9 \\
87.4 \\
86.6 \\
85.4 \\
86.4 \\
84.6 \\
82.0 \\
80.7 \\
82.3 \\
84.0 \\
86.3 \\
91.1 \\
86.7\end{array}$ \\
\hline 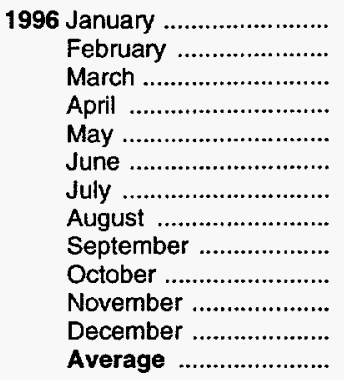 & $\begin{array}{r}87.2 \\
86.8 \\
86.5 \\
95.7 \\
97.1 \\
91.0 \\
92.3 \\
98.4 \\
101.3 \\
97.3 \\
98.1 \\
95.4 \\
93.3\end{array}$ & $\begin{array}{r}99.7 \\
99.6 \\
101.1 \\
109.7 \\
116.7 \\
112.8 \\
103.8 \\
99.8 \\
115.8 \\
116.4 \\
115.3 \\
114.9 \\
108.0\end{array}$ & $\begin{array}{r}90.1 \\
90.9 \\
90.0 \\
101.0 \\
108.6 \\
\text { NA } \\
96.4 \\
94.3 \\
109.1 \\
108.6 \\
107.5 \\
105.1 \\
98.9\end{array}$ & $\begin{array}{l}84.0 \\
83.3 \\
84.5 \\
90.0 \\
97.9 \\
96.2 \\
92.7 \\
92.3 \\
95.7 \\
96.7 \\
96.9 \\
96.4 \\
90.9\end{array}$ & $\begin{array}{r}94.6 \\
95.9 \\
99.1 \\
101.5 \\
97.8 \\
91.0 \\
87.9 \\
88.1 \\
94.5 \\
102.6 \\
105.4 \\
107.5 \\
98.9\end{array}$ \\
\hline 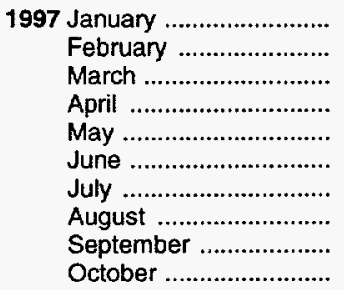 & $\begin{array}{r}94.9 \\
94.5 \\
100.6 \\
98.3 \\
98.4 \\
92.3 \\
90.3 \\
90.5 \\
\text { R } 91.2 \\
93.3\end{array}$ & $\begin{array}{r}117.6 \\
118.8 \\
116.6 \\
114.9 \\
109.1 \\
112.2 \\
108.3 \\
108.8 \\
R 110.9 \\
111.9\end{array}$ & $\begin{array}{r}105.8 \\
106.7 \\
107.5 \\
106.1 \\
104.6 \\
100.2 \\
96.9 \\
99.2 \\
101.5 \\
101.9\end{array}$ & $\begin{array}{r}97.1 \\
97.5 \\
98.7 \\
97.5 \\
96.4 \\
96.0 \\
97.5 \\
96.4 \\
\text { R } 96.6 \\
97.7\end{array}$ & $\begin{array}{r}107.9 \\
105.1 \\
101.6 \\
99.2 \\
96.3 \\
92.3 \\
88.3 \\
86.9 \\
\text { R } 88.5 \\
92.1\end{array}$ \\
\hline
\end{tabular}

$R=$ Revised data. NA=Not available.

Notes: - States are grouped in Tables $9.8 \mathrm{a}, 9.8 \mathrm{~b}$, and $9.8 \mathrm{c}$ by geographic region of the country. - Values for the current month are preliminary.
- Prices prior to 1983 are Energy Information Administration (EIA) estimates. See Note 6 at end of section.

Source: EIA, Petroleum Marketing Monthly, January 1998, Table 18. 
Figure 9.2 Retail Prices of Electricity Sold by Electric Utilities

(Cents per Kilowatthour)

By Sector, 1973-1996

By Sector, Monthly

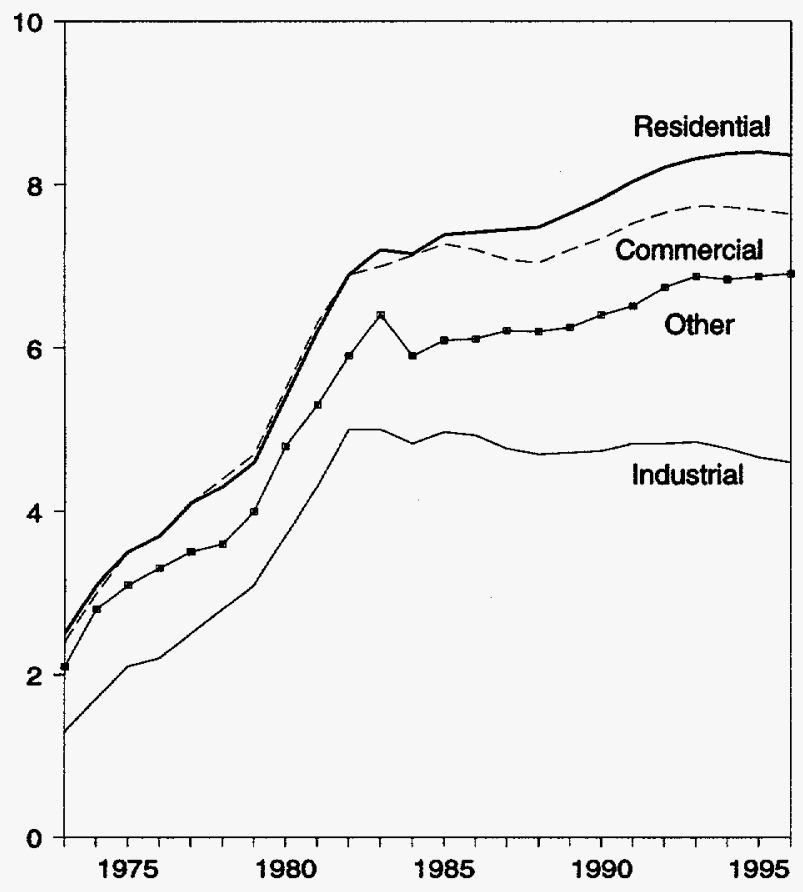

ource: Table 9.9, Monthly Series.

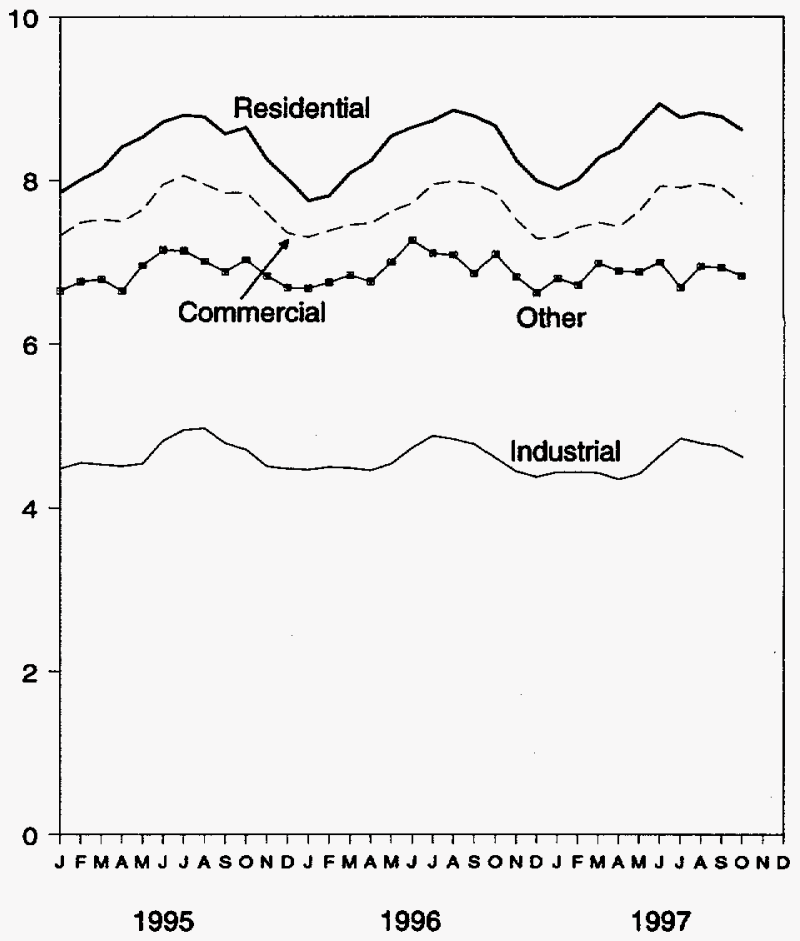

Figure 9.3 Cost of Fossil-Fuel Receipts at Steam-Electric Plants (Dollars per Million Btu)

Costs, 1973-1996

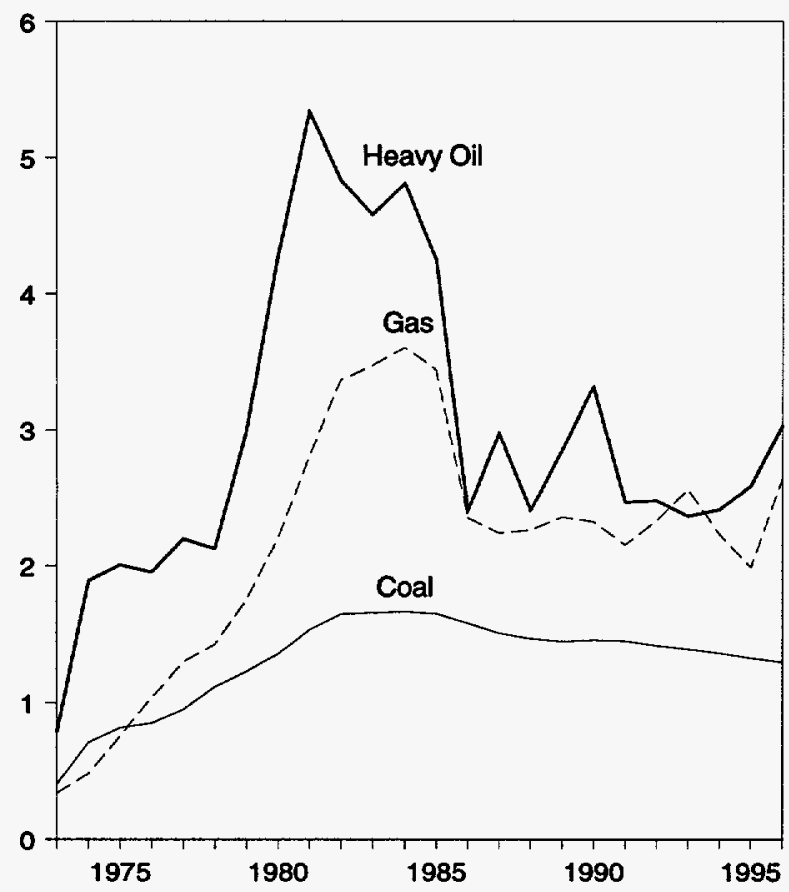

Costs, Monthly

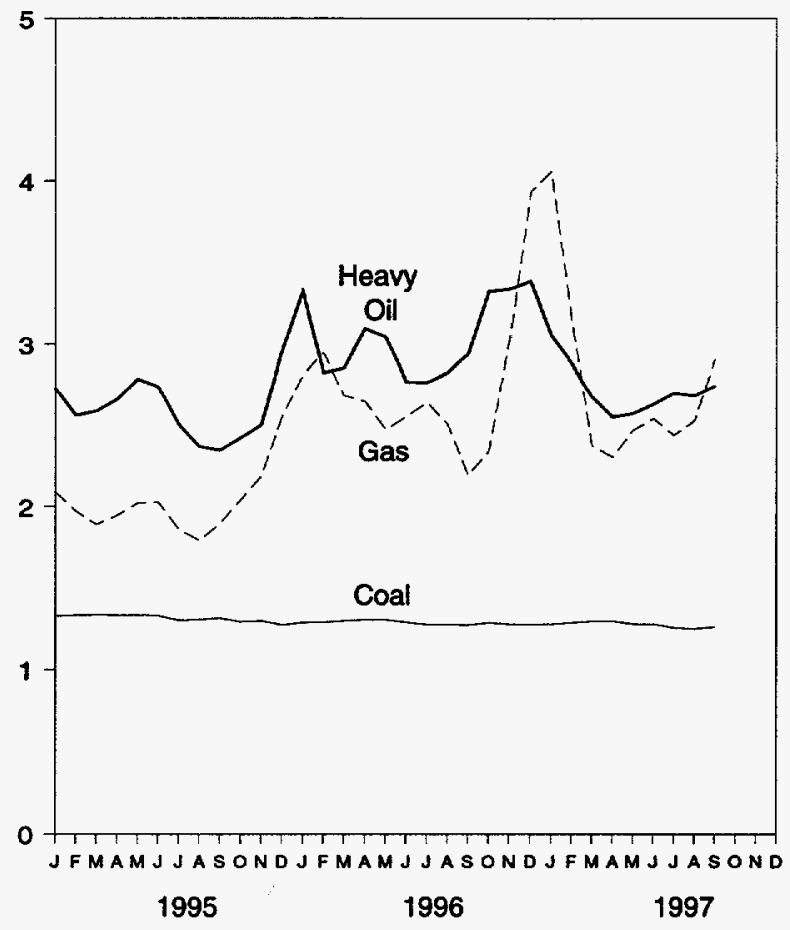

Source: Table 9.10. 
Table 9.9 Retail Prices of Electricity Sold by Electric Utilities

(Cents per Kilowatthour)

\begin{tabular}{|c|c|c|c|c|c|}
\hline - & Residential & Commercial & Industrial & Other & Total \\
\hline $\begin{array}{l}1973 \text { Average } \\
1974 \text { Average } \\
1975 \text { Average } \\
1976 \text { Average } \\
1977 \text { Average } \\
1978 \text { Average } \\
1979 \text { Average } \\
1980 \text { Average } \\
1981 \text { Average } \\
1982 \text { Average } \\
1983 \text { Average } \\
1984 \text { Average } \\
1985 \text { Average } \\
1986 \text { Average } \\
1987 \text { Average } \\
1988 \text { Average } \\
1989 \text { Average } \\
1990 \text { Average } \\
1991 \text { Average } \\
1992 \text { Average }\end{array}$ & $\begin{array}{l}2.5 \\
3.1 \\
3.5 \\
3.7 \\
4.1 \\
4.3 \\
4.6 \\
5.4 \\
6.2 \\
6.9 \\
7.2 \\
7.15 \\
7.39 \\
7.42 \\
7.45 \\
7.48 \\
7.65 \\
7.83 \\
8.04 \\
8.21 \\
8.32 \\
8.38\end{array}$ & $\begin{array}{l}2.4 \\
3.0 \\
3.5 \\
3.7 \\
4.1 \\
4.4 \\
4.7 \\
5.5 \\
6.3 \\
6.9 \\
7.0 \\
7.13 \\
7.27 \\
7.20 \\
7.08 \\
7.04 \\
7.20 \\
7.34 \\
7.53 \\
7.66 \\
7.74 \\
7.73\end{array}$ & $\begin{array}{l}1.3 \\
1.7 \\
2.1 \\
2.2 \\
2.5 \\
2.8 \\
3.1 \\
3.7 \\
4.3 \\
5.0 \\
5.0 \\
4.83 \\
4.97 \\
4.93 \\
4.77 \\
4.70 \\
4.72 \\
4.74 \\
4.83 \\
4.83 \\
4.85 \\
4.77\end{array}$ & $\begin{array}{l}2.1 \\
2.8 \\
3.1 \\
3.3 \\
3.5 \\
3.6 \\
4.0 \\
4.8 \\
5.3 \\
5.9 \\
6.4 \\
5.90 \\
6.09 \\
6.11 \\
6.21 \\
6.20 \\
6.25 \\
6.40 \\
6.51 \\
6.74 \\
6.88 \\
6.84\end{array}$ & $\begin{array}{l}2.0 \\
2.5 \\
2.9 \\
3.1 \\
3.4 \\
3.7 \\
4.0 \\
4.7 \\
5.5 \\
6.1 \\
6.3 \\
6.25 \\
6.44 \\
6.44 \\
6.37 \\
6.35 \\
6.45 \\
6.57 \\
6.75 \\
6.82 \\
6.93 \\
6.91\end{array}$ \\
\hline 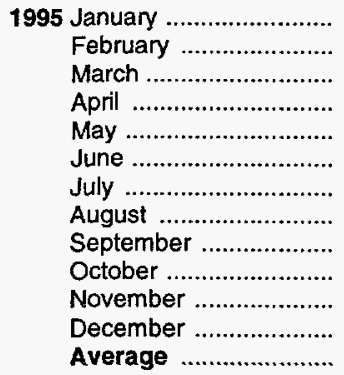 & $\begin{array}{l}7.85 \\
8.01 \\
8.14 \\
8.41 \\
8.53 \\
8.72 \\
8.80 \\
8.78 \\
8.57 \\
8.65 \\
8.26 \\
8.02 \\
8.40\end{array}$ & $\begin{array}{l}7.33 \\
7.49 \\
7.53 \\
7.50 \\
7.64 \\
7.95 \\
8.06 \\
7.95 \\
7.84 \\
7.85 \\
7.60 \\
7.36 \\
7.69\end{array}$ & $\begin{array}{l}4.48 \\
4.55 \\
4.53 \\
4.51 \\
4.54 \\
4.82 \\
4.95 \\
4.97 \\
4.79 \\
4.71 \\
4.51 \\
4.48 \\
4.66\end{array}$ & $\begin{array}{l}6.65 \\
6.76 \\
6.79 \\
6.65 \\
6.96 \\
7.15 \\
7.14 \\
7.01 \\
6.88 \\
7.03 \\
6.83 \\
6.69 \\
6.88\end{array}$ & $\begin{array}{l}6.60 \\
6.68 \\
6.66 \\
6.65 \\
6.74 \\
7.10 \\
7.35 \\
7.34 \\
7.08 \\
6.95 \\
6.70 \\
6.64 \\
6.89\end{array}$ \\
\hline 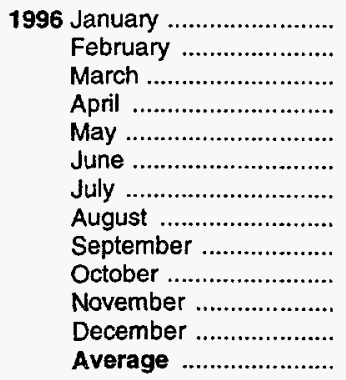 & $\begin{array}{l}\text { R } 7.75 \\
\text { R } 7.81 \\
R 8.09 \\
R 8.24 \\
R 8.54 \\
R 8.65 \\
R 8.73 \\
R 8.86 \\
R 8.79 \\
R 8.67 \\
R 8.25 \\
R 7.99 \\
R 8.36\end{array}$ & $\begin{array}{r}R 7.31 \\
R 7.39 \\
R 7.46 \\
7.48 \\
R 7.62 \\
R 7.72 \\
R 7.95 \\
R 7.99 \\
R 7.96 \\
R 7.84 \\
R 7.52 \\
R 7.29 \\
R 7.64\end{array}$ & $\begin{array}{r}4.47 \\
4.50 \\
4.49 \\
4.46 \\
\text { R } \\
4.54 \\
4.73 \\
4.88 \\
4.84 \\
4.78 \\
4.61 \\
4.45 \\
4.38 \\
4.60\end{array}$ & $\begin{array}{l}R_{6.68} \\
R_{6.75} \\
R_{6.84} \\
R_{6.76} \\
R_{7.00} \\
R_{7.27} \\
R_{7.11} \\
R_{7.09} \\
R_{6.86} \\
R_{7.10} \\
R_{6.82} \\
R_{6.63} \\
R_{6.91}\end{array}$ & $\begin{array}{l}{ }^{R} 6.61 \\
R_{6.60} \\
\text { R }_{6.65} \\
\text { R }_{6.63} \\
\text { R }_{6.77} \\
\text { R }_{7.03} \\
\text { R }_{7.27} \\
\text { R }_{7.30} \\
\text { R }_{7.16} \\
\text { R }_{6.91} \\
\text { R }_{6.65} \\
\text { R }_{6.58} \\
\text { R }_{6.86}\end{array}$ \\
\hline 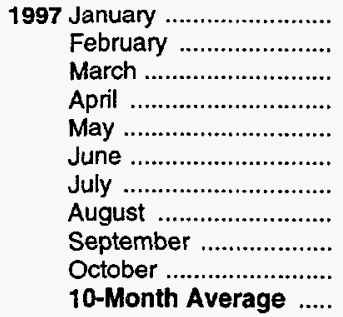 & $\begin{array}{l}7.89 \\
8.01 \\
8.28 \\
8.40 \\
8.68 \\
8.94 \\
8.77 \\
8.83 \\
8.78 \\
8.62 \\
8.52\end{array}$ & $\begin{array}{l}7.31 \\
7.43 \\
7.49 \\
7.44 \\
7.63 \\
7.93 \\
7.91 \\
7.96 \\
7.91 \\
7.71 \\
7.69\end{array}$ & $\begin{array}{l}4.44 \\
4.44 \\
4.43 \\
4.35 \\
4.42 \\
4.64 \\
4.85 \\
4.79 \\
4.75 \\
4.62 \\
4.58\end{array}$ & $\begin{array}{l}6.80 \\
6.72 \\
6.99 \\
6.89 \\
6.88 \\
7.00 \\
6.69 \\
6.95 \\
6.93 \\
6.83 \\
6.87\end{array}$ & $\begin{array}{l}6.64 \\
6.64 \\
6.69 \\
6.61 \\
6.74 \\
7.10 \\
7.27 \\
7.26 \\
7.14 \\
6.91 \\
6.92\end{array}$ \\
\hline $\begin{array}{l}1996 \text { 10-Month Average ..... } \\
1995 \text { 10-Month Average } . . . .\end{array}$ & $\begin{array}{l}8.50 \\
8.46\end{array}$ & $\begin{array}{l}7.73 \\
7.73\end{array}$ & $\begin{array}{l}4.65 \\
4.69\end{array}$ & $\begin{array}{l}6.98 \\
6.91\end{array}$ & $\begin{array}{l}6.94 \\
6.94\end{array}$ \\
\hline
\end{tabular}

$\mathrm{R}=$ Revised data.

Notes: - Prices are calculated by divicling revenue by sales. Revenue may not correspond to sales for a particular month because of electric utility billing and accounting procedures. That lack of correspondence could result in uncharacteristic increases or decreases in the monthly prices. See Note at end of section. - Geographic coverage is the 50 States and the District of Columbia.

Sources: See end of section. 
Table 9.10 Quantity and Cost of Fossil-Fuel Receipts at Steam-Electric Utility Plants

\begin{tabular}{|c|c|c|c|c|c|c|c|c|c|}
\hline & \multicolumn{2}{|c|}{ Coal } & \multicolumn{4}{|c|}{ Petroleum } & \multicolumn{2}{|c|}{ Gas $^{a}$} & \multirow{2}{*}{$\begin{array}{c}\begin{array}{c}\text { All Fossil } \\
\text { Fuels }\end{array} \\
\begin{array}{c}\text { Cost } \\
\text { (cents per } \\
\text { million Btu) }\end{array}\end{array}$} \\
\hline & $\begin{array}{c}\text { Quantity } \\
\text { (thousand } \\
\text { short tons) }\end{array}$ & $\begin{array}{c}\text { Cost } \\
\text { (cents per } \\
\text { million Btu) }\end{array}$ & \multicolumn{2}{|c|}{ Heavy Oilb } & \multicolumn{2}{|c|}{ Total $b, c$} & $\begin{array}{l}\text { Quantity } \\
\text { (million } \\
\text { cubic feet) }\end{array}$ & $\begin{array}{c}\text { Cost } \\
\text { (cents per } \\
\text { million Btu) }\end{array}$ & \\
\hline $\begin{array}{l}1973 \text { Year } \\
1974 \text { Year } \\
1975 \text { Year } \\
1976 \text { Year } \\
1977 \text { Year } \\
1978 \text { Year } \\
1979 \text { Year } \\
1980 \text { Year } \\
1981 \text { Year } \\
1982 \text { Year } \\
1983 \text { Year } \\
1984 \text { Year } \\
1985 \text { Year } \\
1986 \text { Year } \\
1987 \text { Year } \\
1988 \text { Year } \\
1989 \text { Year } \\
1990 \text { Year } \\
1991 \text { Year } \\
1992 \text { Year } \\
1993 \text { Year } \\
1994 \text { Year }\end{array}$ & $\begin{array}{l}374,842 \\
384,868 \\
431,527 \\
454,858 \\
490,415 \\
476,169 \\
556,558 \\
593,995 \\
579,374 \\
601,427 \\
592,728 \\
684,111 \\
666,743 \\
686,964 \\
721,298 \\
727,775 \\
753,217 \\
786,627 \\
769,923 \\
775,963 \\
769,152 \\
831,929\end{array}$ & $\begin{array}{r}40.5 \\
70.9 \\
81.4 \\
84.8 \\
94.7 \\
111.6 \\
122.4 \\
135.1 \\
153.2 \\
164.7 \\
165.6 \\
166.4 \\
164.8 \\
157.9 \\
150.6 \\
146.6 \\
144.5 \\
145.5 \\
144.7 \\
141.2 \\
138.5 \\
135.5\end{array}$ & $\begin{array}{l}512,650 \\
479,166 \\
457,582 \\
495,363 \\
563,685 \\
546,197 \\
479,705 \\
394,159 \\
327,477 \\
228,200 \\
211,705 \\
193,832 \\
156,410 \\
220,585 \\
187,300 \\
230,234 \\
237,668 \\
202,281 \\
163,106 \\
138,537 \\
141,719 \\
135,184\end{array}$ & $\begin{array}{r}78.5 \\
189.0 \\
200.5 \\
195.2 \\
219.8 \\
212.5 \\
298.8 \\
426.7 \\
533.4 \\
483.2 \\
457.8 \\
481.2 \\
424.4 \\
240.1 \\
297.6 \\
240.5 \\
284.6 \\
331.9 \\
246.5 \\
247.5 \\
236.2 \\
240.9\end{array}$ & $\begin{array}{l}535,859 \\
515,217 \\
510,352 \\
549,973 \\
635,556 \\
616,040 \\
515,695 \\
419,140 \\
345,544 \\
239,111 \\
219,652 \\
202,372 \\
164,947 \\
228,522 \\
194,578 \\
236,924 \\
246,422 \\
209,350 \\
169,625 \\
144,390 \\
147,902 \\
142,940\end{array}$ & $\begin{array}{r}80.0 \\
191.0 \\
202.3 \\
199.0 \\
224.9 \\
219.1 \\
307.2 \\
435.1 \\
542.5 \\
492.2 \\
462.8 \\
486.3 \\
431.7 \\
243.7 \\
301.1 \\
243.9 \\
289.3 \\
338.4 \\
254.8 \\
255.1 \\
243.3 \\
248.8\end{array}$ & $\begin{array}{l}3,382,677 \\
3,225,203 \\
3,034,808 \\
2,962,811 \\
3,106,403 \\
3,140,654 \\
3,368,976 \\
3,588,814 \\
3,573,558 \\
3,161,348 \\
2,732,248 \\
2,878,808 \\
2,808,921 \\
2,387,622 \\
2,605,191 \\
2,362,721 \\
2,472,506 \\
2,490,979 \\
2,630,818 \\
2,637,678 \\
2,574,523 \\
2,863,904\end{array}$ & $\begin{array}{r}33.8 \\
48.2 \\
75.2 \\
103.4 \\
129.1 \\
142.2 \\
174.9 \\
219.9 \\
280.5 \\
337.6 \\
347.4 \\
360.3 \\
344.4 \\
235.1 \\
224.0 \\
226.3 \\
235.5 \\
232.1 \\
215.3 \\
232.8 \\
256.0 \\
223.0\end{array}$ & $\begin{array}{r}47.6 \\
91.4 \\
104.4 \\
111.9 \\
129.7 \\
141.1 \\
163.9 \\
192.8 \\
225.6 \\
224.9 \\
220.6 \\
219.1 \\
209.4 \\
175.0 \\
170.6 \\
164.3 \\
167.5 \\
168.9 \\
160.3 \\
159.0 \\
159.5 \\
152.6\end{array}$ \\
\hline 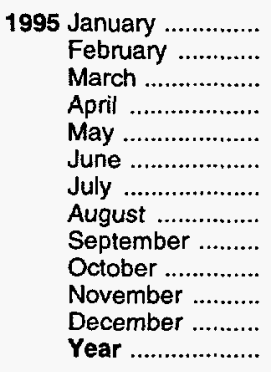 & $\begin{array}{r}70,206 \\
65,789 \\
69,059 \\
66,167 \\
68,564 \\
64,543 \\
67,734 \\
73,242 \\
70,938 \\
70,140 \\
70,196 \\
70,281 \\
\mathbf{8 2 6 , 8 6 0}\end{array}$ & $\begin{array}{l}133.1 \\
133.5 \\
133.8 \\
133.7 \\
133.7 \\
133.3 \\
130.4 \\
130.9 \\
131.8 \\
129.6 \\
130.2 \\
127.7 \\
131.8\end{array}$ & \begin{tabular}{r|}
5,565 \\
6,150 \\
5,040 \\
2,849 \\
5,864 \\
8,476 \\
8,367 \\
9,284 \\
9,036 \\
5,553 \\
4,773 \\
7,259 \\
78,216
\end{tabular} & $\begin{array}{l}273.1 \\
256.2 \\
258.9 \\
266.2 \\
279.0 \\
274.3 \\
250.8 \\
237.0 \\
234.7 \\
242.5 \\
250.5 \\
295.8 \\
258.6\end{array}$ & $\begin{array}{r}6,113 \\
6,535 \\
5,448 \\
3,221 \\
6,213 \\
9,083 \\
8,838 \\
10,029 \\
9,432 \\
6,060 \\
5,414 \\
7,905 \\
\mathbf{8 4 , 2 9 2}\end{array}$ & $\begin{array}{l}282.7 \\
263.1 \\
267.4 \\
280.3 \\
285.8 \\
282.0 \\
257.2 \\
247.7 \\
241.3 \\
253.8 \\
268.8 \\
305.7 \\
267.9\end{array}$ & $\begin{array}{r}188,545 \\
163,665 \\
233,533 \\
222,256 \\
245,676 \\
281,987 \\
376,158 \\
424,284 \\
302,928 \\
228,644 \\
189,641 \\
166,010 \\
3,023,327\end{array}$ & $\begin{array}{l}209.2 \\
197.1 \\
189.0 \\
194.5 \\
202.1 \\
202.8 \\
186.1 \\
179.4 \\
189.5 \\
204.1 \\
218.9 \\
255.3 \\
198.4\end{array}$ & $\begin{array}{l}145.4 \\
143.7 \\
144.3 \\
144.1 \\
147.3 \\
150.4 \\
146.1 \\
145.1 \\
145.1 \\
142.6 \\
143.3 \\
146.1 \\
145.3\end{array}$ \\
\hline 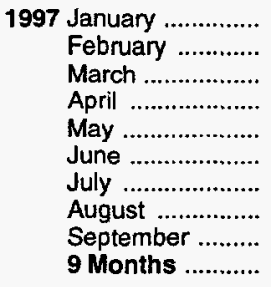 & $\begin{array}{r}71,900 \\
69,089 \\
72,678 \\
69,695 \\
74,909 \\
70,623 \\
74,065 \\
76,342 \\
75,054 \\
654,354\end{array}$ & $\begin{array}{l}128.0 \\
129.0 \\
129.8 \\
129.8 \\
128.0 \\
128.0 \\
125.8 \\
125.2 \\
126.3 \\
127.8\end{array}$ & $\begin{array}{r}8,811 \\
8,958 \\
6,796 \\
6,379 \\
6,476 \\
9,253 \\
10,800 \\
10,994 \\
8,820 \\
77,286\end{array}$ & $\begin{array}{l}305.7 \\
287.5 \\
267.2 \\
254.9 \\
257.1 \\
262.9 \\
269.8 \\
268.2 \\
274.5 \\
272.9\end{array}$ & $\begin{array}{r}9,652 \\
9,346 \\
7,164 \\
6,730 \\
6,967 \\
10,039 \\
11,670 \\
11,563 \\
9,274 \\
82,405\end{array}$ & $\begin{array}{l}321.0 \\
295.3 \\
276.3 \\
264.8 \\
270.5 \\
274.4 \\
280.4 \\
275.4 \\
281.2 \\
283.0\end{array}$ & $\begin{array}{r}133,193 \\
134,946 \\
185,304 \\
184,936 \\
225,899 \\
278,021 \\
373,638 \\
359,977 \\
313,129 \\
2,189,043\end{array}$ & $\begin{array}{l}405.8 \\
315.5 \\
237.1 \\
230.2 \\
246.9 \\
254.0 \\
243.9 \\
252.7 \\
290.5 \\
266.0\end{array}$ & $\begin{array}{l}157.5 \\
150.9 \\
145.4 \\
144.5 \\
146.6 \\
153.2 \\
154.6 \\
154.1 \\
158.3 \\
151.8\end{array}$ \\
\hline $\begin{array}{l}19969 \text { Months ............ } \\
19959 \text { Months ........... }\end{array}$ & $\begin{array}{l}643,045 \\
616,243\end{array}$ & $\begin{array}{l}129.1 \\
132.7\end{array}$ & $\begin{array}{l}78,771 \\
60,630\end{array}$ & $\begin{array}{l}295.4 \\
256.3\end{array}$ & $\begin{array}{l}84,102 \\
64,913\end{array}$ & $\begin{array}{l}305.2 \\
264.6\end{array}$ & $\begin{array}{l}2,096,419 \\
2,439,032\end{array}$ & $\begin{array}{l}256.4 \\
192.5\end{array}$ & $\begin{array}{l}152.2 \\
145.7\end{array}$ \\
\hline
\end{tabular}

a Includes supplemental gaseous fuels.

b Heavy oil includes fuel oil nos. 4,5 , and 6 , and topped crude oil. The weighted averages for petroleum and all fossil fuels include both heavy and light oil (fuel oil nos. 1 and 2, kerosene, and jet fuel) prices. Data do not include petroleum coke.
C Data for 1973-1982 do not include small quantities of rerefined motor oil, bunker oil, and liquefied petroleum gas.

Notes: - See Note 8 at end of section. - Geographic coverage is the 50 States and the District of Columbia.

Sources: See end of section. 
Figure 9.4 Natural Gas Prices

(Dollars per Thousand Cubic Feet)

Selected Prices, 1973-1996

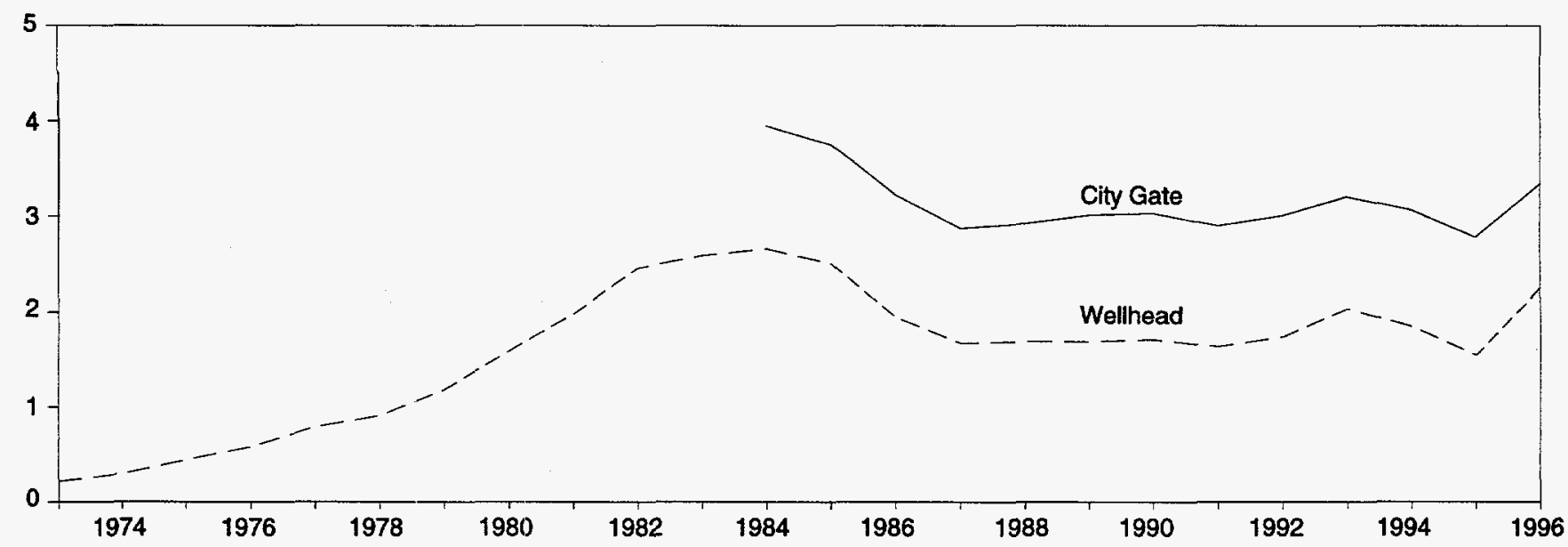

Delivered to Consumers, 1973-1996

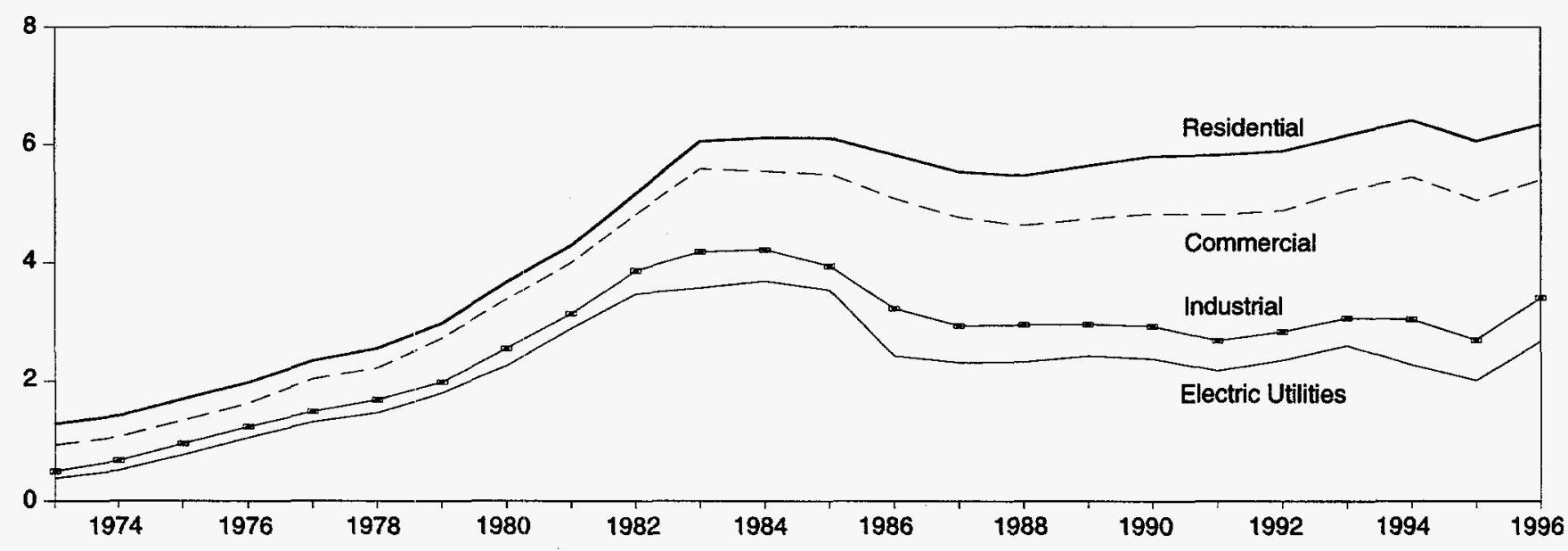

Delivered to Consumers, Mlonthly

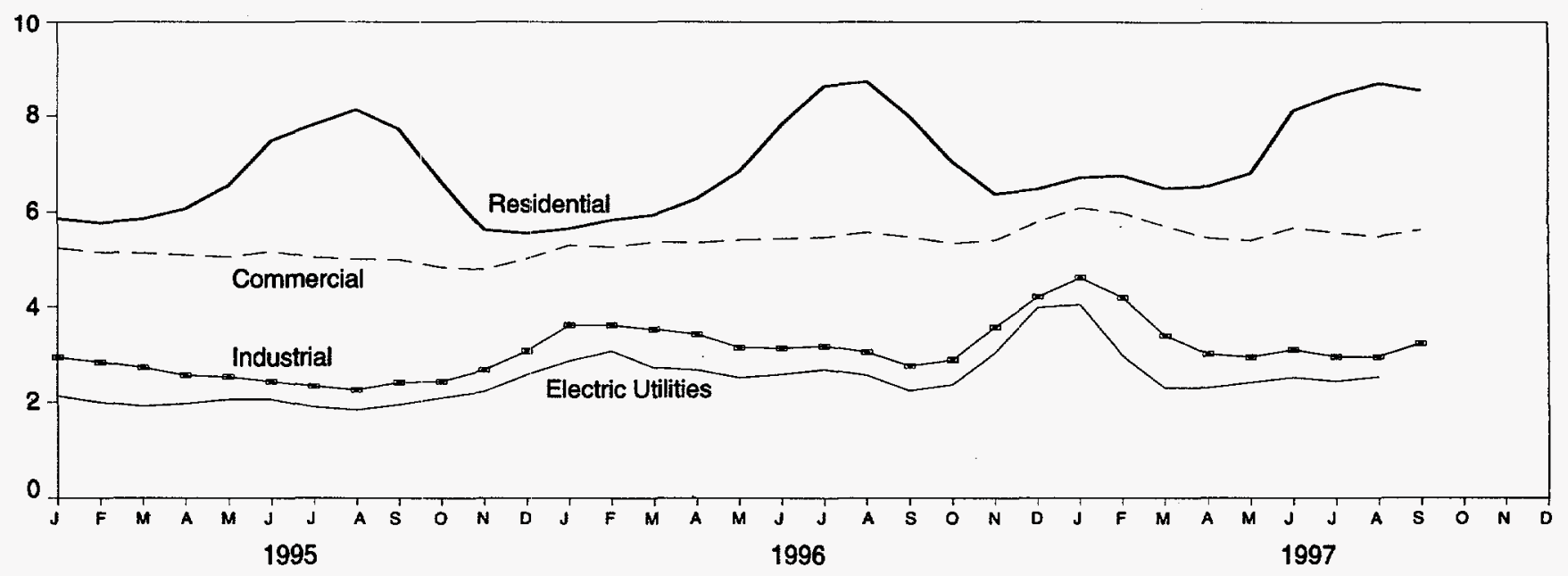

Note: Because vertical scales differ, graphs should not be compared. Source: Table 9.11. 
Table 9.11 Natural Gas Prices

(Prices: Dollars per Thousand Cubic Feet; Share of Volume Delivered: Percentage)

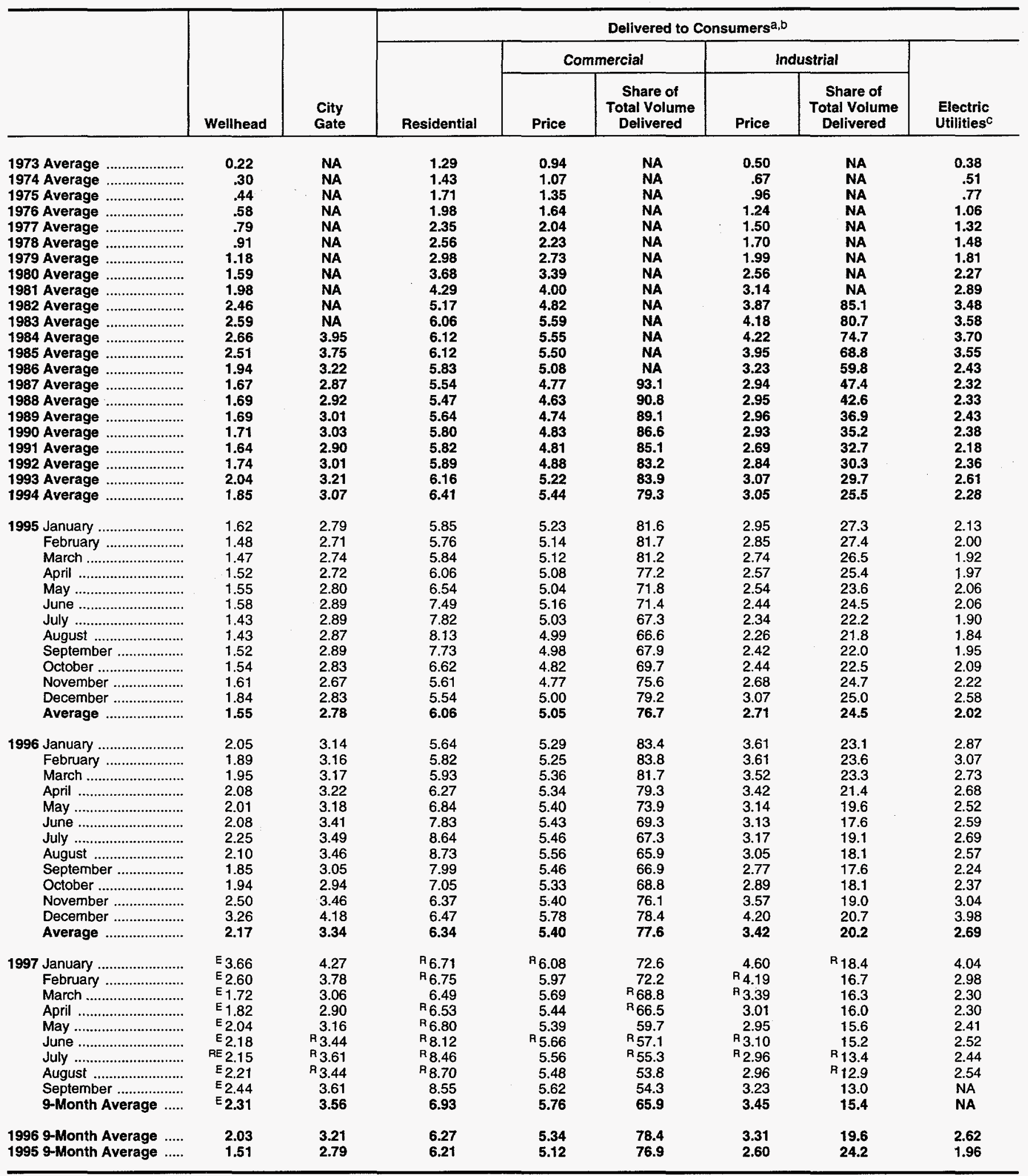

a Includes supplemental gaseous fuels.

Note 9 at end of section. Wellhead annual and year-to-date prices are

See Note 9 at end of section.

c See Note 8 at end of section.

$R=$ Revised data. $N A=$ Not available. E=Estimate.

Notes: - Prices shown on this page are intended to include all taxes. See simple averages of the monthly prices; all other annual and year-to-date prices are volume-weighted averages of the monthly prices. - Geographic coverage is the 50 States and the District of Columbia.

Sources: See end of section. 


\section{Energy Prices Notes}

1. The average domestic first purchase price represents the average price at which all domestic crude oil is purchased. Prior to February 1976, the price represented an estimate of the average of posted prices; beginning with February 1976, the price represents an average of actual first purchase prices. The data series was previously called "Actual Domestic Wellhead Price."

2. F.O.B. literally means "Free on Board." It denotes a transaction whereby the seller makes the product available with an agreement on a given port at a given price; it is the responsibility of the buyer to arrange for the transportation and insurance.

3. The landed cost of imported crude oil from selected countries does not represent the total cost of all imported crude. Prior to April 1975, imported crude costs to U.S. company-owned refineries in the Caribbean were not included in the landed cost, and costs of crude oil from countries that export only small amounts to the United States were also excluded. Beginning in March 1975, however, coverage was expanded to include U.S. company-owned refineries in the Caribbean. Landed costs do not include supplemental fees.

4. Beginning with January 1981 , refiner acquisition costs of crude oil are from data collected on Energy Information Administration (EIA) Form EIA-14, "Refiners' Monthly Cost Report." Those costs were previously published from data collected on Economic Regulatory Administration (ERA) Form ERA-49, "Domestic Crude Oil Entitlements Program Refiners Monthly Report." Form ERA-49 was discontinued with the decontrol of crude oil on January 28, 1981. Crude oil purchases and costs are defined for Form EIA-14 in accordance with conventions used for Form ERA-49. The respondents for the two forms are also essentially the same. However, due to possible different interpretations of the filing requirements and a different method for handling prior period adjustments, care must be taken when comparing the data collected on the two forms.

The refiner acquisition cost of crude oil is the average price paid by refiners for crude oil booked into their refineries in accordance with accounting procedures generally accepted and consistently and historically applied by the refiners concerned. Domestic crude oil is that oil produced in the United States or from the outer continental shelf as defined in 43 USC Section 1331. Imported crude oil is either that oil reported on Form ERA-51, "Transfer Pricing Report," or any crude oil that is not domestic oil. The composite cost is the weighted average of domestic and imported crude oil costs.

Crude oil costs and volumes reported on Form ERA-49 excluded unfinished oils but included the Strategic Pe- troleum Reserve (SPR). Crude oil costs and volumes reported on Federal Energy Administration (FEA) Form FEA-P110-M-1, "Refiners' Monthly Cost Allocation Report," included unfinished oils but excluded SPR. Imported averages derived from Form ERA-49 exclude oil purchased for SPR, whereas the composite averages derived from Form ERA-49 include SPR. None of the prices derived from Form ElA-14 include either unfinished oils or SPR.

5. Several different series of motor gasoline prices are published in this section. U.S. City average retail prices of motor gasoline are calculated monthly by the Bureau of Labor Statistics during the development of the Consumer Price Index (CPI). These prices include all Federal, State, and local taxes paid at the time of sale. From 1974-1977, prices were collected in 56 urban areas. From 1978 forward, prices were collected from a new sample of service stations in 85 urban areas selected to represent all urban consumers-about 80 percent of the total U.S. population. The service stations are selected initially, and on a replacement basis, in such a way that they represent the purchasing habits of the CPI population. Service stations in the current sample include those providing all types of service (i.e., full-, mini-, and self-serve).

Refiner prices of finished motor gasoline for resale and to end users are determined by the EIA in a monthly survey of refiners and gas plant operators (Form EIA-782A). The prices do not include any Federal, State, or local taxes paid at the time of sale. Estimates of prices prior to January 1983 are based on Form FEA-P302-M-1/EIA-460, "Petroleum Industry Monthly Report for Product Prices," and also exclude all Federal, State, or local taxes paid at the time of sale. Sales for resale are those made to purchasers who are other-than-ultimate consumers. Sales to end users are sales made directly to the consumer of the product, including bulk consumers (such as agriculture, industry, and utilities) and residential and commercial consumers.

6. Starting in January 1983, Form EIA-782, "Monthly Petroleum Product Sales Report," replaced 10 previous surveys. Every attempt was made to continue the most important price series. However, prices published through December 1982 and those published since January 1983 do not necessarily form continuous data series due to changes in survey forms, definitions, instructions, populations, samples, processing systems, and statistical procedures. To provide historical data, continuous series were generated for annual data 1978-1982 and for monthly data 1981 and 1982 by estimating the prices that would have been published had Form EIA-782 survey and system been in operation at that time. This form of estimation was performed after detailed adjustment was made for product and sales type matching and for discontinuity due to other factors. An important difference between the previous and present prices is the distinction between wholesale and resale and between retail and end user. The resale category continues 
to sales among resellers. However, sales to bulk consumers, such as utility, industrial, and commercial accounts previously included in the wholesale category are now counted as made to end users. The end-user category continues to include retail sales through company owned and operated outlets but also includes sales to the bulk consumers such as agriculture, industry, and electric utilities. Additional information may be found in "Estimated Historic Time Series for the EIA-782," a feature article reprinted from the December 1983 [3] Petroleum Marketing Monthly, published by EIA.

7. Preliminary monthly data are based on submissions from over 250 publicly and privately owned electric utilities reporting on Form EIA-826 "Monthly Electric Utility Sales and Revenue Report with State Distributions." These utilities are statistically chosen as a cutoff sample from more than 3,000 electric utilities that report annually on Form EIA-861 "Annual Electric Utility Report." Preliminary annual values are the sum of the monthly revenues divided by the sum of the monthly sales. When final Form EIA-861 annual data become available each year, their ratios to the preliminary Form EIA-826 values are used to derive adjusted final monthly values. Prior to January 1986 , only privately owned electric utilities were included in the monthly survey and the sample was chosen using stratification techniques through December 1992.

8. Data for 1973-1982 cover all electric generating plants at which the generator nameplate capacity of all steamelectric units combined totaled 25 megawatts or greater. From 1974-1982, peaking units were included in the data and counted towards the 25-megawatt-or-greater total. Data for 1983-1990 cover all electric generating plants at which the generator nameplate capacity of all steam-electric units combined totaled 50 megawatts or greater. Data for 1991 forward cover all electric generating plants at which the generator nameplate capacity of all steam-electric units and combined-cycle units together totaled 50 megawatts or greater.

9. Natural gas prices are intended to include all taxes. Instructions on the data collection forms specifically direct that all Federal, State, and local taxes, surcharges, and/or adjustments billed to consumers are to be included. However, sales and other taxes itemized on more than 3,000 consumers' bills are sometimes excluded by the reporting utilities. Delivered-to-consumers prices for 1987 forward represent natural gas delivered and sold to residential, commercial, industrial, and electric utility consumers. They do not include the price of natural gas delivered to industrial and commercial consumers on behalf of third parties. Volumes of natural gas delivered on behalf of third parties are included in the consumption data shown in Table 4.4. Additional information is available in the EIA Natural Gas Monthly, Appendix C.

\section{Sources for Table 9.1}

\section{Domestic First Purchase Price}

1973-1976: U.S. Department of the Interior (DOI), Bureau of Mines (BOM), Minerals Yearbook, "Crude Petroleum and Petroleum Products" chapter.

1977: Federal Energy Administration (FEA), based on Form FEA-P124, "Domestic Crude Oil Purchaser's Monthly Report."

1978 forward: Energy Information Administration (EIA), Petroleum Marketing Monthly, January 1998, Table 1.

\section{F.O.B. and Landed Cost of Imports}

November 1973-September 1977: Federal Energy Administration, Form FEA-F701-M-0, "Transfer Pricing Report."

October-December 1977: EIA, Form FEA-F701-M-0, "Transfer Pricing Report."

1978 forward: EIA, Petroleum Marketing Monthly, January 1998, Table 1.

\section{Refine: Acquisition Cost}

1973: EIA estimates. The domestic price was derived by adding estimated transportation costs to the reported domestic first purchase price. The imported price was derived by adding an estimated ocean transport cost to the average "Free Alongside Ship" value published by the U.S. Bureau of the Census.

1974-1976: DOI, BOM, Minerals Yearbook, "Crude Petroleum and Petroleum Products" chapter.

1977: January-September, FEA, based on Form FEAP110-M-1, "Refiners' Monthly Cost Allocation Report." October-December, EIA, based on Form FEA-P110-M-1, "Refiners' Monthly Cost Allocation Report."

1978 forward: EIA, Petroleum Marketing Monthly, January 1998, Table 1.

\section{Sources for Table 9.2}

October 1973-September 1977: Federal Energy Administration, Form FEA-F701-M-0, "Transfer Pricing Report." October 1977-December 1977: Energy Information Administration (EIA), Form FEA-F701-M-0, "Transfer Pricing Report."

1978 forward: EIA, Petroleum Marketing Monthly, January 1998, Table 24 . 


\section{Sources for Table 9.9}

1973-September 1977: Federal Power Commission (FPC), Form FPC-5, "Monthly Statement of Electric Operating Revenues and Income."

October 1977-February 1980: Federal Energy Regulatory Commission (FERC), Form FPC-5, "Monthly Statement of Electric Operating Revenues and Income."

March 1980-1982: FERC, Form FERC-5, "Electric Utility Company Monthly Statement."

1983: Energy Information Administration (EIA), Form EIA-826, "Electric Utility Company Monthly Statement." 1984-1986: EIA, Form EIA-861, "Annual Electric Utility Report."

1987 forward: EIA, Electric Power Monthly, January 1998, Table 26.

\section{Sources for Table 9.10}

1973-1979: Annual data for quantity are simple sums of unrounded monthly values and for cost are averages of monthly values, weighted by quantities of Btu, from the following:

1973-May 1977: Federal Power Commission, Form FPC-423, "Monthly Report on Cost and Quality of Fuels for Electric Utility Plants."

June 1977-December 1977: Federal Energy Regulatory Commission, Form FERC-423, "Monthly Report on Cost and Quality of Fuels for Electric Utility Plants."

1978 and 1979: Energy Information Administration (EIA), Form FERC-423, "Monthly Report on Cost and Quality of Fuels for Electric Utility Plants."

1980-1986: EIA, Electric Power Monthly, April issues.

1987 forward: EIA, Electric Power Monthly, January 1998, Table 26.

\section{Sources for Table $\mathbf{9 . 1 1}$}

\section{Prices, 1973-1989}

Wellhead: Energy Information Administration (EIA), Natural Gas Annual 1994, Volume 1, Table 99.

City Gate, 1984-1986: EIA, Natural Gas Monthly, December 1989, Table 4.

City Gate, 1987-1989: EIA, Natural Gas Monthly, December 1994, Table 4.

Delivered to Consumers, 1973-1990: EIA, Natural Gas Annual 1996, Table 102.

\section{Prices, 1991 forward}

EIA, Natural Gas Monthly, December 1997, Table 4.

\section{Share of Total Volume Delivered, Annual}

Calculated from EIA, Natural Gas Annual, Volume 1, report series, Table 1, "Summary Statistics for Natural Gas in the United States," as total amount of natural gas delivered to the sector's consumers minus the amount delivered for the account of others (to derive the amount on system) divided by the total amount delivered to the sector.

\section{Share of Total Volume Delivered, Monthly}

EIA, table titled, "Percentage of Total Deliveries Represented by Onsystem Sales, by State," in the Natural Gas Monthly issues as follows:

April 1988-March 1989 - Table C-1

April 1989-December 1991 - Table 33

January 1992-February 1993 - Table 32

March 1993-October 1995 - Table 28

November 1995-Present - Table 24 


\section{Section 10. International Energy}

Crude Oil Production. World crude oil production during October 1997 was 67 million barrels per day, up 0.7 million barrels per day from the level in the previous month.

Organization of Petroleum Exporting Countries (OPEC) production during October 1997 averaged 29 million barrels per day, up 0.1 million barrels per day from the level during the previous month. During October 1997, production increased in Iran by 150 thousand barrels per day and Nigeria by 100 thousand barrels per day. Production decreased in Iraq by 110 thousand barrels per day. Production remained unchanged in the other OPEC nations.

Among the non-OPEC nations, production during October 1997 increased in Norway by 282 thousand barrels per day, the United Kingdom by 127 thousand barrels per day, the United States by 47 thousand barrels per day, and Canada by 26 thousand barrels per day. Production decreased in Mexico by 18 thousand barrels per day and Russia by 4 thousand barrels per day. Production remained unchanged in China and Egypt.

Petroleum Consumption. In August 1997, consumption in all Organization for Economic Cooperation and Development (OECD) countries was 40.4 million barrels per day, 2 percent lower than the August 1996 rate. The consumption rate was higher than it was 1 year ago in Italy $(+1$ percent). Consumption rates were lower in Germany ( -10 percent), the United Kingdom ( -4 percent), France and Japan (both -3 percent), the United States ( -1 percent), and Canada (less than -1 percent), compared with the rate 1 year earlier.

Petroleum Stocks. For all OECD countries, petroleum stocks at the end of August 1997 totaled 3.7 billion barrels, 1 percent higher than the ending stock level in August 1996. Stocks were higher in the United Kingdom $(+7$ percent), Germany $(+3$ percent), and Japan and the United States (both +2 percent). Stock levels were lower in Italy $(-5$ percent), Canada ( -2 percent), and France $(-1$ percent), compared with levels 1 year earlier.

Nuclear Electricity Generation. Based on Nucleonics Week ${ }^{2}$ information for October 1997, all reporting countries with nuclear capacity generated 189 gross terawatthours (one terawatthour equals 1 billion kilowatthours) of nuclear-generated electricity.

As of October 31, 1997, there were 435 operable nuclear generating units in the world.

\footnotetext{
${ }^{1}$ Percentage changes are based on unrounded data.

${ }^{2}$ A copyrighted publication of The McGraw-Hill Publishing Companies, Inc. Used with permission.
} 
(Thousand Barrels per Day)

\begin{tabular}{|c|c|c|c|c|c|c|c|c|c|c|c|c|}
\hline & Algeria & Indonesia & Iran & Iraq & Kuwaita & Libya & Nigeria & Qatar & $\begin{array}{c}\text { Saudi } \\
\text { Arabiaa }^{a}\end{array}$ & $\begin{array}{c}\text { United } \\
\text { Arab } \\
\text { Emirates }\end{array}$ & Venezuela & OPEC \\
\hline 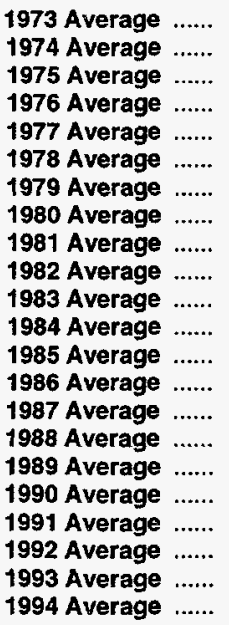 & $\begin{array}{r}1,097 \\
1,009 \\
983 \\
1,075 \\
1,152 \\
1,231 \\
1,224 \\
1,106 \\
1,002 \\
987 \\
968 \\
1,014 \\
1,037 \\
945 \\
1,048 \\
1,040 \\
1,095 \\
1,175 \\
1,230 \\
1,214 \\
1,162 \\
1,180\end{array}$ & $\begin{array}{l}1,339 \\
1,375 \\
1,307 \\
1,504 \\
1,686 \\
1,635 \\
1,591 \\
1,577 \\
1,605 \\
1,339 \\
1,343 \\
1,412 \\
1,325 \\
1,390 \\
1,343 \\
1,342 \\
1,409 \\
1,462 \\
1,592 \\
1,504 \\
1,511 \\
1,510\end{array}$ & $\begin{array}{l}5,861 \\
6,022 \\
5,350 \\
5,883 \\
5,663 \\
5,242 \\
3,168 \\
1,662 \\
1,380 \\
2,214 \\
2,440 \\
2,174 \\
2,250 \\
2,035 \\
2,298 \\
2,240 \\
2,810 \\
3,088 \\
3,312 \\
3,429 \\
3,540 \\
3,618\end{array}$ & $\begin{array}{r}2,018 \\
1,971 \\
2,262 \\
2,415 \\
2,348 \\
2,563 \\
3,477 \\
2,514 \\
1,000 \\
1,012 \\
1,005 \\
1,209 \\
1,433 \\
1,690 \\
2,079 \\
2,685 \\
2,897 \\
2,040 \\
305 \\
425 \\
512 \\
553\end{array}$ & $\begin{array}{r}3,020 \\
2,546 \\
2,084 \\
2,145 \\
1,969 \\
2,131 \\
2,500 \\
1,656 \\
1,125 \\
823 \\
1,064 \\
1,157 \\
1,023 \\
1,419 \\
1,585 \\
1,492 \\
1,783 \\
1,175 \\
190 \\
1,058 \\
1,852 \\
2,025\end{array}$ & $\begin{array}{r}2,175 \\
1,521 \\
1,480 \\
1,933 \\
2,063 \\
1,983 \\
2,092 \\
1,787 \\
1,140 \\
1,150 \\
1,105 \\
1,087 \\
1,059 \\
1,034 \\
972 \\
1,175 \\
1,150 \\
1,375 \\
1,483 \\
1,433 \\
1,361 \\
1,378\end{array}$ & $\begin{array}{l}2,054 \\
2,255 \\
1,783 \\
2,067 \\
2,085 \\
1,897 \\
2,302 \\
2,055 \\
1,433 \\
1,295 \\
1,241 \\
1,388 \\
1,495 \\
1,467 \\
1,341 \\
1,450 \\
1,716 \\
1,810 \\
1,892 \\
1,943 \\
1,960 \\
1,931\end{array}$ & $\begin{array}{l}570 \\
518 \\
438 \\
497 \\
445 \\
487 \\
508 \\
472 \\
405 \\
330 \\
295 \\
394 \\
301 \\
308 \\
293 \\
346 \\
380 \\
406 \\
395 \\
423 \\
413 \\
415\end{array}$ & $\begin{array}{l}7,596 \\
8,480 \\
7,075 \\
8,577 \\
9,245 \\
8,301 \\
9,532 \\
9,900 \\
9,815 \\
6,483 \\
5,086 \\
4,663 \\
3,388 \\
4,870 \\
4,265 \\
5,086 \\
5,064 \\
6,410 \\
8,115 \\
8,332 \\
8,198 \\
8,120\end{array}$ & $\begin{array}{l}1,533 \\
1,679 \\
1,664 \\
1,936 \\
1,999 \\
1,831 \\
1,831 \\
1,709 \\
1,474 \\
1,250 \\
1,149 \\
1,146 \\
1,193 \\
1,330 \\
1,541 \\
1,565 \\
1,860 \\
2,117 \\
2,386 \\
2,266 \\
2,159 \\
2,193\end{array}$ & $\begin{array}{l}3,366 \\
2,976 \\
2,346 \\
2,294 \\
2,238 \\
2,165 \\
2,356 \\
2,168 \\
2,102 \\
1,895 \\
1,801 \\
1,798 \\
1,677 \\
1,787 \\
1,752 \\
1,903 \\
1,907 \\
2,137 \\
2,375 \\
2,371 \\
2,450 \\
2,588\end{array}$ & $\begin{array}{l}30,629 \\
30,351 \\
26,771 \\
30,327 \\
30,893 \\
29,464 \\
30,581 \\
26,606 \\
22,481 \\
18,778 \\
17,497 \\
17,442 \\
16,181 \\
18,275 \\
18,517 \\
20,324 \\
22,071 \\
23,195 \\
23,275 \\
24,398 \\
25,119 \\
25,510\end{array}$ \\
\hline $\begin{array}{c}1995 \text { January ......... } \\
\text { February ....... } \\
\text { March ........... } \\
\text { April ............ } \\
\text { May .............. } \\
\text { June ............ } \\
\text { July ............. } \\
\text { August ......... } \\
\text { Septernber ... } \\
\text { October ........ } \\
\text { November .... } \\
\text { December .... } \\
\text { Average ...... }\end{array}$ & $\begin{array}{l}1,185 \\
1,185 \\
1,185 \\
1,185 \\
1,185 \\
1,185 \\
1,215 \\
1,215 \\
1,215 \\
1,215 \\
1,225 \\
1,225 \\
1,202\end{array}$ & $\begin{array}{l}1,500 \\
1,480 \\
1,490 \\
1,490 \\
1,490 \\
1,490 \\
1,490 \\
1,490 \\
1,490 \\
1,540 \\
1,540 \\
1,540 \\
1,503\end{array}$ & $\begin{array}{l}3,585 \\
3,685 \\
3,485 \\
3,635 \\
3,835 \\
3,585 \\
3,535 \\
3,685 \\
3,635 \\
3,735 \\
3,635 \\
3,685 \\
3,643\end{array}$ & $\begin{array}{l}560 \\
560 \\
560 \\
560 \\
560 \\
560 \\
560 \\
560 \\
560 \\
560 \\
560 \\
560 \\
560\end{array}$ & $\begin{array}{l}2,070 \\
2,070 \\
2,060 \\
2,070 \\
2,050 \\
2,050 \\
2,060 \\
2,075 \\
2,035 \\
2,065 \\
2,070 \\
2,015 \\
2,057\end{array}$ & $\begin{array}{l}1,390 \\
1,390 \\
1,390 \\
1,390 \\
1,390 \\
1,390 \\
1,390 \\
1,390 \\
1,390 \\
1,390 \\
1,390 \\
1,390 \\
1,390\end{array}$ & $\begin{array}{l}1,965 \\
1,946 \\
1,857 \\
2,015 \\
2,044 \\
1,926 \\
1,946 \\
2,000 \\
2,005 \\
2,024 \\
2,074 \\
2,108 \\
1,993\end{array}$ & $\begin{array}{l}455 \\
475 \\
485 \\
485 \\
485 \\
485 \\
485 \\
485 \\
485 \\
485 \\
495 \\
495 \\
483\end{array}$ & $\begin{array}{l}8,120 \\
8,220 \\
8,110 \\
8,220 \\
8,400 \\
8,100 \\
8,410 \\
8,425 \\
8,315 \\
8,315 \\
8,020 \\
8,110 \\
8,231\end{array}$ & $\begin{array}{l}2,285 \\
2,285 \\
2,285 \\
2,285 \\
2,285 \\
2,285 \\
2,285 \\
2,285 \\
2,285 \\
2,285 \\
2,285 \\
2,220 \\
2,279\end{array}$ & $\begin{array}{l}2,600 \\
2,600 \\
2,600 \\
2,670 \\
2,790 \\
2,790 \\
2,790 \\
2,790 \\
2,790 \\
2,840 \\
2,840 \\
2,890 \\
2,750\end{array}$ & $\begin{array}{l}25,715 \\
25,896 \\
25,507 \\
26,005 \\
26,514 \\
25,846 \\
26,166 \\
26,400 \\
26,205 \\
26,454 \\
26,133 \\
26,237 \\
26,092\end{array}$ \\
\hline $\begin{array}{l}1996 \text { January ........ } \\
\text { February ...... } \\
\text { March ........... } \\
\text { April ............ } \\
\text { May .............. } \\
\text { June ............ } \\
\text { July ............ } \\
\text { August ......... } \\
\text { September ... } \\
\text { October ........ } \\
\text { November .... } \\
\text { December .... } \\
\text { Average ...... }\end{array}$ & $\begin{array}{l}1,220 \\
1,220 \\
1,210 \\
1,230 \\
1,245 \\
1,250 \\
1,250 \\
1,250 \\
1,250 \\
1,260 \\
1,260 \\
1,260 \\
1,242\end{array}$ & $\begin{array}{l}1,540 \\
1,540 \\
1,540 \\
1,530 \\
1,530 \\
1,550 \\
1,520 \\
1,540 \\
1,560 \\
1,580 \\
1,570 \\
1,570 \\
1,547\end{array}$ & $\begin{array}{l}3,735 \\
3,685 \\
3,715 \\
3,685 \\
3,635 \\
3,685 \\
3,685 \\
3,715 \\
3,735 \\
3,635 \\
3,685 \\
3,635 \\
3,686\end{array}$ & $\begin{array}{l}555 \\
555 \\
555 \\
555 \\
555 \\
555 \\
555 \\
555 \\
555 \\
555 \\
555 \\
895 \\
584\end{array}$ & $\begin{array}{l}2,038 \\
2,057 \\
2,057 \\
2,067 \\
2,055 \\
2,065 \\
2,065 \\
2,040 \\
2,070 \\
2,075 \\
2,075 \\
2,077 \\
2,062\end{array}$ & $\begin{array}{l}1,400 \\
1,400 \\
1,400 \\
1,400 \\
1,400 \\
1,400 \\
1,400 \\
1,400 \\
1,400 \\
1,400 \\
1,400 \\
1,410 \\
1,401\end{array}$ & $\begin{array}{l}2,160 \\
2,180 \\
2,190 \\
2,160 \\
2,200 \\
2,200 \\
2,170 \\
2,190 \\
2,150 \\
2,210 \\
2,220 \\
2,225 \\
\mathbf{2 , 1 8 8}\end{array}$ & $\begin{array}{l}500 \\
500 \\
500 \\
505 \\
505 \\
505 \\
505 \\
505 \\
525 \\
525 \\
505 \\
545 \\
510\end{array}$ & $\begin{array}{l}8,118 \\
8,248 \\
8,248 \\
8,088 \\
8,135 \\
8,195 \\
8,295 \\
8,220 \\
8,200 \\
8,255 \\
8,255 \\
8,358 \\
8,218\end{array}$ & $\begin{array}{l}2,290 \\
2,265 \\
2,285 \\
2,250 \\
2,275 \\
2,270 \\
2,260 \\
2,260 \\
2,310 \\
2,310 \\
2,250 \\
2,305 \\
2,278\end{array}$ & $\begin{array}{l}2,940 \\
2,940 \\
2,990 \\
2,990 \\
2,990 \\
2,990 \\
3,040 \\
3,090 \\
3,090 \\
3,140 \\
3,190 \\
3,240 \\
3,053\end{array}$ & $\begin{array}{l}26,495 \\
26,590 \\
26,690 \\
26,460 \\
26,525 \\
26,665 \\
26,745 \\
26,765 \\
26,845 \\
26,945 \\
26,965 \\
27,520 \\
26,769\end{array}$ \\
\hline $\begin{array}{l}1997 \text { January ........ } \\
\text { February ...... } \\
\text { March ........... } \\
\text { April ............ } \\
\text { May .............. } \\
\text { June ............ } \\
\text { July ............... } \\
\text { August ......... } \\
\text { Septernber ... } \\
\text { October ........ } \\
\text { 10-Mo. Avg. }\end{array}$ & $\begin{array}{l}1,260 \\
1,270 \\
1,280 \\
1,280 \\
1,280 \\
1,260 \\
1,280 \\
1,280 \\
1,280 \\
1,280 \\
1,275\end{array}$ & $\begin{array}{l}1,570 \\
1,590 \\
1,600 \\
1,560 \\
1,580 \\
1,530 \\
1,530 \\
1,530 \\
1,490 \\
1,490 \\
1,547\end{array}$ & $\begin{array}{l}3,685 \\
3,685 \\
3,685 \\
3,685 \\
3,635 \\
3,735 \\
3,685 \\
3,685 \\
3,485 \\
3,635 \\
3,660\end{array}$ & $\begin{array}{r}1,085 \\
1,125 \\
1,175 \\
1,275 \\
1,325 \\
605 \\
605 \\
1,515 \\
1,735 \\
1,625 \\
1,208\end{array}$ & $\begin{array}{l}2,085 \\
2,077 \\
2,105 \\
2,107 \\
2,027 \\
2,050 \\
2,070 \\
2,070 \\
2,075 \\
2,075 \\
2,074\end{array}$ & $\begin{array}{l}1,430 \\
1,430 \\
1,440 \\
1,450 \\
1,450 \\
1,450 \\
1,450 \\
1,450 \\
1,450 \\
1,450 \\
1,445\end{array}$ & $\begin{array}{l}2,250 \\
2,310 \\
2,240 \\
2,310 \\
2,270 \\
2,330 \\
2,400 \\
2,350 \\
2,300 \\
2,400 \\
2,316\end{array}$ & $\begin{array}{l}585 \\
585 \\
585 \\
585 \\
605 \\
690 \\
685 \\
685 \\
685 \\
685 \\
638\end{array}$ & $\begin{array}{l}8,265 \\
8,408 \\
8,515 \\
8,568 \\
8,548 \\
8,540 \\
8,560 \\
8,660 \\
8,665 \\
8,665 \\
8,540\end{array}$ & $\begin{array}{l}2,300 \\
2,330 \\
2,360 \\
2,360 \\
2,210 \\
2,325 \\
2,325 \\
2,325 \\
2,325 \\
2,325 \\
2,318\end{array}$ & $\begin{array}{l}3,190 \\
3,190 \\
3,200 \\
3,220 \\
3,240 \\
3,260 \\
3,270 \\
3,390 \\
3,430 \\
3,430 \\
3,283\end{array}$ & $\begin{array}{l}27,705 \\
28,000 \\
28,185 \\
28,400 \\
28,170 \\
27,775 \\
27,860 \\
28,940 \\
28,920 \\
29,060 \\
28,304\end{array}$ \\
\hline $\begin{array}{l}1996 \text { 10-Mo. Avg. } \\
1995 \text { 10-Mo. Avg. }\end{array}$ & $\begin{array}{l}1,239 \\
1,197\end{array}$ & $\begin{array}{l}1,543 \\
1,495\end{array}$ & $\begin{array}{l}3,691 \\
3,640\end{array}$ & $\begin{array}{l}555 \\
560\end{array}$ & $\begin{array}{l}2,059 \\
2,060\end{array}$ & $\begin{array}{l}1,400 \\
1,390\end{array}$ & $\begin{array}{l}2,181 \\
1,973\end{array}$ & $\begin{array}{l}508 \\
481\end{array}$ & $\begin{array}{l}8,200 \\
8,264\end{array}$ & $\begin{array}{l}2,278 \\
2,285\end{array}$ & $\begin{array}{l}3,020 \\
2,727\end{array}$ & $\begin{array}{l}26,673 \\
26,073\end{array}$ \\
\hline
\end{tabular}

a Includes about one-half of the production in the Kuwait-Saudi Arabia Neutral Zone from 1973 through July 1990 and in June 1991. Kuwaiti Neutral Zone output was discontinued following Iraq's invasion of Kuwait on August 2, 1990, but was resumed in June 1991. In October 1997, Neutral Zone production by both Kuwait and Saudi Arabia totaled about 530 thousand barrels per day.

b Current members of OPEC are Algeria, Indonesia, Iran, Iraq, Kuwait, Libya, Nigeria, Qatar, Saudi Arabia, the United Arab Emirates, and Venezuela.
Ecuador and Gabon, which withdrew from OPEC membership at the end of 1992 and 1994, respectively, are excluded from all OPEC totals.

Notes: - Crude oil includes lease condensate but excludes natural gas plant liquids. - Monthly data are often preliminary figures and may not average to the annual totals because of rounding or because updates to the preliminary monthly data are not available.

Sources: See end of section. 
Table 10.1b World Oil Production: Persian Gulf Nations, Non-OPEC, and World

(Thousand Barrels per Day)

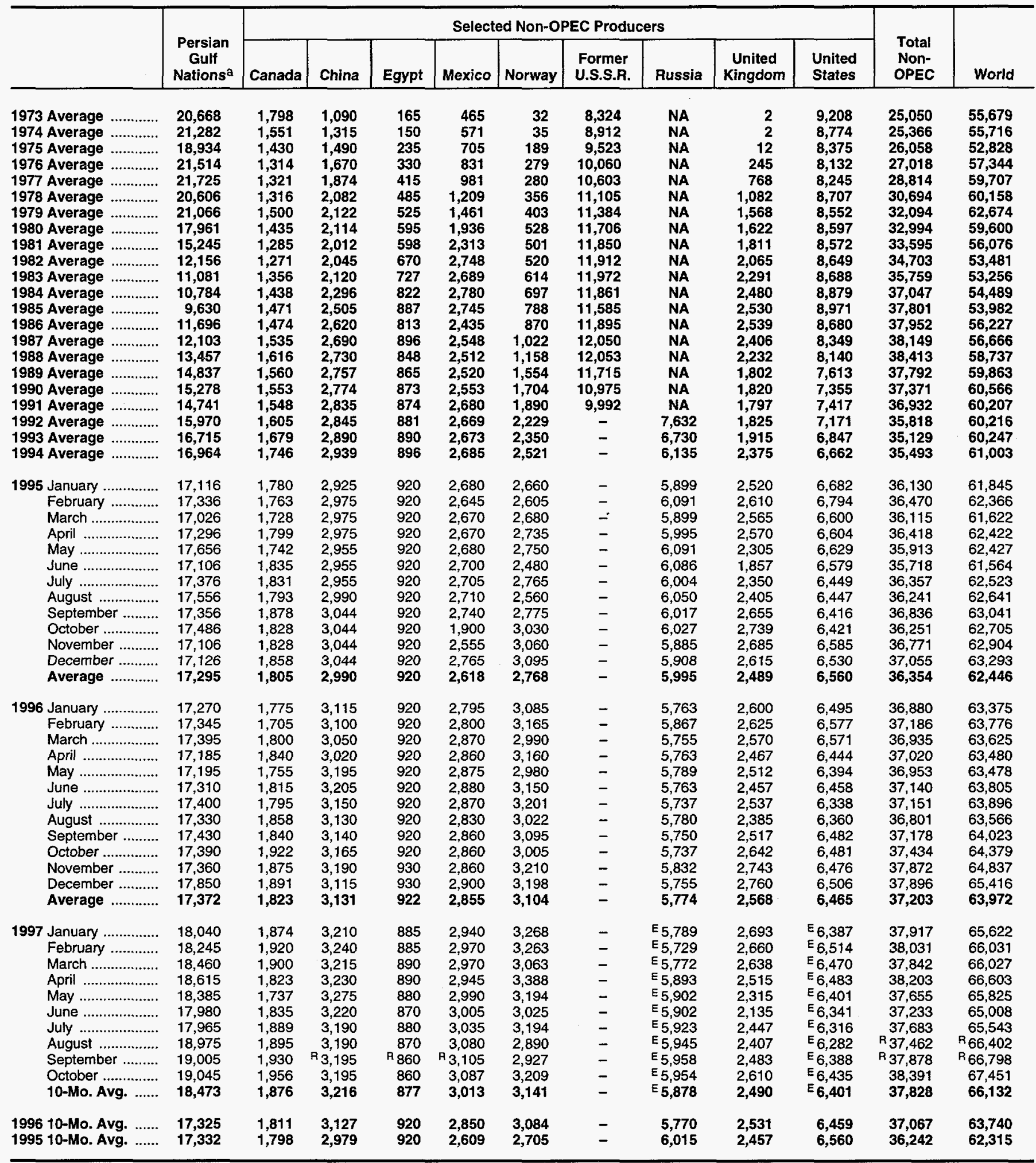

a "The Persian Gulf Nations are Bahrain, Iran, Iraq, Kuwait, Qatar, Saudi Arabia, and the United Arab Emirates. Production from the Neutral Zone between Kuwait and Saudi Arabia is included in "Persian Gulf Nations."

$R=$ Revised data. $N A=$ Not available. $-=$ Not applicable. $E=E s t i m a t e$.

Notes: - Crude oil includes lease condensate but excludes natural gas plant liquids. - Monthly data are often preliminary figures and may not average to the annual totals because of rounding or because updates to the preliminary monthly data are not available. - Data for countries may not sum to World totals due to independent rounding. - U.S. geographic coverage is the 50 States and the District of Columbia.

Sources: See end of section. 
Figure 10.1 Crude Oil Production

(Million Barrels per Day)

World Production, 1973-1996

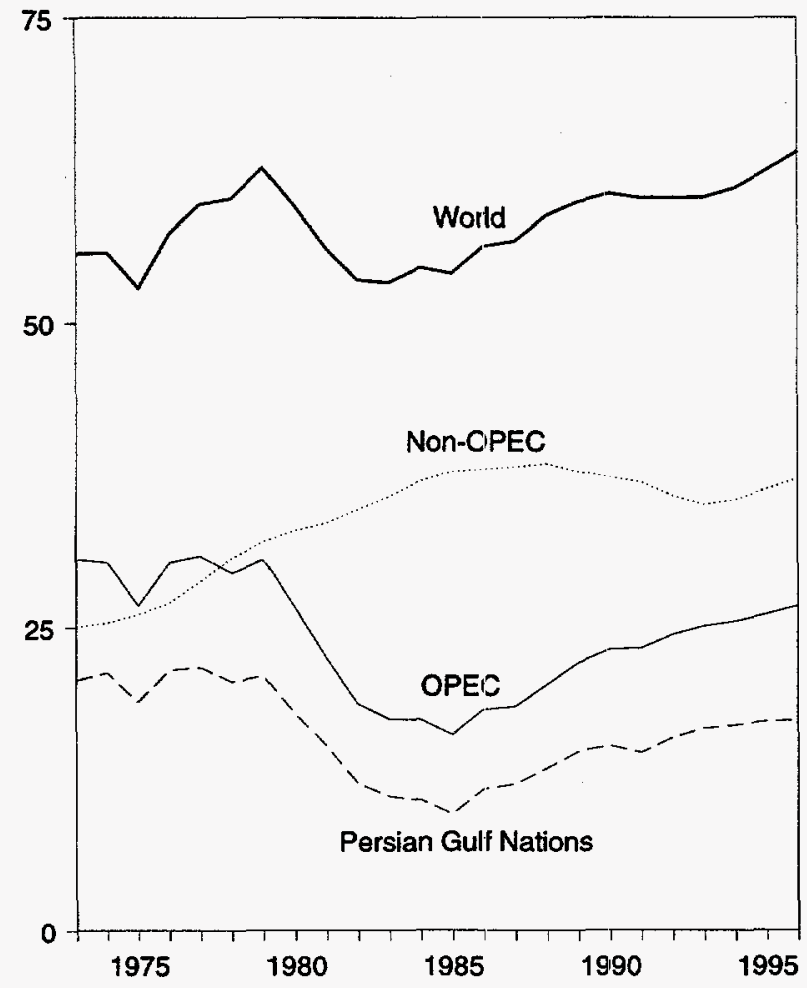

Selected Producers, 1973-1996

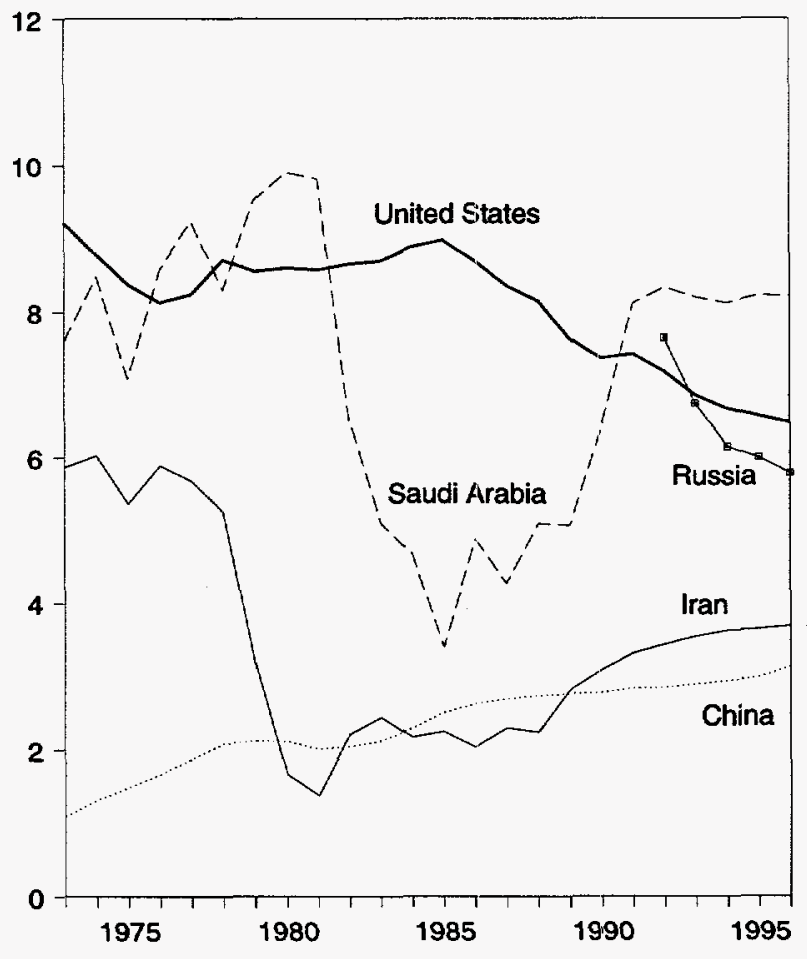

World Production, Monthly

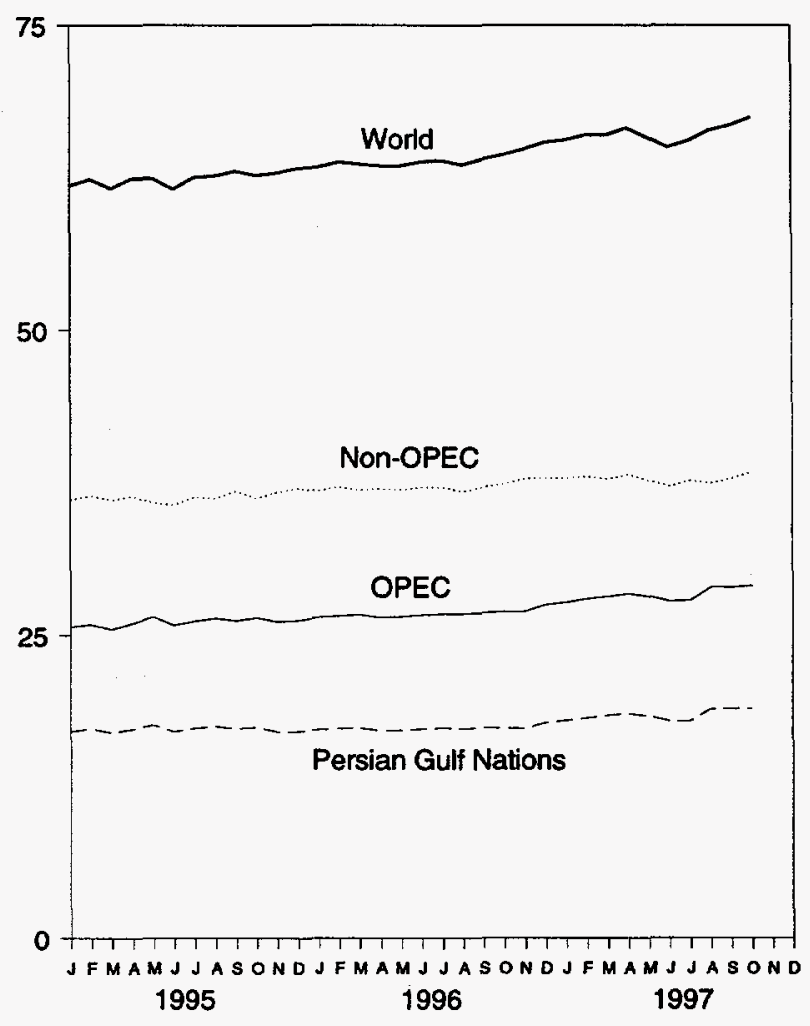

Selected Producers, Monthly

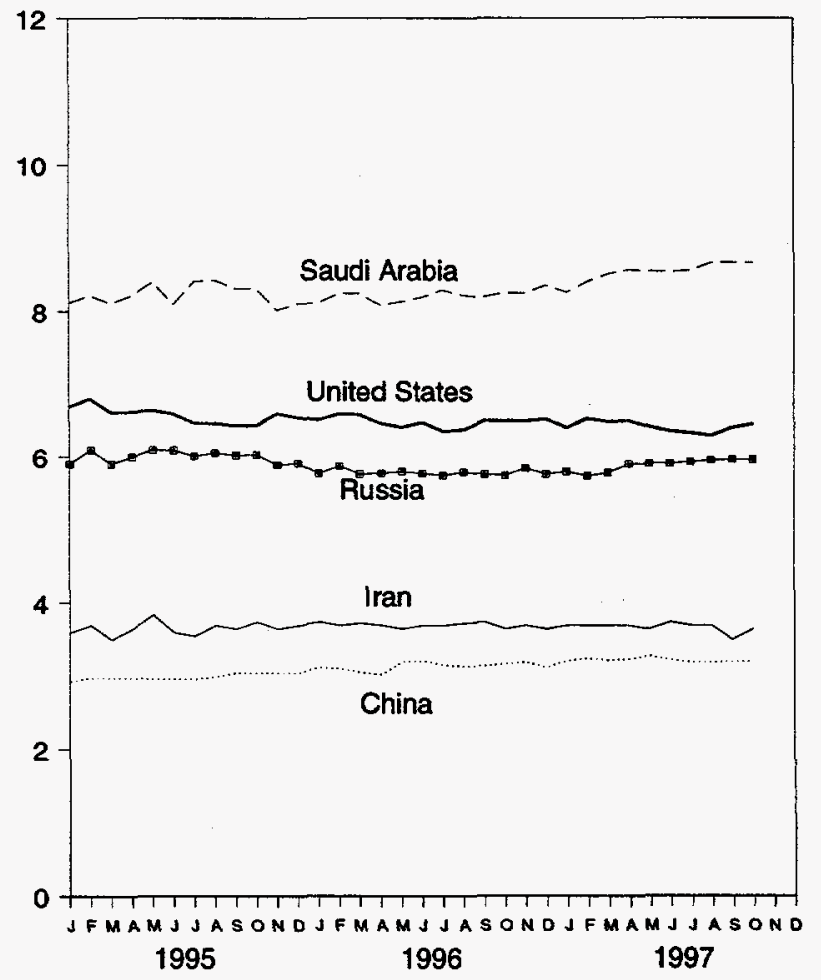

Note: OPEC is the Organization of Petroleum Exporting Countries. Sources: Tables 10.1a and 10.1b. 
Figure 10.2 Crude Oil Production by Selected Country

(Million Barrels per Day)

\begin{tabular}{|c|c|}
\hline & Canada \\
\hline & China \\
\hline & Egypt \\
\hline & Mexico \\
\hline & Norway \\
\hline & Russia \\
\hline & United Kingdom \\
\hline & United States \\
\hline
\end{tabular}

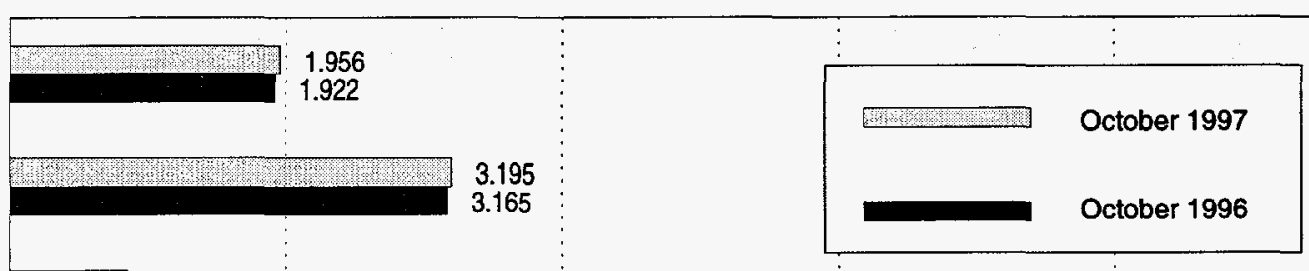

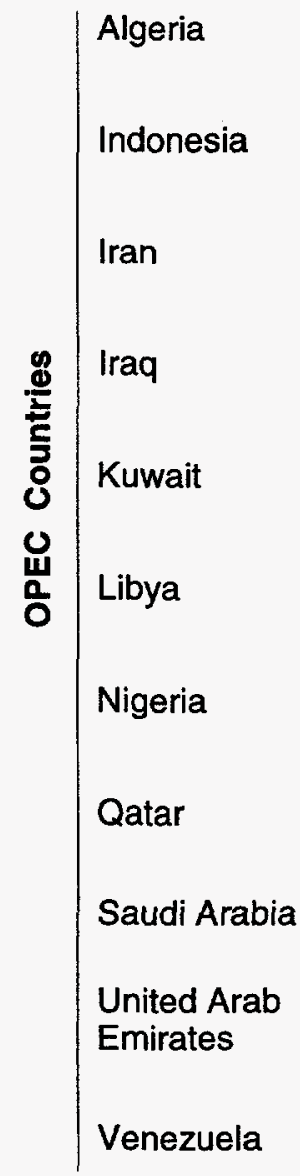
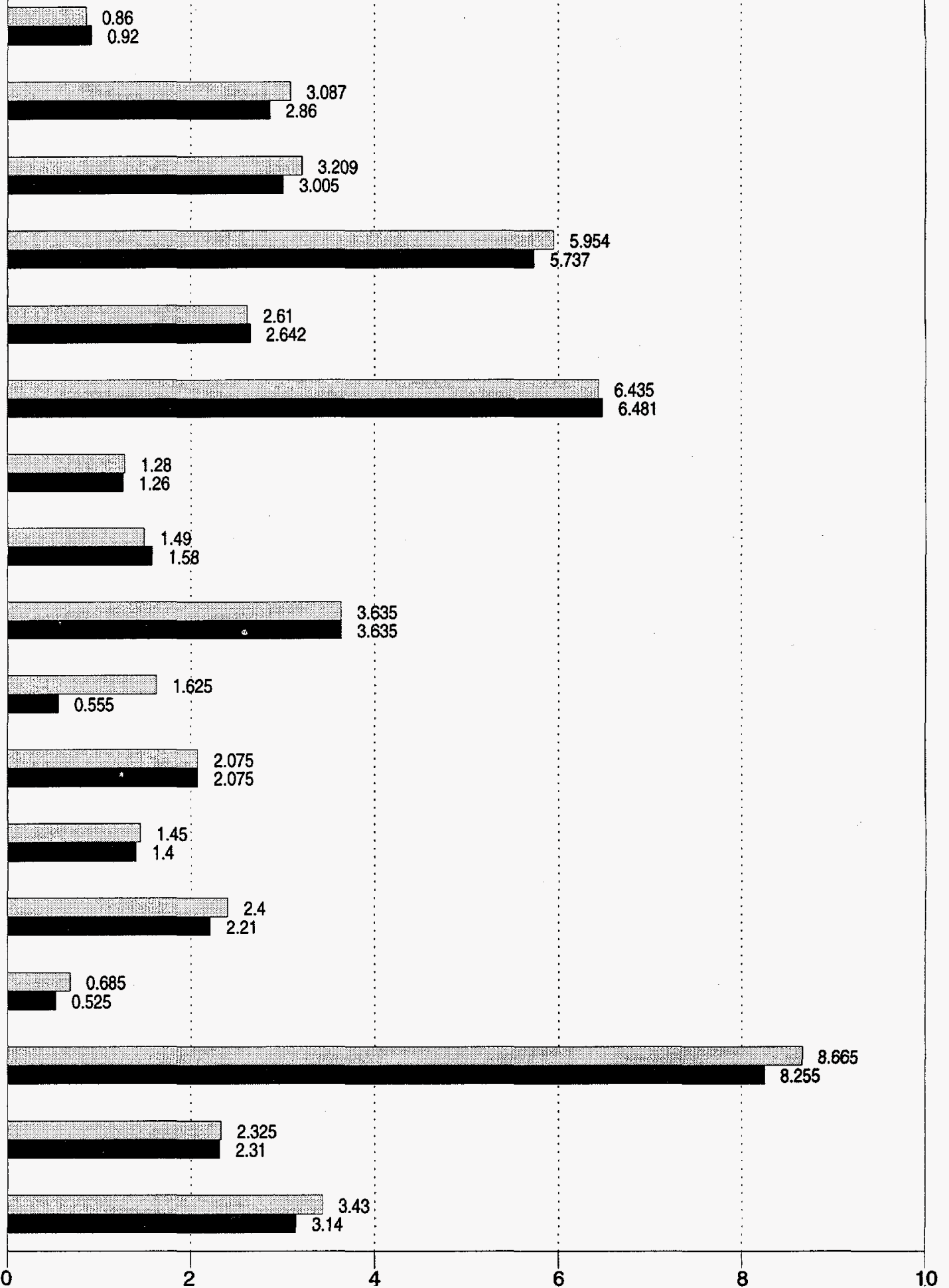

Note: OPEC is the Organization of Petroleum Exporting Countries.

Sources: Tables 10.1a and 10.1b. 
Figure 10.3 Petroleum Consumption in OECD Countries

(Million Barrels per Day)

Overview, 1973-1996

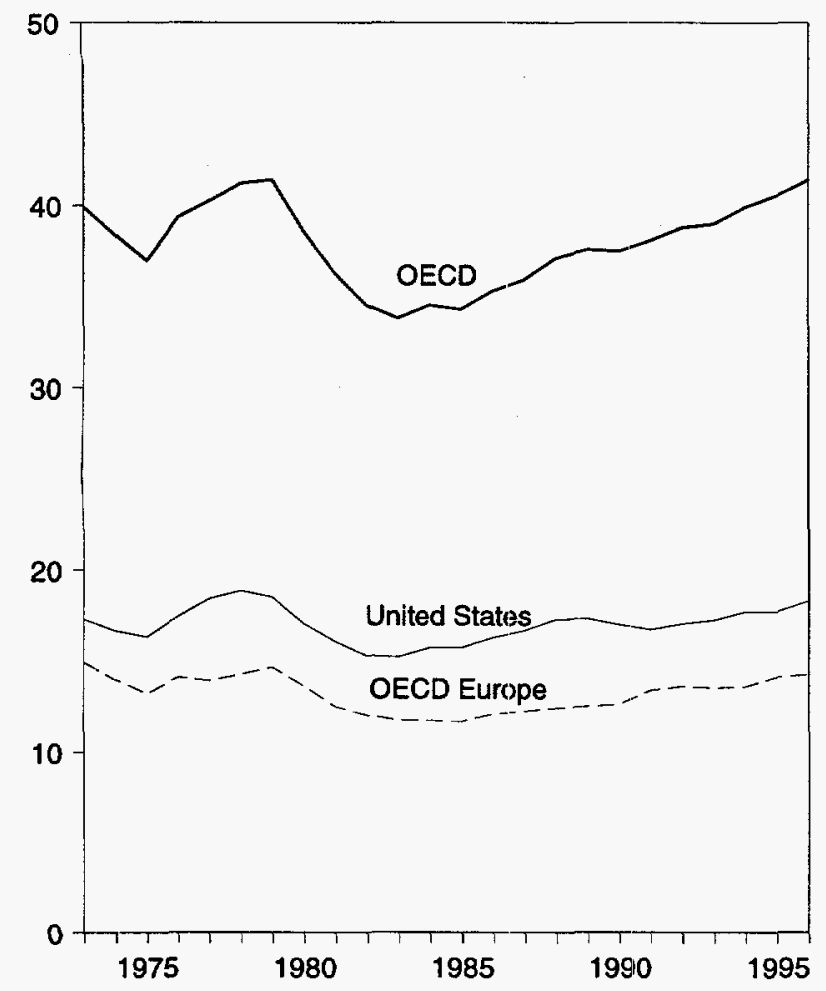

OECD Total, August

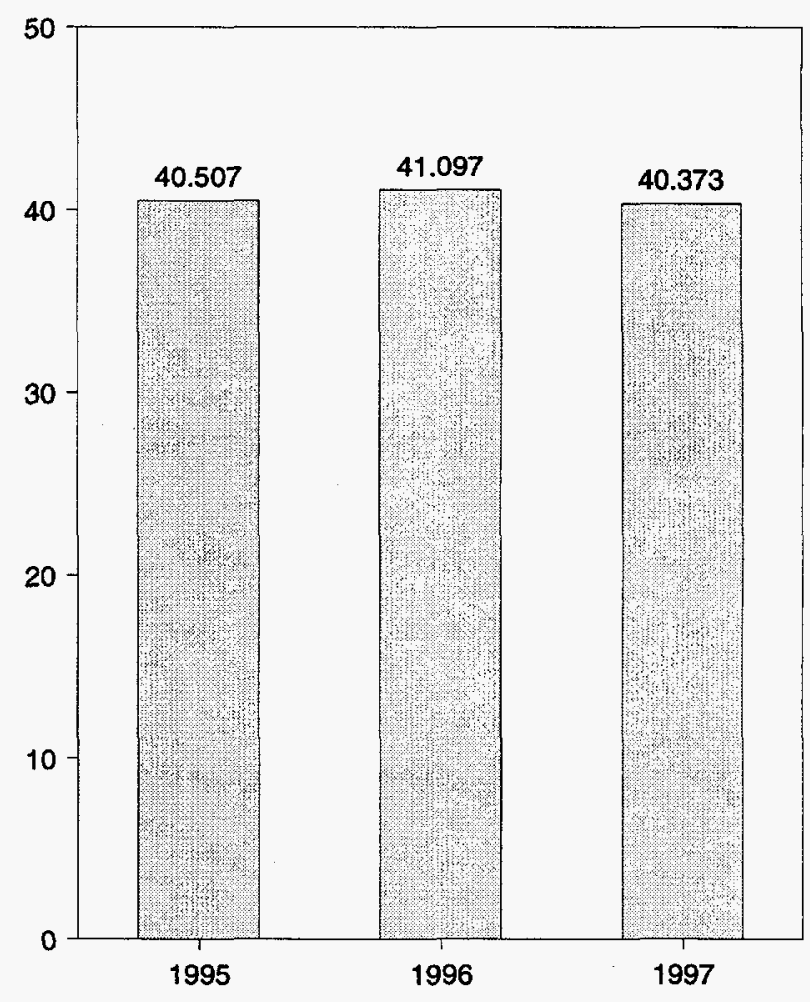

\section{By Selected OECD Country}

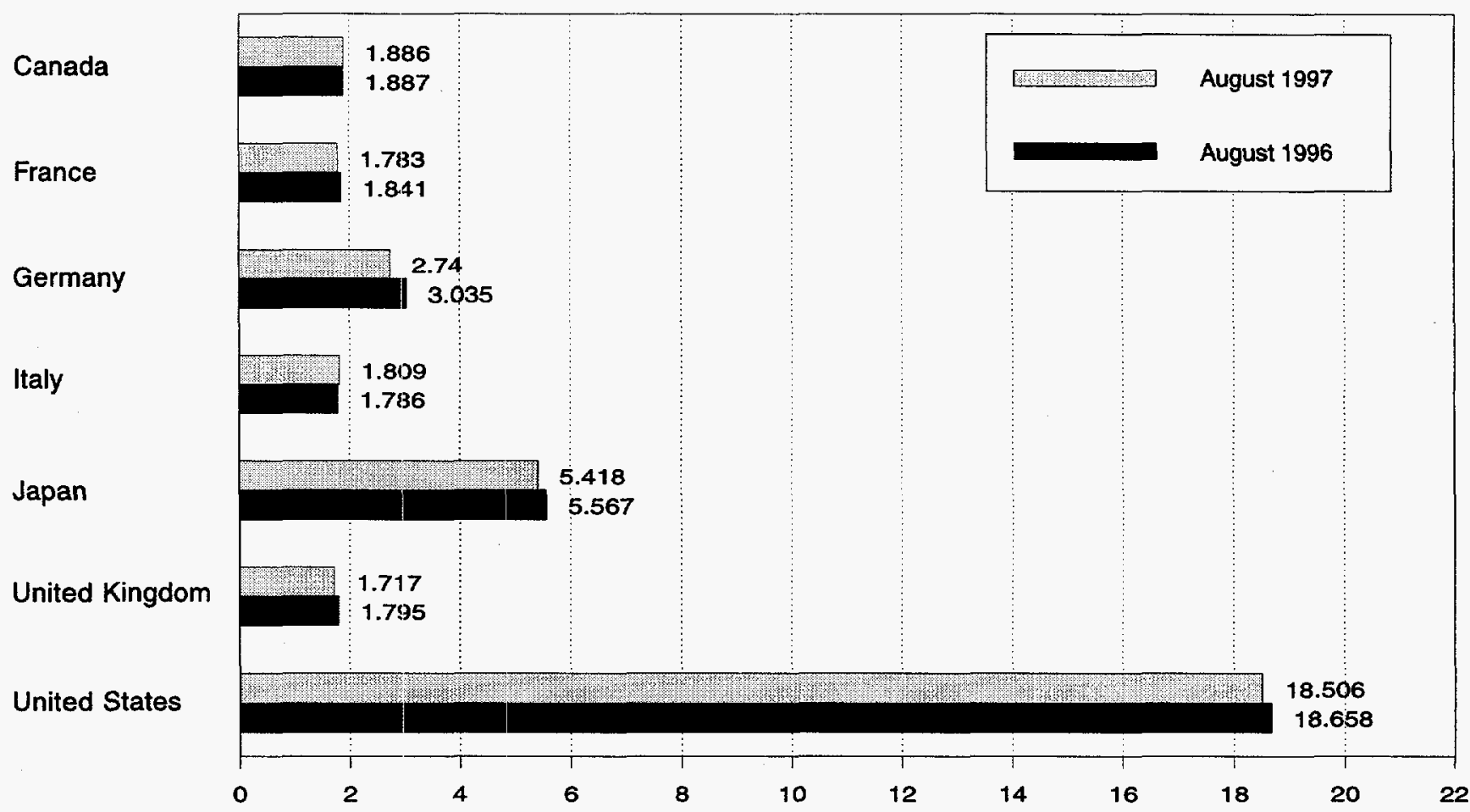

Note: OECD is the Organization for Economic Cooperation and Development.

Source: Table 10.2. 
Table 10.2 Petroleum Consumption in OECD Countries

(Thousand Barrels per Day)

\begin{tabular}{|c|c|c|c|c|c|c|c|c|c|c|}
\hline & Canada & France & Germany ${ }^{a}$ & Italy & Japan & $\begin{array}{l}\text { United } \\
\text { Kingdom }\end{array}$ & $\begin{array}{l}\text { United } \\
\text { States }\end{array}$ & $\begin{array}{l}\text { OECD } \\
\text { Europe }^{b}\end{array}$ & $\begin{array}{l}\text { Other } \\
\text { OECDC }\end{array}$ & OECD $^{d}$ \\
\hline 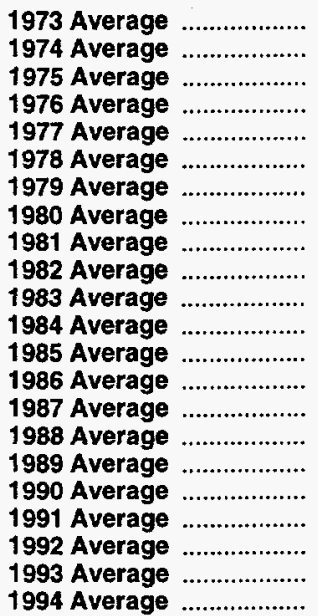 & $\begin{array}{l}1,729 \\
1,779 \\
1,779 \\
1,818 \\
1,850 \\
1,902 \\
1,971 \\
1,873 \\
1,768 \\
1,578 \\
1,448 \\
1,472 \\
1,504 \\
1,506 \\
1,548 \\
1,693 \\
1,733 \\
1,690 \\
1,622 \\
1,643 \\
1,688 \\
1,727\end{array}$ & $\begin{array}{l}2,601 \\
2,447 \\
2,252 \\
2,420 \\
2,294 \\
2,408 \\
2,463 \\
2,256 \\
2,023 \\
1,880 \\
1,835 \\
1,754 \\
1,775 \\
1,772 \\
1,789 \\
1,797 \\
1,857 \\
1,818 \\
1,935 \\
1,926 \\
1,875 \\
1,833\end{array}$ & $\begin{array}{l}3,055 \\
2,748 \\
2,650 \\
2,877 \\
2,865 \\
2,927 \\
3,003 \\
2,707 \\
2,449 \\
2,372 \\
2,324 \\
2,322 \\
2,338 \\
2,498 \\
2,424 \\
2,422 \\
2,280 \\
2,382 \\
2,828 \\
2,843 \\
2,900 \\
2,879\end{array}$ & $\begin{array}{l}2,068 \\
2,004 \\
1,855 \\
1,971 \\
1,897 \\
1,952 \\
2,039 \\
1,934 \\
1,874 \\
1,781 \\
1,750 \\
1,646 \\
1,717 \\
1,738 \\
1,855 \\
1,836 \\
1,930 \\
1,872 \\
1,863 \\
1,937 \\
1,852 \\
1,841\end{array}$ & $\begin{array}{l}\mathbf{4 , 9 4 9} \\
\mathbf{4 , 8 6 4} \\
\mathbf{4 , 6 2 1} \\
\mathbf{4 , 8 3 7} \\
\mathbf{4 , 8 8 0} \\
\mathbf{4 , 9 4 5} \\
\mathbf{5 , 0 5 0} \\
\mathbf{4 , 9 6 0} \\
\mathbf{4 , 8 4 8} \\
\mathbf{4 , 5 8 2} \\
\mathbf{4 , 3 9 5} \\
\mathbf{4 , 5 7 6} \\
\mathbf{4 , 3 8 4} \\
\mathbf{4 , 4 3 9} \\
\mathbf{4 , 4 8 4} \\
\mathbf{4 , 7 5 2} \\
\mathbf{4 , 9 8 3} \\
\mathbf{5 , 1 4 0} \\
\mathbf{5 , 2 8 4} \\
\mathbf{5 , 4 4 6} \\
\mathbf{5 , 4 0 1} \\
\mathbf{5 , 6 7 4}\end{array}$ & $\begin{array}{l}2,341 \\
2,210 \\
1,911 \\
1,892 \\
1,905 \\
1,938 \\
1,971 \\
1,725 \\
1,590 \\
1,590 \\
1,531 \\
1,849 \\
1,634 \\
1,649 \\
1,603 \\
1,697 \\
1,738 \\
1,752 \\
1,801 \\
1,803 \\
1,815 \\
1,837\end{array}$ & $\begin{array}{l}17,308 \\
16,653 \\
16,322 \\
17,461 \\
18,431 \\
18,847 \\
18,513 \\
17,056 \\
16,058 \\
15,296 \\
15,231 \\
15,726 \\
15,726 \\
16,281 \\
16,665 \\
17,283 \\
17,325 \\
16,988 \\
16,714 \\
17,033 \\
17,237 \\
17,718\end{array}$ & $\begin{array}{l}14,925 \\
13,988 \\
13,217 \\
14,124 \\
13,916 \\
14,290 \\
14,667 \\
13,634 \\
12,515 \\
12,053 \\
11,765 \\
11,736 \\
11,681 \\
12,102 \\
12,255 \\
12,427 \\
12,531 \\
12,629 \\
13,391 \\
13,605 \\
13,523 \\
13,597\end{array}$ & $\begin{array}{r}988 \\
1,095 \\
1,041 \\
1,119 \\
1,160 \\
1,204 \\
1,178 \\
1,072 \\
1,080 \\
1,008 \\
954 \\
989 \\
976 \\
951 \\
959 \\
939 \\
998 \\
1,027 \\
1,056 \\
1,041 \\
1,118 \\
1,174\end{array}$ & $\begin{array}{l}39,900 \\
38,379 \\
36,980 \\
39,358 \\
40,237 \\
41,187 \\
41,379 \\
38,595 \\
36,269 \\
34,517 \\
33,793 \\
34,500 \\
34,271 \\
35,279 \\
35,911 \\
37,093 \\
37,570 \\
37,475 \\
38,067 \\
38,768 \\
38,967 \\
39,890\end{array}$ \\
\hline 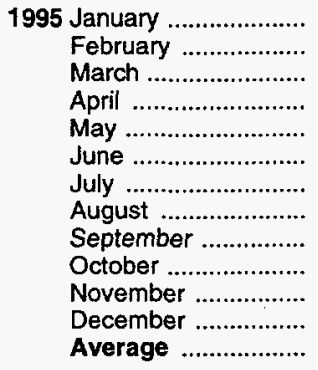 & $\begin{array}{l}1,673 \\
1,856 \\
1,697 \\
1,533 \\
1,706 \\
1,744 \\
1,719 \\
1,847 \\
1,821 \\
1,801 \\
1,814 \\
1,859 \\
1,755\end{array}$ & $\begin{array}{l}1,949 \\
1,895 \\
2,002 \\
1,834 \\
1,763 \\
1,846 \\
1,933 \\
1,787 \\
1,888 \\
1,870 \\
1,957 \\
2,032 \\
1,896\end{array}$ & $\begin{array}{l}2,711 \\
2,789 \\
3,186 \\
2,874 \\
2,942 \\
2,878 \\
2,833 \\
2,925 \\
2,952 \\
2,761 \\
2,913 \\
2,737 \\
2,875\end{array}$ & $\begin{array}{l}2,031 \\
2,225 \\
2,081 \\
1,928 \\
1,917 \\
1,975 \\
1,949 \\
1,810 \\
2,052 \\
2,141 \\
2,286 \\
2,205 \\
2,048\end{array}$ & $\begin{array}{l}6,031 \\
6,773 \\
6,331 \\
5,554 \\
5,027 \\
4,971 \\
5,087 \\
5,567 \\
5,378 \\
5,125 \\
5,884 \\
6,871 \\
5,711\end{array}$ & $\begin{array}{l}1,766 \\
1,965 \\
1,983 \\
1,800 \\
1,789 \\
1,820 \\
1,748 \\
1,806 \\
1,829 \\
1,852 \\
2,021 \\
1,772 \\
1,845\end{array}$ & $\begin{array}{l}17,219 \\
18,279 \\
17,484 \\
17,142 \\
17,293 \\
18,131 \\
17,147 \\
18,044 \\
18,026 \\
17,651 \\
17,979 \\
18,366 \\
17,725\end{array}$ & $\begin{array}{l}13,767 \\
14,136 \\
14,805 \\
13,829 \\
13,586 \\
13,916 \\
13,645 \\
13,795 \\
14,184 \\
14,215 \\
15,010 \\
14,566 \\
14,120\end{array}$ & $\begin{array}{l}1,156 \\
1,211 \\
1,274 \\
1,204 \\
1,295 \\
1,253 \\
1,195 \\
1,255 \\
1,259 \\
1,184 \\
1,198 \\
1,238 \\
1,227\end{array}$ & $\begin{array}{l}39,845 \\
42,255 \\
41,591 \\
39,262 \\
38,908 \\
40,014 \\
38,793 \\
40,507 \\
40,667 \\
39,976 \\
41,885 \\
42,899 \\
40,537\end{array}$ \\
\hline 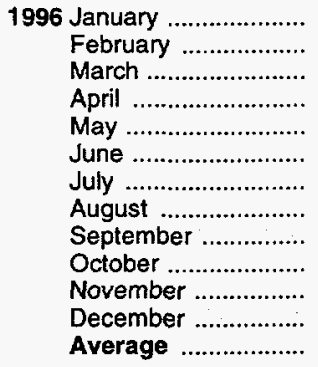 & $\begin{array}{l}1,805 \\
1,874 \\
1,744 \\
1,667 \\
1,715 \\
1,796 \\
1,809 \\
1,887 \\
1,771 \\
1,809 \\
1,941 \\
1,771 \\
1,799\end{array}$ & $\begin{array}{l}1,879 \\
2,183 \\
1,979 \\
1,919 \\
1,810 \\
1,819 \\
1,977 \\
1,841 \\
1,929 \\
1,989 \\
1,880 \\
2,021 \\
1,935\end{array}$ & $\begin{array}{l}2,901 \\
3,030 \\
2,860 \\
2,743 \\
2,864 \\
2,830 \\
2,957 \\
3,035 \\
3,095 \\
2,860 \\
2,975 \\
2,796 \\
2,911\end{array}$ & $\begin{array}{l}2,113 \\
2,259 \\
2,189 \\
1,961 \\
1,880 \\
1,908 \\
2,158 \\
1,786 \\
2,074 \\
2,201 \\
2,083 \\
2,088 \\
2,058\end{array}$ & $\begin{array}{l}6,328 \\
6,886 \\
6,437 \\
5,748 \\
5,147 \\
5,114 \\
5,502 \\
5,567 \\
5,361 \\
5,580 \\
6,114 \\
6,648 \\
\mathbf{5 , 8 6 7}\end{array}$ & $\begin{array}{l}1,762 \\
1,919 \\
1,859 \\
1,853 \\
1,846 \\
1,738 \\
1,790 \\
1,795 \\
1,877 \\
1,910 \\
1,966 \\
1,836 \\
1,845\end{array}$ & $\begin{array}{l}18,261 \\
18,620 \\
18,301 \\
17,885 \\
17,957 \\
18,107 \\
18,211 \\
18,658 \\
17,655 \\
19,171 \\
18,535 \\
18,334 \\
18,309\end{array}$ & $\begin{array}{l}14,036 \\
15,138 \\
14,275 \\
13,676 \\
13,778 \\
13,597 \\
14,245 \\
13,873 \\
14,775 \\
14,723 \\
14,700 \\
14,458 \\
14,269\end{array}$ & $\begin{array}{l}1,165 \\
1,172 \\
1,151 \\
1,154 \\
1,113 \\
1,128 \\
1,084 \\
1,113 \\
1,024 \\
1,133 \\
1,064 \\
1,192 \\
1,124\end{array}$ & $\begin{array}{l}41,595 \\
43,690 \\
41,907 \\
40,130 \\
39,709 \\
39,742 \\
40,850 \\
41,097 \\
40,585 \\
42,415 \\
42,354 \\
42,403 \\
41,368\end{array}$ \\
\hline 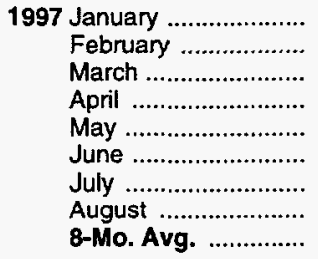 & $\begin{array}{l}1,862 \\
1,862 \\
1,780 \\
1,745 \\
1,823 \\
1,913 \\
1,951 \\
1,886 \\
1,853\end{array}$ & $\begin{array}{l}2,165 \\
2,137 \\
1,796 \\
1,910 \\
1,707 \\
1,882 \\
2,072 \\
1,783 \\
1,929\end{array}$ & $\begin{array}{l}2,901 \\
2,673 \\
2,685 \\
3,225 \\
2,787 \\
3,136 \\
3,098 \\
2,740 \\
2,906\end{array}$ & $\begin{array}{l}2,037 \\
2,126 \\
1,928 \\
1,999 \\
1,898 \\
1,948 \\
2,029 \\
1,809 \\
1,970\end{array}$ & $\begin{array}{l}6,291 \\
6,751 \\
6,146 \\
5,303 \\
5,076 \\
5,131 \\
5,447 \\
5,418 \\
5,686\end{array}$ & $\begin{array}{l}1,828 \\
1,907 \\
1,776 \\
1,823 \\
1,711 \\
1,791 \\
1,766 \\
1,717 \\
1,788\end{array}$ & $\begin{array}{l}18,560 \\
18,308 \\
17,869 \\
18,572 \\
18,244 \\
18,563 \\
19,065 \\
18,506 \\
18,462\end{array}$ & $\begin{array}{r}14,630 \\
14,577 \\
13,576 \\
R_{14,661} \\
R_{13,506} \\
R_{14,289} \\
R_{14,692} \\
13,452 \\
14,165\end{array}$ & $\begin{array}{l}1,138 \\
1,140 \\
1,141 \\
1,174 \\
1,065 \\
1,090 \\
1,142 \\
1,112 \\
1,125\end{array}$ & $\begin{array}{r}42,480 \\
42,638 \\
40,512 \\
R \quad 41,454 \\
R 39,714 \\
40,986 \\
\text { R } 42,297 \\
40,373 \\
41,291\end{array}$ \\
\hline $\begin{array}{l}1996 \text { 8-Mo. Avg. .............. } \\
1995 \text { 8-Mo. Avg. .............. }\end{array}$ & $\begin{array}{l}1,787 \\
1,721\end{array}$ & $\begin{array}{l}1,924 \\
1,876\end{array}$ & $\begin{array}{l}2,902 \\
2,894\end{array}$ & $\begin{array}{l}2,031 \\
1,987\end{array}$ & $\begin{array}{l}5,836 \\
5,657\end{array}$ & $\begin{array}{l}1,820 \\
1,833\end{array}$ & $\begin{array}{l}18,249 \\
17,583\end{array}$ & $\begin{array}{l}14,072 \\
13,933\end{array}$ & $\begin{array}{l}1,134 \\
1,231\end{array}$ & $\begin{array}{l}41,078 \\
40,125\end{array}$ \\
\hline
\end{tabular}

a Through December 1990, the data for Germany are for the former West Germany only. Beginning with January 1991, the data for Germany are for the unified Germany, i.e., the former East Germany and West Germany.

b "OECD Europe" consists of Austria, Belgium, Denmark, Finland, France, Germany, Greece, Iceland, Ireland, Italy, Luxembourg, the Netherlands, Norway, Portugal, Spain, Sweden, Switzerland, Turkey, and the United Kingdom.

"Other OECD" consists of Australia, New Zealand, and the U.S. Territories.

$d$ The Organization for Economic Cooperation and Development (OECD) consists of Canada, Japan, the United States, "OECD Europe" and "Other OECD."

$\mathrm{R}=$ Revised data.

Notes: - Data through 1993 are final. Subsequent data are preliminary. - Totals may not equal sum of components due to independent rounding. - U.S. geographic coverage is the 50 States and the District of Columbia.

Sources: - United States: Table 3.1a. - All Other Data: 1973-1979-International Energy Agency (IEA), Annual Oil and Gas Statistics of OECD Countries. 1980 forward-JEA, quarterly and monthly computer tapes supporting Quarterly Oil Statistics and Energy Balances. 
Figure 10.4 Petroleum Stocks in OECD Countries

(Billion Barrels)

Overview, End of Year, 1973-1996

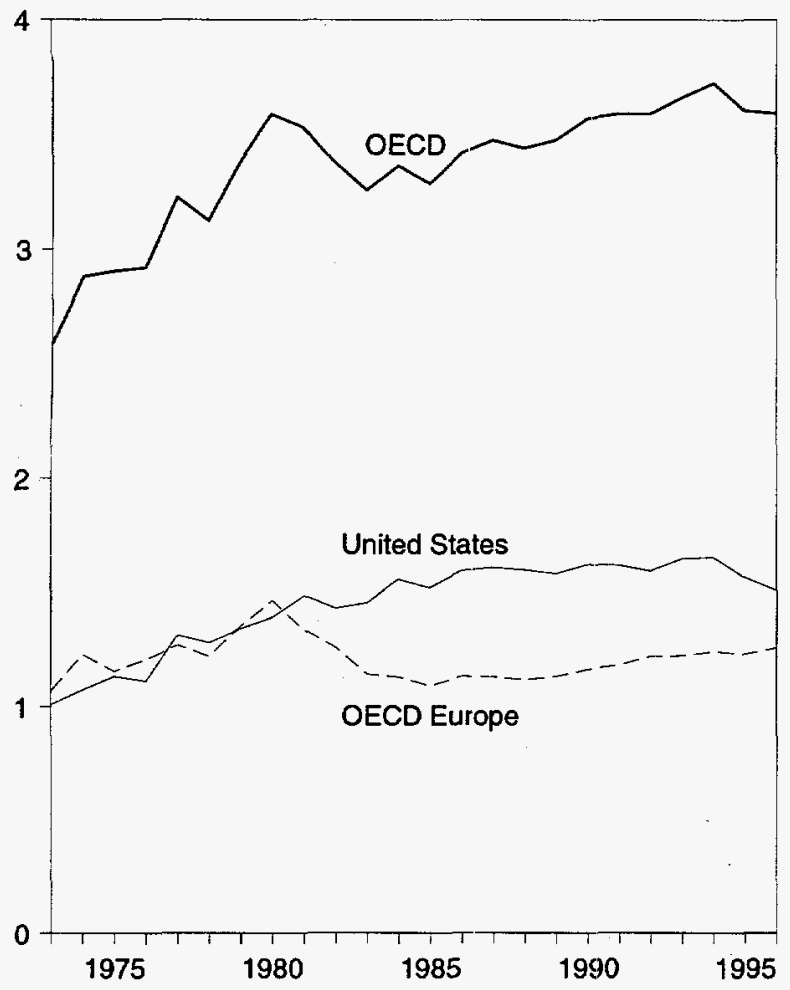

OECD Stocks, End of Month, August

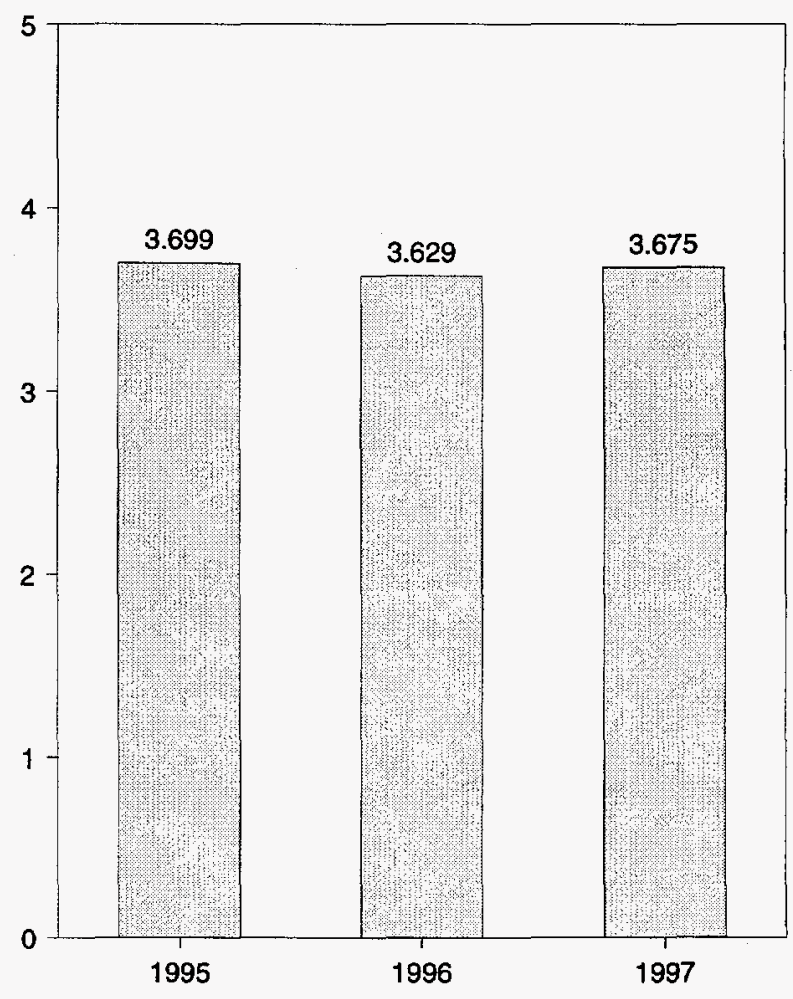

By Selected Country, End of Month

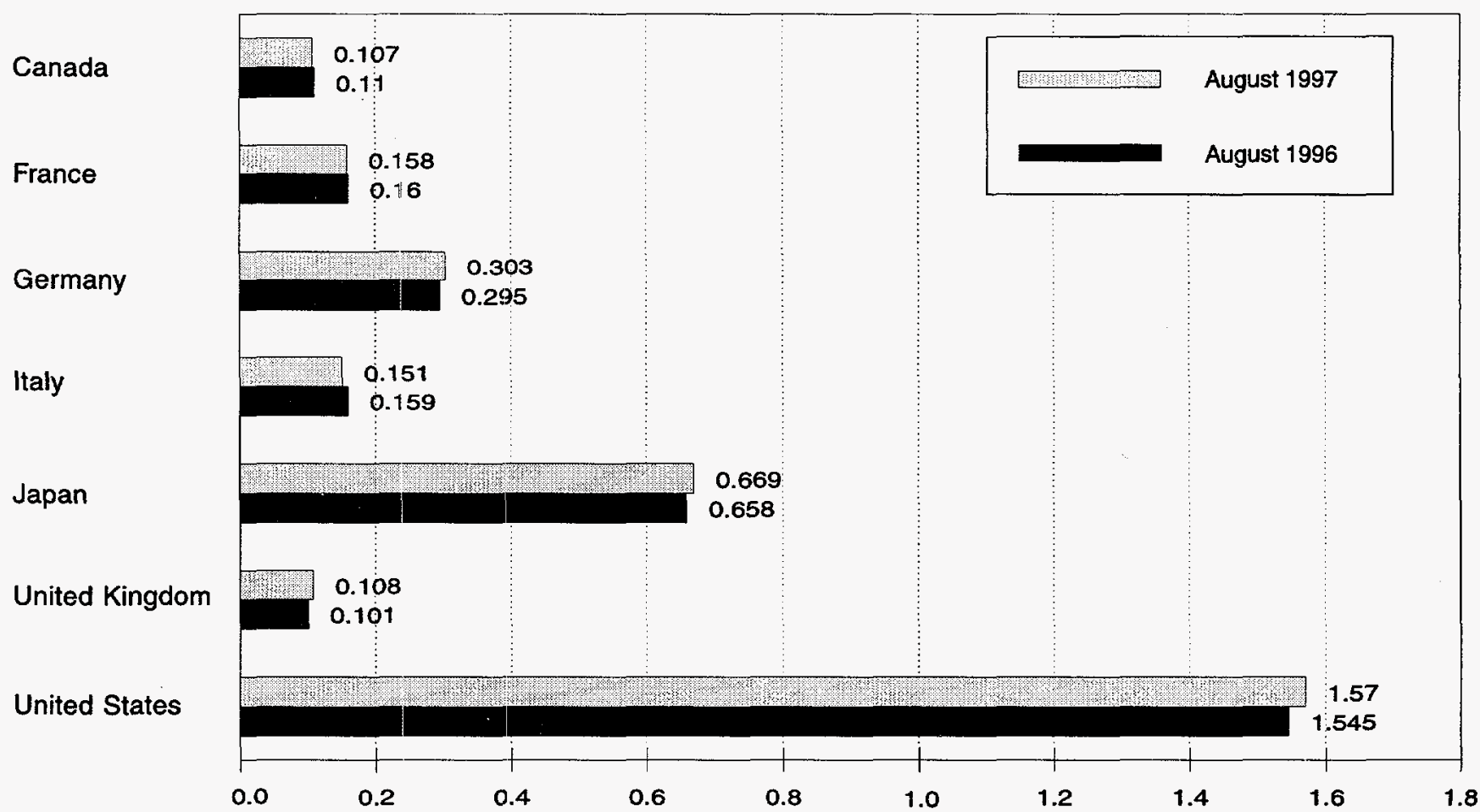

Note: OECD is the Organization for Economic Cooperation and Development.

Source: Table 10.3. 
Table 10.3 Petroleum Stocks in OECD Countries, End of Period (Million Barrels)

\begin{tabular}{|c|c|c|c|c|c|c|c|c|c|c|}
\hline & Canada & France & Germany ${ }^{a}$ & Italy & Japan & $\begin{array}{l}\text { United } \\
\text { Kingdom }\end{array}$ & $\begin{array}{l}\text { United } \\
\text { States }\end{array}$ & $\begin{array}{c}\text { OECD } \\
\text { Europe }^{b}\end{array}$ & $\begin{array}{l}\text { Other } \\
\text { OECDC }\end{array}$ & OECDd \\
\hline 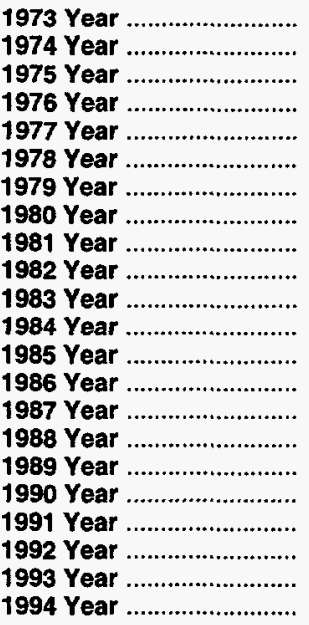 & $\begin{array}{l}140 \\
145 \\
174 \\
153 \\
167 \\
144 \\
150 \\
164 \\
161 \\
136 \\
121 \\
128 \\
113 \\
111 \\
126 \\
116 \\
114 \\
121 \\
119 \\
107 \\
105 \\
119\end{array}$ & $\begin{array}{l}201 \\
249 \\
225 \\
234 \\
239 \\
201 \\
226 \\
243 \\
214 \\
193 \\
153 \\
152 \\
139 \\
127 \\
127 \\
140 \\
138 \\
140 \\
153 \\
146 \\
158 \\
158\end{array}$ & $\begin{array}{l}181 \\
213 \\
187 \\
208 \\
225 \\
238 \\
272 \\
319 \\
297 \\
272 \\
249 \\
239 \\
233 \\
252 \\
259 \\
266 \\
271 \\
265 \\
288 \\
310 \\
309 \\
312\end{array}$ & $\begin{array}{l}152 \\
167 \\
143 \\
143 \\
161 \\
154 \\
163 \\
170 \\
167 \\
179 \\
149 \\
159 \\
157 \\
155 \\
169 \\
155 \\
164 \\
172 \\
160 \\
174 \\
163 \\
164\end{array}$ & $\begin{array}{l}303 \\
370 \\
375 \\
380 \\
409 \\
413 \\
460 \\
495 \\
482 \\
484 \\
470 \\
479 \\
494 \\
509 \\
540 \\
538 \\
577 \\
590 \\
606 \\
603 \\
618 \\
645\end{array}$ & $\begin{array}{l}156 \\
191 \\
165 \\
165 \\
148 \\
157 \\
169 \\
168 \\
143 \\
125 \\
118 \\
112 \\
123 \\
124 \\
121 \\
112 \\
118 \\
112 \\
119 \\
113 \\
118 \\
115\end{array}$ & $\begin{array}{l}1,008 \\
1,074 \\
1,133 \\
1,112 \\
1,312 \\
1,278 \\
1,341 \\
1,392 \\
1,484 \\
1,430 \\
1,454 \\
1,556 \\
1,519 \\
1,593 \\
1,607 \\
1,597 \\
1,581 \\
1,621 \\
1,617 \\
1,592 \\
1,647 \\
1,653\end{array}$ & $\begin{array}{l}1,070 \\
1,227 \\
1,154 \\
1,205 \\
1,268 \\
1,219 \\
1,353 \\
1,464 \\
1,337 \\
1,258 \\
1,142 \\
1,130 \\
1,092 \\
1,133 \\
1,130 \\
1,118 \\
1,133 \\
1,163 \\
1,181 \\
1,219 \\
1,221 \\
1,240\end{array}$ & $\begin{array}{l}67 \\
64 \\
67 \\
68 \\
68 \\
68 \\
75 \\
72 \\
67 \\
68 \\
68 \\
69 \\
66 \\
72 \\
71 \\
71 \\
71 \\
73 \\
65 \\
67 \\
69 \\
69\end{array}$ & $\begin{array}{l}2,588 \\
2,880 \\
2,903 \\
2,918 \\
3,224 \\
3,122 \\
3,379 \\
3,587 \\
3,531 \\
3,376 \\
3,255 \\
3,362 \\
3,284 \\
3,418 \\
3,474 \\
3,440 \\
3,476 \\
3,568 \\
3,588 \\
3,588 \\
3,661 \\
3,726\end{array}$ \\
\hline 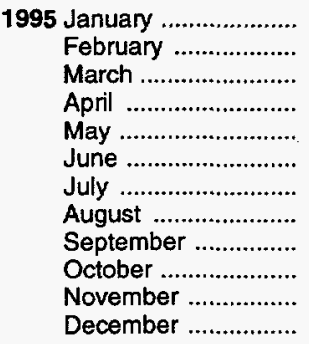 & $\begin{array}{l}121 \\
121 \\
124 \\
122 \\
119 \\
128 \\
130 \\
119 \\
120 \\
123 \\
123 \\
109\end{array}$ & $\begin{array}{l}160 \\
164 \\
152 \\
156 \\
153 \\
166 \\
160 \\
160 \\
162 \\
162 \\
160 \\
159\end{array}$ & $\begin{array}{l}314 \\
316 \\
304 \\
306 \\
304 \\
301 \\
304 \\
303 \\
301 \\
304 \\
297 \\
301\end{array}$ & $\begin{array}{l}167 \\
163 \\
159 \\
159 \\
161 \\
168 \\
171 \\
174 \\
163 \\
165 \\
159 \\
162\end{array}$ & $\begin{array}{l}631 \\
613 \\
619 \\
626 \\
635 \\
640 \\
651 \\
654 \\
658 \\
664 \\
663 \\
630\end{array}$ & $\begin{array}{l}113 \\
114 \\
105 \\
107 \\
112 \\
102 \\
110 \\
109 \\
110 \\
111 \\
110 \\
107\end{array}$ & $\begin{array}{l}1,643 \\
1,608 \\
1,601 \\
1,601 \\
1,612 \\
1,609 \\
1,624 \\
1,614 \\
1,620 \\
1,607 \\
1,604 \\
1,563\end{array}$ & $\begin{array}{l}1,250 \\
1,250 \\
1,189 \\
1,194 \\
1,204 \\
1,208 \\
1,242 \\
1,241 \\
1,232 \\
1,242 \\
1,225 \\
1,228\end{array}$ & $\begin{array}{l}69 \\
64 \\
68 \\
71 \\
72 \\
73 \\
77 \\
72 \\
77 \\
72 \\
72 \\
71\end{array}$ & $\begin{array}{l}3,714 \\
3,655 \\
3,601 \\
3,614 \\
3,641 \\
3,658 \\
3,724 \\
3,699 \\
3,707 \\
3,706 \\
3,685 \\
3,601\end{array}$ \\
\hline 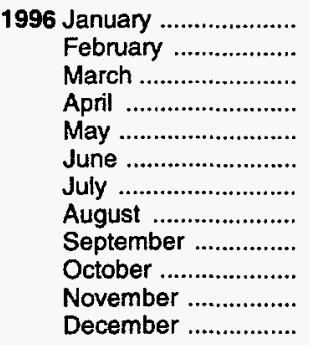 & $\begin{array}{l}104 \\
102 \\
109 \\
109 \\
107 \\
107 \\
110 \\
110 \\
111 \\
111 \\
105 \\
103\end{array}$ & $\begin{array}{l}154 \\
156 \\
156 \\
165 \\
163 \\
160 \\
162 \\
160 \\
152 \\
156 \\
160 \\
158\end{array}$ & $\begin{array}{l}301 \\
298 \\
296 \\
298 \\
295 \\
296 \\
297 \\
295 \\
295 \\
296 \\
297 \\
300\end{array}$ & $\begin{array}{l}157 \\
156 \\
153 \\
150 \\
157 \\
158 \\
155 \\
159 \\
162 \\
155 \\
152 \\
152\end{array}$ & $\begin{array}{l}638 \\
615 \\
627 \\
622 \\
641 \\
640 \\
637 \\
658 \\
664 \\
673 \\
665 \\
651\end{array}$ & $\begin{array}{l}107 \\
103 \\
106 \\
109 \\
105 \\
104 \\
105 \\
101 \\
105 \\
104 \\
106 \\
108\end{array}$ & $\begin{array}{l}1,544 \\
1,500 \\
1,482 \\
1,502 \\
1,520 \\
1,546 \\
1,550 \\
1,545 \\
1,551 \\
1,538 \\
1,522 \\
1,507\end{array}$ & $\begin{array}{l}1,236 \\
1,224 \\
1,212 \\
1,236 \\
1,233 \\
1,229 \\
1,242 \\
1,237 \\
1,229 \\
1,237 \\
1,243 \\
1,256\end{array}$ & $\begin{array}{l}73 \\
69 \\
70 \\
72 \\
75 \\
73 \\
83 \\
79 \\
83 \\
82 \\
81 \\
74\end{array}$ & $\begin{array}{l}3,596 \\
3,511 \\
3,500 \\
3,540 \\
3,575 \\
3,597 \\
3,621 \\
3,629 \\
3,640 \\
3,640 \\
3,616 \\
3,591\end{array}$ \\
\hline 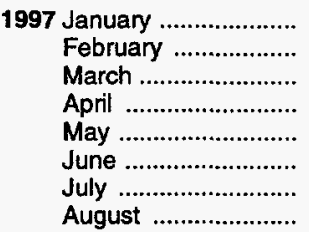 & $\begin{array}{l}104 \\
101 \\
105 \\
108 \\
104 \\
103 \\
105 \\
107\end{array}$ & $\begin{array}{l}156 \\
159 \\
160 \\
159 \\
163 \\
153 \\
153 \\
158\end{array}$ & $\begin{array}{l}304 \\
307 \\
311 \\
300 \\
309 \\
298 \\
304 \\
303\end{array}$ & $\begin{array}{l}158 \\
156 \\
160 \\
151 \\
150 \\
151 \\
150 \\
151\end{array}$ & $\begin{array}{l}650 \\
642 \\
650 \\
665 \\
664 \\
662 \\
670 \\
669\end{array}$ & $\begin{array}{l}107 \\
105 \\
109 \\
108 \\
108 \\
111 \\
106 \\
108\end{array}$ & $\begin{array}{l}1,503 \\
1,482 \\
1,512 \\
1,519 \\
1,562 \\
1,577 \\
1,559 \\
1,570\end{array}$ & $\begin{array}{l}1,279 \\
1,269 \\
1,272 \\
1,247 \\
1,246 \\
1,231 \\
1,226 \\
1,250\end{array}$ & $\begin{array}{l}80 \\
75 \\
76 \\
80 \\
81 \\
83 \\
81 \\
79\end{array}$ & $\begin{array}{l}3,616 \\
3,570 \\
3,616 \\
3,619 \\
3,657 \\
3,656 \\
3,641 \\
3,675\end{array}$ \\
\hline
\end{tabular}

a Through December 1990, the data for Germany are for the former West Germany only. Beginning with January 1991, the data for Germany are for the unified Germany, i.e., the former East Germany and West Germany.

b "OECD Europe" consists of Austria, Belgium, Denmark, Finland, France, Germany, Greece, Iceland, Ireland, Italy, Luxembourg, the Netherlands Norway, Portugal, Spain, Sweden, Switzerland, Turkey, and the United Kingdom.

"Other OECD" consists of Australia, New Zealand, and the U.S. Territories.

$\checkmark$ The Organization for Economic Cooperation and Development (OECD) consists of Canada, Japan, the United States, "OECD Europe" and "Other OECD."

Notes: - Petroleum stocks include crude oil (including strategic resenves) unfinished oils, natural gas plant liquids, and refined products. Petroleum stocks include all nonmilitary petroleum held for storage, regardless of ownership, within each country in bulk terminals, refinery tanks, pipeline tankage, intercoastal tankers, tankers in port, and inland ship bunkers. Data exclude oil held in pipelines (except for those in the United States), rail and truck cars, sea-going ships' bunkers, senvice stations, retail stores, and tankers at sea. - In the United States in January 1975, 1981, and 1983 , numerous respondents were added to bulk terminal and pipeline surveys, thereby affecting subsequent stocks reported. New-basis end-of-year U.S. stocks, in million barrels, would have been 1,121 in 1974, 1,425 in 1980, and 1,461 in 1982. - Data through 1993 are final. Subsequent data are preliminary. - Totals may not equal sum of components due to independent rounding. - U.S. geographic coverage is the 50 States and the District of Columbia.

Sources: " United States: Table 3.1a. - All Other Data: International Energy Agency, quarterly and monthly computer tapes supporting Quarterly Oil Statistics and Energy Balances. 
Figure 10.5 Nuclear Electricity Gross Generation

(Billion Kilowatthours)

U.S. and World, 1973-1996

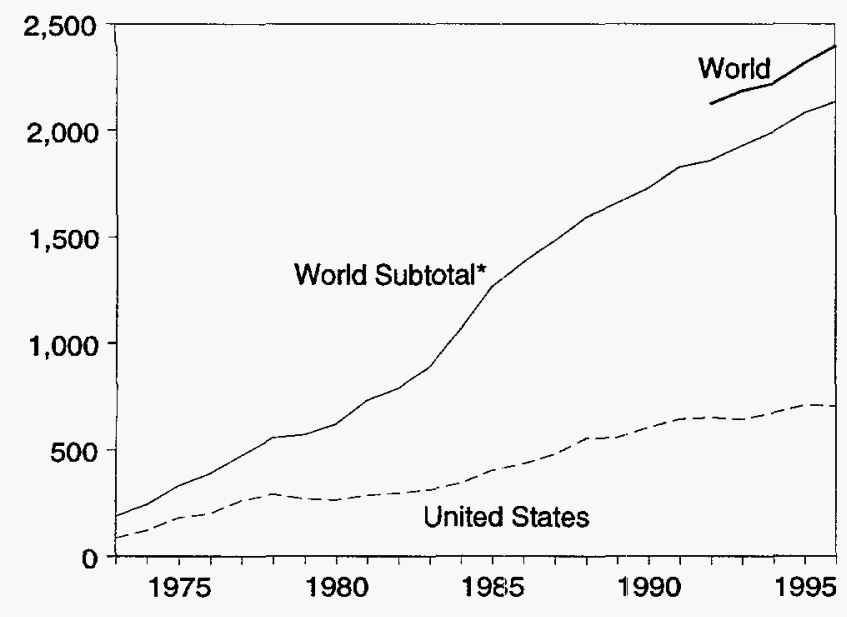

*World excluding Eastern Europe.
By Region, October 1997

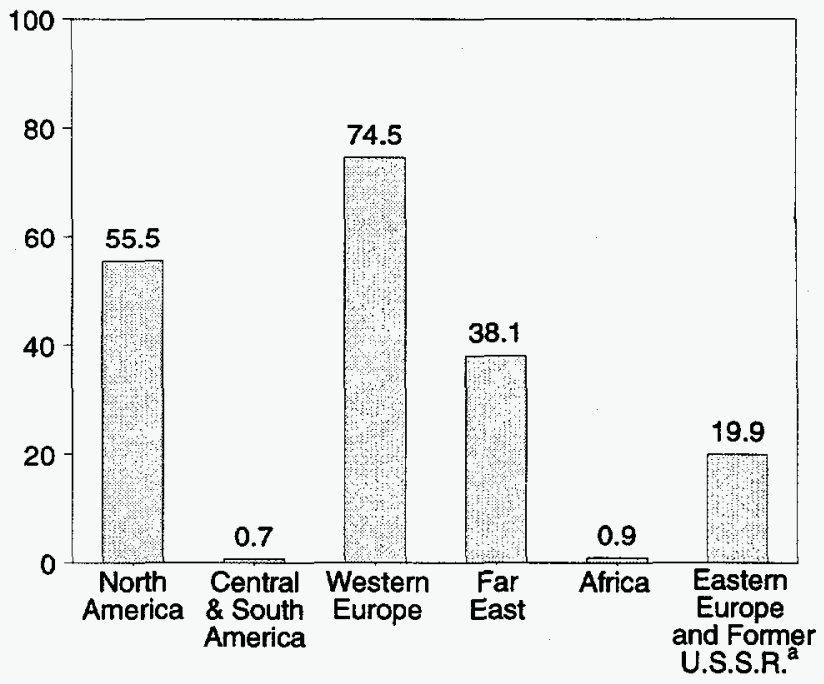

a Does not include Czech Republic or Kazakhstan. See Table 10.4e.

By Selected Country, October 1997

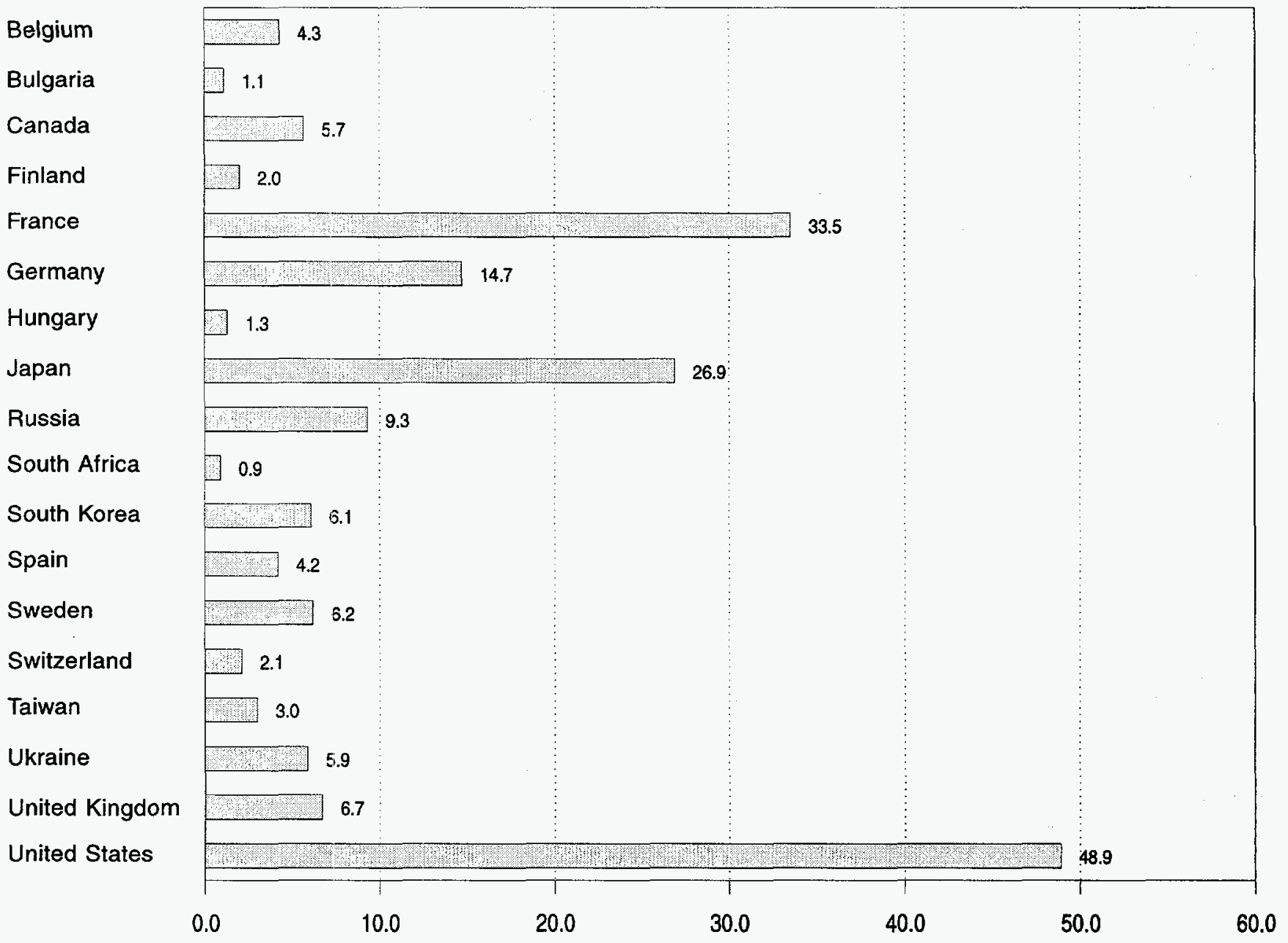

Note: Because vertical scales differ, graphs should not be compared.

Sources: Tables 10.4a-10.4e. 
Table 10.4a Nuclear Electricity Gross Generation: Regions and World

(Billion Kilowatthours)

\begin{tabular}{|c|c|c|c|c|c|c|c|c|}
\hline & $\begin{array}{l}\text { North } \\
\text { America }\end{array}$ & $\begin{array}{c}\text { Central and } \\
\text { South America }\end{array}$ & $\begin{array}{l}\text { Western } \\
\text { Europe }\end{array}$ & Far East & Africa & Subtotal & $\begin{array}{l}\text { Eastern } \\
\text { Europe } \\
\text { and Former } \\
\text { U.S.S.R.a }\end{array}$ & World \\
\hline 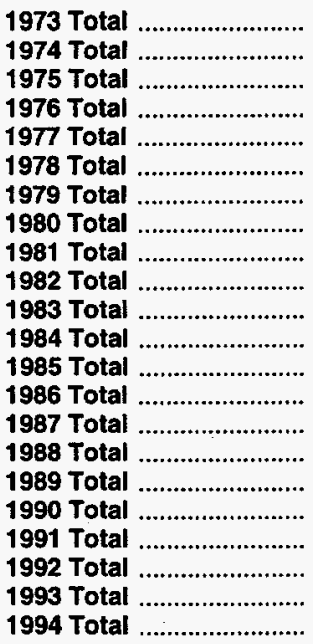 & $\begin{array}{l}103.1 \\
139.7 \\
195.5 \\
219.8 \\
290.8 \\
325.4 \\
309.0 \\
305.8 \\
331.8 \\
341.2 \\
366.6 \\
397.6 \\
465.6 \\
508.8 \\
560.1 \\
639.7 \\
640.2 \\
681.3 \\
733.4 \\
735.2 \\
744.6 \\
787.3\end{array}$ & $\begin{array}{l}- \\
1.0 \\
2.5 \\
2.6 \\
1.6 \\
2.9 \\
2.7 \\
2.3 \\
2.8 \\
1.9 \\
3.6 \\
6.6 \\
9.1 \\
5.8 \\
6.2 \\
5.5 \\
6.6 \\
9.4 \\
9.2 \\
8.8 \\
8.1 \\
8.2\end{array}$ & $\begin{array}{r}73.9 \\
83.9 \\
111.7 \\
126.2 \\
148.1 \\
166.9 \\
184.3 \\
214.2 \\
293.4 \\
321.8 \\
b_{377.2} \\
b_{485.4} \\
b_{582.8} \\
b_{631.5} \\
b_{648.3} \\
b_{688.1} \\
b_{732.2} \\
b_{738.6} \\
b_{769.7} \\
787.8 \\
820.9 \\
820.2\end{array}$ & $\begin{array}{r}12.3 \\
21.4 \\
24.4 \\
40.3 \\
31.5 \\
60.6 \\
74.7 \\
97.4 \\
102.9 \\
123.6 \\
140.1 \\
167.7 \\
202.0 \\
223.6 \\
259.5 \\
248.5 \\
263.4 \\
284.3 \\
303.3 \\
315.2 \\
E 345.2 \\
E 366.7\end{array}$ & $\begin{array}{c}- \\
- \\
- \\
- \\
- \\
- \\
- \\
- \\
- \\
- \\
- \\
4.2 \\
5.9 \\
9.3 \\
6.6 \\
11.1 \\
11.7 \\
8.9 \\
9.7 \\
9.9 \\
7.7 \\
10.3\end{array}$ & $\begin{array}{r}189.3 \\
246.0 \\
334.1 \\
388.9 \\
472.0 \\
555.9 \\
570.7 \\
619.8 \\
730.9 \\
788.5 \\
887.5 \\
1,061.5 \\
1,265.4 \\
1,378.9 \\
1,480.7 \\
1,592.8 \\
1,654.1 \\
1,722.5 \\
1,825.2 \\
1,856.9 \\
\mathbf{1}, 926.6 \\
\mathbf{E}_{\mathbf{1}, 992.6}\end{array}$ & $\begin{array}{l}\text { NA } \\
\text { NA } \\
\text { NA } \\
\text { NA } \\
\text { NA } \\
\text { NA } \\
\text { NA } \\
\text { NA } \\
\text { NA } \\
\text { NA } \\
\text { NA } \\
\text { NA } \\
\text { NA } \\
\text { NA } \\
\text { NA } \\
\text { NA } \\
\text { NA } \\
\text { NA } \\
\text { NA } \\
\text { E 267.5 } \\
\text { E 259.0 } \\
\text { E 227.8 }\end{array}$ & $\begin{array}{c}\text { NA } \\
\text { NA } \\
\text { NA } \\
\text { NA } \\
\text { NA } \\
\text { NA } \\
\text { NA } \\
\text { NA } \\
\text { NA } \\
\text { NA } \\
\text { NA } \\
\text { NA } \\
\text { NA } \\
\text { NA } \\
\text { NA } \\
\text { NA } \\
\text { NA } \\
\text { NA } \\
\text { NA } \\
\text { E }_{2,124.5} \\
\text { E } 2,185.6_{2,220.4}\end{array}$ \\
\hline 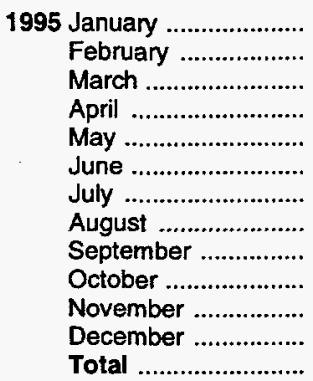 & $\begin{array}{r}75.7 \\
63.1 \\
64.5 \\
59.8 \\
64.2 \\
67.3 \\
75.1 \\
E_{75.6} \\
\text { E }_{68.6} \\
\text { E }_{66.0} \\
\text { E }_{64.2} \\
\text { E }_{72.0} \\
\text { E }_{816.1}\end{array}$ & $\begin{array}{r}1.1 \\
1.0 \\
1.0 \\
.9 \\
.9 \\
.9 \\
1.0 \\
.6 \\
.9 \\
.4 \\
.5 \\
.5 \\
9.6\end{array}$ & $\begin{array}{r}81.9 \\
70.2 \\
74.4 \\
69.6 \\
62.9 \\
61.5 \\
E_{61.1} \\
E_{62.4} \\
E_{63.9} \\
E_{71.5} \\
E_{75.4} \\
E_{81.0} \\
E_{835.7}\end{array}$ & $\begin{array}{r}c_{31.2} \\
c_{29.3} \\
c_{32.1} \\
c_{30.8} \\
c_{31.5} \\
c_{30.2} \\
c_{36.5} \\
c_{39.3} \\
c_{32.4} \\
c_{32.5} \\
c_{32.6} \\
c_{35.6} \\
E_{407.0}\end{array}$ & $\begin{array}{r}1.0 \\
.7 \\
.7 \\
.7 \\
.8 \\
1.1 \\
1.1 \\
1.2 \\
1.3 \\
1.2 \\
1.1 \\
1.0 \\
11.9\end{array}$ & $\begin{array}{r}190.9 \\
164.3 \\
172.6 \\
161.8 \\
160.3 \\
161.0 \\
174.8 \\
179.0 \\
167.2 \\
171.6 \\
173.7 \\
190.1 \\
\text { E }_{2,080.2}\end{array}$ & $\begin{array}{r}b_{22.8} \\
b_{19.6} \\
b_{20.4} \\
b_{17.6} \\
b_{15.1} \\
b_{13.6} \\
b_{14.2} \\
b_{14.9} \\
b_{13.7} \\
b_{16.4} \\
b_{18.3} \\
b_{23.1} \\
E_{234.9}\end{array}$ & $\begin{array}{r}b_{213.7} \\
b_{183.9} \\
b_{193.0} \\
b_{179.3} \\
b_{175.4} \\
b_{174.6} \\
b_{189.0} \\
b_{193.9} \\
b_{180.8} \\
b_{187.9} \\
b_{192.0} \\
b_{213.2} \\
E_{2,315.1}\end{array}$ \\
\hline 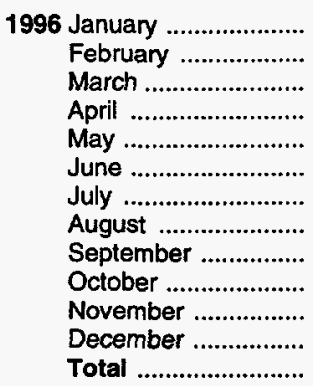 & $\begin{array}{r}E_{76.0} \\
E_{69.0} \\
E_{69.0} \\
61.4 \\
64.7 \\
66.7 \\
72.0 \\
71.5 \\
63.6 \\
61.2 \\
62.4 \\
\text { E } 69.0^{E_{806.4}}\end{array}$ & $\begin{array}{r}1.0 \\
.8 \\
.8 \\
.7 \\
.7 \\
.7 \\
.5 \\
.7 \\
.8 \\
1.0 \\
1.1 \\
1.2 \\
9.8\end{array}$ & $\begin{array}{l}E_{83.4} \\
E_{76.2} \\
E_{77.6} \\
E_{73.2} \\
E_{68.1} \\
E_{63.7} \\
E_{65.9} \\
E_{65.7} \\
E_{69.3} \\
E_{74.4} \\
E_{77.5} \\
E_{84.3} \\
E_{879.5}\end{array}$ & $\begin{array}{r}c_{33.4} \\
c_{30.5} \\
c_{35.0} \\
c_{33.1} \\
c_{33.3} \\
c_{34.2} \\
c_{39.2} \\
c_{39.6} \\
c_{32.7} \\
c_{31.3} \\
c_{33.0} \\
c_{36.9} \\
E_{426.4}\end{array}$ & $\begin{array}{r}.7 \\
.7 \\
1.1 \\
1.1 \\
1.1 \\
.8 \\
.6 \\
1.3 \\
1.3 \\
1.4 \\
1.4 \\
E_{1} 1.1 \\
E_{12.5}\end{array}$ & $\begin{array}{r}194.5 \\
177.1 \\
183.5 \\
169.4 \\
168.0 \\
166.0 \\
178.2 \\
178.8 \\
167.7 \\
169.3 \\
175.4 \\
E_{192.5} \\
E_{2,134.6}\end{array}$ & $\begin{array}{r}b_{24.6} \\
b_{23.3} \\
b_{24.7} \\
b_{20.2} \\
b_{17.2} \\
b_{17.6} \\
b_{16.7} \\
b_{15.4} \\
b_{14.9} \\
b_{17.4} \\
b_{19.9} \\
b_{23.3} \\
E_{261.6}\end{array}$ & $\begin{array}{r}b_{219.1} \\
b_{200.5} \\
b_{208.1} \\
b_{189.6} \\
b_{185.1} \\
b_{183.6} \\
b_{194.9} \\
b_{194.2} \\
b_{182.6} \\
b_{186.7} \\
b_{195.3} \\
b_{215.8} \\
E_{2,396.2}\end{array}$ \\
\hline 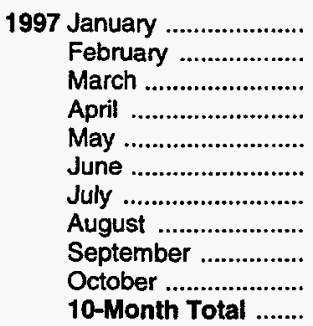 & $\begin{array}{r}E_{70.8} \\
62.1 \\
62.2 \\
E_{56.7} \\
E_{66.8} \\
E_{67.7} \\
E_{71.9} \\
E_{63.2} \\
E_{55.5} \\
\text { E }_{627.4}\end{array}$ & $\begin{array}{r}.9 \\
.9 \\
1.2 \\
1.0 \\
.5 \\
1.1 \\
1.1 \\
1.1 \\
.8 \\
.7 \\
9.4\end{array}$ & $\begin{array}{r}83.3 \\
74.9 \\
E_{79.4} \\
E_{76.7} \\
E_{74.8} \\
E_{66.5} \\
E_{66.2} \\
E_{64.4} \\
E_{67.5} \\
E_{74.5} \\
E_{728.3}\end{array}$ & $\begin{array}{l}c_{36.3} \\
c_{32.6} \\
c_{36.3} \\
E_{35.3} \\
E_{33.7} \\
E_{36.0} \\
E_{42.4} \\
E_{44.8} \\
E_{39.9} \\
E_{38.1} \\
E_{375.5}\end{array}$ & $\begin{array}{r}1.1 \\
.8 \\
.7 \\
1.1 \\
1.4 \\
1.3 \\
1.2 \\
1.2 \\
.7 \\
.9 \\
10.5\end{array}$ & $\begin{array}{r}192.4 \\
171.4 \\
179.7 \\
170.9 \\
167.2 \\
165.7 \\
178.4 \\
183.5 \\
172.2 \\
169.7 \\
1,751.1\end{array}$ & $\begin{array}{l}b_{25.6} \\
b_{23.9} \\
b_{24.6} \\
b_{20.2} \\
b_{18.3} \\
b_{16.7} \\
b_{16.9} \\
b_{17.7} \\
b_{17.9} \\
b_{19.9} \\
b_{201.7}\end{array}$ & $\begin{array}{r}b_{218.0} \\
b_{195.3} \\
b_{204.3} \\
b_{191.2} \\
b_{185.5} \\
b_{182.3} \\
b_{195.3} \\
b_{201.1} \\
b_{190.1} \\
b_{189.6} \\
b_{1,952.8}\end{array}$ \\
\hline $\begin{array}{l}1996 \text { 10-Month Total ....... } \\
1995 \text { 10-Month Total ....... }\end{array}$ & $\begin{array}{l}E_{675.0} \\
E_{679.8}\end{array}$ & $\begin{array}{l}7.6 \\
8.7\end{array}$ & $\begin{array}{l}E_{717.7} \\
E_{679.3}\end{array}$ & $\begin{array}{l}c_{342.1} \\
c_{325.8}\end{array}$ & $\begin{array}{r}10.1 \\
9.8\end{array}$ & $\begin{array}{l}1,752.5 \\
1,703.5\end{array}$ & $\begin{array}{l}b_{192.0} \\
b_{168.2}\end{array}$ & $\begin{array}{l}b_{1,944.4} \\
b_{1,871.7}\end{array}$ \\
\hline
\end{tabular}

a See Table 10.4 e for country-specific estimated annual generation and available monthly generation for Eastern Europe and Former U.S.S.R..

b Sum of available data only.

c Total excluding China.

NA=Not available. $-=$ Not applicable. E=Estimate.

Notes: - Net figures are generally less than gross figures by about 5 percent, the difference being the energy consumed by the generating plants themselves. - Monthly data may not sum to annual toials due to independent rounding and because precommercial generation is included in some annual totals but not in the monthly data. - Data for regions may not sum to totals due to independent rounding.

Source: Based on data from Nucleonics Week, a copyrighted publication of The McGraw-Hill Publishing Companies, Inc. Used with permission. 
Table 10.4b Nuclear Electricity Gross Generation: North, Central, and South America (Billion Kilowatthours)

\begin{tabular}{l|l|l|l|l|l|l|l|}
\hline & Canada & Mexico & United States & North America & Argentina & Central and \\
& Brazil & South America \\
\hline
\end{tabular}

\begin{tabular}{|c|c|c|c|c|c|c|c|}
\hline 1973 Total ................................. & 15.3 & - & 87.8 & 103.1 & - & - & - \\
\hline 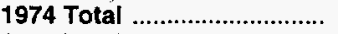 & 15.4 & - & 124.3 & 139.7 & 1.0 & - & 1.0 \\
\hline 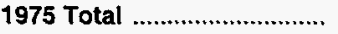 & 13.2 & - & 182.3 & 195.5 & 2.5 & - & 2.5 \\
\hline 1976 Total & 18.0 & - & 201.8 & 219.8 & 2.6 & - & 2.6 \\
\hline 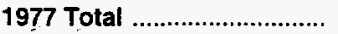 & 26.6 & - & 264.2 & 290.8 & 1.6 & - & 1.6 \\
\hline 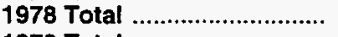 & 33.0 & - & 292.4 & 325.4 & 2.9 & - & 2.9 \\
\hline 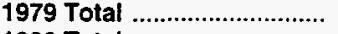 & 38.4 & - & 270.6 & 309.0 & 2.7 & - & 2.7 \\
\hline 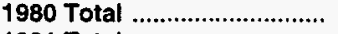 & 40.4 & - & 265.4 & 305.8 & 2.3 & - & 2.3 \\
\hline 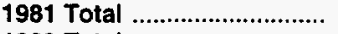 & 43.3 & - & 288.5 & 331.8 & 2.8 & - & 2.8 \\
\hline 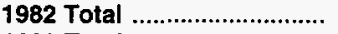 & 42.6 & - & 298.6 & 341.2 & 1.9 & 0.1 & 1.9 \\
\hline 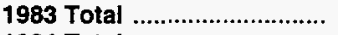 & 53.0 & - & 313.6 & 366.6 & 3.4 & .2 & 3.6 \\
\hline 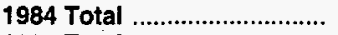 & 53.8 & - & 343.8 & 397.6 & 4.5 & 2.1 & 6.6 \\
\hline 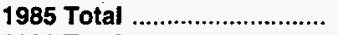 & 62.9 & - & 402.7 & 465.6 & 5.8 & 3.4 & 9.1 \\
\hline 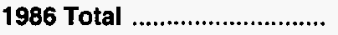 & 74.6 & - & 434.1 & 508.8 & 5.7 & .1 & 5.8 \\
\hline 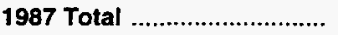 & 80.6 & - & 479.5 & 560.1 & 5.2 & 1.0 & 6.2 \\
\hline 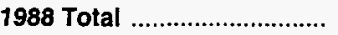 & 85.6 & - & 554.1 & 639.7 & 5.1 & .3 & 5.5 \\
\hline 1989 Total ...................................... & 83.2 & - & 557.0 & 640.2 & 5.0 & 1.6 & 6.6 \\
\hline 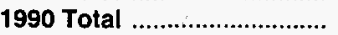 & 75.8 & 2.1 & 603.4 & 681.3 & 7.4 & 2.0 & 9.4 \\
\hline 1991 Total ................................. & 86.1 & 4.2 & 643.0 & 733.4 & 7.7 & 1.4 & 9.2 \\
\hline 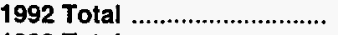 & 81.3 & 3.9 & 650.0 & 735.2 & 7.1 & 1.8 & 8.8 \\
\hline 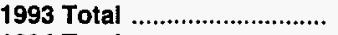 & 97.6 & 4.9 & 642.0 & 744.6 & 7.7 & .4 & 8.1 \\
\hline 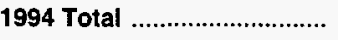 & 110.7 & 4.2 & 672.4 & 787.3 & 8.2 & .0 & 8.2 \\
\hline 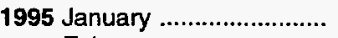 & 9.0 & .3 & 66.4 & 75.7 & .7 & .4 & 1.1 \\
\hline February ............................. & 8.4 & .4 & 54.3 & 63.1 & .6 & .3 & 1.0 \\
\hline 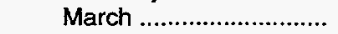 & 9.5 & .4 & 54.6 & 64.5 & .7 & .3 & 1.0 \\
\hline April & 7.6 & .6 & 51.7 & 59.8 & .7 & .2 & :9 \\
\hline 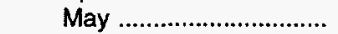 & 6.7 & .5 & 57.1 & 64.2 & .7 & .2 & .9 \\
\hline June & 7.8 & .5 & 59.0 & 67.3 & .7 & .2 & .9 \\
\hline July ……….................. & 9.1 & .9 & 65.1 & 75.1 & .7 & .2 & 1.0 \\
\hline August ............................... & 9.5 & .8 & 65.3 & E75.6 & .6 & .1 & .6 \\
\hline September ........................... & 8.6 & .8 & 59.3 & $E_{68.6}$ & .7 & .2 & .9 \\
\hline October .............................. & 8.1 & .9 & 56.9 & $E_{66.0}$ & .3 & .1 & .4 \\
\hline November ........................... & 8.0 & .8 & 55.4 & $E_{64.2}$ & .2 & .2 & .5 \\
\hline December ........................... & 8.4 & .9 & 62.7 & $E_{72.0}$ & .3 & .2 & .5 \\
\hline 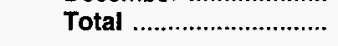 & $E_{100.4}$ & 7.9 & $E_{707.7}$ & $E_{816.1}$ & 7.1 & 2.5 & 9.6 \\
\hline 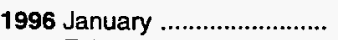 & 9.3 & 1.0 & $E_{65.7}$ & E 76.0 & .7 & .3 & 1.0 \\
\hline February .............................. & 9.3 & .9 & E 58.8 & $E_{69.0}$ & .6 & .2 & .8 \\
\hline March ................................ & 10.2 & .9 & E 57.8 & $E_{69.0}$ & .7 & .1 & .8 \\
\hline April & 8.1 & .9 & 52.4 & 61.4 & .7 & .0 & .7 \\
\hline 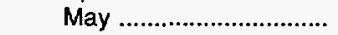 & 6.1 & .9 & 57.7 & 64.7 & .7 & .0 & .7 \\
\hline June & 5.9 & .5 & 60.2 & 66.7 & .7 & .0 & .7 \\
\hline July & 7.7 & .4 & 63.9 & 72.0 & .5 & .0 & .5 \\
\hline August & 8.0 & .3 & 63.2 & 71.5 & .6 & .1 & .7 \\
\hline September .......................... & 6.7 & .5 & 56.4 & 63.6 & 3 & .4 & .8 \\
\hline October ................................. & 7.6 & .5 & 53.1 & 61.2 & .5 & .4 & 1.0 \\
\hline November .......................... & 7.8 & .5 & 54.1 & 62.4 & .7 & .4 & 1.1 \\
\hline December ........................... & 8.5 & .7 & $E_{59.8}$ & $E_{69.0}$ & .7 & .4 & 1.2 \\
\hline 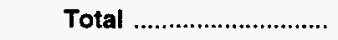 & E 95.2 & 7.9 & $E_{703.3}$ & E 806.4 & 7.4 & 2.4 & 9.8 \\
\hline 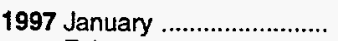 & 8.3 & 1.0 & ${ }^{E} 61.6$ & $E_{70.8}$ & .7 & .3 & .9 \\
\hline February ............................ & 8.3 & .8 & 52.9 & 62.1 & .7 & .3 & .9 \\
\hline 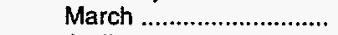 & 8.4 & 1.0 & 52.9 & 62.2 & .7 & .4 & 1.2 \\
\hline April & 8.4 & .9 & 47.4 & 56.7 & .6 & .4 & 1.0 \\
\hline 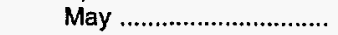 & 5.7 & .9 & $E_{50.2}$ & $E_{56.8}$ & .3 & .3 & .5 \\
\hline June ................................. & 5.7 & .9 & $E_{54.1}$ & ${ }^{E_{60.7}}$ & .7 & .5 & 1.1 \\
\hline July …………………........ & 6.8 & .9 & $E_{59.8}$ & $E_{67.5}$ & .7 & .3 & 1.1 \\
\hline August ............................ & 7.2 & .9 & E 63.8 & $E_{71.9}$ & .7 & .5 & 1.1 \\
\hline September ........................... & 6.1 & .5 & $E_{56.7}$ & ${ }^{E_{63.2}}$ & .7 & .1 & .8 \\
\hline October ................................ & 5.7 & .9 & $E_{48.9}$ & 55.5 & .7 & .0 & .7 \\
\hline 10-Month Total ................ & 70.4 & 8.6 & E548.4 & E 627.4 & 6.5 & 2.9 & 9.4 \\
\hline 1996 10-Month Total ............ & 78.9 & 6.7 & $E_{589.4}$ & $E_{675.0}$ & 6.0 & 1.6 & 7.6 \\
\hline 1995 10-Month Total ............. & 84.1 & 6.2 & E589.6 & E 679.8 & 6.6 & 2.1 & 8.7 \\
\hline
\end{tabular}

$-=$ Not applicable. E=Estimate.

Notes: - Net figures are generally less than gross figures by about 5 percent, the difference being the energy consumed by the generating plants themselves. - Monthly data may not sum to annual totals due to independent rounding and because precommercial generation is included in some annual totals but not in the monthly data. - Data for countries may not sum to regional totals due to independent rounding. - U.S. geographic coverage is the 50 States and the District of Columbia.

Source: Based on data from Nucleonics Week, a copyrighted publication of The McGraw-Hill Publishing Companies, Inc. Used with permission. 
Table 10.4c Nuclear Electricity Gross Generation: Western Europe

(Billion Kilowatthours)

\begin{tabular}{|c|c|c|c|c|c|c|c|c|c|c|c|c|}
\hline & Belgium & Finland & France & Germanya & italy ${ }^{b}$ & $\begin{array}{l}\text { Nether- } \\
\text { lands }\end{array}$ & Slovenia & Spain & Sweden & $\begin{array}{c}\text { Switzer- } \\
\text { land }\end{array}$ & $\begin{array}{c}\text { United } \\
\text { Kingdom }^{c}\end{array}$ & $\begin{array}{l}\text { Western } \\
\text { Europe }\end{array}$ \\
\hline 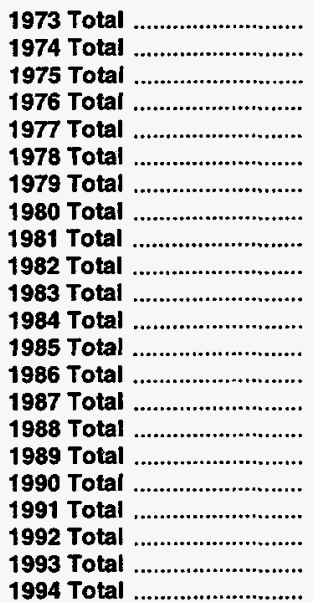 & \begin{tabular}{r|}
0.0 \\
.1 \\
6.8 \\
10.0 \\
11.9 \\
12.5 \\
11.4 \\
12.5 \\
12.8 \\
15.6 \\
24.1 \\
27.7 \\
34.5 \\
38.6 \\
41.9 \\
43.1 \\
41.2 \\
42.7 \\
42.9 \\
43.5 \\
41.9 \\
40.6
\end{tabular} & \begin{tabular}{r|}
- \\
- \\
- \\
- \\
2.7 \\
3.3 \\
6.7 \\
7.0 \\
14.5 \\
16.5 \\
17.4 \\
18.5 \\
18.8 \\
18.8 \\
19.4 \\
19.3 \\
18.8 \\
18.9 \\
19.2 \\
19.0 \\
19.6 \\
19.1
\end{tabular} & \begin{tabular}{|l|}
14.7 \\
14.7 \\
18.3 \\
15.8 \\
17.9 \\
30.6 \\
39.9 \\
61.2 \\
105.2 \\
108.9 \\
144.2 \\
191.2 \\
224.0 \\
254.3 \\
265.5 \\
274.9 \\
302.5 \\
314.1 \\
331.4 \\
337.6 \\
366.7 \\
359.1
\end{tabular} & \begin{tabular}{r|}
11.9 \\
12.0 \\
21.7 \\
24.5 \\
36.0 \\
35.7 \\
42.2 \\
43.7 \\
53.4 \\
63.4 \\
65.8 \\
92.6 \\
125.8 \\
118.9 \\
130.2 \\
145.2 \\
149.6 \\
147.2 \\
147.3 \\
158.8 \\
153.5 \\
151.1
\end{tabular} & $\begin{array}{l}3.1 \\
3.4 \\
3.8 \\
3.8 \\
3.4 \\
4.5 \\
2.6 \\
2.2 \\
2.7 \\
6.8 \\
5.8 \\
6.9 \\
7.0 \\
8.7 \\
.2 \\
.0 \\
.0 \\
.0 \\
.0 \\
.0 \\
.0 \\
.0\end{array}$ & $\begin{array}{l}1.1 \\
3.3 \\
3.3 \\
3.9 \\
3.7 \\
4.1 \\
3.5 \\
4.2 \\
3.7 \\
3.9 \\
3.6 \\
3.8 \\
3.9 \\
4.2 \\
3.6 \\
3.7 \\
4.0 \\
3.4 \\
3.3 \\
3.8 \\
3.9\end{array}$ & $\begin{array}{c}- \\
- \\
- \\
- \\
- \\
- \\
- \\
- \\
- \\
- \\
\text { NA } \\
\text { NA } \\
\text { NA } \\
\text { NA } \\
\text { NA } \\
\text { NA } \\
\text { NA } \\
\text { NA } \\
\text { NA } \\
\text { E.0 } \\
4.0 \\
4.6\end{array}$ & \begin{tabular}{r|}
6.5 \\
7.2 \\
7.5 \\
7.6 \\
6.5 \\
7.6 \\
6.7 \\
5.2 \\
9.4 \\
8.8 \\
10.7 \\
23.1 \\
28.0 \\
37.5 \\
41.2 \\
50.4 \\
56.1 \\
54.3 \\
55.6 \\
$\mathbf{5 5 . 8}$ \\
$\mathbf{5 6 . 1}$ \\
$\mathbf{5 5} .1$
\end{tabular} & \begin{tabular}{r|}
2.1 \\
2.3 \\
12.0 \\
16.0 \\
19.9 \\
23.8 \\
21.0 \\
26.7 \\
37.7 \\
38.8 \\
40.4 \\
51.3 \\
58.6 \\
69.9 \\
67.2 \\
69.4 \\
65.6 \\
68.2 \\
76.8 \\
63.5 \\
61.4 \\
72.8
\end{tabular} & $\begin{array}{r}6.2 \\
7.0 \\
7.7 \\
7.9 \\
8.1 \\
8.3 \\
11.8 \\
14.3 \\
15.2 \\
15.0 \\
15.5 \\
16.3 \\
22.4 \\
22.5 \\
23.0 \\
22.7 \\
22.8 \\
23.6 \\
22.9 \\
23.4 \\
23.3 \\
24.2\end{array}$ & $\begin{array}{l}28.2 \\
33.8 \\
30.5 \\
36.8 \\
38.1 \\
36.6 \\
38.5 \\
37.2 \\
38.9 \\
44.1 \\
49.6 \\
54.1 \\
59.7 \\
58.2 \\
56.2 \\
59.4 \\
71.6 \\
66.1 \\
70.4 \\
78.5 \\
90.4 \\
89.5\end{array}$ & $\begin{array}{r}73.9 \\
83.9 \\
111.7 \\
126.2 \\
148.1 \\
166.9 \\
184.3 \\
214.2 \\
293.4 \\
321.8 \\
d_{377.2} \\
d_{485.4} \\
d_{582.8} \\
d_{631.5} \\
d_{648.3} \\
d_{688.1} \\
d_{732.2} \\
d_{738.6} \\
d_{769.7} \\
787.8 \\
820.9 \\
820.2\end{array}$ \\
\hline 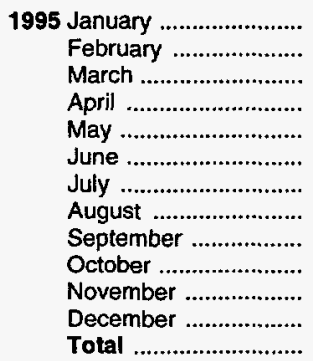 & $\begin{array}{r}4.2 \\
3.7 \\
3.6 \\
4.0 \\
3.4 \\
3.1 \\
2.5 \\
2.5 \\
2.7 \\
3.7 \\
3.8 \\
4.2 \\
41.4\end{array}$ & $\begin{array}{r}1.6 \\
1.5 \\
1.8 \\
1.7 \\
1.3 \\
1.6 \\
1.7 \\
1.4 \\
1.6 \\
1.6 \\
1.4 \\
1.7 \\
18.9\end{array}$ & $\begin{array}{l}38.7 \\
31.7 \\
34.4 \\
30.6 \\
28.3 \\
27.1 \\
28.2 \\
29.0 \\
27.9 \\
31.1 \\
34.4 \\
36.2 \\
377.6\end{array}$ & $\begin{array}{l}15.2 \\
13.1 \\
12.4 \\
12.2 \\
10.2 \\
11.3 \\
11.2 \\
12.1 \\
12.5 \\
13.9 \\
14.8 \\
15.2 \\
154.3\end{array}$ & $\begin{array}{l}.0 \\
.0 \\
.0 \\
.0 \\
.0 \\
.0 \\
.0 \\
.0 \\
.0 \\
.0 \\
.0 \\
.0 \\
.0\end{array}$ & $\begin{array}{l}.3 \\
\text { (s) } \\
.1 \\
.4 \\
.4 \\
.4 \\
.4 \\
.4 \\
.4 \\
.4 \\
.4 \\
.4 \\
4.0\end{array}$ & $\begin{array}{l}.5 \\
.4 \\
.5 \\
.3 \\
.0 \\
.4 \\
.5 \\
.4 \\
.4 \\
.5 \\
.5 \\
.5 \\
4.8\end{array}$ & $\begin{array}{r}5.4 \\
4.6 \\
4.6 \\
4.3 \\
5.0 \\
4.7 \\
4.3 \\
4.3 \\
4.0 \\
4.1 \\
3.8 \\
5.4 \\
54.5\end{array}$ & $\begin{array}{r}7.2 \\
6.2 \\
6.6 \\
6.5 \\
5.6 \\
3.5 \\
4.0 \\
4.5 \\
5.2 \\
6.6 \\
6.8 \\
7.3 \\
69.9\end{array}$ & $\begin{array}{r}2.4 \\
2.2 \\
2.4 \\
2.0 \\
2.1 \\
1.6 \\
1.6 \\
1.3 \\
2.0 \\
2.4 \\
2.3 \\
2.4 \\
24.8\end{array}$ & $\begin{array}{r}6.4 \\
6.8 \\
8.0 \\
7.5 \\
6.5 \\
7.9 \\
E_{6.8} \\
E_{6.4} \\
E_{7.2} \\
E_{7.2} \\
E_{7.2} \\
E_{7.7} \\
E_{85.5}\end{array}$ & $\begin{array}{r}81.9 \\
70.2 \\
74.4 \\
69.6 \\
62.9 \\
61.5 \\
E_{61.1} \\
E_{62.4} \\
E_{63.9} \\
E_{71.5} \\
E_{75.4} \\
E_{81.0} \\
E_{835.7}\end{array}$ \\
\hline 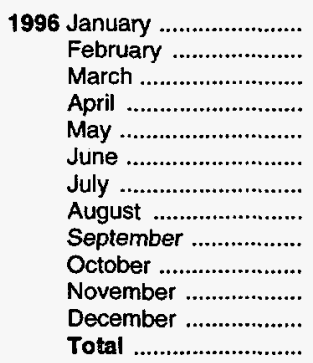 & $\begin{array}{r}4.3 \\
4.1 \\
3.9 \\
3.4 \\
3.4 \\
3.2 \\
3.3 \\
3.1 \\
3.5 \\
3.3 \\
4.0 \\
3.7 \\
43.3\end{array}$ & $\begin{array}{r}1.8 \\
1.7 \\
1.8 \\
1.7 \\
1.4 \\
1.4 \\
1.6 \\
1.4 \\
1.4 \\
1.7 \\
1.8 \\
1.8 \\
19.5\end{array}$ & $\begin{array}{r}38.5 \\
35.5 \\
35.8 \\
33.3 \\
30.6 \\
27.7 \\
30.0 \\
29.9 \\
30.8 \\
34.0 \\
34.8 \\
36.3 \\
397.0\end{array}$ & $\begin{array}{r}15.0 \\
12.7 \\
13.1 \\
12.6 \\
12.4 \\
12.0 \\
12.6 \\
13.1 \\
13.3 \\
13.8 \\
15.1 \\
15.9 \\
161.7\end{array}$ & $\begin{array}{l}.0 \\
.0 \\
.0 \\
.0 \\
.0 \\
.0 \\
.0 \\
.0 \\
.0 \\
.0 \\
.0 \\
.0 \\
.0\end{array}$ & $\begin{array}{l}.4 \\
.1 \\
.2 \\
.4 \\
.4 \\
.4 \\
.4 \\
.4 \\
.4 \\
.4 \\
.4 \\
.4 \\
4.2\end{array}$ & $\begin{array}{r}.5 \\
.5 \\
.5 \\
.5 \\
.3 \\
.0 \\
.1 \\
.5 \\
.5 \\
.5 \\
.5 \\
\text { E.5 } \\
\text { E } 4.6\end{array}$ & $\begin{array}{r}5.4 \\
4.9 \\
4.9 \\
4.6 \\
5.3 \\
4.6 \\
4.6 \\
4.6 \\
4.6 \\
5.1 \\
4.8 \\
5.5 \\
59.1\end{array}$ & $\begin{array}{r}7.4 \\
7.2 \\
7.5 \\
7.3 \\
5.0 \\
5.8 \\
4.7 \\
4.4 \\
5.7 \\
7.0 \\
6.9 \\
7.4 \\
76.2\end{array}$ & $\begin{array}{r}2.4 \\
2.3 \\
2.4 \\
2.3 \\
2.3 \\
1.6 \\
1.6 \\
1.2 \\
2.0 \\
2.2 \\
2.3 \\
2.4 \\
25.0\end{array}$ & $\begin{array}{l}E_{7.7} \\
E_{7.4} \\
E_{7.5} \\
E_{7.0} \\
E_{7.0} \\
E_{7.0} \\
E_{7.0} \\
E_{7.0} \\
E_{7.1} \\
E_{6.6} \\
E_{7.0} \\
E_{10.4} \\
E_{88.8}\end{array}$ & $\begin{array}{r}E_{83.4} \\
E_{76.2} \\
E_{77.6} \\
E_{73.2} \\
E_{68.1} \\
E_{63.7} \\
E_{65.9} \\
E_{65.7} \\
E_{69.3} \\
E_{74.4} \\
E_{77.5} \\
E_{84.3} \\
E_{879.5}\end{array}$ \\
\hline 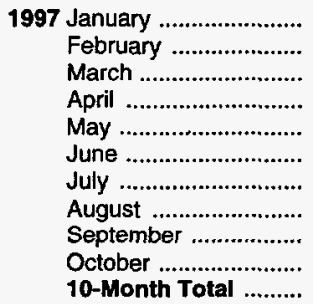 & $\begin{array}{r}4.4 \\
4.0 \\
4.4 \\
3.8 \\
4.3 \\
2.9 \\
2.9 \\
3.6 \\
3.8 \\
4.3 \\
38.6\end{array}$ & $\begin{array}{r}1.8 \\
1.7 \\
1.9 \\
1.8 \\
1.4 \\
1.5 \\
1.9 \\
1.6 \\
1.6 \\
2.0 \\
17.1\end{array}$ & $\begin{array}{r}37.1 \\
32.4 \\
33.8 \\
33.8 \\
\text { E } 33.8 \\
28.0 \\
29.2 \\
28.7 \\
29.7 \\
33.5 \\
\text { E } 319.9\end{array}$ & $\begin{array}{r}16.2 \\
14.2 \\
15.3 \\
15.3 \\
13.4 \\
13.0 \\
12.9 \\
12.4 \\
12.8 \\
14.7 \\
140.1\end{array}$ & $\begin{array}{l}.0 \\
.0 \\
.0 \\
.0 \\
.0 \\
.0 \\
.0 \\
.0 \\
.0 \\
.0 \\
.0\end{array}$ & $\begin{array}{r}.3 \\
.1 \\
.4 \\
.4 \\
\mathrm{E}(\mathrm{s}) \\
.0 \\
.2 \\
\mathrm{E} .2 \\
.3 \\
.3 \\
\mathrm{E}_{2.4}\end{array}$ & $\begin{array}{l}.4 \\
.4 \\
.5 \\
.5 \\
.5 \\
.3 \\
.5 \\
.5 \\
.5 \\
.5 \\
4.4\end{array}$ & $\begin{array}{r}5.2 \\
4.6 \\
3.8 \\
4.2 \\
5.2 \\
4.8 \\
4.9 \\
4.9 \\
4.4 \\
4.2 \\
46.4\end{array}$ & $\begin{array}{r}7.1 \\
6.8 \\
E_{7.3} \\
7.0 \\
5.6 \\
E_{5.0} \\
4.0 \\
E_{4.1} \\
4.5 \\
6.2 \\
E_{57.6}\end{array}$ & $\begin{array}{r}2.4 \\
2.2 \\
2.4 \\
2.3 \\
2.3 \\
1.6 \\
1.9 \\
1.3 \\
2.1 \\
2.1 \\
20.5\end{array}$ & $\begin{array}{r}8.3 \\
8.6 \\
9.6 \\
E_{7.7} \\
E_{8.2} \\
9.3 \\
E_{7.6} \\
E_{7.1} \\
E_{8.0} \\
E_{6.7} \\
E_{81.2}\end{array}$ & $\begin{array}{r}83.3 \\
74.9 \\
E_{79.4} \\
E_{76.7} \\
E_{74.8} \\
E_{66.5} \\
E_{66.2} \\
E_{64.4} \\
E_{67.5} \\
E_{74.5} \\
E_{728.3}\end{array}$ \\
\hline $\begin{array}{l}1996 \text { 10-Month Total .......... } \\
1995 \text { 10-Month Total ......... }\end{array}$ & $\begin{array}{l}35.6 \\
33.4\end{array}$ & $\begin{array}{l}15.9 \\
15.9\end{array}$ & $\begin{array}{l}325.9 \\
306.9\end{array}$ & $\begin{array}{l}130.7 \\
124.3\end{array}$ & $\begin{array}{l}.0 \\
.0\end{array}$ & $\begin{array}{l}3.4 \\
3.2\end{array}$ & $\begin{array}{l}3.7 \\
3.8\end{array}$ & $\begin{array}{l}48.8 \\
45.3\end{array}$ & $\begin{array}{l}61.9 \\
55.8\end{array}$ & $\begin{array}{l}20.3 \\
20.1\end{array}$ & $\begin{array}{l}E_{71.4} \\
E_{70.6}\end{array}$ & $\begin{array}{l}E_{717.7} \\
E_{679.3}\end{array}$ \\
\hline
\end{tabular}

a Through December 1990, the data for Germany are for the former West Germany only. Beginning with January 1991, the data for Germany are for the unified Germany, i.e., the former East Germany and West Germany.

b In 1987, Italy's citizens voted for a nuclear power moratorium, which shut down their nuclear power plants indefinitely.

c Monthly data for the United Kingdom are totals for 4- or 5-week reporting periods, not calendar months.

d Sum of available data only

$\mathrm{NA}=$ Not available. $-=$ Not applicable. $E=$ Estimate. $(\mathrm{s})=$ Less than 0.05 billion kilowatthours.

Notes: - Net figures are generally less than gross figures by about 5 percent, the difference being the energy consumed by the generating plants themselves. - Monthly data may not sum to annual totals due to independent rounding and because precommercial generation is included in some annual totals but not in the monthly data. - Data for countries may not sum to regional totals due to independent rounding.

Source: Based on data from Nucleonics Week, a copyrighted publication of The McGraw-Hill Publishing Companies, Inc. Used with permission. 
Table 10.4d Nuclear Electricity Gross Generation: Far East and Africa

(Billion Kilowatthours)

\begin{tabular}{|c|c|c|c|c|c|c|c|c|}
\hline$\because$ & China ${ }^{a}$ & India & Japan & Pakistan & $\begin{array}{l}\text { South } \\
\text { Korea }\end{array}$ & Taiwan & Far East & $\begin{array}{l}\text { South } \\
\text { Africab }\end{array}$ \\
\hline 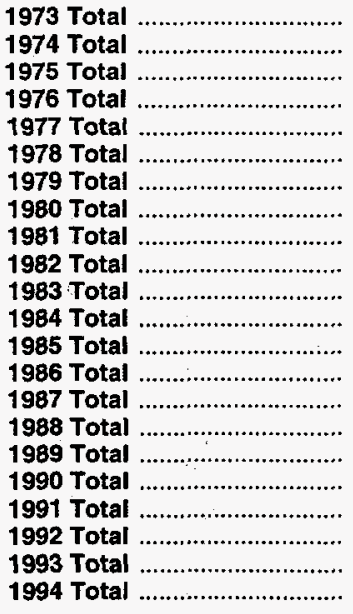 & $\begin{array}{l}- \\
- \\
- \\
\bar{z} \\
- \\
- \\
- \\
- \\
- \\
- \\
- \\
- \\
- \\
- \\
- \\
- \\
\overline{2} .6 \\
14.2\end{array}$ & $\begin{array}{l}2.5 \\
1.9 \\
2.5 \\
3.2 \\
2.8 \\
2.3 \\
3.2 \\
2.9 \\
31.1 \\
2.2 \\
2 . .9 \\
41.1 \\
41.5 \\
51.1 \\
51.5 \\
6 i .1 \\
4.0 \\
61.3 \\
5.4 \\
6 i .3 \\
6.2 \\
51.0\end{array}$ & $\begin{array}{r}9.4 \\
18.9 \\
21.3 \\
36.6 \\
28.2 \\
53.1 \\
62.0 \\
82.8 \\
86.0 \\
104.5 \\
109.1 \\
127.2 \\
152.0 \\
164.8 \\
182.8 \\
173.6 \\
183.7 \\
191.9 \\
205.8 \\
218.0 \\
243.5 \\
253.8\end{array}$ & $\begin{array}{l}0.5 \\
.6 \\
.5 \\
.5 \\
.3 \\
.2 \\
(s) \\
.1 \\
.2 \\
.1 \\
.2 \\
.3 \\
.3 \\
.5 \\
.3 \\
.2 \\
.1 \\
.4 \\
.4 \\
.6 \\
.4 \\
.6\end{array}$ & $\begin{array}{l}- \\
- \\
- \\
- \\
0.1 \\
2.3 \\
3.2 \\
3.5 \\
2.9 \\
3.8 \\
9.0 \\
11.8 \\
16.5 \\
26.1 \\
37.8 \\
38.7 \\
47.2 \\
52.8 \\
56.3 \\
56.4 \\
58.1 \\
58.3\end{array}$ & $\begin{array}{c}- \\
- \\
- \\
- \\
0.1 \\
2.7 \\
6.3 \\
8.2 \\
10.7 \\
13.1 \\
18.9 \\
24.3 \\
28.7 \\
26.9 \\
33.1 \\
29.9 \\
28.3 \\
32.9 \\
35.3 \\
33.8 \\
34.3 \\
34.8\end{array}$ & $\begin{array}{r}12.3 \\
21.4 \\
24.4 \\
40.3 \\
31.5 \\
60.6 \\
74.7 \\
97.4 \\
102.9 \\
123.6 \\
140.1 \\
167.7 \\
202.0 \\
223.6 \\
259.5 \\
248.5 \\
263.4 \\
284.3 \\
303.3 \\
315.2 \\
\text { E } 345.2 \\
\text { E } 366.7\end{array}$ & $\begin{array}{r}- \\
- \\
- \\
- \\
- \\
- \\
- \\
- \\
- \\
- \\
\overline{-} \\
4.2 \\
5.9 \\
9.3 \\
6.6 \\
11.1 \\
11.7 \\
8.9 \\
9.7 \\
9.9 \\
7.7 \\
10.3\end{array}$ \\
\hline 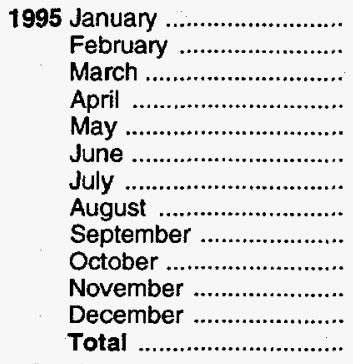 & $\begin{array}{l}E .0 \\
\text { NA } \\
\text { NA } \\
\text { NA } \\
\text { NA } \\
\text { NA } \\
\text { NA } \\
\text { NA } \\
\text { NA } \\
\text { NA } \\
\text { NA } \\
\text { NA } \\
\text { 13.0 }\end{array}$ & $\begin{array}{r}.7 \\
.5 \\
.6 \\
.6 \\
.7 \\
.7 \\
.8 \\
.8 \\
.8 \\
.5 \\
.5 \\
.6 \\
8.0\end{array}$ & $\begin{array}{r}23.1 \\
21.5 \\
23.6 \\
22.6 \\
22.1 \\
20.6 \\
26.3 \\
29.0 \\
23.9 \\
23.8 \\
23.5 \\
26.1 \\
286.1\end{array}$ & $\begin{array}{l}\text { (s) } \\
(\mathrm{s}) \\
(\mathrm{s}) \\
(\mathrm{s}) \\
(\mathrm{s}) \\
.1 \\
.1 \\
.1 \\
(\mathrm{~s}) \\
.1 \\
(\mathrm{~s}) \\
.1 \\
.5\end{array}$ & $\begin{array}{r}4.8 \\
4.9 \\
5.1 \\
4.9 \\
5.4 \\
5.5 \\
6.1 \\
5.9 \\
4.8 \\
5.1 \\
5.5 \\
5.9 \\
64.0\end{array}$ & $\begin{array}{r}2.5 \\
2.3 \\
2.7 \\
2.7 \\
3.2 \\
3.4 \\
3.3 \\
3.4 \\
2.8 \\
3.0 \\
3.0 \\
2.9 \\
35.3\end{array}$ & $\begin{array}{r}c_{31.2} \\
c_{29.3} \\
c_{32.1} \\
c_{30.8} \\
c_{31.5} \\
c_{30.2} \\
c_{36.5} \\
c_{39.3} \\
c_{32.4} \\
c_{32.5} \\
c_{32.6} \\
c_{35.6} \\
\text { E }_{407.0}\end{array}$ & $\begin{array}{r}1.0 \\
.7 \\
.7 \\
.7 \\
.8 \\
1.1 \\
1.1 \\
1.2 \\
1.3 \\
1.2 \\
1.1 \\
1.0 \\
11.9\end{array}$ \\
\hline 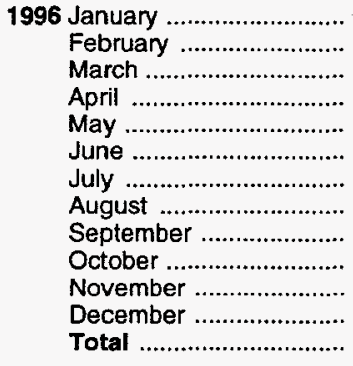 & $\begin{array}{l}\text { NA } \\
\text { NA } \\
\text { NA } \\
\text { NA } \\
\text { NA } \\
\text { NA } \\
\text { NA } \\
\text { NA } \\
\text { NA } \\
\text { NA } \\
\text { NA } \\
\text { NA } \\
\text { 14.3 }\end{array}$ & $\begin{array}{l}.6 \\
.7 \\
.8 \\
.8 \\
.6 \\
.7 \\
.4 \\
.4 \\
.7 \\
.9 \\
.8 \\
.9 \\
8.3\end{array}$ & $\begin{array}{r}24.5 \\
22.2 \\
25.1 \\
24.1 \\
23.5 \\
23.7 \\
27.9 \\
29.0 \\
22.4 \\
21.1 \\
23.0 \\
26.7 \\
293.2\end{array}$ & $\begin{array}{l}\text { (s) } \\
\text { (s) } \\
\text { (s) } \\
\text { (s) } \\
\text { (s) } \\
\text { (s) } \\
\text { (s) } \\
\text { (s) } \\
\text { (s) } \\
(\mathrm{s}) \\
\text { (s) } \\
.0 \\
.4\end{array}$ & $\begin{array}{r}5.2 \\
4.8 \\
6.2 \\
5.6 \\
5.8 \\
6.5 \\
7.3 \\
6.6 \\
6.3 \\
5.8 \\
5.9 \\
6.4 \\
72.5\end{array}$ & $\begin{array}{r}3.0 \\
2.7 \\
2.9 \\
2.5 \\
3.3 \\
3.2 \\
3.7 \\
3.5 \\
3.2 \\
3.4 \\
3.3 \\
3.0 \\
37.8\end{array}$ & $\begin{array}{r}c_{33.4} \\
c_{30.5} \\
c_{35.0} \\
c_{33.1} \\
c_{33.3} \\
c_{34.2} \\
c_{39.2} \\
c_{39.6} \\
c_{32.7} \\
c_{31.3} \\
c_{33.0} \\
c_{36.9} \\
\text { E }_{426.4}\end{array}$ & $\begin{array}{r}.7 \\
.7 \\
1.1 \\
1.1 \\
1.1 \\
.8 \\
.6 \\
1.3 \\
1.3 \\
1.4 \\
1.4 \\
\mathrm{E}_{1} 1.1 \\
\mathrm{E}_{12.5}\end{array}$ \\
\hline 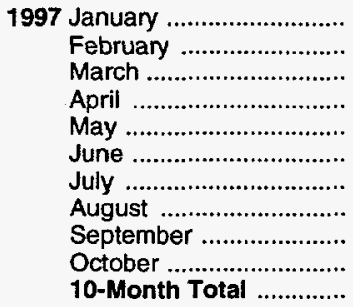 & $\begin{array}{r}\text { NA } \\
\text { NA } \\
\text { NA } \\
.7 \\
1.1 \\
E_{1.1} \\
E_{1.1} \\
E_{1.1} \\
E_{1.1} \\
\text { NA }\end{array}$ & $\begin{array}{r}1.0 \\
.9 \\
.9 \\
\text { E.9 } \\
\text { E.9 } \\
\text { E.9 } \\
\text { E.9 } \\
1.0 \\
1.0 \\
1.0 \\
\text { E.4 }\end{array}$ & $\begin{array}{r}26.1 \\
22.7 \\
26.2 \\
25.4 \\
22.9 \\
24.4 \\
29.0 \\
31.2 \\
27.7 \\
26.9 \\
262.5\end{array}$ & $\begin{array}{l}.0 \\
(\mathrm{~s}) \\
(\mathrm{s}) \\
(\mathrm{s}) \\
(\mathrm{s}) \\
(\mathrm{s}) \\
(\mathrm{s}) \\
(\mathrm{s}) \\
(\mathrm{s}) \\
(\mathrm{s}) \\
.4\end{array}$ & $\begin{array}{r}6.1 \\
6.1 \\
\mathrm{E} 6.1 \\
5.6 \\
5.8 \\
6.7 \\
7.8 \\
7.8 \\
7.1 \\
6.1 \\
\mathrm{E} 65.2\end{array}$ & $\begin{array}{r}3.1 \\
2.9 \\
3.1 \\
2.7 \\
2.9 \\
E_{2.9} \\
3.5 \\
E_{3.5} \\
E_{2.9} \\
3.0 \\
E_{30.4}\end{array}$ & $\begin{array}{r}c_{36.3} \\
c_{32.6} \\
c_{36.3} \\
E_{35.3} \\
E_{33.7} \\
E_{36.0} \\
E_{42.4} \\
E_{44.8} \\
E_{39.9} \\
E_{38.1} \\
E_{375.5}\end{array}$ & $\begin{array}{r}1.1 \\
.8 \\
.7 \\
1.1 \\
1.4 \\
1.3 \\
1.2 \\
1.2 \\
.7 \\
.9 \\
10.5\end{array}$ \\
\hline $\begin{array}{l}199610-M o n t h \text { Total ............. } \\
199510-M o n t h \text { Total ............. }\end{array}$ & $\begin{array}{l}\text { NA } \\
\text { NA }\end{array}$ & $\begin{array}{l}6.7 \\
6.9\end{array}$ & $\begin{array}{l}243.5 \\
236.6\end{array}$ & $\begin{array}{l}.3 \\
.4\end{array}$ & $\begin{array}{l}60.1 \\
52.5\end{array}$ & $\begin{array}{l}31.5 \\
29.4\end{array}$ & $\begin{array}{l}c_{342.1} \\
c_{325.8}\end{array}$ & $\begin{array}{r}10.1 \\
9.8\end{array}$ \\
\hline
\end{tabular}

a The total gross generation estimate for China is calculated as 5 percent more than the annual net nuclear generation reported by the international Atomic Energy Agency (IAEA) and is published in the Energy Information Administration annual reports-1993: World Nuclear Outlook 1994 December 1994, Table 1. 1994: Nuclear Power Generation and Fuel Cycle Report 1996, October 1996, Table 1. 1995 and 1996: Nuclear Power Generation and Fuel Cycle Report 1997, September 1997. Table D4.

b South Africa comprises all of Africa's nuclear electricity generation.

c Total excluding China.

NA=Not available. $-=$ Not applicable. E=Estimate.
Notes: - Net figures are generally less than gross figures by about 5 percent, the difference being the energy consumed by the generating plants themselves. - Monthly data may not sum to annual totals due to independent rounding and because precommercial generation is included in some annual totals but not in the monthly data. - Data for countries may not sum to regional totals due to independent rounding

Source: - China: See footnote a. All Other: Based on data from Nucleonics Week, a copyrighted publication of The McGraw-Hill Publishing Companies, Inc. Used with permission. 
Table 10.4e Nuclear Electricity Gross Generation: Eastern Europe and Former U.S.S.R.

(Billion Kilowatthours)

\begin{tabular}{|c|c|c|c|c|c|c|c|c|c|c|c|}
\hline & Armenia ${ }^{a}$ & Bulgaria & $\begin{array}{c}\text { Czech } \\
\text { Republic }\end{array}$ & Hungary & Kazakhstan ${ }^{b}$ & Lithuaniab & Romania & Russia & Slovakiab & Ukraine & $\begin{array}{c}\text { Eastern } \\
\text { Europe } \\
\text { and } \\
\text { Former } \\
\text { U.S.S.R.b }\end{array}$ \\
\hline 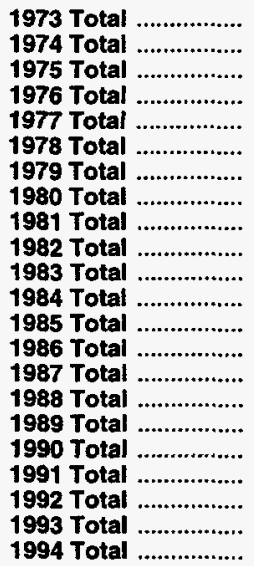 & $\begin{array}{l}\bar{z} \\
\bar{z} \\
\bar{z} \\
\bar{z} \\
\bar{z} \\
\bar{z} \\
\bar{z} \\
\bar{z} \\
\bar{z} \\
\bar{z} \\
\bar{z} \\
\overline{-}\end{array}$ & $\begin{array}{l}\text { NA } \\
\text { NA } \\
\text { NA } \\
\text { NA } \\
\text { NA } \\
\text { NA } \\
\text { NA } \\
\text { NA } \\
\text { NA } \\
\text { NA } \\
\text { NA } \\
\text { NA } \\
\text { NA } \\
\text { NA } \\
\text { NA } \\
\text { NA } \\
\text { NA } \\
\text { NA } \\
\text { 12.2 } \\
14.0 \\
14.9\end{array}$ & $\begin{array}{l}- \\
- \\
- \\
\overline{-} \\
= \\
= \\
- \\
\overline{-} \\
\overline{-} \\
\text { NA } \\
\text { NA } \\
\text { NA } \\
\text { NA } \\
\text { NA } \\
\text { NA } \\
\text { NA } \\
\text { E 12.9 } \\
\text { E 13.2 } \\
\text { 12.7 }\end{array}$ & $\begin{array}{l}\bar{z} \\
\overline{-} \\
\bar{z} \\
\overline{-} \\
\overline{-} \\
\overline{-} \\
\text { NA } \\
\text { NA } \\
\text { NA } \\
\text { NA } \\
\text { NA } \\
\text { NA } \\
\text { NA } \\
\text { NA } \\
\text { NA } \\
\text { E13.8 } \\
13.8 \\
14.0\end{array}$ & $\begin{array}{l}\text { NA } \\
\text { NA } \\
\text { NA } \\
\text { NA } \\
\text { NA } \\
\text { NA } \\
\text { NA } \\
\text { NA } \\
\text { NA } \\
\text { NA } \\
\text { NA } \\
\text { NA } \\
\text { NA } \\
\text { NA } \\
\text { NA } \\
\text { NA } \\
\text { NA } \\
\text { NA } \\
\text { NA } \\
\text { E.5 } \\
\text { E.4 } \\
\text { E.4 }\end{array}$ & $\begin{array}{c}- \\
\overline{-} \\
- \\
- \\
\overline{-} \\
\overline{-} \\
\overline{-} \\
\overline{-} \\
- \\
\overline{-} \\
\text { NA } \\
\text { NA } \\
\text { NA } \\
\text { NA } \\
\text { NA } \\
\text { NA } \\
\text { NA } \\
\text { E 16.4 } \\
\text { E 12.9 } \\
\text { E7.0 }\end{array}$ & $\begin{array}{l}- \\
\overline{-} \\
\overline{-} \\
\overline{-} \\
\overline{-} \\
\overline{-} \\
\overline{-} \\
\overline{-} \\
\overline{-} \\
\overline{-} \\
\overline{-} \\
\overline{-} \\
\overline{-} \\
-\end{array}$ & $\begin{array}{c}\text { NA } \\
\text { NA } \\
\text { NA } \\
\text { NA } \\
\text { NA } \\
\text { NA } \\
\text { NA } \\
\text { NA } \\
\text { NA } \\
\text { NA } \\
\text { NA } \\
\text { NA } \\
\text { NA } \\
\text { NA } \\
\text { NA } \\
\text { NA } \\
\text { NA } \\
\text { NA } \\
\text { NA } \\
\text { E } 125.6 \\
120.4 \\
97.7\end{array}$ & $\begin{array}{l}\text { NA } \\
\text { NA } \\
\text { NA } \\
\text { NA } \\
\text { NA } \\
\text { NA } \\
\text { NA } \\
\text { NA } \\
\text { NA } \\
\text { NA } \\
\text { NA } \\
\text { NA } \\
\text { NA } \\
\text { NA } \\
\text { NA } \\
\text { NA } \\
\text { NA } \\
\text { NA } \\
\text { NA } \\
\text { E 11.7 } \\
\text { E 11.6 } \\
\mathbf{1 2 . 7}\end{array}$ & $\begin{array}{c}- \\
- \\
- \\
- \\
\text { NA } \\
\text { NA } \\
\text { NA } \\
\text { NA } \\
\text { NA } \\
\text { NA } \\
\text { NA } \\
\text { NA } \\
\text { NA } \\
\text { NA } \\
\text { NA } \\
\text { NA } \\
\text { NA } \\
\text { NA } \\
\text { E74.6 } \\
\text { E72.7 } \\
68.4\end{array}$ & $\begin{array}{c}\text { NA } \\
\text { NA } \\
\text { NA } \\
\text { NA } \\
\text { NA } \\
\text { NA } \\
\text { NA } \\
\text { NA } \\
\text { NA } \\
\text { NA } \\
\text { NA } \\
\text { NA } \\
\text { NA } \\
\text { NA } \\
\text { NA } \\
\text { NA } \\
\text { NA } \\
\text { NA } \\
\text { NA } \\
\text { E 267.5 } \\
\text { E 259.0 } \\
\text { E 227.8 }\end{array}$ \\
\hline 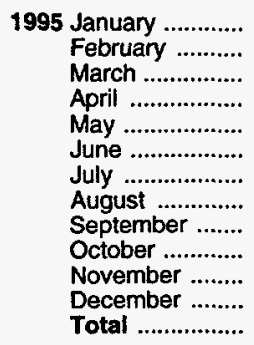 & $\begin{array}{l}- \\
- \\
- \\
- \\
- \\
- \\
- \\
\overline{-} \\
\text { NA } \\
\text { NA } \\
\text { NA }\end{array}$ & $\begin{array}{r}2.2 \\
2.1 \\
1.9 \\
1.5 \\
1.3 \\
.9 \\
1.0 \\
.8 \\
1.0 \\
1.2 \\
1.3 \\
1.9 \\
17.2\end{array}$ & $\begin{array}{l}\text { NA } \\
\text { NA } \\
\text { NA } \\
\text { NA } \\
\text { NA } \\
\text { NA } \\
\text { NA } \\
\text { NA } \\
\text { NA } \\
\text { NA } \\
\text { NA } \\
\text { NA } \\
\text { 12.8 }\end{array}$ & $\begin{array}{r}1.4 \\
1.1 \\
1.3 \\
1.1 \\
1.1 \\
1.0 \\
1.1 \\
1.0 \\
1.1 \\
1.3 \\
1.2 \\
1.4 \\
14.0\end{array}$ & $\begin{array}{l}\text { NA } \\
\text { NA } \\
\text { NA } \\
\text { NA } \\
\text { NA } \\
\text { NA } \\
\text { NA } \\
\text { NA } \\
\text { NA } \\
\text { NA } \\
\text { NA } \\
\text { NA } \\
\text { E.4 }\end{array}$ & $\begin{array}{r}\text { NA } \\
\text { NA } \\
.9 \\
.7 \\
.8 \\
.7 \\
.8 \\
1.0 \\
.9 \\
1.0 \\
1.3 \\
1.7 \\
E 9.7\end{array}$ & $\begin{array}{l}- \\
\overline{-} \\
\overline{-} \\
\bar{z} \\
\overline{-} \\
\overline{-} \\
\overline{-}\end{array}$ & $\begin{array}{r}10.7 \\
8.9 \\
9.0 \\
7.8 \\
7.2 \\
6.6 \\
7.4 \\
7.2 \\
6.5 \\
7.8 \\
8.9 \\
10.5 \\
98.3\end{array}$ & $\begin{array}{l}\text { NA } \\
\text { NA } \\
\text { NA } \\
\text { NA } \\
\text { NA } \\
\text { NA } \\
\text { NA } \\
\text { NA } \\
\text { NA } \\
\text { NA } \\
\text { NA } \\
\text { NA } \\
\text { E 12.0 }\end{array}$ & $\begin{array}{r}8.5 \\
7.5 \\
7.3 \\
6.5 \\
4.8 \\
4.4 \\
4.0 \\
4.8 \\
4.1 \\
5.1 \\
5.7 \\
7.7 \\
70.4\end{array}$ & $\begin{array}{r}c_{22.8} \\
c_{19.6} \\
c_{20.4} \\
c_{17.6} \\
c_{15.1} \\
c_{13.6} \\
c_{14.2} \\
c_{14.9} \\
c_{13.7} \\
c_{16.4} \\
c_{18.3} \\
c_{23.1} \\
E_{234.9}\end{array}$ \\
\hline 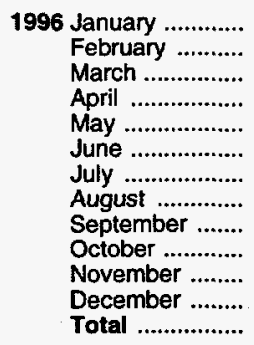 & $\begin{array}{l}\text { NA } \\
\text { NA } \\
\text { NA } \\
\text { NA } \\
\text { NA } \\
\text { NA } \\
\text { NA } \\
\text { NA } \\
\text { NA } \\
\text { NA } \\
\text { NA } \\
\text { NA } \\
\text { NA }\end{array}$ & $\begin{array}{r}2.4 \\
2.1 \\
2.3 \\
1.8 \\
1.0 \\
1.8 \\
.9 \\
1.0 \\
1.0 \\
1.3 \\
1.3 \\
1.7 \\
18.7\end{array}$ & $\begin{array}{l}\text { NA } \\
\text { NA } \\
\text { NA } \\
\text { NA } \\
\text { NA } \\
\text { NA } \\
\text { NA } \\
\text { NA } \\
\text { NA } \\
\text { NA } \\
\text { NA } \\
\text { NA } \\
\text { E 13.5 }\end{array}$ & $\begin{array}{r}1.4 \\
1.3 \\
1.3 \\
1.1 \\
1.2 \\
1.1 \\
1.1 \\
1.0 \\
.9 \\
1.2 \\
1.3 \\
1.4 \\
14.2\end{array}$ & $\begin{array}{l}\text { NA } \\
\text { NA } \\
\text { NA } \\
\text { NA } \\
\text { NA } \\
\text { NA } \\
\text { NA } \\
\text { NA } \\
\text { NA } \\
\text { NA } \\
\text { NA } \\
\text { NA } \\
\text { E.1 }\end{array}$ & $\begin{aligned} 1.6 \\
1.6 \\
1.6 \\
1.0 \\
.8 \\
1.0 \\
.9 \\
.8 \\
.8 \\
1.0 \\
1.0 \\
1.5 \\
\mathbf{1 3} \\
\end{aligned}$ & $\begin{array}{l}- \\
- \\
- \\
- \\
\overline{-} \\
\overline{N A} \\
\text { NA } \\
\text { NA } \\
\text { NA } \\
\text { NA } \\
\text { NA } \\
\text { E } 1.0\end{array}$ & $\begin{array}{r}10.4 \\
10.3 \\
11.2 \\
9.1 \\
8.3 \\
7.7 \\
7.9 \\
8.4 \\
7.3 \\
8.3 \\
9.2 \\
10.5 \\
108.8\end{array}$ & $\begin{array}{c}\text { NA } \\
\text { NA } \\
\text { NA } \\
\text { NA } \\
\text { NA } \\
\text { NA } \\
\text { NA } \\
\text { NA } \\
\text { NA } \\
\text { NA } \\
\text { NA } \\
\text { NA } \\
\text { E 11.8 }\end{array}$ & $\begin{array}{r}8.8 \\
8.0 \\
8.3 \\
7.2 \\
5.8 \\
6.0 \\
6.0 \\
4.3 \\
4.9 \\
5.5 \\
7.0 \\
8.3 \\
80.0\end{array}$ & $\begin{array}{r}c_{24.6} \\
c_{23.3} \\
c_{24.7} \\
c_{20.2} \\
c_{17.2} \\
c_{17.6} \\
c_{16.7} \\
c_{15.4} \\
c_{14.9} \\
c_{17.4} \\
c_{19.9} \\
c_{23.3} \\
\varepsilon_{261.6}\end{array}$ \\
\hline 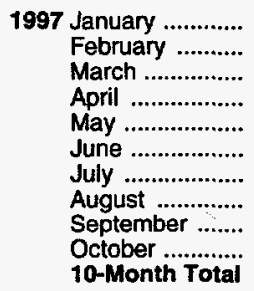 & $\begin{array}{l}.2 \\
.2 \\
.3 \\
.2 \\
.2 \\
.1 \\
.1 \\
.0 \\
.0 \\
.0 \\
1.3\end{array}$ & $\begin{array}{r}1.7 \\
1.7 \\
1.8 \\
1.2 \\
.9 \\
E .9 \\
E .9 \\
1.1 \\
E_{1.1} \\
1.1 \\
\varepsilon_{12.4}\end{array}$ & $\begin{array}{l}\text { NA } \\
\text { NA } \\
\text { NA } \\
\text { NA } \\
\text { NA } \\
\text { NA } \\
\text { NA } \\
\text { NA } \\
\text { NA } \\
\text { NA } \\
\text { NA }\end{array}$ & $\begin{array}{r}1.4 \\
1.2 \\
1.4 \\
1.0 \\
1.0 \\
1.0 \\
1.0 \\
.9 \\
1.0 \\
1.3 \\
11.3\end{array}$ & $\begin{array}{l}\text { NA } \\
\text { NA } \\
\text { NA } \\
\text { NA } \\
\text { NA } \\
\text { NA } \\
\text { NA } \\
\text { NA } \\
\text { NA } \\
\text { NA } \\
\text { NA }\end{array}$ & $\begin{array}{r}1.5 \\
1.3 \\
1.3 \\
.9 \\
.9 \\
.8 \\
.6 \\
.9 \\
.9 \\
1.0 \\
10.0\end{array}$ & $\begin{array}{l}\text { NA } \\
\text { NA } \\
\text { NA } \\
.3 \\
.4 \\
.5 \\
.5 \\
.4 \\
.5 \\
.2 \\
2.8\end{array}$ & $\begin{array}{r}11.2 \\
9.9 \\
10.7 \\
8.5 \\
7.8 \\
6.5 \\
7.2 \\
7.5 \\
7.8 \\
9.3 \\
\mathbf{8 6 . 7}\end{array}$ & $\begin{array}{r}1.2 \\
1.2 \\
.9 \\
.9 \\
.9 \\
.8 \\
.6 \\
.9 \\
.9 \\
.9 \\
9.0\end{array}$ & $\begin{array}{r}8.4 \\
8.4 \\
8.4 \\
7.2 \\
6.2 \\
6.1 \\
6.0 \\
6.0 \\
5.7 \\
5.9 \\
68.2\end{array}$ & $\begin{array}{r}c_{25.6} \\
c_{23.9} \\
c_{24.6} \\
c_{20.2} \\
c_{18.3} \\
c_{16.7} \\
c_{16.9} \\
c_{17.7} \\
c_{17.9} \\
c_{19.9} \\
c_{201.7}\end{array}$ \\
\hline $\begin{array}{l}1996 \text { 10-Month Total } \\
1995 \text { 10-Month Total }\end{array}$ & $\underset{-}{N A}$ & $\begin{array}{l}15.7 \\
14.0\end{array}$ & $\begin{array}{l}\text { NA } \\
\text { NA }\end{array}$ & $\begin{array}{l}11.5 \\
11.5\end{array}$ & $\begin{array}{l}\text { NA } \\
\text { NA }\end{array}$ & $\begin{array}{r}11.1 \\
6.7\end{array}$ & NA & $\begin{array}{l}89.0 \\
79.0\end{array}$ & $\begin{array}{l}\text { NA } \\
\text { NA }\end{array}$ & $\begin{array}{l}64.7 \\
57.0\end{array}$ & $\begin{array}{l}c_{192.0} \\
c_{168.2}\end{array}$ \\
\hline
\end{tabular}

a According to ElA's Nuclear Power Generation and Fuel Cycle Report 1996, Armenia has two units; one came on line in November 1995 but no data are available prior to 1997, and the other is projected to come on line in 2001 .

$b$ The total gross generation estimate for Czech Republic, Kazakhstan, Lithuania, Slovakia, and Eastern European countries is calculated as 5 percent more than the annual net nuclear generation reported by the International Atomic Energy Agency and published in the Energy Information Administration annual reports-1992 and 1993: World Nuclear Outlook 1994, December 1994, Table 1. 1994: Nuclear Power Generation and Fuel Cycle Report 1996, October 1996, Table 1. 1995 and 1996: Nuclear Power Generation and Fuel Cycle Report 1997, September 1997, Table D4.

c Sum of available data only.
NA=Not available. $-=$ Not applicable. $E=$ Estimate

Notes: - Net figures are generally less than gross figures by about 5 percent, the difference being the energy consumed by the generating plants themselves.

- Monthly data may not sum to annual totals due to independent rounding and because precommercial generation is included in some annual totals but not in the monthly data. - Data for countries may not sum to regional totals due to independent rounding.

Source: - Czech Republic, Kazakhstan, Lithuania, Slovakia, and Eastern European Countries: See footnote a. - All Other: Based on data from Nucleonics Week, a copyrighted publication of The McGraw-Hill Publishing Companies, Inc. Used with permission. 


\section{Sources for Tables 10.1a and 10.1b}

\section{United States}

Table 3.1a.

\section{Other Countries: Annual Data}

1973-1979: Energy Information Administration (EIA), International Energy Annual 1981, Table 8.

1980-1995: Office of Energy Markets and End Use, International Database, April 1997.

1996: Average of monthly data.

\section{Other Countries: Monthly Data}

1995-1997: Petroleum Intelligence Weekly, the Oil and Gas Journal, and other industry sources.

\section{World: Annual Data}

1973-1979: EIA, International Energy Annual 1981, Table 8.

1980-1995: Office of Energy Markets and End Use, International Database, April 1997.

1996: Average of monthly data.

\section{World: Monthly Data}

1995-1997: EIA, International Petroleum Statistics Report, sum of all countries' monthly data. 


\section{Appendix A. Thermal Conversion Factors}

The thermal conversion factors presented in the following eight tables can be used to estimate the heat content in British thermal units (Btu) of a given amount of energy measured in physical units, such as barrels or cubic feet. For example, 10 barrels of asphalt have a heat content of approximately 66.36 million Btu ( 10 barrels $\times 6.636$ million $\mathrm{Btu} / \mathrm{barrel}=$ 66.36 million Btu).

Thermal conversion factors for hydrocarbon mixes (Table A1) are weighted averages of the thermal conversion factors for each hydrocarbon included in the mix. For example, in calculating the thermal conversion factor for a 60-40 butane-propane mixture, the thermal conversion factor for butane is weighted 1.5 times more heavily than the thermal conversion factor for propane.

In general, the annual thermal conversion factors presented in Tables A1 through A8 are computed from final annual data. However, if the current year's final data are not available in time for publication, thermal conversion factors for the current year are computed from the best available data and are labeled "preliminary." The source of each factor is described in the section entitled "Thermal Conversion Factor Source Documentation," which follows Table A8 in this appendix.

Table A1. Approximate Heat Content of Petroleum Products (Million Btu per Barrel)

\begin{tabular}{|c|c|c|c|}
\hline Petroleum Product & Heat Content & Petroleum Product & Heat Content \\
\hline Asphalt & 6.636 & Petrochemical Feedstocks & \\
\hline Aviation Gasoline & 5.048 & Naphtha Less Than $401^{\circ} \mathrm{F}$. & 5.248 \\
\hline Butane . . . . . . . . & 4.326 & Other Oils Equal to or Greater Than $401^{\circ} \mathrm{F} \ldots$ & 5.825 \\
\hline Butane-Propane Mixture ${ }^{a}$. & 4.130 & Still Gas . ................. & 6.000 \\
\hline Distillate Fuel Oil........ & 5.825 & Petroleum Coke. ........... & 6.024 \\
\hline Ethane............ & 3.082 & Plant Condensate . . . . . . & 5.418 \\
\hline Ethane-Propane Mixture ${ }^{\mathrm{b}}$. & 3.308 & Propane............ & 3.836 \\
\hline Isobutane. . . . . . . . . & 3.974 & Residual Fuel Oil. . & 6.287 \\
\hline Jet Fuel, Kerosene Type. . & 5.670 & Road Oil. . . . . . . . . . . & 6.636 \\
\hline Jet Fuel, Naphtha Type ... & 5.355 & Special Naphthas ....... & 5.248 \\
\hline Kerosene............ & 5.670 & Still Gas . . . . . . . . . . . . & 6.000 \\
\hline Lubricants ....... & 6.065 & Unfinished Oils ... & 5.825 \\
\hline Motor Gasoline ... . & 5.253 & Unfractionated Stream ... & 5.418 \\
\hline Natural Gasoline and Isopentane........... & 4.620 & 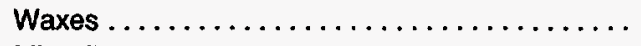 & 5.537 \\
\hline Pentanes Plus. . . . . . . . . . . . . . . . & 4.620 & Miscellaneous $\ldots \ldots \ldots \ldots \ldots \ldots \ldots \ldots$ & 5.796 \\
\hline
\end{tabular}

\footnotetext{
a 60 percent butane and 40 percent propane.

b 70 percent ethane and 30 percent propane.

Source: See "Thermal Conversion Factor Source Documentation," which follows Table A8.
} 
Table A2. Approximate Heat Content of Crude Oil, Crude Oil and Products, and Natural Gas Plant Liquids

(Million Btu per Barrel)

\begin{tabular}{|c|c|c|c|c|c|c|}
\hline & \multicolumn{3}{|c|}{ Crude Oil } & \multicolumn{2}{|c|}{ Crude Oil and Products } & \multirow{2}{*}{$\begin{array}{c}\text { Natural Gas } \\
\text { Plant Liquids } \\
\text { Production }\end{array}$} \\
\hline & Production & Imports & Exports & Imports & Exports & \\
\hline $1973 \ldots \ldots \ldots \ldots \ldots \ldots \ldots \ldots \ldots$ & 5.800 & 5.817 & 5.800 & 5.897 & 5.752 & 4.049 \\
\hline 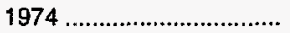 & 5.800 & 5.827 & 5.800 & 5.884 & 5.774 & 4.011 \\
\hline 1975 & 5.800 & 5.821 & 5.800 & 5.858 & 5.748 & 3.984 \\
\hline $1976 \ldots \ldots \ldots \ldots \ldots \ldots \ldots \ldots \ldots \ldots \ldots$ & 5.800 & 5.808 & 5.800 & 5.856 & 5.745 & 3.964 \\
\hline 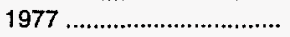 & 5.800 & 5.810 & 5.800 & 5.834 & 5.797 & 3.941 \\
\hline $1978 \ldots \ldots \ldots \ldots \ldots \ldots \ldots \ldots \ldots \ldots \ldots \ldots \ldots$ & 5.800 & 5.802 & 5.800 & 5.839 & 5.808 & 3.925 \\
\hline 1979 & 5.800 & 5.810 & 5.800 & 5.810 & 5.832 & 3.955 \\
\hline 1980 & 5.800 & 5.812 & 5.800 & 5.796 & 5.820 & 3.914 \\
\hline 1981 & 5.800 & 5.818 & 5.800 & 5.775 & 5.821 & 3.930 \\
\hline 1982 & 5.800 & 5.826 & 5.800 & 5.775 & 5.820 & 3.872 \\
\hline $1983 \ldots \ldots \ldots \ldots \ldots \ldots \ldots \ldots$ & 5.800 & 5.825 & 5.800 & 5.774 & 5.800 & 3.839 \\
\hline 1984 & 5.800 & 5.823 & 5.800 & 5.745 & 5.850 & 3.812 \\
\hline 1985 & 5.800 & 5.832 & 5.800 & 5.736 & 5.814 & 3.815 \\
\hline 1986 & 5.800 & 5.903 & 5.800 & 5.808 & 5.832 & 3.797 \\
\hline 1987 & 5.800 & 5.901 & 5.800 & 5.820 & 5.858 & 3.804 \\
\hline 1988 & 5.800 & 5.900 & 5.800 & 5.820 & 5.840 & 3.800 \\
\hline 1989 & 5.800 & 5.906 & 5.800 & 5.833 & 5.857 & 3.826 \\
\hline 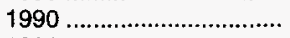 & 5.800 & 5.934 & 5.800 & 5.849 & 5.833 & 3.822 \\
\hline 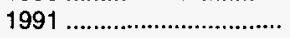 & 5.800 & 5.948 & 5.800 & 5.873 & 5.823 & 3.807 \\
\hline 1992 & 5.800 & 5.953 & 5.800 & 5.877 & 5.777 & 3.804 \\
\hline 1993 & 5.800 & 5.954 & 5.800 & 5.883 & 5.779 & 3.801 \\
\hline 1994 & 5.800 & 5.950 & 5.800 & 5.861 & 5.781 & 3.794 \\
\hline 1995 & 5.800 & 5.924 & 5.800 & 5.849 & 5.751 & 3.796 \\
\hline $1996^{a}$ & 5.800 & 5.935 & 5.800 & 5.843 & 5.745 & 3.777 \\
\hline $1997^{a}$ & 5.800 & 5.935 & 5.800 & 5.843 & 5.745 & 3.777 \\
\hline
\end{tabular}

a Preliminary

Note: Crude oil includes lease condensate.

Source: See "Thermal Conversion Factor Source Documentation," which follows Table A8.

Table A3. Approximate Heat Content of Petroleum Products, Weighted Averages (Million Btu per Barrel)

\begin{tabular}{|c|c|c|c|c|c|c|c|c|}
\hline & \multicolumn{5}{|c|}{ Consumption } & \multirow[b]{2}{*}{ Imports } & \multirow[b]{2}{*}{ Exports } & \multirow{2}{*}{$\begin{array}{l}\text { Liquefied } \\
\text { Petroleum } \\
\text { Gases } \\
\text { Consumption }\end{array}$} \\
\hline & $\begin{array}{l}\text { Residential } \\
\text { and } \\
\text { Commercial }\end{array}$ & Industrial & Transportation & $\begin{array}{l}\text { Electric } \\
\text { Utilities }\end{array}$ & Total & & & \\
\hline 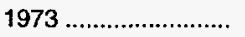 & 5.387 & 5.568 & 5.395 & 6.245 & 5.515 & 5.983 & 5.752 & 3.746 \\
\hline $1974 \ldots \ldots \ldots \ldots \ldots \ldots \ldots$ & 5.377 & 5.538 & 5.394 & 6.238 & 5.504 & 5.959 & 5.773 & 3.730 \\
\hline $1975 \ldots \ldots \ldots \ldots \ldots \ldots \ldots$ & 5.358 & 5.528 & 5.392 & 6.250 & 5.494 & 5.935 & 5.747 & 3.715 \\
\hline 1976 & 5.383 & 5.538 & 5.395 & 6.251 & 5.504 & 5.980 & 5.743 & 3.711 \\
\hline 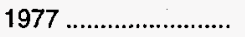 & 5.389 & 5.555 & 5.400 & 6.249 & 5.518 & 5.908 & 5.796 & 3.677 \\
\hline $1978 \ldots \ldots \ldots \ldots \ldots \ldots$ & 5.382 & 5.553 & 5.404 & 6.251 & 5.519 & 5.955 & 5.814 & 3.669 \\
\hline 1979 & 5.471 & 5.418 & 5.428 & 6.258 & 5.494 & 5.811 & 5.864 & 3.680 \\
\hline 1980 & 5.468 & 5.376 & 5.440 & 6.254 & 5.479 & 5.748 & 5.841 & 3.674 \\
\hline 1981 & 5.409 & 5.313 & 5.432 & 6.258 & 5.448 & 5.659 & 5.837 & 3.643 \\
\hline $1982 \ldots \ldots \ldots \ldots \ldots \ldots \ldots$ & 5.392 & 5.263 & 5.422 & 6.258 & 5.415 & 5.664 & 5.829 & 3.615 \\
\hline $1983 \ldots \ldots \ldots \ldots \ldots \ldots \ldots$ & 5.286 & 5.273 & 5.415 & 6.255 & 5.406 & 5.677 & 5.800 & 3.614 \\
\hline 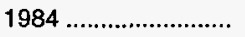 & 5.384 & 5.223 & 5.422 & 6.251 & 5.395 & 5.613 & 5.867 & 3.599 \\
\hline $1985 \ldots \ldots \ldots \ldots \ldots \ldots \ldots$ & 5.326 & 5.221 & 5.423 & 6.247 & 5.387 & 5.572 & 5.819 & 3.603 \\
\hline 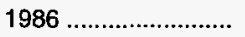 & 5.357 & 5.286 & 5.427 & 6.257 & 5.418 & 5.624 & 5.839 & 3.640 \\
\hline $1987 \ldots \ldots \ldots \ldots \ldots \ldots \ldots$ & 5.316 & 5.253 & 5.430 & 6.249 & 5.403 & 5.599 & 5.860 & 3.659 \\
\hline 1988 & 5.320 & 5.248 & 5.434 & 6.250 & 5.410 & 5.618 & 5.842 & 3.652 \\
\hline 1989 & 5.257 & 5.233 & 5.440 & 6.241 & 5.410 & 5.641 & 5.869 & 3.683 \\
\hline 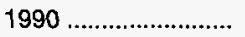 & 5.208 & 5.272 & 5.445 & 6.247 & 5.411 & 5.614 & 5.838 & 3.625 \\
\hline 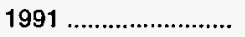 & 5.163 & 5.192 & 5.442 & 6.248 & 5.384 & 5.636 & 5.827 & 3.614 \\
\hline 1992 & 5.169 & 5.188 & 5.445 & 6.243 & 5.378 & 5.623 & 5.774 & 3.624 \\
\hline $1993 \ldots \ldots \ldots \ldots \ldots \ldots \ldots$ & 5.148 & 5.200 & 5.438 & 6.241 & 5.379 & 5.620 & 5.777 & 3.606 \\
\hline 1994 & 5.154 & 5.171 & 5.442 & 6.231 & 5.371 & 5.538 & 5.779 & 3.635 \\
\hline $1995 \ldots \ldots \ldots \ldots \ldots \ldots$ & 5.150 & 5.150 & 5.439 & 6.210 & 5.358 & 5.511 & 5.746 & 3.623 \\
\hline $1996^{a}$ & 5.135 & 5.130 & 5.441 & 6.206 & 5.352 & 5.495 & 5.738 & 3.613 \\
\hline $1997^{a} \ldots \ldots \ldots \ldots \ldots \ldots \ldots$ & 5.135 & 5.130 & 5.441 & 6.206 & 5.352 & 5.495 & 5.738 & 3.613 \\
\hline
\end{tabular}

a Preliminary.

Note: Weighted averages of the products included in each category are calculated by using heat content values shown in Table A1.

Source: See "Thermal Conversion Factor Source Documentation," which follows Table A8. 
Table A4. Approximate Heat Content of Natural Gas

(Btu per Cubic Foot)

\begin{tabular}{|c|c|c|c|c|c|c|c|}
\hline & \multicolumn{2}{|c|}{ Production } & \multicolumn{3}{|c|}{ Consumption } & \multirow[b]{2}{*}{ Imports } & \multirow[b]{2}{*}{ Exports } \\
\hline & Dry & $\begin{array}{l}\text { Marketed } \\
\text { (Wet) }\end{array}$ & $\begin{array}{c}\text { Sectors Other } \\
\text { Than Electric } \\
\text { Utilities }\end{array}$ & $\begin{array}{l}\text { Electric } \\
\text { Utilities }\end{array}$ & Total & & \\
\hline 1973 & 1,021 & 1,093 & 1,020 & 1,024 & 1,021 & 1,026 & 1,023 \\
\hline $1974 \ldots \ldots \ldots \ldots \ldots \ldots \ldots$ & 1,024 & 1,097 & 1,024 & 1,022 & 1,024 & 1,027 & 1,016 \\
\hline 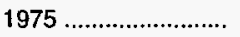 & 1,021 & 1,095 & 1,020 & 1,026 & 1,021 & 1,026 & 1,014 \\
\hline $1976 \ldots \ldots \ldots \ldots \ldots \ldots \ldots$ & 1,020 & 1,093 & 1,019 & 1,023 & 1,020 & 1,025 & 1,013 \\
\hline $1977 \ldots \ldots \ldots \ldots \ldots . . . . . . . . . . . .$. & 1,021 & 1,093 & 1,019 & 1,029 & 1,021 & 1,026 & 1,013 \\
\hline 1978 & 1,019 & 1,088 & 1,016 & 1,034 & 1,019 & 1,030 & 1,013 \\
\hline 1979 & 1,021 & 1,092 & 1,018 & 1,035 & 1,021 & 1,037 & 1,013 \\
\hline 1980 & 1,026 & 1,098 & 1,024 & 1,035 & 1,026 & 1,022 & 1,013 \\
\hline 1981 & 1,027 & 1,103 & 1,025 & 1,035 & 1,027 & 1,014 & 1,011 \\
\hline 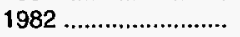 & 1,028 & 1,107 & 1,026 & 1,036 & 1,028 & 1,018 & 1,011 \\
\hline $1983 \ldots \ldots \ldots \ldots \ldots \ldots \ldots$ & 1,031 & 1,115 & 1,031 & 1,030 & 1,031 & 1,024 & 1,010 \\
\hline 1984 & 1,031 & 1,109 & 1,030 & 1,035 & 1,031 & 1,005 & 1,010 \\
\hline 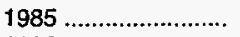 & 1,032 & 1,112 & 1,031 & 1,038 & 1,032 & 1,002 & 1,011 \\
\hline 1986 & 1,030 & 1,110 & 1,029 & 1,034 & 1,030 & 997 & 1,008 \\
\hline $1987 \ldots \ldots \ldots \ldots \ldots \ldots \ldots$ & 1,031 & 1,112 & 1,031 & 1,032 & 1,031 & 999 & 1,011 \\
\hline 1988 & 1,029 & 1,109 & 1,029 & 1,028 & 1,029 & 1,002 & 1,018 \\
\hline 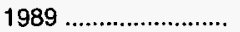 & 1,031 & 1,107 & 1,031 & 1,030 & 1,031 & 1,004 & 1,019 \\
\hline $1990 \ldots \ldots \ldots \ldots \ldots$ & 1,031 & 1,105 & 1,030 & 1,034 & 1,031 & 1,012 & 1,018 \\
\hline $1991 \ldots \ldots \ldots \ldots \ldots \ldots \ldots$ & 1,030 & 1,108 & 1,031 & 1,024 & 1,030 & 1,014 & 1,022 \\
\hline 1992 & 1,030 & 1,110 & 1,031 & 1,022 & 1,030 & 1,011 & 1,018 \\
\hline 1993 & 1,027 & 1,106 & 1,028 & 1,022 & 1,027 & 1,020 & 1,016 \\
\hline 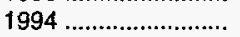 & 1,028 & 1,105 & 1,029 & 1,022 & 1,028 & 1,022 & 1,011 \\
\hline $1995 \ldots \ldots \ldots \ldots \ldots \ldots \ldots$ & 1,027 & 1,106 & 1,027 & 1,025 & 1,027 & 1,021 & 1,011 \\
\hline $1996^{a}$ & 1,027 & 1,109 & 1,027 & 1,024 & 1,027 & 1,022 & 1,011 \\
\hline $1997^{a}$ & 1,027 & 1,109 & 1,027 & 1,024 & 1,027 & 1,022 & 1,011 \\
\hline
\end{tabular}

a Preliminary.

$R=$ Revised data.

Source: See "Thermal Conversion Factor Source Documentation," which follows Table A8.

Table A5. Approximate Heat Content of Coal (Million Btu per Short Ton)

\begin{tabular}{|c|c|c|c|c|c|c|c|c|}
\hline & \multirow[b]{2}{*}{ Production } & \multicolumn{5}{|c|}{ Consumption } & \multirow[b]{2}{*}{ Imports } & \multirow[b]{2}{*}{ Exports } \\
\hline & & $\begin{array}{l}\text { Residential } \\
\text { and } \\
\text { Commercial }\end{array}$ & $\begin{array}{l}\text { Coke } \\
\text { Plants }\end{array}$ & $\begin{array}{c}\text { Other } \\
\text { Industriala }\end{array}$ & $\begin{array}{l}\text { Electric } \\
\text { Utilities }^{b}\end{array}$ & Total & & \\
\hline $1973 \ldots \ldots \ldots \ldots \ldots \ldots \ldots$ & 23.376 & 22.831 & 26.780 & 22.586 & 22.246 & 23.057 & 25.000 & 26.596 \\
\hline $1974 \ldots \ldots$ & 23.072 & 22.479 & 26.778 & 22.419 & 21.781 & 22.677 & 25.000 & 26.700 \\
\hline 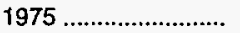 & 22.897 & 22.261 & 26.782 & 22.436 & 21.642 & 22.506 & 25.000 & 26.562 \\
\hline $1976 \ldots \ldots \ldots \ldots \ldots \ldots \ldots$ & 22.855 & 22.774 & 26.781 & 22.530 & 21.679 & 22.498 & 25.000 & 26.601 \\
\hline 1977 & 22.597 & 22.919 & 26.787 & 22.322 & 21.508 & 22.265 & 25.000 & 26.548 \\
\hline $1978 \ldots \ldots \ldots \ldots \ldots \ldots \ldots$ & 22.248 & 22.466 & 26.789 & 22.207 & 21.275 & 22.017 & 25.000 & 26.478 \\
\hline 1979 & 22.454 & 22.242 & 26.788 & 22.452 & 21.364 & 22.100 & 25.000 & 26.548 \\
\hline 1980 & 22.415 & 22.543 & 26.790 & 22.690 & 21.295 & 21.947 & 25.000 & 26.384 \\
\hline 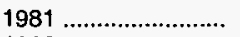 & 22.308 & 22.474 & 26.794 & 22.585 & 21.085 & 21.713 & 25.000 & 26.160 \\
\hline $1982 \ldots \ldots \ldots \ldots \ldots \ldots \ldots$ & 22.239 & 22.695 & 26.797 & 22.712 & 21.194 & 21.674 & 25.000 & 26.223 \\
\hline $1983, \ldots \ldots \ldots \ldots \ldots \ldots \ldots$ & 22.052 & 22.775 & 26.798 & 22.691 & 21.133 & 21.576 & 25.000 & 26.291 \\
\hline 1984 & 22.010 & 22.844 & 26.799 & 22.543 & 21.101 & 21.573 & 25.000 & 26.402 \\
\hline 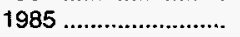 & 21.870 & 22.646 & 26.798 & 22.020 & 20.959 & 21.366 & 25.000 & 26.307 \\
\hline 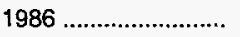 & 21.913 & 22.947 & 26.798 & 22.198 & 21.084 & 21.462 & 25.000 & 26.292 \\
\hline $1987 \ldots \ldots \ldots \ldots \ldots \ldots \ldots$ & 21.922 & 23.404 & 26.799 & 22.381 & 21.136 & 21.517 & 25.000 & 26.291 \\
\hline $1988 \ldots \ldots \ldots \ldots \ldots \ldots \ldots$ & 21.823 & 23.571 & 26.799 & 22.360 & 20.900 & 21.328 & 25.000 & 26.299 \\
\hline 1989 & 21.765 & 23.650 & 26.800 & 22.347 & 20.848 & 21.272 & 25.000 & 26.160 \\
\hline 1990 & 21.822 & 23.137 & 26.799 & 22.457 & 20.929 & 21.331 & 25.000 & 26.202 \\
\hline $1991 \ldots \ldots \ldots \ldots \ldots \ldots \ldots \ldots$ & 21.681 & 23.114 & 26.799 & 22.460 & 20.755 & 21.146 & 25.000 & 26.188 \\
\hline 1992 & 21.646 & 23.105 & 26.799 & 22.250 & 20.787 & 21.143 & 25.000 & 26.161 \\
\hline 1993 & 21.388 & 22.994 & 26.800 & 22.123 & 20.639 & 20.983 & 25.000 & 26.335 \\
\hline 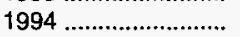 & 21.352 & 23.112 & 26.800 & 22.068 & 20.673 & 21.010 & 25.000 & 26.329 \\
\hline 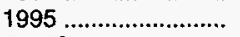 & 21.277 & 23.118 & 26.800 & 21.950 & 20.495 & 20.845 & 25.000 & 26.180 \\
\hline $1996^{\mathrm{C}} \ldots \ldots \ldots \ldots \ldots \ldots$ & 21.277 & 23.118 & 26.800 & 21.950 & 20.495 & 20.845 & 25.000 & 26.180 \\
\hline $1997^{c}$ & 21.277 & 23.118 & 26.800 & 21.950 & 20.495 & 20.845 & 25.000 & 26.180 \\
\hline
\end{tabular}

Includes transportation.

b Data shown in this column are not the same as those shown in the Electric Power Monthly (EPM). The EPM data report coal receipts; the data shown here represent coal consumption.

c Preliminary.

Source: See "Thermal Conversion Factor Source Documentation," which follows Table A8. 
Table A6. Approximate Heat Content of Bituminous Coal and Lignite (Million Btu per Short Ton)

\begin{tabular}{|c|c|c|c|c|c|c|c|c|}
\hline & \multirow[b]{2}{*}{ Production } & \multicolumn{5}{|c|}{ Consumption } & \multirow[b]{2}{*}{ Imports } & \multirow[b]{2}{*}{ Exports } \\
\hline & & $\begin{array}{l}\text { Reisiaential } \\
\text { and } \\
\text { Cornmercial }\end{array}$ & $\begin{array}{l}\text { Coke } \\
\text { Plants }\end{array}$ & $\begin{array}{c}\text { Other } \\
\text { Industriala }\end{array}$ & $\begin{array}{l}\text { Electric } \\
\text { Utilities }\end{array}$ & Total & & \\
\hline $1973 \ldots \ldots \ldots \ldots \ldots \ldots \ldots$ & 23.391 & 22.887 & 26.800 & 22.585 & 22.262 & 23.073 & 25.000 & 26.612 \\
\hline 1974 & 23.087 & 2.523 & 26.800 & 22.420 & 21.799 & 22.694 & 25.000 & 26.716 \\
\hline 1975 & 22.910 & 2.258 & 26.800 & 22.439 & 21.659 & 22.522 & 25.000 & 26.573 \\
\hline 1976 & 22.863 & 22.819 & 26.800 & 22.528 & 21.692 & 22.509 & 25.000 & 26.613 \\
\hline 1977 & 22.597 & 22.594 & 26.800 & 22.290 & 21.521 & 22.266 & 25.000 & 26.561 \\
\hline 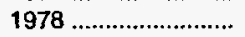 & 22.242 & 22.078 & 26.800 & 22.175 & 21.284 & 22.014 & 25.000 & 26.501 \\
\hline 1979 & 22.449 & 21.884 & 26.800 & 22.436 & 21.372 & 22.100 & 25.000 & 26.570 \\
\hline 1980 & 22.411 & 22.488 & 26.800 & 22.690 & 21.301 & 21.950 & 25.000 & 26.404 \\
\hline 1981 & 22.301 & 22.010 & 26.800 & 22.572 & 21.091 & 21.710 & 25.000 & 26.176 \\
\hline $1982 \ldots \ldots \ldots \ldots \ldots \ldots$ & 22.233 & 22.226 & 26.800 & 22.695 & 21.200 & 21.670 & 25.000 & 26.231 \\
\hline 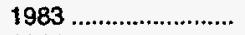 & 22.048 & 22.438 & 26.800 & 22.680 & 21.141 & 21.576 & 25.000 & 26.300 \\
\hline 1984 & 22.005 & 22.406 & 26.800 & 22.525 & 21.108 & 21.570 & 25.000 & 26.410 \\
\hline 1985 & 21.867 & 22.568 & 26.800 & 22.013 & 20.965 & 21.368 & 25.000 & 26.320 \\
\hline 1986 & 21.908 & 22.669 & 26.800 & 22.185 & 21.091 & 21.462 & 25.000 & 26.308 \\
\hline 1987 & 21.918 & 22.800 & 26.800 & 22.360 & 21.143 & 21.514 & 25.000 & 26.304 \\
\hline 1988 & 21.817 & 2.3 .135 & 26.800 & 22.341 & 20.905 & 21.324 & 25.000 & 26.308 \\
\hline 1989 & 21.759 & $2: 2.917$ & 26.800 & 22.324 & 20.854 & 21.268 & 25.000 & 26.166 \\
\hline 1990 & 21.819 & 22.678 & 26.800 & 22.444 & 20.935 & 21.330 & 25.000 & 26.207 \\
\hline $1991 \ldots \ldots \ldots \ldots \ldots \ldots \ldots$ & 21.678 & 22.635 & 26.800 & 22.448 & 20.761 & 21.146 & 25.000 & 26.192 \\
\hline $1992 \ldots \ldots \ldots \ldots \ldots \ldots$ & 21.643 & 22.768 & 26.800 & 22.242 & 20.792 & 21.142 & 25.000 & 26.165 \\
\hline $1993 \ldots \ldots \ldots \ldots \ldots . . . . . . . . . . . .$. & 21.383 & 22.749 & 26.800 & 22.111 & 20.644 & 20.983 & 25.000 & 26.341 \\
\hline $1994 \ldots \ldots \ldots \ldots \ldots \ldots$ & 21.347 & 22.683 & 26.800 & 22.046 & 20.681 & 21.011 & 25.000 & 26.335 \\
\hline $1995 \ldots \ldots \ldots \ldots \ldots \ldots \ldots$ & 21.271 & 22.767 & 26.800 & 21.931 & 20.502 & 20.845 & 25.000 & 26.187 \\
\hline $1996^{b} \ldots \ldots \ldots \ldots \ldots \ldots$ & 21.271 & 22.767 & 26.800 & 21.931 & 20.502 & 20.845 & 25.000 & 26.187 \\
\hline $1997^{b} \ldots \ldots \ldots \ldots \ldots \ldots$ & 21.271 & 22.767 & 26.800 & 21.931 & 20.502 & 20.845 & 25.000 & 26.187 \\
\hline
\end{tabular}

a Includes transportation

breliminary.

Source: See "Thermal Conversion Factor Source Documentation," which follows Table A8.

Table A7. Approximate Heat Content of Anthracite and Coal Coke (Million Btu per Short Ton)

\begin{tabular}{|c|c|c|c|c|c|c|}
\hline & \multirow[b]{3}{*}{ Production } & \multicolumn{4}{|c|}{ Anthracite } & \multirow{3}{*}{$\begin{array}{l}\text { Coal Coke } \\
\text { Imports } \\
\text { and } \\
\text { Exports }\end{array}$} \\
\hline & & \multicolumn{3}{|c|}{ Consumption } & \multirow[b]{2}{*}{$\begin{array}{l}\text { Imports } \\
\text { and } \\
\text { Exports }\end{array}$} & \\
\hline & & $\begin{array}{c}\text { Sectors Other } \\
\text { Than Electric } \\
\text { Utilities }\end{array}$ & Electric Utilities & Total & & \\
\hline 1973 & 22.132 & 22.674 & 17.920 & 21.464 & 25.400 & 24.800 \\
\hline $1974 \ldots \ldots \ldots \ldots \ldots \ldots \ldots$ & 21.711 & 22.330 & 17.200 & 20.919 & 25.400 & 24.800 \\
\hline 1975 & 21.582 & 22.272 & 17.064 & 20.762 & 25.400 & 24.800 \\
\hline 1976 & 22.045 & 22.618 & 17.526 & 21.254 & 25.400 & 24.800 \\
\hline 1977 & 22.661 & 24.101 & 17.244 & 22.066 & 25.400 & 24.800 \\
\hline $1978 \ldots \ldots \ldots \ldots \ldots \ldots \ldots$ & 23.079 & 24.388 & 17.104 & 22.398 & 25.400 & 24.800 \\
\hline 1979 & 23.170 & 24.272 & 17.454 & 22.069 & 25.400 & 24.800 \\
\hline $1980 \ldots \ldots \ldots \ldots \ldots \ldots \ldots$ & 22.869 & 22.719 & 17.652 & 21.405 & 25.400 & 24.800 \\
\hline $1981 \ldots \ldots \ldots \ldots \ldots \ldots \ldots \ldots \ldots \ldots \ldots \ldots \ldots \ldots$ & 23.291 & 23.749 & 18.168 & 22.080 & 25.400 & 24.800 \\
\hline 1982 & 23.289 & 24.578 & 18.160 & 22.518 & 25.400 & 24.800 \\
\hline 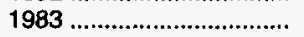 & 22.734 & 24.536 & 16.516 & 21.583 & 25.400 & 24.800 \\
\hline 1984 & 23.107 & 25.128 & 17.018 & 22.322 & 25.400 & 24.800 \\
\hline $1985 \ldots \ldots \ldots \ldots \ldots \ldots \ldots \ldots$ & 22.428 & 23.031 & 16.784 & 20.817 & 25.400 & 24.800 \\
\hline 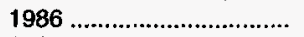 & 23.084 & 24.399 & 15.578 & 21.512 & 25.400 & 24.800 \\
\hline 1987 & 23.108 & 26.293 & 15.962 & 22.435 & 25.400 & 24.800 \\
\hline 1988 & 23.266 & 26.021 & 17.312 & 22.423 & 25.400 & 24.800 \\
\hline 1989 & 23.385 & 27.196 & 16.310 & 22.623 & 25.400 & 24.800 \\
\hline 1990 & 22.574 & 25.199 & 16.140 & 21.668 & 25.400 & 24.800 \\
\hline 1991 & 22.573 & 25.268 & 15.858 & 21.410 & 25.400 & 24.800 \\
\hline $1992 \ldots \ldots \ldots$ & 22.572 & 241.617 & 16.944 & 21.423 & 25.400 & 24.800 \\
\hline 1993 & 22.573 & 24.096 & 16.534 & 21.262 & 25.400 & 24.800 \\
\hline 1994 & 22.572 & 25.037 & 14.680 & 20.828 & 25.400 & 24.800 \\
\hline 1995 & 22.572 & 24.696 & 14.572 & 20.808 & 25.400 & 24.800 \\
\hline $1996^{a}$ & 22.572 & 24,696 & 14.572 & 20.808 & 25.400 & 24.800 \\
\hline $1997^{a}$ & 22.572 & 24.696 & 14.572 & 20.808 & 25.400 & 24.800 \\
\hline
\end{tabular}

a Preliminary.

Source: See "Thermal Conversion Factor Source Documentation," which follows Table A8. 
Table A8. Approximate Heat Rates for Electricity

(Btu per Kilowatthour)

\begin{tabular}{|c|c|c|c|c|}
\hline & \multicolumn{3}{|c|}{ Electricity Generation } & \multirow[b]{2}{*}{$\begin{array}{c}\text { Electricity } \\
\text { Consumption }\end{array}$} \\
\hline & $\begin{array}{c}\text { Fossil-Fueled } \\
\text { Steam-Electric } \\
\text { Plants }^{\mathrm{a}}\end{array}$ & $\begin{array}{c}\text { Nuclear } \\
\text { Steam-Electric } \\
\text { Plants }\end{array}$ & $\begin{array}{l}\text { Geothermal } \\
\text { Energy } \\
\text { Plants }\end{array}$ & \\
\hline 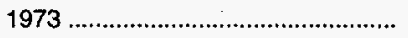 & 10,389 & 10,903 & 21,674 & 3,412 \\
\hline 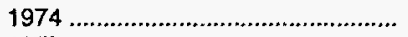 & 10,442 & 11,161 & 21,674 & 3,412 \\
\hline 1975 & 10,406 & 11,013 & 21,611 & 3,412 \\
\hline 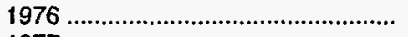 & 10,373 & 11,047 & $21,6 \pm 1$ & 3,412 \\
\hline 1977 & 10,435 & 10,769 & 21,611 & 3,412 \\
\hline 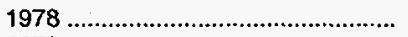 & 10,361 & 10,941 & 21,611 & 3,412 \\
\hline 1979 & 10,353 & 10,879 & 21,545 & 3,412 \\
\hline 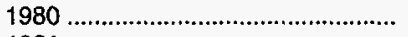 & 10,388 & 10,908 & 21,639 & 3,412 \\
\hline 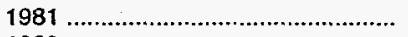 & 10,453 & 11,030 & 21,639 & 3,412 \\
\hline 1982 & 10,454 & 11,073 & 21,629 & 3,412 \\
\hline 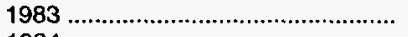 & 10,520 & 10,905 & 21,290 & 3,412 \\
\hline 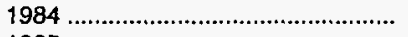 & 10,440 & 10,843 & 21,303 & 3,412 \\
\hline 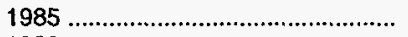 & 10,447 & 10,813 & 21,263 & 3,412 \\
\hline 1986 & 10,446 & 10,799 & 21,263 & 3,412 \\
\hline 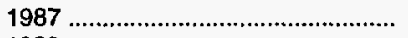 & 10,419 & 10,776 & 21,263 & 3,412 \\
\hline 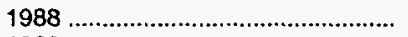 & 10,324 & 10,743 & 21,096 & 3,412 \\
\hline 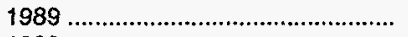 & 10,317 & 10,724 & 21,096 & 3,412 \\
\hline 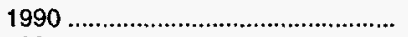 & 10,335 & 10,680 & 21,096 & 3,412 \\
\hline 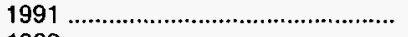 & 10,352 & 10,740 & 20,997 & 3,412 \\
\hline 1992 & 10,302 & 10,678 & 20,914 & 3,412 \\
\hline 1993 & 10,280 & 10,682 & 20,914 & 3,412 \\
\hline 1994 & 10,272 & 10,676 & 20,914 & 3,412 \\
\hline 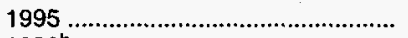 & 10,301 & 10,658 & 20,914 & 3,412 \\
\hline 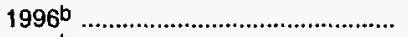 & $E_{10,301}$ & 10,623 & 20,960 & 3,412 \\
\hline 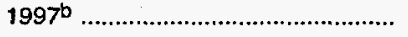 & $E_{10,301}$ & 10,623 & 20,960 & 3,412 \\
\hline
\end{tabular}

a This thermal conversion factor is used for hydroelectric power generation and for biomass fuels, wind, photovoltaic, and solar thermal energy consumed at electric utilities.

b Preliminary.

E=Estimated data.

Source: See "Thermal Conversion Factor Source Documentation," which follows this table.

\section{Thermal Conversion Factor Source Documentation}

\section{Approximate Heat Content of Petroleum and Natural Gas Plant Liquids}

Asphalt. The Energy Information Administration (EIA) adopted the thermal conversion factor of 6.636 million British thermal units (Btu) per barrel as estimated by the Bureau of Mines and first published in the Petroleum Statement, Annual, 1956.

Aviation Gasoline. EIA adopted the Bureau of Mines thermal conversion factor of 5.048 million Btu per barrel for "Gasoline, Aviation" as published by the
Texas Eastern Transmission Corporation in Appendix $\mathrm{V}$ of Competition and Growth in American Energy

Markets 1947-1985, a 1968 release of historical and projected statistics.

Butane. EIA adopted the Bureau of Mines thermal conversion factor of 4.326 million Btu per barrel in the California Oil World and Petroleum Industry, First Issue, April 1942.

Butane-Propane Mixture. EIA adopted the Bureau of Mines calculation of 4.130 million Btu per barrel based on an assumed mixture of 60 percent butane and 40 percent propane. See Butane and Propane.

Crude Oil, Exports. Assumed by EIA to be 5.800 million Btu per barrel or equal to the thermal conversion factor for crude oil produced in the United 
States. See Crude Oil and Lease Condensate, Production.

Crude Oil, Imports. Calculated annually by EIA by weighting the thermal conversion factor of each type of crude oil imported by the quantity imported. Thermal conversion factors for each type were calculated on a foreign country basis, by determining the average American Petroleum Institute (API) gravity of crude imported from each foreign country from Form ERA-60 in 1977 and converting average API gravity to average Btu content by using National Bureau of Standards, Miscellaneous Publication No. 97, Thermal Properties of Petroleum Products, 1933.

Crude Oil and Lease Condensate, Production. EIA adopted the thermal conversion factor of 5.800 million Btu per barrel as reported in a Bureau of Mines internal memorandum, "Bureau of Mines Standard Average Heating Values of Various Fuels, Adopted January 3, 1950."

Crude Oil and Petroleum Products, Exports. Calculated annually by EIA as the average of the thermal conversion factors for each petroleum product exported and crude oil exported weighted by the quantity of each petroleum product and crude oil exported. See Crude Oil, Exports and Petroleum Products, Exports.

Crude Oil and Petroleum Products, Imports. Calculated annually by EIA as the average of the thermal conversion factors for each petroleum product and each type of crude oil imported weighted by the quantity of each petroleum product and each type of crude oil imported. See Crude Oil, Imports and Petroleum Products, Imports.

Distillate Fuel Oil. EIA adopted the Bureau of Mines thermal conversion factor of 5.825 million Btu per barrel as reported in a Bureau of Mines internal memorandum, "Bureau of Mines Standard Average Heating Value of Various Fuels, Adopted January 3, 1950."

Ethane. EIA adopted the Bureau of Mines thermal conversion factor of 3.082 million Btu per barrel in the California Oil World and Petroleum Industry, First Issue, April 1942.

Ethane-Propane Mixture. EIA calculated 3.308 million Btu per barrel based on an assurned mixture of 70 percent ethane and 30 percent propane. See Ethane and Propane.

Isobutane. EIA adopted the Bureau of Mines thermal conversion factor of 3.974 million Btu per barrel in the California Oil World and Petroleum Industry, First Issue, April 1942.
Jet Fuel, Kerosene Type. EIA adopted the Bureau of Mines thermal conversion factor of 5.670 million Btu per barrel for "Jet Fuel, Commercial" as published by the Texas Eastern Transmission Corporation in Appendix $\mathrm{V}$ of Competition and Growth in American Energy Markets 1947-1985, a 1968 release of historical and projected statistics.

Jet Fuel, Naphtha Type. EIA adopted the Bureau of Mines thermal conversion factor of 5.355 million Btu per barrel for "Jet Fuel, Military" as published by the Texas Eastern Transmission Corporation in Appendix $\mathrm{V}$ of Competition and Growth in American Energy Markets 1947-1985, a 1968 release of historical and projected statistics.

Kerosene. EIA adopted the Bureau of Mines thermal conversion factor of 5.670 million Btu per barrel as reported in a Bureau of Mines internal memorandum, "Bureau of Mines Standard Average Heating Values of Various Fuels, Adopted January 3, 1950."

Liquefied Petroleum Gases (LPG) Consumption. Calculated annually by EIA as the average of the thermal conversion factors of each liquefied petroleum gas consumed, weighted by the quantity of each liquefied petroleum gas consumed.

Lubricants. EIA adopted the thermal conversion factor of 6.065 million Btu per barrel as estimated by the Bureau of Mines and first published in the Petroleum Statement, Annual, 1956.

Miscellaneous Products. EIA adopted the thermal conversion factor of 5.796 million Btu per barrel as estimated by the Bureau of Mines and first published in the Petroleum Statement, Annual, 1956.

Motor Gasoline. EIA adopted the Bureau of Mines thermal conversion factor of 5.253 million Btu per barrel for "Gasoline, Motor Fuel" by the Texas Eastern Transmission Corporation in Appendix $\mathrm{V}$ of Competition and Growth in American Energy Markets 1947-1985, a 1968 release of historical and projected statistics.

Natural Gas Plant Liquids, Production. Calculated annually by EIA as the average of the thermal conversion factors of each natural gas plant liquid produced weighted by the quantity of each natural gas plant liquid produced.

Natural Gasoline. EIA adopted the thermal conversion factor of 4.620 million Btu per barrel as estimated by the Bureau of Mines and first published in the Petroleum Statement, Annual, 1956.

Pentanes Plus. EIA assumed the thermal conversion factor to be 4.620 million Btu per barrel or equal to that for natural gasoline. See Natural Gasoline. 
Petrochemical Feedstocks, Naphtha Less Than 401 Degrees Fahrenheit. Assumed by EIA to be 5.248 million Btu per barrel, equal to the thermal conversion factor for special naphthas. See Special Naphthas.

Petrochemical Feedstocks, Oils Equal to or Greater Than 401 Degrees Fahrenheit. Assumed by EIA to be 5.825 million Btu per barrel, equal to the thermal conversion factor for distillate fuel oil. See Distillate Fuel Oil.

Petrochemical Feedstocks, Still Gas. Assumed by EIA to be 6.000 million Btu per barrel, equal to the thermal conversion factor for still gas. See Still Gas.

Petroleum Coke. EIA adopted the thermal conversion factor of 6.024 million Btu per barrel as reported in Btu per short ton in the Bureau of Mines internal memorandum, "Bureau of Mines Standard Average Heating Value of Various Fuels, Adopted January 3, 1950." The Bureau of Mines calculated this factor by dividing 30,120,000 Btu per short ton, as given in the referenced Bureau of Mines internal memorandum, by 5.0 barrels per short ton, as given in the Bureau of Mines Form 6-1300-M and successor EIA forms.

Petroleum Products, Total Consumption. Calculated annually by EIA as the average of the thermal conversion factors for all petroleum products consumed, weighted by the quantity of each petroleum product consumed.

Petroleum Products, Consumption by Electric Utilities. Calculated annually by EIA as the average of the thermal conversion factors for all petroleum products consumed at electric utilities, weighted by the quantity of each petroleum product consumed at electric utilities. The quantity of petroleum consumed is estimated in the State Energy Data System as documented in the State Energy Data Report.

Petroleum Products, Consumption by Industrial Users. Calculated annually by EIA as the average of the thermal conversion factors for all petroleum products consumed in the industrial sector, weighted by the estimated quantity of each petroleum product consumed in the industrial sector. The quantity of petroleum products consumed is estimated in the State Energy Data System as documented in the State Energy Data Report.

Petroleum Products, Consumption by Residential and Commercial Users. Calculated annually by EIA as the average of the thermal conversion factors for all petroleum products consumed by the residential and commercial sector, weighted by the estimated quantity of each petroleum product consumed in the residential and commercial sector. The quantity of petroleum products consumed is estimated in the State Energy Data System as documented in the State Energy Data Report.
Petroleum Products, Consumption by Transportation Users. Calculated annually by EIA as the average of the thermal conversion factor for all petroleum products consumed in the transportation sector, weighted by the estimated quantity of each petroleum product consumed in the transportation sector. The quantity of petroleum products consumed is estimated in the State Energy Data System as documented in the State Energy Data Report.

Petroleum Products, Exports. Calculated annually by EIA as the average of the thermal conversion factors for each petroleum product, weighted by the quantity of each petroleum product exported.

Petroleum Products, Imports. Calculated annually by EIA as the average of the thermal conversion factors for each petroleum product imported, weighted by the quantity of each petroleum product imported.

Plant Condensate. Estimated to be 5.418 million Btu per barrel by EIA from data provided by McClanahan Consultants, Inc., Houston, Texas.

Propane. EIA adopted the Bureau of Mines thermal conversion factor of 3.836 million Btu per barrel in the California Oil World and Petroleum Industry, First Issue, April 1942.

Residual Fuel Oil. EIA adopted the thermal conversion factor of 6.287 million Btu per barrel as reported in the Bureau of Mines internal memorandum, "Bureau of Mines Standard Average Heating Values of Various Fuels, Adopted January 3, 1950."

Road Oil. EIA adopted the Bureau of Mines thermal conversion factor of 6.636 million Btu per barrel, which was assumed to be equal to that of asphalt (see Asphalt) and was first published by the Bureau of Mines in the Petroleum Statement, Annual, 1970.

Special Naphthas. EIA adopted the Bureau of Mines thermal conversion factor of 5.248 million Btu per barrel, which was assumed to be equal to that of total gasoline (aviation and motor) factor and was first published in the Petroleum Statement, Annual, 1970.

Still Gas. EIA adopted the Bureau of Mines estimated thermal conversion factor of 6.000 million Btu per barrel and first published in the Petroleum Statement, Annual, 1970.

Unfinished Oil. EIA assumed the thermal conversion factor to be 5.825 million Btu per barrel or equal to that for distillate fuel oil (see Distillate Fuel Oil) and first published in the Annual Report to Congress, Volume 3, 1977.

Unfractionated Stream. EIA assumed the thermal conversion factor to be 5.418 million Btu per barrel or equal to that for plant condensate (see Plant 
Condensate) and first published in the Annual Report to Congress, Volume 2, 1981.

Waxes. EIA adopted the thermal conversion factor of 5.537 million Btu per barrel as estinated by the Bureau of Mines and first published in the Petroleum Statement, Annual, 1956.

\section{Approximate Heat Content of Natural Gas}

Natural Gas, Total Consumption. 1973-1979: EIA adopted the thermal conversion factor calculated annually by the American Gas Association (AGA) and published in Gas Facts, an AGA annual publication. 1980 forward: Calculated annually by EIA by dividing the total heat content of natural gas consumed by the total quantity of natural gas consumed. The heat content and quantity consumed are from Form EIA-176. Published sources are: 1980-1989: EIA, Natural Gas Annual 1992, Volume 2, Table 15. 1990-1992: EIA, Natural Gas Annual 1992, Volume 2, Table 16. 1993 forward: 1992 value used as an estimate.

Natural Gas, Consumption by Electric Utilities. Calculated annually by EIA by dividing the total heat content of natural gas received at electric utilities by the total quantity received at electric utilities. The heat contents and receipts are from Form FERC-423 and predecessor forms.

Natural Gas, Consumption by Sectors Other Than Electric Utilities. Calculated annually by EIA by dividing the heat content of all natural gas consumed less the heat content of natural gas consumed at electric utilities by the quantity of all natural gas consumed less the quantity of natural gas consumed at electric utilities. Data are from Forms EIA-176, FERC-423, EIA-759, and predecessor forms.

Natural Gas, Exports. Calculated annually by EIA by dividing the heat content of exported natural gas by the quantity of natural gas exported, both reported on Form FPC-14.

Natural Gas, Imports. Calculated annually by EIA by dividing the heat content of imported natural gas by the quantity of natural gas imported, both reported on Form FPC-14.

Natural Gas Production, Dry. Assumed by EIA to be equal to the thermal conversion factor for the consumption of dry natural gas. See Natural Gas Total Consumption.

Natural Gas Production, Marketed (Wet). Calculated annually by EIA by adding the heat content of dry natural gas production and the total heat content of natural gas plant liquids production and dividing this sum by the total quantity of marketed (wet) natural gas production.

\section{Approximate Heat Content of Coal and Coal Coke}

Anthracite, Total Consumption. Calculated annually by EIA by dividing the sum of the heat content of anthracite consumed by electric utilities and all other sectors combined by the total quantity of anthracite consumed.

Anthracite, Consumption by Electric Utilities. Calculated annually by EIA by dividing the heat content of anthracite receipts at electric utilities by the quantity of anthracite received at electric utilities. Heat contents and receipts are from Form FERC-423 and predecessor forms.

Anthracite, Consumption by Sectors Other Than Electric Utilities. Calculated annually by EIA by dividing the heat content of anthracite production less the heat content of the anthracite consumed at electric utilities, net exports, and shipments to U.S. Armed Forces overseas by the quantity of anthracite consumed by sectors other than electric utilities less the quantity of anthracite stock changes, losses, and "unaccounted for."

Anthracite, Imports and Exports. EIA assumed the anthracite imports and exports to be freshly mined anthracite having an estimated heat content of 25.40 million Btu per short ton.

Anthracite, Production. Calculated annually by EIA by dividing the sum of the heat content of freshly mined anthracite (estimated to have an average heat content of 25.400 million Btu per short ton) and the heat content of anthracite recovered from culm banks and river dredging (estimated to have a heat content of 17.500 million Btu per short ton) by the total quantity of anthracite production.

Bituminous Coal and Lignite, Total Consumption. Calculated annually by EIA by dividing the sum of the heat content of bituminous coal and lignite consumed by electric utilities, coal coke plants, other industrial plants, the residential and commercial sector, and the transportation sector by the sum of their respective tonnages.

Bituminous Coal and Lignite, Consumption by Coke Plants. Estimated by EIA to be 26.800 million Btu per short ton on the basis of an input/output analysis of coal carbonization. 
Bituminous Coal and Lignite, Consumption by Electric Utilities. Calculated annually by EIA by dividing the total heat content of bituminous coal and lignite received at electric utilities by the total quantity received at electric utilities. Heat contents and receipts are from Form FERC-423 and predecessor forms.

Bituminous Coal and Lignite, Consumption by Other Industrial and Transportation Users. 1973: Calculated by EIA through regression analysis measuring the difference between the average Btu value of coal consumed by other industrial users and that of coal consumed at electric utilities in the 1974-1982 period. 1974 forward: Calculated annually by EIA by assuming that the bituminous coal and lignite delivered to other industrial users from each coal-producing area (reported on Form EIA-6 and predecessor Bureau of Mines Form 6-1419-Q) contained a heat value equal to that of bituminous coal and lignite received at electric utilities from each of the same coal-producing areas (reported on Form FERC-423). The average Btu value of coal by coal-producing area was applied to the volume of deliveries to other industrial users from each coal-producing area, and the sum total of the heat content was divided by the total volume of deliveries. Coal-producing areas are the Bureau of Mines coal-producing districts for 1974 through 1989 and coal-producing States for 1990 forward.

Bituminous Coal and Lignite, Consumption by Residential and Commercial Users. 1973: Calculated by EIA through regression analysis measuring the difference between the average Btu value of coal consumed by residential and commercial users and that of coal consumed by electric utilities in the 1974-1982 period. 1974 forward: Calculated annually by EIA by assuming that the bituminous coal and lignite delivered to residential and commercial users from each coal-producing area (reported on Form EIA- 6 and predecessor Bureau of Mines Form 6-1419-Q) contained a heat value equal to that of bituminous coal and lignite received at electric utilities from each of the same coal-producing areas (reported on Form FERC-423). The average Btu value of coal by coal-producing area was applied to the volume of deliveries to residential and commercial users from each coal-producing area, and the total of the heat value was divided by the total volume of deliveries. Coal-producing areas are the Bureau of Mines coal-producing districts for 1974 through 1989 and coal-producing States for 1990 forward.

Bituminous Coal and Lignite, Exports. Calculated annually by EIA by dividing the sum of the heat content of exported metallurgical coal (estimated to average 27.000 million Btu per short ton) and the heat content of exported steam coal (estimated to have an average thermal content of 25.000 million Btu per short ton) by the total quantity of bituminous coal and lignite exported.

Bituminous Coal and Lignite, Imports. EIA estimated the average thermal conversion factor to be 25.000 million Btu per short ton.

Bituminous Coal and Lignite, Production. Calculated annually by EIA by dividing the sum of the heat content of bituminous coal and lignite consumption, net exports, stock changes, and unaccounted for by the sum of their respective tonnages. Consumers' stock changes by sectors were assumed to have the same conversion factor as that of the consumption sector. Producers' stock changes and unaccounted for were assumed to have the same conversion factor as that for consumption by all users.

Coal, Consumption. Calculated annually by EIA by dividing the sum of the heat content of bituminous coal and lignite and anthracite consumption by the sum of their respective tonnages.

Coal, Consumption by Electric Utilities. Calculated annually by EIA by dividing the sum of the heat content of bituminous coal and lignite and anthracite received at electric utilities by the sum of their respective tonnages received.

Coal, Consumption by Sectors Other Than Electric Utilities. Calculated annually by EIA by dividing the sum of the heat content of bituminous coal and lignite and anthracite consumed by sectors other than electric utilities by the sum of their respective tonnages.

Coal, Exports. Calculated annually by EIA by dividing the sum of the heat content of bituminous coal and lignite and anthracite exported by the sum of their respective tonnages.

Coal, Imports. Calculated annually by EIA by dividing the sum of the heat content of bituminous coal and lignite and anthracite imported by the sum of their respective tonnages.

Coal, Production. Calculated annually by EIA by dividing the sum of the total heat content of bituminous coal and lignite and anthracite production by the sum of their respective tonnages.

Coal Coke, Imports and Exports. EIA adopted the Bureau of Mines estimate of 24.800 million Btu per short ton.

\section{Approximate Heat Rates for Electricity}

Fossil-Fueled Steam-Electric Plant Generation. There is no generally accepted practice for measuring the thermal conversion rates for power plants that generate electricity from hydroelectric, wood and 
waste, wind, photovoltaic, or solar thermal energy sources. Therefore, EIA uses data from Form EIA-767 to calculate a rate factor that is equal to the prevailing annual average heat rate factor for fossil-fueled steam-electric power plants in the United States. By using that factor, it is possible to evaluate fossil fuel requirements for replacing those sources during periods of interruption such as droughts. The heat content of a kilowatthour of electricity produced, regardless of the generation process, is $3,412 \mathrm{Btu}$ per kilowatthour. 1973-1991: The weighted annual average heat rate for fossil-fueled steam-electric power plants in the United States, as published by EIA in Electric Plant Cost and Power Production Expenses 1991, Table 9. 1992 forward: Unpublished factors calculated on the basis of data from Form EIA-767.

Geothermal Energy Plant Generation. 1973-1981: Calculated annually by EIA by weighting the annual average heat rates of operating geothermal units by the installed nameplate capacities as reported on Form
FPC-12. 1982 forward: Estimated annually by EIA on the basis of an informal survey of relevant plants.

Nuclear Steam-Electric Plant Generation. 1973-1991: Calculated annually by EIA by dividing the total heat content consumed in nuclear generating units by the total (net) electricity generated by nuclear generating units. The heat content and electricity generation are reported on Form FERC-1, Form EIA-412, and predecessor forms. The factors, beginning with 1982 data, are published in the following EIA reports-1982: Historical Plant Cost and Annual Production Expenses for Selected Electric Plants 1982, page 215. 1983-1991: Electric Plant Cost and Power Production Expenses 1991, Table 13. 1992 forward: Calculated annually by EIA by dividing the total heat content of the steam leaving the nuclear generating units to generate electricity by the total (net) electricity generated by nuclear generating units. The heat content and electricity generation data are reported in Nuclear Regulatory. Commission, Licensed Operating Reactors-Status Summary Report. 


\section{Appendix B. Metric and Other Physical Conversion Factors}

Data presented in the Monthly Energy Review and in other Energy Information Administration publications are expressed predominately in units that historically have been used in the United States, such as British thermal units, barrels, cubic feet, and short tons. However, because U.S. commerce involves other nations, most of which use metric units of measure, the U.S. Government is committed to the transition to the metric system, as stated in the Metric Conversion Act of 1975 (Public Law 94-168), amended by the Omnibus Trade and Competitiveness Act of 1988 (Public Law 100-418), and Executive Order 12770 of July 25, 1991.

The metric conversion factors presented in Table B1 can be used to calculate the metric-unit equivalents of values expressed in U.S. customary units. For example, 500 short tons are the equivalent of 453.6 metric tons ( 500 short tons $\mathrm{x} 0.9071847$ metric tons $/$ short ton $=453.6$ metric tons).

In the metric system of weights and measures, the names of multiples and subdivisions of any unit may be derived by combining the name of the unit with prefixes, such as deka, hecto, and kilo, meaning, respectively, 10, 100, 1,000, and deci, centi, and milli, meaning, respectively, one-tenth, one-hundredth, and one-thousandth. Common metric prefixes can be found in Table B2.

The conversion factors presented in Table B3 can be used to calculate equivalents in various physical units commonly used in energy analyses. For example, 10 barrels are the equivalent of 420 U.S. gallons (10 barrels $\times 42$ gallons/barrel $=420$ gallons). 


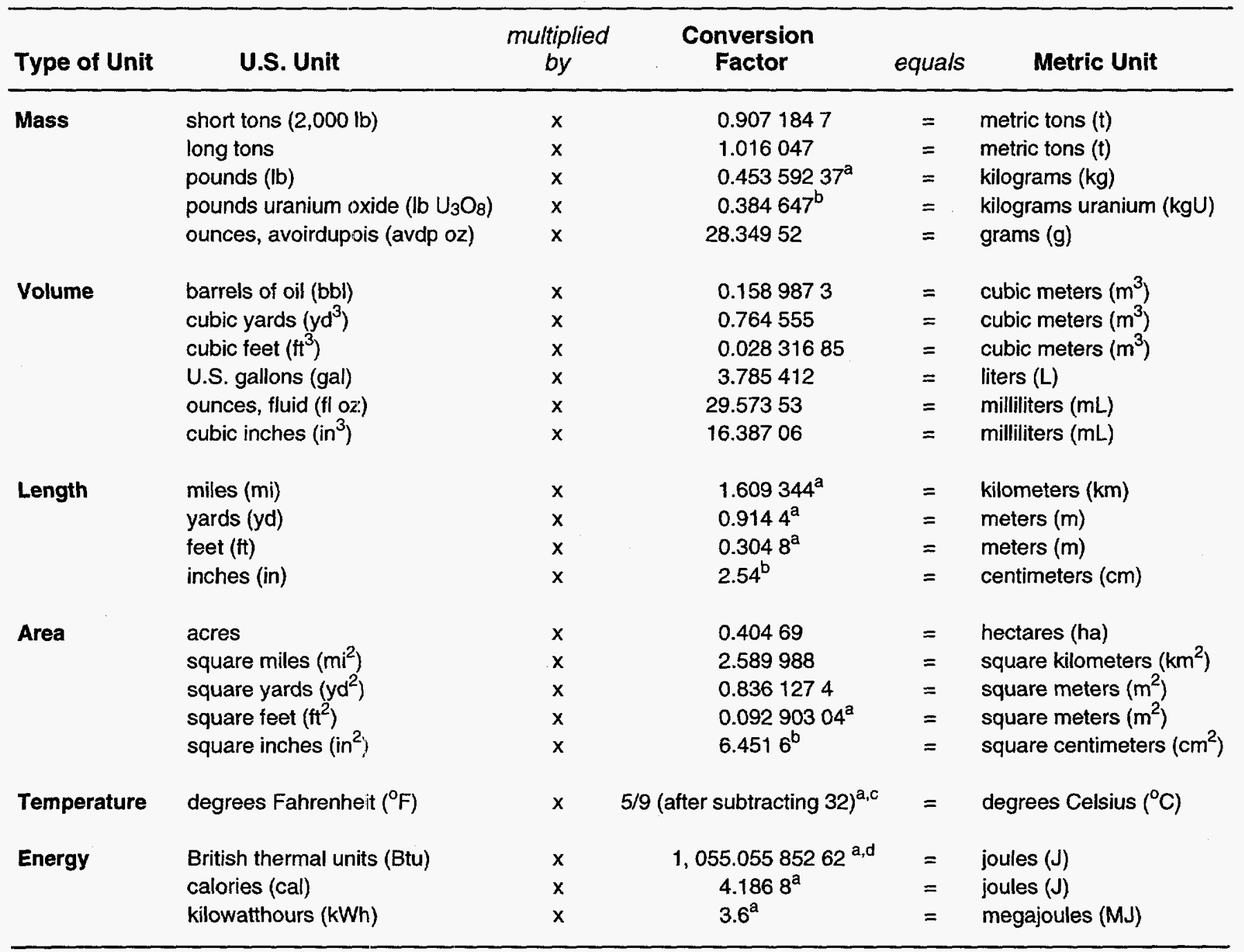

Exact conversion.

${ }^{b}$ Calculated by the Energy information Administration.

${ }^{\circ}$ To convert degrees Celsius $\left({ }^{\circ} \mathrm{C}\right)$ to degrees Fahrenheit $\left({ }^{\circ} \mathrm{F}\right)$ exactly, multiply by $9 / 5$, then add 32 .

The Btu used in this table is the International Table Btu adopted by the Fifth International Conference on Properties of Steam, London, 1956.

Notes: - Spaces have been inserted after avery third digit to the right of the decimal for ease of reading. - Most metric units belong to the International System of Units (SI), and the liter, hectare, and metric ton are accepted for use with the SI units. For more information about the SI units, contact Dr. Barry Taylor at Building 221, Room B610, National Institute of Standards and Technology, Gaithersburg, MD 20899, or on telephone number 301-975-4220.

Sources: - General Services Administration. Federal Standard 376B, Preferred Metric Units for General Use by the Federal Government (Washington, DC, January 27, 1993), pp. 9-11, 13, and 16. - National Institute of Standards and Technology, Special Publications 330, 811 , and 814. American National Standards Institute/Institute of Electrical and Electronic Engineers, ANSI/IEEE Std 268-1992, pp. 28 and 29. 
Table B2. Metric Prefixes

\begin{tabular}{|c|c|c|c|c|c|}
\hline $\begin{array}{l}\text { Unit } \\
\text { Multiple }\end{array}$ & Prefix & Symbol & $\begin{array}{l}\text { Unit } \\
\text { Subdivision }\end{array}$ & Prefix & Symbol \\
\hline $\begin{array}{l}10^{1} \\
10^{2} \\
10^{3} \\
10^{6} \\
10^{9} \\
10^{12} \\
10^{15} \\
10^{18} \\
10^{21} \\
10^{24}\end{array}$ & $\begin{array}{l}\text { deka } \\
\text { hecto } \\
\text { kilo } \\
\text { mega } \\
\text { giga } \\
\text { tera } \\
\text { peta } \\
\text { exa } \\
\text { zetta } \\
\text { yotta }\end{array}$ & $\begin{array}{l}\mathrm{da} \\
\mathrm{h} \\
\mathrm{k} \\
\mathrm{M} \\
\mathrm{G} \\
T \\
P \\
E \\
Z \\
Y\end{array}$ & $\begin{array}{l}10^{-1} \\
10^{-2} \\
10^{-3} \\
10^{-6} \\
10^{-9} \\
10^{-12} \\
10^{-15} \\
10^{-18} \\
10^{-21} \\
10^{-24}\end{array}$ & $\begin{array}{l}\text { deci } \\
\text { centi } \\
\text { milli } \\
\text { micro } \\
\text { nano } \\
\text { pico } \\
\text { femto } \\
\text { atto } \\
\text { zepto } \\
\text { yocto }\end{array}$ & $\begin{array}{l}d \\
c \\
m \\
\mu \\
n \\
p \\
f \\
a \\
z \\
y\end{array}$ \\
\hline
\end{tabular}

Source: U.S. Department of Commerce, National Institute of Standards and Technology, The International System of Units (SI), NIST Special Publication 330, 1991 Edition (Washington, DC, August 1991), p. 10.

Table B3. Other Physical Conversion Factors

\begin{tabular}{|c|c|c|c|c|c|}
\hline Energy Source & Original Unit & $\begin{array}{l}\text { multiplied } \\
\text { by }\end{array}$ & $\begin{array}{l}\text { Conversion } \\
\text { Factor }\end{array}$ & equals & Final Unit \\
\hline Petroleum & barrels (bbl) & $x$ & $42^{a}$ & $=$ & U.S. gallons (gal) \\
\hline Coal & $\begin{array}{l}\text { short tons } \\
\text { long tons } \\
\text { metric tons (t) }\end{array}$ & $\begin{array}{l}x \\
x \\
x\end{array}$ & $\begin{array}{l}2,000^{a} \\
2,240^{a} \\
1,000^{a}\end{array}$ & $\begin{array}{l}= \\
= \\
=\end{array}$ & $\begin{array}{l}\text { pounds (lb) } \\
\text { pounds (lb) } \\
\text { kilograms (kg) }\end{array}$ \\
\hline Wood & $\begin{array}{l}\text { cords }(c d) \\
\text { cords }(c d)\end{array}$ & $\begin{array}{l}x \\
x\end{array}$ & $128^{a .25^{b}}$ & $\begin{array}{l}= \\
=\end{array}$ & $\begin{array}{l}\text { short tons } \\
\text { cubic feet }\left(\mathrm{ft}^{3}\right)\end{array}$ \\
\hline
\end{tabular}

\footnotetext{
${ }^{\mathrm{a}}$ Exact conversion.

${ }^{b}$ Calculated by the Energy Information Administration.

Source: U.S. Department of Commerce, National Institute of Standards and Technology, Specifications, Tolerances, and Other Technical Requirements for Weighing and Measuring Devices, NIST Handbook 44, 1994 Edition (Washington, DC, October 1993), pp. B-10, C-17 and C-21.
} 



\section{Appendix C. Carbon Dioxide Emission Factors for Coal}

The need for accurate estimates of carbon dioxide emissions produced during the combustion of coal has led the Energy Information Administration (EIA) to develop basic emission factors. Basic emission factors reflect the carbon-to-heat-content ratio of coal, a ratio which measures carbon dioxide emissions per unit of energy (pounds per million Btu), assuming complete combustion. These basic factors are derived from 5,426 sample analyses maintained in EIA's Coal Analysis File. Variations in the carbon-to-heat-content ratios of different coals were observed to follow coal rank and geographic origin, leading EIA to develop basic emission factors specific to the rank and the State of origin of the coal.

On the basis of these rank- and State-specific basic emission factors for coal, EIA has also developed emission factors by sector. These sectoral emission factors weight the coal consumed in a given sector by its rank and State of origin. Table $\mathrm{Cl}$ presents the U.S. average carbon dioxide emission factors for coal by sector. Emission factors differ among sectors and within a given sector over time for a number of reasons:
- A higher average emission factor in the residential and commercial sector can be attributed to the steady consumption of bituminous coal and anthracite (presumably for home heating).

- Virtually all of the coal consumed by coke plants comes from only a few States in the Appalachian Coal Basin (West Virginia, Virginia, and eastern Kentucky). Hence, the emission factors for this sector have remained fairly constant.

- Other industrial users of coal (not coke plants) increased consumption of low-rank, high-emission western coals, which has contributed to a rise in their average emission factor.

- Electric utilities, which account for most U.S. coal consumption, have shifted over time away from highrank, low-emission bituminous coal to low-rank, highemission subbituminous coal and lignite as reflected in a gradually rising weighted-average carbon dioxide emission factor.

Table C1. Average Carbon Dioxide Emission Factors for Coal by Coal-Consuming Sector (Pounds of Carbon Dioxide per Million Btu)

\begin{tabular}{|c|c|c|c|c|c|}
\hline \multirow[b]{2}{*}{ Year } & \multirow{2}{*}{$\begin{array}{l}\text { Residential and } \\
\text { Commercial }\end{array}$} & \multicolumn{2}{|c|}{ Industrial } & \multirow{2}{*}{$\begin{array}{l}\text { Electric } \\
\text { Utilities }\end{array}$} & \multirow{2}{*}{$\begin{array}{c}\text { U.S. } \\
\text { Average }^{k}\end{array}$} \\
\hline & & Coke Plants $^{a}$ & Other Coal & & \\
\hline 1980 & 210.6 & 205.8 & 205.9 & 206.7 & 206.5 \\
\hline 1981 & 212.0 & 205.8 & 205.9 & 206.9 & 206.7 \\
\hline 1982 & 210.4 & 205.7 & 206.0 & 207.0 & 206.9 \\
\hline 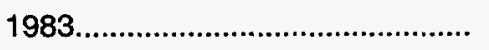 & 209.2 & 205.5 & 205.9 & 207.1 & 207.0 \\
\hline 1984 & 209.5 & 205.6 & 206.2 & 207.1 & 207.0 \\
\hline 1985 & 209.3 & 205.6 & 206.4 & 207.3 & 207.1 \\
\hline 1986 & 209.2 & 205.4 & 206.5 & 207.3 & 207.1 \\
\hline 1987 & 209.4 & 205.2 & 206.4 & 207.3 & 207.2 \\
\hline 1988 & 209.1 & 205.3 & 206.4 & 207.6 & 207.3 \\
\hline 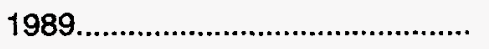 & 209.7 & 205.3 & 206.6 & 207.5 & 207.3 \\
\hline 1990 & 209.5 & 206.2 & 206.8 & 207.6 & 207.4 \\
\hline $1991 \ldots$ & 210.2 & 206.2 & 206.9 & 207.7 & 207.5 \\
\hline 1992 & 211.2 & 206.2 & 207.1 & 207.7 & 207.6 \\
\hline 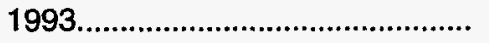 & 209.9 & 206.2 & 207.0 & 207.8 & 207.7 \\
\hline 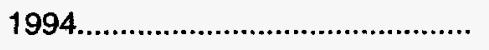 & 209.8 & 206.3 & 207.2 & 207.9 & 207.8 \\
\hline 1995 & 210.2 & 206.4 & 207.2 & 208.1 & 207.9 \\
\hline
\end{tabular}

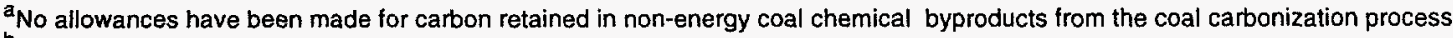

${ }^{b}$ Weighted average. The weights used are consumption values by sector.

Source: Energy information Administration, Office of Coal, Nuclear, Electric and Alternate Fuels.
} 



\section{Appendix D. List of Features}

The following is a complete list of features that have appeared in the Monthly Energy Review since the first issue was published in October 1974. There are several categories of features on the list: "Energy Plugs" are 1-page descriptions of recently released EIA products. "Articles" cover a wide range of energy-related subjects in depth; "Highlights" summarize the most important information presented in the subject Energy
Information Administration (EIA) report; "Energy Previews" provide brief overviews of EIA preliminary energy data on a given topic; "EIA Data News" items present information on recent changes in the scope, design, methodology, and findings of EIA's energy surveys and databases; and "Energy Snapshots" use graphics to set off key data from EIA survey reports.

\section{Feature}

\section{Cover Date}

\section{7}

Energy Plug: Annual Energy Outlook 1997

Energy Plug: The Changing Structure of the Electric Power industry: An Update . . . . . . . . . . . . .

Energy Plug: Performance Profiles of Major Energy Producers $1995 \ldots \ldots \ldots \ldots \ldots \ldots \ldots \ldots$

Energy Plug: The Effects of Title IV of the Clean Air Act Amendments of 1990 on Electric Utilities:

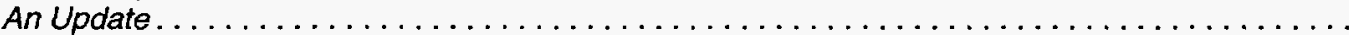

Energy Plug: International Energy Outlook $1997 \ldots \ldots \ldots \ldots \ldots \ldots \ldots \ldots \ldots \ldots \ldots \ldots$

Energy Plug: Restructuring Energy Industries: Lessons From Natural Gas . . . . . . . . . . . .

Energy Plug: An Analysis of U.S. Propane Markets: Winter 1996-97. . . . . . . . . . . . . . . .

Energy Plug: State Energy Price and Expenditure Report $1994 \ldots \ldots \ldots \ldots \ldots \ldots \ldots \ldots$

Energy Plug: Annual Energy Review 1996.

Energy Plug: Motor Gasoline Assessment $1997 \ldots \ldots \ldots \ldots \ldots \ldots \ldots \ldots \ldots \ldots \ldots \ldots \ldots$

Energy Plug: Commercial Buildings Characteristics $1995 \ldots \ldots \ldots \ldots \ldots \ldots \ldots \ldots \ldots \ldots \ldots$

Energy Plug: Household Vehicles Energy Consumption $1994 \ldots \ldots \ldots \ldots \ldots \ldots \ldots \ldots \ldots$

Energy Plug: Electricity Prices in a Competitive Environment . . . . . . . . . . . . . . . .

Energy Plug: Petroleum 1996: Issues and Trends . . . . . . . . . . . . . . . . . . .

Energy Plug: The Intricate Puzzle of Oil and Gas "Reserves Growth" . . . . . . . . . . . . .

Energy Plug: Emissions of Greenhouse Gases in the United States 1996 . . . . . . . . . . . . .

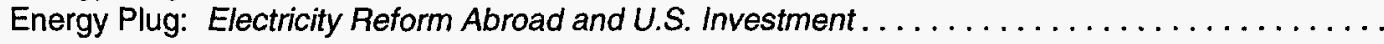

Energy Plug: Annual Energy Outlook $1998 \ldots \ldots \ldots \ldots \ldots \ldots \ldots \ldots \ldots \ldots \ldots \ldots \ldots \ldots$

Energy Plug: Winter Heating Fuels Assessments . . . . . . . . . . . . . . . . . . .

Energy Plug: Oil and Gas Resources of the West Siberian Basin, Russia . . . . . . . . . . . . . .

\section{6}

Energy Plug: Renewable Energy Annual $1995 \ldots \ldots \ldots \ldots \ldots \ldots \ldots \ldots \ldots \ldots \ldots \ldots \ldots$

Energy Plug: State Energy Price and Expenditure Report $1993 \ldots \ldots \ldots \ldots \ldots \ldots \ldots \ldots \ldots$

Energy Plug: Annual Energy Outlook $1996 \ldots \ldots \ldots \ldots \ldots \ldots \ldots \ldots \ldots \ldots \ldots \ldots$

Energy Plug: Alternatives to Traditional Transportation Fuels 1994 , Volume $1 \ldots \ldots \ldots \ldots$

Energy Snapshot: Describing Current and Potential Markets for Alternative-Fuel Vehicles .......

Article: Energy Equipment Choices: Fuel Costs and Other Determinants . . . . . . . . . . .

Energy Plug: Intemational Energy Outlook 1996.

Energy Plug: U.S. Electric Utility Demand-Side Management: Trends and Analysis . . . . . . . . . .

Energy Plug: Country Analysis Brief: Iraq

Energy Plug: Annual Energy Review $1995 \ldots \ldots \ldots \ldots \ldots \ldots \ldots \ldots \ldots \ldots \ldots \ldots \ldots \ldots$

Energy Plug: Voluntary Reporting of Greenhouse Gases 1995 . . . . . . . . . . . . . . . . . .

Energy Plug: Residential Lighting: Use and Potential Savings $\ldots \ldots \ldots \ldots \ldots \ldots \ldots \ldots$

Energy Plug: ElA Electronic Media Meet Customer Needs . . . . . . . . . . . . . . . . . .

Energy Plug: Alternatives to Traditional Transportation Fuels, Volume 2: Greenhouse

Gas Emissions . . . . . . . . . . . . . . . . . . . . . . . . . . .

Energy Plug: State Energy Data Report $1994 \ldots \ldots \ldots \ldots \ldots \ldots \ldots \ldots \ldots \ldots \ldots \ldots$

Energy Plug: Privatization and the Globalization of Energy Markets . . . . . . . . . . . . . . .

Energy Plug: Emissions of Greenhouse Gases in the United States 1995 . . . . . . . . . . . . . .

Energy Plug: Nuclear Power Generation and Fuel Cycle Report 1996 . . . . . . . . . . . . . . .

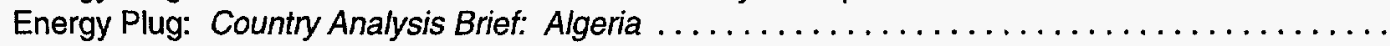

Energy Plug: Denver Clean-City Fleets Survey . . . . . . . . . . . . . . . . . . .

Energy Plug: Natural Gas 1996: Issues and Trends

January 1997

January 1997

January 1997

March 1997

April 1997

May 1997

June 1997

June 1997

July 1997

July 1997

July 1997

August 1997

August 1997

September 1997

September 1997

October 1997

October 1997

November 1997

December 1997

December 1997

January 1996 January 1996 February 1996

February 1996

March 1996

April 1996

May 1996

May 1996

June 1996

July 1996

July 1996

August 1996

August 1996

September 1996

October 1996

October 1996

October 1996

November 1996

November 1996

November 1996

December 1996 


\section{5}

Highlights: Manufacturing Consumption of Energy 1991.

January 1995

Article: U.S. Wind Energy Potential: The Effect of the Proximity of Wind Resources

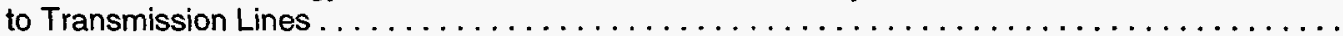

EIA Data News: The Response Analysis Survey: Evaluating Manufacturing Energy

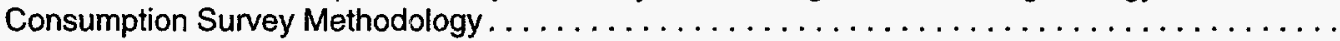

Energy Preview: Electric Utility Fleet Survey 1993, Preliminary Estimates: Assessing the

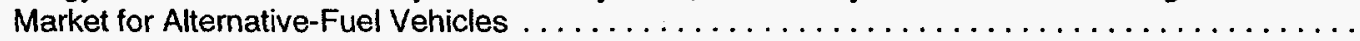

Highlights: Commercial Buildings Energy Consumption and Expenditures 1992 .............

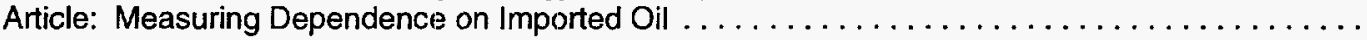

Energy Preview: Household Energy Consumption and Expenditures 1993, Preliminary

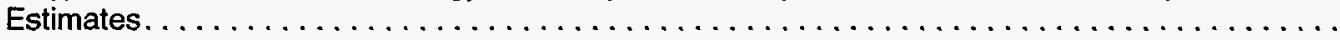

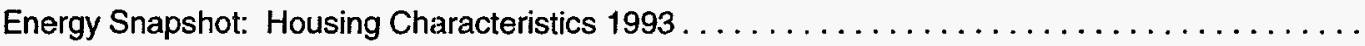

Highlights: State Energy Data Report 1993, Consumption Estimates . ..................

Special Communication: Results of the Monthly Energy Review Features Readership Survey.... Highlights: Annual Energy Review 1994.

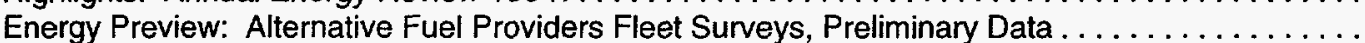
Article: Environmental Externalities in Electric Power Markets: Acid Rain, Urban Ozone, and

Climate Change

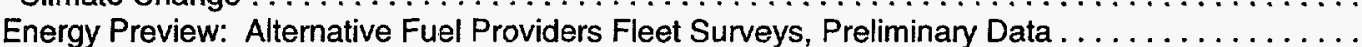

February 1995

March 1995

April 1995

April 1995

August 1995

August 1995

September 1995

October 1995

November 1995

November 1995

November 1995

November 1995

December 1995

\section{4}

Energy Preview: Commercial Buildings Energy Consumption Survey,

Preliminary Estimates, 1992

Highlights: Household Vehicles Energy Cons

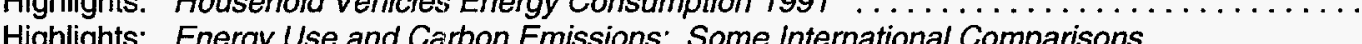

Highlights: Commercial Buildings Characteristics 1992 ...........................

Article: Demand, Supply, and Price Outlook for Reformulated Motor Gasoline 1995 . . . . . . . .

Article: Commercial Nuclear Electric Power in the United States: Problems and Prospects . . . . .

Highlights: Reducing Home Heating and Cooling Costs ........................

Energy Preview: Commercial Buildings Energy Consumption and Expenditures 1992,

Preliminary Estimates . . . . . . . . . . . . . . . . . . . . . . . . . . . . . . . .

Article: Carbon Dioxide Emission Factors for Coal: A Summary.....................

Article: The Impact of Flow Control and Tax Reform on Ownership and Growth in the U.S.

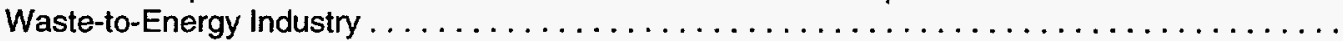

EIA Data News: Data Collection on Alternative-Fuel Vehicles $\ldots \ldots \ldots \ldots \ldots \ldots \ldots \ldots \ldots$

Highlights: Energy End-Use Intensities in Commercial Buildings .................... Article: Change in Method for Estimating Fuel Economy for the Residential Transportation

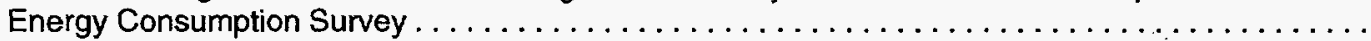

Article: Comparability of Supply- and Consumption-Derived Estimates of Manufacturing

Energy Consumption.

Energy Preview: Housing Characteristics 1993, Selected Preliminary Estimates . . . . . . . . . . . . .

Energy Preview: Propane-Provider Fleet Survey 1993, Preliminary Estimates . . . . . . . . . . . .

Energy Preview: Atlanta Private Fleet Survey 1994, Preliminary Estimates . . . . . . . . . . . . . . .

\section{3}

Energy Preview: Residential Transportation Energy Consumption Survey,

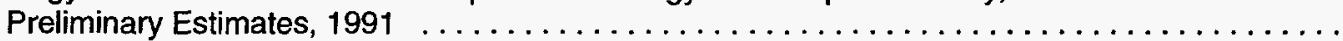

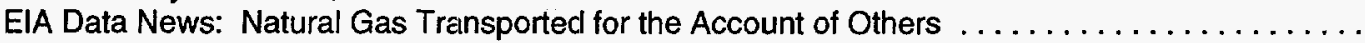
Highlights: Federal Energy Subsidies: Direct and Indirect Intenentions in Energy Markets ...... Highlights: Household Energy Consumption and Expenditures 1990 ................... Article: Demand, Supply, and Price Outlook for Low-Sulfur Diesel Fuel ................. Energy Preview: Manufacturing Energy Consumption Survey, Preliminary Estimates, 1991 ....

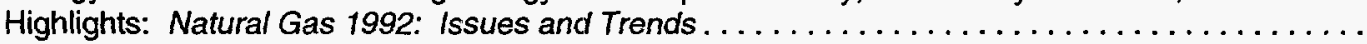

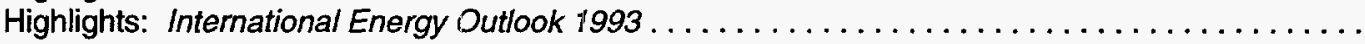
Highlights: The Changing Structure of the U.S. Coal Industry: An Update ................ Highlights: Emissions of Greenhouse Gases in the United States 1985-1990 . . . . . . . . . . . .

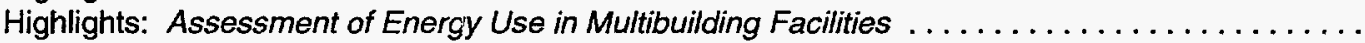

January 1994

February 1994

April 1994

June 1994

July 1994

August 1994

August 1994

September 1994

September 1994

September 1994

October 1994

October 1994

October 1994

October 1994

November 1994

November 1994

December 1994

January 1993

February 1993

July 1993

August 1993

August 1993

September 1993

September 1993

October 1993

November 1993

December 1993

December 1993

April 1992

May 1992

June 1992

August 1992 


\section{2 (Continued)}

EIA Data News: EIA Statistics on Electric Utility Demand-Side Management $\ldots \ldots \ldots \ldots \ldots \ldots$ EIA Data News: EIA Statistics on Nonutility Power Producers ........................ Highlights: Derived Annual Estimates of Manufacturing Energy Consumption, 1974-1988 . . . . . . . Article: Energy Efficiency in the Manufacturing Sector

September 1992 October 1992

November 1992 December 1992

\section{1}

Highlights: U.S. Energy Industry Financial Developments, 1990 Fourth Quarter . . . . . . . . . . .

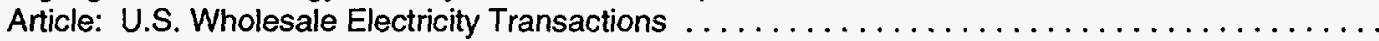

March 1991

April 1991

\section{0}

Article: Refining Results Highlight Energy Companies' First-Half Profit Performance . . . . . . . . . Highlights: U.S. Oil and Gas Resenves by Year of Field Discovery . . . . . . . . . . . . . . . . .

June 1990

August 1990

\section{9}

Article: A Review of Valdez Oil Spill Market Impacts $\ldots \ldots \ldots \ldots \ldots \ldots \ldots \ldots \ldots \ldots \ldots$ Article: Monthly U.S. Crude Oil Production Estimates . . . . . . . . . . . . . . . . . . . Article: Superconductivity and Energy Production and Consumption . . . . . . . . . . . . . Highlights: Commercial Buildings Consumption and Expenditures $1986 \ldots \ldots \ldots \ldots \ldots$ Article: Higher Prices Yield Improved Energy Industry Financial Results

in the First Half of 1989

Article: The Future Structure of the U.S. Commercial Nuclear Power Equipment

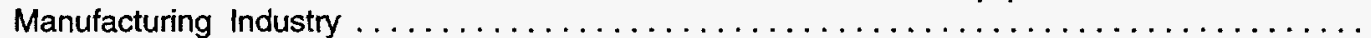
Highlights: Potential Costs of Restricting Chlorofluorocarbon Use . . . . . . . . . . . . . . . . Highlights: Manufacturing Energy Consumption Suney: Changes in Energy Efficiency, 1980-1985 Highlights: Household Energy Consumption and Expenditures 1987, Part 1: National Data ..... Article: Improved Energy Profits Offset by Refining Results in $1989 \ldots \ldots \ldots \ldots \ldots \ldots$. . . . .

March 1989 March 1989

May 1989

May 1989

June 1989

July 1989

September 1989

October 1989

November 1989

December 1989

\section{8}

Article: Measures of Energy Consumption, Expenditures, and Prices . . . . . . . . . . . . . . Article: The U.S. Energy Industry's Financial Recovery Continued in the First Half of $1988 \ldots \ldots \ldots$

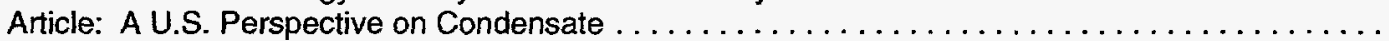
Highlights: Characteristics of Commercial Buildings $1986 \ldots \ldots \ldots \ldots \ldots \ldots \ldots \ldots \ldots \ldots$ Article: State Energy Severance Taxes, $1972-1987 \ldots \ldots \ldots \ldots \ldots \ldots \ldots \ldots \ldots \ldots \ldots \ldots \ldots$ Highlights: Manufacturing Energy Consumption Survey: Consumption of Energy, $1985 \ldots \ldots \ldots$ Highlights: Profiles of Foreign Direct Investment in U.S. Energy $1987 \ldots \ldots \ldots \ldots \ldots$. . . . . . . Highlights: Manufacturing Energy Consumption Survey: Fuel Switching, $1985 \ldots \ldots \ldots \ldots$. . . . . Article: Increased Refining Income Led U.S. Energy Industry Financial Recovery in $1988 \ldots \ldots \ldots$

May 1988 June 1988 June 1988 June 1988 July 1988 September 1988 October 1988 November 1988 December 1988

\section{7}

Article: Manufacturing Sector Energy Consumption, 1985 Provisional Estimates . . . . . . . . . . . Highlights: Consumption and Expenditures, April 1984 Through March 1985,

Part 1: National Data . . . . . . . . . . . . . . . . . . . . . . . . . . . Highlights: Consumption and Expenditures, April 1984 Through March 1985,

Part 2: Regional Data . . . . . . . . . . . . . . . . . . . . . . . . . . . . Article: U.S. Energy Industry Financial Developments, 1987 Second Quarter . . . . . . . . . . . . Article: End-Use Consumption of Residential Energy $\ldots \ldots \ldots \ldots \ldots \ldots \ldots \ldots \ldots \ldots \ldots$

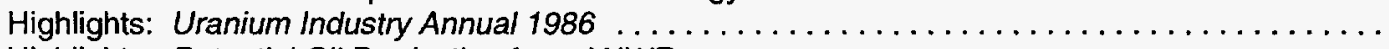
Highlights: Potential Oil Production from ANWR . . . . . . . . . . . . . . . . . . . Highlights: Profiles of Foreign Direct Investment in U.S. Energy $1986 \ldots \ldots \ldots \ldots \ldots \ldots \ldots$ Article: The U.S. Energy Industry in 1987: A Slow Recovery . . . . . . . . . . . . . . .

January 1987

April 1987

May 1987

June 1987

July 1987

September 1987

October 1987

November 1987

December 1987

\section{6}

Article: State Motor Gasoline Taxes, 1960-1985 . . . . . . . . . . . . . . . . . . . . Article: The Impact of Low Oil Prices on Electric Utility Fuel Choice . . . . . . . . . . . . . . Article: U.S. Energy Industry Financial Developments, 1986 Second Quarter . . . . . . . . . . . . . Highlights: International Energy Annual $1985 \ldots \ldots \ldots \ldots \ldots \ldots \ldots \ldots \ldots \ldots \ldots \ldots$ Article: U.S. Energy Industry Financial Developments, $1986 \ldots \ldots \ldots \ldots \ldots \ldots \ldots \ldots \ldots \ldots$

March 1986 June 1986 June 1986 September 1986 December 1986

\section{5}

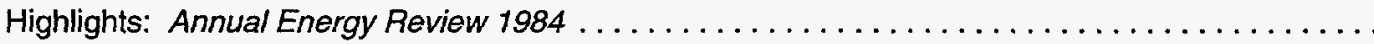
Highlights: Performance Profiles of Major Energy Producers $1983 \ldots \ldots \ldots \ldots \ldots \ldots \ldots \ldots$

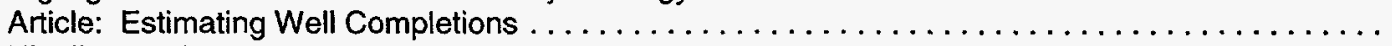
Highlights: State Energy Price and Expenditure Report 1970-1982 . . . . . . . . . . . . . .

January 1985

February 1985

March 1985

March 1985 


\section{5 (Continued)}

Highlights: State Energy Data Report, Consumption Estimates, 1960-1983 . . . . . . . . . . . .

Highlights: Annual Outlook for U.S. Electric Power $1985 \ldots \ldots \ldots \ldots \ldots \ldots \ldots \ldots \ldots$

Highlights: Short-Term Energy Outlook, Volume 1, October $1985 \ldots \ldots \ldots \ldots \ldots \ldots \ldots \ldots$ Highlights: Analysis of Growth in Electricity Demand, 1980-1984 . . . . . . . . . . . . . . . . Highlights: Profiles of Foreign Direct Investment in U.S. Energy $1984 \ldots \ldots \ldots \ldots \ldots \ldots$ Highlights: Performance Profiles of Major Energy Producers $1984 \ldots \ldots \ldots \ldots \ldots \ldots \ldots \ldots$

April 1985

June 1985

August 1985

August 1985

November 1985

December 1985

February 1984

March 1984

March 1984

May 1984

June 1984

September 1984

September 1984

November 1984

December 1984

January 1983

February 1983

April 1983

May 1983

July 1983

July 1983

August 1983

August 1983

Highlights: Port Deepening and User Fees: Impact on U.S. Coal Exports . . . . . . . . . . . . Highlights: U.S. Crude Oil, Natural Gas, and Natural Gas Liquids Reserves,

1982 Annual Report . . . . . . . . . . . . . . . . . . . . . . . . . . . . .

Article: Residential Energy Consumption, 1978 Through $1981 \ldots \ldots \ldots \ldots \ldots \ldots \ldots \ldots \ldots \ldots \ldots$

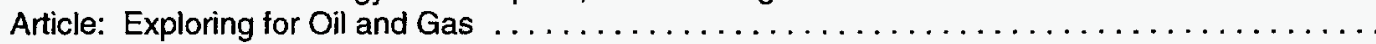
Article: The Influence of Federal Actions on Petroleum Exploration $\ldots \ldots \ldots \ldots \ldots \ldots \ldots \ldots$

Article: Aggregate Statistics: Accurate or Misleading? . . . . . . . . . . . . . . . . .

\section{2}

Article: The Interstate and Intrastate Natural Gas Markets $\ldots \ldots \ldots \ldots \ldots \ldots \ldots \ldots \ldots$ Article: Natural Gas Drilling and Production Under the Natural Gas Policy Act . . . . . . . . . . . . . Highlights: U.S. Crude Oil, Natural Gas, and Natural Gas Liquids Reserves, 1981 Annual Report . Article: Impacts of Financial Constrairits on the Electric Utility Industry . . . . . . . . . . . . . . . Highlights: Energy Company Development Patterns in the Postembargo Era .............

\section{1}

Article: Changes in 1981 Petroleum Data Series $\ldots \ldots \ldots \ldots \ldots \ldots \ldots \ldots \ldots \ldots \ldots \ldots \ldots$ Article: Information Services of the Energy Information Administration . . . . . . . . . . . . Article: An Overview of Natural Gas Markets . . . . . . . . . . . . . . . . . . . . . . . .

\section{0}

Article: The Solar Collector Industry and Solar Energy $\ldots \ldots \ldots \ldots \ldots \ldots \ldots \ldots \ldots \ldots$ Article: Trends in the Installation of Energy Using Equipment in New Residential Buildings ....... Article: The Energy Information Administration's Oil and Gas Reserves

Program-The First Year's Report

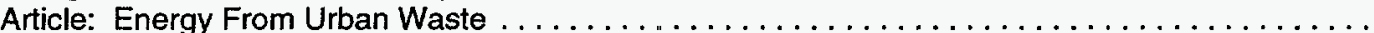
Article: Natural Gas Liquids: Revisions to 1979 Data . . . . . . . . . . . . . . . . . . . . . . . Article: EIA Weekly Petroleum Data: Data Collection and Methods of Estimation . . . . . . . . . . . Article: The Department of Energy Disclosure Policy for Individually Identifiable Information Maintained by the Energy Information Administration

\section{9}

Article: The Energy Requirements of U.S. Agriculture $\ldots \ldots \ldots \ldots \ldots \ldots \ldots \ldots \ldots \ldots$ Article: Three Mile Island-Possible Regulatory Responses and Their Impacts

on the Nation's Short-Term Electric Utility Fuel Outlook . . . . . . . . . . . . . . . . . . Article: Reduction in Natural Gas Requirements Due to Fuel Switching . . . . . . . . . . . .
September 1983 September 1983 November 1983

December 1983[2]

December 1983[3]

January 1982

February 1982

September 1982

October 1982

November 1982

May 1981

September 1981

December 1981

February 1980

March 1980

June 1980

August 1980

October 1980

November 1980

December 1980

July 1979

December 1979
October 1979 


\section{Feature}

Cover Date

1978

Article: Short-Term Petroleum Supply and Demand

May 1978

1977

Article: Crude Oil Entitlements Program ...

January 1977

Article: Motor Gasoline Supply and Demand

July 1977

\section{6}

Article: Curtailments of Natural Gas Service

Article: Home Heating Conservation Alternatives and the Solar Collector Industry ...

January 1976

Article: Trends in United States Petroleum Imports

March 1976

September 1976

\section{5}

Article: Energy Consumption .

March 1975

Article: Nuclear Power .

April 1975

Article: The Price of Crude Oil

June 1975

Article: U.S. Coal Resources and Reserves .

July 1975

Article: Propane-A National Energy Resource

September 1975

Article: Short-Term Energy Supply and Demand Forecasting at FEA

October 1975 



\section{Glossary}

Anthracite: A hard, black, lustrous coal containing a high percentage of fixed carbon and a low percentage of volatile matter. Often referred to as hard coal. It conforms to ASTM Specification D388-84 for anthracite, meta-anthracite, and semianthracite.

Asphalt: A dark-brown-to-black cement-like material containing bitumens as the predominant constituents obtained by petroleum processing. The definition includes crude asphalt as well as the following finished products: cements, fluxes, the asphalt content of emulsions (exclusive of water), and petroleum distillates blended with asphalt to make cutback asphalts.

ASTM: The American Society for Testing and Materials.

Aviation Gasoline Blending Components: Naphthas that are used for blending or compounding into finished aviation gasoline (e.g., straight-run gasoline, alkylate, and reformate). Excludes oxygenates (alcohols and ethers), butane, and pentanes plus.

Aviation Gasoline, Finished: All special grades of gasoline for use in aviation reciprocating engines, as given in ASTM Specification D910 and Military Specification MIL-G-5572. Excludes blending components that will be used in blending or compounding into finished aviation gasoline.

Barrel (petroleum): A unit of volume equal to 42 U.S. gallons.

Base (Cushion) Gas: The volume of gas needed as a permanent inventory to maintain adequate underground storage reservoir pressures and deliverability rates throughout the withdrawal season. All native gas is included in the base gas volume.

Bituminous Coal: A dense black coal, often with well-defined bands of bright and dull material, with a moisture content usually less than 20 percent. Often referred to as soft coal. It is the most common coal and is used primarily for generating electricity, making coke, and space heating. It conforms to ASTM Specification D388-84 for bituminous coal. In this report, bituminous coal includes subbituminous coal.

British Thermal Unit (Btu): The quantity of heat needed to raise the temperature of 1 pound of water by $1^{\circ} \mathrm{F}$ at or near $39.2^{\circ} \mathrm{F}$. See Heat Content of a Quantity of Fuel, Gross and Heat Content of a Quantity of Fuel, Net.
Butane: A normally gaseous straight-chain or branched-chain hydrocarbon $\left(\mathrm{C}_{4} \mathrm{H}_{10}\right)$. It is extracted from natural gas or refinery gas streams. It includes isobutane and normal butane and is designated in ASTM Specification D1835 and Gas Processors Association Specifications for commercial butane.

- Isobutane: A normally gaseous branched-chain hydrocarbon. It is a colorless paraffinic gas that boils at a temperature of $10.9^{\circ} \mathrm{F}$. It is extracted from natural gas or refinery gas streams.

- Normal Butane: A normally gaseous straight-chain hydrocarbon. It is a colorless paraffinic gas that boils at a temperature of $31.1^{\circ} \mathrm{F}$. It is extracted from natural gas or refinery gas streams.

Butylene: An olefinic hydrocarbon $\left(\mathrm{C}_{4} \mathrm{H}_{8}\right)$ recovered from refinery processes.

Capacity Factor: The ratio of the electrical energy produced by a generating unit for a given period of time to the electrical energy that could have been produced at continuous full-power operation during the same period.

\section{CIF: See Cost, Insurance, Freight.}

City Gate: A point or measuring station at which a distribution gas utility receives gas from a natural gas pipeline company or transmission system.

Coal: A black or brownish-black solid, combustible substance formed by the partial decomposition of vegetable matter without access to air. The rank of coal, which includes anthracite, bituminous coal, subbituminous coal, and lignite, is based on fixed carbon, volatile matter, and heating value. Coal rank indicates the progressive alteration, or coalification, from lignite to anthracite. Lignite contains approximately 9 to 17 million Btu per ton. The heat contents of subbituminous and bituminous coal range from 16 to 24 million Btu per ton, and from 19 to 30 million Btu per ton, respectively. Anthracite contains approximately 22 to 28 million Btu per ton.

Coal Coke: A hard, porous product made from baking bituminous coal in ovens at temperatures as high as $2,000^{\circ} \mathrm{F}$. It is used both as a fuel and as a reducing agent in smelting iron ore in a blast furnace.

Commercial Sector: The commercial sector, as defined economically, consists of business establishments that are not engaged in transportation or in manufacturing or other types of industrial activity (agriculture, mining, or construction). Commercial establishments include hotels, motels, restaurants, 
wholesale businesses, retail stores, laundries, and other service enterprises; religious and nonprofit organizations; health, social, and educational institutions; and Federal, State, and local governments. Street lights, pumps, bridges, and public services are also included if the establishment operating them is considered commercial.

Completion: The installation of permanent equipment for the production of oil or gas. If a well is equipped to produce only oil or gas from one zone or reservoir, the definition of a well (classified as an oil well or gas well) and the definition of a completion are identical. However, if a well is equipped to produce oil and/or gas separately from more than one reservoir, a well is not synonymous with a completion.

Conversion Factor: A number that translates units of one system into corresponding values of another system. Conversion factors can be used to translate physical units of measure for various fuels into Btu equivalents.

Cost, Insurance, Freight (CIF): A type of sale in which the buyer of the product agrees to pay a unit price that includes the f.o.b. value of the product at the point of origin plus all costs of insurance and transportation. This type of transaction differs from a "delivered" purchase in that the buyer accepts the quantity as determined at the loading port (as certified by the Bill of Loading and Quality Report) rather than pay on the basis of the quantity and quality ascertained at the unloading port. It is similar to the terms of an f.o.b. sale, except that the seller, as a service for which he is compensated, arranges for transportation and insurance.

Crude Oil f.o.b. Price: The crude oil price actually charged at the oil-producing country's port of loading. Includes deductions for any rebates and discounts or additions of premiums, where applicable. It is the actual price paid with no adjustment for credit terms.

Crude Oil (Including Lease Condensate): A mixture of hydrocarbons that exists in liquid phase in underground reservoirs and remains liquid at atmospheric pressure after passing through surface separating facilities. Included are lease condensate and liquid hydrocarbons produced from tar sands, gilsonite, and oil shale. Drip gases are also included, but topped crude oil (residual oil) and other unfinished oils are excluded. Where identifiable, liquids produced at natural gas processing plants and mixed with crude oil are likewise excluded.

Crude Oil Landed Cost: The price of crude oil at the port of discharge, including charges associated with the purchase, transporting, and insuring of a cargo from the purchase point to the port of discharge. The cost does not include charges incurred at the discharge port (e.g., import tariffs or fees, wharfage charges, and demurrage).
Crude Oil Refinery Input: The total crude oil put into processing units at refineries.

Crude Oil Stocks: Stocks of crude oil and lease condensate held at refineries, in pipelines, at pipeline terminals, and on leases.

Crude Oil Used Directly: Crude oil consumed as fuel by crude oil pipelines and on crude oil leases.

Cubic Foot (natural gas): A unit of volume equal to 1 cubic foot at a pressure base of 14.73 pounds standard per square inch absolute and a temperature base of $60^{\circ} \mathrm{F}$.

Degree-Day Normals: Simple arithmetic averages of monthly or annual degree-days over a long period of time (usually the 30-year period 1961-1990). The averages may be simple degree-day normals or population-weighted degree-day normals.

Degree-Days, Cooling (CDD): The number of degrees per day that the daily average temperature is above $65^{\circ} \mathrm{F}$. The daily average temperature is the mean of the maximum and minimum temperatures for a 24-hour period.

Degree-Days, Heating (HDD): The number of degrees per day that the daily average temperature is below $65^{\circ} \mathrm{F}$. The daily average temperature is the mean of the maximum and minimum temperatures for a 24-hour period.

Degree-Days, Population-Weighted: Heating or cooling degree-days weighted by the population of the area in which the degree-days are recorded. To compute State population-weighted degree-days, each State is divided into from one to nine climatically homogeneous divisions, which are assigned weights based on the ratio of the population of the division to the total population of the State. Degree-day readings for each division are multiplied by the corresponding population weight for each division and those products are then summed to arrive at the State population-weighted degree-day figure. To compute national population-weighted degree-days, the Nation is divided into nine Census regions, each comprising from three to eight States, which are assigned weights based on the ratio of the population of the region to the total population of the Nation. Degree-day readings for each region are multiplied by the corresponding population weight for each region and those products are then summed to arrive at the national population-weighted degree-day figure.

Design Electrical Rating, Net: The nominal net electrical output of a nuclear unit as specified by the electric utility for the purpose of plant design.

Development Well: A well drilled within the proved area of an oil or gas reservoir to the depth of a stratigraphic horizon known to be productive. 
Distillate Fuel Oil: A general classification for one of the petroleum fractions produced in conventional distillation operations. Included are products known as No. 1, No. 2, and No. 4 fuel oils and No. 1, No. 2, and No. 4 diesel fuels. It is used primarily for space heating, on- and off-highway diesel engine fuel (including railroad engine fuel and fuel for agricultural machinery), and electric power generation.

Dry Hole: An exploratory or development well found to be incapable of producing either oil or gas in sufficient quantities to justify completion as an oil or gas well.

Dry Natural Gas Production (as a decrement from gas reserves): The volume of natural gas withdrawn from reservoirs during the report year less (1) the volume returned to such reservoirs in cycling, repressuring of oil reservoirs, and conservation operations; (2) shrinkage resulting from the removal of lease condensate and plant liquids; and (3) nonhydrocarbon gases, where they occur in sufficient quantity to render the gas unmarketable. Volumes of gas withdrawn from gas storage reservoirs and native gas that has been transferred to the storage category are not considered production. This is not the same as marketed production, since the latter also excludes vented and flared gas but contains liquids.

Dry Natural Gas Production (as an increment to gas supply): Gross withdrawals from production reservoirs less gas used in reservoir repressuring, amounts vented and flared, nonhydrocarbons removed, and various natural gas constituents, such as ethane, propane, and butane, removed at natural gas processing plants. The parameters for measurement are $60^{\circ} \mathrm{F}$ and 14.73 pounds standard per square inch absolute.

Electrical System Energy Losses: The amount of energy lost during generation, transmission, and distribution of electricity, including plant and unaccounted-for uses.

Electricity Generation: The process of producing electric energy or transforming other forms of energy into electric energy. Also the amount of electric energy produced or expressed in watthours (Wh).

Electricity Generation, Gross: The total amount of electric energy produced by the generating station or stations, measured at the generator terminals.

Electricity Generation, Net: Gross generation less electricity consumed at the generating plant for station use. Electricity required for pumping at pumped-storage plants is regarded as plant use and is deducted from gross generation.

Electricity Production: Net electricity (gross electricity output measured at generator terminals minus power plant use) generated by publicly and privately owned electric utilities. Excludes industrial electricity generation (except autogeneration of hydroelectric power).

Electricity Sales: The amount of kilowatthours sold in a given period of time; usually grouped by classes of service, such as residential, commercial, industrial, and other. "Other" sales include sales for public street and highway lighting and other sales to public authorities, sales to railroads and railways, and interdepartmental sales.

Electric Power Plant: A station containing prime movers, electric generators, and auxiliary equipment for converting mechanical, chemical, and/or fission energy into electric energy.

Electric Utility: A corporation, person, agency, authority, or other legal entity or instrumentality that owns and/or operates facilities for the generation, transmission, distribution, or sale of electric energy, primarily for use by the public, and that files forms listed in the Code of Federal Regulations, Title 18, Part 141. Facilities that qualify as cogenerators or small power producers under the Public Utility Regulatory Policies Act are not considered electric utilities.

Electric Utility Sector: The electric utility sector consists of privately and publicly owned establishments that generate, transmit, distribute, or sell electricity primarily for use by the public and that meet the definition of an electric utility. Nonutility power producers are not included in the electric utility sector.

End-Use Sectors: The residential, commercial, industrial, and transportation sectors of the economy.

Energy: The capacity for doing work as measured by the capability of doing work (potential energy) or the conversion of this capability to motion (kinetic energy). Energy has several forms, some of which are easily convertible and can be changed to another form useful for work. Most of the world's convertible energy comes from fossil fuels that are burned to produce heat that is then used as a transfer medium to mechanical or other means in order to accomplish tasks. Electrical energy is usually measured in kilowatthours, while heat energy is usually measured in British thermal units.

Energy Consumption: The use of energy as a source of heat or power or as an input in the manufacturing process.

Energy Consumption, End-Use: Primary end-use energy consumption is the sum of fossil fuel consumption by the four end-use sectors (residential, commercial, industrial, and transportation) and generation of hydroelectric power by nonelectric utilities. Net end-use energy consumption includes electric utility sales to those sectors but excludes 
electrical system energy losses. Total end-use energy consumption includes both electric utility sales to the four end-use sectors and electrical system energy losses.

Energy Consumption, Total: The sum of fossil fuel consumption by the five sectors (residential, commercial, industrial, transportation, and electric utility) plus hydroelectric power, nuclear electric power, net imports of coal coke, and electricity generated for distribution from wood, waste, geothermal, wind, photovoltaic, and solar thermal energy.

Energy Source: A substance, such as petroleum, natural gas, or coal, that supplies heat or power. In Energy Information Administration reports, electricity and renewable forms of energy, such as biomass, geothermal, wind, and solar, are considered to be energy sources.

Ethane: A normally gaseous straight-chain hydrocarbon $\left(\mathrm{C}_{2} \mathrm{H}_{6}\right)$. It is a colorless, paraffinic gas that boils at a temperature of $-127.48^{\circ} \mathrm{F}$. It is extracted from natural gas and refinery gas streams.

Ethylene: An olefinic hydrocarbon $\left(\mathrm{C}_{2} \mathrm{H}_{4}\right)$ recovered from refinery processes or petrochemical processes.

Exploratory Well: A well drilled to find and produce oil or gas in an unproved area, to find a new reservoir in a field previously found to be productive of oil or gas in another reservoir, or to extend the limit of a known oil or gas reservoir.

Exports: Shipments of goods from the 50 States and the District of Columbia to foreign countries and to Puerto Rico, the Virgin Islands, and other U.S. possessions and territories.

\section{f.a.s.: See Free Alongside Ship.}

Federal Energy Regulatory Commission (FERC): The Federal agency with jurisdiction over interstate electricity sales, wholesale electric rates, hydroelectric licensing, natural gas pricing, oil pipeline rates, and gas pipeline certification. FERC is an independent regulatory agency within the Department of Energy and is the successor to the Federal Power Commission.

Federal Power Commission (FPC): The predecessor agency of the Federal Energy Regulatory Commission. The Federal Power Commission was created by an Act of Congress under the Federal Water Power Act on June 10,1920 . It was charged originally with regulating the electric power and natural gas industries. It was abolished on September 30, 1977, when the Department of Energy was created. Its functions were divided between the Department of Energy and the Federal Energy Regulatory Commission, an independent regulatory agency.
First Purchase Price: The marketed first sales price of domestic crude oil, consistent with the removal price defined by the provisions of the Windfall Profits Tax on Domestic Crude Oil (Public Law 96-223, Sec. 4998 (c)).

Flared Natural Gas: Natural gas burned in flares on the base site or at gas processing plants.

\section{f.o.b.: See Free on Board.}

Footage Drilled: Total footage for wells in various categories, as reported for any specified period, includes (1) the deepest total depth (length of well bores) of all wells drilled from the surface, (2) the total of all bypassed footage drilled in connection with reported wells, and (3) all new footage drilled for directional sidetrack wells. Footage reported for directional sidetrack wells does not include footage in the common bore, which is reported as footage for the original well. In the case of old wells drilled deeper, the reported footage is that which was drilled below the total depth of the old well.

\section{Former U.S.S.R.: See U.S.S.R.}

Fossil Fuel: Any naturally occurring organic fuel, such as petroleum, coal, and natural gas.

Fossil Fuel Steam-Electric Power Plant: An electricity generation plant in which the prime mover is a turbine rotated by high-pressure steam produced in a boiler by heat from burning fossil fuels.

Free Alongside Ship (f.a.s.): The value of a commodity at the port of exportation, generally including the purchase price, plus all charges incurred in placing the commodity alongside the carrier at the port of exportation.

Free on Board (f.o.b.): A transaction whereby the seller makes the product available within an agreed-on period at a given port at a given price. It is the responsibility of the buyer to arrange for the transportation and insurance.

Fuel Ethanol: An anhydrous, denatured aliphatic alcohol $\left(\mathrm{C}_{2} \mathrm{H}_{5} \mathrm{OH}\right)$ intended for motor gasoline blending. See Oxygenates.

Full-Power Operation: Operation of a nuclear generating unit at 100 percent of its design capacity. Full-power operation precedes commercial operation.

Gasohol: A blend of finished motor gasoline (leaded or unleaded) and alcohol (generally ethanol but sometimes methanol) limited to 10 percent by volume of alcohol. Gasohol is included in finished leaded and unleaded motor gasoline. 
Gas-Turbine Electric Power Plant: A plant in which the prime mover is a gas turbine. A gas turbine typically consists of an axial-flow air compressor, one or more combustion chambers where liquid or gaseous fuel is burned and the hot gases expand to drive the generator and then are used to run the compressor.

Gas Well: A well completed for the production of natural gas from one or more gas zones or reservoirs. (Wells producing both crude oil and natural gas are classified as oil wells.)

Geothermal Energy: Energy from the internal heat of the Earth, which may be residual heat, friction heat, or a result of radioactive decay. The heat is found in rocks and fluids at various depths and can be extracted by drilling and/or pumping.

Geothermal Energy (as used at electric utilities): Hot water or steam extracted from geothermal reservoirs in the Earth's crust and supplied to steam turbines at electric utilities that drive generators to produce electricity.

Gross Domestic Product (GDP): The total value of goods and services produced by labor and property located in the United States. As long as the labor and property are located in the United States, the supplier (that is, the workers and, for property, the owners) may be either U.S. residents or residents of foreign countries.

Heat Content of a Quantity of Fuel, Gross: The total amount of heat released when a fuel is burned. Coal, crude oil, and natural gas all include chemical compounds of carbon and hydrogen. When those fuels are burned, the carbon and hydrogen combine with oxygen in the air to produce carbon dioxide and water. Some of the energy released in burning goes into transforming the water into steam and is usually lost. The amount of heat spent in transforming the water into steam is counted as part of gross heat content but is not counted as part of net heat content. Also referred to as the higher heating value. Btu conversion factors typically used in EIA represent gross heat content.

Heat Content of a Quantity of Fuel, Net: The amount of usable heat energy released when a fuel is burned under conditions similar to those in which it is normally used. Also referred to as the lower heating value. Btu conversion factors typically used in EIA represent gross heat content.

Heavy Oil: The fuel oils remaining after the lighter oils have been distilled off during the refining process. Except for start-up and flame stabilization, virtually all petroleum used in steam-electric power plants is heavy oil.

Hydrocarbon: An organic chemical compound of hydrogen and carbon in the gaseous, liquid, or solid phase. The molecular structure of hydrocarbon compounds varies from the simplest (methane, the primary constituent of natural gas) to the very heavy and very complex.

Hydroelectric Power: The production of electricity from the kinetic energy of falling water.

Hydroelectric Power Plant: A plant in which the turbine generators are driven by falling water.

Imports: Receipts of goods into the 50 States and the District of Columbia from foreign countries and from Puerto Rico, the Virgin Islands, and other U.S. possessions and territories.

Industrial Sector: The industrial sector comprises manufacturing industries, which make up the largest part of the sector, along with mining, construction, agriculture, fisheries, and forestry. Establishments in this sector range from steel mills, to small farms, to companies assembling electronic components.

Internal Combustion Electric Power Plant: A power plant in which the prime mover is an internal combustion engine. Diesel or gas-fired engines are the principal types used in electric power plants. The plant is usually operated during periods of high demand for electricity.

Jet Fuel: The term includes kerosene-type jet fuel and naphtha-type jet fuel. Kerosene-type jet fuel is a kerosene-quality product used primarily for commercial turbojet and turboprop aircraft engines. Naphtha-type jet fuel is a fuel in the heavy naphthas range used primarily for military turbojet and turboprop aircraft engines.

Kerosene: A petroleum distillate that has a maximum distillation temperature of $401^{\circ} \mathrm{F}$ at the 10-percent recovery point, a final boiling point of $572^{\circ} \mathrm{F}$, and a minimum flash point of $100^{\circ} \mathrm{F}$. Included are the two grades designated in ASTM D3699 (No. 1-K and No. $2-\mathrm{K}$ ) and all grades of kerosene called range or stove oil. Kerosene is used in space heaters, cook stoves, and water heaters; it is suitable for use as an illuminant when burned in wick lamps.

Lease and Plant Fuel: Natural gas used in well, field, and lease operations (such as gas used in drilling operations, heaters, dehydrators, and field compressors) and used as fuel in natural gas processing plants.

Lease Condensate: A natural gas liquid recovered from gas well gas (associated and non-associated) in lease separators or natural gas field facilities. Lease condensate consists primarily of pentanes and heavier hydrocarbons.

Light Oil: Lighter fuel oils distilled off during the refining process. Virtually all petroleum used in 
internal combustion and gas-turbine engines is light oil.

Lignite: A brownish-black coal of low rank with a high content of moisture and volatile matter. Often referred to as brown coal. It is used almost exclusively for electric power generation. It conforms to ASTM Specification D388-84 for lignite.

Liquefied Natural Gas (LNG): Natural gas (primarily methane) that has been liquefied by reducing its temperature to $-260^{\circ} \mathrm{F}$ at atmospheric pressure.

Liquefied Petroleum Gases (LPG): Ethane, ethylene, propane, propylene, normal butane, butylene, and isobutane produced at refineries or natural gas processing plants, including plants that fractionate new natural gas plant liquids.

Low-Power Testing: The period of time between a nuclear generating unit's initial fuel loading date and the issuance of its operating (full-power) license. The maximum level of operation during that period is 5 percent of the unit's design thermal rating.

Lubricants: Substances used to reduce friction between bearing surfaces or as process materials either incorporated into other materials used as processing aids in the manufacturing of other products or as carriers of other materials. Petroleum lubricants may be produced either from distillates or residues. Other substances may be added to impart or improve certain required properties. Excluded are byproducts of lubricating oil refining, such as aromatic extracts derived from solvent extraction or tars derived from deasphalting. Included are all grades of lubricating oils from spindle oil to cylinder oil and those used in greases. Lubricant categories are paraffinic and naphthenic.

Marketed Production: Gross withdrawals less gas used for repressuring, quantities vented and flared, and nonhydrocarbon gases removed in treating or processing operations. Includes all quantities of gas used in field and processing operations.

Methanol: A light, volatile alcohol $\left(\mathrm{CH}_{3} \mathrm{OH}\right)$ eligible for motor gasoline blending. See Oxygenates.

Miscellaneous Petroleum Products: All finished petroleum products not classified elsewhere-for example, petrolatum, lube refining byproducts (aromatic extracts and tars), absorption oils, ram-jet fuel, petroleum rocket fuels, synthetic natural gas feedstocks, and specialty oils.

Motor Gasoline Blending Components: Naphthas that will be used for blending or compounding into finished motor gasoline (e.g., straight-run gasoline, alkylate, reformate, benzene, toluene, and zylene). Excluded are oxygenates (alcohols and ethers), butane, and pentanes plus.
Motor Gasoline, Finished: A complex mixture of relatively volatile hydrocarbons, with or without small quantities of additives, that has been blended to form a fuel suitable for use in spark-ignition engines. Motor gasoline, as given in ASTM Specification D439 or Federal Specification VV-G-1690B, includes a range in distillation temperatures from 122 to $158^{\circ} \mathrm{F}$ at the 10 -percent recovery point and from 365 to $374^{\circ} \mathrm{F}$ at the 90-percent recovery point. Motor gasoline includes reformulated motor gasoline, oxygenated motor gasoline, and other finished motor gasoline. Blendstock is excluded until blending has been completed.

- Reformulated Motor Gasoline: Motor gasoline, formulated for use in motor vehicles, the composition and properties of which are certified as "reformulated motor gasoline" by the Environmental Protection Agency.

- Oxygenated Motor Gasoline: Motor gasoline, formulated for use in motor vehicles, that has an oxygen content of 1.8 percent or higher by weight.

- Other Finished Motor Gasoline: Motor gasoline that is not included in the reformulated or oxygenated categories.

Motor Gasoline, Finished Gasohol: A blend of finished motor gasoline (leaded or unleaded) and alcohol (generally ethanol, but sometimes methanol) in which 10 percent or more of the product is alcohol.

Motor Gasoline, Finished Leaded: Motor gasoline that contains more than 0.05 gram of lead per gallon or more than 0.005 gram of phosphorus per gallon. Premium and regular grades are included, depending on the octane rating. Includes leaded gasohol. Blendstock is excluded until blending has been completed. Alcohol that is to be used in the blending of gasohol is also excluded.

Motor Gasoline, Finished Leaded Premium: Motor gasoline having an antiknock index, calculated as $(\mathrm{R}+\mathrm{M}) / 2$, greater than 90 and containing more than 0.05 gram of lead per gallon or more than 0.005 gram of phosphorus per gallon.

Motor Gasoline, Finished Leaded Regular: Motor gasoline having an antiknock index, calculated as $(\mathrm{R}+\mathrm{M}) / 2$, greater than or equal to 87 and less than or equal to 90 and containing more than 0.05 gram of lead or 0.005 gram of phosphorus per gallon.

Motor Gasoline, Finished Unleaded: Motor gasoline containing not more than 0.05 gram of lead per gallon and not more than 0.005 gram of phosphorus per gallon. Premium and regular grades are included, depending on the octane rating. Includes unleaded gasohol. Blendstock is excluded until blending has been completed. Alcohol that is to be used in the blending of gasohol is also excluded. 
Motor Gasoline, Finished Unleaded Midgrade: Motor gasoline having an antiknock index, calculated as $(\mathrm{R}+\mathrm{M}) / 2$, greater than or equal to 88 and less than or equal to 90 and containing not more than 0.05 gram of phosphorus per gallon.

Motor Gasoline, Finished Unleaded Premium: Motor gasoline having an antiknock index, calculated as $(\mathrm{R}+\mathrm{M}) / 2$, greater than 90 and containing not more than 0.05 gram of lead or 0.005 gram of phosphorus per gallon.

Motor Gasoline, Finished Unleaded Regular: Motor gasoline having an antiknock index, calculated as $(\mathrm{R}+\mathrm{M}) / 2$, of 87 containing not more than 0.05 gram of lead per gallon and not more than 0.005 gram of phosphorus per gallon.

Motor Gasoline Retail Prices: Motor gasoline prices calculated each month by the Bureau of Labor Statistics (BLS) in conjunction with the construction of the Consumer Price Index (CPI). Those prices are collected in 85 urban areas selected to represent all urban consumers-about 80 percent of the total U.S. population. The service stations are selected initially, and on a replacement basis, in such a way that they represent the purchasing habits of the CPI population. Service stations in the current sample include those providing all types of service (i.e., full-, mini-, and self-service).

Motor Gasoline, Total: Includes finished leaded motor gasoline (premium and regular), finished unleaded motor gasoline (premium, midgrade, and regular), motor gasoline blending components, and gasohol.

MTBE (Methyl Tertiary Butyl Ether): An ether, $\left(\mathrm{CH}_{3}\right)_{3} \mathrm{COCH}_{3}$, intended for motor gasoline blending. See Oxygenates.

Naphtha: A genetic term applied to a petroleum fraction with an approximate boiling range between 122 and $400^{\circ} \mathrm{F}$.

Natural Gas: A mixture of hydrocarbons (principally methane) and small quantities of various nonhydrocarbons existing in the gaseous phase or in solution with crude oil in underground reservoirs.

Natural Gas, Dry: The marketable portion of natural gas production, which is obtained by subtracting extraction losses, including natural gas liquids removed at natural gas processing plants, from total production.

Natural Gas Marketed Production: Gross withdrawals of natural gas from production reservoirs, less gas used for reservoir repressuring; nonhydrocarbon gases removed in treating and processing operations; and quantities vented and flared.
Natural Gas Plant Liquids (NGPL): Natural gas liquids recovered from natural gas in processing plants and, in some situations, from natural gas field facilities, as well as those extracted by fractionators. Natural gas plant liquids are defined according to the published specifications of the Gas Processors Association and the American Society for Testing and Materials as follows: ethane, propane, normal butane, isobutane, pentanes plus, and other products from natural gas processing plants (i.e., products meeting the standards for finished petroleum products produced at natural gas processing plants, such as finished motor gasoline, finished aviation gasoline, special naphthas, kerosene, distillate fuel oil, and miscellaneous products).

Natural Gas Wellhead Price: The wellhead price of natural gas is calculated by dividing the total reported value at the wellhead by the total quantity produced as reported by the appropriate agencies of individual producing States and the U.S. Minerals Management Service. The price includes all costs prior to shipment from the lease, including gathering and compression costs, in addition to State production, severance, and similar charges.

Natural Gas, Wet: Natural gas prior to the extraction of liquids and other miscellaneous products.

\section{Net Consumption: See Energy Consumption, End-Use.}

Nonhydrocarbon Gases: Typical nonhydrocarbon gases that may be present in reservoir natural gas are carbon dioxide, helium, hydrogen sulfide, and nitrogen.

Nuclear Electric Power: Electricity generated by an electric power plant whose turbines are driven by steam generated in a reactor by heat from the fissioning of nuclear fuel.

Nuclear Electric Power Plant: A single-unit or multiunit facility in which heat produced in one or more reactors by the fissioning of nuclear fuel is used to drive one or more steam turbines.

Nuclear Reactor: An apparatus in which the nuclear fission chain can be initiated, maintained, and controlled so that energy is released at a specific rate. The reactor includes fissionable material (fuel), such as uranium or plutonium; fertile material; moderating material (unless it is a fast reactor); a heavy-walled pressure vessel; shielding to protect personnel; provision for heat removal; and control elements and instrumentation.

Offshore: That geographic area that lies seaward of the coastline. In general, the coastline is the line of ordinary low water along with that portion of the coast that is in direct contact with the open sea or the line marking the seaward limit of inland water. 
Oil: See Crude Oil (Including Lease Condensate).

Oil Well: A well completed for the production of crude oil from one or more oil zones or reservoirs. Wells producing both crude oil and natural gas are classified as oil wells.

Operable (nuclear): A U.S. nuclear generating unit is considered operable after it completes low-power testing and is issued a full-power operating license by the Nuclear Regulatory Commission. A foreign nuclear generating unit is considered operable once it has generated electricity to the grid.

Organization for Economic Cooperation and Development (OECD): Members are Australia, Austria, Belgium, Canada, Denmark, Faroe Islands, Finland, France, Germany, Greece, Greenland, Hawaiian Trade Zone, Iceland, Ireland, Italy, Japan, Luxembourg, Mexico, Netherlands, New Zealand, Norway, Portugal, Spain, Sweden, Switzerland, Turkey, United Kingdom, and United States and its territories (Guam, Puerto Rico, and the Virgin Islands). In addition, Czech Republic, Hungary, Poland, and South Korea joined the OECD in 1996.

Organization of Petroleum Exporting Countries (OPEC): Countries that have organized for the purpose of negotiating with oil companies on matters of oil production, prices, and future concession rights. Current members are Algeria, Indonesia, Iran, Iraq, Kuwait, Libya, Nigeria, Qatar, Saudi Arabia, the United Arab Emirates, and Venezuela.

Oxygenated Motor Gasoline: See Motor Gasoline, Finished.

Oxygenates: Any substance which, when added to motor gasoline, increases the amount of oxygen in that motor gasoline blend. Through a series of waivers and interpretive rules, the Environmental Protection Agency (EPA) has determined the allowable limits for oxygenates in unleaded gasoline. The "Substantially Similar" Interpretive Rules (56 FR [February 11, 1991]) allows blends of aliphatic alcohols other than methanol and aliphatic ethers, provided the oxygen content does not exceed 2.7 percent by weight. The "Substantially Similar" Interpretive Rules also provide for blends of methanol up to 0.3 percent by volume exclusive of other oxygenates, and butanol or alcohols of a higher molecular weight up to 2.75 percent by weight. Individual waivers pertaining to the use of oxygenates in unleaded motor gasoline have been issued by the EPA. They include:

- Fuel Ethanol. Blends of up to 10 percent by volume anhydrous ethanol (200 proof).

- Methanol. Blends of methanol and gasoline-grade tertiary butyl alcohol (GTBA) such that the total oxygen content does not exceed 3.5 percent by weight and the ratio of methanol to GTBA is less than or equal to 1 .
It is also specified that this blended fuel must meet ASTM volatility specifications.

Blends of up to 5.0 percent by volume methanol with a minimum of 2.5 percent by volume cosolvent alcohols having carbon number of 4 or less (i.e., ethanol, propanol, butanol, and/or GTBA). The total oxygen must not exceed 3.7 percent by weight, and the blend must meet ASTM volatility specifications as well as phase separation and alcohol purity specifications.

- MTBE (Methyl tertiary butyl ether). Blends up to 15.0 percent by volume MTBE that must meet the ASTM D4814 specifications. Blenders must take precautions that the blends are not used as base gasolines for other oxygenated blends.

Pentanes Plus: A mixture of hydrocarbons, mostly pentanes and heavier, extracted from natural gas. Includes isopentane, natural gasoline, and plant condensate.

Petrochemical Feedstocks: Chemical feedstocks derived from petroleum principally for the manufacture of chemicals, synthetic rubber, and a variety of plastics.

Petroleum: A generic term applied to oil and oil products in all forms, such as crude oil, lease condensate, unfinished oils, petroleum products, natural gas plant liquids, and nonhydrocarbon compounds blended into finished petroleum products.

Petroleum Coke: A residue that is the final product of the condensation process in cracking. The product is either marketable petroleum coke or catalyst petroleum coke.

Petroleum Coke, Catalyst: The carbonaceous residue that is deposited on and deactivates the catalyst used in many catalytic operations (e.g., catalytic cracking). Carbon is deposited on the catalyst, thus deactivating the catalyst. The catalyst is reactivated by burning off the carbon, which is used as a fuel in the refining process. That carbon or coke is not recoverable in a concentrated form.

Petroleum Coke, Marketable: Those grades of coke produced in delayed or fluid cokers that may be recovered as relatively pure carbon. Marketable petroleum coke may be sold as is or may be further purified by calcining.

Petroleum Consumption: The sum of all refined petroleum products supplied. For each refined petroleum product, the amount supplied is calculated by adding production and imports, then subtracting changes in primary stocks (net withdrawals are a plus quantity and net additions are a minus quantity) and exports. 
Petroleum Imports: Imports of petroleum into the 50 States and the District of Columbia from foreign countries and from Puerto Rico, the Virgin Islands, and other U.S. territories and possessions. Included are imports for the Strategic Petroleum Reserve and withdrawals from bonded warehouses for onshore consumption, offshore bunker use, and military use. Excluded are receipts of foreign petroleum into bonded warehouses and into U.S. territories and U.S. Foreign Trade Zones.

Petroleum Products: Products obtained from the processing of crude oil (including lease condensate), natural gas, and other hydrocarbon compounds. Petroleum products include unfinished oils, liquefied petroleum gases, pentanes plus, aviation gasoline, motor gasoline, naphtha-type jet fuel, kerosene-type jet fuel, kerosene, distillate fuel oil, residual fuel oil, petrochemical feedstocks, special naphthas, lubricants, waxes, petroleum coke, asphalt, road oil, still gas, and miscellaneous products.

Petroleum Products Supplied: Se e Petroleum Consumption.

Petroleum Stocks, Primary: For individual products, quantities that are held at refineries, in pipelines, and at bulk terminals that have a capacity of 50,000 barrels or more, or that are in transit thereto. Stocks held by product retailers and resellers, as well as tertiary stocks held at the point of consumption, are excluded. Stocks of individual products held at gas processing plants are excluded from individual product estimates but are included in other oils estimates and total.

Photovoltaic and Solar Thermal Energy (as used at electric utilities): Energy radiated by the sun as electromagnetic waves (electromagnetic radiation) that is converted at electric utilities into electricity by means of solar (photovoltaic) cells or concentrating (focusing) collectors.

Pipeline Fuel: Gas consumed in the operation of pipelines, primarily in compressors.

Primary Consumption: See Energy Consumption, End-Use.

Propane: A normally gaseous straight-chain hydrocarbon $\left(\mathrm{C}_{3} \mathrm{H}_{8}\right)$. It is a colorless paraffinic gas that boils at a temperature of $-43.67^{\circ} \mathrm{F}$. It is extracted from natural gas or refinery gas streams. It includes all products designated in ASTM Specification D1835 and Gas Processors Association Specifications for commercial propane and HD-5 propane.

Propylene: An olefinic hydrocarbon $\left(\mathrm{C}_{3} \mathrm{H}_{6}\right)$ recovered from refinery or petrochemical processes.

Refiner Acquisition Cost of Crude Oil: The cost of crude oil to the refiner, including transportation and fees. The composite cost is the weighted average of domestic and imported crude oil costs.

Refinery (petroleum): An installation that manufactures finished petroleum products from crude oil, unfinished oils, natural gas liquids, other hydrocarbons, and alcohol.

Renewable Energy: Energy obtained from sources that are essentially inexhaustible (unlike, for example, the fossil fuels, of which there is a finite supply). Renewable sources of energy include wood, waste, photovoltaic, and solar thermal energy.

Repressuring: The injection of a pressurized fluid (such as air, gas, or water) into oil and gas reservoir formations to effect greater ultimate recovery.

Residential Sector: The residential sector is considered to consist of all private residences, whether occupied or vacant, owned or rented, including single-family homes, multifamily housing units, and mobile homes. Secondary homes, such as summer homes, are also included. Institutional housing, such as school dormitories, hospitals, and military barracks, generally are not included in the residential sector; they are included in the commercial sector.

Residual Fuel Oil: The heavier oils that remain after the distillate fuel oils and lighter hydrocarbons are distilled away in refinery operations and that conform to ASTM Specifications D396 and 975. Included are No. 5, a residual fuel oil of medium viscosity; Navy Special, for use in steam-powered vessels in government service and in shore power plants; and No. 6 , which includes Bunker $C$ fuel oil and is used for commercial and industrial heating, for electricity generation, and to power ships. Imports of residual fuel oil include imported crude oil burned as fuel.

Road Oil: Any heavy petroleum oil, including residual asphaltic oil used as a dust palliative and surface treatment on roads and highways. It is generally produced in six grades, from 0 , the most liquid, to 5 , the most viscous.

Rotary Rig: A machine used for drilling wells that employs a rotating tube attached to a bit for boring holes through rock.

Short Ton (coal): A unit of weight equal to 2,000 pounds.

\section{SIC: See Standard Industrial Classification.}

Solar Energy: The radiant energy of the sun, which can be converted into other forms of energy, such as heat or electricity.

Standard Industrial Classification (SIC): A set of codes developed by the Office of Management and 
Budget which categorizes industries into groups with similar economic activities.

Startup Test Phase of Nuclear Power Plant: A nuclear power plant that has been licensed by the Nuclear Regulatory Commission to operate but is still in the initial testing phase, during which the production of electricity may not be continuous. In general, when the electric utility is satisfied with the plant's performance, it formally accepts the plant from the manufacturer and places it in commercial operation status. A request is then submitted to the appropriate utility rate commission to include the power plant in the rate base calculation.

Steam-Electric Power Plant: A plant in which the prime mover is a steam turbine. The steam used to drive the turbine is produced in a boiler where fossil fuels are burned.

Strategic Petroleum Reserve (SPR): Petroleum stocks maintained by the Federal Government for use during periods of major supply interruption.

Supplemental Gaseous Fuels: Any gaseous substance that, introduced into or commingled with natural gas, increases the volume available for disposition. Such substances include, but are not limited to, propane-air, refinery gas, coke oven gas, still gas, manufactured gas, biomass gas, or air or inert gases added for Btu stabilization.

Synthetic Natural Gas (SNG): A manufactured product chemically similar in most respects to natural gas, resulting from the conversion or reforming of petroleum hydrocarbons. It may easily be substituted for, or interchanged with, pipeline quality natural gas. Also referred to as substitute natural gas.

Total Consumption: See Energy Consumption, End-Use.

Transportation Sector: The transportation sector consists of private and public vehicles that move people and commodities. Included are automobiles, trucks, buses, motorcycles, railroads and railways (including streetcars), aircraft, ships, barges, and natural gas pipelines.

Unaccounted-for Crude Oil: Arithmetic difference between the calculated supply and the calculated disposition of crude oil. The calculated supply is the sum of crude oil production and imports, less changes in crude oil stocks. The calculated disposition of crude oil is the sum of crude oil input to refineries, crude oil exports, crude oil burned as fuel, and crude oil losses.
Underground Storage: The storage of natural gas in underground reservoirs at a different location from which it was produced.

United States: Unless otherwise noted, "United States" in this publication means the 50 States and the District of Columbia. U.S. exports include shipments to U.S. territories, and imports include receipts from U.S. territories.

U.S.S.R.: The Union of Soviet Socialist Republics consisted of 15 constituent republics: Armenia, Azerbaijan, Belarus, Estonia, Georgia, Kazakhstan, Kyrgyzstan, Latvia, Lithuania, Moldova, Russia, Tajikistan, Turkmenistan, Ukraine, and Uzbekistan. As a political entity, the U.S.S.R. ceased to exist as of December 31, 1991.

Vented Natural Gas: Gas released into the air on the base site or at processing plants.

Wellhead Price: The value of crude oil or natural gas at the mouth of the well.

Well Servicing Unit: Truck-mounted equipment generally used for downhole services after a well is drilled. Services include well and recompletions, maintenance, repairs, workovers, and well plugging and abandonments. Jobs range from minor operations, such as pulling the rods and rod pumps out of an oil well, replacing the pump and rerunning the assemblage into the well, to major workovers, such as milling out and repairing collapsed casing. Well depth and characteristics determine the type of equipment used.

Wind Energy (as used at electric utilities): The kinetic energy of wind converted at electric utilities into mechanical energy by wind turbines (i.e., blades rotating from a hub) that drive generators to produce electricity for distribution.

Wood and Waste (as used at electric utilities): Wood energy, garbage, bagasse, sewerage gas, and other industrial, agricultural, and urban refuse used to generate electricity for distribution.

Wood Energy: Wood and wood products used as fuel, including round wood (cord wood), limb wood, wood chips, bark, sawdust, forest residues, charcoal, pulp waste, and spent pulping liquor.

Working Gas: The gas in a reservoir that is in addition to the base (cushion) gas. It may or may not be completely withdrawn during any particular withdrawal season. Conditions permitting, the total working capacity could be used more than once during any given season. 
OPC8144

\section{Publication Order Form}

\section{Performance Profiles of Major Energy Producers 1996}

Published: January 1998

Energy Information Administration

GPO Stock No. 061-003-01025-3

Price per copy: $\$ 16.00$

Personal Name or Attention Line:

Company Name, if applicable:

Street Address:

City, State, Zip Code:

Daytime Phone Number (area code first):

Purchase Order No:

May we make your name and address available to other mailers? yes no

Please include payment with this order form. Allow a minimum of 4 weeks for domestic delivery and an additional 6 weeks for international delivery.

Quantity $X \$ 16.00=\$$ (total due). (International customers add 25\%)

Check payable to Superintendent of Documents

GPO Deposit Account No.

VISA or MasterCard Account No.

Authorizing Signature Credit Card Expiration Date $-$

Note: Price includes regular domestic postage and handling. It is subject to change.

Mail order form to: U.S. Government Printing Office

P.O. Box 371954

Pittsburgh, PA 15250-7954

Or fax order form to: $202-512-2250$ 


\section{State Energy Profiles}

Click on State's Two-Letter Code

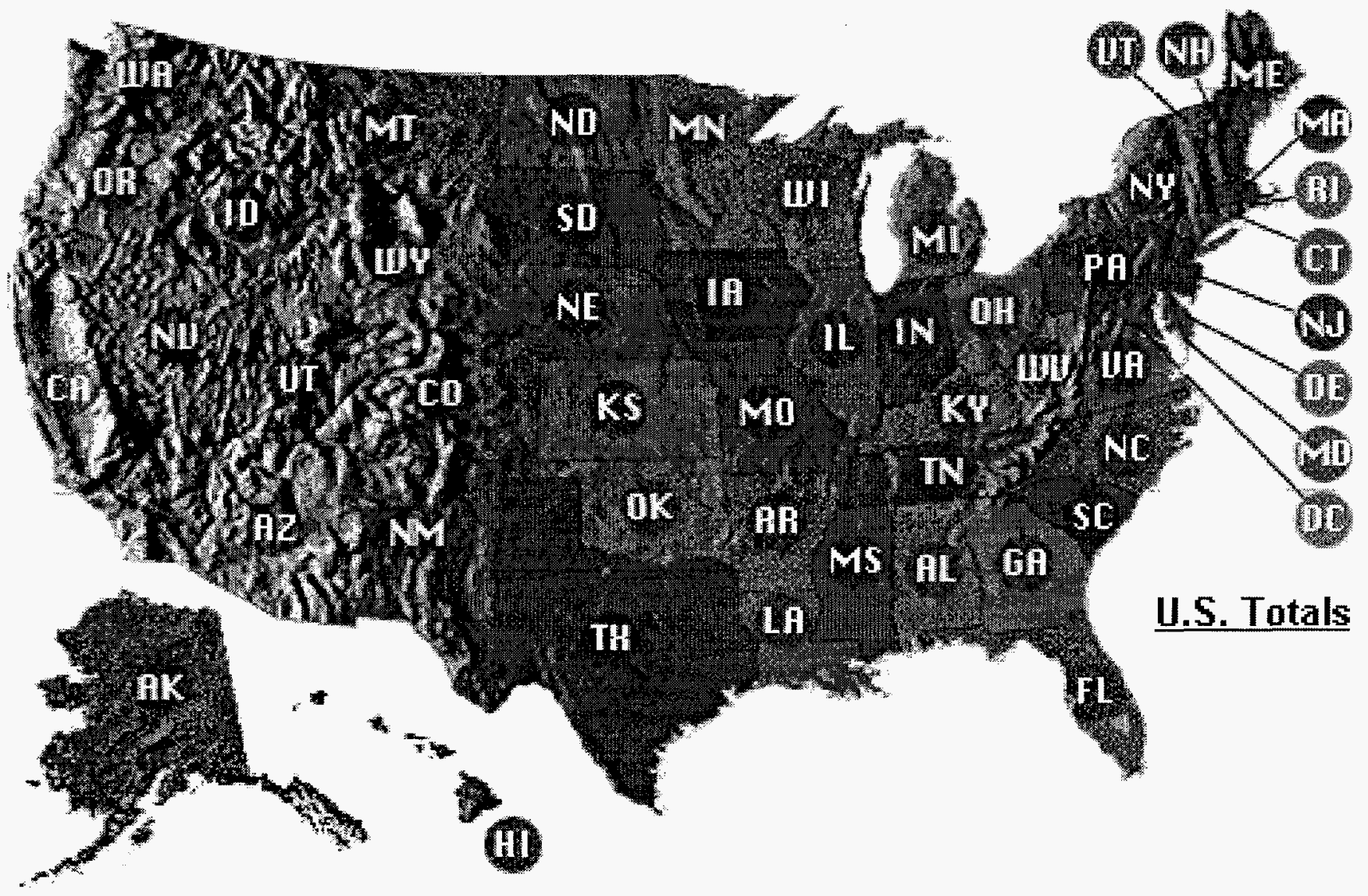

1995 Consumption Data Now Available

The new State Energy Profiles interactive map on the Energy Information Administration World Wide Web site provides energy consumption, price, and expenditure data on a Stateby-State basis. Selected summary graphs, tables, and rankings are available, as well as direct access to the detailed tables from the State Energy Data Report. (Consumption Estimates) and the State Energy Price and Expenditure Report.

Go to http://www.eia.doe.gov and click on "State Energy Data."

The site also provides both reports in their entirety in viewable .pdf format, as well as data files in ASCII comma-delimited format for use in spreadsheets and databases. 